\title{
Small Molecule Activation of Copper and Iron Complexes with Bis(oxazoline) Ligands
}

\author{
Dissertation \\ for the award of the degree \\ "Doctor of Philosophy" (Ph.D.)
}

Division of Mathematics and Natural Sciences

of the Gerog-August-Universität Göttingen

within the doctoral program BioMetals

of the Georg-August University School of Science (GAUSS)

\author{
submitted by \\ Vandana Esther Goswami \\ from India
}

Göttingen, 2016 


\section{Thesis Committee}

Prof. Dr. Franc Meyer, Institute of Inorganic Chemistry, Georg-August University, Göttingen

Dr. Inke Siewert, Institute of Inorganic Chemistry, Georg-August University, Göttingen

Prof. Dr. Ebbe Nordlander, Department of Chemical Physics, Lund University, Sweden

Members of the Examination Board

Reviewer: Prof. Dr. Franc Meyer, Institute of Inorganic Chemistry, Georg-August University, Göttingen

Second Reviewer: Dr. Inke Siewert, Institute of Inorganic Chemistry, Georg-August University, Göttingen

Further members of the Examination Board

Prof. Dr. Dietmar Stalke, Institute of Inorganic Chemistry, Georg-August University, Göttingen

Prof. Dr. Claudia Höbartner, Institute of Organic and Biomolecular Chemistry, Georg-August University, Göttingen

Jun.-Prof. Dr. Selvan Demir, Institute of Inorganic Chemistry, Georg-August University, Göttingen

Dr. Franziska Thomas, Institute of Organic and Biomolecular Chemistry, GeorgAugust University, Göttingen

Date of the oral examination: 17.10.2016 


\section{TABLE OF CONTENTS}

Chapter 1: General Introduction to Diiron, Dicopper Metalloenzymes and Bis(oxazoline) Ligands............1

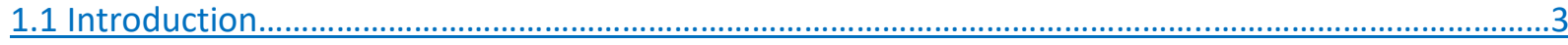

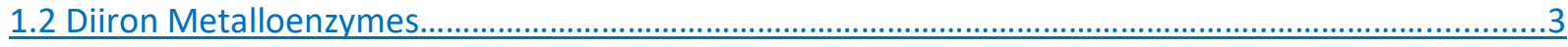

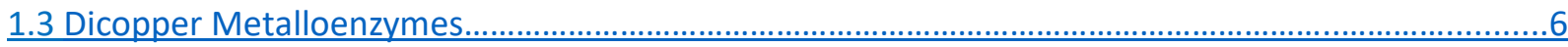

1.4 A General Introduction to Bis(oxazoline) Ligands ..................................................................................

Chapter 2: An overview of Copper in Biological and Synthetic Systems.............................................11

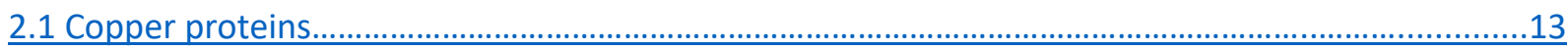

2.2 Type 3 Copper proteins: Hemocyanin, Tyrosinase and Catechol Oxidase..........................................14

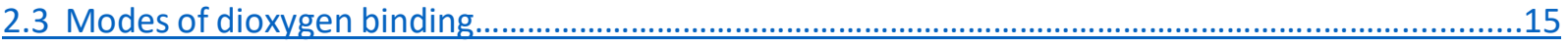

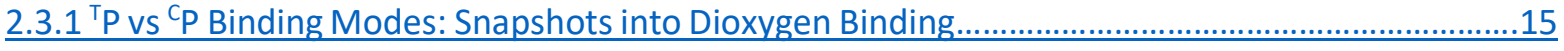

2.3.2 The ${ }^{\mathrm{S} P}$ Binding Mode: A Hemocyanin Model..................................................................................17

2.3.3 The Bis $(\mu$-oxo) Motif $(0)$ : Breaking the O-O Bond........................................................................18

2.3.4 ${ }^{\mathrm{S}} \mathrm{P}$ vs O: Insights into the O-O bond Scission and Tuning the Equilibrium.................................. 20

2.4 Particulate Methane Monoxygenase: pMMO................................................................................22

$\underline{2.5 \text { Catalytic reactivity of Tyrosinase Models towards external monophenolic substrates......................23 }}$

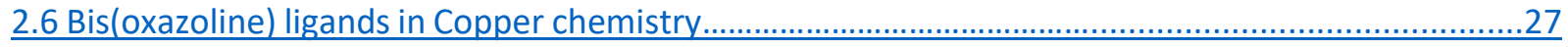

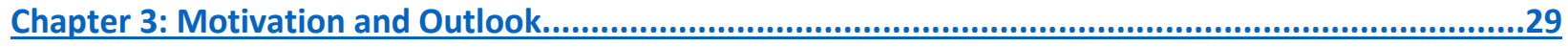

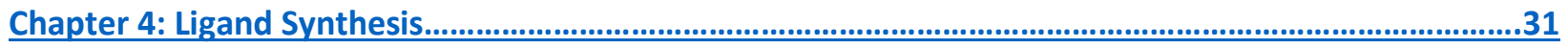

Chapter 5: Dicopper(II) Side on Peroxo Complexes with BOX ligands...................................................37

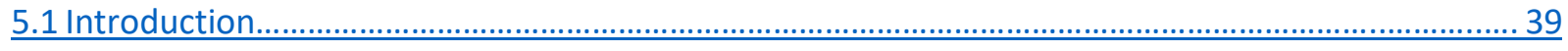

5.2 Synthesis and characterization of $\mathrm{Cu}(\mathrm{I}) \mathrm{BOX}$ complexes with proton responsive ligands

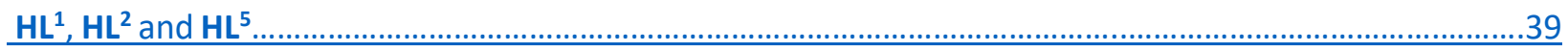

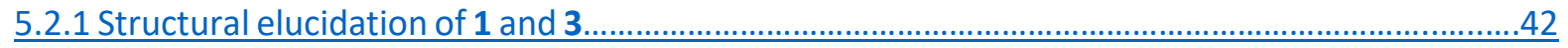

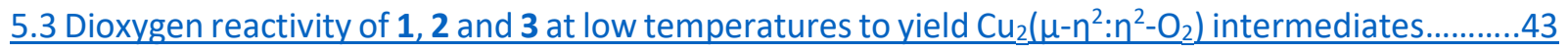

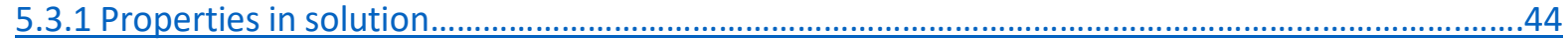

5.3.2 Structural elucidation of a $\mathrm{Cu}_{2}\left(\mu-\eta^{2}: n^{2}-\mathrm{O}_{2}\right)$ dicopper(II) complex (6) with an unusually

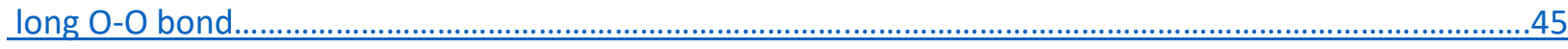

5.4 Synthesis and Characterization of a $\mathrm{Cu}(\mathrm{I}) \mathrm{BOX}$ complex with a Non-Proton Responsive

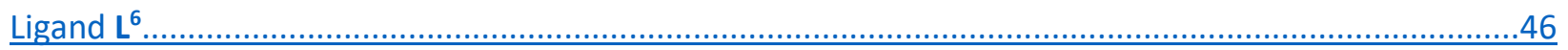

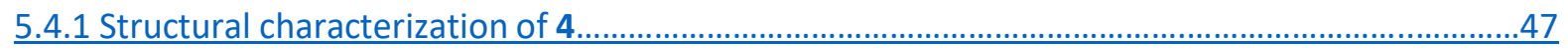

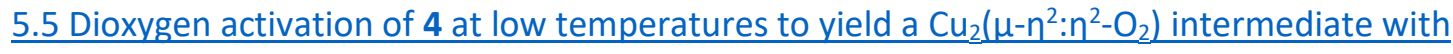




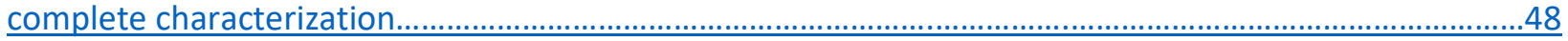

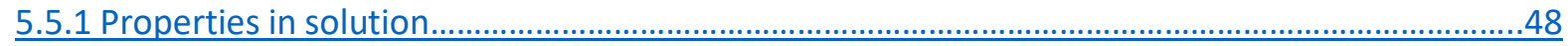

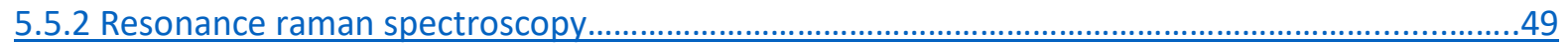

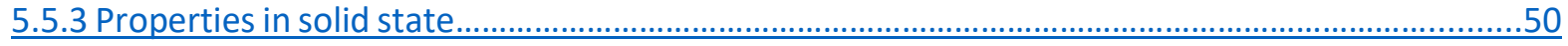

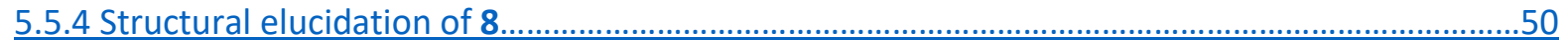

5.5.5 Magnetic measurements................................................................................................................... 51

5.6 Comparison of 6 and 8 with other structurally characterized $\mathrm{Cu}_{2}\left(\mu-\eta^{2}: \eta^{2}-\mathrm{O}_{2}\right)$ complexes...............53

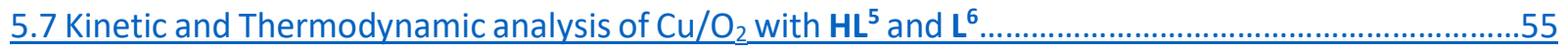

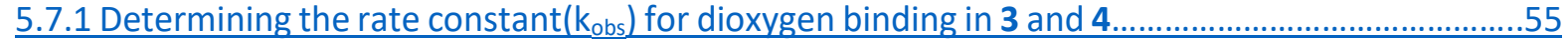

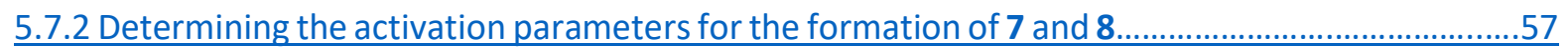

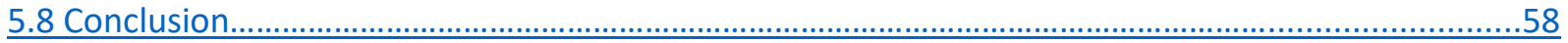

Chapter 6: Dicopper(III) bis( $\mu$-oxo) complexes with BOX Ligands....................................................61

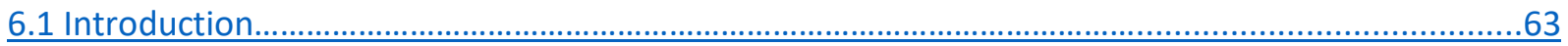

6.2 Synthesis and Characterization of Copper(I) complexes with Mono-anionic BOX ligands

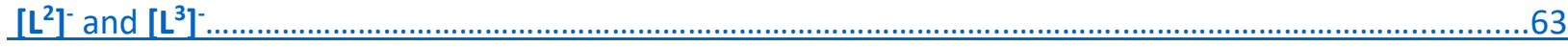

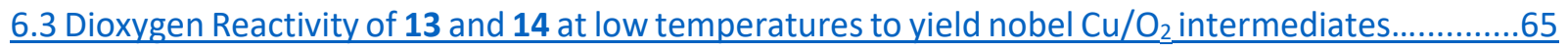

6.3.1 Structural elucidation of a rare neutral Dicopper(III) bis( $\mu$-oxo) complex 10 ................................66

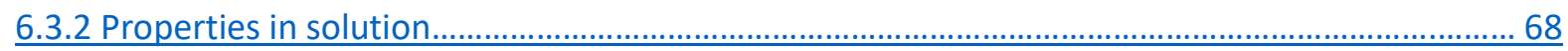

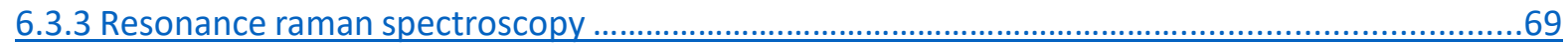

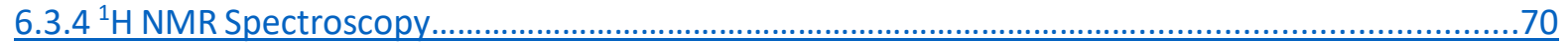

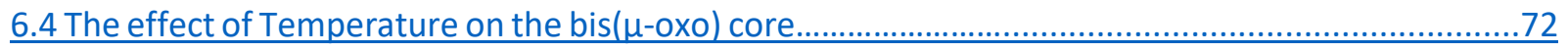

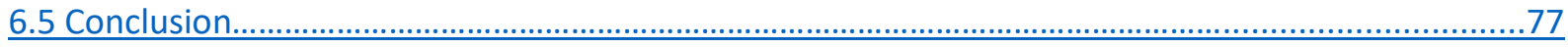

Chapter 7: Equilibrium Studies between ${ }^{\mathrm{S}} \mathrm{P} / \mathrm{O}$ Complexes with BOX Ligands......................................79

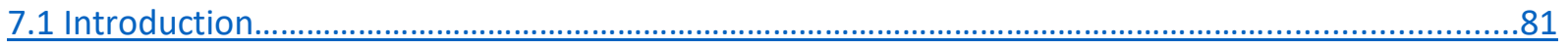

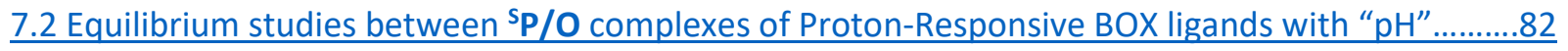

7.2.1 Tuning the equilibrium by virtue of a base........................................................................................

7.2.2 Reverting the equilibrium with an acid.........................................................................................

7.2.3 Cleavage of the $\mathrm{O}-\mathrm{O}$ bond and insights into conversion ................................................................87

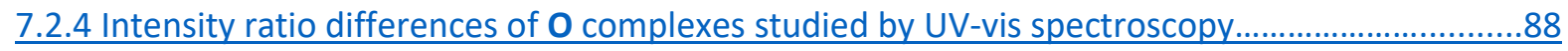

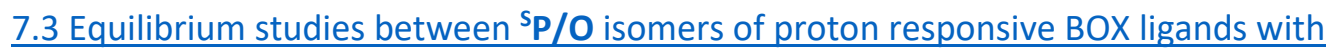

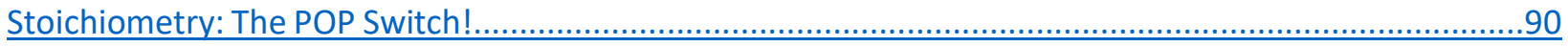

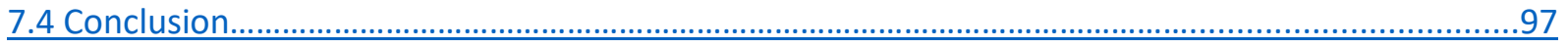

Chapter 8: Stability of ${ }^{\mathrm{S}} \mathrm{P}$ vs O Complexes of BOX Ligands and their Substrate Reactivity.......................99

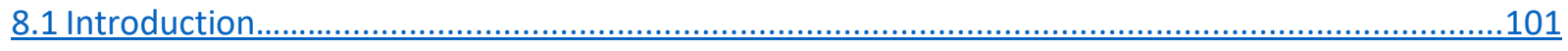




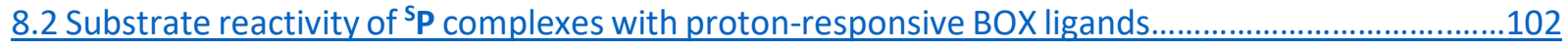

8.2.1 Potential PCET Reactions ...................................................................................................

8.3 Substrate reactivity exhibited by ${ }^{\mathrm{S}} \mathbf{P}$ complex of non-proton-responsive BOX ligands...................104

8.4 Substrate reactivity exhibited by $\mathbf{O}$ complexes of monoanionic BOX ligands................................107

8.5 Stability of ${ }^{\text {SP }}$ complexes vs O complexes.......................................................................................... 108

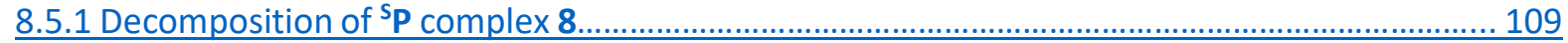

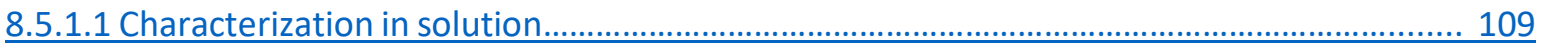

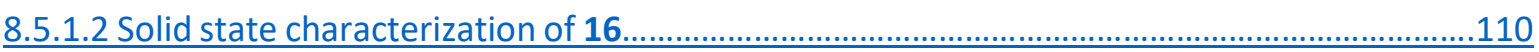

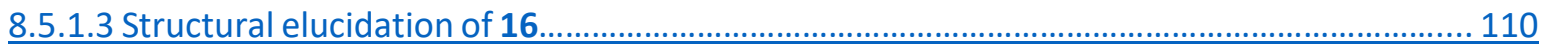

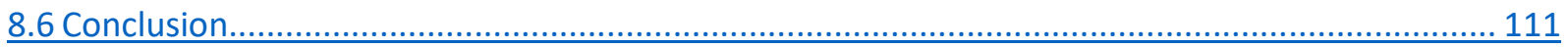

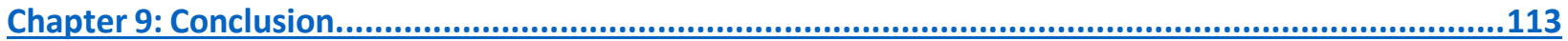

Chapter 10: An overview of Iron in biological and synthetic systems.......................................................117

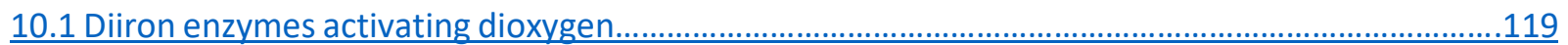

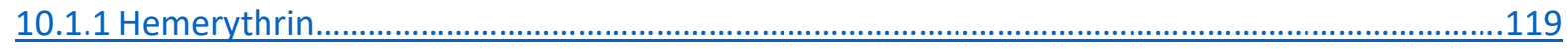

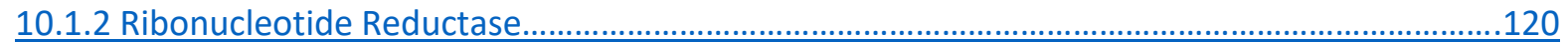

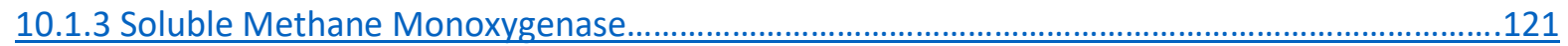

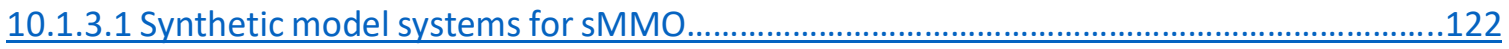

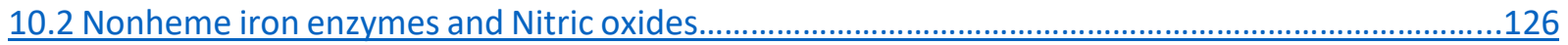

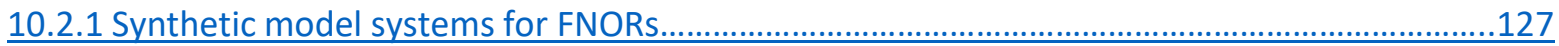

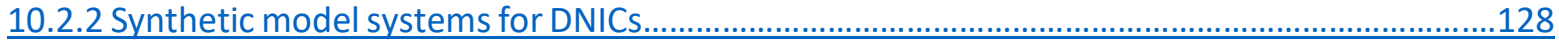

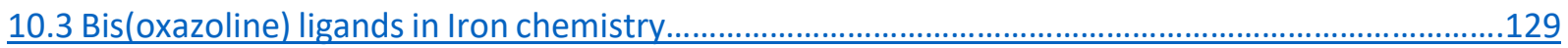

Chapter 11: Mononuclear and Dinuclear Fe(II) Complexes with BOX ligands............................................132

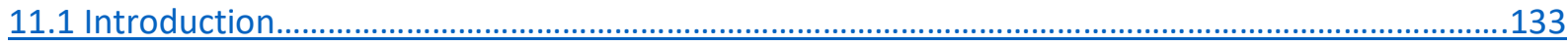

11.2 Synthesis and characterization of mononuclear Fe(II) complexes with neutral BOX ligands..........133

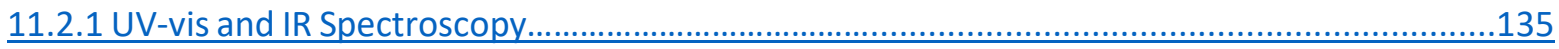

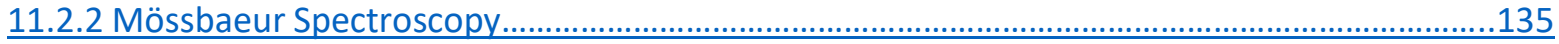

11.2.3 Structural elucidation of mononuclear Fe(II)BOX compelxes...................................................137

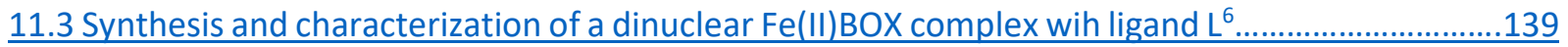

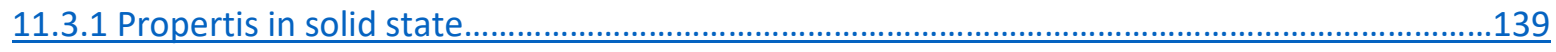

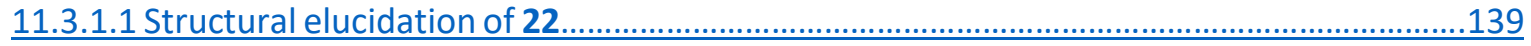

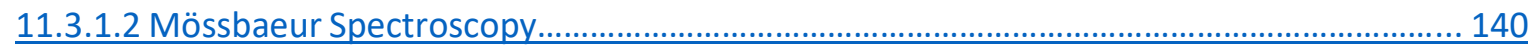

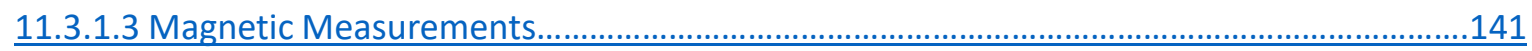

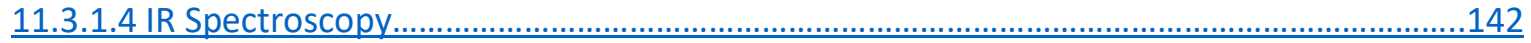

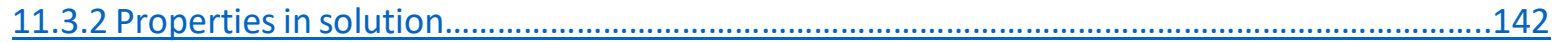


12.2 Dioxygen reactivity of mononuclear and dinuclear Fe(II)BOX systems..........................................147

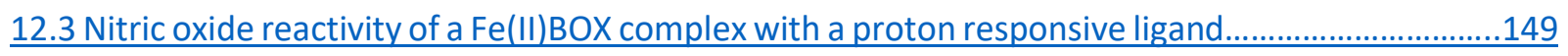

12.3.1 Preliminary 'NO' reactivity of a dinuclear Fe(II)BOX complex....................................................149

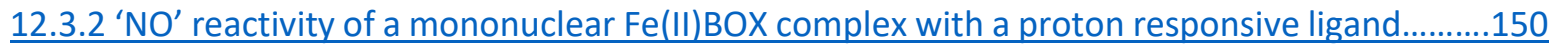

12.3.2.1 Structural elucidation of a DNIC 23 ........................................................................... 150

12.3.2.2 Properties in solution: IR and UV-vis Spectroscopy ...................................................151

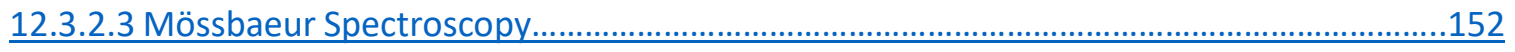

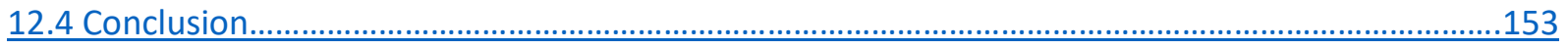

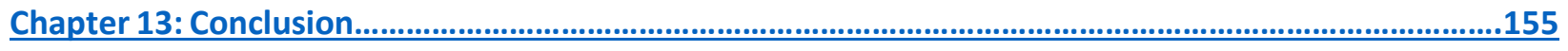

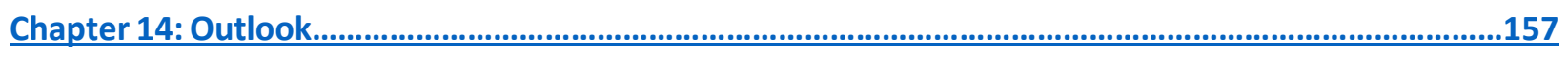

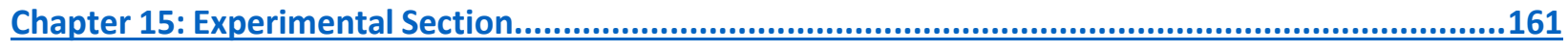

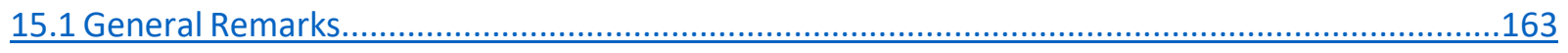

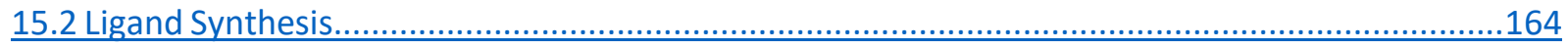

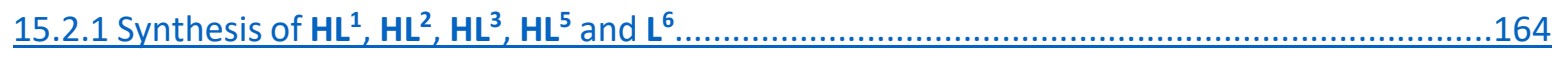

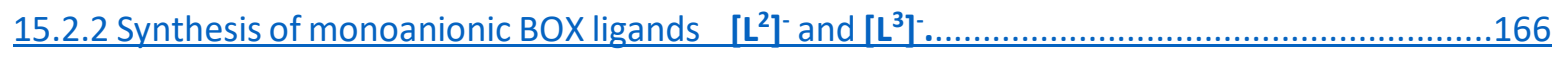

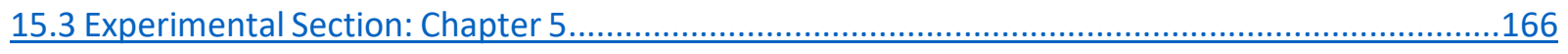

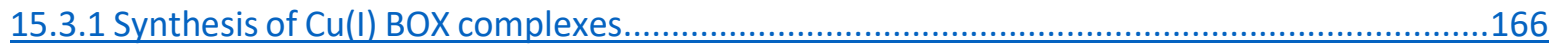

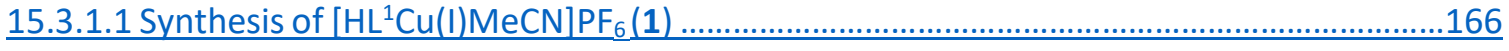

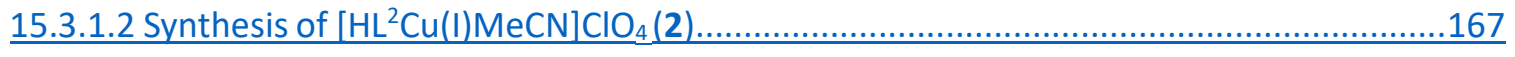

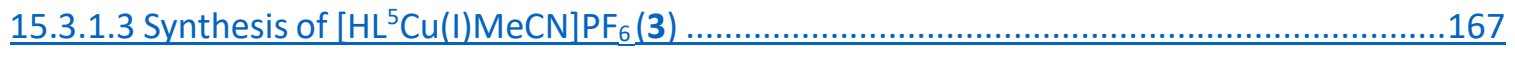

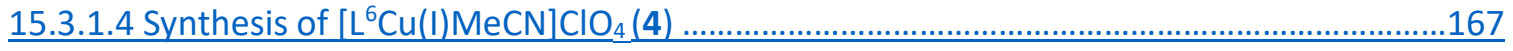

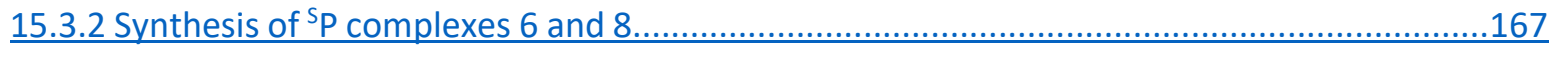

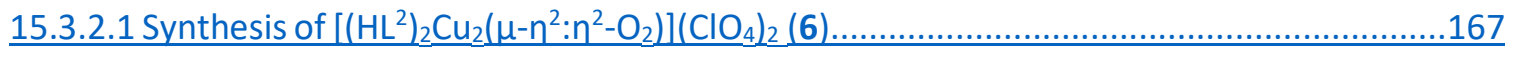

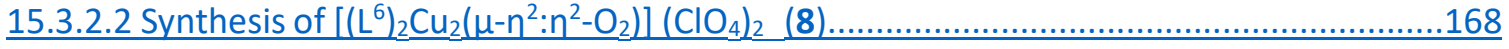

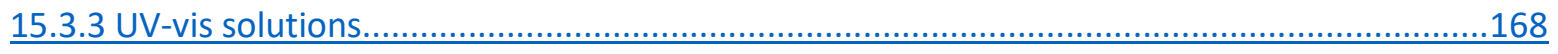

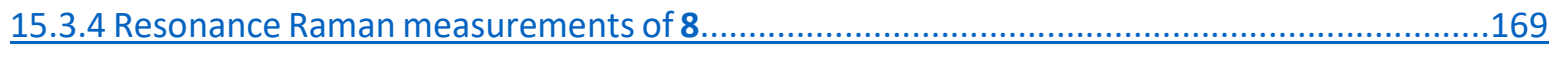

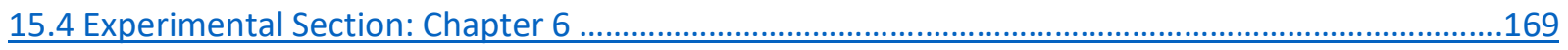

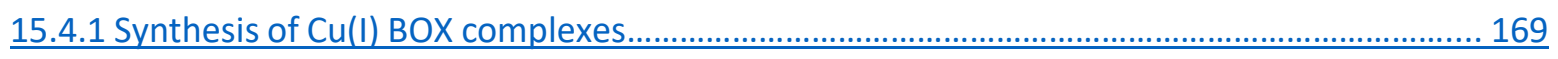

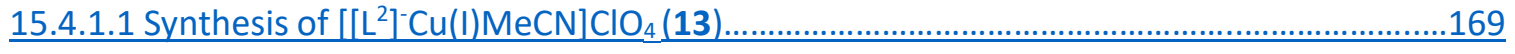

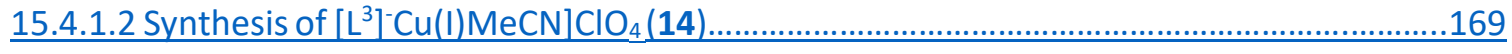

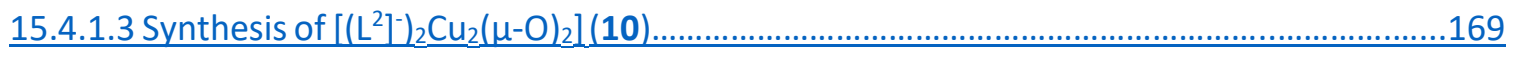

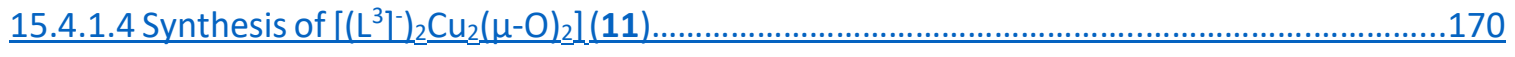

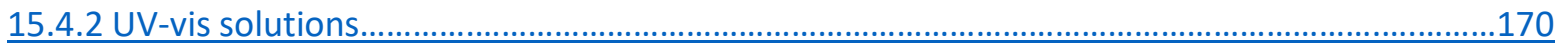

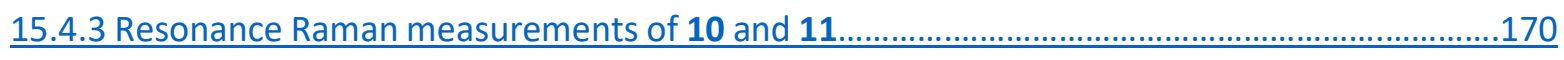


15.5 Experimental Section: Chapter 7 ....................................................................................171

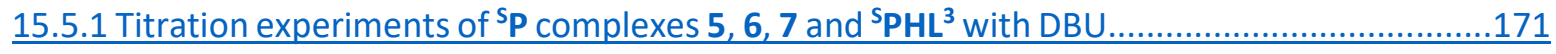

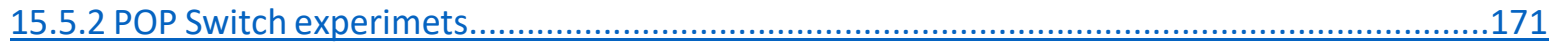

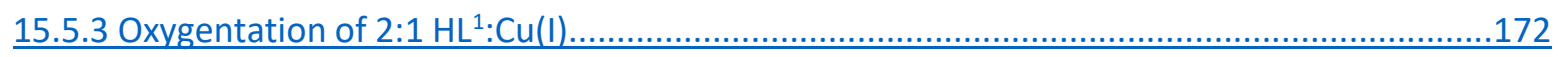

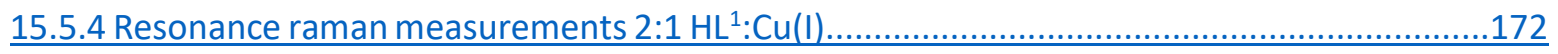

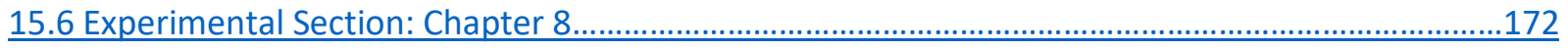

15.6.1 Substrate reactivity of DTBP/DTBP-H: $\mathrm{NEt}_{3}$ with ${ }^{\mathrm{S}} \mathrm{P}$ complexes $5,6,{ }^{\mathrm{S}} \mathrm{PHL}^{3}$ and $7 \ldots \ldots \ldots \ldots \ldots \ldots \ldots . .172$

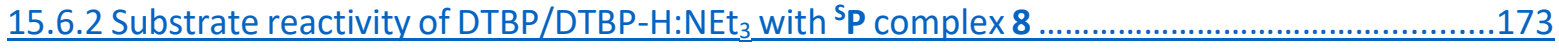

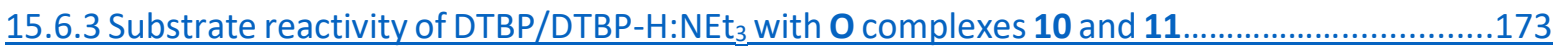

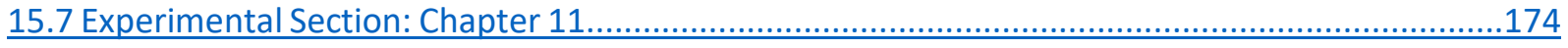

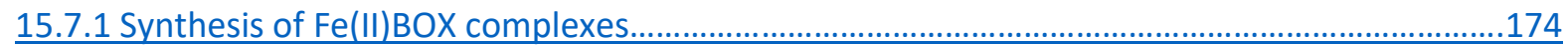

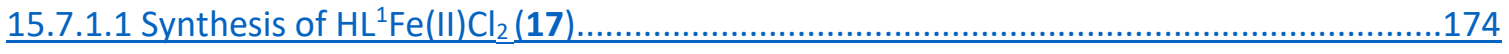

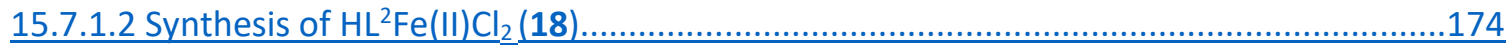

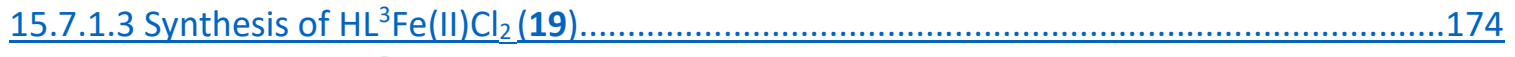

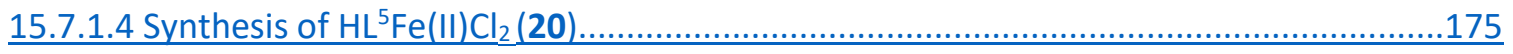

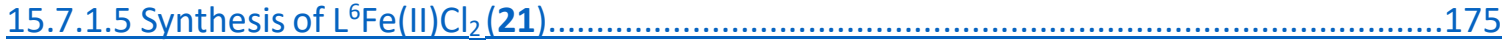

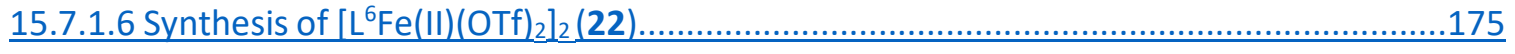

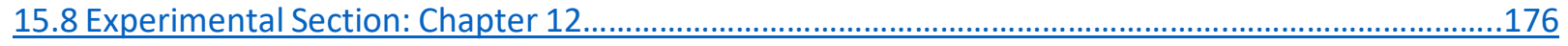

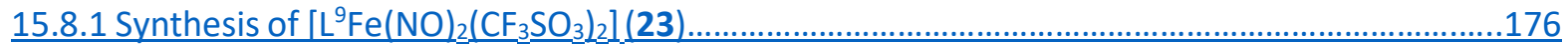

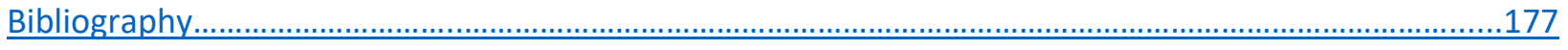

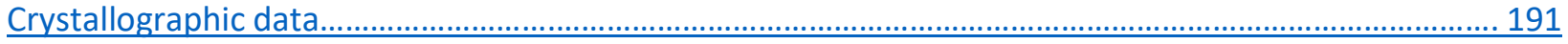

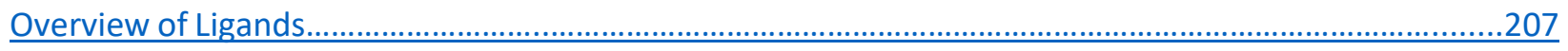

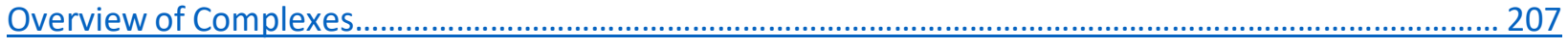

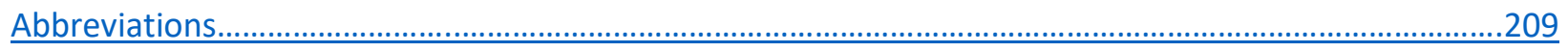





\section{Chapter 1}

General Introduction to Diiron,

Dicopper Metalloenzymes and

Bis(oxazoline) Ligands 


\section{Overview}

1.1 Introduction

1.2 Diiron Metalloenzymes

1.3 Dicopper Metalloenzymes

1.4 An Introduction to Bis(oxazoline) Ligands 


\subsection{Introduction}

Metalloenzymes can simply be defined as enzymes that contain one or more metal ions as an integral part of their active site, or as a structural cofactor. They are responsible for catalyzing various physiological reactions. ${ }^{[1,2]} \mathrm{A}$ large number of these catalytic reactions are carried out in conjunction with small molecules such as $\mathrm{O}_{2}, \mathrm{~N}_{2}, \mathrm{H}_{2}, \mathrm{CO}_{2}, \mathrm{~N}_{2} \mathrm{O}$, and $\mathrm{CH}_{4}$. These elementary reactions are pertinent to energy storage and release, in the form of making and breaking of chemical bonds which is important for viable fuel and chemical feedstock formation. ${ }^{[3]}$ While some of these small molecules have shown to be significant to the environment and climate change, others are notably important to the chemical industry. Despite being readily abundant in nature, these small molecules are quite thermodynamically stable which makes their direct usage rather difficult. The activation step required for these small molecules is associated with reaction pathways involving multielectron redox processes and proton transfer reactions, which are thermodynamically demanding and mechanistically complex. ${ }^{[4-11]}$ A deeper understanding of these processes puts forward a major challenge to the scientific community.

In contrast, bioinorganic chemistry is a field that inspects the role of metals in biology. Gaining an insight into how these natural enzymes function with the ultimate goal to exploit catalytic activity is a key endeavor of this field. Hence, substantial efforts have been applied to generate model systems capable of emulating the fascinating activity of these biological systems in order to design and develop efficient catalysts.

Of the different metals present in nature, Copper and Iron have proved to be of high importance and are present in the active sites of several metalloenzymes. ${ }^{[12]}$ The following sections give a brief overview of the different dimetallic copper and iron containing metalloenzymes, and their biological functions that are carried out by activating various small molecules present in nature.

\subsection{Diiron metalloenzymes}

Iron plays an important role in various metabolic processes and is present in the active site of various enzymes. A few examples are Nitrogenase, Hemerythrin, FNOR's, Hydrogenase and sMMO.

Nitrogenase : The enzyme Nitrogenase is commonly produced by certain bacteria and cyanobacteria and plays a crucial role in the process of nitrogen fixation. ${ }^{[13-16]}$ A homodimeric Fe protein and a heterodimeric MoFe protein together form the Nitrogenase motif (Fig. 1.1). The former unit consists of an identical Fe $\mathrm{S}_{4}$ clusters responsible for the supply of electrons, while the latter and larger unit consists of an alpha subunit, a beta subunit, and an iron-sulphur cluster which uses these electrons to convert nitrogen to ammonia.

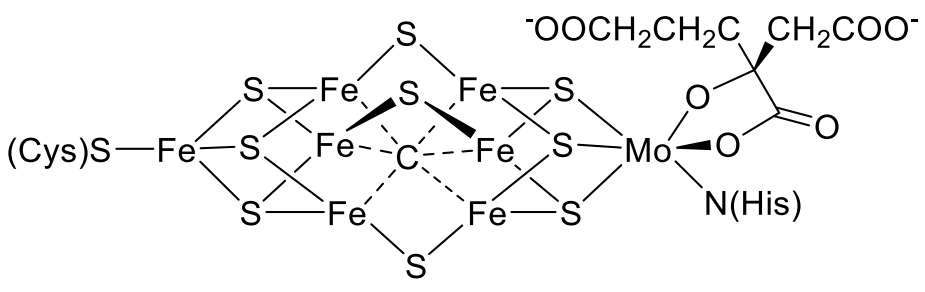

Figure 1.1 Schematic representation of the FeMoco of molybdenum dependent nitrogenase. Recent studies have elucidated the presence of a carbide core within the cluster. ${ }^{[16]}$ 
The overall enthalpy of this reaction for the equilibrium formation of ammonia from molecular nitrogen and hydrogen is negative, but with a high activation energy. ${ }^{[17]}$ The enzyme Nitrogenase behaves as a catalyst reducing this energy barrier and allowing the reaction to occur at feasible temperatures.

Today, the famous Haber-Bosch process which was developed in the first half of the early $20^{\text {th }}$ century is the main artificial nitrogen-fixation process used in industry for the generation of ammonia. Iron surfaces promoted by alkali metals in the presence of high temperature and pressure replicate the biological process on a large scale. ${ }^{[18]}$

Hydrogenase: Hydrogenases are divided into three subcategories depending on the metal ion present within their active site. ${ }^{[19]}$ They are responsible for the reversible catalytic oxidation of molecular hydrogen which is essential for energy release. ${ }^{[20]}$ The three subcategories of Hydrogenases are the [FeNi], [FeFe] and the [Fe] only hydrogenase (Fig. 1.2). The active sites of the first two resemble each other and have iron-sulfur clusters, along with carbon monoxide and cyanide as ligand systems. ${ }^{[1,22]}$ The $[\mathrm{Fe}]$ only hydrogenase however, has no iron-sulfur clusters bound to it. ${ }^{[23,24]}$

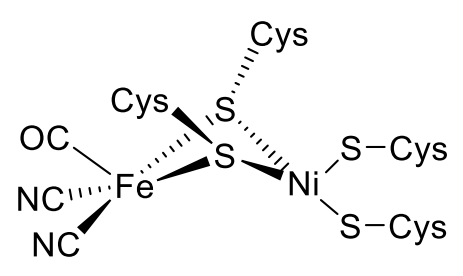

$[\mathrm{NiFe}] \mathrm{H}_{2}$ ase

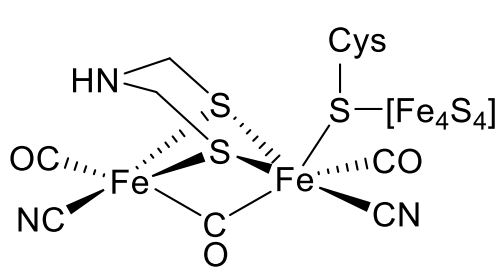

$[\mathrm{FeFe}] \mathrm{H}_{2}$ ase<smiles></smiles>

$[\mathrm{Fe}] \mathrm{H}_{2}$ ase

Figure. 1.2 Schematic representations of the three subcategories of Hydrogenases. ${ }^{[21-23]}$

Of all three subcategories, the [FeFe] hydrogenase is most active in molecular hydrogen production. ${ }^{[22]}$ Hydrogenases have been found in deep sea settings where due to the lack of other sources of energy, the hydrogenase is used to generate energy. Mimicking biological hydrogenases in order to generate systems capable of capturing and storing renewable energy as fuel, where the production of hydrogen is used as a fuel source, is an extensive ongoing area of research.

Of the large number of Iron-oxygen proteins/enzymes found in nature, Hemerythrin and sMMO are dinuclear. With the aid of dioxygen, sMMO carries out catalytic activity whereas Hemerythrin behaves as an oxygen transporter.

Hemerythrin is an oxygen transporter found in marine invertebrates. It consists of a pair of iron centers where the iron atoms are bound to the protein matrix via a carboxylate side chain of glutamate, aspartate and 5 Histidine residues (Scheme 1.1). ${ }^{[25-28]}$ Unlike Hemoglobin, which has per protein subunit a heme group containing only a single iron center and is responsible for oxygen transport in vertebrates, Hemerythrin does not contain any heme unit. ${ }^{[29]}$ 


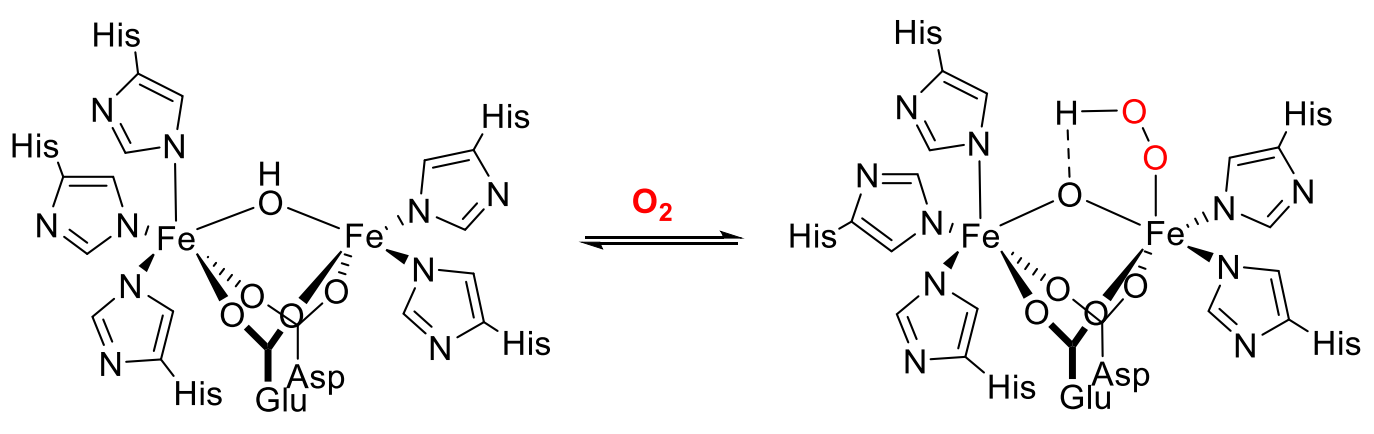

Scheme 1.1 Schematic representation of dioxygen binding in Hemerythrin. ${ }^{[28]}$

SMMO: Methanotrophic bacteria use carbon as their only source of energy. They convert methane to methanol, the BDE of methane being quite high, $105 \mathrm{kcalmol}^{-1} .^{[30,31]}$ This impressive catalysis is carried out by soluble Methane Monoxygenase (sMMO), a non heme diiron protein having 2 iron atoms within its active site coordinated by histidine and glutamate residues. ${ }^{[32]}$ The two iron centers are bridged by a carboxylate group. Dioxygen binds to the sMMO forming the active iron-oxygen intermediate which then catalyzes $\mathrm{C}-\mathrm{H}$ bond cleavage to generate methanol (Scheme 1.2). ${ }^{[33]}$

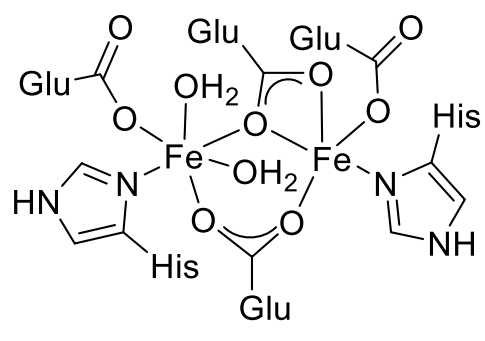

$\mathrm{MMOH}_{\text {red }}$

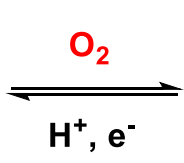

Scheme 1.2 Active site of the hydroxylase unit of sMMO in its active reduced form (MMOHred), and its resting state $\left(\mathrm{MMOH}_{\mathbf{0 x}}\right) \cdot{ }^{[32]}$

As methane forms one of the major components of natural gas and is produced as a side product in a large number of industrial reactions, significant efforts are being made in order to replicate a functional sMMO model that can be used industrially to benefit optimal conversion of natural gas into fuels and chemicals.

Nitric Oxide reductases: NOR's are a class of enzymes present in various bacteria that help in catalyzing the reduction of nitric oxide to nitrous oxide. ${ }^{[34,35]}$ Nitric oxide is an important molecule which plays a 
crucial role not only in muscle contraction/relaxation, vasodilation, and intracellular messaging but also in immune defense against tumor cells. When present in high concentration nitic oxide is toxic and can be fatal.

Flavo-diiron Nitric Oxide reductases (FNOR's) are dinuclear iron containing enzymes. The iron centers are linked to glutamate, histidine and aspartate residues, bridged by the carboxylate group of an aspartate linker and a hydroxide bridge ${ }^{[36,37]}$ Evolved in certain pathogenic microbes, these FNORs detoxify NO by a two electron process to nitrous oxide, thus avoiding what is known as nitrosative stress (Scheme 1.3).

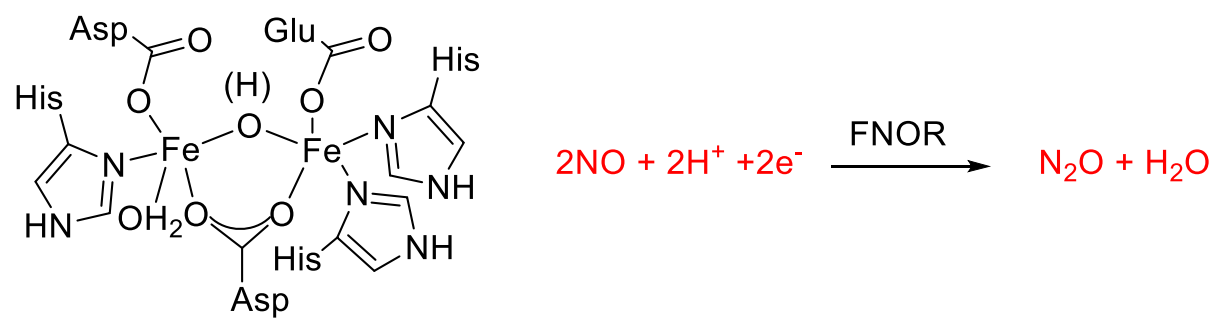

Scheme 1.3 Active site of FNOR in Desulfovibrio gigas and the catalyzed reaction.

\subsection{Dicopper metalloenzymes}

Of the different copper proteins present in nature, $\mathrm{Cu}_{\mathrm{A}}$, Type 3 and Type 4 all contain an active site with dinuclear copper centers.

$\mathbf{C u}_{\mathrm{A}}$ : Copper ${ }_{\mathrm{A}}$ proteins contain two cooper ions and are found in cytochrome c oxidase and nitrous oxide reductase. The copper atoms are coordinated by two histidine, one methionine, a protein backbone carbonyl oxygen and two bridging cysteine residues. ${ }^{[38]}$

Cytochrome c oxidase, found in various bacteria and mitochondria is a large transmembrane protein. ${ }^{[39]} \mathrm{A}$ large and complex structure made up of several subunits, the binuclear $\mathrm{Cu}_{\mathrm{A}}$ centers play a vital role in transport of electrons to and from the different subunits, effectively reducing dioxygen to two molecules of water.

Nitrous oxide reductase, a copper containing enzyme catalyzes the final denitrification step wherein nitrous oxide is reduced to dinitrogen. Composed of $\mathrm{Cu}_{\mathrm{z}}$ site made up of 4 copper atoms and a $\mathrm{Cu}_{\mathrm{A}}$ unit, the $\mathrm{Cu}_{\mathrm{A}}$ unit undergoes one electron redox changes, and the $\mathrm{Cu}_{\mathrm{z}}$ site is responsible for catalytic processes. ${ }^{[40]}$

Type 3: These copper proteins comprise of Hemocyanin (Hc), Catechol Oxidase (CO) and Tyrosinase (Ty). ${ }^{[41]}$ They each contain a pair of copper centers bound by three histidine residues. Hemocyanin reversibly binds dioxygen and serves as an oxygen transporter in arthropods and mollusks (Scheme 1.4). ${ }^{[42,43]}$ Catechol oxidase and Tyrosinase bind dioxygen to carry out the catalytic conversion of monophenols to catechols (only Ty) and the two electron oxidation of catechol to quinone (CO and Ty), which is significant in the formation of melanin in the body. ${ }^{[44,45]}$ 

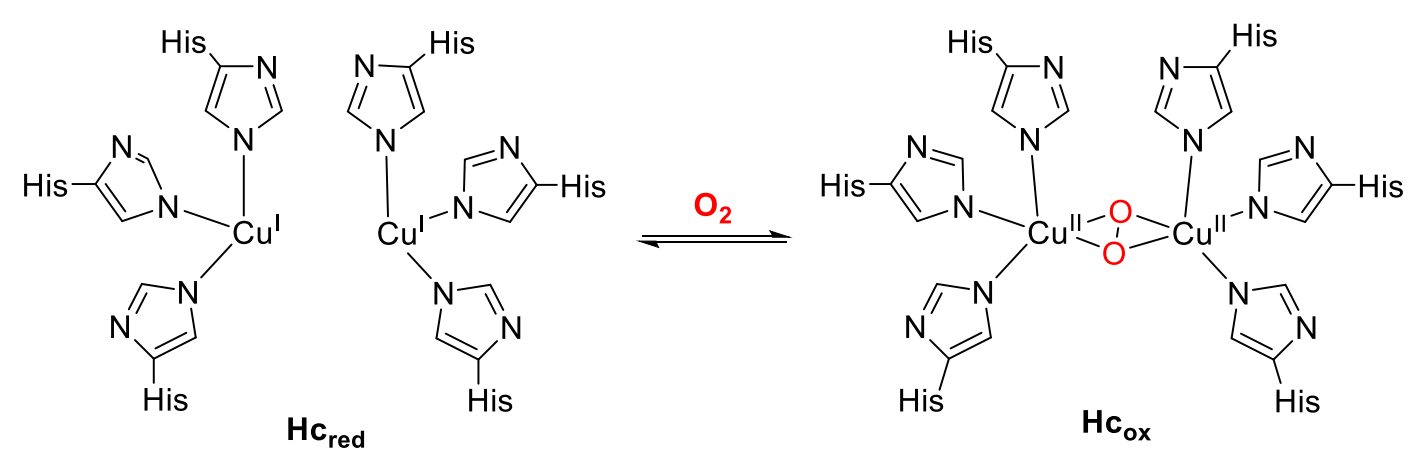

Scheme 1.4 Active site of Hemocyanin $(\mathrm{Hc})$ in its reduced form $\mathbf{H} \mathbf{c}_{\mathbf{r e d}}$ and oxidized form $\mathbf{H} \mathbf{c}_{\mathbf{o x}}$ after binding dioxygen. ${ }^{[42]}$

pMMO: Similar to sMMO mentioned earlier, pMMO is a methane monooxygenase but is known to have a dinuclear copper center within its active site instead of iron. ${ }^{[46,47]}$ One copper is coordinated by two histidine imidazoles while the other is ligated by a histidine imidazole and a primary amine of an $\mathrm{N}$ terminal histidine (Fig. 1.3) ${ }^{[46]}$ Present in methanotrophic bacteria and formed in the presence of iron starvation, pMMO binds dioxygen to form an active copper dioxygen intermediate which is responsible for converting methane to methanol, where methane is used as the primary source of energy by microorganisms. ${ }^{[48-50]}$

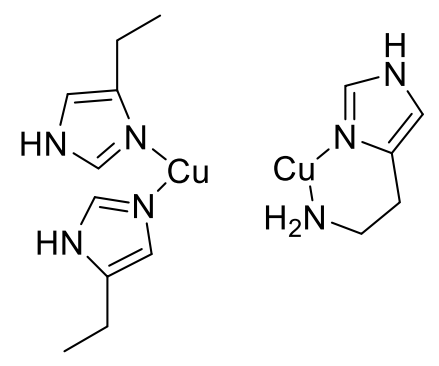

Figure 1.3 Schematic representation of the active site of pMMO. ${ }^{[46]}$

In conclusion, it is evident that diiron and dicopper metalloenzymes in conjunction with small molecules carry out a variety of biological processes in different organisms vital for their existence. The following chapters shall focus on dicopper and diiron mentalloenzymes activating dioxygen and nitric oxide. A detailed introduction to these systems in comparison with synthetic analogues is described in Chapter 2 (for copper) and Chapter 10 (for iron). The primary focus of this work is based on these systems. 


\subsection{An Introduction to Bis(oxazoline) ligands}

Bis(oxazoline) ligands commonly referred to as BOX ligands have been investigated since $1990 .{ }^{[51,52]}$ This class of ligands contain two oxazoline rings linked by a methylene spacer. They are $C_{2}$ - symmetric when the substituents at the backbone are identical and are often termed as privileged chiral ligands. Exploitation of the chiral nature of these ligands in combination with suitable metals have proved that metal-BOX complexes are one of the most resourceful class of chiral catalysts capable of promoting a large number of organic reactions. ${ }^{[51]}$ The electronic properties and coordination geometry of metal-BOX's are the key factors responsible for asymmetric induction. With the aid of techniques such as X-ray and NMR spectroscopy, over time rules have been devised to understand the mechanism and function of these catalysts. ${ }^{[53]}$ As a result, their impact in the field of asymmetric catalysis and coordination chemistry has attracted much attention and contributed largely to the general approach of organic synthesis. Dating back to 1991, Evans et al. reported the catalytic asymmetric cyclopropanation reaction of olefins with a $\mathrm{Cu}(\mathrm{I})-$ BOX complex with ligand A (Fig. 1.4). ${ }^{[54]}$ Shortly after, Corey et al. designed a Fe(III)-BOX complex with ligand $\mathbf{B}$ suitable for enantioselective catalysis of Diels-Alder additions (Fig 1.4). ${ }^{[55]}$ This followed the development of several other BOX ligands due to their feasible and flexible synthesis and an extensive usage thereafter in a wide range of other reactions. ${ }^{[51]}$

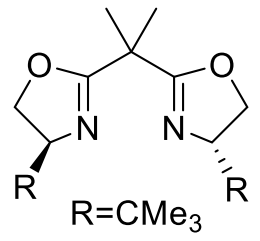

A

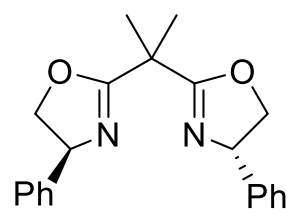

B

Figure 1.4. BOX ligands used in $\mathbf{A}$ and $\mathbf{B}$ used with Copper(I) and Iron (III) respectively for asymmetric catalysis. ${ }^{[54][55]}$

Besides the classical BOX ligands, derivatives such as Pyridine BOX (PyBOX) and aza-BOX are also used in enantioselective catalysis (Fig.1.5). ${ }^{[56,57]}$ Recently it was shown by Sibi et al. that the aminoindanol derived $\mathrm{BOX}$ ligand $(\mathbf{C})$ along with $\mathrm{Cu}(\mathrm{OTf})_{2}$ proved to be a promising precursor for the kinetic resolution of racemic $\alpha, \beta$ unsaturated pyrazolidinone imides. ${ }^{[58]}$ This emphasizes the advantage of this class of ligands proving indeed their flexibility and privilege.

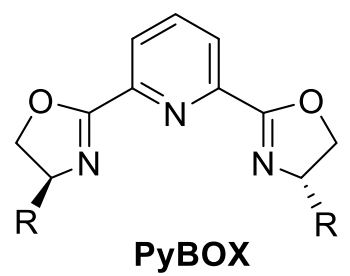

PyBOX

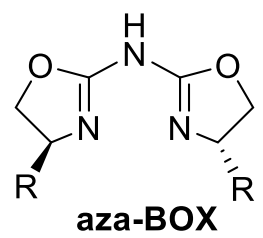

aza-BOX

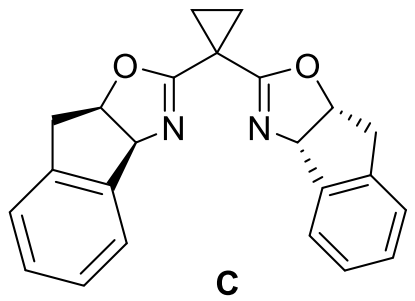

C

Figure 1.5. Derivatives of BOX ligands, $\mathrm{PyBOX}{ }^{[56]}$, aza BOX ${ }^{[57]}$ and ligand $\mathbf{C}^{[58]}$ ( $\mathbf{C}$ has been used for kinetic resolution). 
Bis(oxazoline)s are primarily expressed as dimine systems. For ligand systems having only a single $\mathrm{R}$ substituent in the backbone, tautomerism of the ligand may occur. A shift of the $\mathrm{H}$ atom from the bridging carbon to one of the nitrogen atoms of the oxazoline rings gives the iminoenamine form which may have either an $E$ or a $Z$ configuration (Fig 1.6). This is quite the opposite to $\beta$-diketones, where the enol form is more stable than the keto form. Similar tautomerism also occurs in case of semicorrins ${ }^{[59]}$ when there exists a strong electron withdrawing group at the bridging carbon, eg in ${ }^{\mathrm{CN}} \mathrm{BOX}$. Some aza-semicorrins are known to exist in the iminoenamine form as well (Fig.1.6). ${ }^{[60]}$

A<smiles>[R]C(C1=NCCO1)C1=NCCO1</smiles>

dimine<smiles>CC(C1=NCCO1)=C1NCCO1</smiles>

(E)-iminoenamine<smiles>CC(C1=NCCO1)=C1NCCO1</smiles>

(Z)-iminoenamine

B

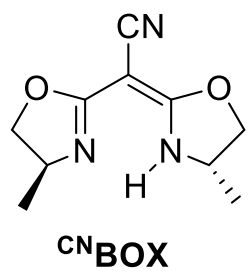<smiles></smiles>

aza-semicorrins

Figure 1.6. A: BOX ligand shown in the dimine form, and its tautomeric isomers. B: Ligand classes related to bis(oxazoline)s represented in their sole tautomeric form.

Evidence of the iminoenamine tautomer of BOXs, in solution or in solid state is rare and little is known. Ligands $\left[\mathrm{H}\left\{{ }^{\mathrm{Me}, \mathrm{H}} \mathrm{BOX}-\mathrm{Me}_{2}\right\}\right]^{+[61]}$ and $\left[\mathrm{H}\left\{{ }^{\mathrm{Me}, \mathrm{H}} \mathrm{BOX}-\mathrm{Ph}, \mathrm{H}\right\}\right]^{+}{ }^{[62]}$ ( ${ }^{\mathrm{x}, \mathrm{B}} \mathrm{BOX}-\mathrm{AB}$ :where $\mathrm{x}$ and y represent the substituents on the bridging carbon, and $\mathrm{A}$ and $\mathrm{B}$ represent the substituents on the ring) are amongst the few that were isolated in the solid state and shown to exist in the $(Z)$ - iminoenamine form. The ${ }^{\mathrm{H}, \mathrm{H}} \mathrm{BOX}$ $t \mathrm{Bu}-\mathrm{H}$ ligand was shown to exhibit a small amount of the iminoenamine isomer in solution which was confirmed by UV-vis spectroscopy ${ }^{[63]}$. The ${ }^{p \mathrm{CF}_{3}\left(\mathrm{C}_{6} \mathrm{H}_{4}\right) \mathrm{CH}_{2}, \mathrm{H}} \mathrm{BOX}-\mathrm{H}_{2}$ ligand system was recently isolated in the solid state as the iminoenamine tautomer ${ }^{[64]}$ More recently, investigation on the ${ }^{\mathrm{H}, \mathrm{Ph}} \mathrm{BOX}-\mathrm{Me}_{2}$ ligand by Meyer et al. revealed the ligand to exist in the iminoenamine form in the solid state, but as a tautomeric mixture in solution (Fig.1.7). ${ }^{[65]}$ This tautomeric equilibrium was studied via temperature dependent ${ }^{1} \mathrm{H}$ NMR spectroscopy which showed at low temperatures the iminoenamine form was preferred, whereas at room temperature a mixture of the two existed. The phenyl residue present in the backbone accounted for stabilization. Such tautomeric equilibrium is uncommon and seldom reported.<smiles>CC1(C)COC(C=CNN2C(=C(C3=NC(C)(C)CO3)C3=NC(C)(C)CO3)OCC2(C)C)=N1</smiles>

Figure 1.7 Tautomeric isomers of the ${ }^{\mathrm{H}, \mathrm{Ph}} \mathrm{BOX}-\mathrm{Me}_{2}$ ligand, known to be in equilibrium at room temperature in solution. 
The deprotonation of the neutral bidentate BOX ligands leads to monoanionic bis(oxazolinate)s (D) which are similarly bidentate but anionic, and are similar to the $\beta$-diketiminate (NacNac) ligands (Fig.1.8). ${ }^{[66]}$ This suggests that ${ }^{\mathrm{R}, \mathrm{H}} \mathrm{BOXs}$ are proton responsive in nature, which represents an add-on advantage to this ligand class. The monoanionic bis(oxazolinate)s have been well established as well and have proved to be equally versatile and to effectively chelate various metal centers such as Magensium, Aluminium, Copper, Zinc and even lanthanides such at Yttrium and Lanthanum. ${ }^{[66]}$ Intramolecular hydroamination, ${ }^{[67,68]}$ ring opening polymerization $^{[69]}$ and cyclopropanation of styrene ${ }^{[70]}$ are among the few organic reactions where these metal complexes are used. This robust and stable class of ligands are capable of a substantial degree of assymetric induction in enantioselective catalysis.
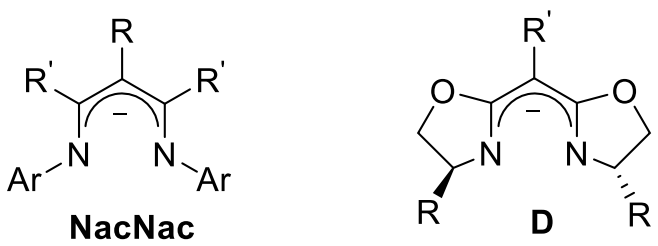

Figure 1.8. NacNac ligand shown on the left. Monoanionc-bis(oxazolinate) D represented on the right.

Though BOX ligands have been vastly used in the field of organic synthesis, their application as suitable scaffolds for small molecule activation with appropriate metals is less common. Very recently, work by Meyer et al. revealed that this privileged ligand class is well suited for supporting biomimetic $\mathrm{Cu} / \mathrm{O}_{2}$ chemistry. ${ }^{[71]}$

With that in mind, the main focus of this work was to synthesize new, well suited, neutral and anionic ligands capable of mimicing biological scaffolds and study in further detail their recctivity towards small molecules such as dioxygen and nitric oxide with their corresponding copper and iron complexes. This shall be discussed in further detail in the upcoming chapters. 


\section{Chapter 2}

An Overview of Copper in Biological and Synthetic Systems 


\section{Overview}

\subsection{Copper Proteins}

2.2 Type 3 Copper Proteins: Hemocyanin, Tyrosinase and Catechol Oxidase

2.3 Modes of Dioxygen Binding

2.3.1 ${ }^{\mathbf{T}} \mathbf{P}$ vs ${ }^{\mathbf{C}} \mathbf{P}$ Binding Modes: Snapshots into Dioxygen Binding

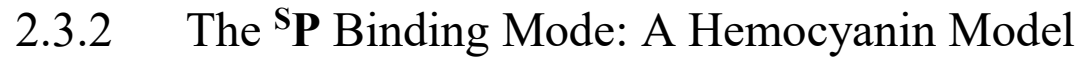

2.3.3 The Bis $\mu$-oxo Motif $(\mathbf{O})$ : Breaking the O-O Bond

2.3.4 $\quad{ }^{\mathbf{S}} \mathbf{P}$ vs $\mathbf{O}$ : Insights into the $\mathrm{O}-\mathrm{O}$ bond Scission and Tuning the Equilibrium

2.4 Particulate Methane Monoxygenase: pMMO

2.5 Catalytic Reactivity of Tyrosinase models towards External Monophenolic Substrates

2.6 Bis(oxazoline) ligands in Copper chemistry 


\subsection{Copper Proteins}

The easily attainable (I)/(II) redox couple and biological abundance contributes to copper's diverse role in nature. A large range of metalloenzymes contain copper within their active site and can broadly be classified into seven different categories, based on structural and spectroscopic properties (Fig.2.1). ${ }^{[72-74]}$ These enzymes are involved in $\mathrm{O}_{2}$ binding, activation and reduction, substrate activation, $\mathrm{NO}_{2}{ }^{-}$and $\mathrm{N}_{2} \mathrm{O}$ reduction, and electron transfer(ET) reactions. ${ }^{[38]}$ The mononuclear blue copper sites (Type 1) and the binuclear $\mathbf{C} \mathbf{u}_{\mathbf{A}}$ sites, wherein the copper centers are ligated to sulphur donors of cysteine residues, carry out ET reactions. ${ }^{[75]}$ Mononuclear $\mathbf{C} \mathbf{u}_{\mathbf{B}}$ centers catalyze the one electron reduction of nitrite to NO in bacterial denitrification, while $\mathbf{C} \mathbf{u}_{\mathbf{z}}$, a novel catalytic site comprising of a cluster of four copper ions bonded by seven histidine and three other ligands, catalyzes the final step of bacterial denitrification in which nitrous oxide is reduced to dinitrogen. ${ }^{[38]}$ The remaining three classes, Type 2, Type 3 and Type 4 use dioxygen as an electron acceptor for oxidation and oxygenation of organic substrates. ${ }^{[41]}$ The following sections focus on biological and synthetic model complexes of Type 3 and Type 4 dicopper proteins that activate and bind dioxygen

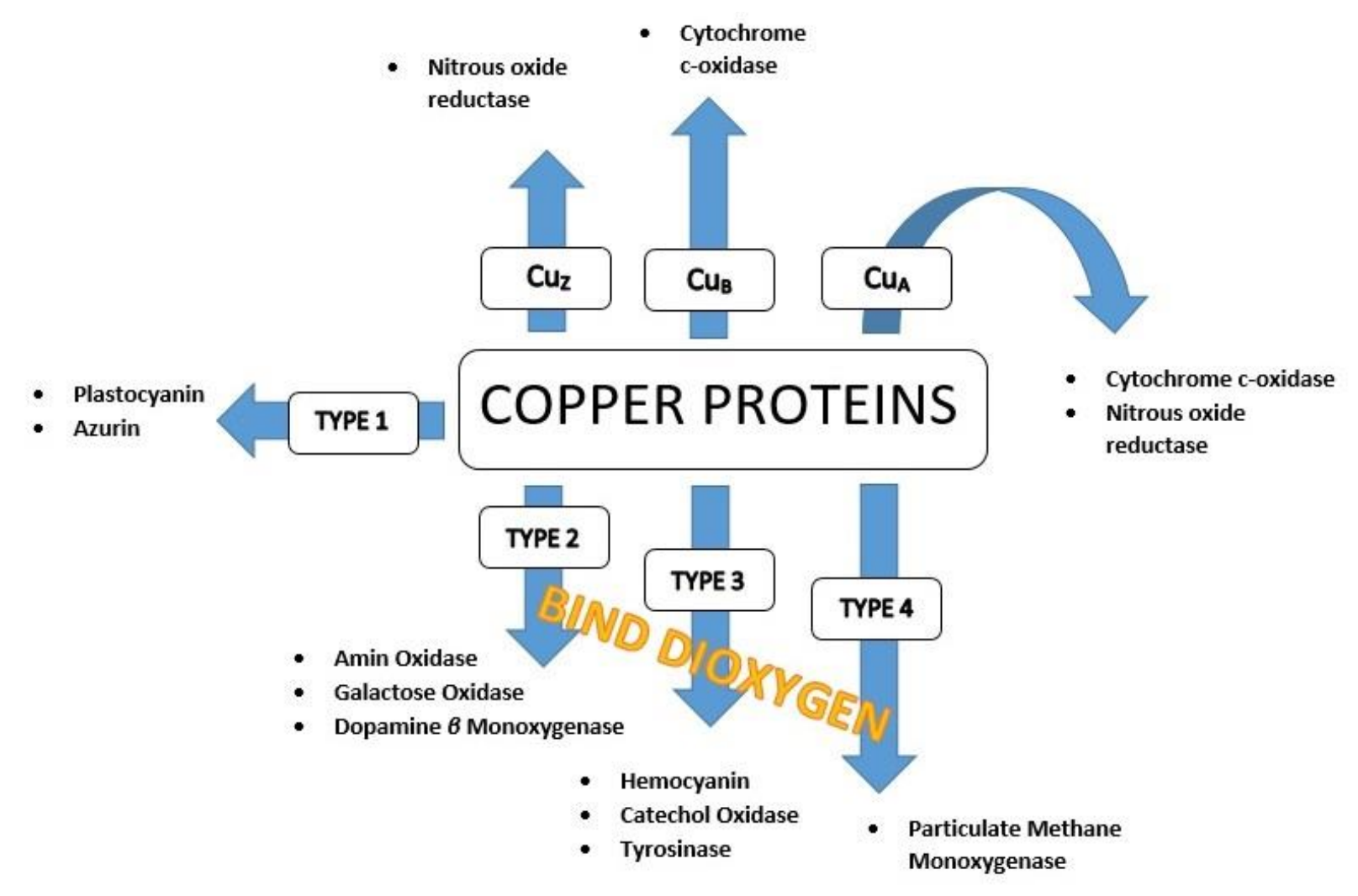

Figure 2.1 Different types of copper proteins found in nature. Type 3 and Type 4 copper proteins and their synthetic analogues form the focal point of this work. ${ }^{[38]}$ 


\subsection{Type 3 Copper Proteins: Hemocyanin, Catechol oxidase and Tyrosinase}

The Type 3 copper proteins, Haemocyanin ( $\mathrm{Hc}$ ), Catechol oxidase (CO), and Tyrosinase (Ty), comprise a pair of copper(I) centers within their active site, each coordinated by three histidine residues. ${ }^{[76]}$ They exhibit distinct spectroscopic features upon dioxygen binding, and have been extensively investigated. ${ }^{[77-}$ ${ }^{79]}$ All bind dioxygen in a characteristic side-on bridging form, whereby the deoxy $\mathrm{Cu}^{\mathrm{I}}$ center is converted to $\mathrm{Cu}^{\mathrm{II}}$ in the oxy state (Fig. 2.2) ${ }^{\left[{ }^{80]}\right.}$ This results in their active sites bearing a close resemblance in geometric and electronic properties, and hence a similarity in their crystallographic structures and spectroscopic parameters. ${ }^{[81,82]}$ The dioxygen binding to the copper centers in the deoxy state of these enzymes is spin forbidden. Extensive work by Solomon et al. has shown that this spin forbiddeness is overcome by a delocalization of the unpaired electrons onto the metal centers, and superexchange coupling between the metal centers via the ligand bridge (Scheme 2.1). ${ }^{[41,83,84]}$<smiles>Sc1cn(C2(n3cnc(S)c3)OC2(n2cnc(S)c2)n2cnc(S)c2)cn1</smiles>

Figure 2.2. Schematic representation of dioxygen binding in Hemocyanin, Catechol Oxidase and Tyrosinase. ${ }^{[42]}$

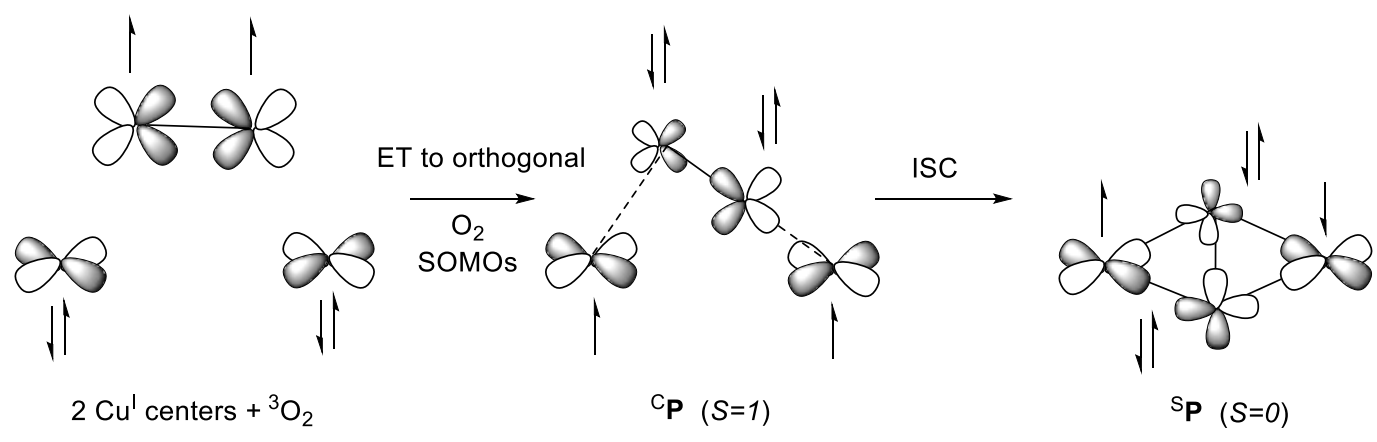

Scheme 2.1. Schematic representation of the dioxygen binding pathway in Type-3 copper proteins. ${ }^{[83]}$

Though similarly bound to dioxygen, these enzymes differ with respect to their functions. Hc behaves as an oxygen transporter in arthropods and mollusks, reversibly binding dioxygen and transporting it. ${ }^{[85]}$ Ty on binding dioxygen mediates the $o$-hydroxylation of monophenols to catechols, and both $\mathrm{CO}$ and $\mathrm{Ty}$ perform two electron oxidation of catechols to quinone. ${ }^{[86]}$ This has physiological importance in the 
synthesis of melanin in the body, where Ty is the rate limiting enzyme that converts L-tyrosine to Ldopaquinone, which finally undergoes several further reactions to form melanin (Scheme 2.2). ${ }^{[87]}$<smiles>N[C@@H](Cc1ccc(O)cc1)C(=O)[O-]</smiles>

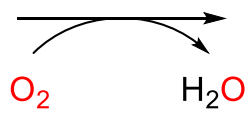<smiles>[NH3+][C@@H](CC1=CC(=O)C(=O)C=C1)C(=O)[O-]</smiles>

Scheme 2.2. Hydroxylation and oxidation of tyrosine to dopaquinone mediated by Tyrosinase. ${ }^{[87]}$

The intermediate responsible for the functioning of the enzymes Hc, Ty and more recently CO, is known to be a $\mu-\eta^{2}: \eta^{2}$ peroxo dicopper(II) ( $\left.{ }^{\mathbf{S}} \mathbf{P}\right)$ species. ${ }^{[42]}$ Interconversion of the ${ }^{\mathbf{S}} \mathbf{P}$ to the bis $\mu$-oxo dicopper(III) (O) isomer has recently been observed in several synthetic analogues and raises a pertinent question in context to the significance of the $\mathbf{O}$ isomer in biological systems. ${ }^{[88-94]}$ The impressive selective catalytic oxidation of phenols in Ty has mechanistically been elucidated to follow an electrophilic aromatic substitution (EAS) mechanism, where the phenolic substrate binds to one of the copper centers in the active site, and is then hydroxylated. ${ }^{[95,96]}$ Wether isomerization between the ${ }^{\mathbf{S}} \mathbf{P}$ and $\mathbf{O}$ species occurs during this bio-catalytical cycle is still under speculation. Several synthetic analogues have been developed to gain further insight into the functioning of this class of enzymes. ${ }^{[97-107]}$ These are discussed in the following sections.

\subsection{Modes of Dioxygen Binding}

Over the last few decades, a variety of synthetic analogues have been uncovered and have helped gain insights into the different binding modes of dioxygen to copper sites. ${ }^{[88,108-110]}$ The $2: 1 \mathrm{Cu} / \mathrm{O}_{2}$ ratio which parallels the stoichiometry in the Type $\mathbf{3}$ and Type $\mathbf{4}$ enzymes suggests that dioxygen could bind either ${ }^{\mathrm{S}} \mathbf{P}$, $\mathbf{O},{ }^{\mathrm{T}} \mathbf{P}$, or ${ }^{\mathrm{C}} \mathbf{P}$ to the copper ions (Fig. 2.3).
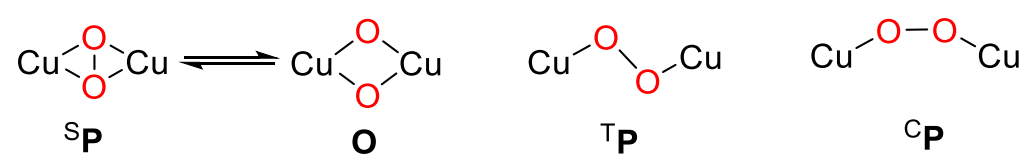

Figure 2.3 Different binding modes of dioxygen to copper in a 2:1 stoichiometric ratio of $\mathrm{Cu} / \mathrm{O}_{2}$.

\subsection{1 ${ }^{\mathrm{T}} \mathrm{P}$ vs ${ }^{\mathrm{C}} \mathrm{P}$ binding mode: Snapshots into Dioxygen Binding}

Dating back to 1988, the first strusturally elucidated copper-dioxygen system was reported by Karlin et $a l .{ }^{[110,111]}$ With assistance of the TPA ligand, the dioxygen was shown to be bound in a trans $\mu-1,2$ mode resulting in the $\mathrm{Cu} \cdots \mathrm{Cu}$ distance of $4.4 \AA$. LMCT from the peroxide ligand to the copper centers leads to absorption features at $500 \mathrm{~nm}$ (strong, in plane, $\pi_{\sigma}{ }^{*}$ to d), and $600 \mathrm{~nm}$ (shoulder, out of plane, $\pi_{\mathrm{v}}{ }^{*}$ to d) in ${ }^{\mathrm{T}} \mathbf{P}$ complexes, and the $\mathrm{O}-\mathrm{O}$ stretch herein occurs around $800-830 \mathrm{~cm}^{-1}$ when investigated by resonance Raman 
spectroscopy (rR). ${ }^{[11-131]}$ The large dihedral angle of $180^{\circ}$ of the Cu-O-O-Cu core in these ${ }^{\mathbf{T}} \mathbf{P}$ complexes causes large magnetic overlap of the $\mathrm{Cu} / \mathrm{O}_{2}$ orbitals, resulting in strong antiferromagnetic coupling between the two copper centers and a singlet $S=0$ ground state. ${ }^{[132]}$ Tetradentate ligand systems with four strongly coordinating donors, such as tetb ${ }^{[133]}$ and tren, ${ }^{[124,125,131]}$ have proved to be successful scaffolds for the ${ }^{\mathbf{T}} \mathbf{P}$ binding mode (Fig. 2.4). Though several synthetic ${ }^{\mathbf{T}} \mathbf{P}$ complexes have been characterized over time, this binding mode is not considered biologically relevant but is pertinent to dioxygen coordination to the dinuclear copper sites in Type 3 copper enzymes, and dicopper sites in general.

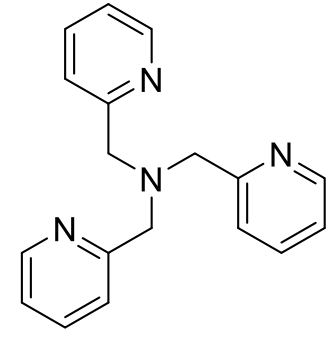

TPA

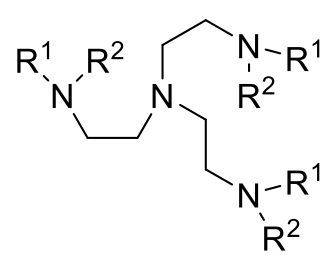

$$
\begin{aligned}
& \mathrm{R}^{1}=\mathrm{R}^{2}=\mathrm{Me}=M \mathrm{e}_{6} \text { tren } \\
& \mathrm{R}^{1}=\mathrm{R}^{2}=\mathrm{H}=\mathrm{Bz_{3 }} \text { tren }
\end{aligned}
$$

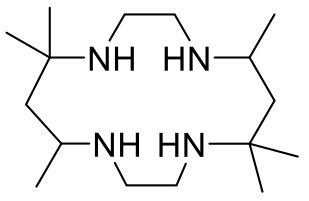

tetb

Figure 2.4. Ligand systems shown to bind dioxygen in a ${ }^{\mathbf{T}} \mathbf{P}$ fashion. ${ }^{[110,124,131,133]}$

Very recently, Meyer et al. showed that an end-on cis binding mode of dioxygen to the copper centers was possible. With aid of a pyrazolate/tacn ligand system, the first ${ }^{\mathbf{C}} \mathbf{P}$ was crystallographically characterized, and exhibited surprisingly diminished antiferromagnectic coupling (Fig.2.5 left). ${ }^{[109]}$ This resulted from the reduced magnetic overlap of the $\mathrm{Cu} / \mathrm{O}_{2}$ orbitals due to the ligand system supporting a $\mathrm{Cu}-\mathrm{O}-\mathrm{O}-\mathrm{Cu}$ torsion of $65^{\circ}$ (as opposed to $180^{\circ}$ observed in ${ }^{\mathrm{T}} \mathbf{P}$ complexes). This ${ }^{\mathbf{C}} \mathbf{P}$ motif was previously considered a transient intermediate in spin forbidden dioxygen binding and is the first of its kind to be structurally characterized. Though it differs in terms of magnetic properties with respect to the ${ }^{\mathbf{T}} \mathbf{P}$ motif, its spectroscopic features are similar. Soon after, the first ferromagnetically coupled dicopper(II) peroxo system with a triplet ground state $(S=1)$ was reported in the same group, wherein the previously used pyrazolate/tacn ligand system was modified to constrain the $\mathrm{Cu}-\mathrm{O}-\mathrm{O}-\mathrm{Cu}$ to $104^{\circ}$ thus resulting in a vanishing overlap of the magnetic orbitals (Fig. 2.5 right). ${ }^{[134]}$ This ferromagnetically coupled peroxo system was assigned as neither cis nor trans, as it differed with respect to the coordination chemistry when compared to other crystallographically characterized trans systems, and in terms of torsion angles and $\mathrm{Cu}-\mathrm{Cu}$ distances when compared to the cis peroxo system. Till date, this is the only reported dicopper peroxo system that does not exhibit any antiferromagnetic coupling, and can be considered a snapshot in the initial stages of dioxygen binding in Type 3 copper proteins.
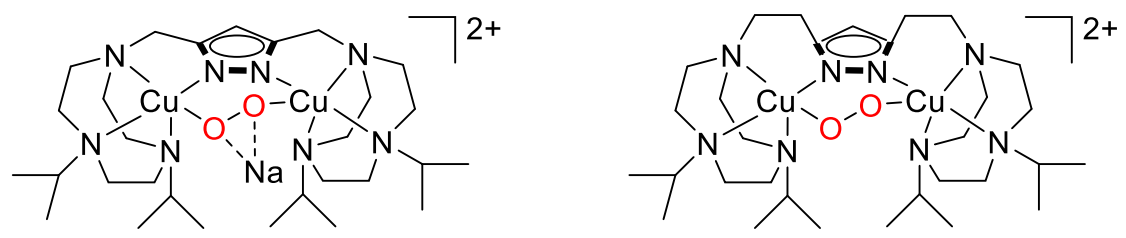

Figure 2.5. Left: Copper complex with a TACN ligand system shown to exhibit an end-on cis binding mode of dioxygen. Right: Copper complex with a TACN system shown to form the first ferromagnetically coupled dicopper(II) peroxo species. ${ }^{[109,134]}$ 


\subsubsection{The SP Binding Mode: A Hemocyanin Model}

The first ${ }^{\mathbf{S}} \mathbf{P}$ complex with the tridentate $\mathrm{HB}\left(3,5-\mathrm{i} \mathrm{Pr}_{2} \mathrm{pz}\right)_{3}$ ligand system was structurally characterized by Kitajima et al. in 1989. ${ }^{[108]}$ The $\mu-\eta^{2}: \eta^{2}$ binding mode of dioxygen to the copper centers results in diagnostic CT features divergent from the ${ }^{\mathrm{T}} \mathbf{P}$ motif. A strong band at $350 \mathrm{~nm}$ and a weak band at $500 \mathrm{~nm}$ arises due to an in plane $\left(\pi_{\sigma}{ }^{*}\right.$ to $\left.\mathrm{d}\right)$ and out of plane $\left(\pi_{\mathrm{v}}{ }^{*}\right.$ to $\left.\mathrm{d}\right)$ overlap of the peroxide and copper orbitals respectively (Fig. 2.6) ${ }^{\left[{ }^{[0,108,135-161]}\right.}$ The $\mathrm{Cu} \cdots \mathrm{Cu}$ bond length typical for ${ }^{\mathbf{S}} \mathbf{P}$ complexes lies in the range of $\sim 3.5 \AA$ and the $\mathrm{O}-\mathrm{O}$ distance lies in the range of $\sim 1.5 \AA$. The $\mathrm{rR}$ observed $\mathrm{O}-\mathrm{O}$ stretching frequency for this motif is $\sim 750$ $\mathrm{cm}^{-1}$, being much lower than for other dicopper-peroxo intermediates. ${ }^{[108,136,141,142,144,146,162-165]}$ Theoretical calculations on the bonding description of these complexes rationalized the weak O-O bond as a result of back donation of the electron density from the $\mathrm{Cu}(\mathrm{II}) \mathrm{d}_{\mathrm{x} 2-\mathrm{y} 2}$ orbitals to the peroxide $\sigma^{*}$ orbitals in the HOMO. Strong antiferromagnetic coupling that results from the large overlap between the $\mathrm{Cu} / \mathrm{O}_{2}$ magnetic orbitals makes these ${ }^{\mathbf{S}} \mathbf{P}$ complexes EPR silent, with an $S=0$ ground state. ${ }^{[93]}$

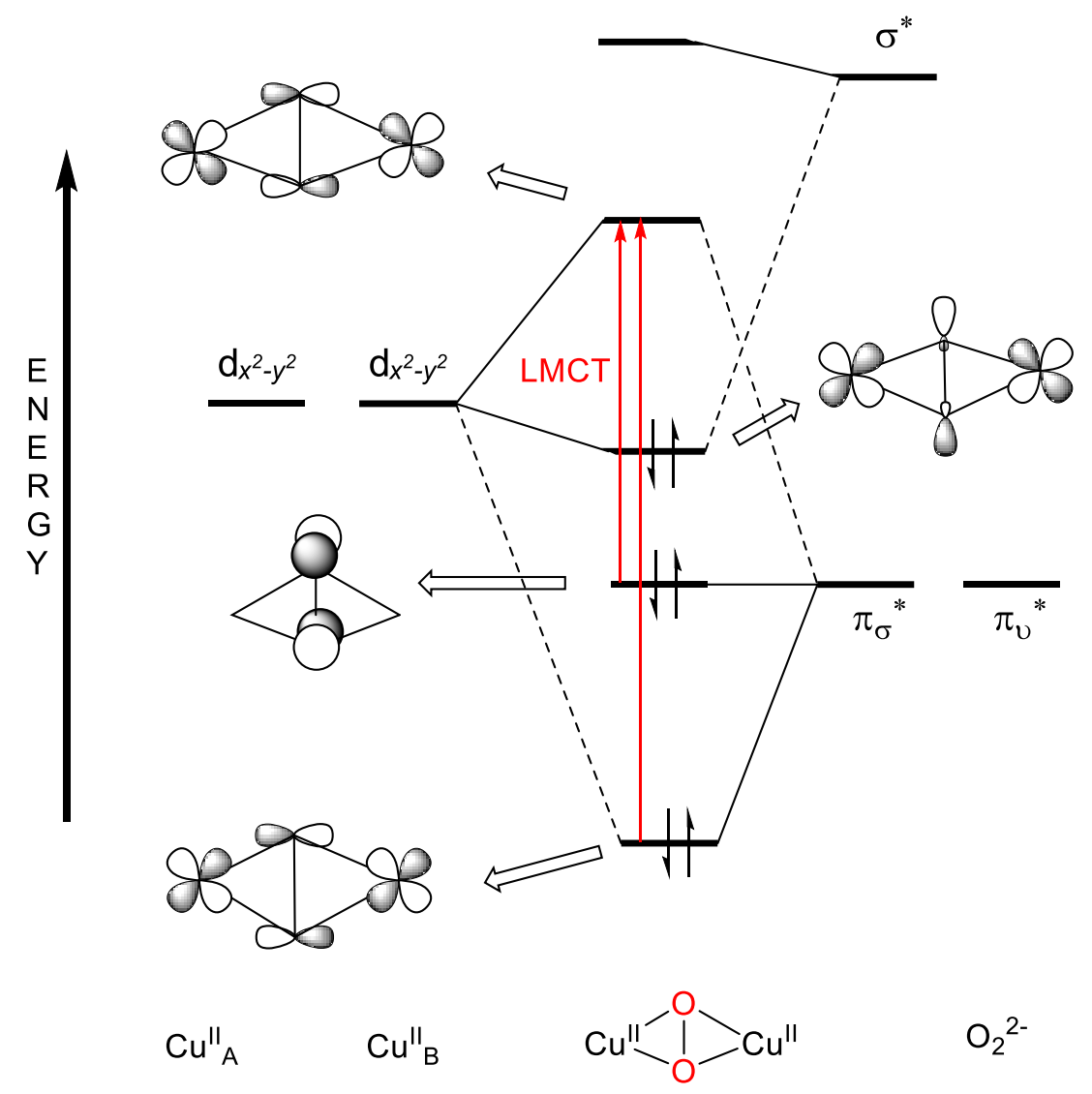

Figure 2.6. Schematic molecular orbital diagram for the side-on $\left(\mu-\eta^{2}: \eta^{2}\right)$ peroxide bridged dicopper(II) species $\mathbf{S} \mathbf{P}$. The red arrows indicate the assigned $\mathrm{UV}$-vis features in ${ }^{\mathbf{S}} \mathbf{P}$, in plane $\pi_{\sigma}{ }^{*}$ to $\mathrm{d}$ and out of plane $\pi_{\mathrm{v}}{ }^{*}$ to $\mathrm{d}$ (peroxo to $\mathrm{Cu}^{\mathrm{II}}{ }_{2}$ ) $\mathrm{CT}$ transitions at $\sim 350 \mathrm{~nm}$ and $\sim 500 \mathrm{~nm}$ respectively. ${ }^{[41,132]}$ 
Though a large number of ${ }^{\mathbf{S}} \mathbf{P}$ complexes have been characterized, structural characterization still remains a challenge for this binding motif. Tridentate N-donor ligands capable of facial coordination, such as $i \mathrm{Pr}_{3}$ tacd, and ${ }^{t} \mathrm{Bu}_{3}$ tacn have proved to be most beneficial in this case. ${ }^{[142,144]}$ This stems from these type of ligands being bulky in nature, capable of shielding the reactive unit from subsequent decomposition. Recently, Karlin et al. isolated a new ${ }^{\mathbf{S}} \mathbf{P}$ complex with the tridentate MeAN ligand, having the longest O$\mathrm{O}$ bond reported to date. ${ }^{[164]}$ The weak $\mathrm{O}-\mathrm{O}$ bond however did not reflect an increase in backbonding into the $\sigma^{*}$ orbital of the peroxide, and further did not result in $\mathrm{O}-\mathrm{O}$ cleavage. Till date, only one bidentate ligand system, $\alpha-S_{\mathrm{p}}$, has been reported to isolate a ${ }^{\mathbf{S}} \mathbf{P}$ complex with structural elucidation (Fig. 2.7). ${ }^{[162]}$

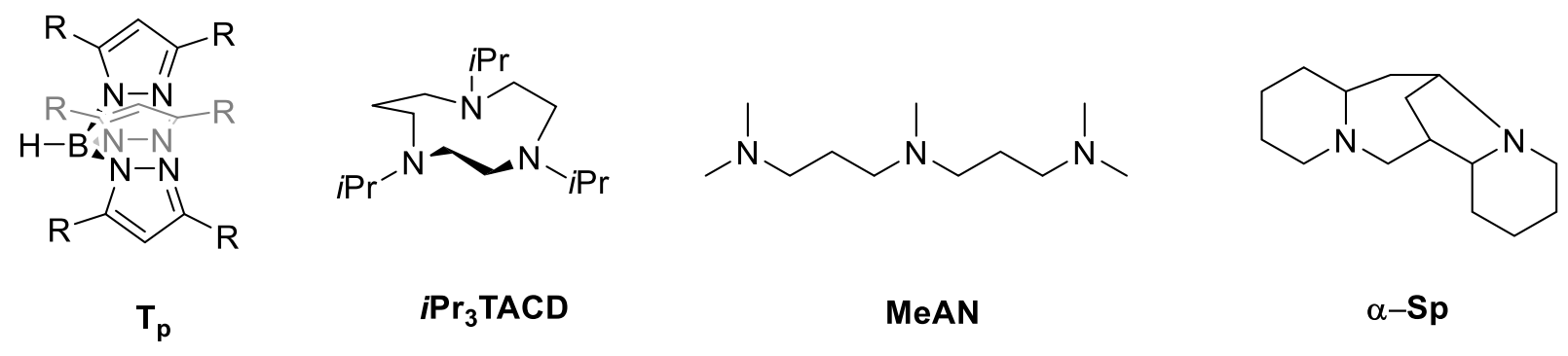

Figure 2.7. Different ligand systems shown to bind dioxygen in an $\mathbf{S} P$ fashion. ${ }^{[108,144,162,164]}$

Soon after the first ${ }^{\mathbf{S}} \mathbf{P}$ complex was structurally characterized, this motif was identified as the novel binding mode in the Type 3 copper protein oxyHc, which marks a pioneering achievement in bioinorganic chemistry. ${ }^{[42]}$ The analogy of structural and spectroscopic features of these model complexes with that of the native system forms the focal point of fundamental ideas that emphasize bioinorganic modeling attempts. For example, simple model complexes that are easily attainable in aprotic solvents under abiological conditions could in turn put forward possible intermediates involved in the mechanism of these biochemical systems, thus leading us closer to a better understanding of these important enzymes.

\subsubsection{The Bis $\mu$-oxo Motif: Breaking the O-O Bond}

The previously unknown bis( $\mu$-oxo) dicopper (III) system (O), a $\mathrm{Cu}_{2} \mathrm{O}_{2}$ motif lacking an $\mathrm{O}-\mathrm{O}$ bond was initially reported by Tolman et al. with the 1,4,7-triazocyclononane system. ${ }^{[88]}$ The weak O-O bond in ${ }^{\mathbf{S}} \mathbf{P}$ species arising from the backbonding of copper to the oxygen orbitals, helps to rationalize this O-O bond scission, justifying easy isomerization of the two forms. ${ }^{[79,80,90,93]}$ The $\mathbf{O}$ form differs significantly from its ${ }^{\text {SP }}$ isomer as being more compact and having a shorter $\mathrm{Cu} \cdots \mathrm{Cu}$ bond distance of $\sim 2.8 \AA .^{[88,138,166-171]}$ The two CT bands are more intense due to a higher degree of covalency. The band at $\sim 300 \mathrm{~nm}$ has similar origins to that of $350 \mathrm{~nm}$ observed in the ${ }^{\mathbf{S}} \mathbf{P}$ form $\left(\pi_{\sigma}{ }^{*}\right.$ to $\left.\mathrm{d}\right)$, however, the second band at $\sim 400 \mathrm{~nm}$ is characteristic of the bis $\mu$-oxo core, and results from overlap of the $\sigma^{*}$ orbital of oxo to $\mathrm{d}$ orbitals of copper (Fig.2.9). ${ }^{[167,172,173]}$ The $\mathbf{O}$ isomer is EPR silent and the diamagnetic nature of these complexes gives rise to NMR spectral features in the range of 0 to $10 \mathrm{ppm} \cdot{ }^{[138]} \mathrm{rR}$ observed stretching frequency indicates an oxygen isotope sensitive feature at $\sim 600 \mathrm{~cm}^{-1}$, due to the symmetric vibration of the $\mathrm{Cu}_{2} \mathrm{O}_{2}$ core. ${ }^{[161,174,175]}$ 


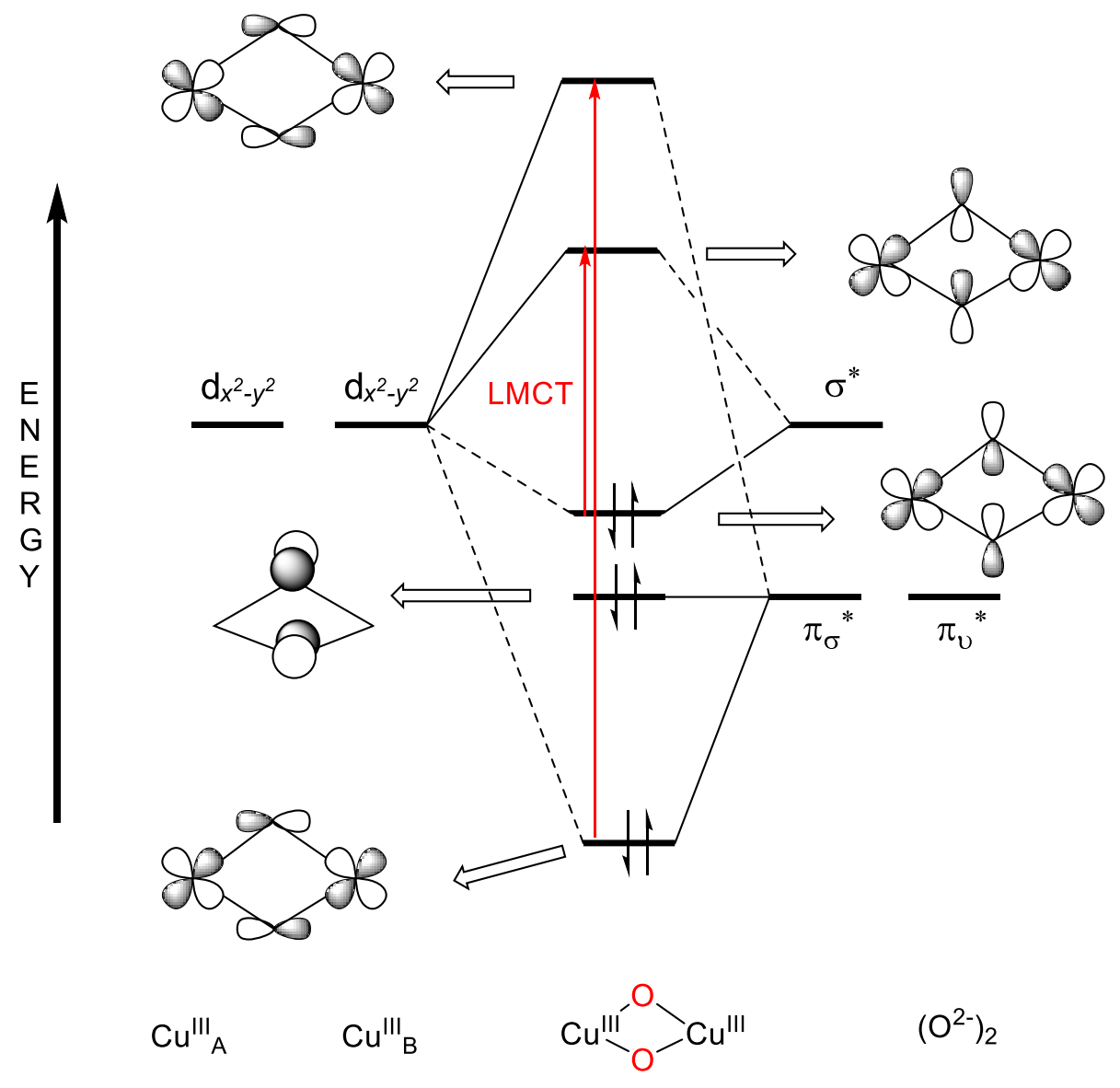

Figure 2.9 Schematic molecular orbital diagram for the bis( $\mu$-oxo) bridged dicopper(III) species $\mathbf{O}$. The red arrows indicate the assigned $\mathrm{UV}$-vis features in $\mathbf{O}, \sigma^{*}$ to $\mathrm{d}$ and $\pi_{\sigma}{ }^{*}$ to $\mathrm{d}$ (oxo to $\mathrm{Cu}^{\mathrm{III}}$ ) $\mathrm{CT}$ transitions at $\sim 400 \mathrm{~nm}$ and $\sim 300 \mathrm{~nm}$ respectively. [41][132]

A large number of ligand systems have been employed in generating the $\mathbf{O}$ species making this one of the most recognized $\mathrm{Cu} / \mathrm{O}_{2}$ forms. Structural characterization with bidentate ligands such as $\mathrm{R}_{2}{ }^{1} \mathrm{R}_{2}{ }^{2} \mathrm{eda},{ }^{[161,168,173,174,176-179]}$ tridentate ligands such as R-PYAN, ${ }^{[88,138,143,175]}$ and tetradentate ligands such as 6-R-tpa ${ }^{[167,180]}$ have revealed a rather square planar geometry of the bridging oxide $\left(\mathrm{O}^{2-}\right)$ and the two copper centers, making the core more close packed in comparison to the $\mathbf{P}$ binding motif. Moreover, the $\mathrm{Cu}$ centers bear an oxidation state of +3 as opposed to +2 which is observed in all other $\mathrm{Cu} / \mathrm{O}_{2}$ forms described above. Most of the structurally characterized $\mathbf{O}$ species are cationic in nature with neutral ligands. ${ }^{[88,138,166-170]}$ Monoanionic ligands forming neutral $\mathbf{O}$ species are uncommon and rare, with only one system comprising the $\left[\mathrm{Bu}_{2}^{\mathrm{t}} \mathrm{P}\left(\mathrm{NSiMe}_{3}\right)_{2}\right]^{-}$ligand being reported till date (Fig.2.10) . ${ }^{[171]}$ 
As has been initially shown by Tolman et al., and investigated by several groups thereafter, there exists an equilibrium between the $\mathbf{O}$ and the ${ }^{\mathbf{S}} \mathbf{P}$ species. ${ }^{[38,91]}$ The weak O-O bond in ${ }^{\mathbf{S}} \mathbf{P}$ species helps to rationalize this $\mathrm{O}-\mathrm{O}$ bond scission, accounting for easy isomerization of the two forms. Though the $\mathbf{O}$ motif has yet to be biologically observed, its importance is under serious speculation.

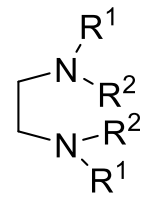

$\mathbf{R}_{\mathbf{2}}{ }^{1} \mathbf{R}_{\mathbf{2}}{ }^{2}$ eda<smiles>[R]c1cccc(CCNCCCN(C)C)c1</smiles>

R-PYAN

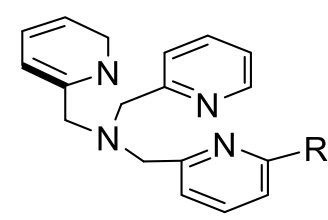

6-R-tpa<smiles>CN=P(C)(C)C</smiles>

$\left[\mathrm{Bu}_{2}^{\mathrm{t}} \mathrm{P}\left(\mathrm{NSiMe}_{3}\right)_{2}\right]^{-}$

$$
\mathrm{R}=\mathrm{NMe}_{2}, \mathrm{OMe}, \mathrm{H}, \mathrm{Cl}
$$

Figure 2.10 Ligand systems shown to bind dioxygen in an $\mathbf{O}$ fashion. ${ }^{[168][143][180]}$

\subsubsection{SP vs O: Insights into the O-O bond Scission and Tuning the Equilibrium.}

The ubiquitous ${ }^{\mathbf{S}} \mathbf{P}$ and $\mathbf{O}$ isomers have been shown to exist in equilibrium with each other in measurable quantities. ${ }^{[181,182]}$ The facile isomerization of the two stems from easy cleavage of the O-O bond. On elongation of the $\mathrm{O}-\mathrm{O}$ bond, the $\sigma^{*}$ orbital in ${ }^{\mathrm{S}} \mathbf{P}$ involved in backbonding decreases in energy, oxidizing the copper HOMO and becoming fully occupied (Fig.2.11). ${ }^{[93]}$ A number of systems wherein this equilibrium has been well characterized have shown that the $\mathbf{O}$ species is enthalpically stabilized, whereas the ${ }^{\mathbf{S}} \mathbf{P}$ species is stabilized entropically. ${ }^{[92,166,183]}$ Thermodynamic parameters determined for the isomerization revealed a small entropy difference between these two binuclear $\mathrm{Cu} / \mathrm{O}_{2}$ species, justifying easy interconversion of the two. Though not always observed, it is assumed that rapid equilibrium exists between the two isomers.

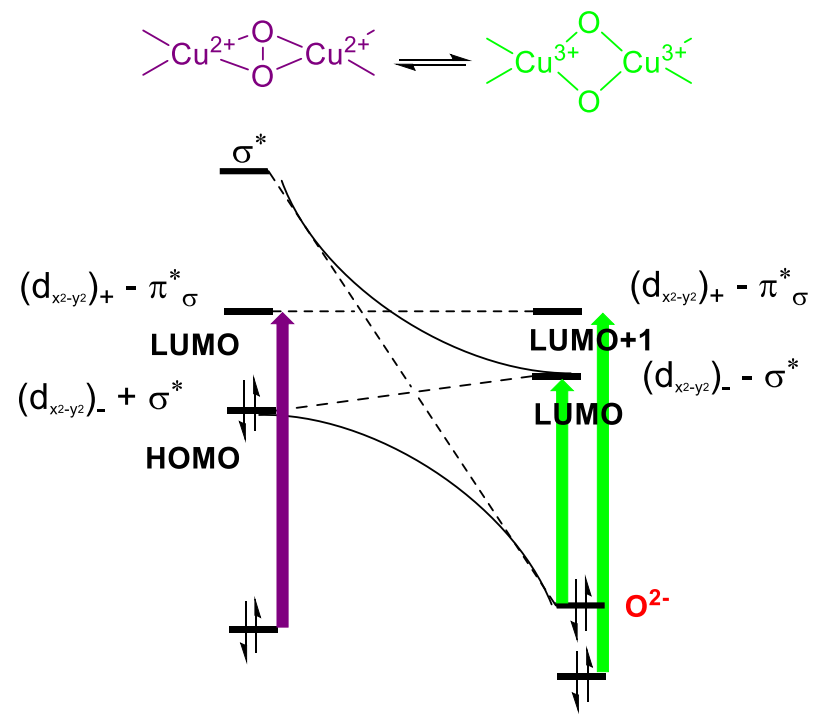

Figure 2.11 Orbital correlation diagram for the ${ }^{\mathbf{S}} \mathbf{P} / \mathbf{O}$ isomerization. ${ }^{[93]}$ 
Several groups have investigated in detail factors controlling the $\mathbf{S} \mathbf{P} / \mathbf{O}$ equilibrium. Thermodymanic parameters determined for this equilibrium reveal that temperature dependence is only weak. Ligand structural effects have proved to be most dominant in controlling the formation of the two isomers. Studies with different types of ligands demonstrated that increasing the steric demand of a particular ligand facilitates the formation of the ${ }^{\mathbf{S}} \mathbf{P}$ form rather than the $\mathbf{O}$ form. ${ }^{[88,138,140,184-186]}$ The rationale being that intramolecular interactions are higher in the $\mathbf{O}$ as compared to the ${ }^{\mathbf{S}} \mathbf{P}$ isomer due to its close packed structure. This was also in accordance with theoretical calculations on space filled models conducted by Stack et al. ${ }^{[184]}$

Besides ligand structural effects, the coordinating ability of the counterion too has an influence on isomeric preference. Solomon et al. demonstrated with aid of the ${ }^{\mathrm{Me}, \mathrm{Me}} \mathrm{pd}$ ligand (Fig. 2.12) that using the triflate counterion favored the formation of the ${ }^{\mathrm{S}} \mathbf{P}$ isomer as opposed to $\mathbf{O} .{ }^{[183]}$ The more exposed $\mathrm{Cu}_{2} \mathrm{O}_{2}$ core of the ${ }^{\mathbf{S}} \mathbf{P}$ complex, in conjunction with the $\mathrm{Cu}$ (II) being able to axially coordinate ligands makes axial counterion coordination feasible, which in some cases leads to extra stability. The nature of the solvent suggests that for bidentate and tridentate amine ligands, more polar solvents favor the formation of the $\mathbf{O}$ species while less polar solvents shift the equilibrium in the direction of the ${ }^{\mathbf{S}} \mathbf{P}$ species.${ }^{[2,138,183,184]}$ However, in the first ever reported equilibrium case by Tolman et al. using the $i \operatorname{Pr}_{3} \operatorname{tacd}$ ligand system, the ratio of two solvents, THF: $\mathrm{CH}_{2} \mathrm{Cl}_{2}$ determined which isomer preferentially formed. This was accounted for by solvent coordination to the complex exhibiting an additional stabilization. ${ }^{[88]}$

The MePy2 ligand family as shown by Karlin and Fukuzumi independently has demonstrated that electronic effects of the ligand also control the isomerization in addition to structural effects. ${ }^{[140,185]}$ Increasing the electron donating ability of the ligand stabilizes the +3 oxidation state of the copper, favoring the $\mathbf{O}$ formation over the ${ }^{\mathbf{S}} \mathbf{P}$ isomer.

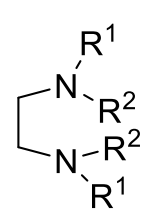

$\mathbf{R}_{\mathbf{2}}{ }^{1} \mathbf{R}_{\mathbf{2}}{ }^{2}$ eda

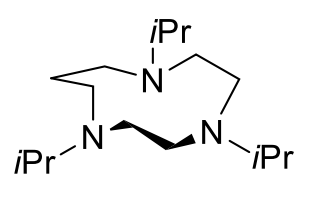

$\operatorname{iPr}_{3} \mathrm{TACD}$

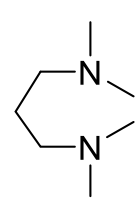

Me,Mepd

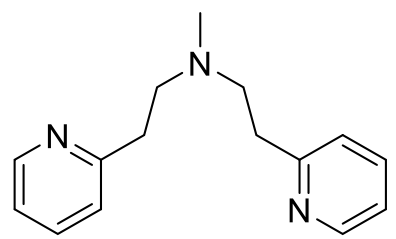

MePY2

Figure 2.12 Ligand systems known to form equilibrium mixtures of ${ }^{\mathbf{S}} \mathbf{P}$ and $\mathbf{O}$ isomers. ${ }^{[88,140,183,184]}$

Though a large number of factors have successfully been uncovered in influencing the equilibrium of these two $2: 1 \mathrm{Cu} / \mathrm{O}_{2}$ species, biologically it has yet not been observed. Interestingly, the biological scaffolds that host the cooper sites in these novel enzymes are comprised mainly of histidine imidazole residues, with available protons in the vicinity. This raises the credibility of interconversion being triggered by (de)protonation events by a change in local $\mathrm{pH}$, which may lead to further insights into the biochemical pathway. 


\subsection{Particulate Methane Monoxygenase: pMMO}

The most extensively investigated dicopper protein presently is pMMO. This integral membrane protein present in methanotrophic bacteria converts methane into methanol. ${ }^{[49]}$ On account of its difficulty to isolate, it falls behind with reference to insights into its active site and biological mechanism compared to other copper proteins. ${ }^{[187]}$ Till only very recently, the resting phase of pMMO was determined to have a dicopper site, which was located in one of the three subunits of the enzyme. ${ }^{[46]}$ Rather unique as compared to other copper enzymes, in pMMO one copper is coordinated by two histidine imidazoles and the other is ligated by a histidine imidazole and a primary amine of an $\mathrm{N}$ terminal histidine, making the active site non symmetrical in nature. ${ }^{[47,48]}$ The two copper ions are roughly at a distance of $2.5 \AA$. Investigations by Rosenzweig et al. of the pMMO enzyme with oxidants suggested plausible binding of dioxygen to the active site. A $\mu-\eta^{2}: \eta^{2}$ peroxo species was observed as the intermediate, whose spectroscopic features disappeared on reaction with methane. ${ }^{[188]}$ This highlighted the relevance of this dioxygen binding motif which could potentially help identify the nature of the active intermediate in pMMO. Till date however, no certain characterization of the dioxygen bonded adduct or possible intermediate for methane oxygenation has been identified.

Solomon and Schoonheydt suggested the copper loaded aluminosilicate zeolite, Cu-ZSM-5, to be most similar to pMMO. ${ }^{[189]}$ The $\mathrm{Cu}(\mathrm{I})$ centers herein were oxidized to $\mathrm{Cu}(\mathrm{II})$ by dioxygen, and a $\mathrm{Cu}_{2}{ }_{2}(\mu$-oxo) species was identified. Independent DFT studies in conjunction with experimental findings suggested this motif to replicate similar activity of the enzyme, when modeled into the inorganic Cu-ZSM-5 and pMMO active site.
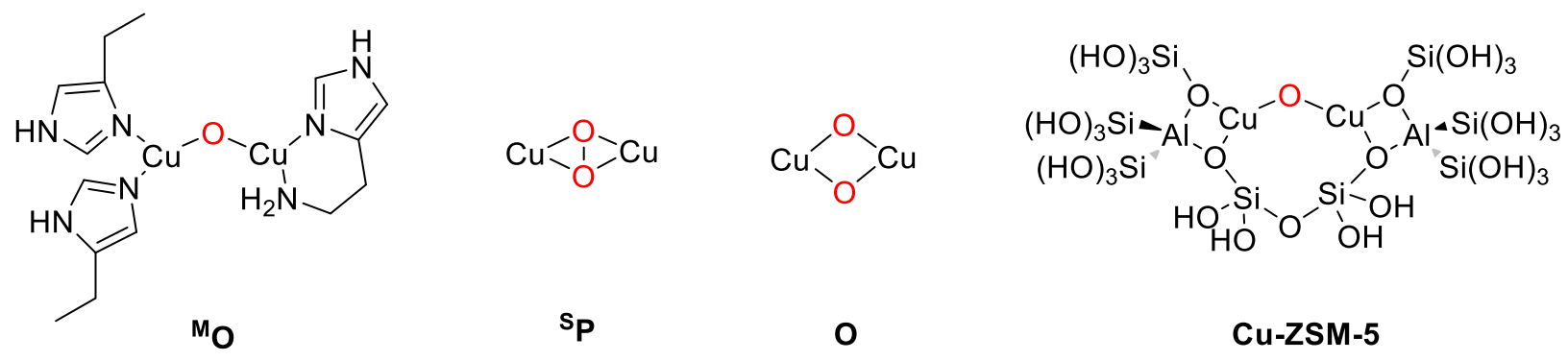

Figure 2.13 Schematic representation of different dioxygen binding modes proposed for pMMO. Shown on the right Cu-ZSM5 , an inorganic model proposed for the enzyme active site. ${ }^{[189]}$

Recently, Stack et al. proposed a dicopper(III) bis ( $\mu$-oxide) to be a potential intermediate for pMMO. ${ }^{[190]}$ With low temperature ligand exchange as a method of assembling the $\mathrm{O}$ motif, a complex similar to the coordination sphere of $\mathrm{pMMO}$ was produced suggesting histidine imidazole ligands to replicate this method of core capture to attain a $\mathrm{Cu}$ (III) bis $(\mu$-oxo) motif as an active intermediate (Fig. 2.14). The $\mathrm{Cu}$ (III) complexes also mediated $\mathrm{C}-\mathrm{H}$ activation of exogenous substrates. These findings present $\mathrm{pMMO}$ as the only copper enzyme to have a potential $\mathrm{Cu}$ (III) intermediate within its active site.

Computational calculations jointly with molecular mechanics conducted by Yoshizawa et al. recently have put forward the active intermediate to be a $\mu-\eta^{2}: \eta^{2}$ peroxo species, similar to the proposal of Rosenzweig et. al.. ${ }^{[191]}$ TD-DFT calculations on the resting phase of pMMO and dioxygen suggested the intermediate resembled that of $\mathrm{Hc}$ and Ty, a side on peroxo motif. The calculated $\mathrm{Cu} \cdots \mathrm{Cu}$ and $\mathrm{O} \cdots \mathrm{O}$ bond lengths were in agreement with analogous and native models. 


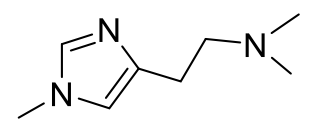

$\mathrm{L}_{3}$

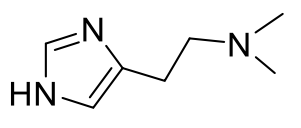

$\mathrm{L}_{4}$

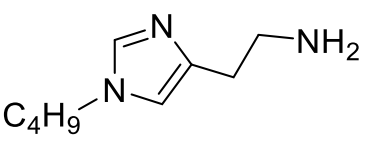

$\mathbf{L}_{5}$

Figure 2.14 Ligand systems employed by Stack et al. that propose dioxygen to bind as an $\mathbf{O}$ motif in pMMO. ${ }^{[190]}$

Optimal conversion of natural gas of which methane forms a major component is still an ongoing challenge. Though a large number of industrial catalysts have been developed, they have proved to be costly and insufficient. In contrast, pMMO uses methane as its sole form of carbon and energy to convert it into methanol. A better understanding of this enzyme in its biological pathway would be a seminal achievement in bioinorganic chemistry. Scientific findings have proposed both the $\mathbf{O}$ and ${ }^{\mathbf{S}} \mathbf{P}$ form to be potential key intermediates of this enzyme. Taking into account the biological histidine scaffolds with amenable protons in the vicinity, credibility of conversion of the two by protonation/deprotonation is of significant biological importance.

\subsection{Catalytic Reactivity of Tyrosinase models towards external monophenolic substrates}

The catalytic reactivity executed by Type 3 copper proteins has aroused large interest over the last few decades. All three Type 3 copper proteins bind dioxygen to form a ${ }^{\mathbf{S}} \mathbf{P}$ intermediate. Though the oxy forms of these enzymes resemble each other spectroscopically, they differ in terms of their reactivity. ${ }^{[42,44,81,86]}$

The enzyme Hemocyanin behaves as an oxygen transporter in arthropods and mollusks, reversibly binding dioxygen, but exhibits no catalytic activity. This lack of dioxygen reactivity towards external substrates is associated with the active site of the enzyme being deeply buried within the protein matrix, making it inaccessible to external substrates. ${ }^{[192]}$ Comparatively, the active sites of Catechol oxidase and Tyrosinase after activation are easily accessible to substrates for catalytic activity. ${ }^{[44,86]}$

Tyrosinase catalyzes the ortho-hydroxylation of tyrosine to DOPA, and subsequent two electron oxidation of DOPA to dopaquinone which forms the first step of melanin synthesis. ${ }^{[43,76,193,194]}$ This catalytic activity exhibited by similar model complexes has been investigated in detail by several groups. Based on kinetic data and different intermediates that have been successfully isolated during the course of study, a reactive scheme containing two interpenetrating cycles (monophenolase cycle and diphenolase cycle) has been established. ${ }^{[76,95]}$ The monophenolase cycle (Scheme 2.3 blue cycle) starts from the oxy form of Tyrosinase, where monophenols are converted to $o$-diphenols and subsequently to $o$-quinones. The deoxy site is formed again, regenerating the $\mathrm{Cu}(\mathrm{I})$ centers for subsequent $\mathrm{O}_{2}$ binding and catalytic activity. The diphenolase activity expressed by both $\mathrm{CO}$ and Ty (Scheme 2.3 green cycle) accounts for the conversion of external catechols to $o$-quinone. During the course of this cycle, the met-derivative of Tyrosinase is generated which is responsible for the two electron oxidation of catechol, such that two molecules of diphenol are converted to $o$-quinone. The general reactivity observed in Tyrosinase as well as other synthetic analogues is in agreement with an electrophilic substitution mechanism. ${ }^{[4,45,195,196]}$ Hammett type experiments conducted with small molecule model systems support this pathway, though radical pathways have also been proposed. ${ }^{[197]}$ 


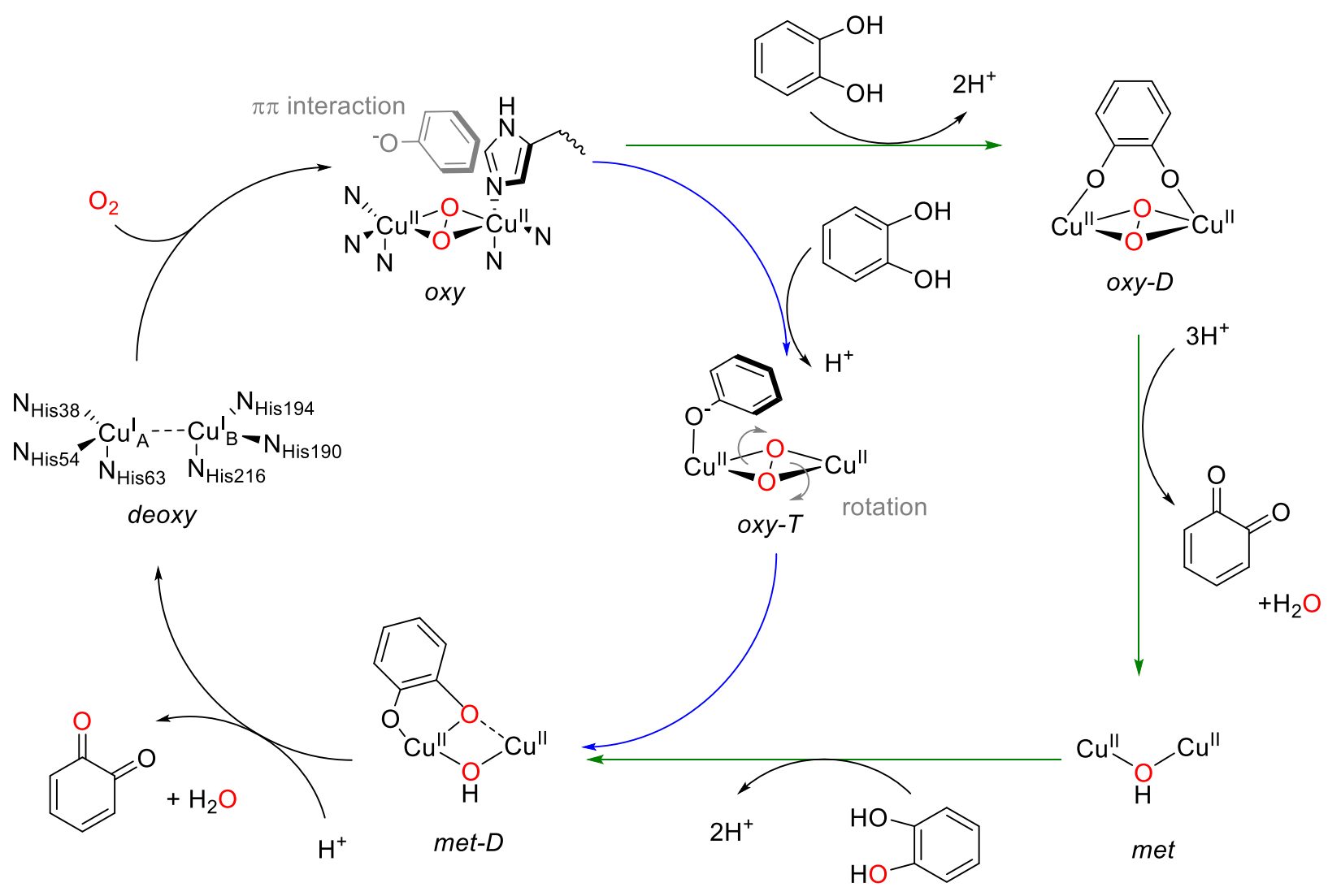

Scheme 2.3. Generally accepted mechanism displaying two interpenetrating cycles for the ortho-hydroxylation of tyrosine to DOPA exhibited by Tyrosinase. Monophenolase activity is depicted in blue and diphenolase activity depicted in green. ${ }^{\text {[76][95] }}$

A large number of binuclear copper complexes mimicking the active site of the Tyrosinase enzyme have been synthesized and spectroscopically characterized. ${ }^{[95,100,103,105-107,145,198]}$ Investigations of their reactivity towards organic substrates have helped in elucidating the mechanism of the analogous biochemical reaction. The first conformation of this kind of reactivity was reported by Karlin et al. with the help of a binucleating XYL ligand system (Fig. 2.15). ${ }^{[199]}$ The system was shown to form a ${ }^{\mathbf{S}} \mathbf{P}$ complex which was detected by $\mathrm{rR}$ spectroscopy. Thermal decomposition of the complex resulted in endogenous arene hydroxylation analogous to intermolecular phenol hydroxylation.

Apart from this, individual ${ }^{\mathbf{S}} \mathbf{P}, \mathbf{O}$ and ${ }^{\mathrm{T}} \mathbf{P}$ systems have also been found to exhibit monophenolic substrate activity. The first small molecule model of Tyrosinase forming a stable dioxygen intermediate and mediating $o$ - hydroxylation of phenols, was reported by Casella et al. with the ligand system L66 (Fig. 2.15). ${ }^{[157,200]}$ Initially shown in 1991 that the $\mathrm{Cu}(\mathrm{I})$ complex mediated the catalytic conversion of sodium 2,4-di-tert-butyl phenolate (DTBP) to 3,5-di-tert-butyl quinone(DTBQ) in the presence of dioxygen with a TON of 1.2, reinvestigation of the same $\mathrm{Cu}(\mathrm{I})$ complex a decade later revealed that a ${ }^{\mathrm{S}} \mathbf{P}$ intermediate was responsible for catalytic conversion. ${ }^{[157]}$ Following this, several other small molecule models have been synthesized, emulating catalytic activity of the enzyme. Most recently, a ${ }^{\mathbf{S}} \mathbf{P}$ system developed by HerresPawlis and Stack with aid of the bis(pyrazolyl)(2-pyridyl)methane $\left(\mathrm{HC}(3-t \mathrm{BuPz})_{2}(\mathrm{Py})\right)_{2}$ mononucleating ligand (Fig. 2.15), showed impressive catalytic activity with a TON of 15. A range of para substituted 
substrates were also investigated with the complex which resulted in a negative slope of the Hammett correlation, agreeing with the electrophilic aromatic substitution mechanism followed by these reactions. ${ }^{[201]}$

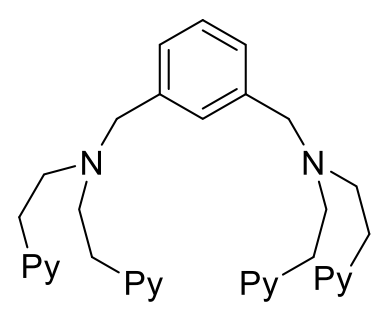

XYL<smiles>Cn1c(CCN(CCc2nc3ccccc3n2C)Cc2cccc(CN(CCc3nc4ccccc4n3C)CCc3nc4ccccc4n3C)c2)nc2ccccc21</smiles>

L66<smiles>CC(C)(C)c1ccn(Cc2ccccn2)n1</smiles>

$\mathrm{HC}(3-\mathrm{tBuPz})_{2}(\mathrm{Py})$

Figure 2.15. Ligand systems shown to bind dioxygen in an ${ }^{\mathbf{S}} \mathbf{P}$ binding mode that display similar catalytic activity as Tyrosinase. ${ }^{[199-201]}$

$\mathrm{Cu}(\mathrm{I})$ systems wherein the active intermediates were not successfully isolated have also shown to be competent in monoxygenation reactions. ${ }^{[101,202,203]}$ Reglier et al. first reported reactivity of $\mathrm{Cu}(\mathrm{I}) \mathrm{BiPh}(\mathrm{impy})_{2}$ ) (Fig. 2.16), successfully oxidizing DTBP to DTBQ in the presence of triethyl amine and dioxygen with a TON of $16 .{ }^{[202]}$ Tuczek et al. have demonstrated a series of mononuclear $\mathrm{Cu}(\mathrm{I})$ complexes and simple bidentate ligands exhibiting impressive TON's for catalytic activity. Most recently, a $\mathrm{Cu}(\mathrm{I})$ model system containing a benzimidazole moiety $\mathrm{L}_{\text {bzm }}$ within the ligand, reported a highest TON of 31 (Fig. 2.16). ${ }^{[204]}$ The reactivity studies were carried out similar to that of Reglier et al. and formation of the quinone was monitored by UV-vis spectroscopy.

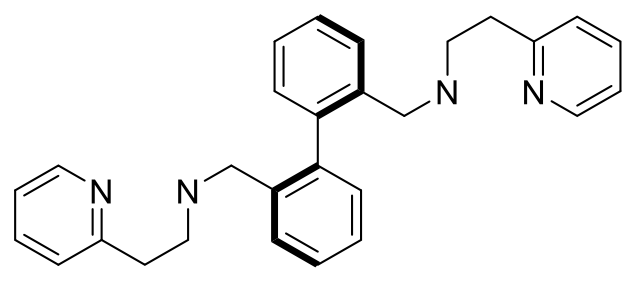

$B i P h(i m p y)_{2}$

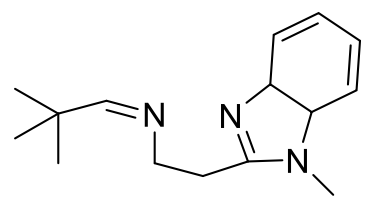

$\mathrm{L}_{\text {bzm }}$

Figure 2.16. Ligand systems that form $\mathrm{Cu}(\mathrm{I})$ complexes and successfully carry out monoxygenation reactions. ${ }^{[202,204]}$ 
In addition, the $\mathbf{O}$ isomer which is known to exist in rapid equilibrium with the ${ }^{\mathbf{S}} \mathbf{P}$ isomer, and has yet to be biologically detected, has too demonstrated catalytic activity with external substrates ${ }^{[12]}$ In most cases however, the unphysiological radical based C-C and C-O coupling products are observed. ${ }^{[12,79,205]}$ Stack et al. first illustrated $\mathbf{O}$ activity with the $\mathrm{Cu}(\mathrm{I}) \mathrm{DBED}$ system (Fig. 2.17). ${ }^{[206]}$ The complex formed the ${ }^{\mathbf{S}} \mathbf{P}$ isomer with dioxygen at low temperatures. Addition of substrate resulted in the cleavage of the O-O bond forming the $\mathbf{O}$ isomer, which was shown to be responsible for activity. In 2008, the $\mathrm{Cu}(\mathrm{I})_{2}\left(\mathrm{M}-\mathrm{XYL} \mathrm{L}^{\mathrm{MeAN}}\right)$ (Fig. 2.17) system published by Company et al. was the first example of an independent $\mathbf{O}$ intermediate demonstrating catalytic activity in $67 \%$ yield. ${ }^{[207]}$ Soon after, Herres-Pawlis and Stack reported similar activity for an $\mathbf{O}$ complex with the ligand system ${ }^{2} \mathrm{~L}$ (Fig. 2.17), exhibiting catecholase activity in $>95 \%$ yield. ${ }^{[208]}$ The evidence of catalytic activity exhibited by $\mathbf{O}$ complexes raises a pertinent question in context to weather the ${ }^{\mathbf{S}} \mathbf{P}$ species is responsible for catalysis, or if substrate binding to the ${ }^{\mathbf{S}} \mathbf{P}$ core triggers $\mathbf{O}$ formation which then carries out the monoxygenation. This issue is presently under investigation.

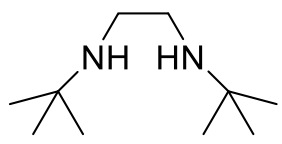

DBED

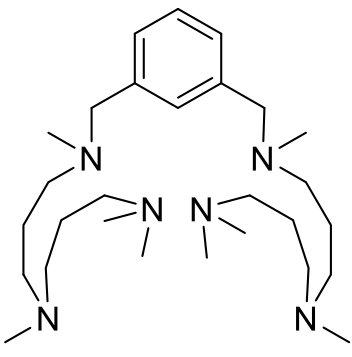

$M-X Y L^{M e A N}$

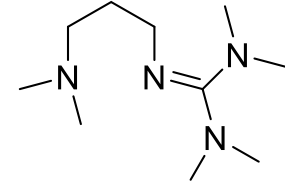

${ }^{2} \mathrm{~L}$

Figure 2.17 Ligand systems shown to bind dioxygen in an $\mathbf{O}$ fashion that display similar catalytic activity as Tyrosinase. ${ }^{[206-}$ 208]

The ${ }^{\mathbf{T}} \mathbf{P}$ binding mode which has been considered as an initial dioxygen binding motif for Type 3 copper proteins, has recently reported monophenolic oxygenation. ${ }^{[89,209,210]}$ Garcia-Bosch et al. demonstrated a ${ }^{\mathbf{T}} \mathbf{P}$ complex with the $\mathrm{m}-\mathrm{Xyl}{ }^{\mathrm{N} 3 \mathrm{~N} 4}$ ligand system, capable of hydroxylating external phenols. ${ }^{[211]}$ The oxygenated species was characterized by UV-vis and rR spectroscopy along with DFT calculations. 39\% conversion of the substrate was observed after workup, and a Hammett plot indicated an electrophilic attack on the phenolic substrate similar to that observed in ${ }^{\mathbf{S}} \mathbf{P}$ synthetic analogues and the biological system. However, further investigations on this system proved the active species to be an $\mathbf{O}$ intermediate which was shown to be in equilibrium with the ${ }^{\mathbf{T}} \mathbf{P}$ form. ${ }^{[212]}$ 


\subsection{Bis(oxazoline) ligands in Copper chemistry}

Though BOX ligands have been vastly used in the field of organic synthesis and their complexes have proved to be the most resourceful class of chiral catalysts capable of promoting a large number of organic reactions, their application as suitable scaffolds for small molecule activation with appropriate metals is poorly developed. ${ }^{[53]}$

Previously, several ligands of the type ${ }^{\mathrm{R}, \mathrm{H}} \mathrm{BOX}$ with modifications in the backbone residues showed to have an influence on ligand redox non-innocence, which was unexpectedly encountered. Upon coordination with $\mathrm{CuCl}_{2}$, the ligands were oxidised, and during the course of the reaction were shown to undergo consecutive reactions such as dimerization by $\mathrm{C}-\mathrm{C}$ radical coupling and mono-oxygenation. The selectivity of these reactions were dependent on the bulkiness of the backbone substituent demonstrating that more sterically demanding ligands prevent this reaction pathway (Scheme 2.4).

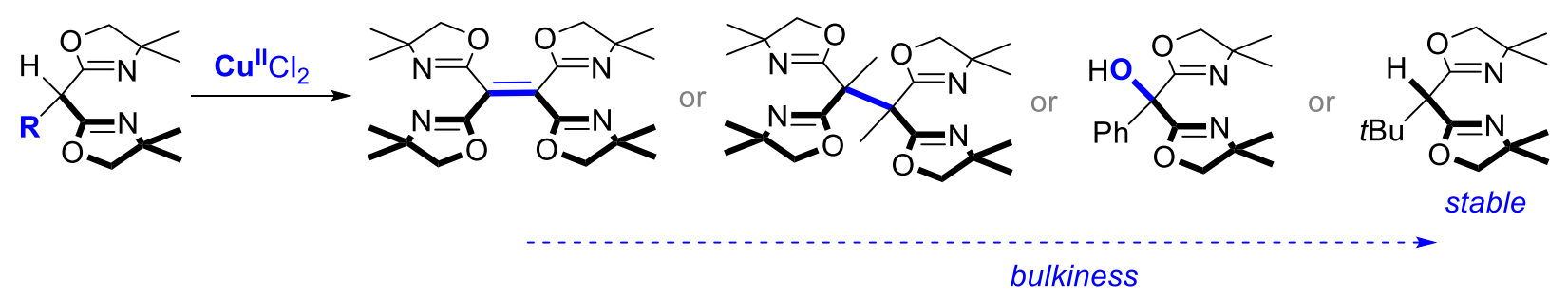

Scheme 2.4: Schematic representation of the non-innocence displayed by $\mathrm{Cu}(\mathrm{II})$ complexes of BOX ligands investigated in previous work.

In contrast to the copper(II) complexes of these ligand systems, the air sensitive copper(I) complexes demonstrated reversible dioxygen binding at low temperatures. Bulky dimethyl residues at the oxazoline rings were beneficial in sterically shielding the sensitive copper oxygen moiety. Spectroscopic characterisation of the adducts identified them as $\mu-\eta^{2}: \eta^{2}$-peroxodicopper(II) complexes (Scheme 2.5). ${ }^{\text {[71] }}$ Though the $\mu-\eta^{2}: \eta^{2}$-peroxodicopper(II) complexes were identified with these ligands, characterization of theses complexes via X-ray crystallography still remianed a challange, and none of the compelxes showed reactivity similar to those of the parent enzymes.<smiles></smiles>

$\mathrm{HL}^{1}$

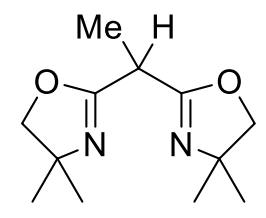

$\mathrm{HL}^{2}$

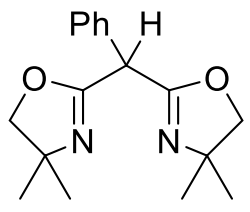

$\mathrm{HL}^{3}$<smiles>CC1(C)COC(C(C)(C)C)=N1</smiles>

$\mathrm{HL}^{4}$

Figure 2.18 Set of BOX ligands used in previous work. 
2

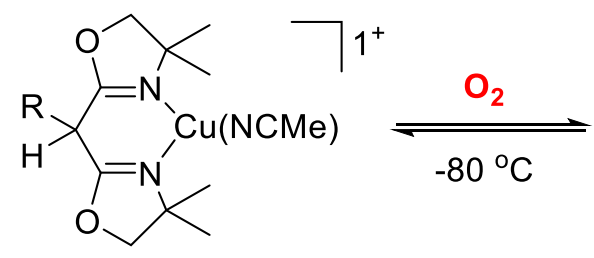

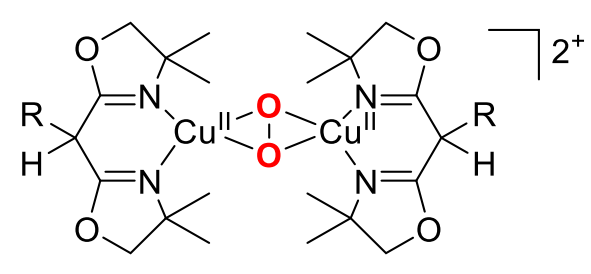

Scheme 2.5: Side on binding of dioxygen to Copper(I)BOX complexes to yield $\mu-\eta^{2}: \eta^{2}$-peroxodicopper(II) complexes.

[71]

As was mentioned earlier in Chapter 1, BOX ligands are known to exist in their tautomeric forms ${ }^{[61,64]} \mathrm{A}$ detailed investigation of the free bis(oxazoline) ${ }^{\mathrm{Ph}, \mathrm{H}} \mathrm{BOX}$ (used in previous work) evidenced an equilibrium mixture between the diimine and iminoenamine tautomers. ${ }^{[65]}$ The latter are reminiscent of $\beta$-diketimines that are extensively used as anionic ligands after deprotonation, suggesting that ${ }^{\mathrm{R}, \mathrm{H}} \mathrm{BOXs}$ may serve as proton responsive ligands. ${ }^{[66]}$ We have now exploited this concept in bioinspired $\mathrm{Cu} / \mathrm{O}_{2}$ chemistry which shall further be discussed in Chapters 6 and 7. 
Chapter 3

Motivation 
Dioxygen binding to copper in the active site of metalloenzymes has attracted much attention in the past few decades. These metalloenzymes stand as ideal prototypes for selective oxidation and oxygenation of $\mathrm{C}-\mathrm{H}$ bonds which is essentially relevant for viable fuel and chemical feedstock formation. Gaining an insight into how these enzymes function, and synthetically mimicking their active sites to reproduce catalytic activity is a challenge in the field of bio-inorganic chemistry.

An array of $\mathrm{Cu}_{\mathrm{x}} / \mathrm{O}_{2}$ intermediates with different dioxygen binding modes have meanwhile been uncovered, and their diagnostic spectroscopic features and distinct reactivities are reasonably well understood ${ }^{[38]}$. The copper centers in these impressive copper proteins are usually ligated to $\mathrm{N}$ donors of either histidine residues or amines, making the biological scaffold of these enzymes prone to protonation and deprotonation. Binuclear complexes with a $\mu-\eta^{2}: \eta^{2}$ peroxo dicopper(II) core, as found in the oxygenated forms of type 3 copper proteins such as Haemocyanin or Tyrosinase, are among the most prominent species. As discussed earlier, it has been shown that the ${ }^{\mathbf{S}} \mathbf{P}$ core can be transformed into the bis $\mu$-oxido dicopper(III) core (O) that lacks the $\mathrm{O}-\mathrm{O}$ bond. While the relevance of the copper(III) state has mostly been excluded for biological systems, recent studies addressing the $\mathrm{O}_{2}$ activation in bacterial particulate methane monoxygenase $(\mathrm{pMMO})^{[50]}$ have emphasized the potential importance of both the ${ }^{\mathbf{S}} \mathbf{P}$ and $\mathbf{O}$ cores in the enzyme. ${ }^{[12,13,14,15]}$ More generally, interconversions between different $\mathrm{Cu}_{2} / \mathrm{O}_{2}$ species are increasingly recognized as potential scenarios for modulating and elaborating the reactivity of these intermediates. ${ }^{[88,92][214][215]}$

The BOX ligands previously used in $\mathrm{Cu} / \mathrm{O}_{2}$ chemistry were all neutral and bidentate in nature. Dioxygen binding of their copper(I) complexes yielded their $\mu-\eta^{2}: \eta^{2}$-peroxodicopper(II) complexes. The first objective of this work was to extend the range of BOX ligand scaffolds by modifying their electronic properties and backbone substituents, and investigate the impact on their corresponding copper complexes. This study should then comprise of various techniques such as UV-vis, IR, Raman, X-ray crystallography and ESI-MS in order to gain insights into the reactive intermediates formed.

Furthermore, the evidence that certain free bis(oxazoline)s ${ }^{\mathrm{R}, \mathrm{H}} \mathrm{BOX}$ could exist as an equilibrium mixture between the diimine and iminoenamine tautomers suggested that the ${ }^{\mathrm{R}, \mathrm{H}} \mathrm{BOXs}$ may serve as proton responsive ligands. The iminoenamine form bears a close resemblance to $\beta$-diketimines that are extensively used as anionic ligands after deprotonation. The second objective therefore was to examine if this feature of the neutral ligand could be exploited, in analogy to the biological ligand scaffold being prone to protonation/deprotonation, and applied to interconversion of reactive intermediates.

The final goal would be to study the effect of these reactive intermediates on small organic molecules similar to those encountered in the biological system, and analyse the potential of these synthetic analogues in comparison to novel metalloenzymes found in nature. 


\section{Chapter 4 \\ Ligand Synthesis}




\section{Overview}

4.1 Introduction

4.2 Synthesis of neutral BOX ligands

4.3 Synthesis of monoanionic BOX ligands

[Note: Experimental procedures for this chapter are described in detail under Chapter 15, section 15.2] 


\subsection{Introduction}

Bis(oxazoline) ligands have been investigated in detail over more than the last two decades, and their application in organic synthesis has been well established. Ligands $\mathbf{H L}^{1}, \mathbf{H L}^{2}, \mathbf{H L}^{3}, \mathbf{H L}^{4}$ (Fig. 2.18), previously used in $\mathrm{Cu} / \mathrm{O}_{2}$ chemistry were all neutral and bidentate in nature. In this work, four new ligand systems $\left.\mathbf{H} \mathbf{L}^{5}, \mathbf{L}^{6}, \mathbf{L}^{2}\right]^{-}$and $\left[\mathbf{L}^{3}\right]^{-}$, with modifications in electronic properties and backbone substituents have been introduced, to extend the range of BOX ligand scaffolds and study their impact on $\mathrm{Cu} / \mathrm{O}_{2}$ chemistry.

The ligands differ with respect to being neutral-bidentate, $\left(\mathbf{H L}^{5}\right)$ and $\left(\mathbf{L}^{6}\right)$, and monoanionic-bidenatate $\left(\left[\mathbf{L}^{2}\right]^{-},\left[\mathbf{L}^{3}\right]^{-}\right)$in nature. Though $\mathbf{H L}^{1}, \mathbf{H L}^{2}$ and $\mathbf{H L}^{3}$, were previously used to generate their $\mu-\eta^{2}: \eta^{2}-$ peroxodicopper(II) complexes, they have further been used in this work to study in detail the equilibrium between their respective ${ }^{\mathbf{S}} \mathbf{P}$ and $\mathbf{O}$ isomers (this shall be discussed in detail in Chapter 7 ). A detailed study of substrate reactivity of their corresponding $\mu-\eta^{2}: \eta^{2}$-peroxodicopper(II) complexes with suitable organic molecules has also been performed (this shall be discussed in further detail in Chapter 8). Scheme 4.1 illustrates the set of BOX ligands used in the present work. Described below is the synthetic procedure of ligands used in this work.

A<smiles></smiles>

$\mathrm{HL}^{1}$

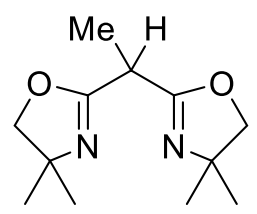

$\mathrm{HL}^{2}$<smiles>CC1(C)COC(C(C2=NC(C)(C)CO2)c2ccccc2)=N1</smiles>

$\mathrm{HL}^{3}$

B

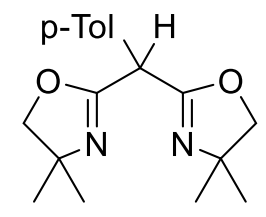

$\mathrm{HL}^{5}$

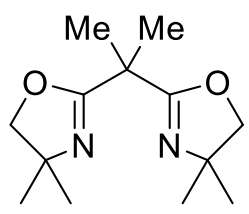

$\mathrm{L}^{6}$

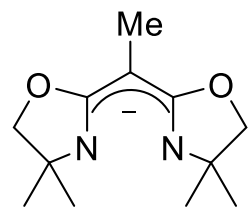

$\left[\mathrm{L}^{2}\right]^{-}$

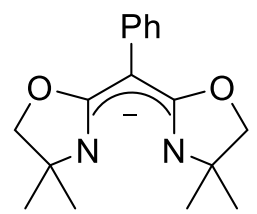

$\left[\mathrm{L}^{3}\right]^{-}$

Scheme 4.1. A: Set of ligands used in previous work as well as this work. B: New ligands that have been synthesized and used in this work.

\subsection{Synthesis of neutral BOX ligands}

Ligands $\mathbf{H L}^{1}, \mathbf{H L}^{2}, \mathbf{H L}^{3}, \mathbf{H L}^{5}$, and $\mathbf{L}^{6}$ were prepared in a three step synthetic procedure. First the bishydroxy malonamide precursors $\mathbf{X}$ were synthesized from their respective diethylmalonates, which were obtained from commercial suppliers, and an amino alcohol (2-amino-2-methyl-1-propanol). This mixture, together with a catalytic amount of sodium hydride was heated to $413 \mathrm{~K}$ for four hours, under an atmosphere of Argon. There was no solvent used during this step. The ethanol produced was removed under vaccum, and the malonamides were yielded quantitatively. The obtained malonamide could be used in the following step without further purification (Scheme 4.2). 


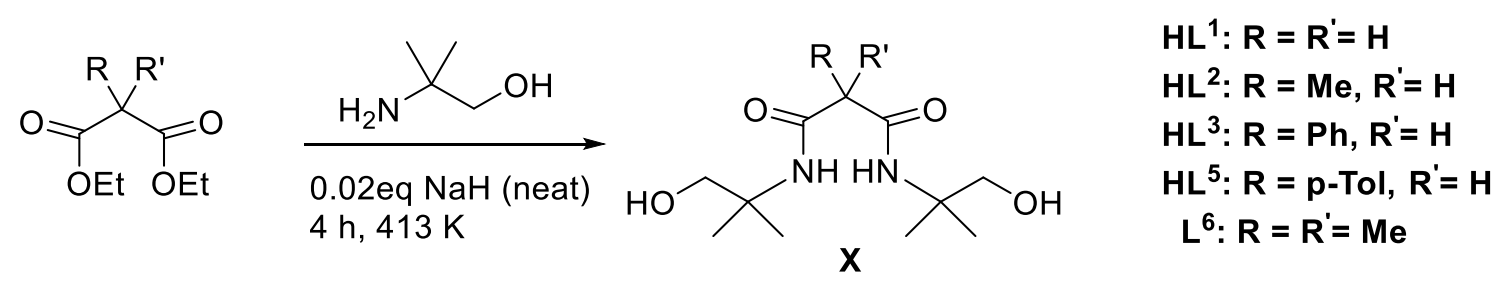

Scheme 4.2. Synthesis of the respective bishydroxy malonamides $\mathbf{X}$.

The corresponding bishydroxymalonamides were then treated with methanesulfonyl chloride $(\mathrm{MsCl})$ in the presence of triethylamine in dichloromethane to obtain their respective mesylates $\mathbf{Y}$. This was done in order to facilitate ring closure in the final step (Scheme 4.3).<smiles>[X]C(C)(CO)NC(=O)C([R])([R])C(=O)NC(C)(C)CO</smiles>

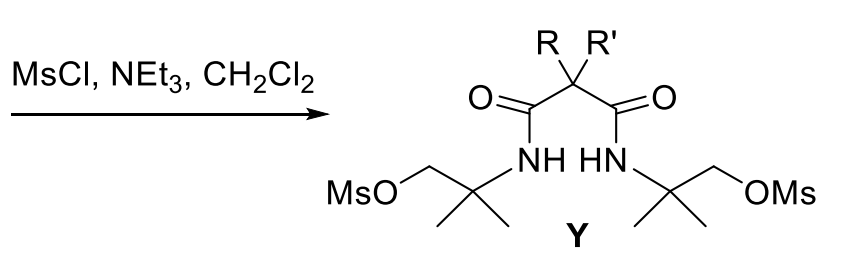

$H L^{1}: R=R^{\prime}=H$

$H L^{2}: R=M e, R^{\prime}=H$

$H L^{3}: R=P h, R^{\prime}=H$

$H L^{5}: R=p-T o l, R^{\prime}=H$

$L^{6}: R=R^{\prime}=M e$

Scheme 4.3. Generation of the mesylates $\mathbf{Y}$ from their corresponding bishydroxy malonamides $\mathbf{X}$.

For ring closure, the corresponding mesylates $\mathbf{Y}$ were treated with sodium hydroxide in a 1:1 mixture of water and methanol and refluxed for two hours. Ligands $\mathbf{H} \mathbf{L}^{1}, \mathbf{H L}^{2}$, and $\mathbf{L}^{6}$ were obtained as colorless oils after purification by bulb-to-bulb kugelhohr vacuum distillation. Ligands $\mathbf{H L}^{3}$ and $\mathbf{H L}^{\mathbf{5}}$ precipitated in the final synthesis step and were obtained as white and pale yellow powders, respectively. All ligands were characterized by ${ }^{1} \mathrm{H} \mathrm{NMR},{ }^{13} \mathrm{C}$ NMR spectroscopy, and ESI-MS.
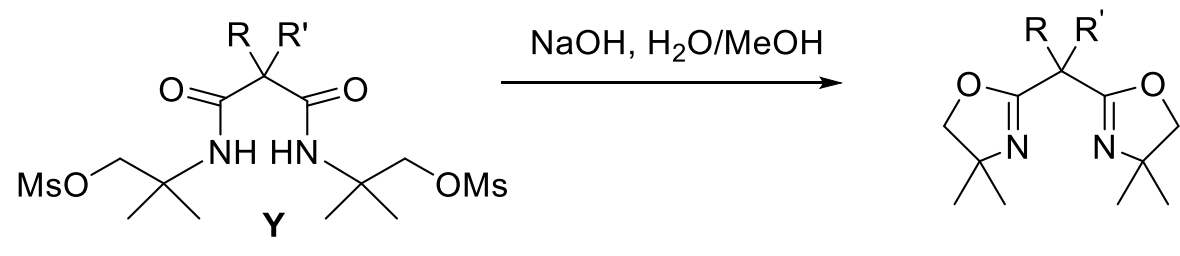

Scheme 4.4. Synthetic route for ring closure to obtain the desired BOX ligand. 


\subsection{Synthesis of monoanionic BOX ligands}

The monoanionic-bis(oxazolinate) ligands $\left[\mathbf{L}^{2}\right]^{-}$and $\left[\mathbf{L}^{3}\right]^{-}$were prepared as their lithium salts in a one step synthetic procedure. The corresponding protonated ligands $\mathbf{H L}^{2}, \mathbf{H L}^{3}$ were taken up in hexane and cooled to $233 \mathrm{~K}$, under an atmosphere of argon. 1 equivalent of $1.6 \mathrm{M} n$-BuLi in hexane was added dropwise, and immediately a colorless solid precipitated. The pure ligands were obtained as white solids by washing with cold pentane and drying under vacuum.
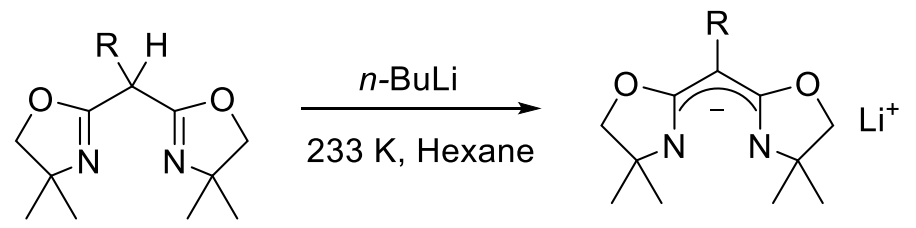

$\left[\mathrm{L}^{2}\right]^{-}: \mathbf{R}=\mathbf{M e}$

$\left[L^{3}\right]^{-}: \mathbf{R}=\mathbf{P h}$

Scheme 4.5. Synthetic route for generating monoanionic BOX ligands. 
[Note: This page has intentionally been left blank] 


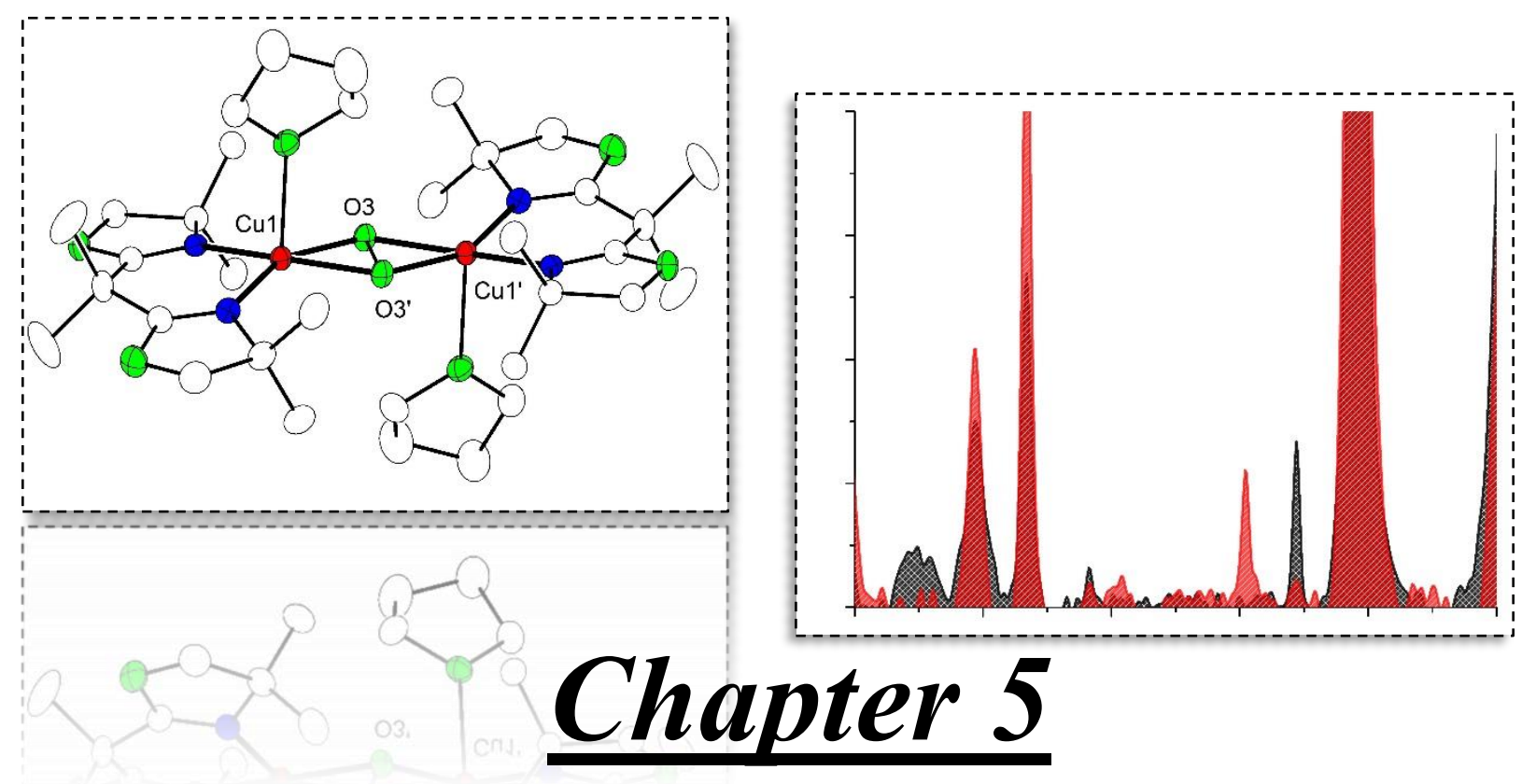

Dicopper(II) Side on Peroxo Complexes

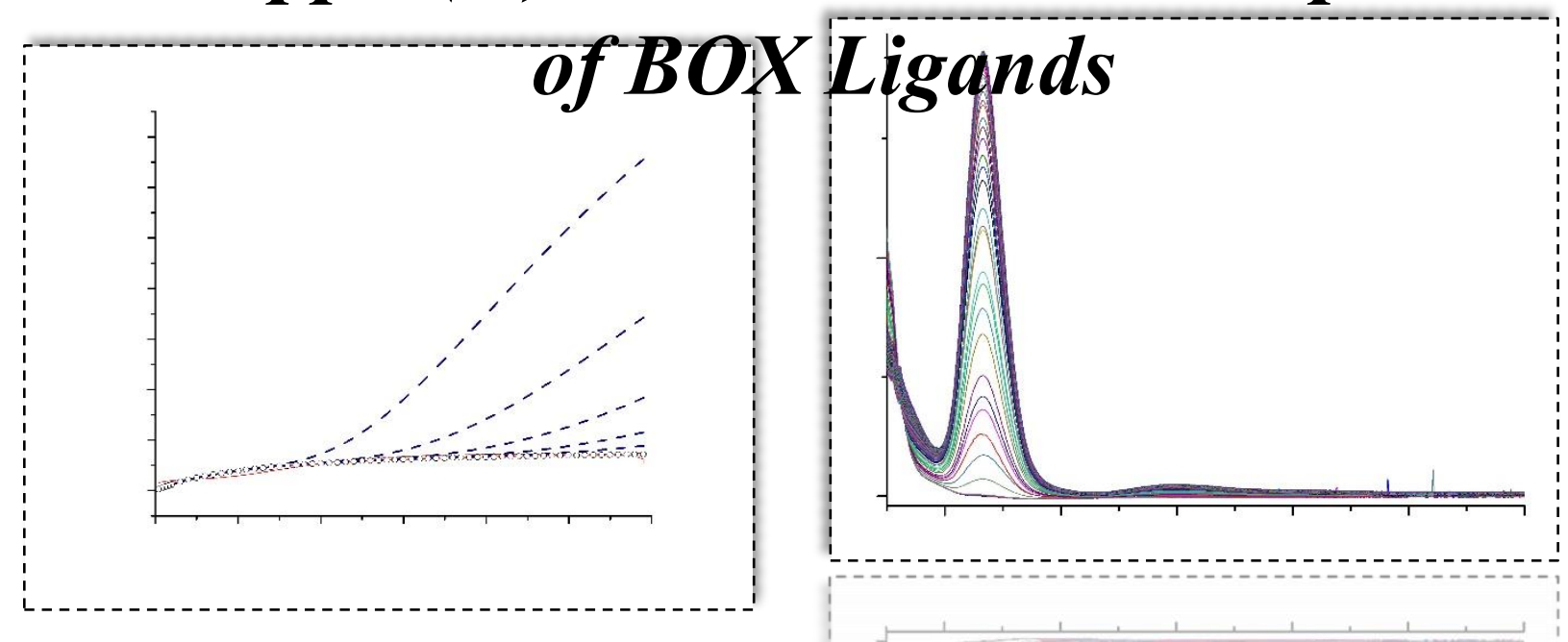




\section{Overview}

5.1 Introduction

5.2 Synthesis and characterization of $\mathrm{Cu}(\mathrm{I}) \mathrm{BOX}$ complexes with proton responsive ligands $\mathbf{H L}^{1}, \mathbf{H L}^{2}$ and $\mathbf{H L}^{\mathbf{5}}$

5.2.1 Structural elucidation of $\mathbf{1}$ and $\mathbf{3}$

5.3 Dioxygen reactivity of $\mathbf{1}, \mathbf{2}$ and $\mathbf{3}$ at low temperatures to yield $\mathrm{Cu}_{2}\left(\mu-\eta^{2}: \eta^{2}-\mathrm{O}_{2}\right)$ intermediates 5.3.1 Properties in solution

5.3.2 Structural elucidation of a $\mathrm{Cu}_{2}\left(\mu-\eta^{2}: \eta^{2}-\mathrm{O}_{2}\right)$ dicopper(II) complex (6) with an unusually long $\mathrm{O}-\mathrm{O}$ bond

5.4 Synthesis and characterization of a $\mathrm{Cu}(\mathrm{I}) \mathrm{BOX}$ complex with a non-proton responsive ligand $\mathbf{L}^{\mathbf{6}}$

5.4.1 Structural characterization of 4

5.5 Dioxygen activation of 4 at low temperatures to yield a $\mathrm{Cu}_{2}\left(\mu-\eta^{2}: \eta^{2}-\mathrm{O}_{2}\right)$ intermediate with complete characterization $(\mathbf{8})$

5.5.1 Properties in solution

5.5.2 Resonance Raman Spectroscopy

5.5.3 Properties in solid state

5.5.4 Structural elucidation of 8

5.5.5 Magnetic measurements

5.6 Comparison of $\mathbf{6}$ and $\mathbf{8}$ with other structurally characterized $\mathrm{Cu}_{2}\left(\mu-\eta^{2}: \eta^{2}-\mathrm{O}_{2}\right)$ complexes

5.7 Kinetic and Thermodynamic analysis of $\mathrm{Cu} / \mathrm{O}_{2}$ complexes with $\mathbf{H L}^{\mathbf{5}}$ and $\mathbf{L}^{\mathbf{6}}$

5.7.1 Determining the rate constant $\left(k_{o b s}\right)$ for dioxygen binding in $\mathbf{3}$ and $\mathbf{4}$

5.7.2 Determining the activation parameters for the formation of $\mathbf{7}$ and $\mathbf{8}$

\subsection{Conclusion}

[Note: Experimental procedures for this chapter are described in detail under Chapter 15, section $15.3]$ 


\subsection{Introduction}

The synthesis of $\mathrm{Cu}(\mathrm{I})$ complexes with BOX ligands and their reactivity towards dioxygen has been investigated in an earlier project. ${ }^{[71]}$ Reactions with suitable $\mathrm{Cu}(\mathrm{I})$ salts were shown to generate air sensitive mononuclear $\mathrm{Cu}(\mathrm{I}) \mathrm{BOX}$ complexes which demonstrated reversible dioxygen binding at low temperatures. The $\mathrm{Cu} / \mathrm{O}_{2}$ species were identified as $\mu-\eta^{2}: \eta^{2}$-peroxodicopper(II) complexes, where the $\mathrm{Cu}(\mathrm{I})$ centers were oxidized to $\mathrm{Cu}(\mathrm{II})$, and thermodynamic and kinetic parameters for $\mathrm{O}_{2}$ binding were determined. ${ }^{[71]}$ The ligand systems employed previously were all proton responsive in nature, and while their ${ }^{\mathbf{S}} \mathbf{P}$ complexes were characterized in the solid state and in solution, structural elucidation still remained a challenge.

The following section describes the $\mathrm{Cu}(\mathrm{I})$ complexes of $\mathbf{H} \mathbf{L}^{\mathbf{1}}, \mathbf{H} \mathbf{L}^{2}, \mathbf{H} \mathbf{L}^{\mathbf{5}}$ and $\mathbf{L}^{6}$ (Fig. 5.1) and focuses particularly on the dioxygen reactivity with $\mathbf{H L}^{2}, \mathbf{H L}^{\mathbf{5}}$ and $\mathbf{L}^{6}$. The two new ligand systems, $\mathbf{H L}^{\mathbf{5}}$ and $\mathbf{L}^{6}$ introduced herein, have been modified to study electronic effects on $\mathrm{Cu} / \mathrm{O}_{2}$ complexes generated by BOX ligands. In contrast to the previously used $\mathbf{H L}^{3}, \mathbf{H L}^{5}$ was designed to have an additional methyl group on the phenyl ring in order to investigate inductive effects. On the other hand, $\mathbf{L}^{\mathbf{6}}$, unlike the other ligand systems was designed to be non-proton responsive in nature. As mentioned earlier, the ligand systems previously used were prone to deprotonation by virtue of a relatively acidic proton within the ligand backbone. $\mathbf{L}^{6}$ however, hosts two methyl groups within its backbone with no protons available for deprotonation, making this system unique in comparison to the rest.

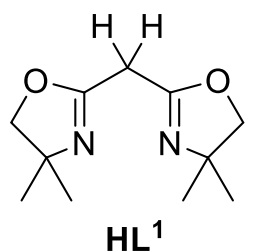

$\mathrm{HL}^{1}$

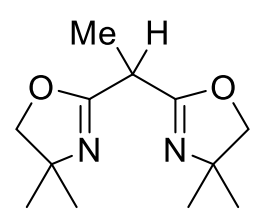

$\mathrm{HL}^{2}$

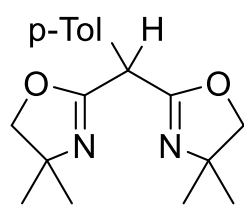

$\mathrm{HL}^{5}$

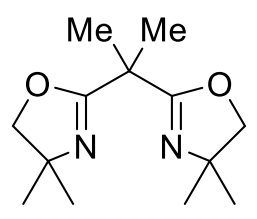

$\mathrm{L}^{6}$

Figure 5.1 Ligand systems employed in the present study of copper dioxygen chemistry. $\mathbf{H L}^{\mathbf{5}}$ and $\mathbf{L}^{\mathbf{6}}$ are the new systems introduced.

\subsection{Synthesis and characterization of $\mathrm{Cu}(\mathrm{I}) \mathrm{BOX}$ complexes with proton responsive ligands $\mathrm{HL}^{1}$, $\mathrm{HL}^{2}$ and $\mathrm{HL}^{5}$}

The general scheme employed for the generation of $\mathrm{Cu}(\mathrm{I})$ complexes with proton responsive ligands is depicted in Scheme 5.1. Ligands $\mathbf{H L}^{1}$ and $\mathbf{H L}^{5}$ were individually treated with 1.1 equivalents of tetrakis(acetonitrile)copper(I) hexaflourophosphate in THF to yield colorless solutions of their $\mathrm{Cu}(\mathrm{I})$ complexes respectively ( $\mathbf{1}$ and $\mathbf{3}$ ). In the case of $\mathbf{H L}^{\mathbf{2}}$, tetrakis(acetonitrile) copper(I) perchlorate was used as the $\mathrm{Cu}(\mathrm{I})$ source to generate the $\mathrm{Cu}(\mathrm{I})$ complex $\mathbf{2}$. ESI-MS analysis of the colorless solution of $\mathbf{1}$ in THF (Fig 5.2 left) revealed one major peak, namely $\left[\mathbf{H L}^{1} \mathrm{Cu}(\mathrm{I}) \mathrm{MeCN}\right]^{+}(\mathrm{m} / z=314)$. The two other peaks could be assigned to $\left[\mathbf{H L}^{1} \mathrm{Cu}(\mathrm{I})\right]^{+}(\mathrm{m} / z=273)$ and $\left[\left(\mathbf{H L}^{1}\right)_{2} \mathrm{Cu}(\mathrm{I})\right]^{+}(\mathrm{m} / z=483.1)$. ESI-MS analysis of the colorless solution of 2 (Fig 5.2 right) revealed a major peak which corresponded to the monomer $\left[\mathbf{H L}^{2} \mathrm{Cu}(\mathrm{I}) \mathrm{MeCN}\right]^{+}$ $(m / z=328.1)$ and a second peak corresponding to the dimer $\left[\left(\mathbf{H L}^{2}\right)_{2} \mathrm{Cu}_{2} \mathbf{C N}\right]^{+}(\mathrm{m} / z=600.2)$. 

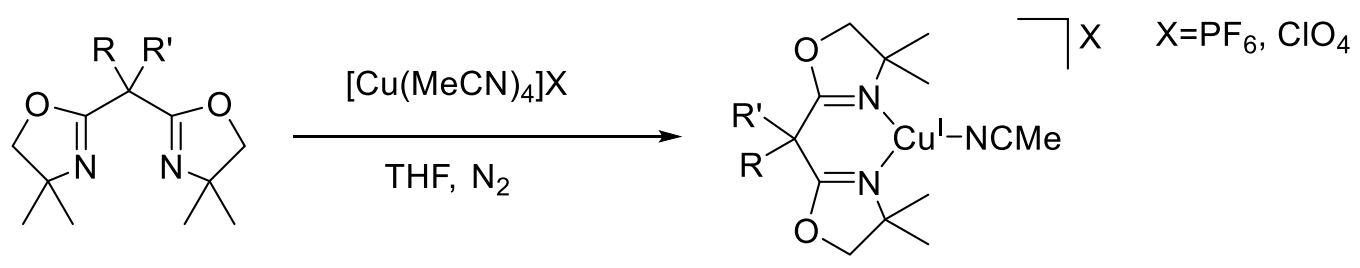

$$
\begin{aligned}
& R=R^{\prime}=H=H L^{1} \\
& R=H, R^{\prime}=M e=H L^{2} \\
& R=H, R^{\prime}=p-T o l=H L^{5} \\
& R=R^{\prime}=M e=L^{6}
\end{aligned}
$$

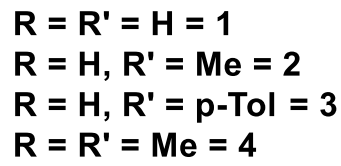

Scheme 5.1 General scheme for the synthesis of $\mathrm{Cu}(\mathrm{I}) \mathrm{BOX}$ complexes.
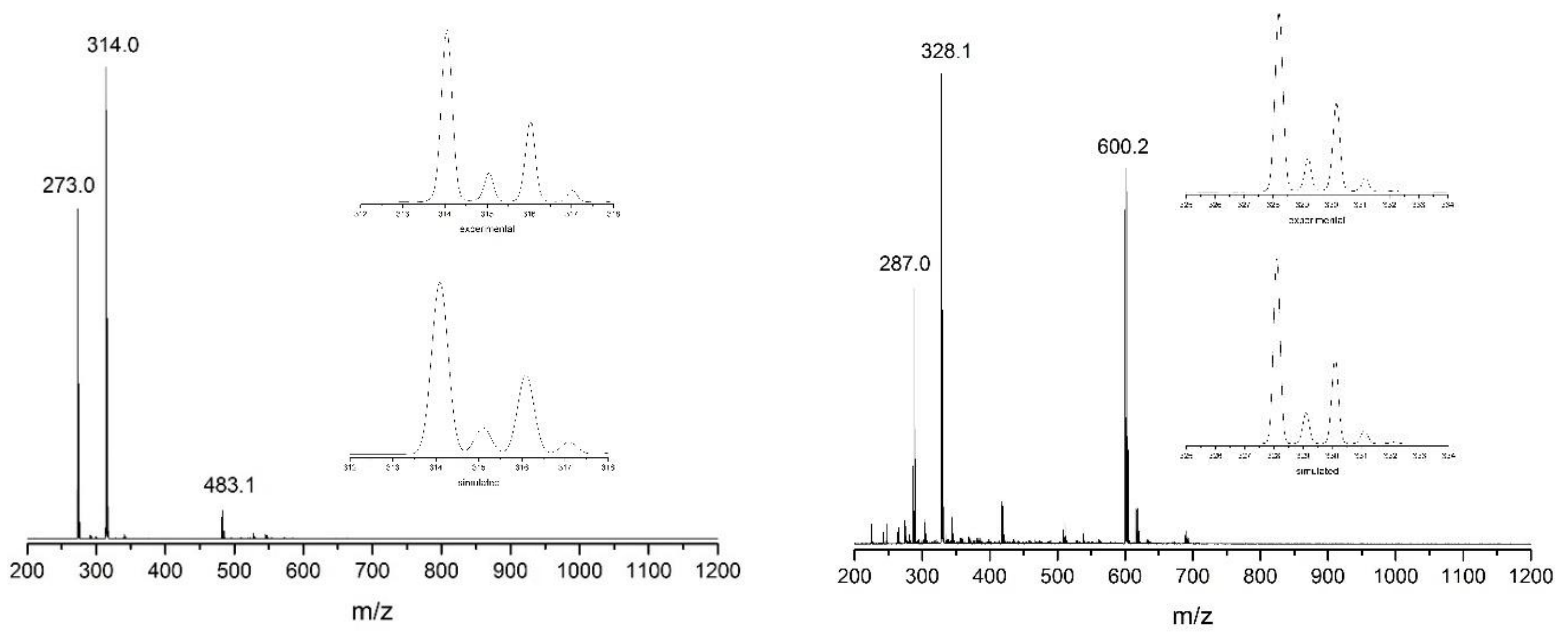

Figure 5.2 Left: ESI-MS of $\mathbf{1}$ in THF. The two major peaks are assigned to the copper(I) complex of $\mathbf{H L}^{\mathbf{1}}$, differing by an acetonitrile molecule. The inset shows an enlargement of the peak at $m / z=314$, together with a simulation of the isotopic pattern corresponding to $\left[\mathbf{H L}^{\mathbf{1}} \mathrm{Cu}(\mathrm{I}) \mathrm{MeCN}\right]^{+}$. Right: ESI-MS spectrum of $\mathbf{2}$ in THF. The two major peaks could be assigned to the monomer and dimer of the copper(I) complex of $\mathbf{H L}^{2}$. The inset shows an enlargement of the peak at $\mathrm{m} / z=328.1$ together with a simulation of the isotopic pattern corresponding to $\left[\mathbf{H L}^{2} \mathrm{Cu}(\mathrm{I}) \mathrm{MeCN}\right]^{+}$.

ESI-MS analysis of the crude copper(I) complex in THF with $\mathbf{H L}^{5}$ (Fig 5.3) revealed one major peak consistent with the mass of $\left[\mathrm{HL}^{5} \mathrm{Cu}(\mathrm{I}) \mathrm{MeCN}\right]^{+}(\mathrm{m} / z=404.1)$. The two other peaks could be assigned to $\left[\mathbf{H L}^{5} \mathrm{Cu}(\mathrm{I})\right]^{+}(\mathrm{m} / \mathrm{z}=363.1)$ and $\left[\left(\mathbf{H L}^{5}\right)_{2} \mathrm{Cu}(\mathrm{I})\right]^{+}(\mathrm{m} / \mathrm{z}=663.2)$. 


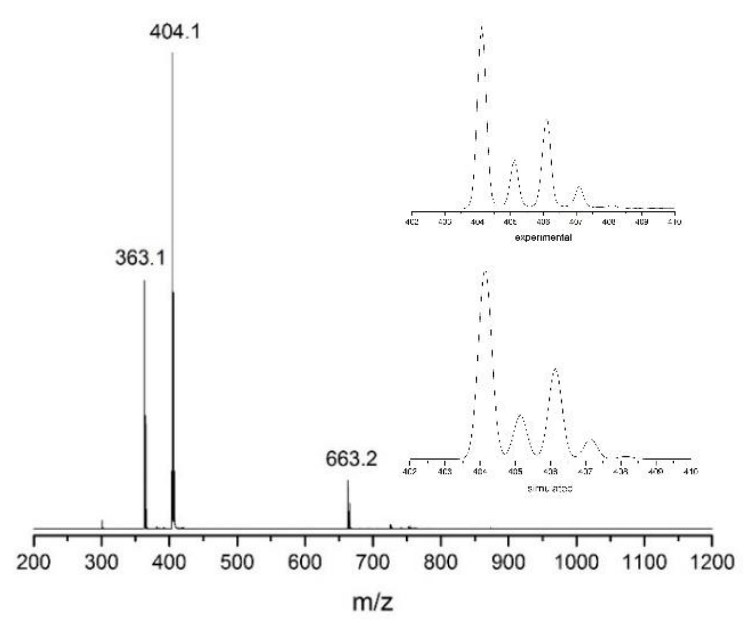

Figure 5.3 ESI-MS of $\mathbf{3}$ in THF. The two major peaks could be assigned to the copper(I) complex of $\mathbf{H L}^{\mathbf{5}}$, differing by an acetonitrile molecule. The inset shows an enlargement of the peak at $\mathrm{m} / \mathrm{z}=404.1$ with a simulation of the isotopic pattern, corresponding to $\left[\mathbf{H L}^{5} \mathrm{Cu}(\mathrm{I}) \mathrm{MeCN}\right]^{+}$.

The diamagnetic nature of the $\mathrm{Cu}(\mathrm{I})$ complexes enabled ${ }^{1} \mathrm{H}-\mathrm{NMR}$ spectroscopy to be used as a means of characterization. The analysis of $\mathbf{1}$ in a solution of $\mathrm{CD}_{3} \mathrm{CN}$ (Fig 5.4) is in agreement with the solid state structure (Fig 5.6 left) and indicates a high $C_{2 v}$ symmetry due to the $\mathrm{CH}_{2}$ moiety present in the ligand backbone. Complex 3 was also characterized by ${ }^{1} \mathrm{H}-\mathrm{NMR}$ spectroscopy (Fig 5.5).
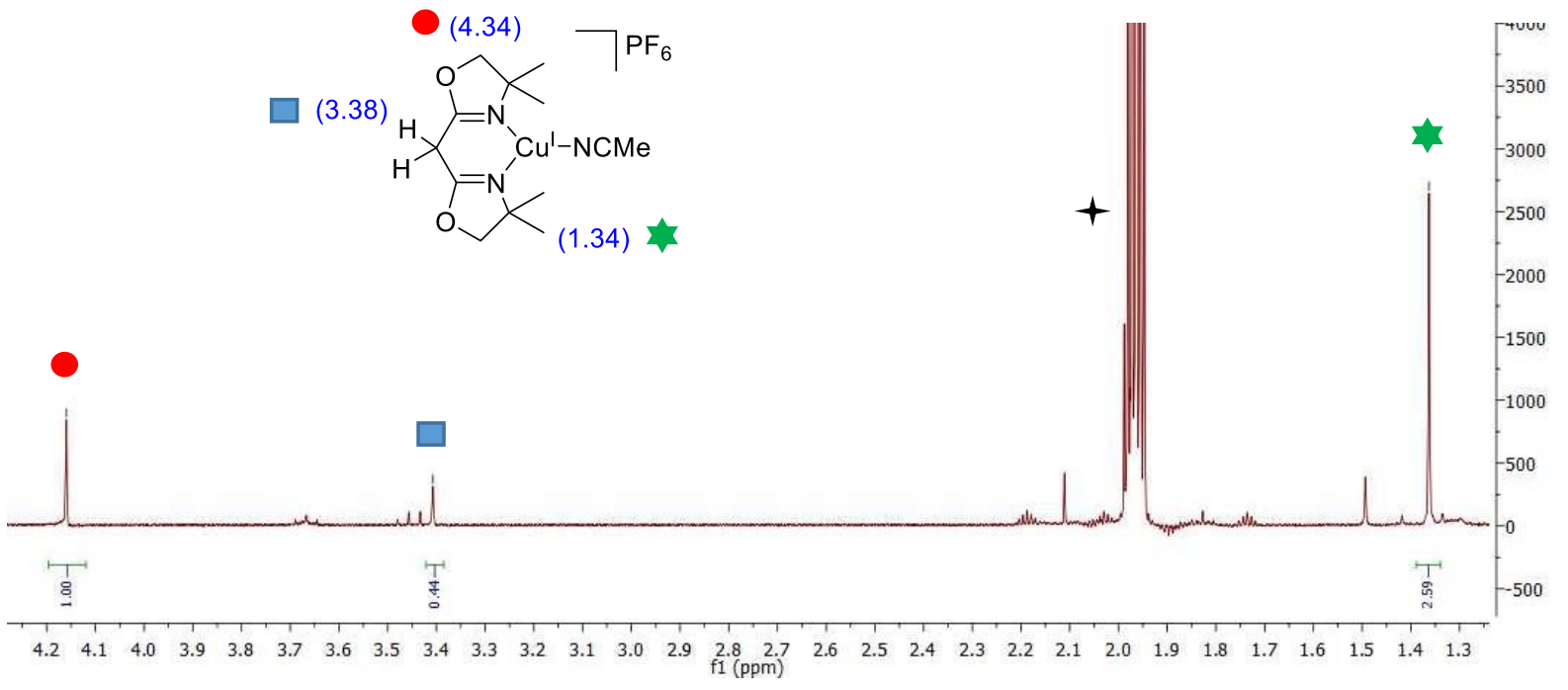

Figure 5.4: ${ }^{1} \mathrm{H}$ NMR spectrum of 1 in $\mathrm{CD}_{3} \mathrm{CN}$ at $293 \mathrm{~K}$. Solvent peaks marked with ${ }^{+}$. 


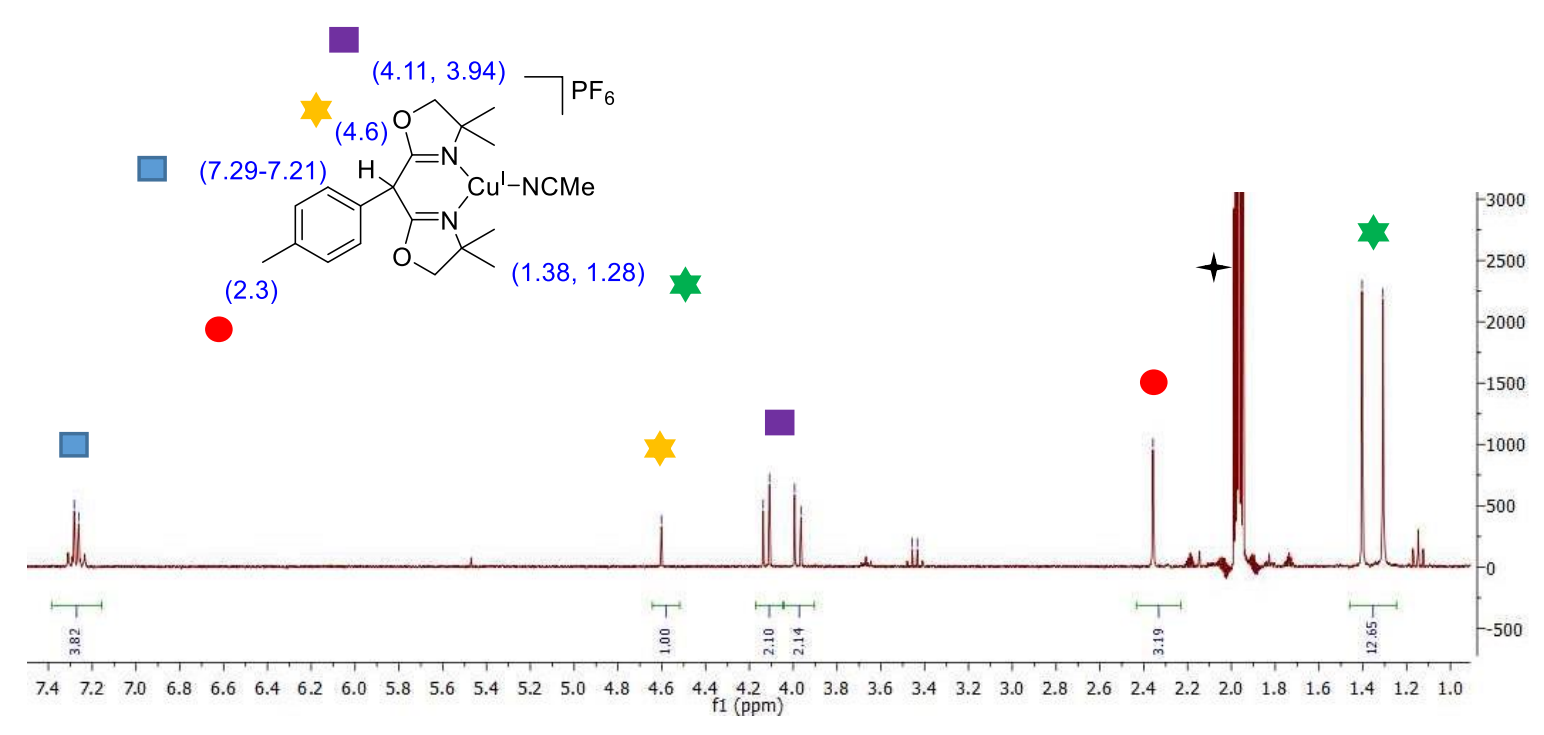

Figure 5.5: ${ }^{1} \mathrm{H}$ NMR spectrum of 3 in $\mathrm{CD}_{3} \mathrm{CN}$ at $293 \mathrm{~K}$. Solvent peaks marked with + .

\subsubsection{Structural elucidation of 1 and 3}

The $\mathrm{Cu}(\mathrm{I})$ complexes $\mathbf{1}$ and $\mathbf{3}$ were also characterized via X-ray crystallography. Addition of $\mathrm{Et}_{2} \mathrm{O}$ to a solution of 1 resulted in precipitation of the crude complex as a white powder. Recrystallization from THF and $\mathrm{Et}_{2} \mathrm{O}$ under inert conditions yielded single crystals of $\left[\mathbf{H L}^{1} \mathrm{Cu}(\mathrm{I}) \mathrm{MeCN} \mathrm{PF}_{6}(\mathbf{1})\right.$ (Fig 5.6 left) suitable for X-ray diffraction in 50\% yield. Slow diffusion of $\mathrm{Et}_{2} \mathrm{O}$ into a 1:1 (THF: $\left.\mathrm{CHCl}_{3}\right)$ solution of 3 afforded colorless single crystals of $\left[\mathrm{HL}^{5} \mathrm{Cu}(\mathrm{I}) \mathrm{MeCN}\right] \mathrm{PF}_{6}(3)$ suitable for X-ray diffraction in $60 \%$ yield (Fig 5.6 right). 1 and 3 crystallize in the monoclinic space groups $P 2_{1}$ and $P 2_{1 / c}$ respectively. The copper ions are coordinated in a trigonal planar fashion to the bidentate $\mathbf{H L}^{\mathbf{1}}$ and $\mathbf{H} \mathbf{L}^{5}$ ligands with an exogenous acetonitrile solvent molecule. The boat shaped coordination taken up by these $\mathrm{Cu}(\mathrm{I})$ complexes places the oxazoline rings with the copper and acetonitrile moiety on a single plane, while the two methyl groups of the $\mathrm{CMe}_{2}$ groups lie above and below the plane. The ideal trigonal planar geometry of $120^{\circ}$ is slightly deviated in both cases due to the small bite angle of $\mathrm{N} 2-\mathrm{Cu}-\mathrm{N} 1,94.9^{\circ}$ in $\mathbf{1}$ and $94.4^{\circ}$ in $\mathbf{3}$. In comparison to the $\mathrm{Cu}(\mathrm{I})$ complex of $\mathbf{H L}^{3}$ which was crystallized in a previous study ${ }^{[71]}$ the bite angle formed by N2-Cu-N1 in $\mathbf{3}$ as well as the angle between the residue $\mathrm{R}$ and the $\mathrm{N}_{3} \mathrm{Cu}$ coordination plane is much larger $\left(94.4^{\circ}>93.4^{\circ}\right.$ and $109^{\circ}>80^{\circ}$, respectively ). 

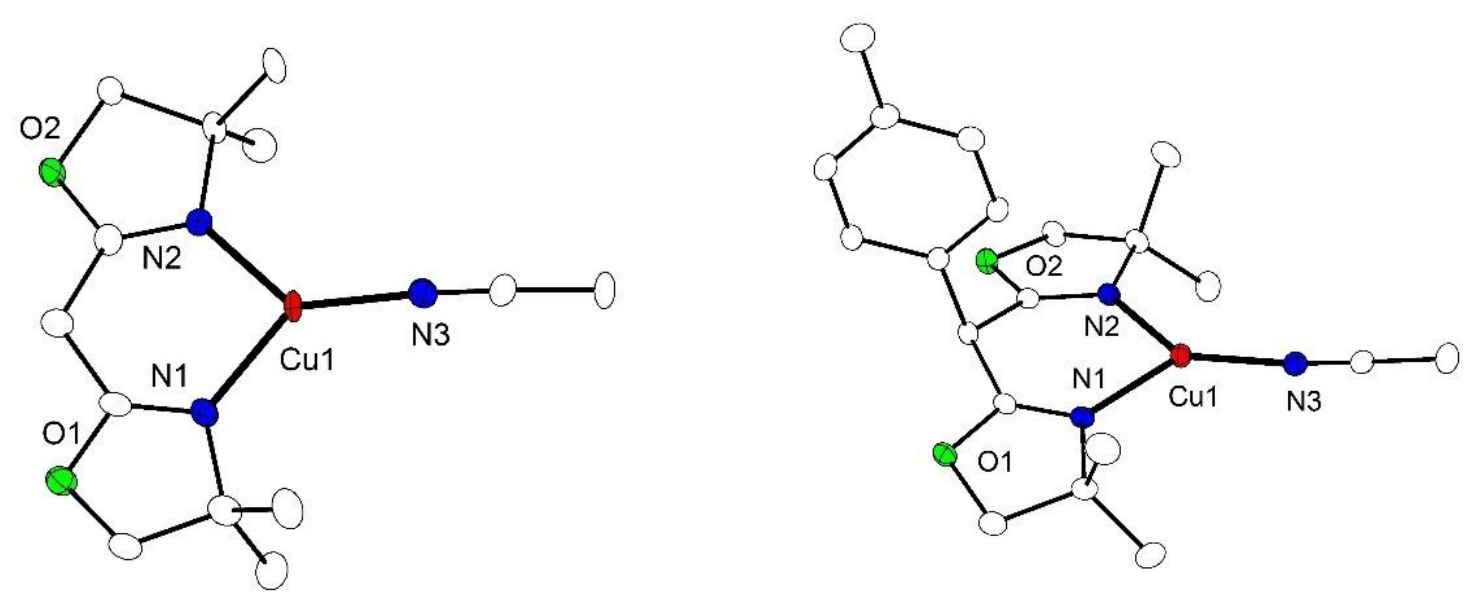

Figure 5.6 Left: Molecular structure of 1 in the crystal together with a partial labelling scheme. Thermal displacement ellipsoids are drawn at $30 \%$. Anions and hydrogen atoms are omitted for clarity. Selected bond lengths $[\AA]$ and angles $\left[^{\circ}\right]: \mathrm{Cu} 1-$ N1 1.9700(17), Cu1-N2 1.9869(18), Cu1-N3 1.8787(19); N3-Cu1-N1 131.654(8), N3-Cu1-N2 133.337(8), N1-Cu1-N2 94.978(7). Right: Molecular structure of 3 in the crystal together with a partial labelling scheme. Thermal displacement ellipsoids are drawn at $30 \%$. Anions and hydrogen atoms are omitted for clarity. Selected bond lengths $[\AA]$ and angles $\left[^{\circ}\right]: \mathrm{Cu} 1-$ N1 2.0037(17), Cu1-N2 1.9745(18), Cu1-N3 1.8664(19); N3-Cu1-N1 126.018(8), N3-Cu1-N2 139.469(8), N1-Cu1-N2 94.499(7).

\subsection{Dioxygen reactivity of 1,2 and 3 at low temperatures to yield $\mathrm{Cu}_{2}\left(\mu-\eta^{2}: \eta^{2}-\mathrm{O}_{2}\right)$ intermediates}

Dioxygenation of $\mathrm{Cu}(\mathrm{I}) \mathrm{BOX}$ complexes $\mathbf{1}, \mathbf{2}$ and $\mathbf{3}$ at $193 \mathrm{~K}$ gave rise to deep purple colored solutions of their $\mathrm{Cu}_{2}\left(\mu-\eta^{2}: \eta^{2}-\mathrm{O}_{2}\right)$ complexes according to Scheme 5.2.

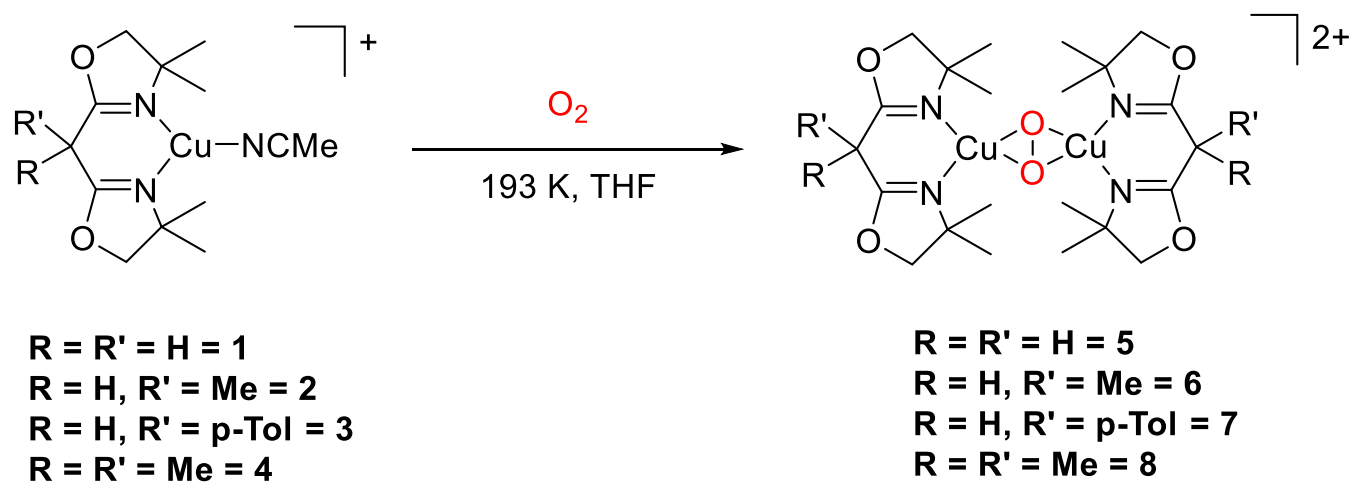

Scheme 5.2 Activation of dioxygen with $\mathrm{Cu}(\mathrm{I}) \mathrm{BOX}$ complexes in solution at $193 \mathrm{~K}$ to yield $\mathbf{S} \mathbf{P}$ complexes 5,6,7, and 8. 


\subsubsection{Properties in solution}

Purple colored solutions of the formation of $\left[\left(\mathbf{H L}^{\mathbf{1}}\right)_{2} \mathrm{Cu}_{2}\left(\mu-\eta^{2}: \eta^{2}-\mathrm{O}_{2}\right)\right](\mathbf{5})$ (Fig 5.7 left), $\left[\left(\mathbf{H L}^{\mathbf{5}}\right)_{2} \mathrm{Cu}_{2}\left(\mu-\eta^{2}: \eta^{2}-\right.\right.$ $\mathrm{O}_{2}$ ) (7) (Fig 5.7 right) and $\left[\left(\mathbf{H L}^{2}\right)_{2} \mathrm{Cu}_{2}\left(\mu-\eta^{2}: \eta^{2}-\mathrm{O}_{2}\right)\right]$ (6) (Fig 5.8) were monitored using UV-vis spectroscopy, where two intense optical features at $\sim 350 \mathrm{~nm}$ and $\sim 500 \mathrm{~nm}$ were observed, which are typical for a dicopper $\mu-\eta^{2}: \eta^{2}$-peroxo species and comparable to the spectroscopic features of oxyhemocyanin and oxytyrosinase. ${ }^{[216]}$ The distinctive spectrocopic features for ${ }^{\mathbf{S}} \mathbf{P}$ complexes are in direct correlation with the side-on peroxide binding mode, ${ }^{[93]}$ and theoretical studies conducted by Solomon et al. have made them well understood (Refer to sec. 2.3.2 of introduction, fig. 2.6). The highest occoupied peroxide orbitals are composed of degenerate $\pi^{*}$ levels. Approaching the metal center, these levels split up into $\pi_{\sigma}{ }^{*}$ and $\pi_{\mathrm{v}}{ }^{*}$ orbitals. The $\pi_{\sigma}{ }^{*}$ orbital lies in plane with the $\mathrm{Cu}_{2} \mathrm{O}_{2}$ core, which results in a $\sigma$ overlap with the half filled $\mathrm{Cu}(\mathrm{II}) \mathrm{d}_{\mathrm{xy}}$ orbitals. This leads to an intense LMCT band obsereved around $300-350 \mathrm{~nm}$, assigned as $\mathrm{O}_{2}{ }^{2-} \pi_{\sigma}{ }^{*}$ to $\mathrm{Cu}(\mathrm{II}) \mathrm{d}_{\mathrm{xy}}$ orbitals; $330 \mathrm{~nm}\left(\varepsilon=7422 \mathrm{M}^{-1} \mathrm{~cm}^{-1}\right)$ for $5,330 \mathrm{~nm}\left(\varepsilon=19113 \mathrm{M}^{-1} \mathrm{~cm}^{-1}\right)$ for 6 and $333 \mathrm{~nm}(\varepsilon=$ $4403 \mathrm{M}^{-1} \mathrm{~cm}^{-1}$ ) for 7 . The second $\pi_{\mathrm{v}}{ }^{*}$ orbital however, lies perpendicular to the $\mathrm{Cu}_{2} \mathrm{O}_{2}$ plane allowing only small $\pi$ bonding interaction with the copper centers. This leads to the second LMCT band observed around $500 \mathrm{~nm}$, assigned to transitions between $\mathrm{O}_{2}{ }^{2-} \pi_{\mathrm{v}}{ }^{*}$ to $\mathrm{Cu}(\mathrm{II}) \mathrm{d}_{\mathrm{xy}}$ orbitals; $501 \mathrm{~nm}\left(\varepsilon=454 \mathrm{M}^{-1} \mathrm{~cm}^{-1}\right)$ for 5, 500 $\mathrm{nm}\left(\varepsilon=1530 \mathrm{M}^{-1} \mathrm{~cm}^{-1}\right)$ for 6 and $504 \mathrm{~nm}\left(\varepsilon=357 \mathrm{M}^{-1} \mathrm{~cm}^{-1}\right)$ for 7 . The intensity of the two bands are ususally in a ratio of 20:1 with the band around $500 \mathrm{~nm}$ appearing as a shoulder in most cases.
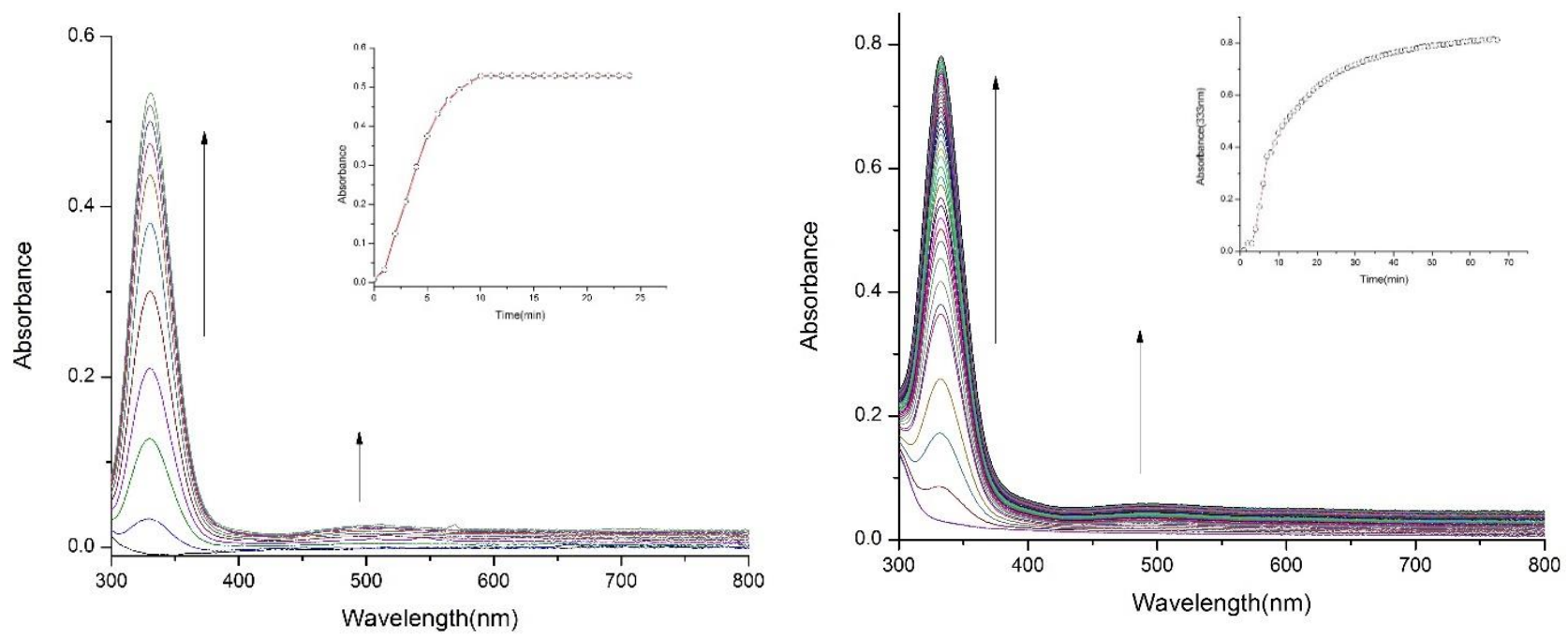

Figure 5.7 Left: Formation of $\left[\left(\mathbf{H L}^{\mathbf{1}}\right)_{2} \mathrm{Cu}_{2}\left(\mu-\eta^{2}: \eta^{2}-\mathrm{O}_{2}\right)\right](\mathbf{5})$ with bands at $330 \mathrm{~nm}$ and $501 \mathrm{~nm}$ in THF at $193 \mathrm{~K}$ monitored by UV/Vis spectroscopy. The inset displays formation of the band at $330 \mathrm{~nm}$ with time. Right: Formation of $\left[\left(\mathbf{H L}^{5}\right)_{2} \mathrm{Cu}_{2}\left(\mu-\eta^{2}: \eta^{2}-\mathrm{O}_{2}\right)\right](7)$ with bands at $333 \mathrm{~nm}$ and $504 \mathrm{~nm}$ in THF at $193 \mathrm{~K}$ monitored by UV/Vis spectroscopy. The inset displays formation of the band at 333 nm with time. 


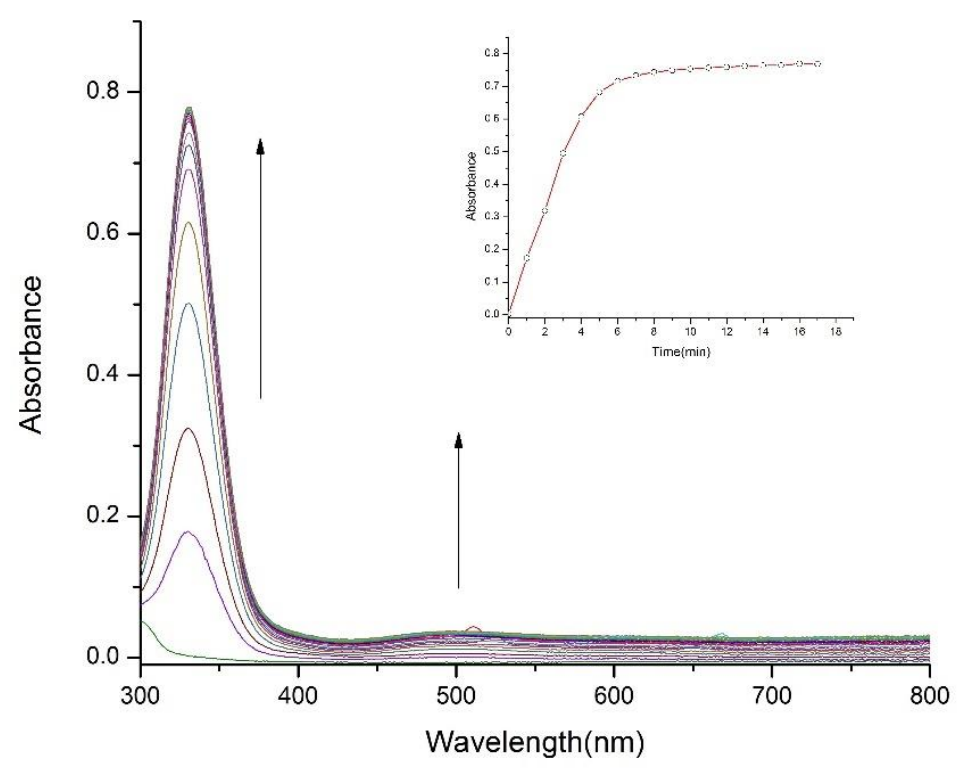

Figure 5.8: Formation of $\left[\left(\mathbf{H L}^{2}\right)_{2} \mathrm{Cu}_{2}\left(\mu-\eta^{2}: \eta^{2}-\mathrm{O}_{2}\right)\right](\mathbf{6})$ with bands at $333 \mathrm{~nm}$ and $500 \mathrm{~nm}$ in THF at $193 \mathrm{~K}$ monitored by UV/Vis spectroscopy. The inset shows formation of the band at $333 \mathrm{~nm}$ with time.

\subsubsection{Structural elucidation of a $\mathrm{Cu}_{2}\left(\mu-\eta^{2}: \eta^{2}-\mathrm{O}_{2}\right)$ dicopper(II) complex (6) with an unusually long $\mathrm{O}-\mathrm{O}$ bond}

The stability of 6 at $193 \mathrm{~K}$ made it possible to store solutions of the complex at low temperatures over several months. Formation of $\left[\left(\mathbf{H L}^{2}\right)_{2} \mathrm{Cu}_{2}\left(\mu-\eta^{2}: \eta^{2}-\mathrm{O}_{2}\right)\right]$ was confirmed by X-ray crystallography. Single crystals sutiable for $\mathrm{X}$-ray diffraction were grown by diffusion of $\mathrm{Et}_{2} \mathrm{O}$ into a 1:1 THF/acetone solution of $\mathbf{6}$ at $193 \mathrm{~K}$. X-ray diffraction analysis revealed a centrosymmetric molecular structure of the cation $\mathbf{6}$

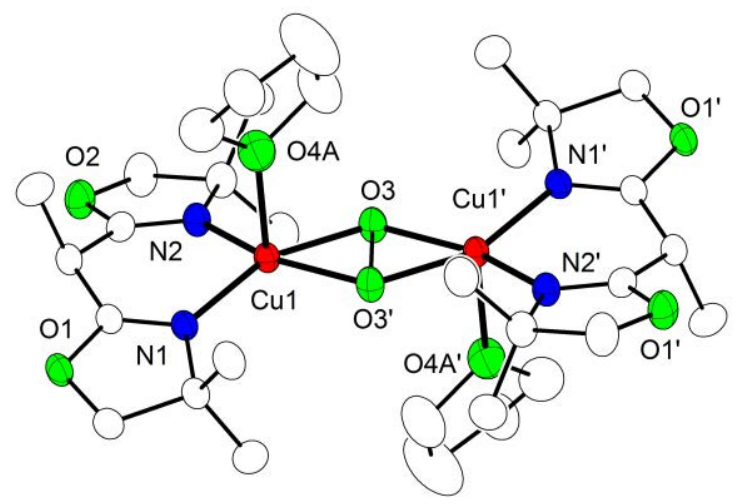

Fig 5.9 Plot (30\% probability thermal ellipsoids) of the molecular structure of one of the two crystallographically independent cations in 6 (hydrogen atoms and disorder omitted for clarity). Selected distances $[\AA]$ and angles $\left[{ }^{\circ}\right]$ : $\mathrm{Cu} 1-\mathrm{O} 31.916(3), \mathrm{Cu} 1-$ O3' 1.919(3), Cu1-N2 1.942(4), Cu1-N1 1.959(3), Cu1-O4A 2.328(6), O3-O3' 1.583(6), Cu1 ‥Cu1' 3.4921(8); O3-Cu1-O3' 48.78(15), O3-Cu1-N2 105.38(14), O3'-Cu1-N2 152.73(14), O3-Cu1-N1 157.01(14), O3'-Cu1-N1 109.49(14), N2-Cu1N1 94.36(15), O3-Cu1-O4A 91.4(3), O3'-Cu1-O4A 90.0(4), N2-Cu1-O4A 100.5(4), N1-Cu1-O4A 96.5(3), Cu1-O3-Cu1' 131.22(15). Symmetry transformation used to generate equivalent atoms: (') $1-x, 1-y,-z$. 
(Fig 5.9) with two perchlorate anions. The molecule crystallizes as two crystallographically independent units.

Each copper ion was found to be coordinated in a slightly distorted square pyramidal (SP-5) environment ( $\tau=0.14$; where $\tau_{5}$ is the geometry index for SP-5 complexes defined as $(\beta-\alpha) / 60^{\circ}$, where $\beta$ and $\alpha$ are defined as the largest bond angles for the coordinated ion) constituted by the $\mathbf{H L}^{2}$ BOX capping ligand, the peroxide moiety and an additional THF solvent molecule bound axially. Exemplary complexes bearing copper and ethylene diamine ligand systems with comparable transitions to that of $\mathbf{6}$ have also shown to contain an additional weakly bound axial ligand which could either be the counterion or a solvent molecule, ${ }^{[137,217]}$ similar to this case. The $\mathrm{Cu}-\mathrm{O}$ distance of the bridging side-on $\mu-\eta^{2}: \eta^{2}$ peroxide was determined to be 1.91 - $1.92 \AA$. The $\mathrm{Cu}-\mathrm{O}$ distance of the THF molecule bound in the apical position was $2.33 \AA$. The large difference in these $\mathrm{Cu}-\mathrm{O}$ distances suggests that the solvent molecule is only weakly bound to the copper centers compared to the peroxide ligand. The $\mathrm{Cu} \cdots \mathrm{Cu}$ separation of $3.49-3.51 \AA$ is typical for a ${ }^{\mathbf{S}} \mathbf{P}$ core, ${ }^{[41]}$ and the $\mathrm{O}-\mathrm{O}$ bond length of the central peroxide at $1.58 \AA$ is the longest $\mathrm{O}-\mathrm{O}$ bond reported so far for any synthetic or biological $\mathrm{Cu}_{2}\left(\mu-\eta^{2}: \eta^{2}-\mathrm{O}_{2}\right)$ system. ${ }^{[108,136,141,142,144,162-165]}$ The additional acetonitrile molecule bound to the copper(I) complex observed by ESI-MS does not appear in the crystal structure of the copper(II) peroxide counterpart.

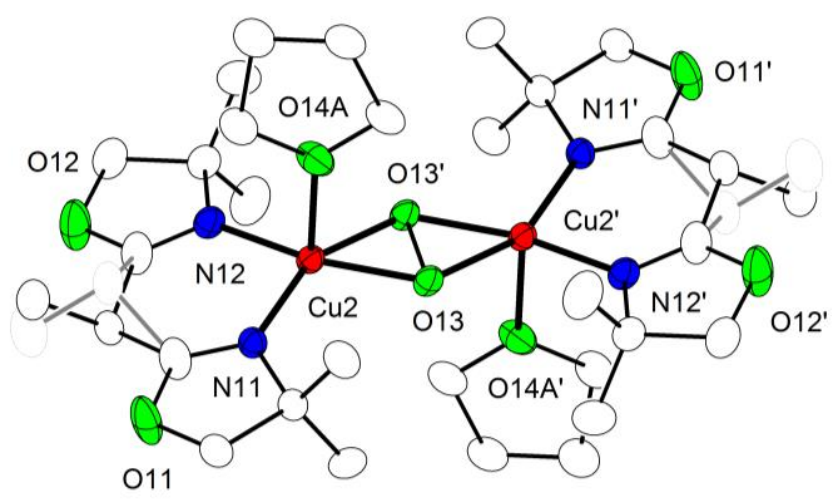

Fig 5.10 Plot (30\% probability thermal ellipsoids) of the molecular structure of one of the two crystallographically independent cations in 6 (hydrogen atoms and the THF disorder are omitted for clarity). Selected distances $[\AA]$ and angles $\left[^{\circ}\right]$ : Cu2-O13'

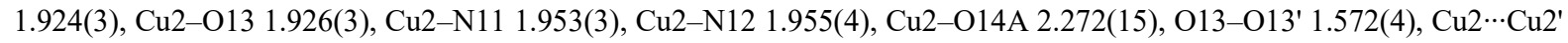
3.5148(7); O13'-Cu2-O13 48.20(14), O13'-Cu2-N11 154.34(14), O13-Cu2-N11 107.60(13), O13'-Cu2-N12 107.74(14), O13-Cu2-N12 154.48(14), N11-Cu2-N12 94.23(15), O13'-Cu2-O14A 93.3(13), O13-Cu2-O14A 92.5(10), N11-Cu2O14A 96.7(17), N12-Cu2-O14A 98.0(14), Cu2'-O13-Cu2 131.80(14). Symmetry transformation used to generate equivalent atoms: (') $-x, 1-y, 1-z$.

\subsection{Synthesis and characterization of a $\mathrm{Cu}(\mathrm{I}) \mathrm{BOX}$ complex with a non-proton responsive ligand $\mathrm{L}^{6}$}

Ligand $\mathbf{L}^{6}$ was suitably designed to be non-proton responsive in nature, with two methyl groups present in the ligand backbone. The copper(I) complex of $\mathbf{L}^{6}(\mathbf{4})$ was prepared by treating a 1:1 equivalent of $\mathbf{L}^{6}$ with tetrakis(acetonitrile) copper(I) perchlorate in THF (Scheme 5.1). ESI-MS analysis of the crude reaction mixture in THF (Fig 5.11 left) revealed one major peak, namely $\left[\mathbf{L}^{6} \mathrm{Cu}(\mathrm{I}) \mathrm{MeCN}\right]^{+}(\mathrm{m} / z=342.1)$. The two other peaks could be assigned to $\left[\mathbf{L}^{6} \mathrm{Cu}(\mathrm{I})\right]^{+}(\mathrm{m} / z=301)$ and $\left[\left(\mathbf{L}^{6}\right)_{2} \mathrm{Cu}_{2}(\mathrm{I}) \mathrm{CN}\right]^{+}(\mathrm{m} / z=628.2)$. The diamagnetic nature of $\mathbf{4}$ enabled the usage of ${ }^{1} \mathrm{H}$ NMR for characterization in solution. 


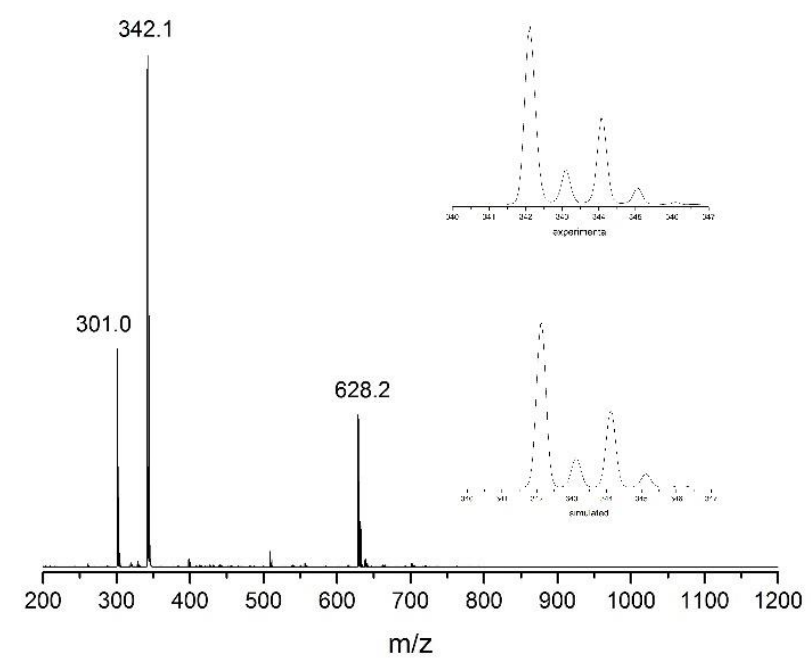

Figure 5.11 Left: ESI-MS of 4 in THF. The two major peaks could be assigned to the copper(I) complex of $\mathbf{L}^{6}$, differing by an acetonitrile coligand. The inset shows an enlargement of the peak at $m / z=342.1$, together with a simulation of the isotopic pattern corresponding to $\left[\mathrm{L}^{6} \mathrm{Cu}(\mathrm{I}) \mathrm{MeCN}\right]^{+}$.

\subsubsection{Structural characterization of 4}

Single crystals suitable for X-ray diffraction were obtained in $50 \%$ yield by slow diffusion of $\mathrm{Et}_{2} \mathrm{O}$ into a THF solution of 4. X-ray diffraction analysis revealed $\mathbf{4}$ to coexist with its dimer in the solid state. Both the monomer and dimer cocrystallize in the triclinic space group $P \overline{1}$. The monomer (Fig 5.12 left), similar to $\mathbf{1}$ and $\mathbf{3}$, has a copper ion coordinated to the ligand and an acetonitrile molecule in a slightly distorted trigonal planar geometry. The boat shaped coordination is maintained, with the oxazoline rings being flat and planar. The methyl groups of the $\mathrm{CMe}_{2}$ units present in the ligand backbone and in the oxazoline rings orient themselves above and below the plane. The bite angle formed by $\mathrm{N} 2-\mathrm{Cu}-\mathrm{N} 1$ is the smallest, $92.8^{\circ}$, compared to the other copper(I) complexes $\mathbf{1}$ and $\mathbf{3}$ reported above.

The dimer (Fig 5.12 right) crystallizes with two copper ions and two ligand molecules per single unit with each copper ion coordinated to two nitrogen atoms of different ligands. Unlike the monomer, the copper ions exhibit a linear coordination, with no exogenous acetonitrile molecule. The metal-ligand $\mathrm{Cu}-\mathrm{N}$ bond lengths herein are slightly shorter compared to the monomer, which could be accounted for by a lower coordination number of the metal center. 1H NMR studies in combination with DOSY analysis of 4 in THF- $\mathrm{d}_{8}$ revealed the presence of only a single species, the monomer within solution. No peaks corresponding to the dimer were observed. This suggests that though $\mathbf{4}$ exists as a monomer and dimer in the sold state, within solution, only the monomeric species is retained. 

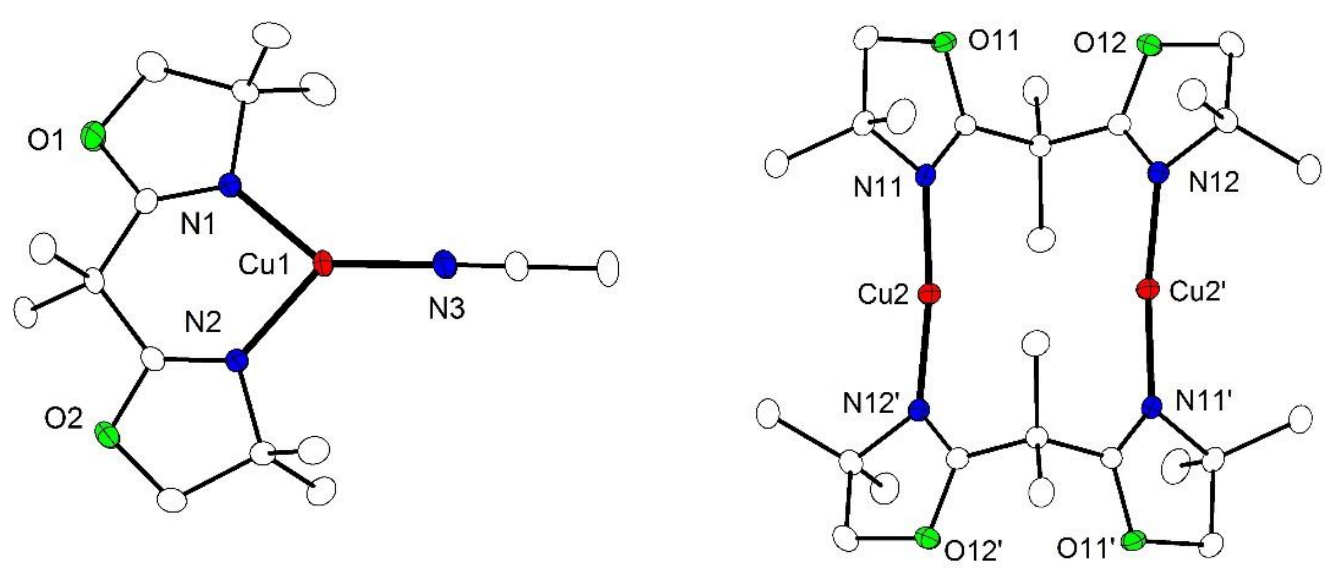

Figure 5.12 Left: Plot (30\% probability thermal ellipsoids) of the molecular structure of one of the two crystallographically independent cations of 4 (hydrogen atoms omitted for clarity). Selected bond lengths $[\AA]$ and angles $\left[{ }^{\circ}\right]$ : $\mathrm{Cu} 1-\mathrm{N} 1$ 1.9469(17), Cu1-N2 1.9909(18), Cu1-N3 1.8540(19); N3-Cu1-N1 141.13(8), N3-Cu1-N2 125.90(8), N1-Cu1-N2 92.83(7). Right: Plot (30\% probability thermal ellipsoids) of the molecular structure of one of the two crystallographically independent cations of 4 (hydrogen atoms omitted for clarity). Selected distances $[\AA]$ and angles $\left[^{\circ}\right]$ : Cu2-N11 1.8699(17), Cu2-N12' 1.8738(17), Cu2 $\cdots$ $\mathrm{Cu} 2$ ' 3.4313(5); N11-Cu2-N12' 171.43(8). Symmetry transformation used to generate equivalent atoms: (') $1-x, 1-y, 1-z$.

\subsection{Dioxygen activation of 4 at low temperatures to yield a $\mathrm{Cu}_{2}\left(\mu-\eta^{2}: \eta^{2}-\mathrm{O}_{2}\right)$ intermeditae with complete characterization}

\subsubsection{Properties in Solution}

Oxygenation of a solution of 4 at $193 \mathrm{~K}$ in THF yielded the $\left[\left(\mathbf{L}^{6}\right)_{2} \mathrm{Cu}_{2}\left(\mu-\eta^{2}: \eta^{2}-\mathrm{O}_{2}\right)\right](\mathbf{8})$ complex according to Scheme 5.2. Monitoring the reaction by UV-vis spectroscopy (Fig5.13) led to intense optical features at

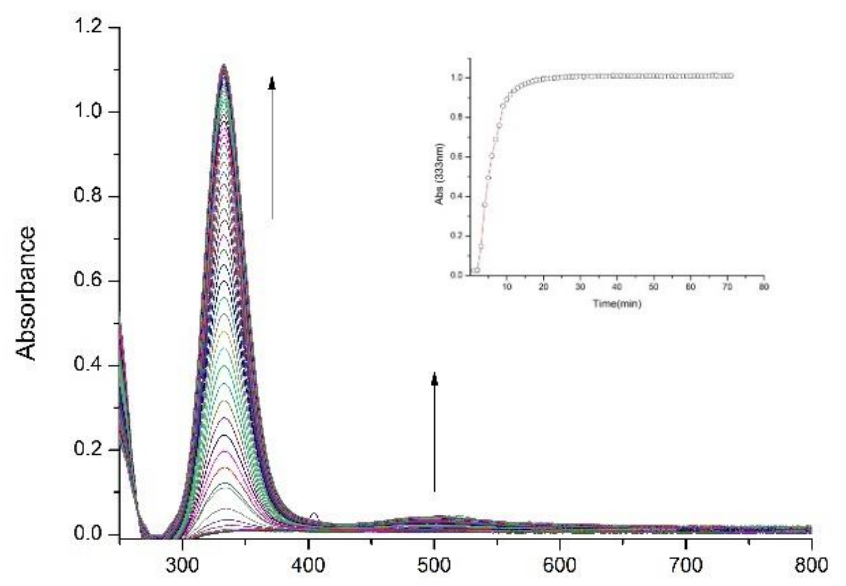

Fig 5.13 Formation of $\left[\left(\mathbf{L}^{6}\right)_{2} \mathrm{Cu}_{2}\left(\mu-\eta^{2}: \eta^{2}-\mathrm{O}_{2}\right)\right](\mathbf{8})$ with bands at $333 \mathrm{~nm}$ and $500 \mathrm{~nm}$ in THF at $193 \mathrm{~K}$ monitored by UV/Vis spectroscopy. Inset shows the formation of the band at $333 \mathrm{~nm}$ with time. 
$333 \mathrm{~nm}\left(\varepsilon \approx 21440 \mathrm{M}^{-1} \mathrm{~cm}^{-1}\right)$ and $500 \mathrm{~nm}\left(\varepsilon \approx 895 \mathrm{M}^{-1} \mathrm{~cm}^{-1}\right)$, typical for the LMCT transitions of dicopper $\mu-\eta^{2}: \eta^{2}$-peroxo species.

\subsubsection{Resonance Raman spectroscopy}

A deeper insight into the dioxygen binding mode of $\mathbf{8}$ was obtained from resonance Raman (rR) spectroscopy. An excitation wavelength of $633 \mathrm{~nm}$ was used due to diminished fluorescence in comparison to the $457 \mathrm{~nm}$ laser. THF solutions of 4 were exposed to either naturally abundant or isotopically labelled dioxygen $\left({ }^{18} \mathrm{O}_{2}\right)$ at $193 \mathrm{~K}$ in young NMR tubes. Solutions of 8 in a dry-ice/acetone bath at $193 \mathrm{~K}$ showed an oxygen isotope sensitive feature at $740 \mathrm{~cm}^{-1}$ which shifts to $700 \mathrm{~cm}^{-1}$ on isotopic labelling $\left(\Delta^{16} \mathrm{O}_{2}-\Delta^{18} \mathrm{O}_{2}\right.$ $=40 \mathrm{~cm}^{-1}$ ) (Figure 5.14). This was assigned to the $\mu-\eta^{2}: \eta^{2}$ coordinated peroxide, and is comparable to the side on $\mu-\eta^{2}: \eta^{2}$ copper(II) dioxygen complexes having $v_{\mathrm{O}-\mathrm{O}}=730-760 \mathrm{~cm}^{-1}\left(\Delta\left[{ }^{18} \mathrm{O}_{2}\right] \mathrm{ca} .40 \mathrm{~cm}^{-1}\right), \mathrm{rR} \mathrm{cm}^{-1}$ $\left(\Delta^{18} \mathrm{O}_{2}\right)=-744(-39)$ for oxy-hemocyanin ${ }^{[218]}$, and -755(-41) for oxy-tyrosinase $\left.{ }^{[219]}\right)$. In the case of $\mu-\eta^{2}: \eta^{2}-$ peroxodicopper(II) complexes, due to backbonding which occurs from the HOMO of $\mathrm{Cu}$ (II) $\mathrm{d}_{\mathrm{xy}}$ orbitals to the in-plane, unoccupied high energy $\sigma^{*}$ orbital of the peroxide, the O-O stretch is weakened compared to the O-O stretch observed with $\mathrm{H}_{2} \mathrm{O}_{2}\left(v_{\mathrm{O}-\mathrm{O}}=880 \mathrm{~cm}^{-1}\right)$, 1,2-trans-peroxo $\left(v_{\mathrm{O}-\mathrm{O}}=830 \mathrm{~cm}^{-1}\left(\Delta\left[{ }^{18} \mathrm{O}_{2}\right] 46\right)\right)$, 1,2cis-peroxo $\left(v_{\mathrm{O}-\mathrm{O}}=800 \mathrm{~cm}^{-1}\left(\Delta\left[{ }^{18} \mathrm{O}_{2}\right] 45\right)\right)$, and bis $(\mu$-oxo $)\left(v_{\mathrm{Cu}-\mathrm{O}}=600 \mathrm{~cm}^{-1}\left(\Delta\left[{ }^{18} \mathrm{O}_{2}\right] 28\right)\right)$ dicopper systems. rR spectroscopy confirmed the absence of a MeCN molecule bound to $\left[\left(\mathbf{L}^{6}\right)_{2} \mathrm{Cu}_{2}\left(\mu-\eta^{2}: \eta^{2}-\mathrm{O}_{2}\right)\right]$ which was in accordance with X-ray crystallography (Fig. 5.16). There was no evidence of a bis( $\mu$-oxo) dicopper(III) species which is expected to exhibit a vibrational mode at $580-650 \mathrm{~cm}^{-1}$ with a $\Delta\left[{ }^{18} \mathrm{O}_{2}\right]$ shift of $23-30 \mathrm{~cm}^{-1}$ due to the oxo core.

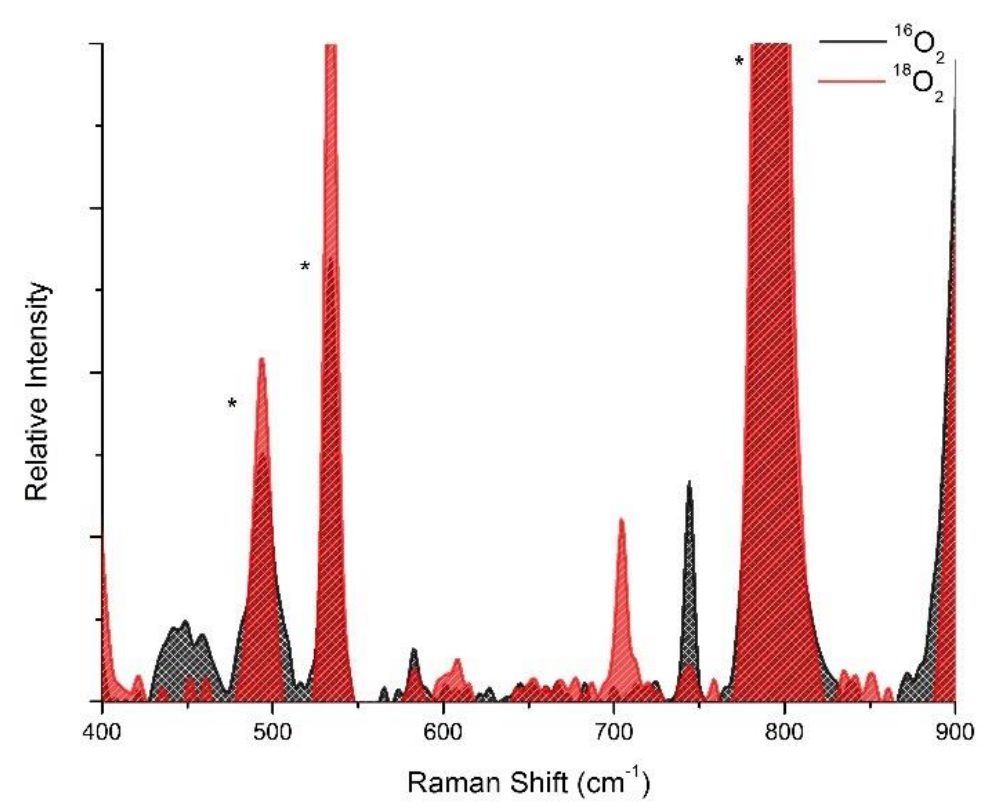

Figure 5.14. Resonance raman spectrum of 8 in THF at $193 \mathrm{~K} .{ }^{16} \mathrm{O}_{2}$ spectrum indicated in black and ${ }^{18} \mathrm{O}_{2}$ spectrum indicated in red. Residual solvent signals are marked with an asterisk $(*)$. 


\subsubsection{Properties in solid state}

Isolated solid purple material of complex $\mathbf{8}$ was also characterized by solid state UV-vis and IR spectroscopy. When compared to the solution spectrum, the reflectance spectrum (Fig 5.15 left) of a ground sample of the dioxygen adduct showed similar distinctive peaks. Two intense bands at $337 \mathrm{~nm}$ and $480 \mathrm{~nm}$ similar to those in solution were observed indicating that the ${ }^{\mathbf{S}} \mathbf{P}$ structure is present both in solution and in the solid state. The IR spectrum of the naturally abundant dioxygen adduct of 8 (Fig 5.15 right) was measured in a $\mathrm{KBr}$ pellet, which revealed a stretch at $747 \mathrm{~cm}^{-1}$ which could possibly be assigned to the OO stretch, similar to that observed by rR studies $\left({ }^{16} \mathrm{O}_{2}\right)$.
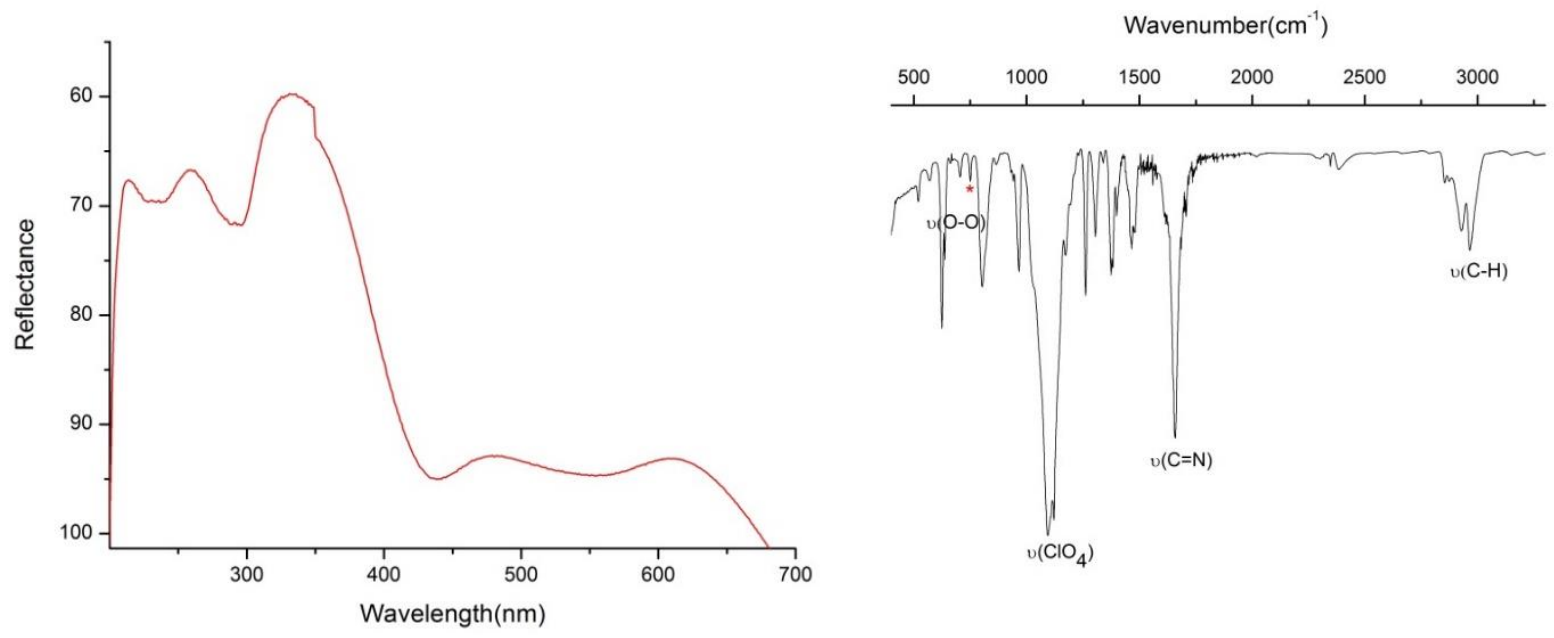

Fig 5.15 Left: UV-vis features of 8 in the solid state. Bands at $337 \mathrm{~nm}$ and $480 \mathrm{~nm}$ are also present in UV-vis solution. Right: IR spectra of $\mathbf{8}$ measured with a $\mathrm{KBr}$ pellet. Possible $v(\mathrm{O}-\mathrm{O})$ for naturally abundant dioxygen adduct of $\mathbf{8}$ marked with *.

\subsubsection{Structural elucidation of $\boldsymbol{8}$}

Single crystals of $\mathbf{8}$ were sucessfully grown from a 1:1 THF:acetone $/ \mathrm{Et}_{2} \mathrm{O}$ solution at $193 \mathrm{~K}$ confirming the $\mu-\eta^{2}: \eta^{2}$-peroxo binding mode. X-ray diffraction analysis (Figure 5.16) revealed a centrosymmetric molecular structure of the cation $\mathbf{8}$, similar to that of $\mathbf{6}$ with two perchlorate anions and a disordered acetone molecule. Each copper ion displays a slightly distorted square pyramidal geometry $(\tau=0.16)$ constituted by the ligand $\mathbf{L}^{6}$, the peroxide moiety, and the apical bound THF solvent molecule. The two $\mathrm{Cu}-\mathrm{N}$ equatorial

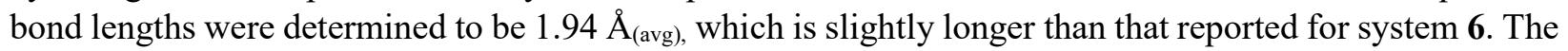
apical bound THF solvent molecules displayed a $\mathrm{Cu}-\mathrm{O}$ distance of $2.324 \AA$ and $\mathrm{Cu}-\mathrm{O}$ equatorial bonds contributed by the peroxide were $1.924 \AA$ and $1.929 \AA$. The bond length between the two copper centers was $3.52 \AA$ and the O-O bond length of the peroxide was $1.56 \AA$, which is fairly long compared to other structurally reported ${ }^{\mathbf{S}} \mathbf{P}$ complexes. The absence of $\mathrm{MeCN}$ bound to $\mathbf{8}$, as confirmed by rR spectroscopy was in accordance with X-ray structural analysis. 


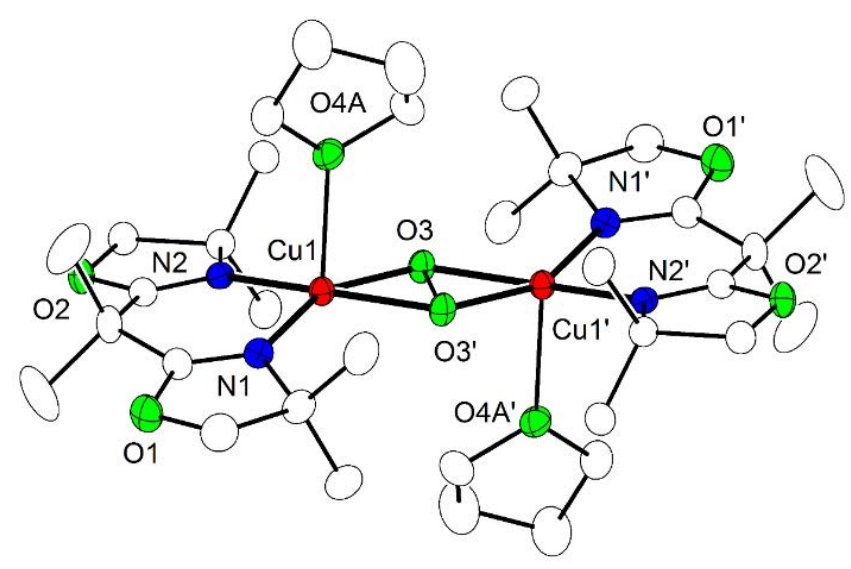

Fig 5.16 Plot (30\% probability thermal ellipsoids) of the molecular structure of the cationic part of $\mathbf{8}$ (hydrogen atoms and disorder omitted for clarity). Selected distances $[\AA]$ and angles $\left[{ }^{\circ}\right]$ : Cu1-O3 1.929(3), Cu1-O3' 1.924(3), Cu1-N1 1.942(3), Cu1-N2 1.950(3), Cu1-O4A 2.318(5), O3-O3' 1.564(5), Cu1Cu1' 3.5214(7); O3'-Cu1-O3 47.90(13), O3'-Cu1-N1 107.96(13), O3-Cu1-N1 153.99(13), O3'-Cu1-N2 154.15(13), O3-Cu1-N2 108.16(12), N1-Cu1-N2 93.09(14), O3'-Cu1O4A 92.02(18), O3-Cu1-O4A 89.55(19), N1-Cu1-O4A 102.4(2), N2-Cu1-O4A 98.03(18), Cu1'-O3-Cu1 132.10(13). Symmetry transformation used to generate equivalent atoms: (') $1-x, 1-y,-z$.

\subsubsection{Magnetic measurements}

The thermal stability of $\mathbf{8}$ allowed its magnetic properties to be determined. Magnetic susceptibility measurements were carried out using a Quantum Design MPM-5S superconducting quantum interference device (SQUID magnetometer). The temperature dependence of $\chi_{\text {mol }} T$ within the temperature range of 2$295 \mathrm{~K}$ for crystalline material of $\mathbf{8}$ was negligible, suggestive of very strong antiferromagnetic coupling between the two cupric ions and maximum population of the singlet ground state (Fig 5.17.) Simulations of the data using the Heisenberg-Dirac-van Vleck Hamiltonian; $H=-2 J S_{1}: S_{2}$, showed the lower limit of the exchange coupling to be $-2 J \geq 1600 \mathrm{~cm}^{-1}$, which is rather high for reported ${ }^{\mathbf{S}} \mathbf{P}$ systems. In order to confirm this lower limit, simulations with fixed $J$ values were performed (black dashed lines; Fig. 5.17). These indicated the molar susceptibility to be far higher than what was experimentally observed, thus conforming the lower limit. Decomposition of 8 with temperature did not permit high temperature $(>300 \mathrm{~K})$ magnetic measurements to be conducted.

Experimental data for magnetic coupling in $\mathrm{Cu}_{2} / \mathrm{O}_{2}$ systems by SQUID magnetometry are still relatively scarce due to the thermal lability of such intermediates. Oxyhemocyanin also exhibits a large singlet-triplet splitting of $-2 J \geq 600 \mathrm{~cm}^{-1}$. In the system reported by Karlin et al. ${ }^{[220]}$, a $\mu-\eta^{2}: \eta^{2}$ peroxo bridged model system, the singlet-triplet splitting was reported at $-2 J \geq 600 \mathrm{~cm}^{-1}$, and $-2 J \geq 800 \mathrm{~cm}^{-1}$ for the system reported by Kitajima and Solomon et al. ${ }^{[221]}$. In the case of trans-1,2-peroxo coordinated model systems, the splitting was reported to be $-2 J \geq 600 \mathrm{~cm}^{-1}$. Recently in 2014, the first cis-peroxo binding geometry was reported by Meyer et al. where the peroxo exhibits only a weak antiferromagnetic coupling of $-2 J \geq 144$ $\mathrm{cm}^{-1}$ with a Cu-O-O-Cu torsion of $65^{\circ} .{ }^{[109]}$ Subsequently, the first ferromagnetically coupled dicopper(II) peroxo system with a triplet ground state $(S=1)$ was reported in the same group, wherein the $\mathrm{Cu}-\mathrm{O}-\mathrm{O}-\mathrm{Cu}$ torsion was constrained to close to $90^{\circ} .{ }^{[134]}$ To date, this is the only reported dicopper peroxo system that 
does not exhibit any antiferromagnetic coupling. The experimental data for the magnetic measurements of 8 confirms a singlet $S=0$ ground state, with strong antiferromagnetic coupling of $-2 J \geq 1600 \mathrm{~cm}^{-1}$ between the copper(II) ions and lies well within the range of other ${ }^{\mathbf{S}} \mathbf{P}$ systems.

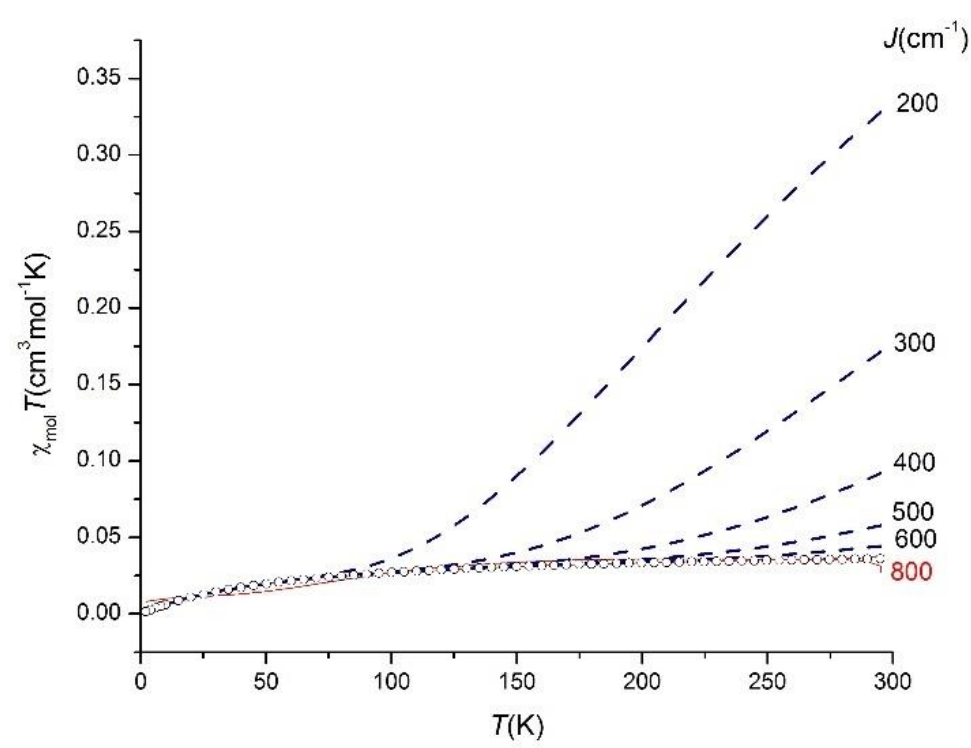

Figure 5.17. $\chi \mathrm{m}$ T vs $\mathrm{T}$ measurement of $\mathbf{8}$ obtained from SQUID measurements at $0.5 \mathrm{~T}$. The red solid line corresponds to the best fit for two anitferromagnetically coupled $S=1 / 2$ ions with $g=2.0$ Additional fit parameters: temperature independent paramagnetism and paramagnetic impurity. The fit gives lower limit of $-J \geq 800 \mathrm{~cm}^{-1}$. The black lines represent simulations assuming weaker coupling (smaller $J$ values). 


\subsection{Comparison of 6 and 8 with other structurally characterized $\mathrm{Cu}_{2}\left(\mu-\eta^{2}: \eta^{2}-\mathrm{O}_{2}\right)$ complexes}

The first structurally elucidated synthetic ${ }^{\mathbf{S}} \mathbf{P}$ complex was demonstrated by Kitajima et al. with sterically demanding $f a c$, monoanionic, tris(pyrazolyl) borate ligands $\mathrm{Tp}^{\mathrm{R} 3, \mathrm{R} 5}(\mathrm{R} 3=\mathrm{Me}, i \mathrm{Pr}, \mathrm{Ph} ; \mathrm{R} 5=\mathrm{Me}, i \mathrm{Pr}) .{ }^{[108]}$ The spectroscopic features exhibited by these complexes were similar to that of oxyHc, which put forward the idea that dioxygen binds similarly in the enzyme. Soon after, the structural characterization of oxyHc from horseshoe crab (Limilus polyphemus) $)^{[42,222,223]}$ and octopus (Octopus dofleini) ${ }^{[224]}$ confirmed the sideon binding mode. The octopus oxyHc structure measured at $2.3 \AA$ revealed a symmetric core with a $\mathrm{Cu} \cdots \mathrm{Cu}$ distance of $3.54 \AA$ and an O-O bond length of $1.38 \AA$. This differed slightly from the horseshoe crab oxyHc, where the $\mathrm{Cu}$ centers were bound in a distorted ${ }^{\text {S}} \mathbf{P}$-geometry, with $\mathrm{Cu} \cdots \mathrm{Cu}$ and $\mathrm{O}-\mathrm{O}$ bond lengths being $3.60 \AA$ and $1.41 \AA$ respectively. These parameters however, were quite similar to the synthetic system reported by Kitajima $(\mathrm{Cu} \cdots \mathrm{Cu} 3.56 \AA$ and $\mathrm{O}-\mathrm{O} 1.41 \AA)$. Following this, only a handful of ${ }^{\mathrm{S}} \mathbf{P}$ complexes have successfully been characterized within the last two decades, proving structural elucidation to be a challenging task for these complexes. Most ${ }^{\mathbf{S}} \mathbf{P}$ systems have been crystallographically characterized with tridentate bulky ligand systems capable of encapsulating the $\left(\mu-\eta^{2}: \eta^{2}-\mathrm{O}_{2}\right)$ unit within the copper centers. The sterically demanding binucleating bitripy ligand, ${ }^{[142]}$ sterically hindered hexapyridine system, ${ }^{[163]}$ as well as macrocyclic ligands such as $i \operatorname{Pr}_{3}$ tacd and $t \mathrm{Bu}_{3}$ tacn belong to this ligand class. ${ }^{[144,165]}{ }^{\mathrm{The}}{ }^{\mathbf{S}} \mathbf{P}$ complex reported by Gorun et al. which was structurally characterized with the tris(pyrazolyl)borate $\mathrm{Tp}^{\mathrm{CF} 3, \mathrm{Me}}$ ligand, demonstrated enhanced stability for several days when compared to other ${ }^{\mathbf{S}} \mathbf{P}$ systems, which are inherently known to be thermally labile. ${ }^{[146]}$ This was accounted for by the inertness and the electron withdrawing nature of the $\mathrm{CF}_{3}$ groups making this complex more thermally stable. The ${ }^{\mathbf{S}} \mathbf{P}$ complex reported by Masuda et al. with the aid of $(-)$ - $\alpha$-isosparteine capping ligands, demonstrated a carboxylate bridged $\mu-\eta^{2}: \eta^{2}$ butterfly structure constraining the planarity between the copper centers and the peroxide ligand. ${ }^{[162]}$ This led to the $\mathrm{Cu} \cdots \mathrm{Cu}$ bond distance (3.26 $\AA$ ) within the complex to be the shortest reported so far. The O-O bond distance of $1.49 \AA$ remained within the normal realm of ${ }^{\mathbf{S}} \mathbf{P}$ complexes. Moreover, this is the only bidentate ligand system reported to date that is known to isolate a ${ }^{\mathbf{S}} \mathbf{P}$ complex. Recently Karlin et. al. isolated a new ${ }^{\mathbf{S}} \mathbf{P}$ complex with the tridentate MeAN ligand which has the longest O-O bond reported to date $(1.54 \AA) \cdot{ }^{[164]}$ However, this did not reflect in an increase in backbonding nor result in O-O bond cleavage.

In contrast to the ligand systems mentioned above, BOX systems are simple bidentate ligands. This ligand class has proved efficient in isolating ${ }^{\mathbf{S}} \mathbf{P}$ complexes. Of the ${ }^{\mathbf{S}} \mathbf{P}$ complexes isolated during the course of this work, complex $\mathbf{6}$ which was generated with the proton responsive $\mathbf{H L}^{2}$ ligand now demonstrates the longest O-O bond (1.58 $\AA$ ) reported in any synthetic or biological system. Complex $\mathbf{8}$, which was obtained with the non-proton responsive $\mathbf{L}^{6}$ ligand also demonstrates an unusually long $\mathrm{O}-\mathrm{O}$ bond (1.56 $\AA$ ). This too did not affect the backbonding as the $\mathrm{rR}$ values for $\mathbf{8}$ lie within the normal reported range of ${ }^{\mathbf{S}} \mathbf{P}$ complexes. In analogy to the system reported by Karlin et. al., the long $\mathrm{O}-\mathrm{O}$ bond did not result in any O-O bond cleavage.

Table 5.1 gives an overview of all biologically and synthetically structurally characterized ${ }^{\mathrm{S}} \mathbf{P}$ complexes. 
Table 5.1. Geometric and Spectroscopic features of $\mu-\eta^{2}: \eta^{2-}$ peroxodicopper(II) (BOX)complexes, and all $\mu-\eta^{2}: \eta^{2-}$ peroxodicopper(II) complexes characterized by XRD so far.

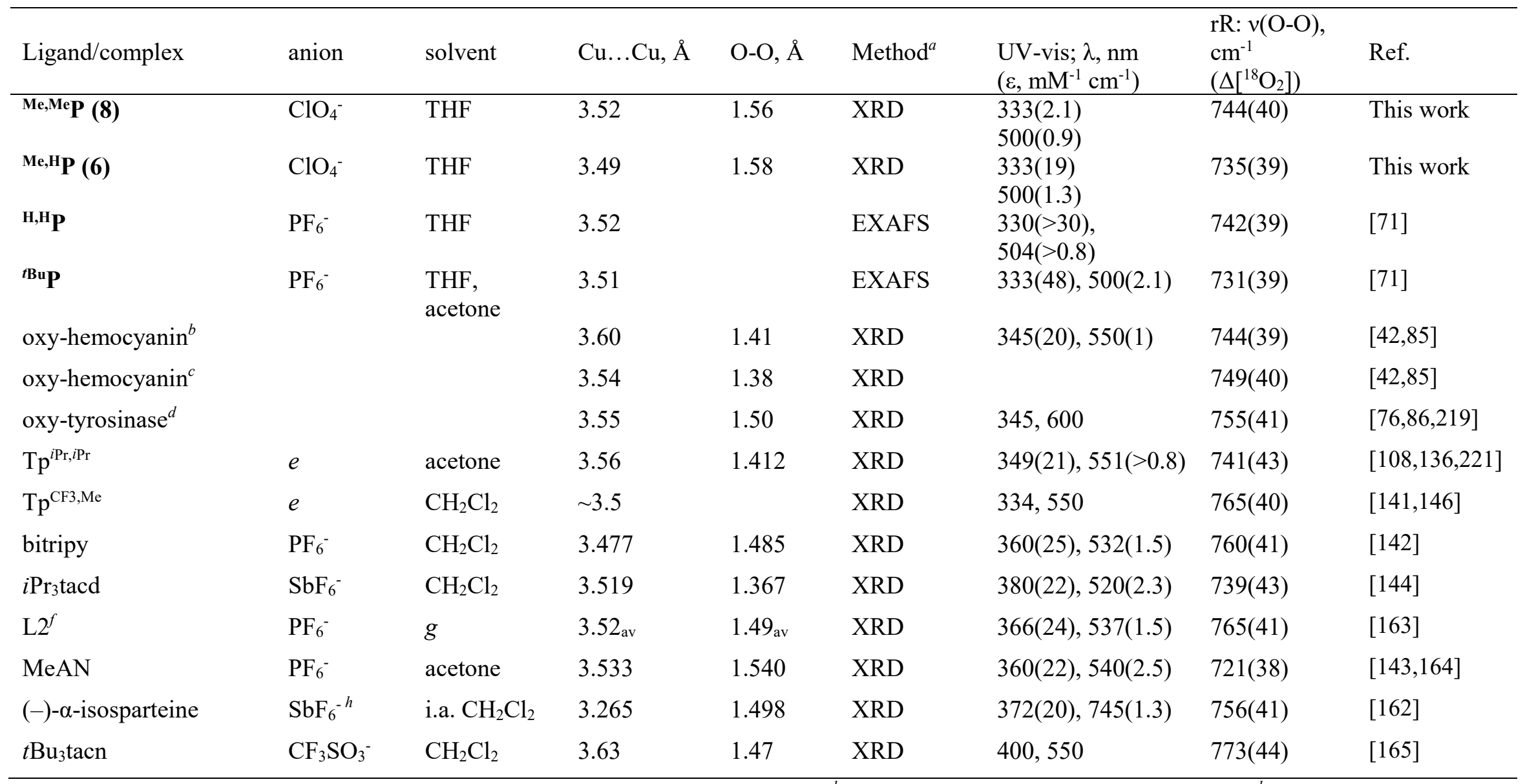

${ }^{a}$ Structural data from EXAFS or from X-ray diffraction(XRD) measurements. ${ }^{b}$ Limulus Polyphemus. ${ }^{c}$ Octopus dof leini. ${ }^{d}$ Mushroom (Agaricus bisporus). ${ }^{e}$ Neutral complex. ${ }^{f}$ Two independent molecules in the unit cell. ${ }^{g} \mathrm{CH}_{2} \mathrm{Cl}_{2} /$ acetone, 3:0.002, mixture. ${ }^{h} \mathrm{An}$ additional bridging $\mu$-Bz- ligand is present 


\subsection{Kinetic and Thermodynamic analysis of $\mathrm{Cu} / \mathrm{O}_{2}$ binding with $\mathrm{HL}^{5}$ and $\mathrm{L}^{6}$}

The kinetic and thermodynamic parameters for a range of $\mathrm{Cu} / \mathrm{O}_{2}$ systems has led to a well-accepted scheme for the mechanism of dioxygen activation (Scheme 5.3). ${ }^{[132]}$

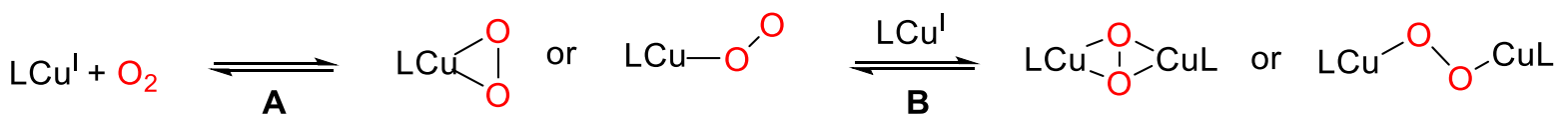

Scheme 5.3 Mechanistic framework for dioxygen activation of $\mathrm{Cu}(\mathrm{I})$ complexes.

As evident from the processes $\mathrm{A}$ and $\mathrm{B}$, a total of three cases arise for the formation of a $\mathrm{Cu} / \mathrm{O}_{2}$ complex. In case(I) the rates of both $\mathrm{A}$ and $\mathrm{B}$ are slow and well defined, which would allow for kinetic investigations of the individual processes. In case (II), A $>>B$ in terms of the rates of reaction, and B forms the observable rate determining step. In case (III), B >>A, and A forms the observable rate determining step.

In correlation to a previous study of $\mathrm{Cu} / \mathrm{O}_{2}$ complexes with $\mathrm{BOX}$ ligands, and in concordance to the present work $^{[71]}$, case (III) is operative with $\mathrm{Cu}(\mathrm{I}) \mathrm{BOX}$ complexes for dioxygen activation. No accumulation of any intermediates was observed during the formation of $\mathrm{Cu}_{2}\left(\mu-\eta^{2}: \eta^{2}-\mathrm{O}_{2}\right)$ complexes by UV-vis spectroscopic analysis, which suggested the formation of a single species from the $\mathrm{Cu}(\mathrm{I})$ complex and dioxygen. Moreover, stopped flow analysis and oxygen-concentration dependent experiments conducted on a $\mathrm{Cu}(\mathrm{I})^{t} \mathrm{Bu}$ system previously (where ${ }^{t} \mathrm{Bu}$ represents ligand $\mathbf{H L}^{4}$, refer to Chapter 2, fig. 2.18), led to established pseudo first order reaction conditions, where the rate is independent of the concentration of dioxygen when present in excess. The following section describes the kinetic and thermodynamic parameters for oxygenation of $\mathbf{3}$ and $\mathbf{4}$, and compares them to the previously investigated $\mathrm{Cu}(\mathrm{I})^{t} \mathrm{Bu}$ system.

\subsubsection{Determination of the rate constant $\left(k_{o b s}\right)$ for dioxygen binding in 3 and 4}

Dioxygen reactivity for $\mathbf{3}$ and $\mathbf{4}$ to yield $\mathbf{7}$ and $\mathbf{8}$ was shown to follow first order kinetics. The traces at $\sim 333$ $\mathrm{nm}$ and $\sim 500 \mathrm{~nm}$ were fitted with a single exponential function to obtain the rate constants for their respective reactions. While the previously described systems, ${ }^{{ }^{t} \mathbf{B u}, \mathrm{H}} \mathbf{P}$ and ${ }^{\mathbf{P h}, \mathrm{H}} \mathbf{P}$ ( ${ }^{\mathrm{x}, \mathrm{y}} \mathbf{P}$ : where $\mathrm{x}$ and y represent the residue of the ligand backbone, and $\mathbf{P}$ respresents ${ }^{\mathbf{S}} \mathbf{P}$ complexes) were described to be sufficiently soluble under reaction conditions, and ${ }^{\mathbf{H}, \mathbf{H}} \mathbf{P}$ and ${ }^{\mathrm{Me}, \mathbf{H}} \mathbf{P}$ were found to precipitate upon formation, the two new systems introduced herein were found to be readily soluble and did not precipitate within short timescales. The formation of ${ }^{\mathbf{M e}, \mathbf{M e}} \mathbf{P}(\mathbf{8})$ from its copper(I) complex was completed after approximately 12 minutes, while the formation of ${ }^{\mathbf{P}-\mathbf{T o l}, \mathbf{H}} \mathbf{P}$ (7) was observed to be much slower compared to all the other systems and full formation required over 50 minutes to an hour (Table 5.2). The reaction kinetic profiles were deconvoluted with SPECFIT/32 global analysis software. Time resolved spectra of both complexes ${ }^{\mathrm{Me}, \mathrm{Me}} \mathbf{P}$ and ${ }^{\mathbf{p}-\mathbf{T o l}, \mathbf{H}} \mathbf{P}$ gave rise to a single exponential growth with the corresponding logarithmic plot bearing a linear relationship. The calculated $k_{o b s}$ values for ${ }^{\mathbf{M e}, \mathbf{M e}} \mathbf{P}$ was $0.545 \mathrm{~min}^{-1}$ (Fig 5.18) and that for ${ }^{\mathbf{p}-\mathrm{Tol}, \mathbf{H}} \mathbf{P}$ was $0.098 \mathrm{~min}^{-1}$ (Fig. 5.19), both determined at $195 \mathrm{~K}$ from the non-linear curve fits. Table 5.2 gives an overview of the rate constant of all $\mathrm{Cu}(\mathrm{BOX}){ }^{\mathbf{S}} \mathbf{P}$ complexes at $195 \mathrm{~K}$ synthesized so far. ${ }^{[225]}$ 

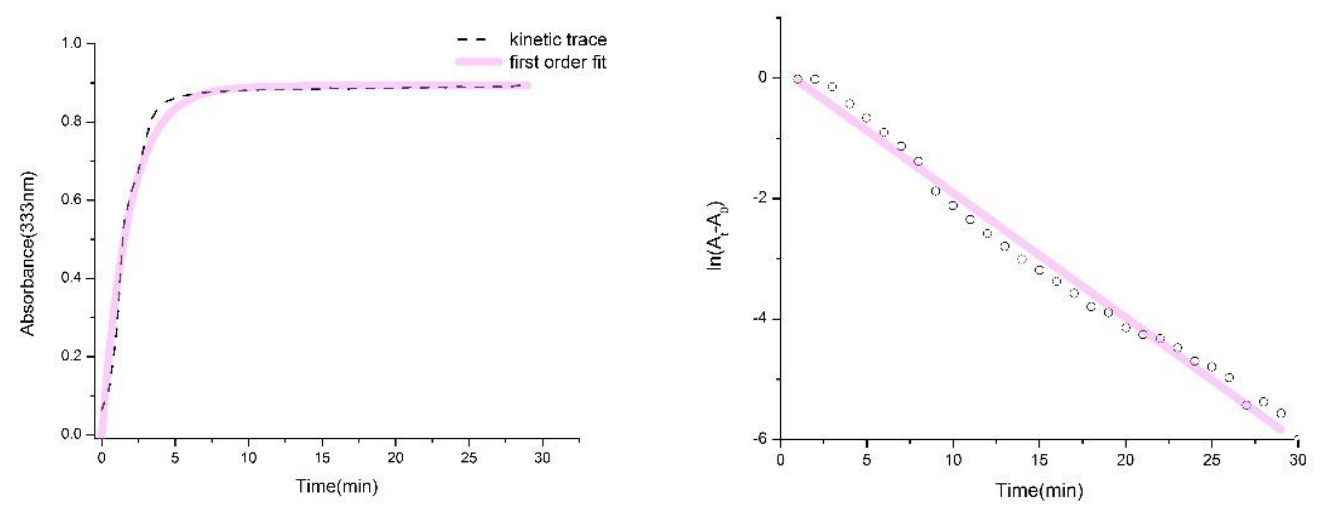

Fig 5.18 Left: Kinetic time trace of Absorbance $(333 \mathrm{~nm})$ and a non-linear first order fit at $195 \mathrm{~K}$ for formation of ${ }^{\mathbf{M e}, \mathbf{M e}} \mathbf{P}(\mathbf{8})$. Right: First order plot of $\ln \left(\mathrm{A}_{\mathrm{t}}-\mathrm{A}_{0}\right)$ vs $t$ based on the absorbance changes at $333 \mathrm{~nm}$ with linear regression. $k_{o b s}=0.545 \mathrm{~min}^{-1}$.
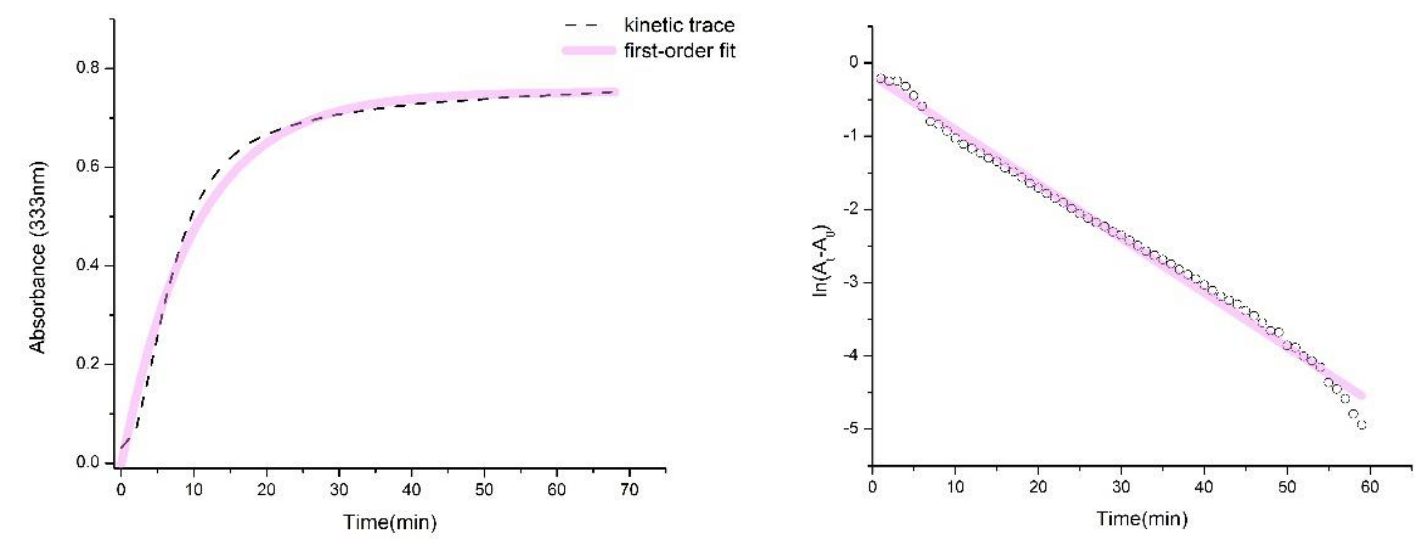

Fig 5.19 Left: Kinetic time trace of Absorbance $(333 \mathrm{~nm})$ and a non-linear first order fit at $195 \mathrm{~K}$ for the formation of p${ }_{\text {Tol,H}} \mathbf{P}(7)$. Right: First order plot of $\ln \left(\mathrm{A}_{\mathrm{t}}-\mathrm{A}_{0}\right)$ vs $t$ based on the absorbance changes at $333 \mathrm{~nm}$ with linear regression. $k_{o b s}=$ $0.098 \mathrm{~min}^{-1}$.

Table 5.2 Overview of rate constants of formation at $195 \mathrm{~K}$ for all $\mathrm{Cu}(\mathrm{BOX}){ }^{\mathrm{S}} \mathbf{P}$ complexes synthesized so far.

\begin{tabular}{cccc}
\hline${ }^{\mathbf{S}} \mathbf{P}$-Complex & Ligand & $\mathrm{k}_{\text {obs }}\left(\mathrm{min}^{-1}\right)$ & \\
\hline${ }^{\mathbf{H}, \mathbf{H}} \mathbf{P}$ & $\mathbf{H L}^{\mathbf{1}}$ & $\geq 1$ & Previous work \\
${ }^{\mathbf{M e}, \mathbf{H}} \mathbf{P}$ & $\mathbf{H L}^{2}$ & $\sim 1.5$ & Previous work \\
${ }_{\mathbf{P h}, \mathbf{H}} \mathbf{P}$ & $\mathbf{H L}^{3}$ & 0.71 & Previous work \\
${ }^{{ }_{\mathbf{B u}, \mathbf{H}}} \mathbf{P}$ & $\mathbf{H L}^{4}$ & 0.25 & Previous work \\
${ }^{\mathbf{p}-\mathbf{T o l}, \mathbf{H}} \mathbf{P}$ & $\mathbf{H L}^{\mathbf{5}}$ & 0.098 & This work \\
${ }^{\mathbf{M e}, \mathbf{M e}} \mathbf{P}$ & $\mathbf{L}^{\mathbf{6}}$ & 0.545 & This work \\
\hline
\end{tabular}




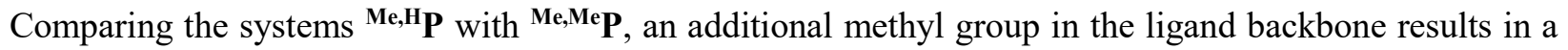
decrease of $k_{o b s}$ by a factor of 3 . Similarly, the ${ }^{\mathbf{p}-\mathbf{T o l}, \mathbf{H}} \mathbf{P}$ system containing a methyl group on the phenyl ring has a lower $k_{o b s}$ than ${ }^{\mathbf{P h}, \mathbf{H}} \mathbf{P}$. Thus an increase in steric bulk of the ligand system tends to slow down the oxygenation reaction process leading to a decrease of the rate constants. This observation is in agreement to that reported in a previous study. ${ }^{[71]}$

\subsubsection{Determining the activation parameters for the formation of 7 and $\boldsymbol{8}$}

Kinetic parameters for dioxygen activation for the formation of $\mathbf{7}$ and $\mathbf{8}$ were obtained from Eyring plots of $\ln (\mathrm{k} / \mathrm{T})$ vs $1 / \mathrm{T}$ from experiments conducted at $203 \mathrm{~K}, 193 \mathrm{~K}, 183 \mathrm{~K}$ and $173 \mathrm{~K}$ for 7 (Fig. 5.20 left) and $193 \mathrm{~K}, 183 \mathrm{~K}$ and $173 \mathrm{~K}$ for $\mathbf{8}$ (Fig. $5.20 \mathrm{right}$ ). The generally reported activation enthalpy for oxygenation of copper(I) complexes is rather small $\left(\Delta H^{*}\right.$ on $\approx 5$ to $\left.10 \mathrm{kcal} \mathrm{mol}^{-1}\right)$, and usually in conjunction with an unfavorable activation entropy $\left(\Delta S_{\text {on }}^{\ddagger} \approx-14\right.$ to $\left.2 \mathrm{cal} \mathrm{K}^{-1} \mathrm{~mol}^{-1}\right) \cdot{ }^{[79]}$
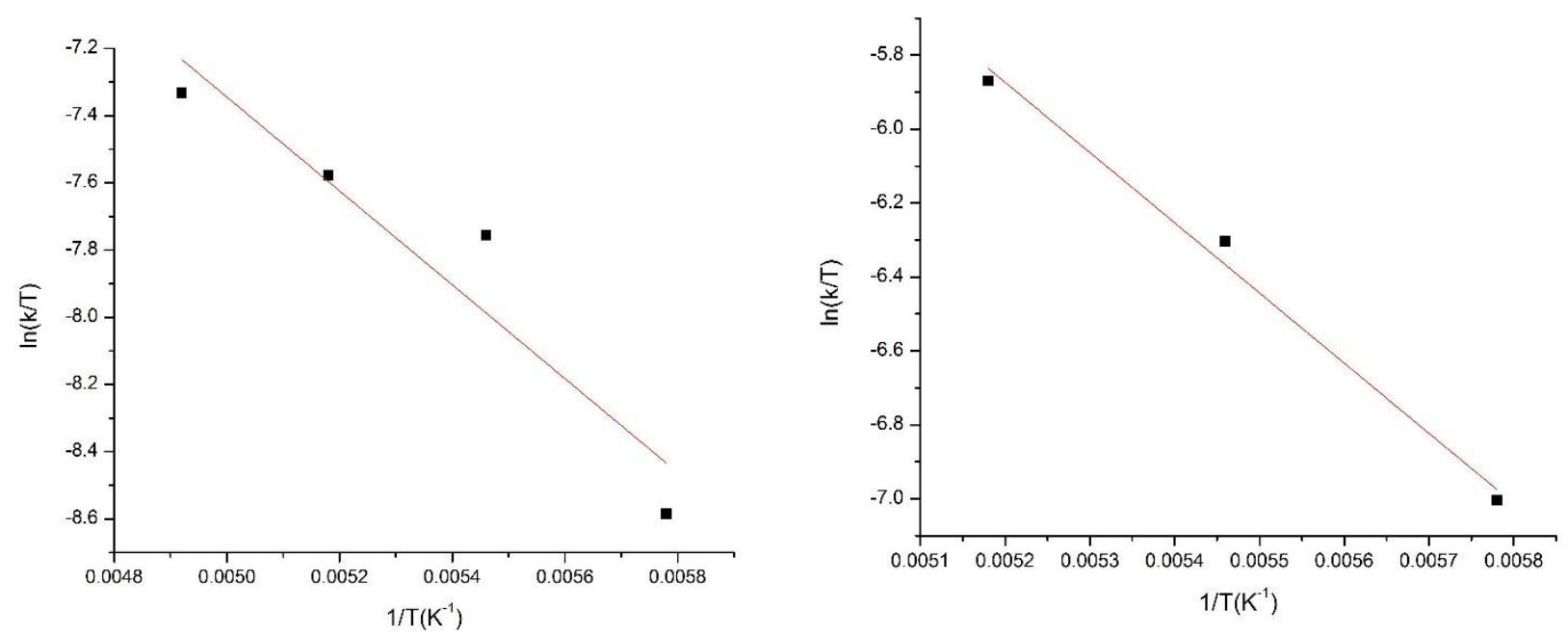

Fig 5.20 Left: Eyring plot of $\ln (\mathrm{k} / \mathrm{T})$ vs $1 / \mathrm{T}$ for the formation of 7 . Right: Eyring plot of $\ln (\mathrm{k} / \mathrm{T})$ vs $1 / \mathrm{T}$ for the formation of 8 .

The activation enthalpies, $\Delta H^{*}$ for the formation of 7 and $\mathbf{8}$ were calculated at $2.77 \pm 0.64 \mathrm{kcal} \mathrm{mol}^{-1}$ (Fig. 5.20 left) and $3.68 \pm 0.3 \mathrm{kcal} \mathrm{mol}^{-1}$ (Fig. 5.20 right) respectively. These rather small values are indicative of a low barrier, which is representative of a diffusion controlled process. Structural elucidation of the copper(I) complexes, $\mathbf{3}$ and $\mathbf{4}$ display the copper(I) centers to be coordinated by the capping ligand and an acetonitrile moiety, with low steric hindrance around the metal. This would permit direct attack of dioxygen on the vacant coordination sites around the copper center with high feasibility and low inhibition, thus resulting in an almost barrier free process. The slightly higher $\Delta H^{*}$ values that have been reported for synthetic $\mathrm{Cu} / \mathrm{O}_{2}$ systems mainly comprise tridentate ligands, which are bulkier in nature in comparison to the bidentate BOX ligands. This results in a higher steric bulk around the metal, leading to larger magnitudes of activation enthalpy. 
The unfavorable activation entropies $\Delta S^{\ddagger}$ of $-47.9 \pm 3.4 \mathrm{calK}^{-1} \mathrm{~mol}^{-1}$ for the formation of 7 , and $-39.2 \pm 2$ $\mathrm{calK}^{-1} \mathrm{~mol}^{-1}$ for the formation of $\mathbf{8}$ can be explained by the thermolability of the system, along with the steric bulk of the methyl groups on the oxazoline ring. The strong negative entropies result in the rates being entropically controlled. Thus, the rate of dioxygen binding to the $\mathrm{Cu}(\mathrm{I})$ complexes in solution $\left(0.098 \mathrm{~min}^{-1}\right.$ for 7 and $0.54 \mathrm{~min}^{-1}$ for 8 ) is rather slow. The associative mechanism reflected in the large negative magnitudes of $\Delta S^{\sharp}$ is suggestive of fewer degrees of freedom available for energy distribution after dioxygen binds to the copper site. Table 5.3 gives an overview of the activation parameters for the formation of ${ }^{\mathbf{S}} \mathbf{P}$ complexes $\mathbf{7}$ and $\mathbf{8}$.

Table 5.3 Activation parameters for the formation of $\mathbf{7}$ and $\mathbf{8}$ at low temperatures in THF.

\begin{tabular}{cccc}
\hline${ }^{\text {SP }}$-Complex & $\begin{array}{c}\Delta H^{\dagger} \\
\mathrm{kcal} \mathrm{mol}^{-1}\end{array}$ & $\begin{array}{c}\Delta S^{\ddagger} \\
\mathrm{cal} \mathrm{K}^{-1} \mathrm{~mol}^{-1}\end{array}$ & \\
\hline${ }^{\mathrm{p}-\mathrm{Tol} P} \mathrm{P}(\mathbf{7})$ & $2.77 \pm 0.64$ & $-47.9 \pm 3.4$ & This work \\
${ }^{\mathrm{Me}, \mathrm{Me} P(8)}$ & $3.68 \pm 0.3$ & $-39.2 \pm 2$ & This work \\
& & & \\
\hline
\end{tabular}

The entropically most disfavored reaction for ${ }^{\mathbf{p}-\mathbf{T o l}, \mathbf{H}} \mathbf{P}(7)$ is reflected in its $k_{o b s}$, which is the smallest in comparison to all other $\mathrm{Cu}(\mathrm{BOX}){ }^{\mathbf{S}} \mathbf{P}$ complexes.

\subsection{Conclusion}

In summary, the $\mathrm{Cu}(\mathrm{I})$ complexes of ligands $\mathbf{H} \mathbf{L}^{1}, \mathbf{H L}^{2}, \mathbf{H} \mathbf{L}^{5}$ and $\mathbf{L}^{6}$ were prepared and characterized with structural elucidation in case of $\mathbf{H L}^{1}, \mathbf{H L}^{5}$ and $\mathbf{L}^{6}$. The former two exist solely in their monomeric forms in the solid state, while the latter coexists with its dimer. The dioxygen reactivity of all $\mathrm{Cu}(\mathrm{I})$ complexes mentioned above generated their dicopper(II) $\left(\mu-\eta^{2}: \eta^{2}-\mathrm{O}_{2}\right)$ complexes. This was investigated in detail in solution. The ${ }^{\mathbf{S}} \mathbf{P}$ complex of $\mathbf{H L}^{2}$ was successfully characterized via X-ray crystallography, which unambiguously determined the binding mode of dioxygen within the complex. Moreover, the O-O bond length measured herein is the longest in any biological or synthetic analogue.

The new ligand system $\mathbf{L}^{6}$ which was introduced to extend the range of BOX ligand scaffolds and study their impact on $\mathrm{Cu} / \mathrm{O}_{2}$ chemistry, proved to be quite successful. The dicopper(II) $\left(\mu-\eta^{2}: \eta^{2}-\mathrm{O}_{2}\right)$ complex was characterized both in solution and in the solid state. UV-vis spectroscopic features along with data obtained from $\mathrm{rR}$ studies were shown to be consistent with other reported ${ }^{\mathbf{S}} \mathbf{P}$ complexes. Magnetic measurements revealed very strong antiferromagnetic coupling between the two cupric ions, as is expected for ${ }^{\mathbf{S}} \mathbf{P}$ complexes. Final determination of the dioxygen binding mode was elucidated by X-ray crystallography measurements. The O-O bond length of ${ }^{5} \mathbf{P}$ complex with $\mathbf{L}^{6}$ was also unusually long, but this did not translate into any unusual $\mathrm{O}-\mathrm{O}$ stretching vibration (as observed in $\mathrm{rR}$ measurements). 
Thus, two new ${ }^{\mathbf{S} P}$ complexes were structurally characterized during the course of this work. It should be noted that structural characterization of synthetic ${ }^{\mathbf{S}} \mathbf{P}$ analogues is a challenging task, and only a hand-full of examples are known in literature.

The kinetic and activation parameters for the formation of ${ }^{\mathbf{S}} \mathbf{P}$ complexes with the new ligand systems $\mathbf{H L}^{\mathbf{5}}$ and $\mathbf{L}^{6}$ were also determined. The electronic effect of the methyl group introduced in the design of $\mathbf{H L}^{\mathbf{5}}$ was found to have a significant impact on the $\mathrm{Cu} / \mathrm{O}_{2}$ system, which was observed in its $k_{o b s}$ value.

In conclusion, bis-oxazoline ligands are simple and bidentate in nature. Termed as a privileged class of ligands, they have been extensively used in asymmetric catalysis but not explored in small molecule activation. Their use in $\mathrm{Cu} / \mathrm{O}_{2}$ chemistry has proved to be of significant benefit, which is reflected in their capacity to lead to crystalline ${ }^{\mathbf{S}} \mathbf{P}$ complexes, a challenging task. This makes this class of ligands privileged in the field of bio-inorganic chemistry as well. Moreover, the simplicity and proton responsive nature of the ligand systems has led to insights into the plausible mechanism of substrate reactivity and equilibrium studies. This shall be discussed in Chapters 7 and 8 . 
[Note: This page has intentionally been left blank] 


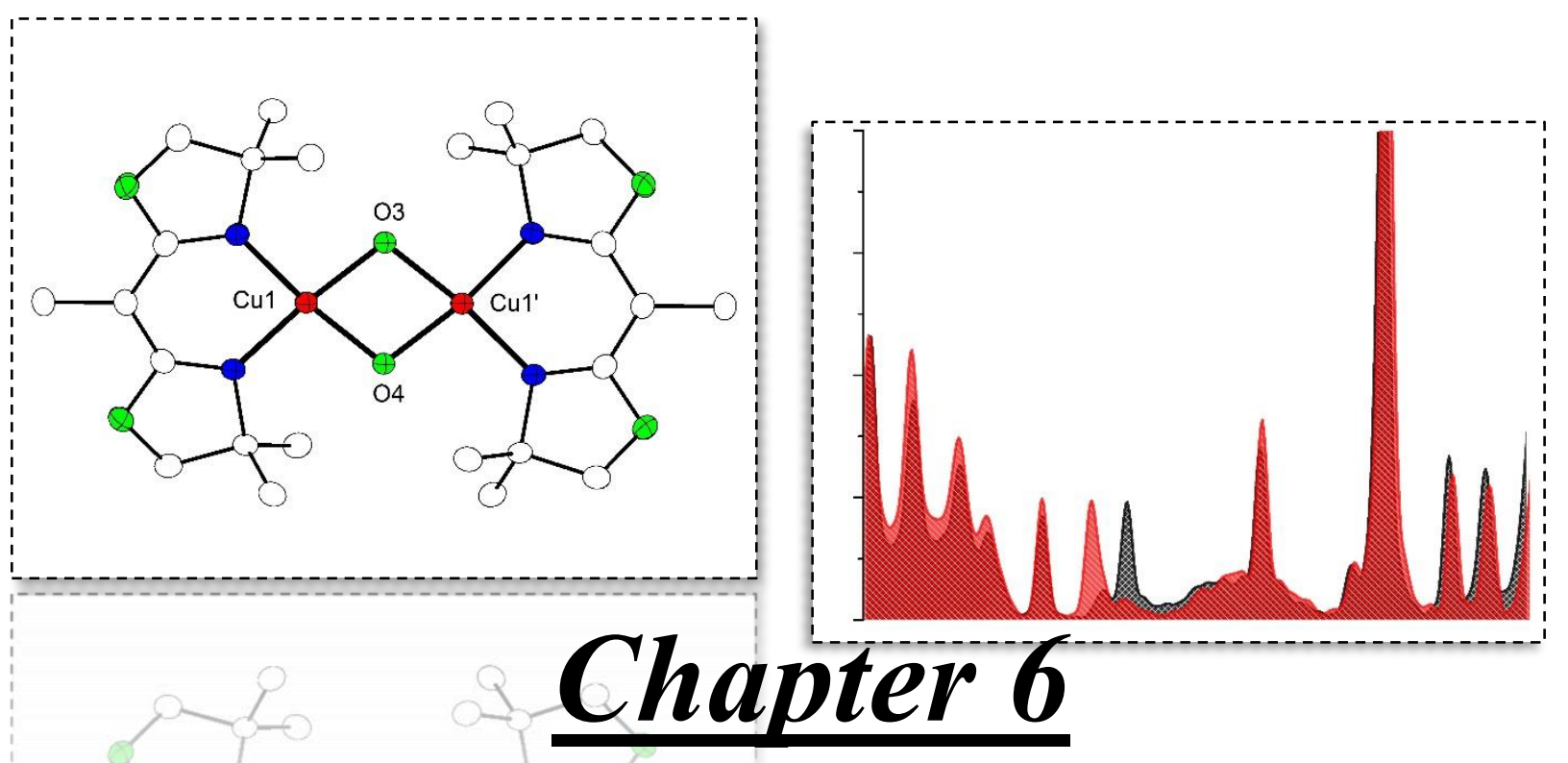

\section{Dicopper(III) bis( $\mu$-oxo) complexes of}

\section{BOX Ligands}




\section{Overview}

6.1 Introduction

6.2 Synthesis and characterization of copper(I) complexes with mono-anionic BOX ligands $\left[\mathbf{L}^{2}\right]^{-}$and $\left[\mathbf{L}^{3}\right]^{-}$

6.3 Dioxygen reactivity of $\mathbf{1 3}$ and $\mathbf{1 4}$ at low temperatures to yield nobel $\mathrm{Cu} / \mathrm{O}_{2}$ intermediates 6.3.1 Structural elucidation of a rare neutral dicopper(III) bis $(\mu$-oxo) complex (10)

6.3.2 Properties in solution

6.3.3 Resonance Raman Spectroscopy

6.3.4 ${ }^{1} \mathrm{H}$ NMR Spectroscopy

6.4 The effect of temperature on the bis( $\mu$-oxo) core

6.5 Conclusion

[Note: Experimental procedures for this chapter are described in detail under Chapter 15, section $15.4]$ 


\subsection{Introduction}

The proton responsive BOX ligands that were previously synthesized demonstrated reversible dioxygen binding to form their $\mu-\eta^{2}: \eta^{2}$-peroxodicopper(II) complexes. Spectroscopic characterization of the adducts in solution and in the solid state confirmed these to be ${ }^{\mathbf{S}} \mathbf{P}$ systems. ${ }^{[71]}$ Furthermore, evidence that certain free bis(oxazoline)s, ${ }^{\mathrm{R}, \mathrm{H}} \mathrm{BOX}$ exist as an equilibrium mixture between the diimine and iminoenamine tautomers, suggested that ${ }^{\mathrm{R}, \mathrm{H}} \mathrm{BOXs}$ may serve as proton responsive ligands. ${ }^{[65]}$ The iminoenamine tautomer is reminiscent of $\beta$ diketiminates that have been extensively used as anionic ligands after deprotonation. ${ }^{[174]}$ With that in mind, two new monoanionic-bidentate ligands, $\left[\mathbf{L}^{2}\right]^{-}$and $\left[\mathbf{L}^{3}\right]^{-}$, were synthesized from their protonated counterparts as lithium salts to study the electronic effects on $\mathrm{Cu} / \mathrm{O}_{2}$ chemistry.

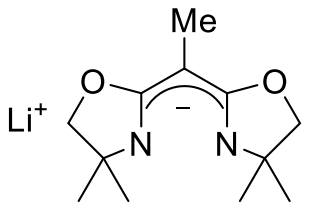

$\left[\mathrm{L}^{2}\right]^{-}$

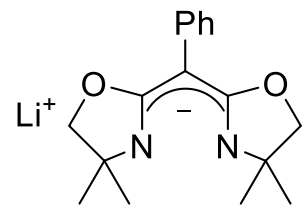

$\left[\mathrm{L}^{3}\right]^{-}$

Figure 6.1 New monoanionic-bidentate ligand systems $\left[\mathbf{L}^{2}\right]^{-}$and $\left[\mathbf{L}^{3}\right]^{-}$employed in the present study of copper dioxygen chemistry.

\subsection{Synthesis and characterization of Copper(I) complexes with mono-anionic BOX ligands $\left[\mathrm{L}^{2}\right]^{-}$and $\left[\mathbf{L}^{3}\right]^{-}$}

Ligands $\left[\mathbf{L}^{2}\right]^{-}$and $\left[\mathbf{L}^{3}\right]^{-}$were individually treated with 1.1 equivalents of tetrakis(acetonitrile)copper(I) perchlorate in THF to yield air sensitive yellow and blue green colored solutions of their $\mathrm{Cu}(\mathrm{I})$ complexes (13 and 14) respectively (Scheme 6.1). ESI-MS analysis of the yellow copper(I) complex 13 in THF (Fig 6.2 left), revealed one major peak belonging to $\left(\left[\mathbf{L}^{2}\right]^{-} \mathrm{Cu}(\mathrm{I})(\mathrm{MeCN})_{2} \mathrm{Li}\right)^{+}(\mathrm{m} / z=376.1)$. The two other peaks could be assigned to $\left(\left[\mathbf{L}^{2}\right]^{-} \mathrm{Cu}(\mathrm{I})(\mathrm{MeCN}) \mathrm{H}\right)^{+}(\mathrm{m} / z=328.1)$ and $\left[\left(\left[\mathbf{L}^{2}\right]^{-}\right)_{2} \mathrm{Cu}(\mathrm{I})\right]^{+}(\mathrm{m} / z=509.1)$. ESI-MS analysis of the blue-green copper(I) complex 14 in THF (Fig 6.2 right), revealed one major peak belonging to $\left(\left[\mathbf{L}^{3}\right]^{-} \mathrm{Cu}(\mathrm{I})(\mathrm{MeCN}) \mathrm{H}\right)^{+}(\mathrm{m} / z=390.1)$ and a second peak belonging to $\left[\left[\mathbf{L}^{3}\right]^{-} \mathrm{Cu}(\mathrm{I}) \mathrm{H}\right]^{+}(\mathrm{m} / z=349.1)$
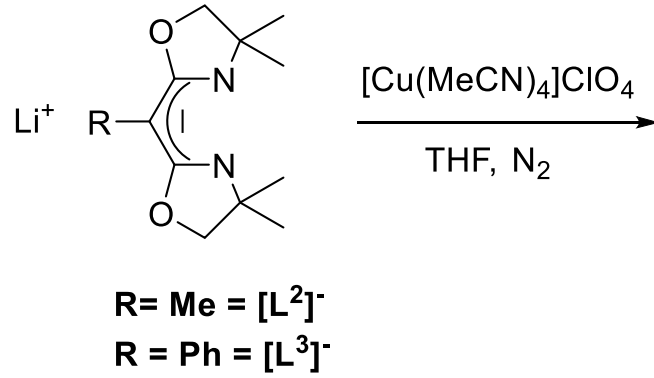<smiles>[R]C1=C2OCC(C)(C)N2CN(C)C1(C)C</smiles>

$$
\begin{aligned}
& R=M e=13 \\
& R=P h=14
\end{aligned}
$$

Scheme 6.1 General scheme for the synthesis of $\mathbf{1 3}$ and $\mathbf{1 4 .}$ 

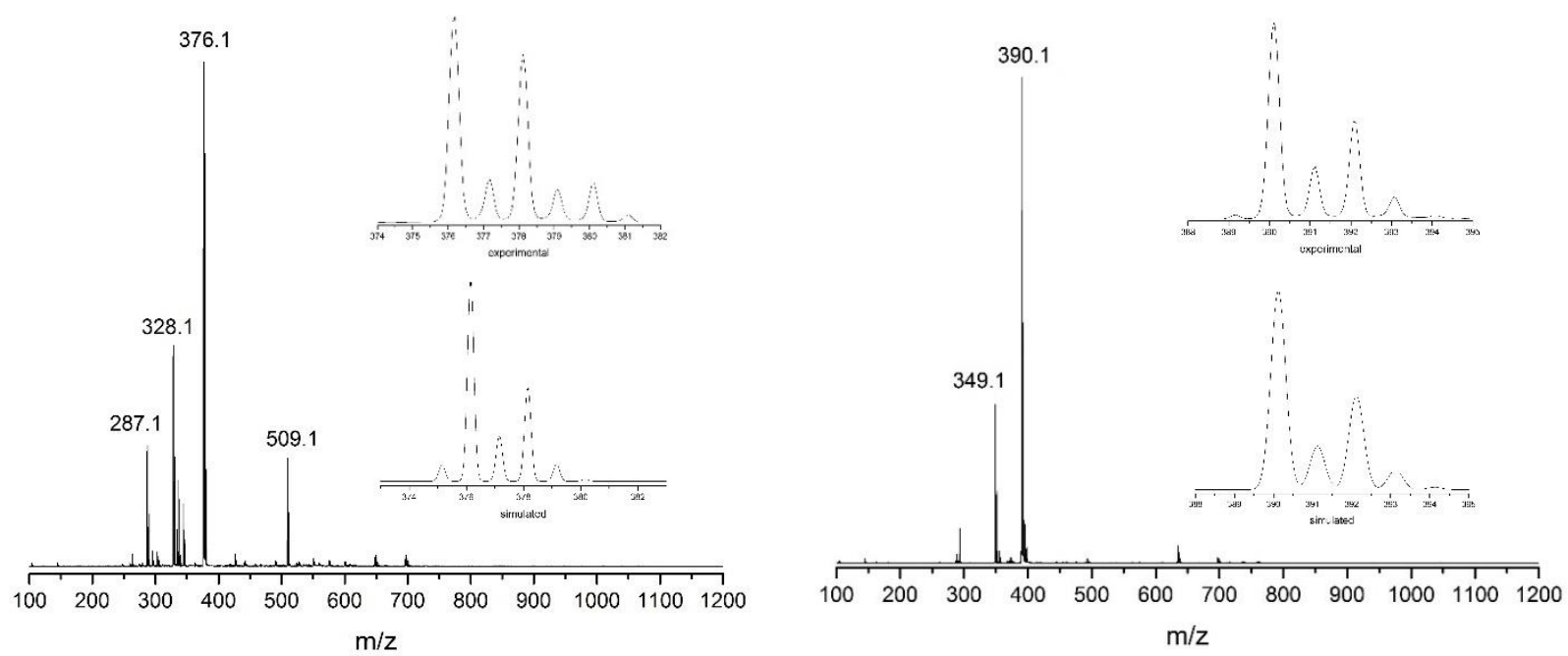

Figure 6.2 Left: ESI-MS of 13 in THF. The two major peaks could be assigned to the copper(I) complex of [ $\left.\mathbf{L}^{2}\right]^{-}$, differing by an acetonitrile molecule, a lithium ion and a proton. The inset shows an enlargement of the peak at $\mathrm{m} / z=376.1$, together with a simulation of the isotopic pattern corresponding to $\left[\left[\mathbf{L}^{2}\right]^{-} \mathrm{Cu}(\mathrm{I})(\mathrm{MeCN})_{2} \mathrm{Li}\right]^{+}$. Right: ESI-MS of 14 in THF. The two major peaks could be assigned to the copper(I) complex of $\left[\mathbf{L}^{3}\right]^{-}$and a proton, differing by an acetonitrile molecule. The inset shows an enlargement of the peak at $m / z=390.1$, together with a simulation of the isotopic pattern, corresponding to [[ $\left.\mathbf{L}^{3}\right]$ $\mathrm{Cu}(\mathrm{I}) \mathrm{MeCNH}]^{+}$.

The diamagnetic nature of complexes 13 and 14 enabled ${ }^{1} \mathrm{H}-\mathrm{NMR}$ spectroscopy as a means of characterization, Fig. 6.3 (for 13) and Fig 6.4 (for 14). The well resolved signals with corresponding integrals indicate a high symmetry for both the molecules in solution. The complexes were also analyzed by ${ }^{13} \mathrm{C}\left\{{ }^{1} \mathrm{H}\right\}$ NMR spectroscopy.

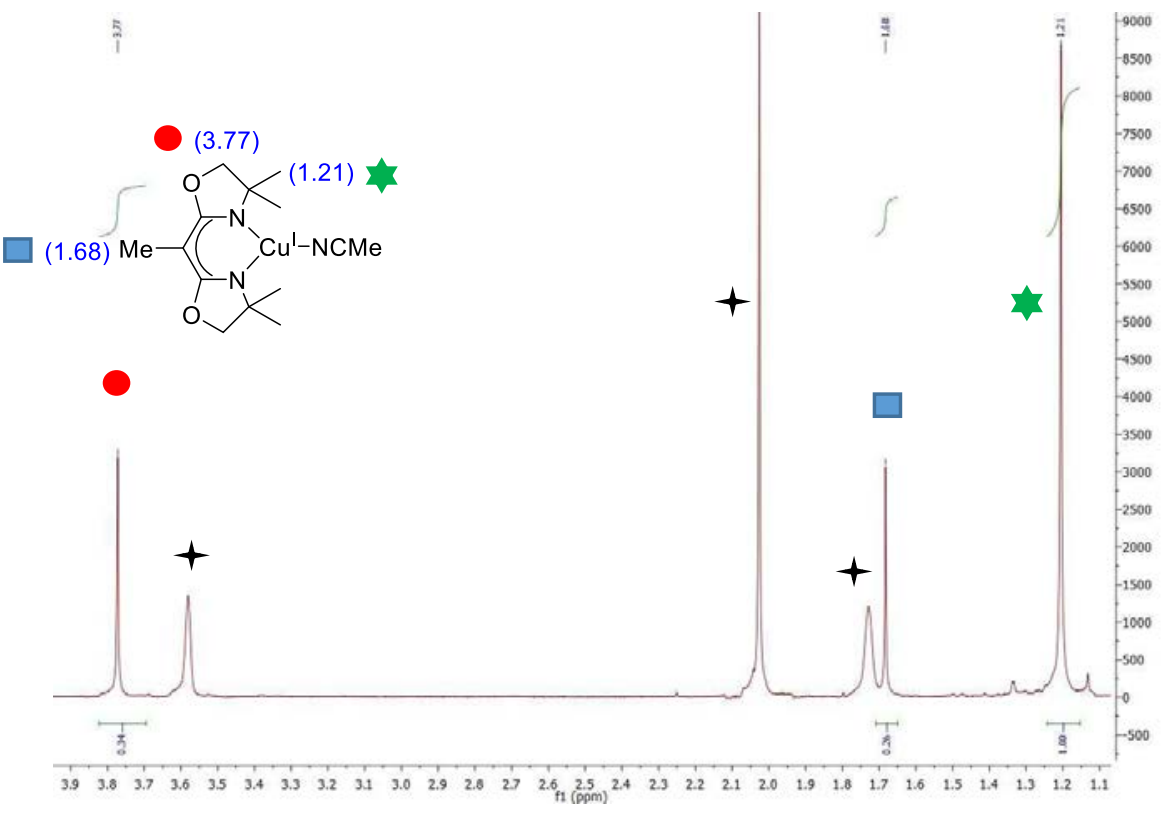

Figure 6.3 ${ }^{1} \mathrm{H}$ NMR spectrum of 13 in THF-d 8 at 293 K. Solvent molecules marked with + . 


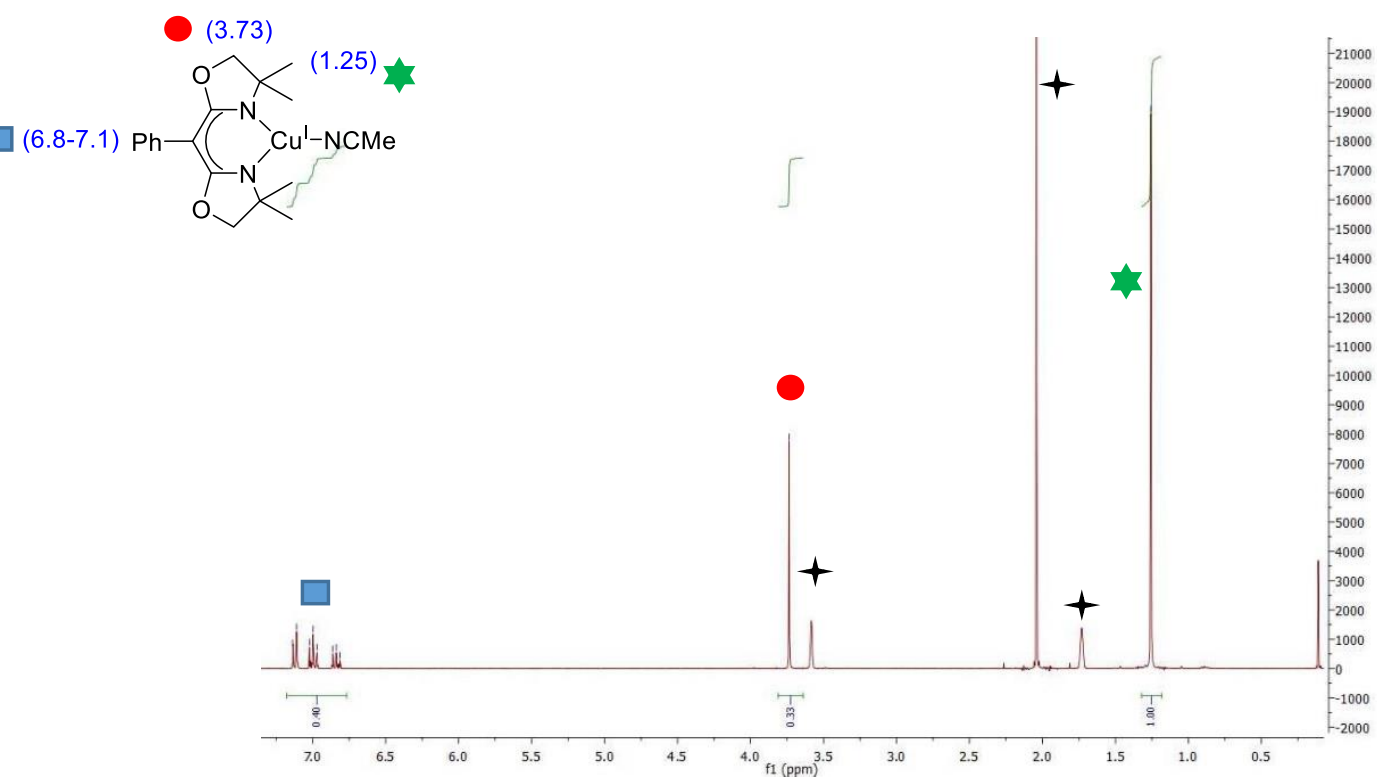

Figure 6.4 ${ }^{1} \mathrm{H}$ NMR spectrum of $\mathbf{1 4}$ in THF-d 8 at 293 K. Solvent molecules marked with + .

\subsection{Dioxygen reactivity of 13 and 14 at low temperatures to yield $\mathrm{Cu} / \mathrm{O}_{2}$ intermediates}

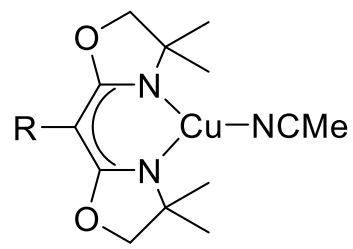

$$
\begin{aligned}
& R=M e=13 \\
& R=P h=14
\end{aligned}
$$

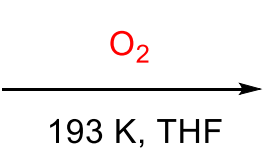

$193 \mathrm{~K}, \mathrm{THF}$

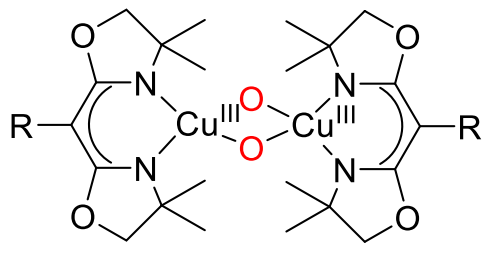

$R=M e=10$

$R=P h=11$

Scheme 6.2 General scheme for the activation of dioxygen with $\mathbf{1 3}$ and $\mathbf{1 4}$ to form $\mathbf{1 0}$ and $\mathbf{1 1}$, respectively, in THF solution at $193 \mathrm{~K}$. 


\subsubsection{Structural elucidation of a rare neutral dicopper(III) bis( $\mu$-oxo) complex (10)}

Single crystals of $\mathbf{1 0}$ suitable for X-ray diffraction were grown by slow diffusion of $\mathrm{Et}_{2} \mathrm{O}$ into a 1:1 Me THF:pentane solution at $193 \mathrm{~K}$ which unambiguously confirmed $\mathrm{O}_{2}$ to bind in a bis( $\mu$-oxo) fashion. 10 reveals a molecular structure with a $D_{2}$ symmetry in the solid state. Each copper ion is bonded to one bidentate ligand $\left[\mathbf{L}^{2}\right]^{-}$and two bridging oxo atoms (Figure 6.5 left), making the complex neutral. Though most bis( $\mu$-oxo) dicopper (III) complexes display a rather square planar geometry, in case of 10, the N1$\mathrm{Cu}-\mathrm{N} 2$ and $\mathrm{O} 3-\mathrm{Cu}-\mathrm{O} 4$ coordination planes (of each coppe center) are twisted at an angle of $24.5^{\circ}$, causing a tilt between the two copper coordination planes (Fig. 6.5 right). This tilt deviates the overall complex from bearing an ideal square planar geometry, as would be expected for $\mathrm{Cu}(\mathrm{III})$ ions bearing a coordination number of four. Of the few crytallographically characterized bis( $\mu$-oxo) dicopper (III) complexes, most of them are dicationic or monocationic in nature, with only one neutral complex being reported till date (Table 6.1). The $\mathrm{Cu} \cdots \mathrm{Cu}$ bond distance of $2.86 \AA$ in $\mathbf{1 0}$ is rather long compared to other synthetic analouges, with the $\mathrm{O} \cdots \mathrm{O}$ non bonding interaction being $2.23 \AA$, the shortest ever reported. In comparison to the ${ }^{\mathbf{S} P}$ isomers (4 and 8), which were also sucessfully crystallized during the course of this work, the two Cu-N bonds (1.91 $\AA$ ) and the two $\mathrm{Cu}-\mathrm{O}$ bonds $(1.82 \AA)$ are significantly shorter, as expected for the higher metal oxidation state (1.95 $\AA$ and $1.91 \AA$ for $4.1 .94 \AA$ and $1.92 \AA$ for $\mathbf{8})$. Theoretical studies by Solomon et al. on moleculer orbitals of the ${ }^{\mathbf{S}} \mathbf{P} / \mathbf{O}$ isomers has shown that scission of the $\mathrm{O}-\mathrm{O}$ bond from the ${ }^{\mathrm{S}} \mathbf{P}$ isomer forming the $\mathbf{O}$ congener results in the formation of a filled low energy bonding $\sigma^{*}$ orbital (refer to figure 2.11 of introduction). Interaction of this orbital with the $\mathrm{Cu}-\mathrm{d}_{\mathrm{xy}}$ orbitals is the source of increased bond strength and covalency in $\mathbf{O}$ complexes compared to their ${ }^{\mathrm{S}} \mathbf{P}$ analogues. ${ }^{[226,227]} \mathrm{The} \mathrm{Cu} \cdots \mathrm{Cu}$ bond length $(2.86 \AA)$, and the $\mathrm{O} \cdots \mathrm{O}$ separation $\left(2.23 \AA\right.$ ) in $\mathbf{1 0}$ also suggests the $\mathbf{O}$ form to be more compact in comparison to the ${ }^{\mathbf{S}} \mathbf{P}$ form. Structural evidences revealed the absence of any axially bound ligand to the copper centers, unlike that observed in the ${ }^{\mathbf{S}} \mathbf{P}$ counterpart. This implies that the anionic scaffold in conjunction with the oxo ligand suffice the coordination sphere of the copper centers with respect to number, and electronic requirements. The combined data clearly indicate that the intermediate is indeed $\left[\left(\left[\mathbf{L}^{2}\right]^{-}\right)_{2} \mathrm{Cu}_{2}(\mu-\mathrm{O})_{2}\right]$, the second structurally characterized 'neutral' bis( $\mu$-oxo) dicopper(III) complex reported to date. ${ }^{[171]}$
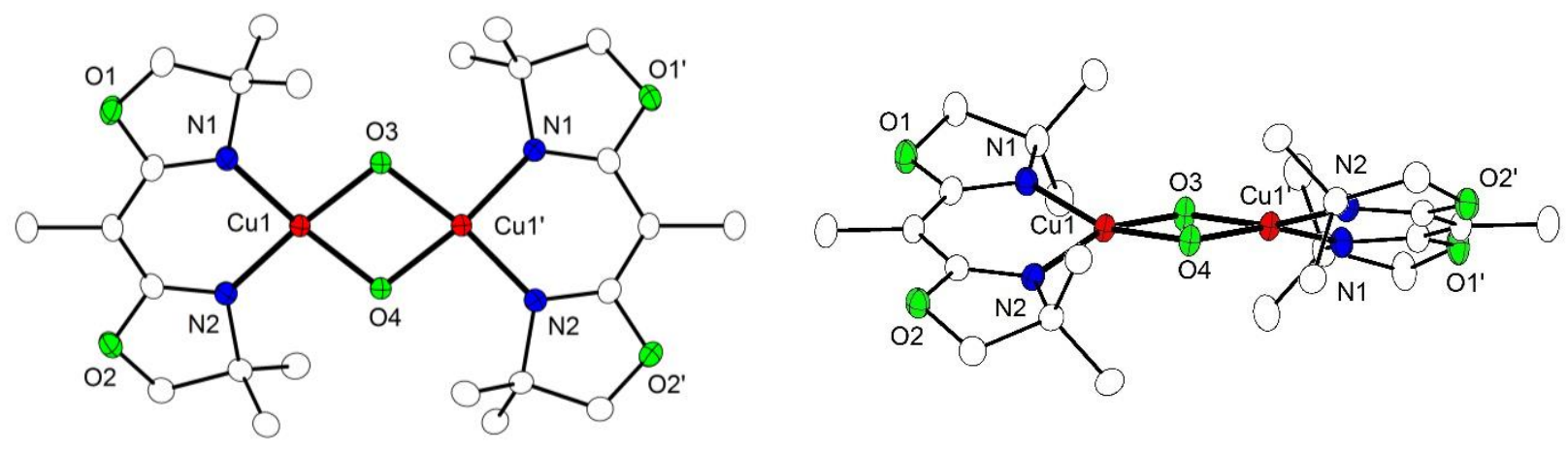

Figure 6.5 Left: Plot (30\% probability thermal ellipsoids) of the molecular structure of $\mathbf{1 0}$ (hydrogen atoms and solvent molecules omitted for clarity). Selected distances $[\AA]$ and angles $\left[{ }^{\circ}\right]$ : Cu1-O3 1.8196(16), Cu1-O4 1.8204(15), Cu1-N1

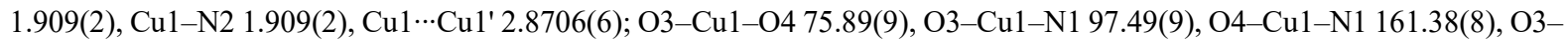
Cu1-N2 161.19(7), O4-Cu1-N2 97.30(9), N1-Cu1-N2 93.98(9), Cu1-O3-Cu1' 104.15(13), Cu1-O4-Cu1' 104.08(12). Symmetry transformation used to generate equivalent atoms: (') $x, 1 / 2-y, 1 / 2-z$. Right: Side view of $\mathbf{1 0}$ demonstrating the tilt between the two copper coordination planes. 
Table 6.1. Geometric and Spectroscopic features of all bis( $\mu$-oxo) dicopper(III) complexes characterized by XRD so far

\begin{tabular}{|c|c|c|c|c|c|c|c|c|}
\hline Ligand/complex & anion & solvent & $\mathrm{Cu} . . \mathrm{Cu}, \AA$ & $\mathrm{O}-\mathrm{O}, \AA \AA$ & Method $^{a}$ & $\begin{array}{l}\text { UV-vis; } \lambda, \mathrm{nm} \\
\left(\varepsilon, \mathrm{mM}^{-1} \mathrm{~cm}^{-1}\right)\end{array}$ & $\begin{array}{l}\text { rR: } v(\mathrm{O}-\mathrm{O}), \\
\mathrm{cm}^{-1} \\
\left(\Delta\left[{ }^{18} \mathrm{O}_{2}\right]\right)\end{array}$ & Ref. \\
\hline$\left[\mathrm{L}^{2}\right]^{-}(10)$ & $a$ & THF & 2.86 & 2.230 & XRD & $\begin{array}{l}297(26.8) \\
335(7.4) \\
395(10.1)\end{array}$ & $598(26)$ & $\begin{array}{l}\text { This } \\
\text { work }\end{array}$ \\
\hline $\mathrm{L}^{\mathrm{Bn} 3}$ & $\mathrm{ClO}_{4}^{-}$ & $\mathrm{CH}_{2} \mathrm{Cl}_{2}$ & 2.794 & 2.287 & XRD & $\begin{array}{l}318(12) \\
430(14)\end{array}$ & $\begin{array}{l}602- \\
608+b(23)\end{array}$ & {$[88]$} \\
\hline$i \operatorname{Pr}_{4} \mathrm{dtne}$ & $\mathrm{SbF}_{6}^{-}$ & $\mathrm{CH}_{2} \mathrm{Cl}_{2}$ & 2.783 & 2.351 & $\mathrm{XRD}$ & $316(13), 414(14)$ & $600(582)$ & {$[169]$} \\
\hline $\mathrm{L}_{\mathrm{ME}}$ & $\mathrm{CF}_{3} \mathrm{SO}_{3}^{-}$ & $\mathrm{CH}_{2} \mathrm{Cl}_{2}$ & 2.743 & 2.344 & $\mathrm{XRD}$ & $306(21), 401(28)$ & $610(23)$ & {$[168]$} \\
\hline$\left[\mathrm{Bu}_{2}^{\mathrm{t}} \mathrm{P}\left(\mathrm{NSiMe}_{3}\right)_{2}\right]^{-}$ & $a$ & Pentane & 2.906 & 2.338 & $\mathrm{XRD}$ & $315(\mathrm{sh}), 444(10)$ & & {$[171]$} \\
\hline $\mathrm{Me}_{2}$ tpa & $\mathrm{PF}_{6}^{-}$ & Acetone & 2.758 & 2.32 & XRD & $\begin{array}{l}378(22) \\
494(0.33)\end{array}$ & $590(26)$ & {$[167]$} \\
\hline $\mathrm{iPr}_{2} / \mathrm{TMPD}^{c}$ & $\mathrm{CF}_{3} \mathrm{SO}_{3}$ & THF & 2.849 & 2.30 & XRD & $398(17)$ & $653(28)$ & {$[176]$} \\
\hline $\mathrm{D}_{4}$-Me $\mathrm{Me}_{2}$-etpy & $\mathrm{ClO}_{4}^{-}$ & Acetone & 2.866 & 2.287 & XRD & $\begin{array}{l}390(19), \\
526(0.33), \\
765(0.06)\end{array}$ & $579(28)$ & {$[170]$} \\
\hline
\end{tabular}

${ }^{a}$ Neutral complex, ${ }^{b}$ Fermi doublet, ${ }^{c}$ Assembeled in a stepwise process from $\left[\left(\mathrm{HMe}_{2} \mathrm{~L}^{i \mathrm{Pr} 2}\right) \mathrm{Cu}\left(\mathrm{O}_{2}\right)\right]$ and $\left[\left(\mathrm{L}^{\mathrm{TMPD}}\right) \mathrm{Cu}(\mathrm{MeCN})\right]^{+}$ 


\subsubsection{Properties in solution}

Oxygenation of $\mathbf{1 3}$ and $\mathbf{1 4}$ at $193 \mathrm{~K}$ in THF resulted in dark green colored solutions of $\left[\left(\left[\mathbf{L}^{2}\right]^{-}\right)_{2} \mathrm{Cu}_{2}(\mu-\mathrm{O})_{2}\right]$ (10) and $\left[\left(\left[\mathbf{L}^{3}\right]^{-}\right)_{2} \mathrm{Cu}_{2}(\mu-\mathrm{O})_{2}\right](\mathbf{1 1})$ (Scheme 6.2) in a matter of seconds. Optical features at $297 \mathrm{~nm}(\varepsilon \approx 26800$ $\left.\mathrm{M}^{-1} \mathrm{~cm}^{-1}\right), 333 \mathrm{~nm}\left(\varepsilon \approx 7440 \mathrm{M}^{-1} \mathrm{~cm}^{-1}\right)$ and $395 \mathrm{~nm}\left(\varepsilon \approx 10140 \mathrm{M}^{-1} \mathrm{~cm}^{-1}\right)$ for 10 (Fig. 6.6 left) and $287 \mathrm{~nm}$ $\left(\varepsilon \approx 36720 \mathrm{M}^{-1} \mathrm{~cm}^{-1}\right), 337 \mathrm{~nm}\left(\varepsilon \approx 13620 \mathrm{M}^{-1} \mathrm{~cm}^{-1}\right)$ and $397 \mathrm{~nm}\left(\varepsilon \approx 10740 \mathrm{M}^{-1} \mathrm{~cm}^{-1}\right)$ for 11 (Fig 6.6 right) were observed on monitoring via $\mathrm{UV}$-vis spectroscopy.
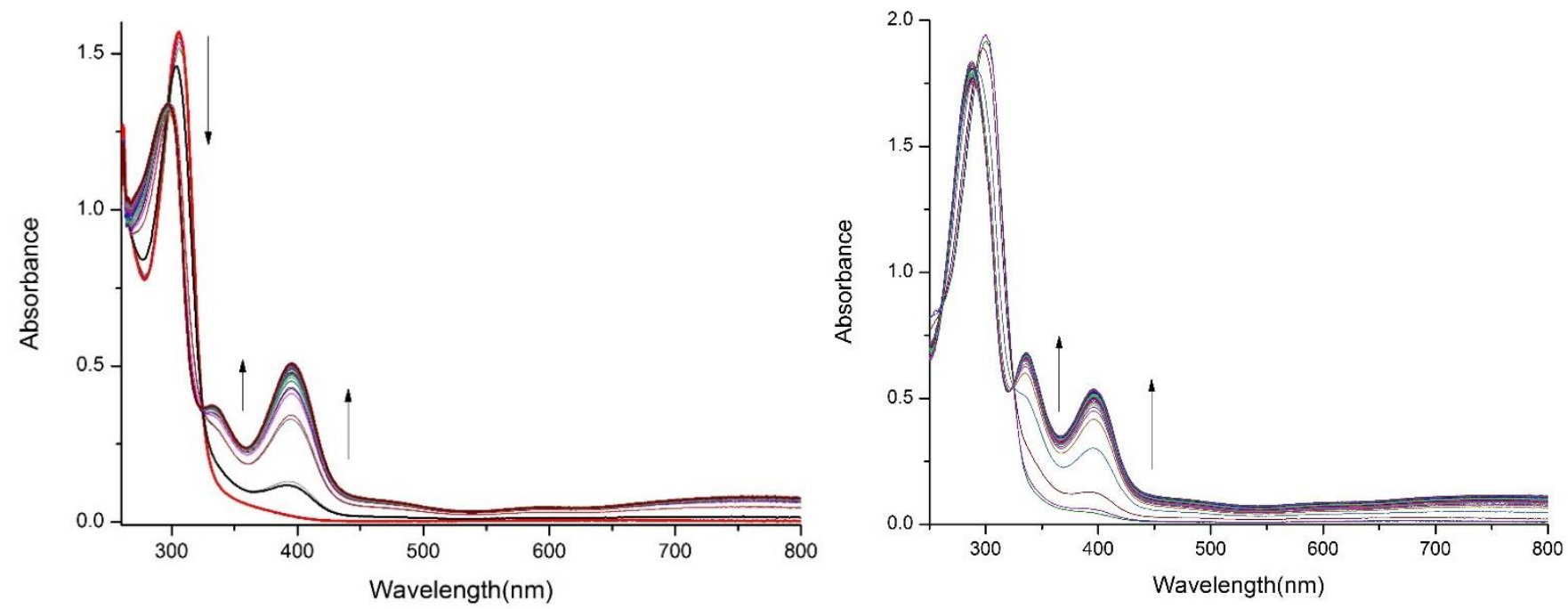

Figure 6.6 Left: Formation of $\left[\left(\left[\mathbf{L}^{2}\right]^{-}\right)_{2} \mathrm{Cu}_{2}(\mu-\mathrm{O})_{2}\right](\mathbf{1 0})$ in THF at $193 \mathrm{~K}$ monitored by UV/Vis spectroscopy. Formation of bands at $333 \mathrm{~nm}$ and $395 \mathrm{~nm}$. Right: Formation of $\left[\left(\left[\mathbf{L}^{3}\right]^{-}\right)_{2} \mathrm{Cu}_{2}(\mu-\mathrm{O})_{2}\right](\mathbf{1 1})$ in THF at $193 \mathrm{~K}$ monitored by UV/Vis spectroscopy. Formation of bands at $335 \mathrm{~nm}$ and $397 \mathrm{~nm}$.

These high intensity features are qualitatively similar to those of bis( $\mu$-oxo) dicopper(III) species. ${ }^{[88,166,168]}$ The former band at $\sim 290 \mathrm{~nm}$ is most likely a ligand based transition. The latter two at $\sim 333 \mathrm{~nm}$ and $\sim 400$ $\mathrm{nm}$ resemble LMCT transitions. The band at $\sim 333 \mathrm{~nm}$ has a similar origin to ${ }^{\mathbf{S}} \mathbf{P}$ complexes and arises from an in plane overlap of the oxo $\pi_{\sigma}{ }^{*}$ to $\mathrm{Cu}(\mathrm{III}) \mathrm{d}_{\mathrm{xy}}$ orbital. ${ }^{\left[{ }^{[3]}\right.}$ In most cases, the intensity is far lower compared to the ${ }^{\mathrm{S}} \mathbf{P}$ counterpart. The feature at $\sim 400 \mathrm{~nm}$ is characteristic for the bis $(\mu$-oxo $)$ core, and is present when the $\mathrm{O}-\mathrm{O}$ bond is cleaved. Absent in ${ }^{\mathbf{S}} \mathbf{P}$ complexes, it originates from an in plane overlap of the new bonding $\sigma^{*}$ orbital of the bis( $\mu$-oxo) ligand and the $d_{x y}$ orbital of copper (refer to sec. 2.3 .3 of introduction, figure 2.9). ${ }^{[93]}$ In comparsion to other bis( $\mu$-oxo) dicopper(III) complexes with both bidentate and tridentate ligand systems, ${ }^{[138,168,169,179,228]}$ the molar absorbtivites exhibited by $\mathbf{1 0}$ and $\mathbf{1 1}$ are rather low. The dicopper(III) bis $\left(\mu\right.$-oxo) complex synthesized by Tolman et al. with beta-diketiminate ligands ${ }^{[174]}$ exhibits a band at 380 $\mathrm{nm}\left(\varepsilon \sim 20,000 \mathrm{M}^{-1} \mathrm{~cm}^{-1}\right)$ and $420 \mathrm{~nm}\left(5000 \mathrm{M}^{-1} \mathrm{~cm}^{-1}\right)$ with the feature at $380 \mathrm{~nm}$ being far more intense. An interesting trend in these CT transitions suggests that longer intermetal distances correlate with lowerintensity transitions. ${ }^{[138,166,168,169,179,228]}$ The crystallographically characterized motif of $\mathbf{1 0}$ displays a fairly 
long $\mathrm{Cu} \cdots \mathrm{Cu}$ bond distance of $2.86 \AA$ (Fig 6.5) and is in accordance with this observation. In analogy, the only other neutral bis( $\mu$-oxo) system apart from 10, reported by Hoffman et al. ${ }^{[171]}$ with the aid of anionic imidophosphanamide ligands (refer to sec. 2.3.3 of introduction), displays similarly low intensities for the two bands. A reason for $\mathbf{1 0}$ and $\mathbf{1 1}$ to exhibit the same trend could be the high basicity of the monoanionic BOX liagnds with nitrogen donors compared to other ligands employed in $\mathrm{Cu} / \mathrm{O}_{2}$ chemistry. Both CT bands are relatively low in energy for LMCT transitions. ${ }^{[171]}$ This fact along with the intensities is suggestive of a high degree of $\mathrm{Cu}-\mathrm{O}$ covalency. In comparison to their protonated counterparts $\mathbf{H L}^{2}$ and $\mathbf{H L}^{3},\left[\mathbf{L}^{2}\right]^{-}$ and $\left[\mathbf{L}^{3}\right]^{-}$essentially have the same ligand environment but lack a proton in the backbone. This peripheral deprotonation causes electronic differences in the ligand scaffold making these deprotonated forms more anionic and suitable to support the +3 oxidation state of copper. Thus preferably the bis $(\mu$-oxo) intermediates form with these basic anionic ligands as opposed to the $\mu-\eta^{2}: \eta^{2}$ peroxo intermediates. Exchanging the solvent to $\mathrm{CH}_{2} \mathrm{Cl}_{2}$ or acetone did not result in the formation of the bis( $\mu$-oxo) species.

\subsubsection{Resonance Raman Spectroscopy}

Further evidence confirming the formation of a dicopper(III) bis( $\mu$-oxo) species was obtained by resonance Raman (rR) spectroscopy with a laser excitation of $633 \mathrm{~nm}$. 1:1 THF:pentane solutions of 13 and THF solutions of 14 were exposed to naturally abundant $\left({ }^{16} \mathrm{O}_{2}\right)$ or isotopically labelled dioxygen $\left({ }^{18} \mathrm{O}_{2}\right)$ at 193 $\mathrm{K}$ and $77 \mathrm{~K}$ respectively in young NMR tubes. Solutions of $\mathbf{1 0}$ in a dry-ice/acetone bath revealed only one oxygen isotope sensitive feature seen with a single peak at $598 \mathrm{~cm}^{-1}$ which shifted to $572 \mathrm{~cm}^{-1}$ on labelling $\left(\Delta^{16} \mathrm{O}_{-}{ }^{18} \mathrm{O}=26 \mathrm{~cm}^{-1}\right)$ (Fig $6.7 \mathrm{left}$ ); the corresponding feature for 11 was found at $601 \mathrm{~cm}^{-1}$ and $573 \mathrm{~cm}^{-1}$ $\left(\Delta^{16} \mathrm{O}-{ }^{18} \mathrm{O}=28 \mathrm{~cm}^{-1}\right)$ (Fig 6.7 right). These features have been assigned to the $\mathrm{Cu}_{2}(\mu-\mathrm{O})_{2}$ core vibration and are signature breathing modes of the $\mathbf{O}$ core. ${ }^{[145]}$ In several other bis( $\mu$-oxo) dicopper (III) systems, having bidentate and tridentate ligands, often more than one oxygen isotope sensitive feature is obsereved on $\mathrm{rR}$ analysis. ${ }^{[88,168,179]}$ Normal modes of vibration for a $D_{2 h} \mathrm{Cu}_{2} \mathrm{O}_{2}$ core from normal coordinate analysis reveal two $a_{\mathrm{g}}$ modes being Raman active in nature $\left(118.6 \mathrm{~cm}^{-1}\left(v_{1}\right)\right.$ and $\left.611.5 \mathrm{~cm}^{-1}\left(v_{3}\right)\right)$. ${ }^{[93]}$ The additional isotope responsive features have been assigned as difference and combination bands between the fundamentals $v_{1}$ and $v_{3}$. In case of complexe $\mathbf{1 0}$ however, though it exhibits a $D_{2 h} \mathrm{Cu}_{2} \mathrm{O}_{2}$ core, most probably the difference and combination bands have a rather low intensity and are thus not observed in the rR spectra. In comparison to other related $\left[\mathrm{M}_{2}(\mu-\mathrm{O})_{2}\right]^{3+}$ units $(\mathrm{M}=\mathrm{Fe}$ or $\mathrm{Mn}),{ }^{[229-231]}$ the vibrational mode of the oxo core is observed around $660-700 \mathrm{~cm}^{-1}$. These slightly higher values result from a combination of factors such as the small mass and high charge of the metal centers, and changes in the M-O-M angles. Several $\mathrm{O}_{2}$ isotope independent peaks were also observed in the rR spectra of complexes 10 and 11 in the $450-550 \mathrm{~cm}^{-1}$ region. These could be assigned to the $\mathrm{Cu}-\mathrm{N}$ stretching modes and have shown to be highly ligand dependent. ${ }^{[3]}$ There was no evidence of the side on peroxo dicopper(II) species which is expected to exhibit the O-O stretching vibration at $720-765 \mathrm{~cm}^{-1}$ with an $\Delta^{18} \mathrm{O}$ shift of $40-50 \mathrm{~cm}^{-1} \cdot[136,218,220]$ 

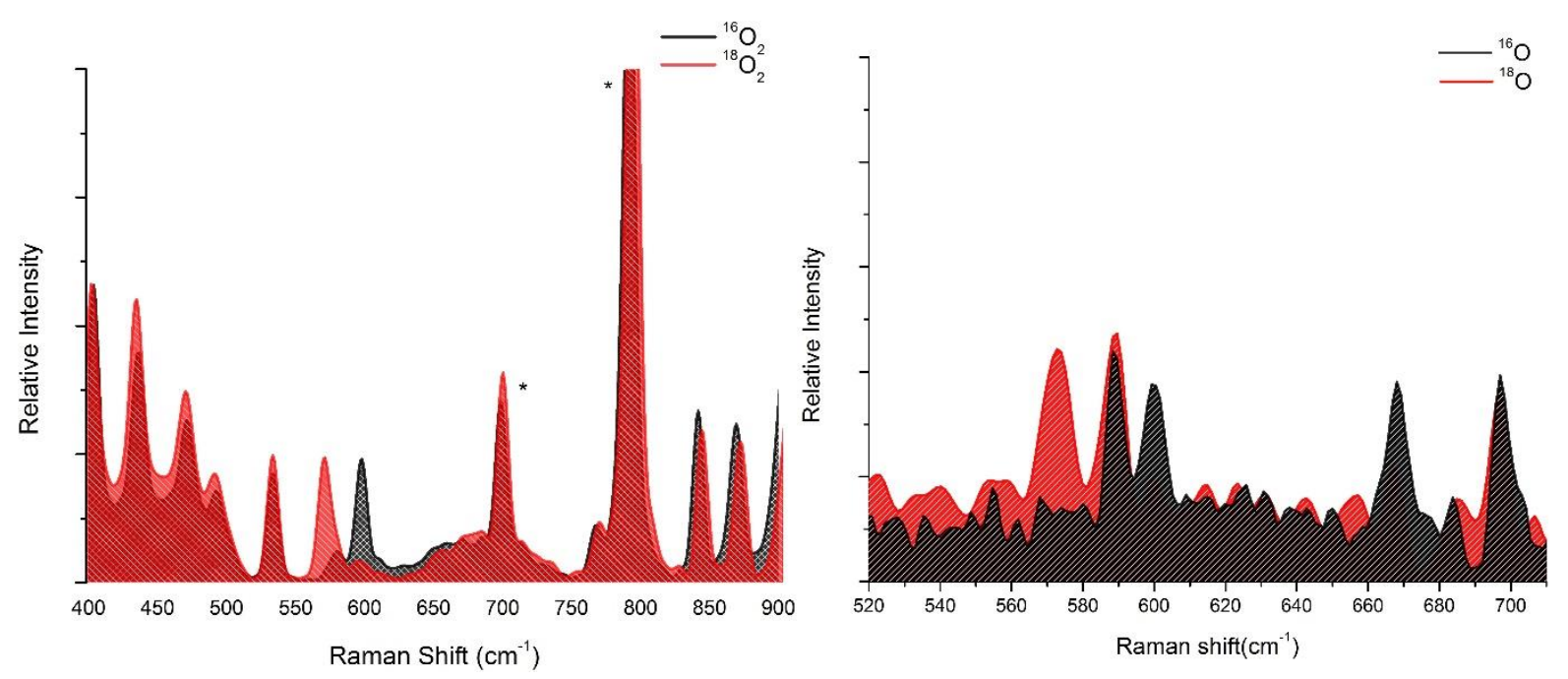

Figure 6.7. Left: Resonance Raman spectrum of 10 in a 1:1 THF:pentane solution at $193 \mathrm{~K} .{ }^{16} \mathrm{O}_{2}$ spectrum indicated in black and ${ }^{18} \mathrm{O}_{2}$ indicated in red. Residual solvent signals are marked with an asterisk (*). Right: Resonance Raman spectrum of 11 in a THF solution at $77 \mathrm{~K} .{ }^{16} \mathrm{O}_{2}$ spectrum indicated in black and ${ }^{18} \mathrm{O}_{2}$ indicated in red. Residual solvent signals are marked with an asterisk $(*)$.

\subsection{4 ${ }^{1} H$ NMR Spectroscopy}

The +3 oxidation state of the copper centers in dicopper(III) bis( $\mu$-oxo) complexes makes them EPR silent and diamagnetic in nature. ${ }^{[138]}$ This enabled ${ }^{1} \mathrm{H}-\mathrm{NMR}$ spectroscopy as a means of characterization for $\mathbf{1 0}$ and $\mathbf{1 1}$ in solution. Solutions of $\mathbf{1 3}$ and $\mathbf{1 4}$ were individually prepared in THF- $\mathrm{d}_{8}$ and exposed to dioxygen at $193 \mathrm{~K}$ in Young NMR tubes. Low temperature ${ }^{1} \mathrm{H}-\mathrm{NMR}$ spectra were measured at $193 \mathrm{~K}$ which enabled assignment of the peaks as shown in figure 6.8 for $\mathbf{1 0}$ and figure 6.9 for $\mathbf{1 1}$. The chemical shifts associated with the starting material, the $\mathrm{Cu}(\mathrm{I})$ complexes are absent in these solutions, suggesting total conversion to the dicopper(III) bis( $\mu$-oxo) species. The small change in shifts signify the absence of paramagnetic contributions ruling out the formation of potential ${ }^{\mathbf{S}} \mathbf{P}$ complexes. For complex 10, the copper bis $(\mu$-oxo) complex of ligand $\left[\mathbf{L}_{2}^{-}\right]$, the signals at $1.21 \mathrm{ppm}$ assigned to the $\mathrm{CMe}_{2}$ groups, and $3.77 \mathrm{ppm}$ assigned to the Me group in the ligand backbone (Fig. 6.3) shift downfield by $0.17 \mathrm{ppm}$ in complex $13 \mathrm{Cu}$ (I) complex of ligand $\left[\mathbf{L}_{2}^{-}\right]$(Fig. 6.8). The protons for the $\mathrm{CH}_{2}$ groups however shift upfield from $1.68 \mathrm{ppm}$ to $1.58 \mathrm{ppm}$. These changes in $\delta$ values could be explained by the change in electron density over the ligand scaffold. The external binding of the bis( $\mu$-oxo $)$ ligand to the copper centers increases their oxidation state from +1 to +3 , resulting in an increase in electron deficiency over the metal centers. This stimulates an increase in the extent of overlap between the anionic ligands and the copper centers, causing a shift in the overall electron distribution and a change in $\delta$ values. The sharp clean peaks present with chemical shifts between 0-10 ppm clearly indicate the compounds to be diamagnetic bis( $\mu$-oxo $)$ dicopper(III) complexes. 


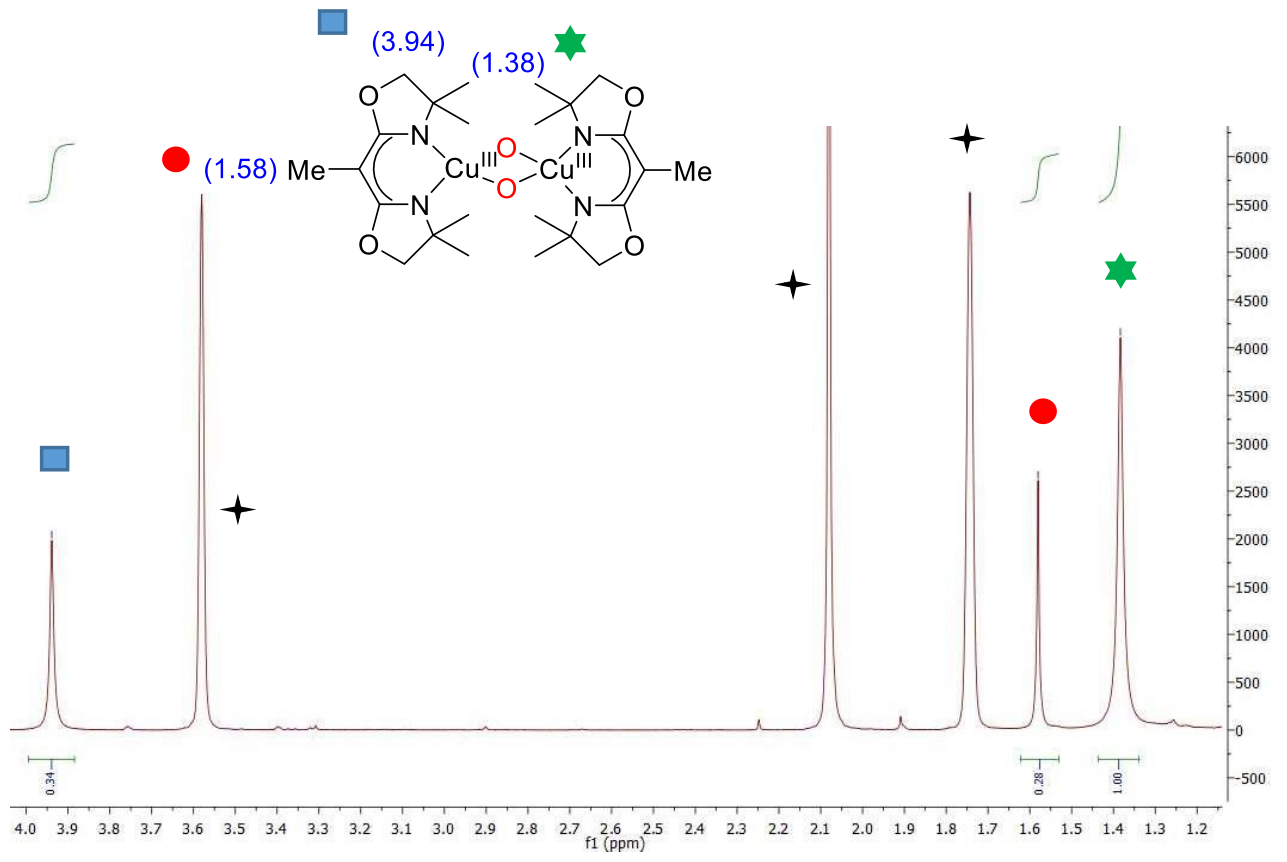

Figure 6.8 ${ }^{1} \mathrm{H}$ NMR spectrum of $\mathbf{1 0}$ in THF-d 8 at $193 \mathrm{~K}$. Solvent molecules marked with + .

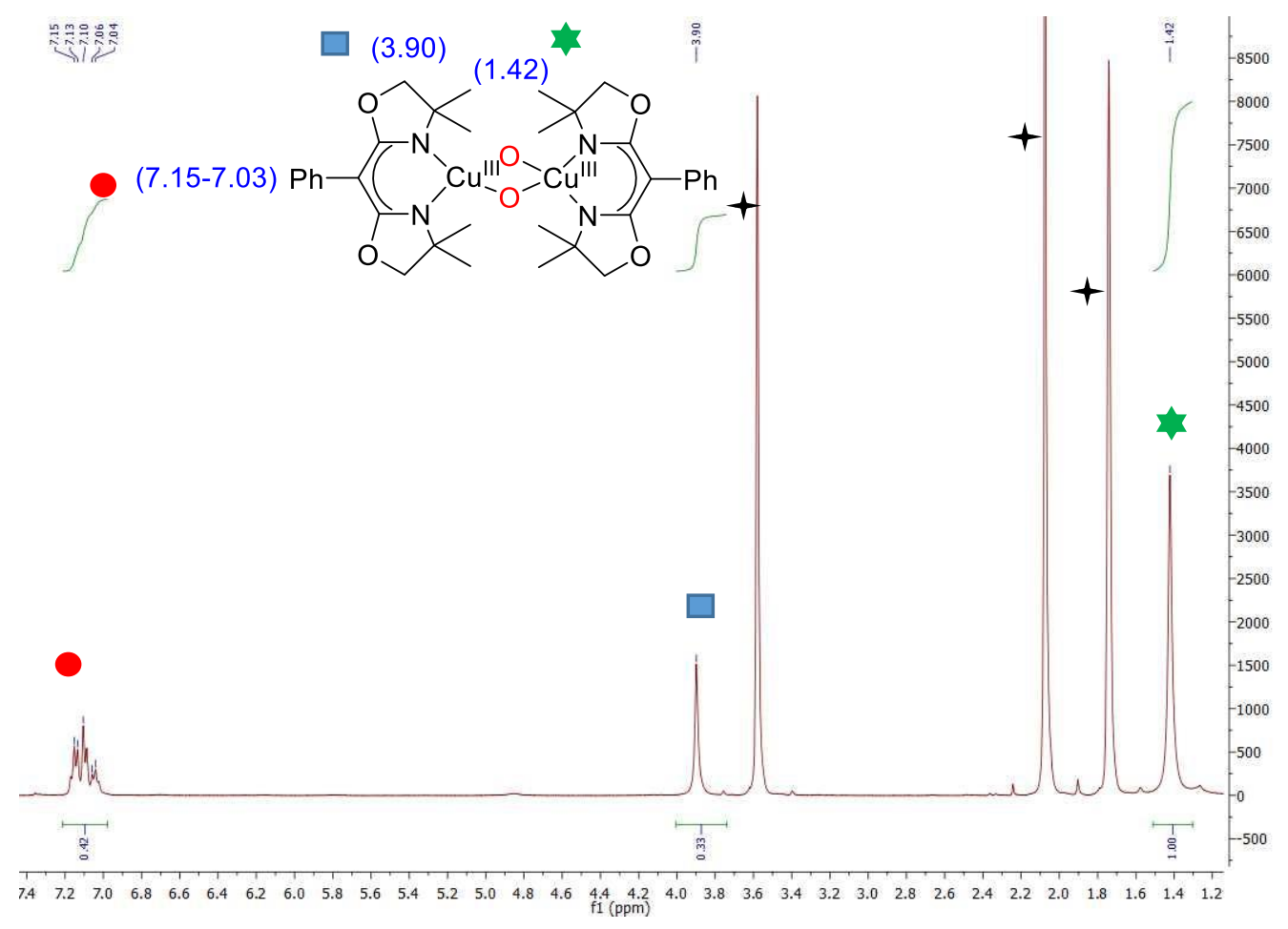

Figure 6.9 ${ }^{1} \mathrm{H}$ NMR spectrum of 11 in THF-d 8 at 193 K. Solvent molecules marked with ${ }^{+}$. 


\subsection{The effect of temperature on the bis( $\mu$-oxo) core}

The CT features executed by bis( $\mu$-oxo) dicopper(III) complexes makes it convenient to characterize these intermediates in solution. The transition arising from $\pi_{\sigma}{ }^{*} \operatorname{bis}(\mu$-oxo $)$ to the $\mathrm{Cu}(\mathrm{III}) \mathrm{d}_{\mathrm{xy}}$ orbital is expressed between $300-350 \mathrm{~nm}$, and that of $\sigma^{*}$ bis $\left(\mu\right.$-oxo) to $\mathrm{Cu}(\mathrm{III}) \mathrm{d}_{\mathrm{xy}}$ is expressed around $400 \mathrm{~nm}$, the latter being characteristic of the bis( $\mu$-oxo) core. Complexes 10 and 11 were characterized in THF solutions at $193 \mathrm{~K}$, where both expressed similar transitions. In case of 10, the transitions at $333 \mathrm{~nm}$ and $395 \mathrm{~nm}$ had an intensity ratio of 1:1.3 (333:395, Fig 6.6 left) and for 11 at $337 \mathrm{~nm}$ and $397 \mathrm{~nm}$ with an intensity ratio of 1:0.7 (337:397, Fig 6.6 right). These bis( $\mu$-oxo) species revealed an interesting effect with temperature variation.

On warming a solution of $\mathbf{1 0}$ from $193 \mathrm{~K}$ to $243 \mathrm{~K}$, the band at $333 \mathrm{~nm}$ started to increase in intensity whereas that at $395 \mathrm{~nm}$ started to decrease as obsereved by UV-vis spectroscopy (Fig 6.10 left). The ratio of the two bands changed from 1:1.3 to 1:0.8 (333:395), with the band at $333 \mathrm{~nm}$ being higher than that of $395 \mathrm{~nm}$ (Fig 6.10 left inset). Maintaing the solution at $243 \mathrm{~K}$ for a substantial duration of time revealed no further changes in the spectra. Successive cooling of the solution from $243 \mathrm{~K}$ back to $193 \mathrm{~K}$ revealed the spectra to regain its original form (Fig. 6.10 right). The ratio of the two bands reverted back to 1:1.3 (Fig. 6.10 right inset) and the spectrum obsereved on initial formation of the complex was restored.
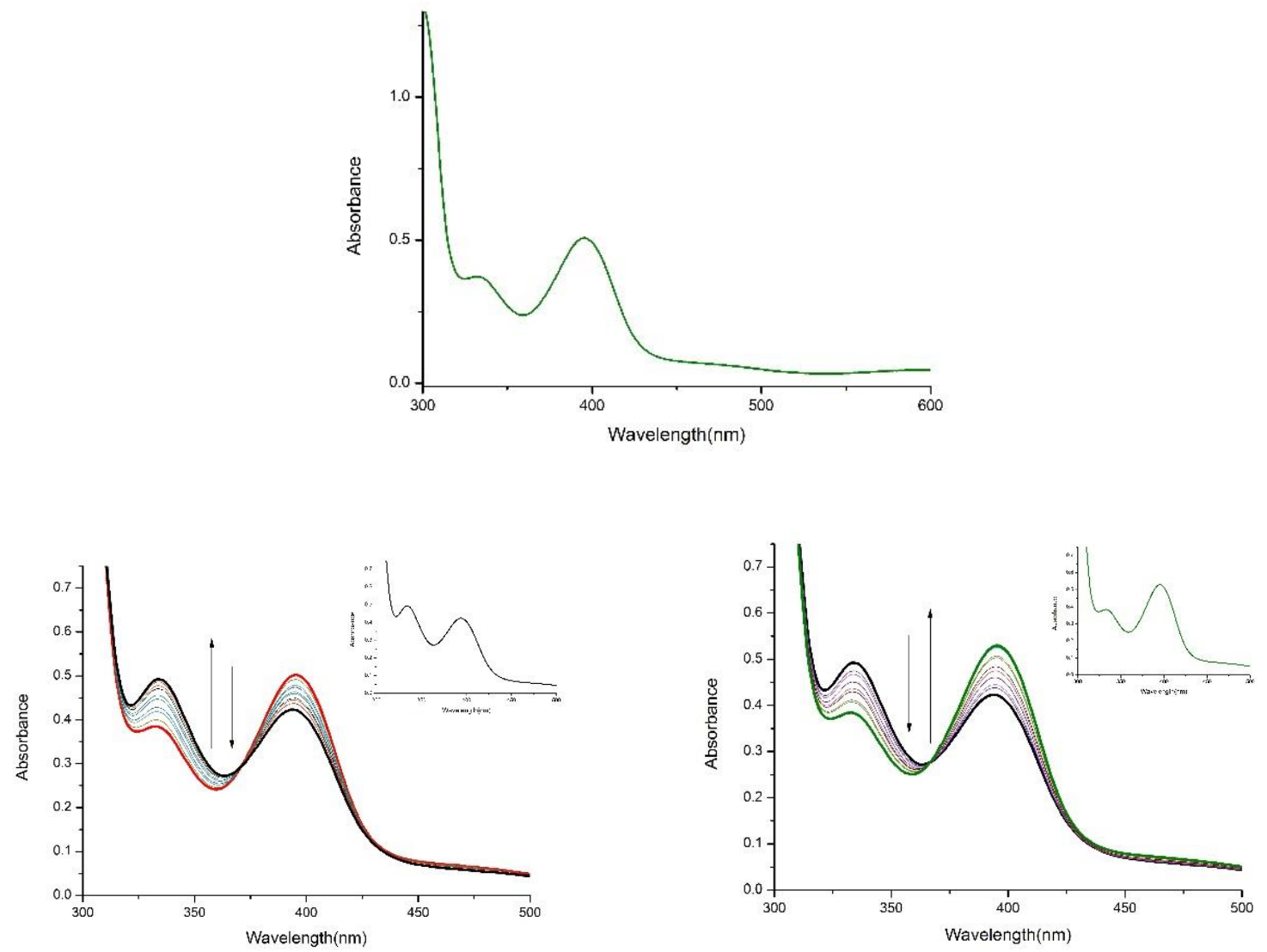

Figure 6.10 Top: UV-vis spectroscopic features of $\mathbf{1 0}$ with characteristic bands at $333 \mathrm{~nm}$ and $395 \mathrm{~nm}$ at $193 \mathrm{~K}$. Left: Temperature variation from $193 \mathrm{~K}$ to $243 \mathrm{~K}$ depicting a gain in intensity at $333 \mathrm{~nm}$, with a loss in intensity at $395 \mathrm{~nm}$. Inset shows the final spectra at $243 \mathrm{~K}$. Right: Spectra obtained on cooling down the solution from $243 \mathrm{~K}$ to $193 \mathrm{~K}$. Resultant spectra obtained at $193 \mathrm{~K}$ is shown in the inset. 


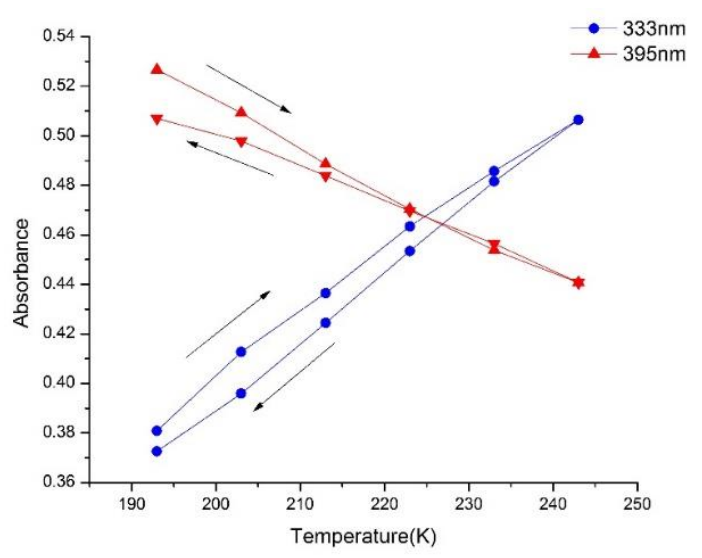

Figure 6.11 Plot of the absorbance of bands at $333 \mathrm{~nm}$ (blue) and $395 \mathrm{~nm}$ (red) for $\mathbf{1 0}$ on warming up (193 K to $243 \mathrm{~K}$ ) and cooling down ( $243 \mathrm{~K}$ to $193 \mathrm{~K})$.

The change in the intensity of the two bands with temperature (333 nm and $395 \mathrm{~nm}$ ) for complex $\mathbf{1 0}$ is depicted in figure 6.11, also showing the reversibility. A slight loss in overall absorbance of both the bands when reverting back from $243 \mathrm{~K}$ to $193 \mathrm{~K}$ indicates a possible onset of decomposition of the complex on warming the solution.

The same phenomenon was observed for 11, as well as for bis( $\mu$-oxo $)$ complexes formed from their respective ${ }^{\mathrm{S}} \mathbf{P}$ counterparts with a base (complexes 9 and 12, refer to Chapter 7). In case of $\mathbf{1 0}$ and 11, rR spectra of their frozen solutions revealed only one oxygen sensitive isotope feature belonging solely to the $\mathbf{O}$ isomer (Fig. 6.7). Moreover, characterization of these complexes via low temperature ${ }^{1} \mathrm{H}$ NMR spectroscopy gave rise to clean sharp signals between 0-10 ppm indicative of diamagnetic species within solution (Fig. 6.8 and 6.9). Additionally, DOSY NMR of $\mathbf{1 0}$ at $193 \mathrm{~K}$ in THF-d $\mathrm{d}_{8}$ revealed only a single species in solution. These results suggest the absence of any ${ }^{\mathbf{S}} \mathbf{P}$ species.

In order to investigate weather solvent binding/dissociation occurs with temperature, a VT- ${ }^{1} \mathrm{H}$ NMR studies of complex 10 between $193 \mathrm{~K}$ and $243 \mathrm{~K}$ was analyzed (Fig 6.12 and 6.13). The spectra obtained demonstrated no major shift in peaks of the bis( $\mu$-oxo) dicopper (III) complex, except for a slight broadening as a result of change in viscosity of the deuterated solvent at low temperatures. Additionally, no new peaks appeared on warming or cooling of the solution. This implied that spectroscopic changes with temperature variation did not result from solvent binding/dissociation and corroborates the absence of any ${ }^{\mathbf{S}} \mathbf{P}$ species forming with temperature change. 


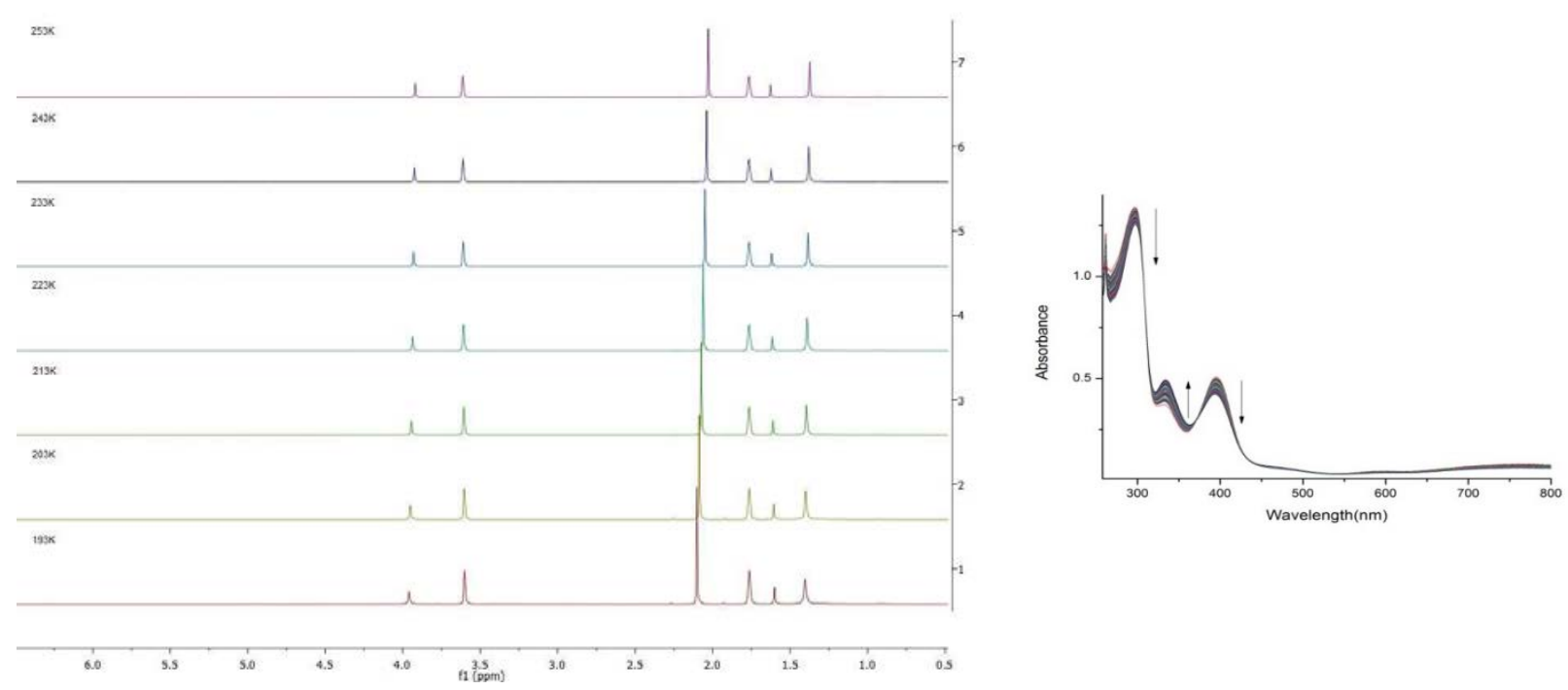

Figure 6.12 Left: VT- ${ }^{1} \mathrm{H}$ NMR spectra of $\mathbf{1 0}$ on warming up from $193 \mathrm{~K}$ to $253 \mathrm{~K}$. As evident no new peaks or shifts in peaks were observed. Right: UV-vis monitoring of $\mathbf{1 0}$ on warming up.
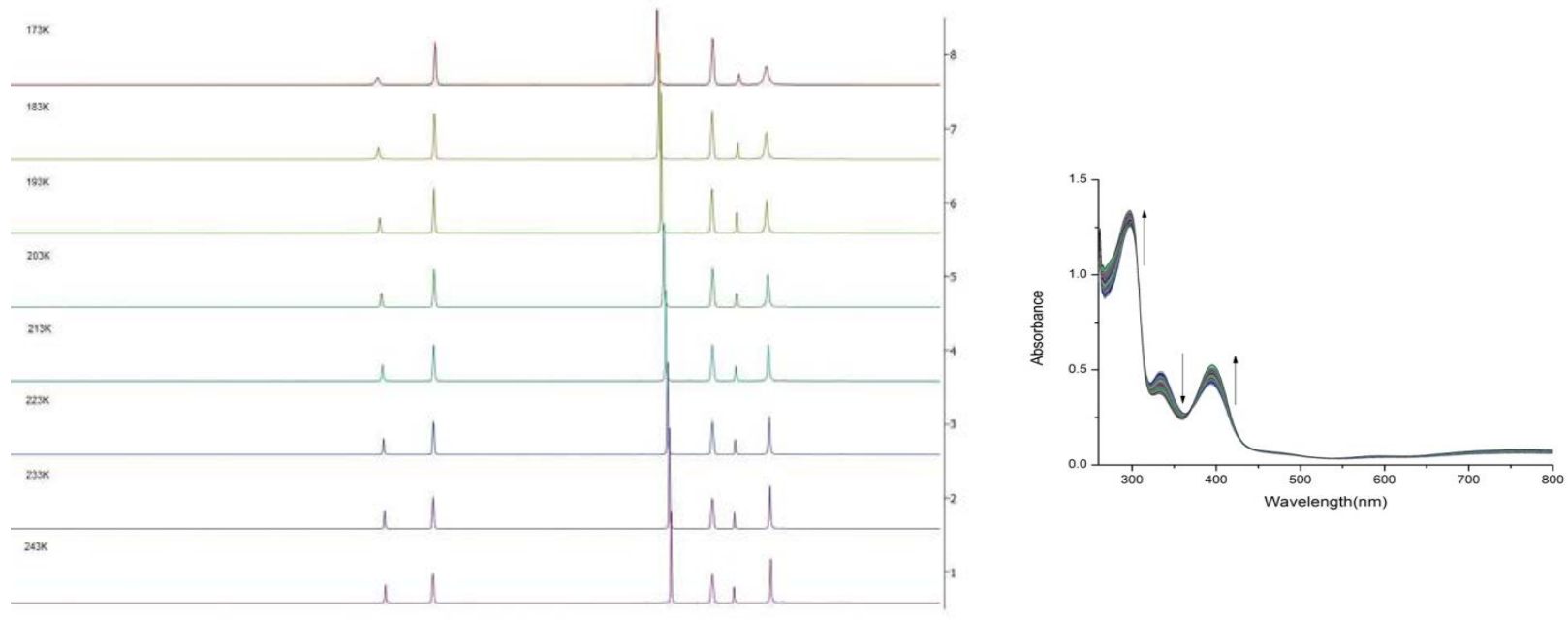

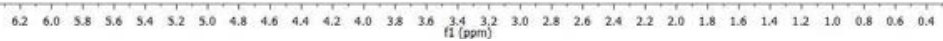

Figure 6.13 Left: VT- ${ }^{1} \mathrm{H}$ NMR spectra of 10 on cooling from $243 \mathrm{~K}$ to $173 \mathrm{~K}$. As evident no new peaks or shifts in peaks were observed. Right: UV-vis monitoring of $\mathbf{1 0}$ on cooling down. 
For the $\mathbf{O}$ complex 12 that was formed from its ${ }^{\mathbf{S}} \mathbf{P}$ counterpart by a base (Scheme 6.3; fig 6.14 left; refer also to chapter 7), a similar change in intensity of the bands at $337 \mathrm{~nm}$ and $398 \mathrm{~nm}$ was observed with temperature variation (Fig 6.15). A plot of the change in absorbance of both the bands (337 $\mathrm{nm}$ and 398 $\mathrm{nm}$ ) showed no alteration with excess base (Fig 6.14 right). This demonstrates complete deprotonation of the ${ }^{\mathbf{S}} \mathbf{P}$ species with the resultant spectra belonging solely to the $\mathbf{O}$ complex. Both these findings from systems 10 and $\mathbf{1 1}$ of monoanionic ligands, and $\mathbf{9}$ and $\mathbf{1 2}$ obtained by deprotonating their ${ }^{\mathbf{S}} \mathbf{P}$ complexes imply that within solution there exists only the $\mathbf{O}$ isomer. Thus one can rule out the effect of temperature variation to be a result of the equilibrium between the $\mathbf{O}$ and ${ }^{\mathbf{S}} \mathbf{P}$ isomers.
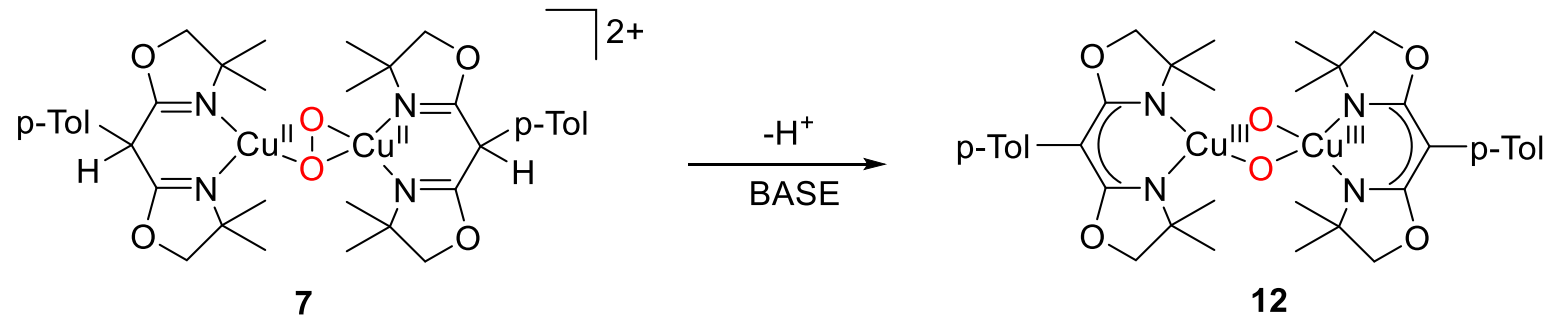

Scheme 6.3. $\mathrm{O}$ complex 12 being formed from its ${ }^{S} \mathbf{P}$ counterpart 7 by a base.
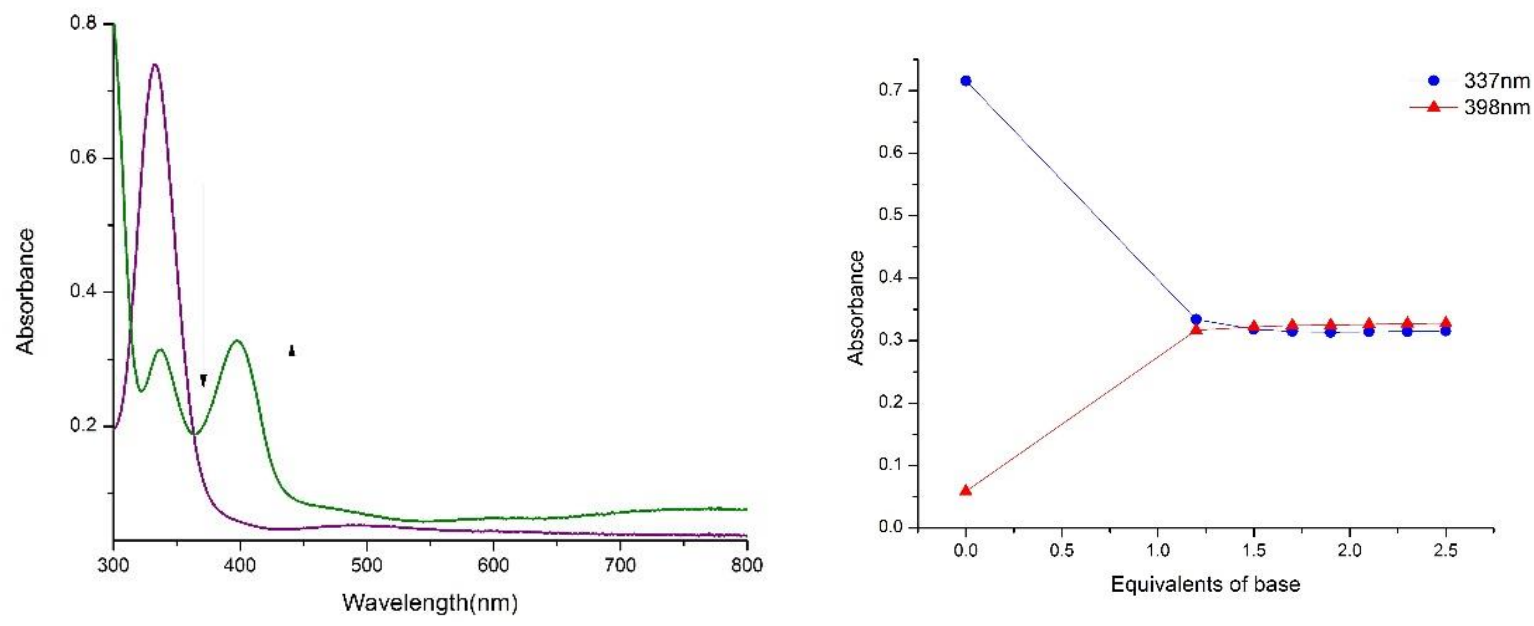

Figure 6.14 Left: UV-vis spectroscopic features on the $\mathbf{O}$ complex 12 (green) being formed from it ${ }^{\mathbf{S}} \mathbf{P}$ counterpart 7 (purple) in solution by a base. Right: Plot of change in absorbance of bands at $337 \mathrm{~nm}$ (blue) and $398 \mathrm{~nm}$ (red) with addition of equivalents of base, depicting no further change in the presence of excess base. 

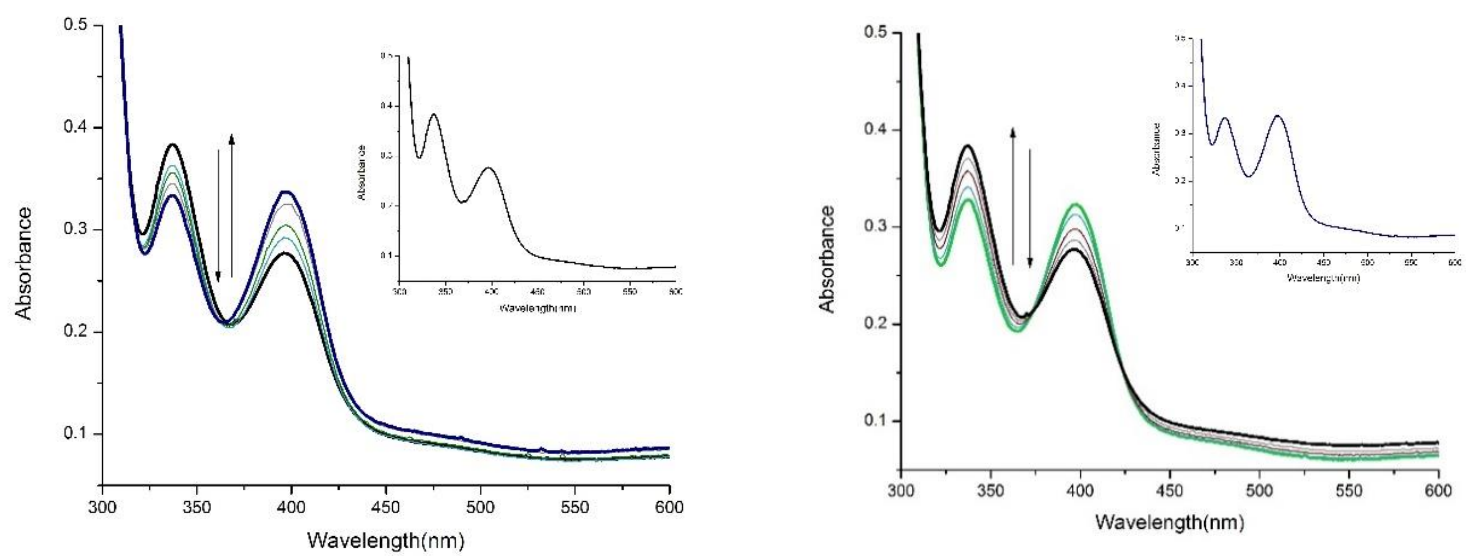

Figure 6.15 Left: Temperature variation of 12 in solution from $193 \mathrm{~K}$ to $243 \mathrm{~K}$ depicting a gain in intensity at $337 \mathrm{~nm}$, with a loss in intensity at $398 \mathrm{~nm}$. Inset, final spectra at $243 \mathrm{~K}$ Right: Spectra obtained on cooling down the solution again from 243 K to 193 K. Resultant spectra obtained at $193 \mathrm{~K}$ depicted inset.

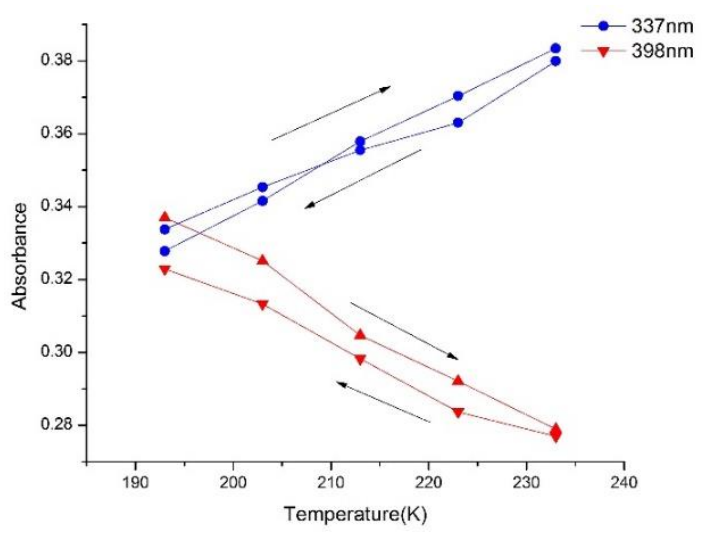

Figure 6.16 Plot of the absorbance of bands at $337 \mathrm{~nm}$ (blue) and $398 \mathrm{~nm}$ (red) for 12 on warming up (193 K to $243 \mathrm{~K}$ ) and cooling down (243 K to $193 \mathrm{~K})$.

Since the effect of temperature has been ruled out as an extension of equilibrium between ${ }^{\mathbf{S}} \mathbf{P}$ and $\mathbf{O}$ isomers, the change in absorbance of the bands could be explained as follows. X ray characterization of complex $\mathbf{1 0}$ (Fig 6.5) reveals it to be rather twisted than planar. Plausibly, the average degree of this twist could depend upon temperature variation. On warming the solution from $193 \mathrm{~K}$ to $243 \mathrm{~K}$, a change in the degree of twist leads to a change in the extent of overlap between the bis( $\mu$-oxo) ligand and metal orbitals (Refer to fig. 2.9 of introduction). The $\pi_{\sigma}{ }^{*}$ orbital of the oxo ligand decreases in energy at $243 \mathrm{~K}$ relative to that at $193 \mathrm{~K}$, resulting in a higher degree of overlap with the $\mathrm{Cu}-\mathrm{d}_{\mathrm{xy}}$ orbitals. This increase in orbital overlap results in a higher intensity of transition at $333 \mathrm{~nm}$. Contrastingly, the low energy bonding $\sigma *$ orbital, which shows a high-intensity transition at $400 \mathrm{~nm}$ in $\mathbf{O}$ complexes $\mathbf{1 0}$ and 11, tends to increase in energy. This results in a decrease in orbital overlap between the $\mathrm{Cu}-\mathrm{d}_{\mathrm{xy}}$ and $\sigma *$ orbital of the oxo ligand, leading to a loss in intensity of the band at $400 \mathrm{~nm}$. On cooling the solution down again to $193 \mathrm{~K}$, the initial degree of twist within the complex is regained, with the ligand and metal orbitals reorienting themselves to their original forms. Thus the spectra obtained on initial formation of the complex is restored. It is noteworthy to mention that though a large number of studies have been conducted on several $\mathbf{O}$ complexes with different ligand classes, the 
effect of temperature on the bis( $\mu$-oxo) core has not yet been reported.

\subsection{Conclusion}

In conclusion, the two new ligands $\left[\mathbf{L}^{2}\right]^{-}$and $\left[\mathbf{L}^{3}\right]^{-}$, which had been introduced to extend the range of BOX ligand scaffolds on $\mathrm{Cu} / \mathrm{O}_{2}$ chemistry could indeed find application in this research. As was demonstrated in chapter 5, the protonated counterparts of $\left[\mathbf{L}^{2}\right]^{-}$and $\left[\mathbf{L}^{3}\right]^{-}$gave rise to their respective ${ }^{\mathbf{S}} \mathbf{P}$ complexes with their $\mathrm{Cu}(\mathrm{I})$ analogues and dioxygen at low temperatures. However, the deprotonated form of these ligand generate their respective $\mathbf{O}$ complexes from their $\mathrm{Cu}(\mathrm{I})$ analogues and dioxygen at low temperatures. Peripheral deprotonation of the ligands forms scaffolds that are strong donors, capable of supporting the +3 oxidation state of copper as opposed to +2 . This increased Lewis basicity of the ligands leads to their preferential formation of bis( $\mu$-oxo) dicopper(III) intermediates.

The two new $\mathrm{Cu}(\mathrm{I})$ complexes 13 and $\mathbf{1 4}$ were characterized in solution via ${ }^{1} \mathrm{H}$ NMR spectroscopy and ESIMS. Their corresponding $\mathbf{O}$ complexes $\mathbf{1 0}$ and $\mathbf{1 1}$ were fully characterized in solution. UV-vis features, rR data and low temperature ${ }^{1} \mathrm{H}$ NMR studies evidence the formation of dicopper(III) bis( $\mu$-oxo) complexes. The unambiguous binding mode of dioxygen was confirmed by the structural characterization of complex 10. The measured copper-ligand bond lengths were observed to be shorter than in its corresponding ${ }^{\mathbf{S}} \mathbf{P}$ analogue 6, as is expected. Moreover, $\mathbf{1 0}$ is a rare example of a neutral bis( $\mu$-oxo) dicopper(III) synthetic system that has been structurally characterized, only the second of its kind to be reported to date.

In addition to the characterization of these novel $\mathrm{Cu} / \mathrm{O}_{2}$ intermediates in solution and in the solid state (for 10), an additional effect of temperature on the bis( $\mu$-oxo) core was investigated. Temperature variation as analyzed by UV-vis spectroscopy revealed a change in intensity of LMCT bands for both the bis( $\mu$-oxo) dicopper(III) complexes generated from their deprotonated ligands (10 and 11) as well from their ${ }^{\mathbf{S}} \mathbf{P}$ analogues by virtue of a base ( $\mathbf{9}$ and $\mathbf{1 2}$, refer to Chapter 7). This was explained by a plausible fluxional twist of the complex with temperature. A temperature change may result in a change of the average degree of twist, resulting in a change of the extent of orbital overlap between the oxo ligand and the copper centers. Moreover, this change is reversible in nature. $\mathrm{rR}$ data, VT- ${ }^{1} \mathrm{H}-\mathrm{NMR}$ spectroscopy as well as analysis of the UV-vis spectra after deprotonation (for $\mathbf{O}$ complexes generated from their ${ }^{\mathbf{S}} \mathbf{P}$ congeners), suggests the absence of any potential ${ }^{\mathbf{S}} \mathbf{P}$ complex within the solution, thus ruling out temperature variation to effect the equilibrium between the ${ }^{\mathrm{S}} \mathbf{P} / \mathbf{O}$ isomers.

Though the $\mathbf{O}$ isomer has yet shown to be of biological relevance, its existence in equilibrium with the ${ }^{\mathbf{S}} \mathbf{P}$ counterpart has raised pertinent questions with respect to the active intermediate responsible for catalytic activity in enzymes such as CO and Ty. Most recently, a bis( $\mu$-oxo) dicopper(III) intermediate has been proposed for the active site of the pMMO enzyme responsible for methane monoxygenation, highlighting the importance of this $\mathrm{Cu} / \mathrm{O}_{2}$ binding mode. 
[Note: This page has intentionally been left blank] 


\section{Chapter 7 \\ Equilibrium Studies between ${ }^{P} / O$ Complexes of BOX Ligands}

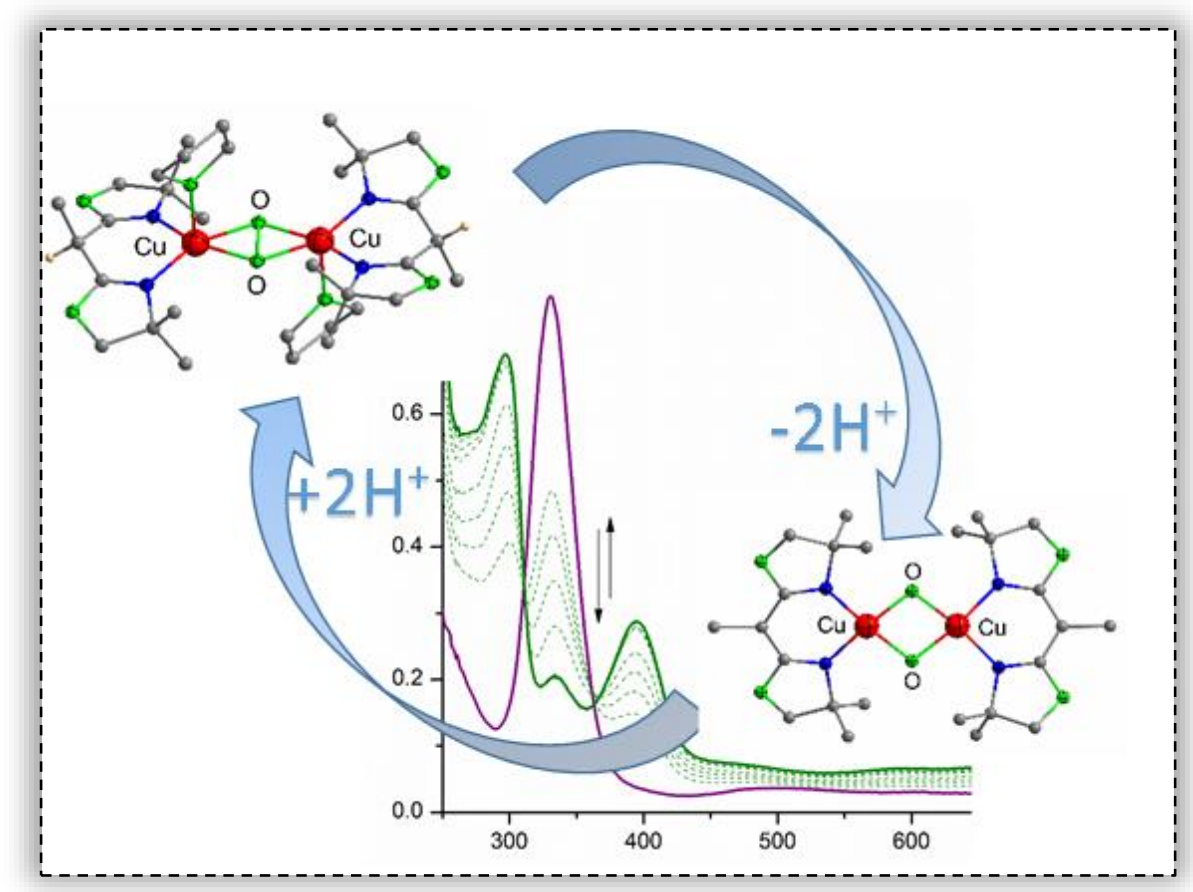




\section{Overview}

7.1 Introduction

7.2 Equilibrium studies between ${ }^{\mathbf{S}} \mathbf{P} / \mathbf{O}$ complexes of proton-responsive BOX ligands with $\mathrm{pH}$ 7.2.1 Tuning the equilibrium by virtue of a base

7.2.2 Reverting the equilibrium with an acid

7.2.3 Cleavage of the $\mathrm{O}-\mathrm{O}$ bond and insights into conversion

7.2.4 Intensity ratio differences of $\mathbf{O}$ complexes

7.3 Equilibrium studies between ${ }^{\mathbf{S}} \mathbf{P} / \mathbf{O}$ isomers of proton responsive BOX ligands with stoichiometry: The POP Switch!

\subsection{Conclusion}

[Note: Experimental procedures for this chapter are described in detail under Chapter 15, section 15.5] 


\subsection{Introduction}

In comparison to the ${ }^{\mathbf{S}} \mathbf{P}$ isomer that has been synthetically investigated in great detail by a large number of groups ${ }^{[38]}$ and also proved to be the $\mathrm{O}_{2}$ binding mode of the active site of Hemocyanin, ${ }^{[42]}$ the $\mathbf{O}$ isomer, a second $\mathrm{Cu} / \mathrm{O}_{2}$ intermediate has yet to be biologically discovered. Its first observation in 1996 by Tolman et al. led to further investigations of this binding motif which revealed it to be in rapid equilibrium with the ${ }^{\text {SP }} \mathbf{\text { isomer (Fig. 7.1) }}{ }^{[88,132]}$ Detailed theoretical calculations by Solomon et al. on various systems has established a rapid equilibrium between the two isomers to be attributed to a flat potential energy surface of interconversion. ${ }^{[93]}$ Moreover, the large extent of backbonding in the ${ }^{\mathbf{S}} \mathbf{P}$ isomer that is observed in its fairly low $\mathrm{rR}$ stretching frequency triggers the reductive cleavage of the $\mathrm{O}-\mathrm{O}$ bond, en route to the $\mathbf{O}$ species. Several factors such as solvent, counterion, ligand steric demands, and temperature have proved to control and affect this equilibrium. ${ }^{[92,138,140,183-185]}$

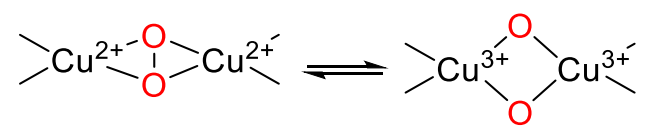

Fig 7.1 Equilibrium demonstrated by ${ }^{\mathbf{S}} \mathbf{P} / \mathbf{O}$ isomers.

In Chapter 5, the $\mathrm{Cu}(\mathrm{I})$ complexes of proton responsive ligands $\mathbf{H} \mathbf{L}^{\mathbf{1}}, \mathbf{H L}^{\mathbf{2}}$, and $\mathbf{H} \mathbf{L}^{\mathbf{5}}$ were shown to bind dioxygen at low temperatures in a $\mu-\eta^{2}: \eta^{2}$-peroxo binding mode. These complexes were characterized in solution as well as in the solid state (6), which confirmed them to be ${ }^{\mathbf{S}} \mathbf{P}$ complexes. The following section describes two new factors previously unknown to have an influence on the equilibrium between these ${ }^{\mathbf{S}} \mathbf{P}$ and their corresponding $\mathbf{O}$ complexes. All equilibrium studies have been conducted with proton responsive ligands $\mathbf{H L}^{1}, \mathbf{H L}^{2}, \mathbf{H L}^{\mathbf{5}}$ and ligand system $\mathbf{H L}^{3}$ (Fig. 7.2, the dioxygen reactivity of $\mathrm{Cu}(\mathrm{I}) \mathbf{H L}^{3}$ was investigated in a previous project and was confirmed to form its ${ }^{\mathbf{S}} \mathbf{P}$ complex at low temperatures). ${ }^{[71]}$

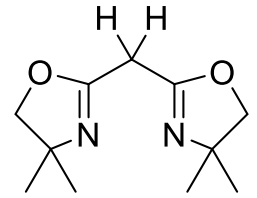

$\mathrm{HL}^{1}$

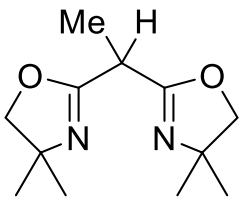

$\mathrm{HL}^{2}$

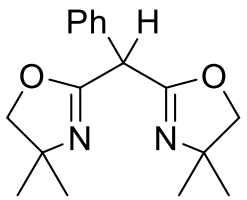

$\mathrm{HL}^{3}$

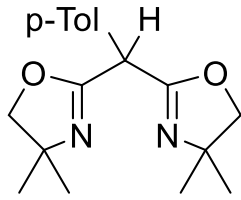

$\mathrm{HL}^{5}$

Fig 7.2 Proton responsive ligands $\mathbf{H L}^{1}, \mathbf{H L}^{2}, \mathbf{H L}^{3}$ and $\mathbf{H L}^{5}$ that have been employed in equilibrium studies between ${ }^{\mathbf{S}} \mathbf{P} / \mathbf{O}$ complexes. 


\subsection{Equilibrium studies between ${ }^{\mathrm{P}} / \mathrm{O}$ complexes of proton-responsive BOX ligands with "pH"}

\subsubsection{Tuning the equilibrium by virtue of a base}

Addition of dioxygen to $\mathrm{Cu}(\mathrm{I})$ complexes of ligands $\mathbf{H L}^{1}, \mathbf{H L}^{2}, \mathbf{H L}^{3}$ and $\mathbf{H L}^{\mathbf{5}}$ at $193 \mathrm{~K}$ resulted in purple colored solutions of their ${ }^{\mathbf{S}} \mathbf{P}$ complexes within a matter of seconds (Refer to Chapter 5, Sections 5.3 for $\mathbf{H L}^{1}, \mathbf{H L}^{2}$ and $\mathbf{H L}^{5}{ }^{5}{ }^{\mathrm{S}} \mathbf{P}$ complex of ligand system $\mathbf{H L}^{3}$ was characterized in a previous project $\left.{ }^{[71]}\right)$. Addition of an external base to these purple colored solutions resulted in an immediate color change from purple to dark green. The reactions were conducted at $193 \mathrm{~K}$ and the green colored solutions persisted with time. On UV-vis monitoring of these reactions, interesting changes in the ${ }^{\mathbf{S}} \mathbf{P}$ spectra were observed. With gradual addition of the base, the band at $333 \mathrm{~nm}$ gradually started to decrease in intensity and two completely new bands at $\sim 290 \mathrm{~nm}$ and $\sim 400 \mathrm{~nm}$ started to form. The weak band at $\sim 500 \mathrm{~nm}$ characteristic of ${ }^{\mathbf{S}} \mathbf{P}$ complexes no longer persisted. On completion of the reaction the resulting spectra exhibited three bands at $\sim 290 \mathrm{~nm}$, $\sim 333 \mathrm{~nm}$ and $\sim 400 \mathrm{~nm}$, which differed in intensity depending upon the ligand system used (Figs. 7.3-7.6). Addition of excess base to these reaction mixtures led to no further changes in the spectra.

On conducting the similar reaction with the ${ }^{\mathbf{S}} \mathbf{P}$ complex of the non-proton responsive ligand $\mathbf{L}^{\mathbf{6}}$, addition of a base resulted in a color change from purple to yellow rather than purple to green (contrary to what was observed with proton responsive ligands). Monitoring this reaction via UV-vis spectroscopy did not result in any new band formation at $400 \mathrm{~nm}$ but resulted in decomposition of the complex (Fig. 7.7).

Several bases such as $\mathrm{DBU}, \mathrm{NEt}_{3}, n$-BuLi, $\mathrm{KO} t \mathrm{Bu}$, and $\mathrm{NaO} t \mathrm{Bu}$ were screened to investigate the reaction for ${ }^{\mathbf{S}} \mathbf{P}$ complexes with proton responsive ligands. All bases resulted in similar spectral changes indicating the reaction to be independent of the nature of the base. The difference in reactivity between the systems $\mathbf{H L}^{1}, \mathbf{H L}^{2}, \mathbf{H L}^{3}, \mathbf{H L}^{5}$ with $\mathbf{L}^{6}$ was attributed to their proton responsive and non-proton responsive nature to an external base and essentially the process of deprotonation of the ligands resulted in spectral changes.

On detailed analysis of the UV-vis spectra formed after deprotonation and in comparison to the spectral features of other $\mathrm{Cu} / \mathrm{O}_{2}$ intermediates at low temperatures, the new species were assigned to as $\mathbf{O}$ intermediates of the deprotonated BOX ligands. The spectral features at $\sim 333 \mathrm{~nm}$ and $\sim 400 \mathrm{~nm}$ are similar to those of other $\mathbf{O}$ complexes reported in literature. The band at $\sim 400 \mathrm{~nm}$ that forms on the process of deprotonation is characteristic of $\mathbf{O}$ complexes and present only when the O-O bond is cleaved.

The rationale behind this process of interconversion is as follows. Addition of an external base to ${ }^{\mathbf{S}} \mathbf{P}$ complexes of proton responsive ligands leads to deprotonation within the ligand backbone. This peripheral deprotonation causes a change in the electronic properties of the ligand scaffold resulting in the ligand to attain a more Lewis basic character. The additional negative charge over the ligand triggers the cleavage of the O-O bond within the peroxide complex, converting the peroxide ligand to an oxide motif with simultaneous change in the copper oxidation state from +2 to +3 . This cleavage of the $\mathrm{O}-\mathrm{O}$ bond results in the formation of a completely new band at $\sim 400 \mathrm{~nm}$. The strong $\mathrm{Cu}-\mathrm{O}$ bond, and the absence of backbonding within the $\mathbf{O}$ isomer stabilizes these complexes. This hypothesis in conjunction with the fact that the interconversion fails with the non-proton responsive ligand system $\mathbf{L}^{6}$, supports the new species to be $\mathbf{O}$ intermediates. Final confirmation of this binding mode was attained on systems wherein the proton responsive ligands $\mathbf{H} \mathbf{L}^{2}$ and $\mathbf{H} \mathbf{L}^{3}$ were already deprotonated to generate ligands $\left[\mathbf{L}^{2}\right]^{-}$and $\left[\mathbf{L}^{3}\right]^{-}$. Addition of dioxygen to the $\mathrm{Cu}(\mathrm{I})$ complexes of $\left[\mathbf{L}^{2}\right]^{-}$and $\left[\mathbf{L}^{3}\right]^{-}$at low temperatures resulted in direct formation of their $\mathbf{O}$ isomers. These were characterized in detail along with structural elucidation for the $\mathbf{O}$ complex with $\left[\mathbf{L}^{2}\right]^{-}$ , which unambiguously confirmed the oxygen to bind as a bis( $\mu$-oxo) motif (refer to Chapter 6). 
Figures 7.3 - 7.6 demonstrate the spectroscopic changes of the ${ }^{\mathrm{S}} \mathbf{P}$ complexes of $\mathbf{H L} \mathbf{L}^{1}, \mathbf{H L}^{2}, \mathbf{H L}^{3}$ and $\mathbf{H L}^{5}$ with DBU as an external base (Scheme 7.1). A total of around 1.2 equivalents of base per dinuclear copper species was required for complete deprotonation of all ${ }^{\mathbf{S}} \mathbf{P}$ complexes. A plot of the change in absorbance of the two bands at $\sim 333 \mathrm{~nm}$ and $\sim 400 \mathrm{~nm}$ with excess base is shown in the inset for each system. Table 7.1 describes the optical features with molar absorbtivities and intensity ratios for the ${ }^{\mathbf{S}} \mathbf{P}$ and $\mathbf{O}$ complexes with proton responsive ligands $\mathbf{H L}^{1}, \mathbf{H L}^{2}, \mathbf{H L}^{3}$ and $\mathbf{H L}^{5}$.

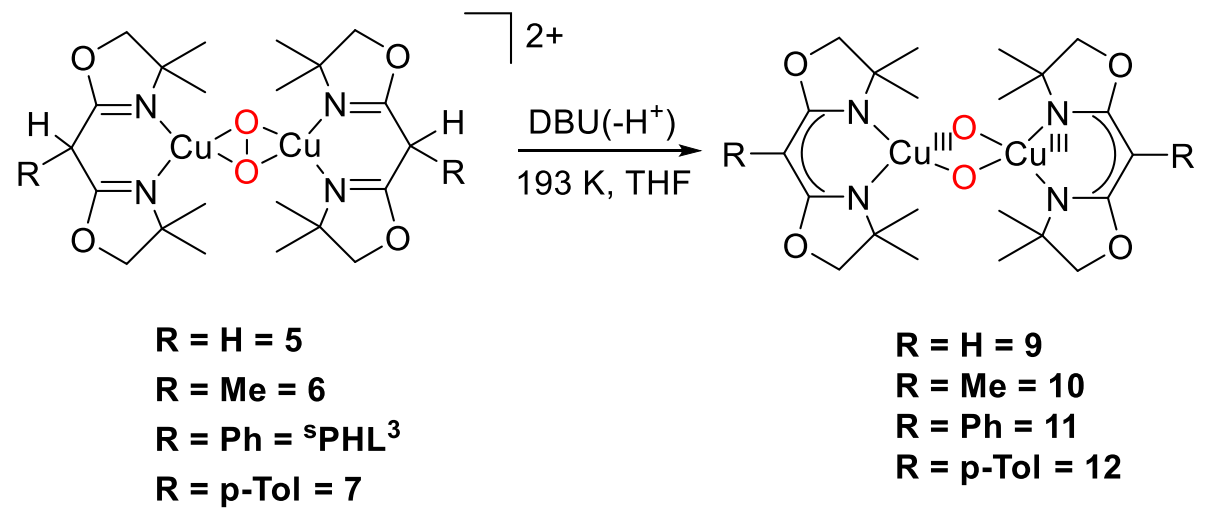

Scheme 7.1 Schematic representation of the interconversion between ${ }^{\mathbf{S}} \mathbf{P}$ and $\mathbf{O}$ complexes of proton responsive ligands by virtue of a base (DBU).
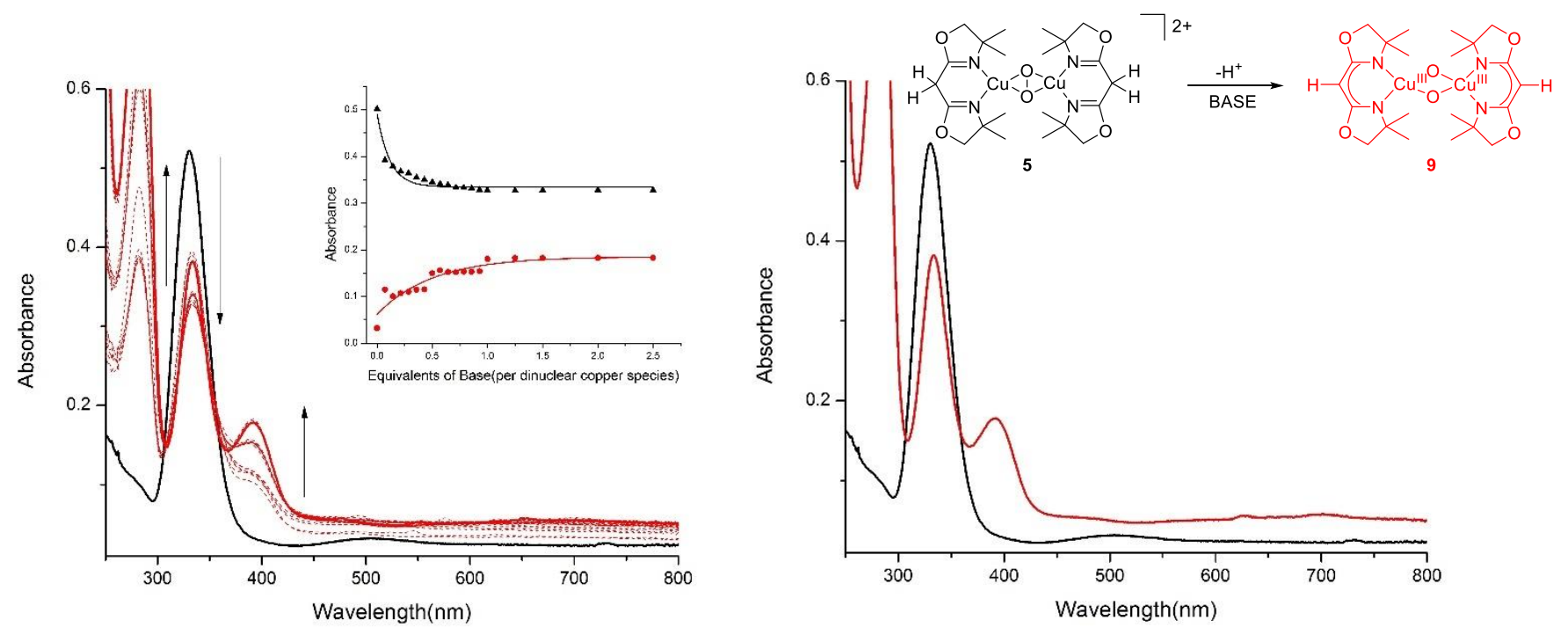

Figure 7.3 Left: ${ }^{\mathbf{S}} \mathbf{P}$ complex of $\mathbf{H L}^{\mathbf{1}}$ (5) in black; gradual titration of DBU resulting in the formation of $\mathbf{O}$ complex 9 with bands at $392 \mathrm{~nm}$ and $282 \mathrm{~nm}$ arising, and that at $335 \mathrm{~nm}$ decreasing in intensity. Shown in the inset is a plot of the change in intensity of bands at $335 \mathrm{~nm}$ and $392 \mathrm{~nm}$ with equivalents of base. Right: ${ }^{\mathbf{S}} \mathbf{P}$ complex of $\mathbf{H L}^{\mathbf{1}}$ (5) in black with its $\mathbf{O}$ complex (9) in red after complete deprotonation. Shown in the inset is the schematic representation of the interconversion from $\mathbf{5}$ to $\mathbf{9}$. 

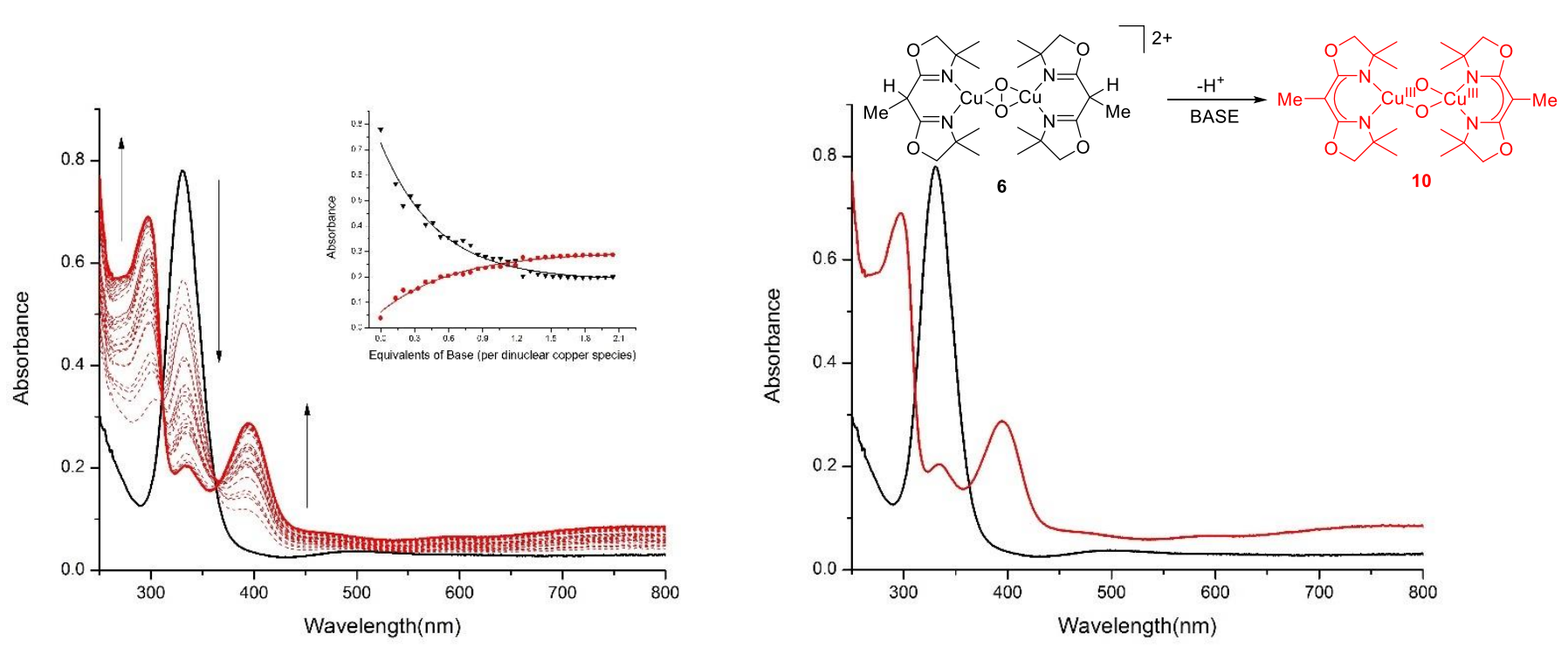

Figure 7.4 Left: ${ }^{\mathbf{S}} \mathbf{P}$ complex of $\mathbf{H L}^{2}$ (6) in black; gradual titration of DBU resulting in the formation of $\mathbf{O}$ complex 10 with bands at $395 \mathrm{~nm}$ and $296 \mathrm{~nm}$ arising, and that at $335 \mathrm{~nm}$ decreasing in intensity. Shown in the inset is a plot of the change in intensity of bands at $335 \mathrm{~nm}$ and $395 \mathrm{~nm}$ with equivalents of base. Right: ${ }^{\mathbf{S}} \mathbf{P}$ complex of $\mathbf{H L}^{2}$ (6) in black with its $\mathbf{O}$ complex (10) in red after complete deprotonation. Shown in the inset is the schematic representation of the interconversion from 6 to 10.
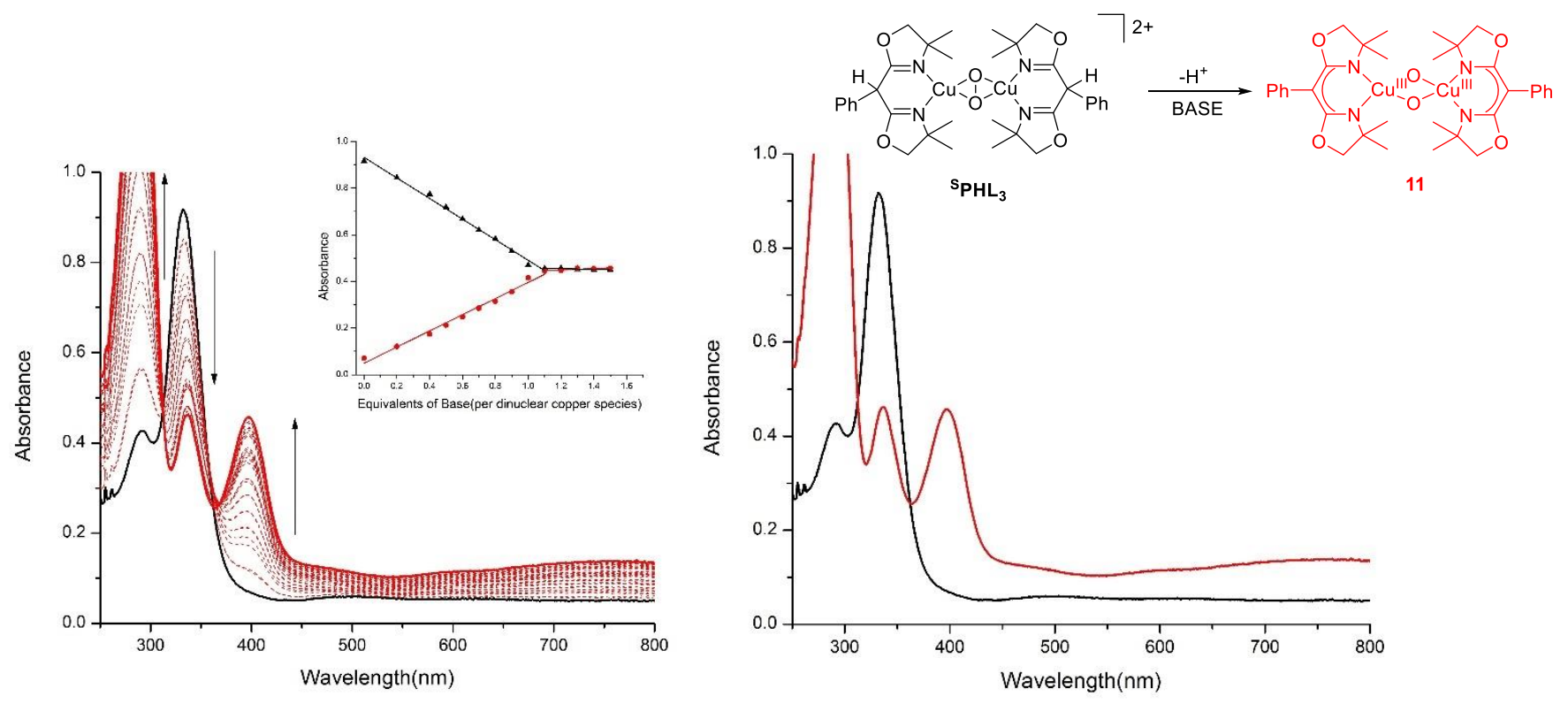

Figure 7.5 Left: ${ }^{\mathrm{S}} \mathbf{P}$ complex of $\mathbf{H L}^{3}$ in black; gradual titration of DBU resulting in the formation of $\mathbf{O}$ complex 11 with bands at $397 \mathrm{~nm}$ and $288 \mathrm{~nm}$ arising, and that at $336 \mathrm{~nm}$ decreasing in intensity. Shown in the inset is a plot of the change in intensity of bands at $336 \mathrm{~nm}$ and $397 \mathrm{~nm}$ with equivalents of base. Right: ${ }^{\mathbf{S}} \mathbf{P}$ complex of $\mathbf{H L}^{\mathbf{3}}$ in black with its $\mathbf{O}$ complex (11) in red after complete deprotonation. Shown in the inset is the schematic representation of the interconversion from ${ }^{\mathbf{S}} \mathbf{P H L}{ }^{3}$ to $\mathbf{1 1}$. 

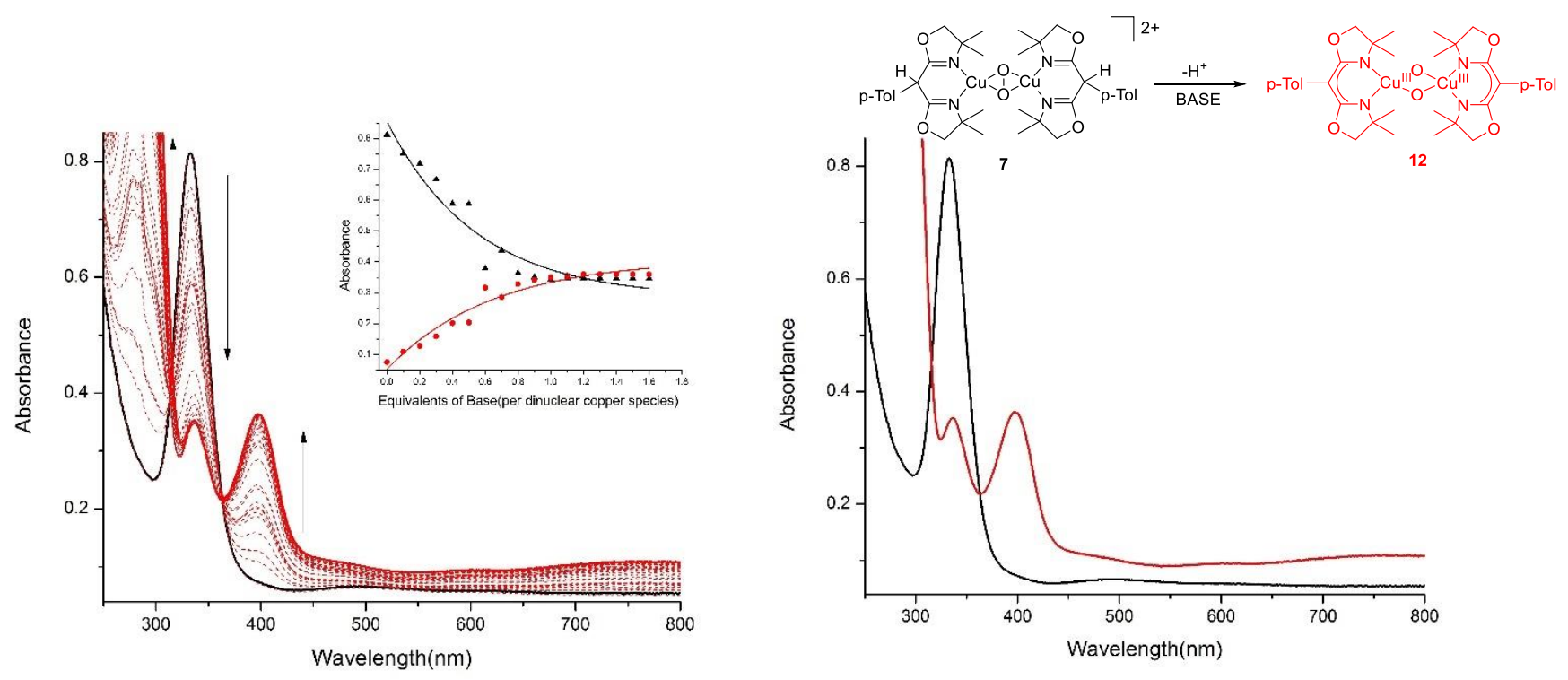

Figure 7.6 Left: ${ }^{\mathbf{S}} \mathbf{P}$ complex of $\mathbf{H L}^{\mathbf{5}}$ (7) in black; gradual titration of DBU resulting in the formation of $\mathbf{O}$ complex 12 with bands at $398 \mathrm{~nm}$ and $293 \mathrm{~nm}$ arising, and that at $337 \mathrm{~nm}$ decreasing in intensity. Shown in the inset is a plot of the change in intensity of bands at $337 \mathrm{~nm}$ and $398 \mathrm{~nm}$ with equivalents of base. Right: ${ }^{\mathbf{S}} \mathbf{P}$ complex of $\mathbf{H L}^{\mathbf{5}}$ (7) in balck with its $\mathbf{O}$ complex (12) in red after complete deprotonation. Shown in the inset is the schematic representation of the interconversion from 7 to 12.

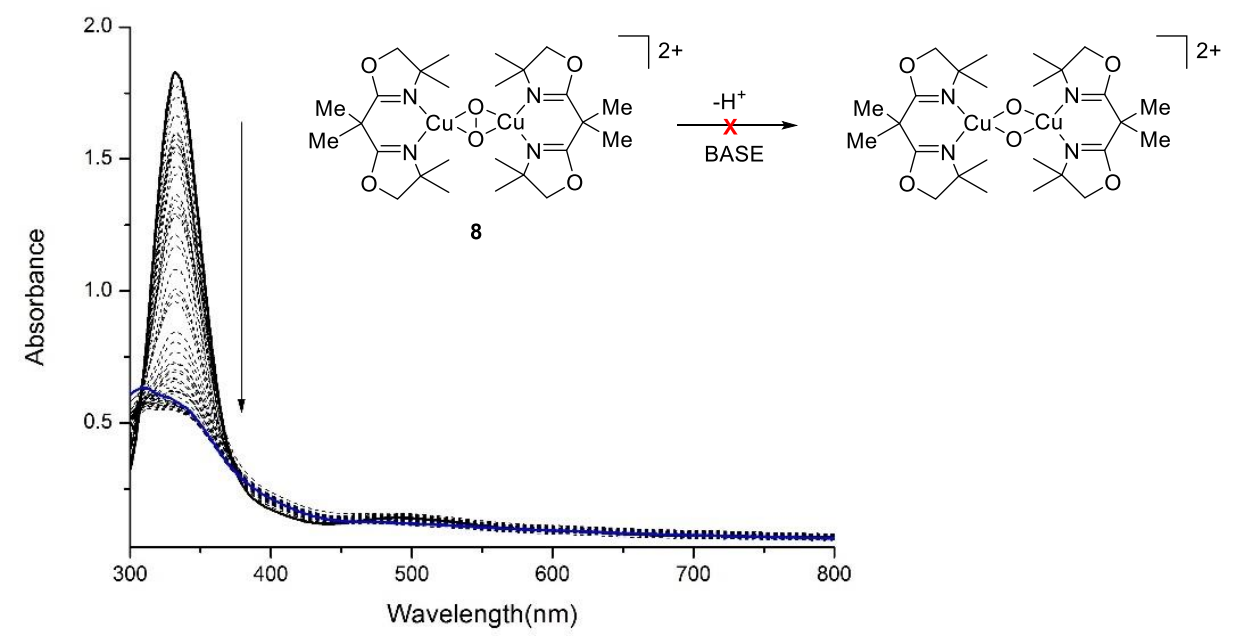

Figure 7.7 Addition of DBU to ${ }^{\mathbf{S}} \mathbf{P}$ complex of non-proton responsive ligand $\mathbf{L}^{\mathbf{6}}$, leading to decomposition of the complex with no new band formation at $\sim 290 \mathrm{~nm}$ or $\sim 400 \mathrm{~nm}$. 


\subsubsection{Reverting the equilibrium with an acid}

In order to test the reversibilty of the equilibrium demonstrated above, various Lewis acids such as HLutOTf, $\mathrm{HLutBF}_{4}$, [HLut: lutidinium], $\mathrm{HBF}_{4} \cdot \mathrm{Et}_{2} \mathrm{O},\left[\mathrm{Et}_{2} \mathrm{OH}\right] \mathrm{BArF}^{-}$were titrated into the $\mathbf{O}$ solutions of complexes 9-12 (Fig. 7.8 and 7.9). Unfortunately, addition of an external acid $/ \mathrm{H}^{+} \mathrm{did}$ not revert the equilibrium back to the ${ }^{\mathbf{S}} \mathbf{P}$ isomer, but led to gradual decomposition of the corresponding $\mathbf{O}$ complexes. The reactions were monitored via UV-vis spectroscopy which demonstrated a gradual decrease in the intensity of the $\mathbf{O}$ bands at $\sim 333 \mathrm{~nm}$ and $\sim 400 \mathrm{~nm}$, but no new band formation at $\sim 500 \mathrm{~nm}$.

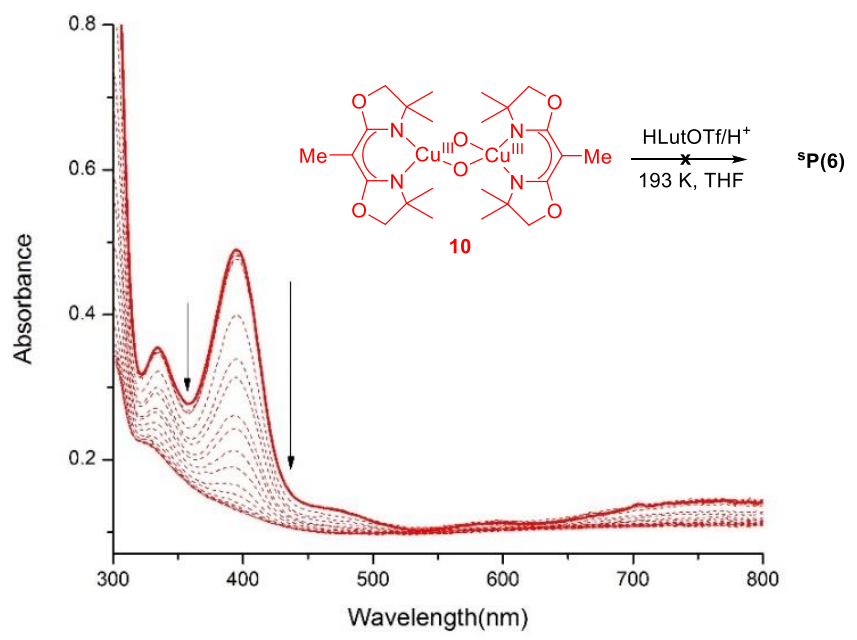

Figure 7.8 UV-vis monitoring of the adddition of HLutOTf to $\mathbf{O}$ complex $\mathbf{1 0}$ demonstrating decomposition.
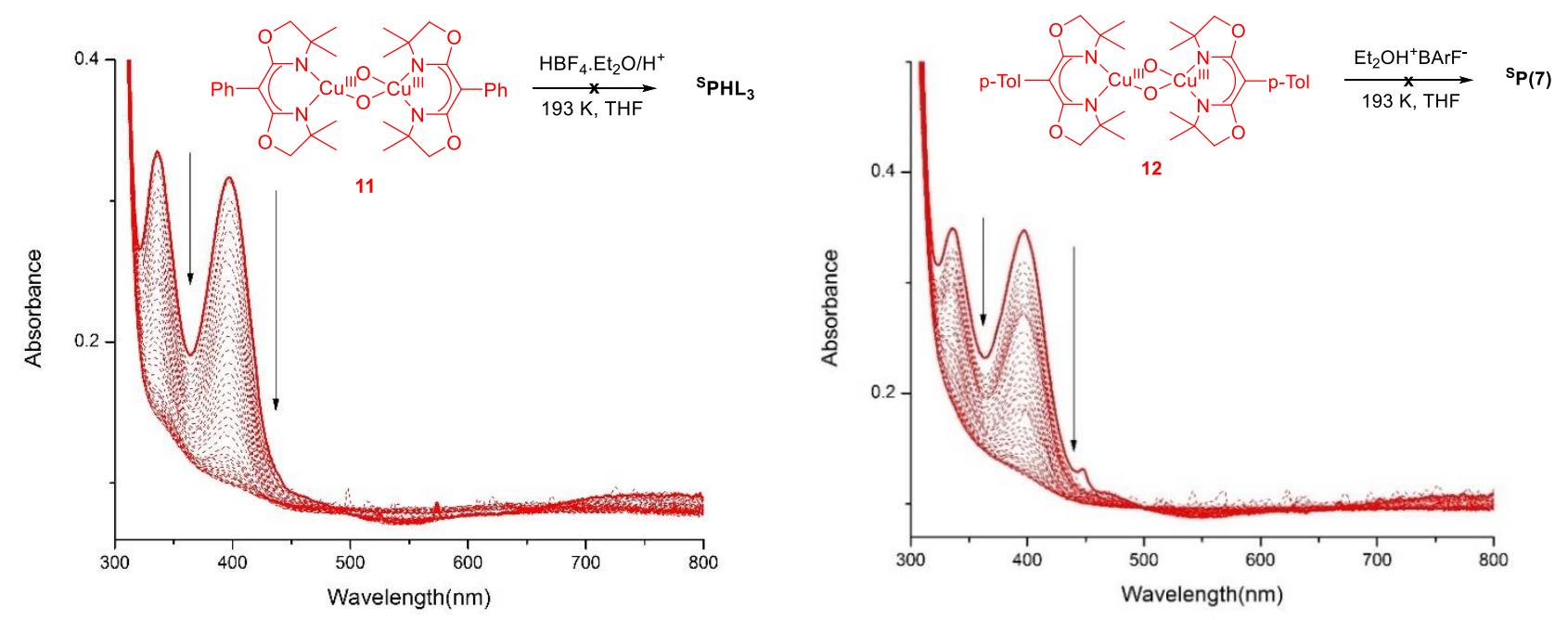

Figure 7.9 Left: UV-vis monitoring of the adddition of $\mathrm{HBF}_{4} \cdot \mathrm{Et}_{2} \mathrm{O}$ to $\mathbf{O}$ complex 11 demonstrating decomposition. Right: $\mathrm{UV}$-vis monitoring of the adddition of $\mathrm{Et}_{2} \mathrm{OH}^{+} \mathrm{BArF}^{-}$to $\mathbf{O}$ complex 12 demonstrating decomposition. 
Analysis of the solution after the addition of an acid via ESI-MS did not yield major insights into the nature of the decomposed product. In most cases, the dominant major peaks corresponded to the base used for deprotonation and the ligand system. No peaks corresponding to a dicopper(II) bis( $\mu$-hydroxo) species were observed. A potential hypothesis to this observation suggests that perhaps the proton preferentially attacks the oxygen centers of the bis( $\mu$-oxo) core due to its high nucleophilicity, as opposed to the ligand backbone (Scheme 7.2). This inhibits the equilibrium to revert back from the $\mathbf{O}$ to the ${ }^{\mathbf{S}} \mathbf{P}$ isomer. Protonation of the $\mathbf{O}$ species then leads to the formation of a dicopper(III) bis( $\mu$-hydroxo) complex as a kinetic product, which, due to its high reactivity makes it difficult to isolate and observe. This instability leads to gradual decomposition as is observed when the bis( $\mu$-oxo) complexes are protonated in solution.

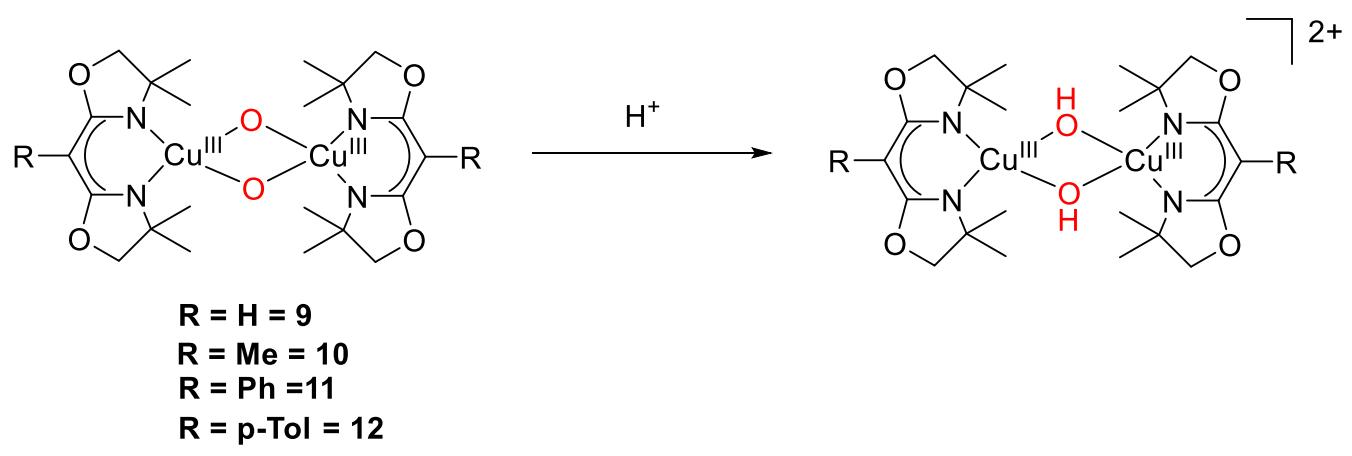

Scheme 7.2 Plausible hypothesis for the decomposition of $\mathbf{O}$ complexes with proton responsive ligands with an acid. Protonation of the $\mathbf{O}$ intermediates forms a reactive bis( $\mu$-hydroxo) dicopper(III) species that decomposes with time.

\subsubsection{Cleavage of the $O-O$ bond and insights into conversion}

Detailed theoretical investigation has led to insights into the mechanism of interconversion between ${ }^{\mathbf{S}} \mathbf{P} / \mathbf{O}$ complexes. ${ }^{[93]}$ This explains comprehensively the differences in spectroscopic features between the two isomers. As described in the MO diagram in figure 7.10, the UV-vis spectral features for ${ }^{\mathbf{S}} \mathbf{P}$ complexes arise from an in plane and out of plane overlap of the $\pi^{*}$ peroxide and $\mathrm{Cu} \mathrm{d}_{\mathrm{xy}}$ orbitals. Within the molecular orbitals of ${ }^{\mathbf{S}} \mathbf{P}$ complexes, there exists an unoccupied high energy $\sigma^{*}{ }_{u}$ orbital. This orbital is antibonding in nature and participates in backcbonding with the filled $\mathrm{Cu} \mathrm{d} \mathrm{d}_{\mathrm{xy}}$ orbitals. On cleavage of the $\mathrm{O}-\mathrm{O}$ bond, this high energy unoccupied antibonding orbital becomes a new, low energy bonding orbital of the oxide ligand. This new donor orbital which is present only when the $\mathrm{O}-\mathrm{O}$ bond is cleaved, gives rise to the characteristic $\sigma^{*}{ }_{u}$ to $\mathrm{Cu} \mathrm{d} \mathrm{d}_{\mathrm{xy}}$ transition that is exhibited at $\sim 400 \mathrm{~nm}$ for $\mathbf{O}$ complexes. As previously described in chapter 5 , the rR features exhibited by ${ }^{\mathbf{S}} \mathbf{P}$ complexes are rather low in comparison to what is expected, which is a result of backbonding between the $\mathrm{Cu} \mathrm{d}$ xy and the $\sigma^{*}{ }_{u}$ oribital. The process of O-O cleavage is an extension of this back-donation of electron density from the $\mathrm{Cu} \mathrm{dxy}$ into the $\mathrm{O}_{2}{ }^{2-} \sigma^{*}{ }_{u}$ orbitals. 


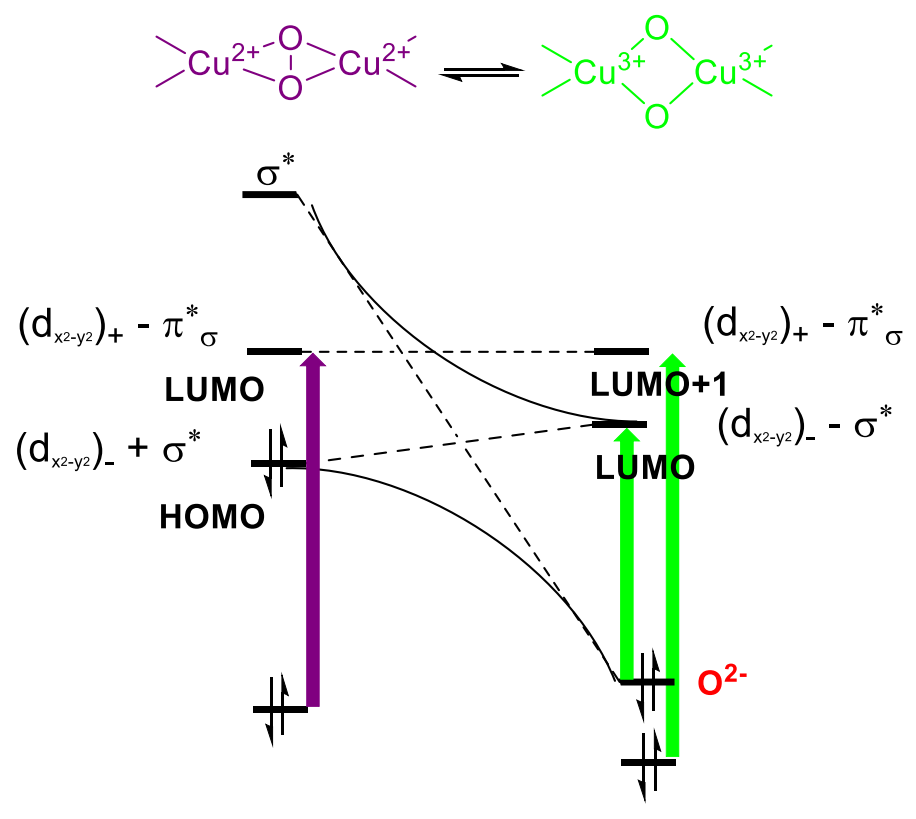

Figure 7.10 Orbital correlation diagram for the ${ }^{\mathbf{S}} \mathbf{P} / \mathbf{O}$ isomerization. ${ }^{[93]}$

The high intensity optical features of the $\mathbf{O}$ complexes formed on deprotonation of their ${ }^{\mathbf{S}} \mathbf{P}$ counterparts with proton responsive ligands are qualitatively similar to those of bis $(\mu$-oxo) dicopper(III) species reported in literature. ${ }^{[88,166,168]}$ Each of the $\mathbf{O}$ complexes, 9, 10, 11 and 12 exhibits three bands at $\sim 290 \mathrm{~nm}, \sim 333 \mathrm{~nm}$, and $\sim 400 \mathrm{~nm}$ (Table 7.1). For a detailed molecular orbital explanation, refer to Chapter 6, section 6.3.2.

\subsubsection{Intensity ratio differences of $\boldsymbol{O}$ complexes}

The spectra of $\mathbf{O}$ complexes formed on deprotonation of their ${ }^{\mathbf{S}} \mathbf{P}$ counterparts differ in intensity ratio of the bands at $\sim 333 \mathrm{~nm}$ and $\sim 400 \mathrm{~nm}$ (Table 7.1)

The MO diagram (Fig. 7.10) for the isomerization of ${ }^{\mathbf{S}} \mathbf{P} / \mathbf{O}$ complexes reported by Solomon et al., describes the energetic crossover of the $\sigma^{*}{ }_{u}$ orbital from the ${ }^{\mathbf{S}} \mathbf{P}$ to the $\mathbf{O}$ binding motif, resulting in the reductive cleavage of the $\mathrm{O}-\mathrm{O}$ bond within the peroxde ligand, giving rise to two $\mu$-oxo bridges ${ }^{\left[{ }^{[3]}\right.}$ This results in an increase in $\mathrm{O} \cdots \mathrm{O}$ bond length, with a simultaneous shortening of the $\mathrm{Cu}-\mathrm{O}$ and $\mathrm{Cu}-\mathrm{N}$ bond lengths. The shortening of these two bonds however, leads to a destabiliazation of the $\mathrm{Cu} \mathrm{d}_{\mathrm{xy}}$ orbitals.

In case of the bis( $\mu$-oxo) MO energy levels, these two orbitals have crossed with the $\sigma^{*}{ }_{u}$ orbital being lower in energy compared to $\mathrm{Cu} \mathrm{d}_{\mathrm{xy}}$. Moreover, the ligand substituents for each system differ and this results in an overall electronic difference over their $\mathbf{O}$ systems. A possibility for the different intensities of transition could be the relative difference in the crossover of the $\mathrm{Cu} \mathrm{d}_{\mathrm{xy}}$ and the $\sigma^{*}{ }_{u}$ orbital in each case, in conjunction with a variation of orbital overlap between the metal and the bis( $\mu$-oxo) ligand. 
Table 7.1 Optical features of ${ }^{\mathrm{S}} \mathbf{P}$ and $\mathbf{O}$ complexes with proton responsive BOX ligands.

\begin{tabular}{cccc}
\hline Ligand & \multicolumn{1}{c}{${ }^{\mathbf{P}}$} & \multicolumn{1}{c}{$\mathbf{O}$} & $\begin{array}{c}\text { Intensity ratio } \\
\sim 333 \mathrm{~nm}: \sim 400 \mathrm{~nm}\end{array}$ \\
\hline \multirow{2}{*}{$\mathbf{H L}^{1}$} & $330 \mathrm{~nm}\left(\varepsilon=7420 \mathrm{M}^{-1} \mathrm{~cm}^{-1}\right)$ & $282 \mathrm{~nm}\left(\varepsilon=11980 \mathrm{M}^{-1} \mathrm{~cm}^{-1}\right)$ & \\
& $501 \mathrm{~nm}\left(\varepsilon=450 \mathrm{M}^{-1} \mathrm{~cm}^{-1}\right)$ & $335 \mathrm{~nm}\left(\varepsilon=5420 \mathrm{M}^{-1} \mathrm{~cm}^{-1}\right)$ & $1: 0.468$ \\
& & $392 \mathrm{~nm}\left(\varepsilon=2550 \mathrm{M}^{-1} \mathrm{~cm}^{-1}\right)$ & \\
\hline \multirow{2}{*}{$\mathbf{H L}^{2}$} & $330 \mathrm{~nm}\left(\varepsilon=19113 \mathrm{M}^{-1} \mathrm{~cm}^{-1}\right)$ & $296 \mathrm{~nm}\left(\varepsilon=9850 \mathrm{M}^{-1} \mathrm{~cm}^{-1}\right)$ & \\
& $500 \mathrm{~nm}\left(\varepsilon=1350 \mathrm{M}^{-1} \mathrm{~cm}^{-1}\right)$ & $335 \mathrm{~nm}\left(\varepsilon=2910 \mathrm{M}^{-1} \mathrm{~cm}^{-1}\right)$ & $1: 1.40$ \\
& & $395 \mathrm{~nm}\left(\varepsilon=4110 \mathrm{M}^{-1} \mathrm{~cm}^{-1}\right)$ & \\
\hline \multirow{2}{*}{$\mathbf{H L}^{3}$} & $332 \mathrm{~nm}\left(\varepsilon=18920 \mathrm{M}^{-1} \mathrm{~cm}^{-1}\right)$ & $288 \mathrm{~nm}\left(\varepsilon=28680 \mathrm{M}^{-1} \mathrm{~cm}^{-1}\right)$ & \\
& $501 \mathrm{~nm}\left(\varepsilon=1220 \mathrm{M}^{-1} \mathrm{~cm}^{-1}\right)$ & $336 \mathrm{~nm}\left(\varepsilon=9520 \mathrm{M}^{-1} \mathrm{~cm}^{-1}\right)$ & $1: 0.98$ \\
& & $397 \mathrm{~nm}\left(\varepsilon=9420 \mathrm{M}^{-1} \mathrm{~cm}^{-1}\right)$ & \\
\hline \multirow{2}{*}{$\mathbf{H L}^{5}$} & $333 \mathrm{~nm}\left(\varepsilon=4400 \mathrm{M}^{-1} \mathrm{~cm}^{-1}\right)$ & $293 \mathrm{~nm}\left(\varepsilon=6850 \mathrm{M}^{-1} \mathrm{~cm}^{-1}\right)$ & \\
& $504 \mathrm{~nm}\left(\varepsilon=360 \mathrm{M}^{-1} \mathrm{~cm}^{-1}\right)$ & $337 \mathrm{~nm}\left(\varepsilon=1910 \mathrm{M}^{-1} \mathrm{~cm}^{-1}\right)$ & $1: 1.03$ \\
\hline
\end{tabular}




\subsection{Equilibrium studies between ${ }^{\mathrm{S}} \mathrm{P} / \mathrm{O}$ isomers of proton responsive $\mathrm{BOX}$ ligands with stoichiometry}

\section{Stoichiometric effects on $\mathrm{Cu} / \mathrm{O}_{2}$ chemistry: The POP Switch!}

Described in the previous section, addition of an external base to ${ }^{\mathbf{S}} \mathbf{P}$ solutions of proton responsive BOX ligands yields their corresponding $\mathbf{O}$ isomers. The reactions were monitored via UV-vis spectroscopy. In addition, deprotonated forms of the ligands $\mathbf{H L}^{2}$ and $\mathbf{H L}^{3}$ generate directly their $\mathbf{O}$ complexes with dioxygen and $\mathrm{Cu}(\mathrm{I})$ at low temperatures (described in Chapter 6), which further corroborates the new species to be $\mathbf{O}$ complexes indeed.

Besides tuning the equilibrium between the ${ }^{\mathbf{S}} \mathbf{P} / \mathbf{O}$ isomers of proton responsive BOX ligands with " $\mathrm{pH}$ " (addition of a base), the stoichiometry of the ligand also seemed to have an effect on this equilibrium. Surprisingly, addition of the ligand $\mathbf{H L}^{\mathbf{1}}$ to its purple colored ${ }^{\mathbf{S}} \mathbf{P}$ complex $\mathbf{5}$ at low temperatures resulted in a color change from purple to green. The reactions were monitored via UV-vis spectroscopy and the resultant spectra matched exactly that when an external base was used with this ${ }^{\text {SP }} \mathbf{P}$ system (Fig.7.11 left). Similar changes in spectroscopic features were observed with the band at $335 \mathrm{~nm}$ decreasing in intensity, and a new band at $392 \mathrm{~nm}$ forming. A total of 1.5 equivalents of ligand $\mathbf{H L}^{\mathbf{1}}$ per dinuclear copper species was required to bring about total conversion of $\mathbf{P}_{\mathbf{1}}$ (5) to $\mathbf{O}(\mathbf{9})$ (Scheme 7.3).

Subsequent addition at low temperatures of a $\mathrm{Cu}(\mathrm{I})$ source $\left(\left[\mathrm{Cu}(\mathrm{I})(\mathrm{MeCN})_{4}\right] \mathrm{ClO}_{4} ; 5\right.$ equivalents per dinuclear copper species) to the green colored $\mathbf{O}$ solution $\mathbf{9}$, resulted in a color change back to purple. On analysis via UV-vis spectroscopy, the band at $392 \mathrm{~nm}$ started to gradually decrease in intensity with a shoulder at $500 \mathrm{~nm}$ being formed (Fig.7.11 right). The $335 \mathrm{~nm}$ band started to increase in intensity and the resulting spectra $\mathbf{P}_{\mathbf{2}}$ resembled that of $\mathbf{P}_{\mathbf{1}}$ (Fig. 7.12). However, the overall intensity of the bands in $\mathbf{P}_{\mathbf{2}}$ were much higher compared to $\mathbf{P}_{\mathbf{1}}$ due to an increase in the concentration of the solution (addition of external ligand and $\mathrm{Cu}(\mathrm{I})$ solutions). Scheme 7.3 describes the subsequent interconversion of ${ }^{\mathbf{S}} \mathbf{P} / \mathbf{O}$ species with the effect of stoichiometry for ligand system $\mathbf{H L}^{\mathbf{x}}(\mathrm{x}=1,2,3,5)$.

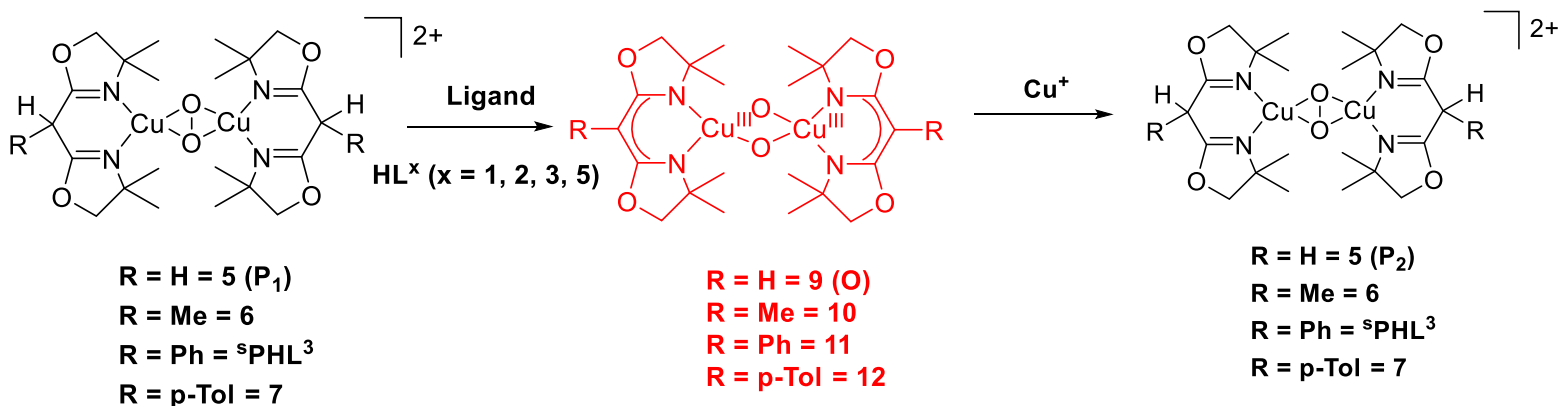

Scheme 7.3 Schematic representation of the interconversion between ${ }^{\mathrm{S}} \mathbf{P} / \mathbf{O}$ isomers of ligand system $\mathbf{H L}^{1}, \mathbf{H L}^{2}, \mathbf{H L}^{3}$ and $\mathbf{H L}^{5}$ with stoichiometry. Addition of the ligands to their ${ }^{\mathbf{S}} \mathbf{P}$ solutions to form their $\mathbf{O}$ counterparts. Subsequent addition of a $\mathrm{Cu}(\mathrm{I})$ source in solution reforms the respective ${ }^{\mathbf{S}} \mathbf{P}$ complex.

Scheme 7.3 demonstrates the addition of the proton responsive ligand $\mathbf{H L}^{\mathbf{1}}$ to a solution of its ${ }^{\mathbf{S}} \mathbf{P}$ complex $5\left(\mathbf{P}_{1}\right)$ to generate the $\mathbf{O}$ complex $\mathbf{9}$. Ligand $\mathbf{H L}^{1}$ behaves as a base to capture the released proton from $\mathbf{5}$, generating 9. In solution therefore there exists the protonated form of $\mathbf{H L}^{\mathbf{1}}\left(\mathrm{H}_{2} \mathrm{~L}^{1+}\right)$ and its deprotonated complex. Addition of a $\mathrm{Cu}(\mathrm{I})$ source to this mixture, wherein excess ligand is present regenerates $5\left(\mathbf{P}_{2}\right)$. 
Coordination with the copper ions and reaction with dioxygen liberates the proton from $\mathrm{H}_{2} \mathrm{~L}_{1}^{+}$, reprotonating its deprotonated form. This procedure avoids the potentially detrimental presence of any exogenous base in the reaction mixture. UV-vis monitoring of the reaction sequence (Fig. 7.12) confirmed that 1 equivalent of $\mathbf{9}$ is formed during the first step, and a total of 1.8 equivalents of $\mathbf{5}$ is present after complete addition of the $\mathrm{Cu}(\mathrm{I})$ source.
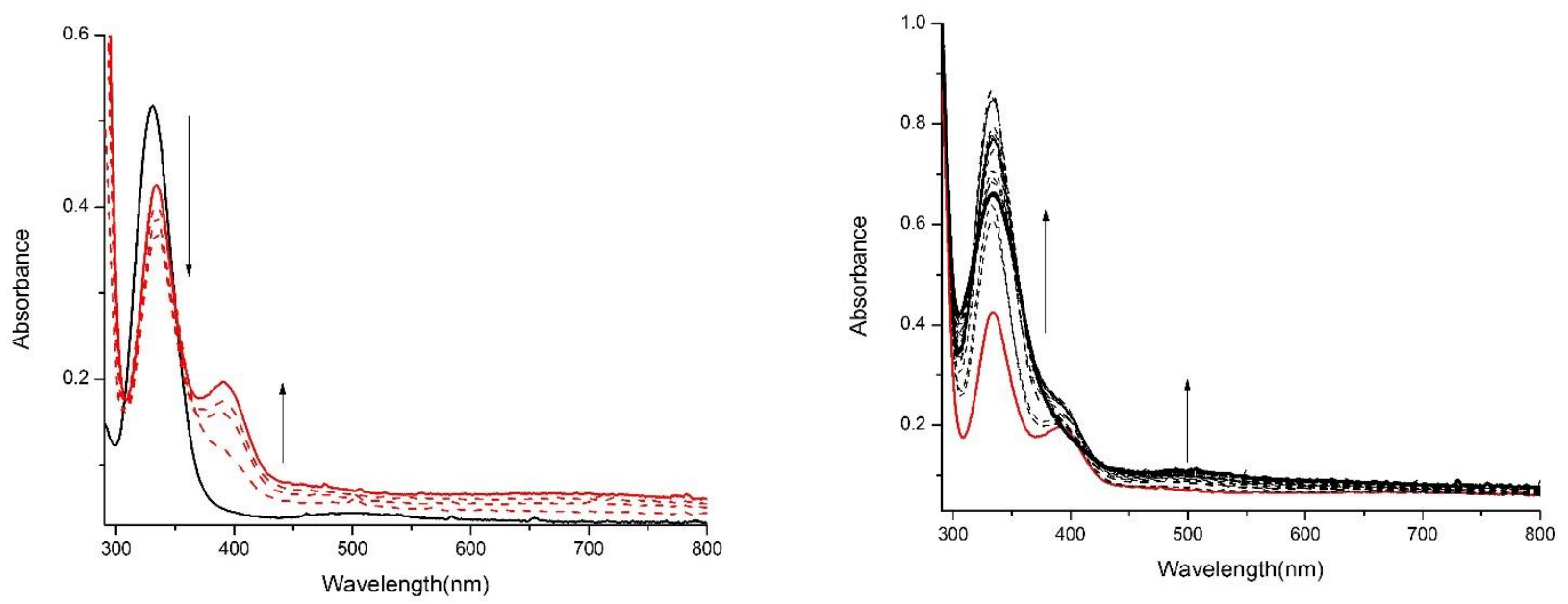

Figure 7.11 Left: Titration of $\mathbf{H L}^{\mathbf{1}}$ to a solution of $\mathbf{5}$ in THF at $193 \mathrm{~K}$ resulting in the formation of $\mathbf{9}$, displaying the ligand to behave as a base. Right: Subsequent titration of a $\left[\mathrm{Cu}(\mathrm{I})(\mathrm{MeCN})_{4}\right] \mathrm{ClO}_{4}$ solution to 9 to reform $\mathbf{5}$.

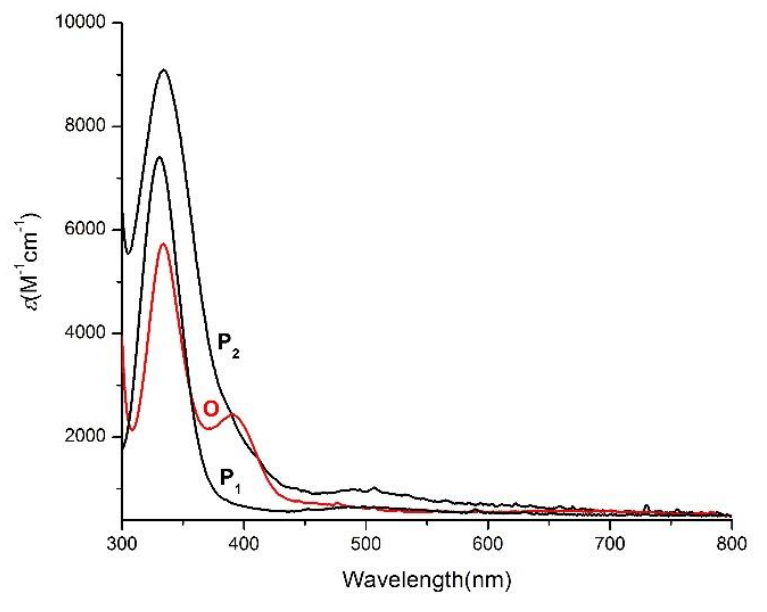

Figure 7.12 UV-vis monitoring of the reaction sequence demonstrating the interconversion of 5 to 9 using $\mathbf{H L}^{\mathbf{1}}$ as a base and subsequent conversion of $\mathbf{9}$ to $\mathbf{5}$ with $\left[\mathrm{Cu}(\mathrm{MeCN})_{4}\right] \mathrm{ClO}_{4}$ (baseline correction and dilution factor applied)

As described above, it was possible to tune the equilibrium between the ${ }^{\mathbf{S}} \mathbf{P} / \mathbf{O}$ isomers with stoichiometry, starting from the ${ }^{\mathbf{S}} \mathbf{P}(\mathbf{5})$ complex. Tuning this equilibrium beginning from the $\mathbf{O}(\mathbf{9})$ isomer was also possible (Scheme 7.4). Besides the ligand behaving as an external base, its basic character was retained when used 
in excess with a $\mathrm{Cu}(\mathrm{I})$ salt. Oxygenation of 2 equivalents of $\mathbf{H L}^{1}$ with 1 equivalent of $\left[\mathrm{Cu}(\mathrm{I})(\mathrm{MeCN})_{4}\right] \mathrm{PF}_{6}$ at low temperatures yielded a dark green colored solution, as opposed to the expected purple colored ${ }^{\mathbf{S}} \mathbf{P}$ complex 5. Spectroscopic analysis revealed the direct formation of the $\mathbf{O}$ complex $\mathbf{9}$, with the formation of bands at $335 \mathrm{~nm}$ and $392 \mathrm{~nm}$ on oxygenation (Fig.7.13 left). Subsequent addition of a $\left[\mathrm{Cu}(\mathrm{I})(\mathrm{MeCN})_{4}\right] \mathrm{PF}_{6}$ solution resulted in formation of $\mathbf{5}$, with the band at $392 \mathrm{~nm}$ decreasing in intensity and absorbtion bands for the ${ }^{\mathbf{S}} \mathbf{P}$ complex $\mathbf{5}$ persisting with duration of time (Fig. 7.13 right).

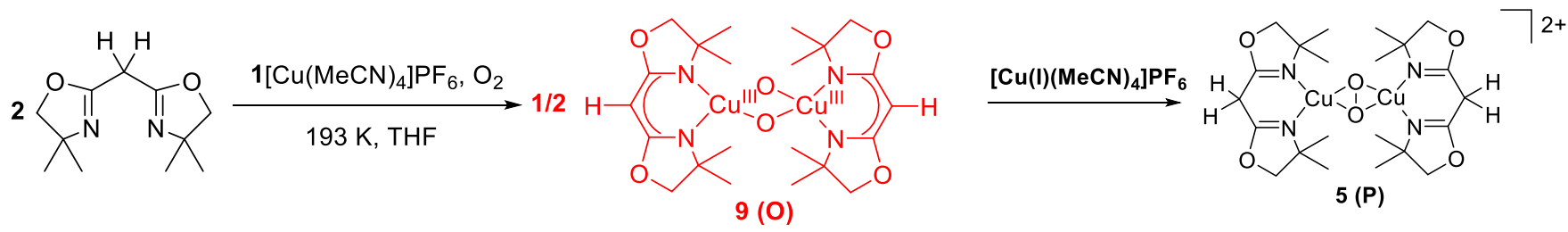

Scheme 7.4 Schematic representation of oxygenation of a 2:1 solution of $\mathrm{HL}^{1}:\left[\mathrm{Cu}(\mathrm{I})(\mathrm{MeCN})_{4}\right] \mathrm{PF} 6$ in $\mathrm{THF}$ at $193 \mathrm{~K}$ to form 9. Subsequent addition of $\left[\mathrm{Cu}(\mathrm{I})(\mathrm{MeCN})_{4}\right] \mathrm{PF}_{6}$ leading to the formation of $\mathbf{5}$.
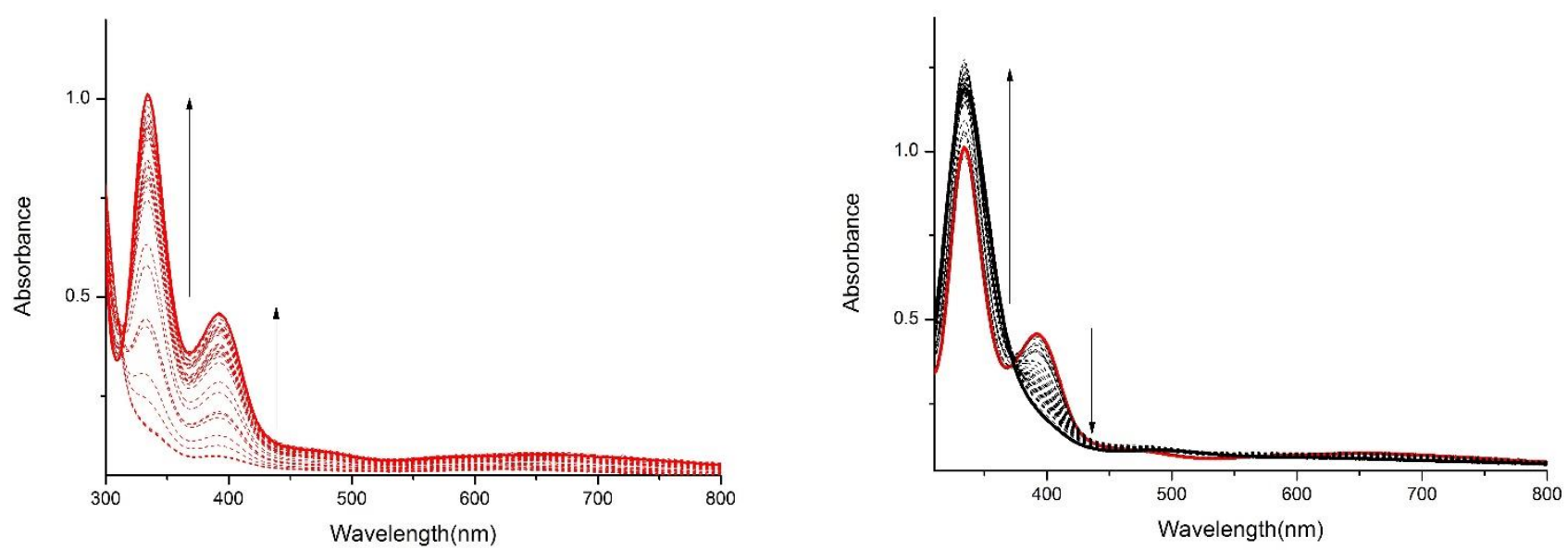

Figure 7.13 Left: Oxygenation of a 2:1 $\mathrm{HL}^{1}: \mathrm{Cu}(\mathrm{I})$ solution in $\mathrm{THF}$ at $193 \mathrm{~K}$ with formation of bands at $335 \mathrm{~nm}$ and $392 \mathrm{~nm}$, demonstrating formation of $\mathbf{O}$ complex 9. Right: Titration of a $[\mathrm{Cu}(\mathrm{I}) \mathrm{MeCN}] \mathrm{PF}_{6}$ solution into a solution of $\mathbf{9}$ to form the ${ }^{\mathbf{S}} \mathbf{P}$ complex 5.

In order to confirm the formation of the $\mathbf{O}$ complex 9 when a 2:1 stoichiometric mixture of $\mathbf{H L}^{1}: \mathrm{Cu}(\mathrm{I})$ was used, resonance Raman studies with a laser excitation of $633 \mathrm{~nm}$ were carried out. THF solutions of 2:1 $\mathrm{HL}^{1}: \mathrm{Cu}(\mathrm{I})$ were exposed to naturally abundant $\left({ }^{16} \mathrm{O}_{2}\right)$ or isotopically labelled dioxygen $\left({ }^{18} \mathrm{O}_{2}\right)$ at $193 \mathrm{~K}$ in young NMR tubes. Frozen solutions in liquid nitrogen at $77 \mathrm{~K}$ revealed only one oxygen isotope sensitive feature seen with a single peak at $598 \mathrm{~cm}^{-1}$ which shifted to $571 \mathrm{~cm}^{-1}$ on labelling $\left(\Delta^{16} \mathrm{O}-{ }^{18} \mathrm{O}=27 \mathrm{~cm}^{-1}\right)($ Fig. 7.14). These features have been assigned to the $\mathrm{Cu}_{2}(\mu-\mathrm{O})_{2}$ core vibration and are signature breathing modes of the $\mathbf{O}$ core. In comparison to the other $\mathbf{O}$ complexes $\mathbf{1 0}$ and 11, with the deprotonated ligands $\left[\mathbf{L}^{2}\right]^{-}$and $\left[\mathbf{L}^{3}\right]^{-}$respectively (refer to Chapter 7), rR studies revealed similar stretching frequencies at $598 \mathrm{~cm}^{-1}$ which shifted to $572 \mathrm{~cm}^{-1}$ on labelling, for the system $\left[\mathbf{L}^{2}\right]^{-}\left(\Delta^{16} \mathrm{O}-{ }^{18} \mathrm{O}=26 \mathrm{~cm}^{-1}\right)($ Fig 6.7 left $)$; and $601 \mathrm{~cm}^{-1}$ which shifted to $573 \mathrm{~cm}^{-1}\left(\Delta^{16} \mathrm{O}^{18}{ }^{18} \mathrm{O}=28 \mathrm{~cm}^{-1}\right)$ (Fig 6.7 right) for the system $\left[\mathbf{L}^{3}\right]^{-}$. Complexes $\mathbf{1 0}$ and $\mathbf{1 1}$ were charcterized in detail in solution as well as crystallographically (in case of 10), which confirmed 
unambigously the dioxygen to bind as a bis( $\mu$-oxo) motif. The similarities in the rR features of $\mathbf{1 0}$ and $\mathbf{1 1}$ with an oxygenated 2:1 solution of $\mathbf{H L}^{\mathbf{1}}: \mathrm{Cu}(\mathrm{I})$ confirms the formation of the $\mathbf{O}$ complex $\mathbf{9}$ at low temperatures. Furthermore, there was no evidence of the side on peroxo dicopper(II) species which is expected to exhibit the O-O stretching vibration at $720-765 \mathrm{~cm}^{-1}$ and with an $\Delta^{18} \mathrm{O}$ shift of $40-50 \mathrm{~cm}^{-1}$ [136,218,220]

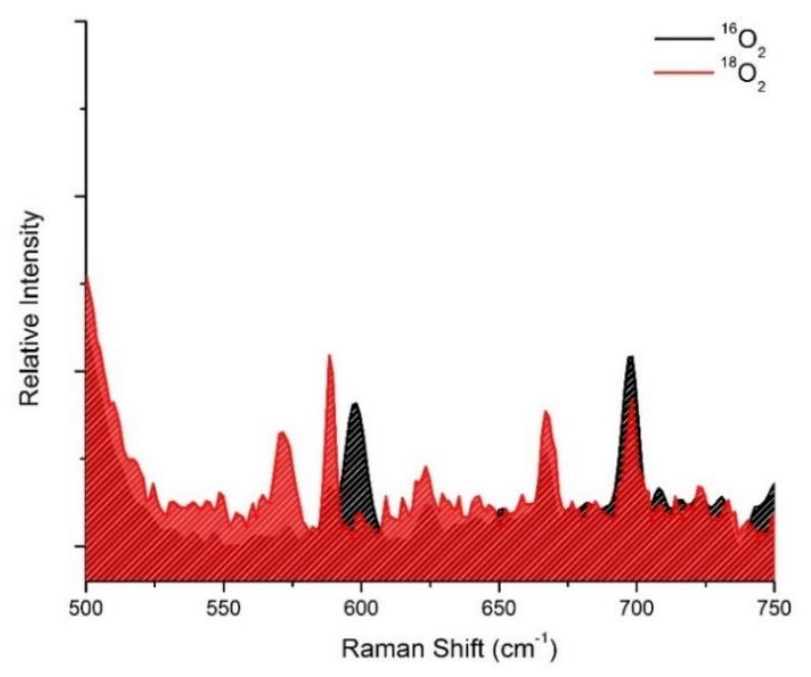

Figure 7.14 Resonance Raman spectrum of an oxygenated 2:1 $\mathrm{HL}_{1}:\left[\mathrm{Cu}(\mathrm{I})(\mathrm{MeCN})_{4}\right] \mathrm{PF}_{6}$ frozen solution in $\mathrm{THF}$ at $77 \mathrm{~K}$, showing the formation of the $\mathbf{O}$ complex $9 .{ }^{16} \mathrm{O}_{2}$ spectrum indicated in black and ${ }^{18} \mathrm{O}_{2}$ indicated in red.

In addition to the proton responsive ligand $\mathbf{H L}^{\mathbf{1}}$ behaving as a base, the same was demonstrated for ligands $\mathbf{H L}^{2}, \mathbf{H L}^{3}$ and $\mathbf{H L}^{5}$.

For the ${ }^{\mathbf{S}} \mathbf{P}$ complex $\mathbf{6}$ with ligand system $\mathbf{H L}^{2}, 8$ equivalents of the ligand per dicopper species was required for conversion of $\mathbf{6}$ to its $\mathbf{O}$ complex 10 (Fig. 7.15 left). Addition of a $\mathrm{Cu}(\mathrm{I})$ source (10 equivalents per dinuclear copper species) did indeed revert back the equilibrium to $\mathbf{6}$, however the absorbance bands were fairly high due to large increase in the concentration of the UV-vis solutions (Fig. 7.15 right; Scheme 7.3). 

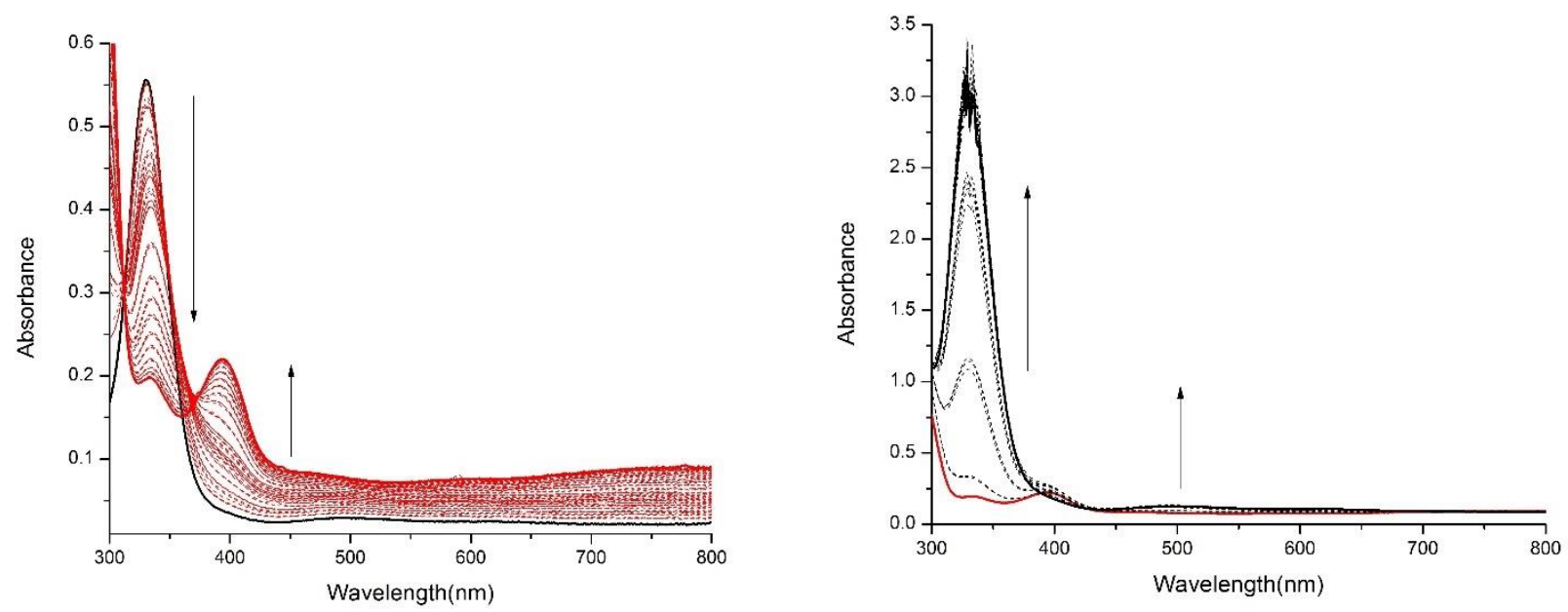

Figure 7.15 Left: Titration of $\mathbf{H L}^{2}$ to a solution of $\mathbf{6}$ in THF at $193 \mathrm{~K}$ resulting in the formation of 10, displaying the ligand to behave as a base. Right: Subsequent titration of a $\left[\mathrm{Cu}(\mathrm{I})(\mathrm{MeCN})_{4}\right] \mathrm{ClO}_{4}$ solution to $\mathbf{1 0}$ to reform $\mathbf{6}$.

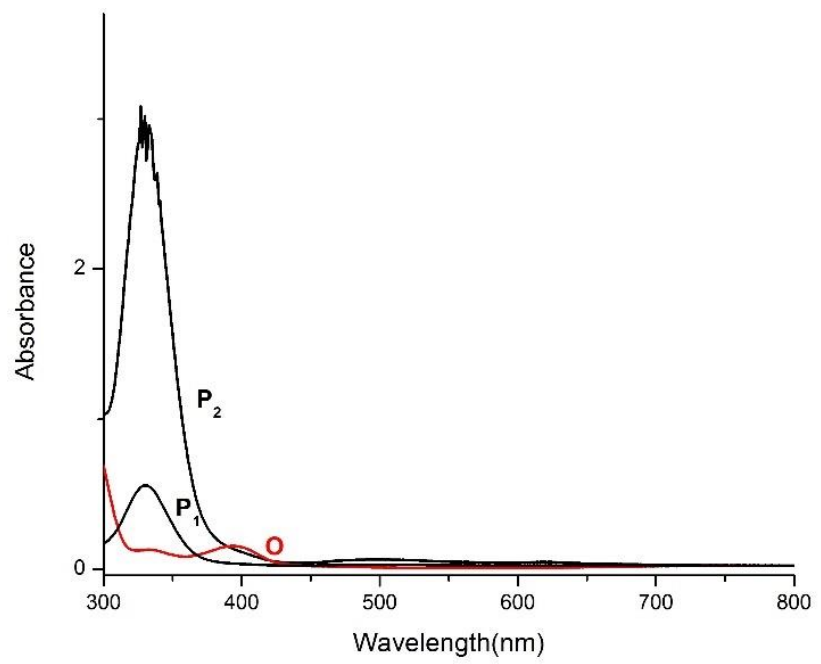

Figure 7.16 UV-vis monitoring of the reaction sequence demonstrating the interconversion of $\mathbf{6}$ to $\mathbf{1 0} \mathrm{using}_{\mathbf{H L}} \mathbf{H L}^{2}$ a base, and subsequent conversion of $\mathbf{1 0}$ to $\mathbf{6}$ with $\left[\mathrm{Cu}(\mathrm{MeCN})_{4}\right] \mathrm{ClO}_{4}$ (baseline correction and dilution factor applied)

For ligand systems $\mathbf{H L}^{3}$ and $\mathbf{H} \mathbf{L}^{\mathbf{5}}, 2.5$ and 2 equivalents of ligand per dinuclear copper species were required for conversion of their ${ }^{\mathbf{S}} \mathbf{P}$ to $\mathbf{O}$ forms respectively (Fig. 7.17 left and 7.19 left). Addition of a $\mathrm{Cu}(\mathrm{I})$ source, 5 equivalents per dinuclear copper species for $\mathbf{1 1}$ and 2 equivalents per dinuclear copper species for 12, resulted in the distinct formation of the ${ }^{\mathrm{S}} \mathbf{P}$ band at $\sim 500 \mathrm{~nm}$. However, in both cases, the band at $\sim 333 \mathrm{~nm}$ appeared only as a shoulder (Fig. 7.17 right and Fig.7.19 right). 

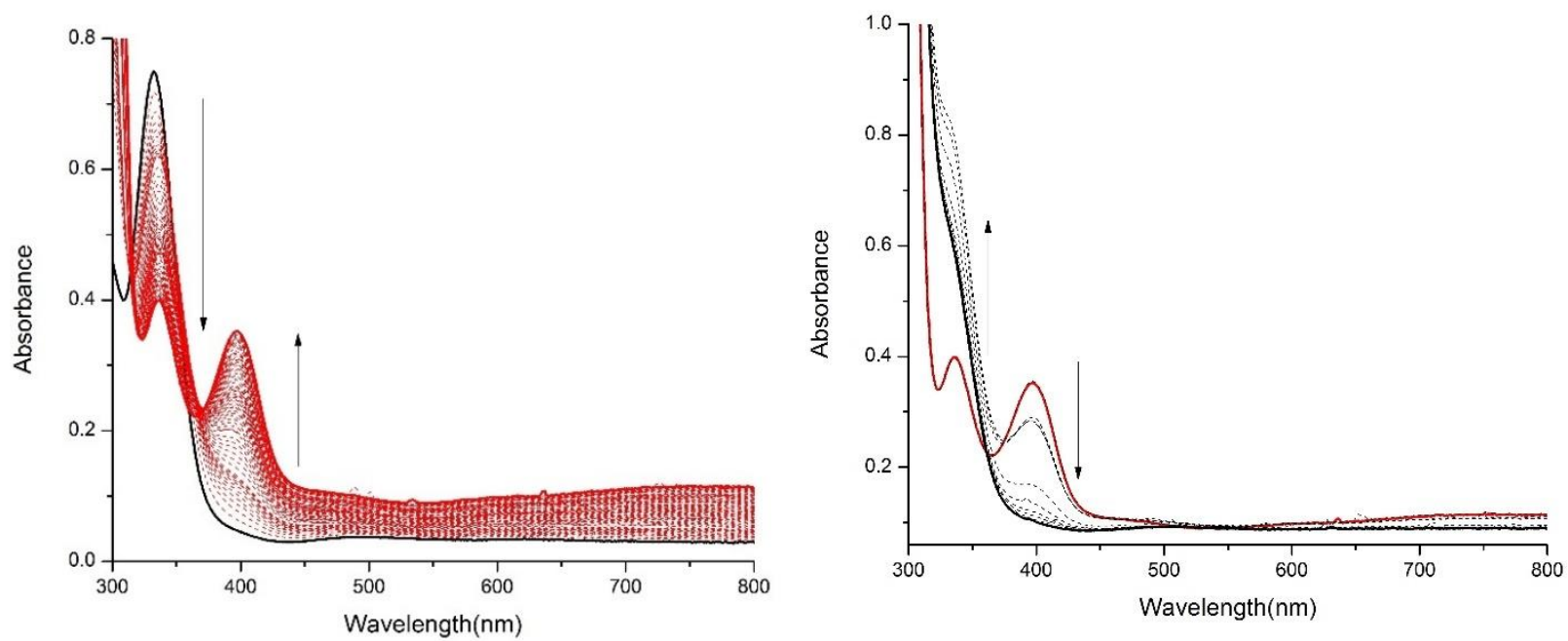

Figure 7.17 Left: Titration of $\mathbf{H L}^{3}$ to a solution of ${ }^{\mathbf{S}} \mathbf{P H L}{ }^{3}$ in THF at $193 \mathrm{~K}$ resulting in the formation of $\mathbf{1 1}$, displaying the ligand to behave as a base. Right: Subsequent titration of a $\left[\mathrm{Cu}(\mathrm{I})(\mathrm{MeCN})_{4}\right] \mathrm{ClO}_{4}$ solution to $\mathbf{1 1}$ to reform ${ }^{\mathbf{S}} \mathbf{P H L}^{\mathbf{3}}$.

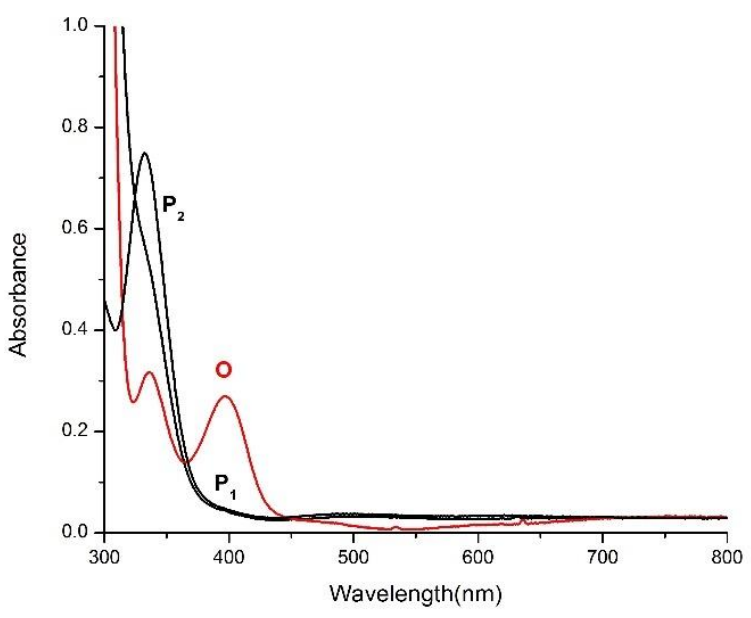

Figure 7.18 UV-vis monitoring of the reaction sequence demonstrating the interconversion of ${ }^{\mathrm{S}} \mathbf{P H L}$ to $\mathbf{1 1} \mathbf{u s i n g}^{\mathbf{H}} \mathbf{H L}^{3}$ as a base and subsequent conversion of 11 to ${ }^{5} \mathbf{P H L}^{3}$ with $\left[\mathrm{Cu}(\mathrm{MeCN})_{4}\right] \mathrm{ClO}_{4}$ (baseline correction and dilution factor applied). 

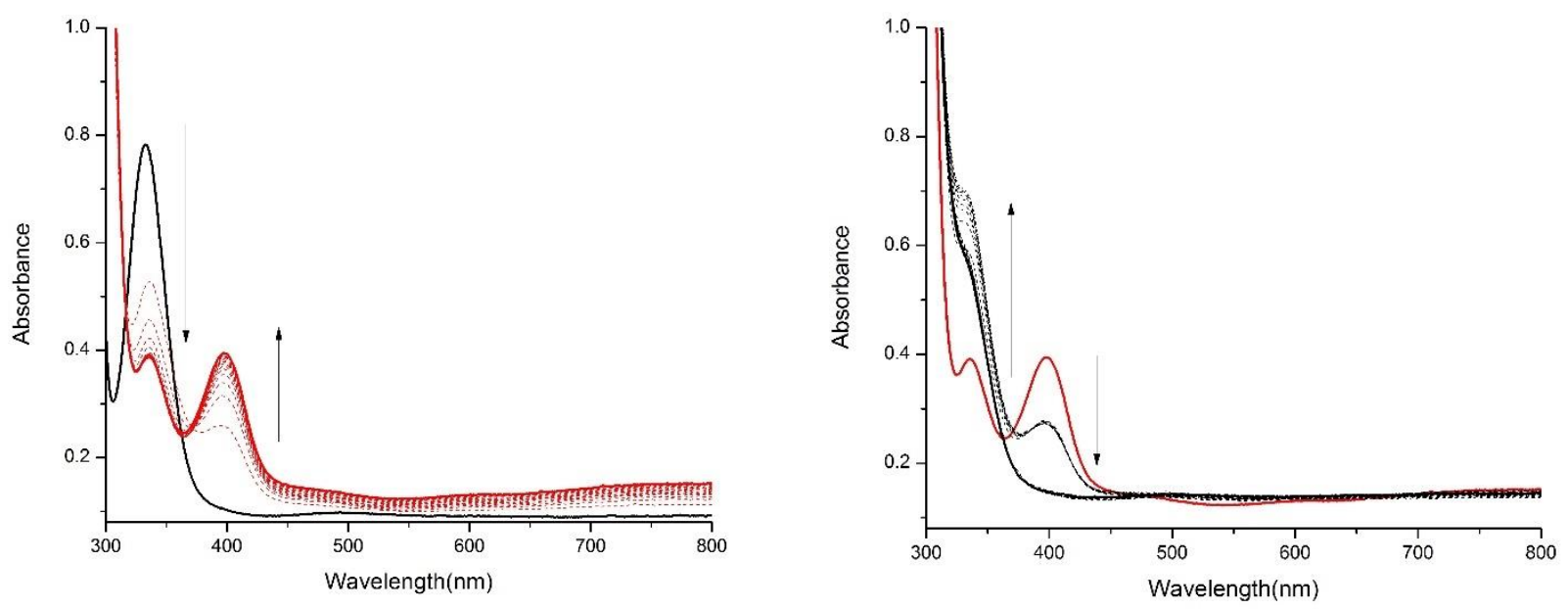

Figure 7.19 Left: Titration of $\mathbf{H L}^{\mathbf{5}}$ to a solution of 7 in THF at $193 \mathrm{~K}$ reuslting in the formation of 12, displaying the ligand to behave as a base. Right: Subsequent titration of a $\left[\mathrm{Cu}(\mathrm{I})(\mathrm{MeCN})_{4}\right] \mathrm{ClO}_{4}$ solution to 12 to reform 7.

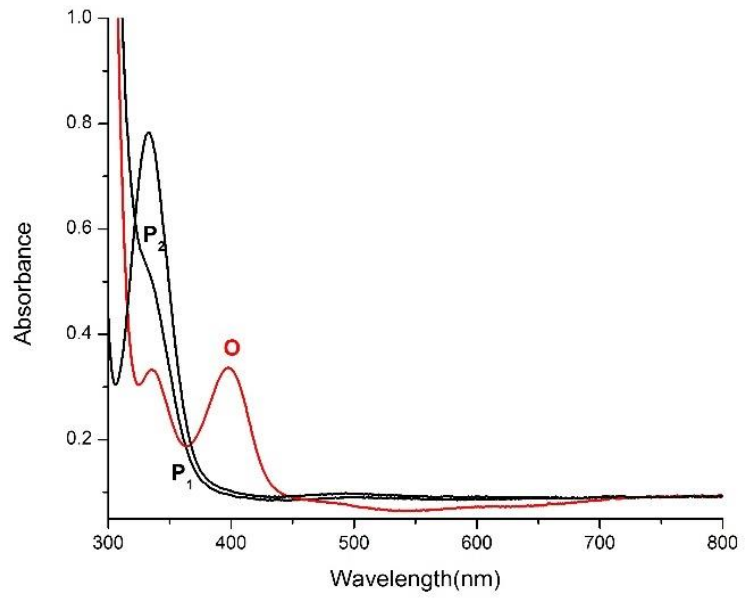

Figure 7.20 UV-vis monitoring of the reaction sequence demonstrating the interconversion of 7 to $\mathbf{1 2}$ using $\mathbf{H L}^{\mathbf{5}}$ as a base and subsequent conversion of $\mathbf{1 2}$ to 7 with $\left[\mathrm{Cu}(\mathrm{MeCN})_{4}\right] \mathrm{ClO}_{4}$ (baseline correction and dilution factor applied) 


\subsection{Conclusion}

In conclusion, during the course of this work, a new factor "pH" was shown to affect the equilibrium between ${ }^{\mathbf{S}} \mathbf{P} / \mathbf{O}$ complexes of proton responsive BOX ligands.

By virtue of a base, it was possible to successfully shift the equilibrium from the ${ }^{\mathbf{S}} \mathbf{P}$ to the $\mathbf{O}$ isomers of ligands $\mathbf{H L}^{1}, \mathbf{H L}^{2}, \mathbf{H L}^{3}$ and $\mathbf{H L}^{5}$. All reactions were monitored via $\mathrm{UV}$-vis spectroscopy wherein addition of an external base led to changes in spectroscopic features from the ${ }^{\mathbf{S}} \mathbf{P}$ to the $\mathbf{O}$ complexes. However, the same was not observed for the non-proton responsive ligand system $\mathbf{L}^{6}$. The deprotonated ligands form suitable scaffolds to stabilize the +3 oxidation state of copper which facilitates a cleavage of the $\mathrm{O}-\mathrm{O}$ bond, shifting the equilibrium from the dicopper(II) $\left(\mu-\eta^{2}: \eta^{2}\right)$-peroxo isomer to the dicopper(III) bis( $\mu$-oxo) isomer. Addition of an acid did not revert the equilibrium back to the ${ }^{\mathbf{S}} \mathbf{P}$ complexes but lead to their decomposition. This was attributed to the high nucleophilicity of the bis( $\mu$-oxo) core. As discussed in Chapter 6, the $\mathrm{Cu}(\mathrm{I})$ complexes of the deprotonated ligand systems $\left[\mathbf{L}^{2}\right]^{-}$and $\left[\mathbf{L}^{3}\right]^{-}$directly form their $\mathbf{O}$ complexes with $\mathrm{O}_{2}$ at low temperatures, which have been characterized in detail confirming the dioxygen binding mode to be the bis( $\mu$-oxo) motif. This further confirms the new intermediate formed by addition of a base to be an $\mathbf{O}$ species.

The stoichiometry of the proton responsive ligands was shown to affect the $\mathrm{Cu} / \mathrm{O}_{2}$ intermediate that formed at low temperatures and resulted in the POP (peroxo-oxo-peroxo) switch. For ligand $\mathbf{H L}^{\mathbf{1}}$, a $1: 1$ stoichiometric mixture of $\mathrm{HL}^{1}: \mathrm{Cu}(\mathrm{I})$ lead to the formation of the ${ }^{\mathbf{S}} \mathbf{P}$ intermediate (Chapter 5), whereas a 2:1 stoichiometric mixture of $2 \mathrm{HL}^{1}: \mathrm{Cu}(\mathrm{I})$ favored the $\mathbf{O}$ intermediate. This was confirmed by UV-vis spectroscopy as well as rR studies, and was attributed to the ligand behaving as a base. The ligands $\mathbf{H L}^{2}$, $\mathbf{H L}^{3}$ and $\mathbf{H L}^{\mathbf{5}}$ also displayed base like character wherein addition of these ligands to their respective ${ }^{\mathbf{S}} \mathbf{P}$ complexes resulted in their $\mathbf{O}$ isomers. In all cases the equilibrium could be reverted by the external addition of a $\mathrm{Cu}(\mathrm{I})$ source.

The external affect of "pH" is a new factor that contributes to tuning the equilibrium between ${ }^{\mathbf{S}} \mathbf{P} / \mathbf{O}$ intermdiates. It is interesting to note that in type III dicopper proteins as well as in the dicopper active site of pMMO, all copper ions are coordinated by histidine imidazoles. These offer a backside $\mathrm{N}$ atom amenable to potential (de)protonation equilibria in response to local $\mathrm{pH}$ changes. In fact, (de)protonation of histidine imidazole ligands in metalloproteins is widely used for tuning redox potentials and electronic structures of the metallocofactors. ${ }^{[232-234]}$ The ability to tune the equilibrium between these novel intermediates in a synthetic system via $\mathrm{pH}$ puts forward a plausible theory that perhaps, $\mathrm{pH}$ forms a pertinent factor in controlling the active species responsible for catalytic activity in these biological systems. 
[Note: This page had intentionally been left blank 


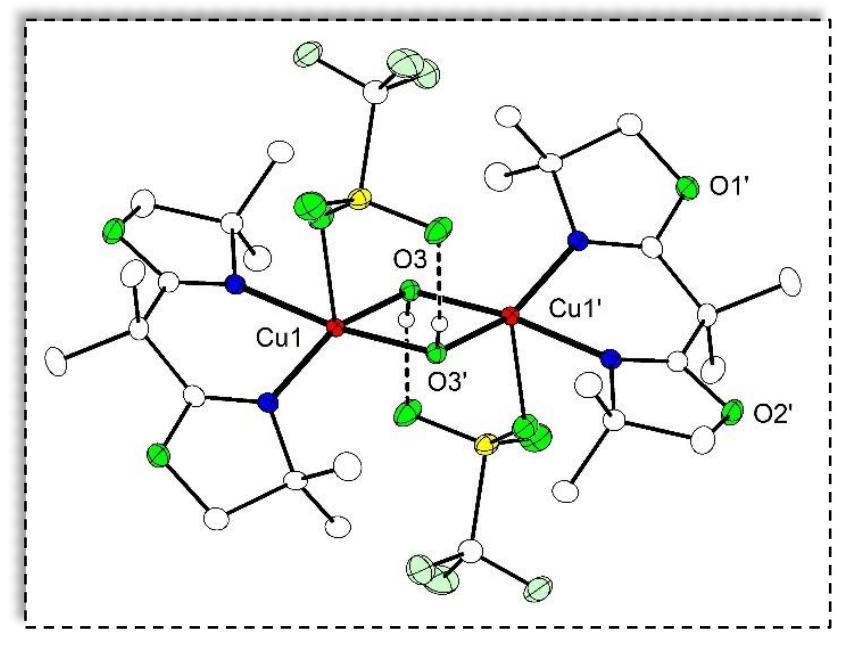

\section{Chapter 8}

Stability of ${ }^{S} P$ vs O Complexes of BOX Ligands and their Substrate. Reactivity

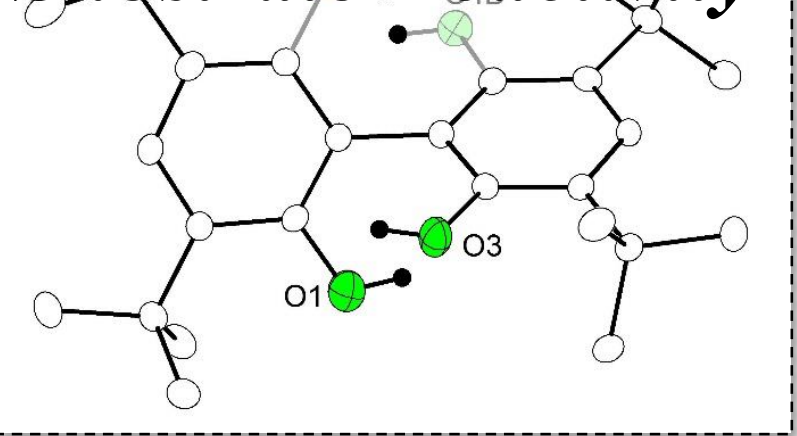




\section{Overview}

8.1 Introduction

8.2 Substrate reactivity of ${ }^{\mathbf{S}} \mathbf{P}$ complexes with proton-responsive BOX ligands

8.2.1 Potential PCET Reactions

8.3 Substrate reactivity exhibited by ${ }^{\mathbf{S}} \mathbf{P}$ complex of non-proton-responsive BOX ligands

8.4 Substrate reactivity exhibited by $\mathbf{O}$ complexes of monoanionic BOX ligands

8.5 Stability of ${ }^{\mathbf{S}} \mathbf{P}$ complexes vs $\mathbf{O}$ complexes

8.5.1 Decomposition of ${ }^{\mathbf{S}} \mathbf{P}$ complex $\mathbf{8}$

8.5.1.1 Characterization in solution

8.5.1.2 Solid state characterization of $\mathbf{1 6}$

8.5.1.3 Structural elucidation of $\mathbf{1 6}$

8.6 Conclusion

[Note: Experimental procedures for this chapter are described in detail under Chapter 15, section 15.6] 


\subsection{Introduction}

The contrasting reactivity demonstrated by different $\mathrm{Cu} / \mathrm{O}_{2}$ intermediates, $\left({ }^{\mathrm{S}} \mathbf{P}, \mathbf{O},{ }^{\mathrm{T}} \mathbf{P}\right.$, and $\left.{ }^{\mathbf{C}} \mathbf{P}\right)$ towards organic substrates is attributed to the nature of their dioxygen binding modes (Fig. 8.1). ${ }^{[196]}$ The end-on bound peroxo moieties ${ }^{\mathbf{T}} \mathbf{P}$ and ${ }^{\mathbf{C}} \mathbf{P}$, which are not considered to be biologically relevant but contribute to understanding the mechanism of dioxygen binding, tend to be more basic and nucleophilic in nature. Though in 2010, Garcia-Bosch et al. proposed a ${ }^{\mathbf{T}} \mathbf{P}$ model system to demonstrate electrophilic catalytic behavior, ${ }^{[211]}$ further analysis of this $\mathrm{Cu} / \mathrm{O}_{2}$ synthetic system revealed it to be in equilibrium with an $\mathbf{O}$ species which was responsible for catalytic reactivity, with the nucleophilic reactivity of the ${ }^{\mathbf{T}} \mathbf{P}$ binding mode being retained. ${ }^{[212]}$ A number of ${ }^{\mathbf{S}} \mathbf{P}$ model systems that parallel the dioxygen binding mode of Ty have shown electrophilic reactivity towards organic substrates emulating the enzyme (refer to sec. 2.5 of introduction). ${ }^{[95,200,201]}$ Synthetic $\mathbf{O}$ complexes known to be in equilibrium with their ${ }^{\mathrm{S}} \mathbf{P}$ congeners in the majority of cases carry out $\mathrm{H}$-atom abstraction reactions to form unphysiological radical based products. Though in a few cases, $\mathbf{O}$ complexes have also demonstrated electrophilic catalytic reactivity. ${ }^{[207,208]}$

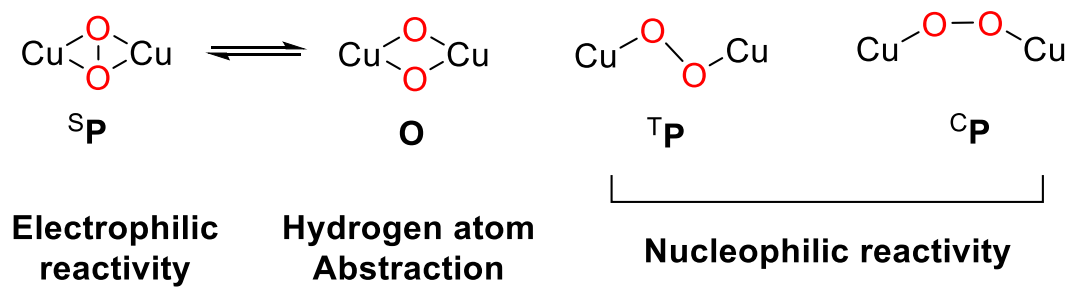

Figure 8.1 Different reactivity patterns observed in $\mathrm{Cu}_{2} \mathrm{O}_{2}$ adducts.

The following sections describe the reactivity patterns displayed by $\mathrm{Cu}_{2} \mathrm{O}_{2}$ complexes that have been synthesized and described in Chapters 5 and 6. A series of external substrates (Fig. 8.2), such as the sodium salt of 2,4-di-tert-butyl phenolate (DTBP-Na), 2,4-di-tert-butyl-phenol (DTBP-H), triphenyl phosphine $\left(\mathrm{PPh}_{3}\right)$, and thioanisole $(\mathrm{PhSMe})$ were screened for reactivity. In addition, radical substrates were also screened with ${ }^{\mathrm{S}} \mathbf{P}$ complexes of proton responsive BOX ligands to investigate potential PCET reactions.<smiles>CC(C)(C)c1ccc(O[Na])c(C(C)(C)C)c1</smiles>

DTBP-Na<smiles>CC(C)(C)c1ccc(O)c(C(C)(C)C)c1</smiles>

DTBP-H<smiles>CC1(C)CCCC(C)(C)N1[O-]</smiles>

TEMPO<smiles>CC(C)(C)c1cc(C(C)(C)C)c([O])c(C(C)(C)C)c1</smiles>

2,4,6-tri-tert-butyl phenoxy radical<smiles>c1ccc(P(c2ccccc2)c2ccccc2)cc1</smiles>

$\mathrm{PPh}_{3}$

PhSMe

Figure 8.2 Substrates that have been screened for reactivity during the course of this work. 


\subsection{Substrate reactivity of ${ }^{\mathrm{S}} \mathrm{P}$ complexes with proton-responsive BOX ligands}

Of the substrates mentioned above, $\mathrm{PPh}_{3}$ and $\mathrm{PhSMe}$ showed no external reactivity with ${ }^{\mathbf{S}} \mathbf{P}$ complexes of proton responsive BOX ligands. Addition of these substrates to ${ }^{\mathrm{S}} \mathbf{P}$ solutions $\mathbf{5}, \mathbf{6},{ }^{\mathrm{S}} \mathbf{P} \mathbf{H L}^{\mathbf{3}}$, and $\mathbf{7}$ at $193 \mathrm{~K}$ in THF resulted in no spectral changes when analyzed by UV-vis spectroscopy, and analysis of the organic products by ${ }^{1} \mathrm{H}-\mathrm{NMR}$ spectroscopy after workup revealed peaks corresponding only to the starting material. In contrast, addition of the external substrates DTBP-Na and DTBP-H:NEt ${ }_{3}$ (1:2) to ${ }^{\mathbf{S}} \mathbf{P}$ solutions of these ligands demonstrated interesting reactivity.

Gradual titration of DTBP-Na or DTBP-H:NEt 3 (1:2) $193 \mathrm{~K}$ in THF to ${ }^{\mathrm{S}} \mathbf{P}$ complexes $\mathbf{5}, \mathbf{6},{ }^{\mathbf{S}} \mathbf{P H L}$, and 7 resulted in a change of their characteristic LMCT bands (refer to Chapter 5). The band at $333 \mathrm{~nm}$ started to decrease in intensity with a new band forming at $\sim 400 \mathrm{~nm}$. The shoulder at $\sim 500 \mathrm{~nm}$ diminished with the resulting spectra resembling the $\mathbf{O}$ complexes $\mathbf{9 , 1 0}, \mathbf{1 1}, \mathbf{1 2}$ respectively (refer to Chapter 7). On comparing the resultant UV-vis spectra of ${ }^{\mathbf{S}} \mathbf{P}$ complexes with external substrates DTBP-Na or DTBP-H:NEt 3 (1:2) with those formed by deprotonation of their ${ }^{\mathbf{S}} \mathbf{P}$ congeners, the spectra were identical suggesting that these external substrates behave as a base. Figure 8.3 (left) demonstrates the reactivity of ${ }^{\mathbf{S}} \mathbf{P}$ complex $\mathbf{6}$ with DTBP-Na where a total of 4 equivalents of substrate were required for full conversion of 6 to 10. The figure on the right, (Fig. 8.3 right) demonstrates the deprotonation of complex $\mathbf{6}$ with DBU to form $\mathbf{1 0}$. As is evident from these figures, the resulting spectra in both cases have similar bands at $333 \mathrm{~nm}$ and $395 \mathrm{~nm}$. Further addition of the substrate, $\sim 300$ equivalents, led to gradual fading of the $\mathbf{O}$ bands at $333 \mathrm{~nm}$ and 395 $\mathrm{nm}$. The same was observed for ${ }^{\mathbf{S}} \mathbf{P}$ complexes $\mathbf{5},{ }^{\mathbf{S}} \mathbf{P H L}_{3}$ and $\mathbf{7}$. In case of DTBP-H:NEt ${ }_{3,}(1: 2)$ a neutral phenol in the presence of a base, similar spectral changes were observed.

On workup of the reaction mixtures according to standard procedures and analysis of the organic products by ${ }^{1} \mathrm{H}-\mathrm{NMR}$ spectroscopy, a $33 \%$ conversion of substrate to the unphysiological radical based C-C coupled product was observed (Scheme 8.1). No catalytic conversion of the external phenols to catechols or quinones resulted from ${ }^{\mathbf{S}} \mathbf{P}$ complexes of proton responsive BOX ligands.
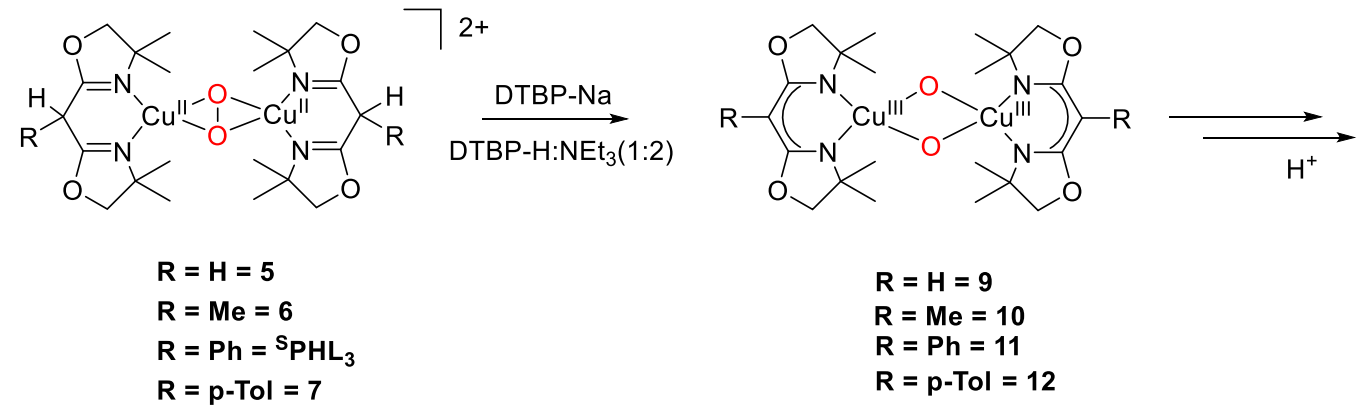<smiles>CC(C)(C)c1cc(-c2cc(C(C)(C)C)cc(C(C)(C)C)c2O)c(O)c(C(C)(C)C)c1</smiles>

$33 \%$

Scheme 8.1 Reactivity of ${ }^{\mathbf{S}} \mathbf{P}$ complexes $\left(\mathbf{5}, \mathbf{6},{ }^{\mathbf{S}} \mathbf{P H L}^{\mathbf{3}}, \mathbf{7}\right)$ of proton responsive BOX ligands with DTBP-Na or DTBP-H:NEt 3 $(1: 2)$ to generate their $\mathbf{O}$ isomers $\mathbf{9}, \mathbf{1 0}, \mathbf{1 1}$ and 12, which after acidic workup results in the formation of $33 \% \mathrm{C}-\mathrm{C}$ coupled product. 

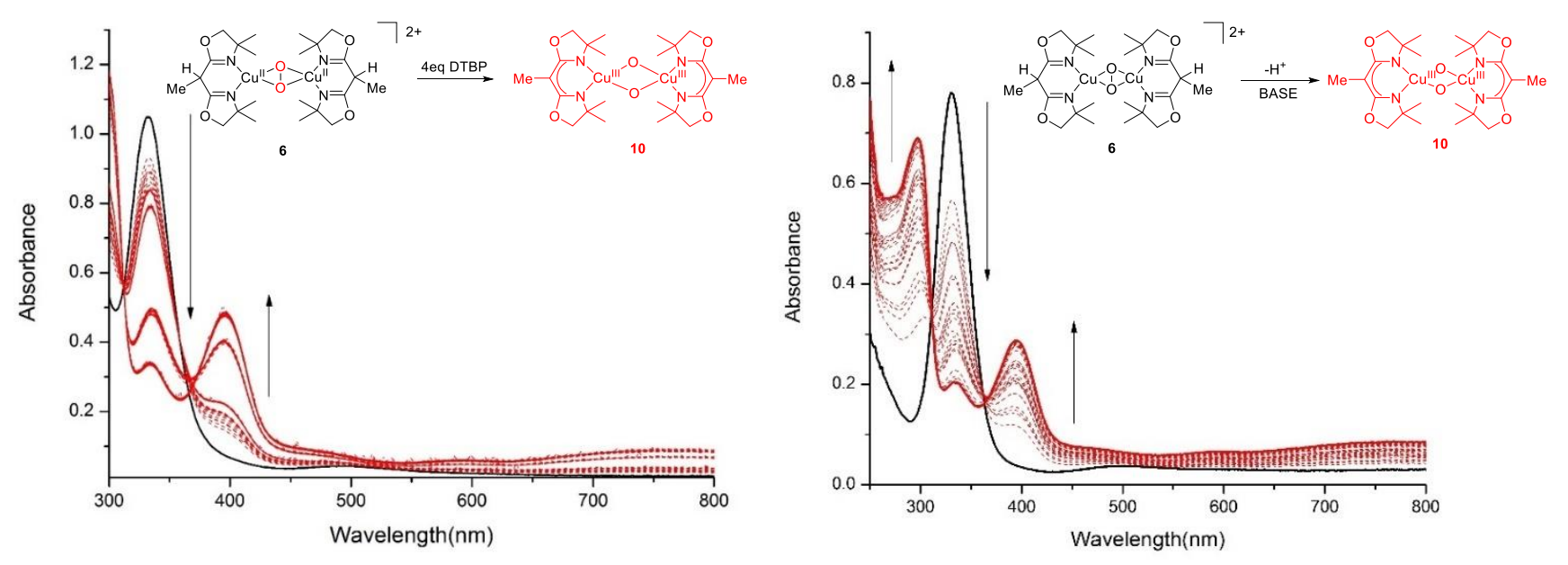

Figure 8.3 Left: UV-vis monitoring of ${ }^{\mathrm{S} P}$ complex 6 with DTBP to generate the $\mathbf{O}$ isomer 10. Right: UV-vis monitoring of 6 with DBU to generate $\mathbf{1 0 .}$

Thus the proton responsive nature of BOX ligands inherently forbids reactivity of their corresponding ${ }^{\mathbf{S}} \mathbf{P}$ complexes with basic substrates such as phenolates. These substrates trigger formation of the $\mathbf{O}$ complexes by deprotonation of the ligand backbone, facilitating $\mathrm{O}-\mathrm{O}$ bond cleavage. Further reactivity of these $\mathbf{O}$ complexes in the presence of an excess of substrate leads to formation of unphysiological products.

\subsubsection{Potential PCET Reactions}

As PCET reactions form an integral part of various biological processes, reactivity of ${ }^{\mathbf{S}} \mathbf{P}$ complex $\mathbf{6}$ with suitable radical reactors such as TEMPO and the 2,4,6 tri-tert-butyl phenoxy radical were screened to test their feasibility (Scheme 8.2). However, addition of these substrates did not result in any spectral changes corresponding to formation of any mixed-valent peroxo species. The only changes in UV-vis features were caused by the substrate itself upon addition. Though TEMPO/TEMPOH redox couple stands as an excellent example of a PCET reagent, due its feasible BDFE and low O-H bond strength, ${ }^{[235]}$ the inability to meadiate PCET reactions on ${ }^{\mathbf{S}} \mathbf{P}$ systems discussed in Scheme 8.2 sugessts that the BDFE of the C-H bond within the ${ }^{\text {SP }}$ complex is much higher than that of radical substarte. The same was demonstrated by the 2,4,6 tri-tertbutyl phenoxy radical.

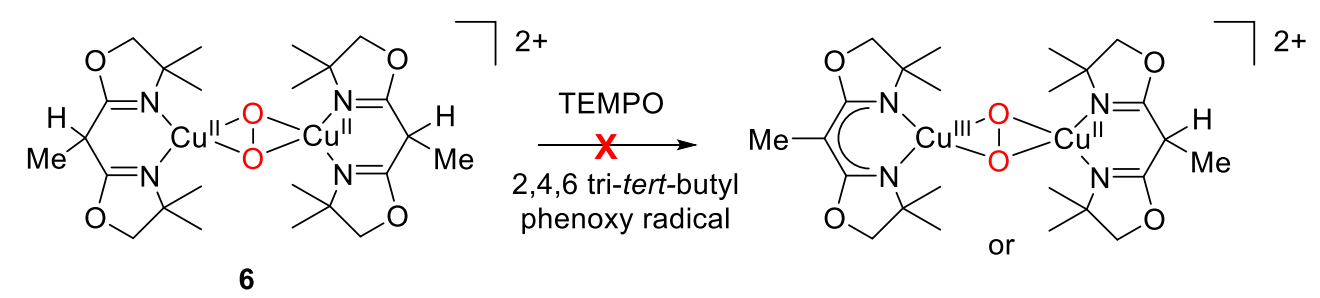

Scheme 8.2 Reactivity scheme to test the feasibility of PCET reactions with ${ }^{\mathrm{S}} \mathrm{P}$ complex 6.

As is discussed in Chapter 7, the $\mathbf{O}$ complexes of proton responsive ligands were formed on deprotonating their ${ }^{\mathbf{S}} \mathbf{P}$ congeners. A second route to generate these was by reacting the $\mathrm{Cu}(\mathrm{I})$ analogues of the monoanionic 
ligands $\left[\mathbf{L}^{2}\right]^{-}$and $\left[\mathbf{L}^{3}\right]^{-}$with dioxygen at low temperatures (Chapter 6). For both these cases, a bis $(\mu$-oxo) dicopper(III) complex resulted. A third route to generate a bis( $\mu$-oxo) dicopper(II) complexe from its bis ( $\mu$-hydroxo) counterpart with the assistance of a base was attempted. The $\mathrm{Cu}(\mathrm{I})$ complex of ligand $\mathbf{H L}^{2}$ was exposed to dioxygen at room temperatures to generate directly the bis( $\mu$-hydroxo) species. This solution was cooled to $193 \mathrm{~K}$ and an external base, $\mathrm{DBU} / n \mathrm{BuLi}$ was gradually titrated into the solution. However, no spectral changes corresponding to an $\mathbf{O}$ complex was observed (Scheme 8.3). Generating the ${ }^{\mathbf{S}} \mathbf{P}$ complex 6 with the TEMPO radical (Scheme 8.3) from the bis( $\mu$-hydroxo) complex was also unattainable. On UV-vis monitoring, addition of TEMPO to a bis( $\mu$-hydroxo) solution of $\mathbf{H L}^{2}$ resulted in no spectral changes corresponding to ${ }^{\mathrm{S}} \mathbf{P}$ complex $\mathbf{6}$.

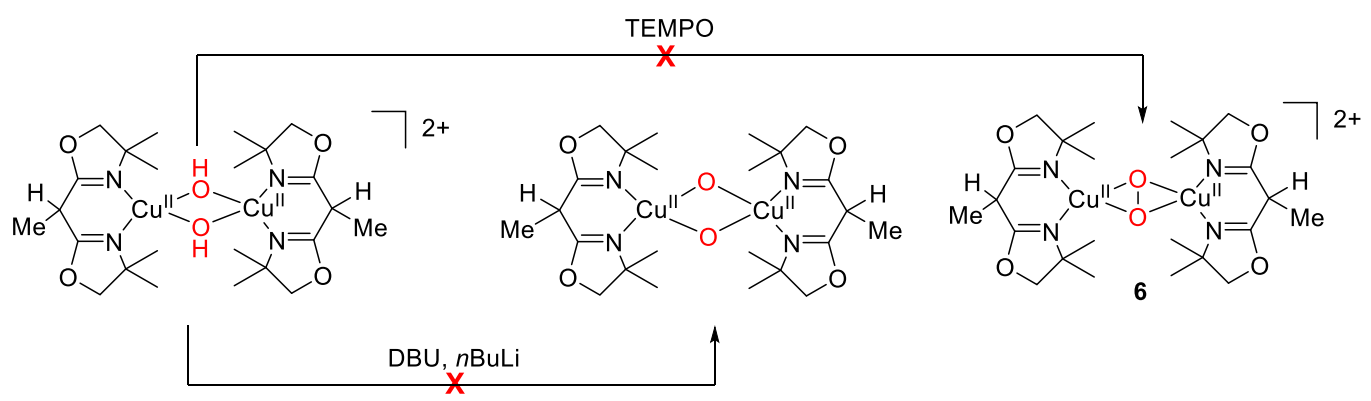

Scheme 8.3 Reactivity scheme to test the feasibility of generating ${ }^{\mathbf{S}} \mathbf{P}$ complex $\mathbf{6}$ and a bis( $\mu$-oxo) dicopper(II) complex from the respective bis( $\mu$-hydroxo) complex.

The unfeasibility of generating the ${ }^{\mathbf{S}} \mathbf{P}$ and $\mathbf{O}$ complexes from their bis( $\mu$-hydroxo) counterparts in Scheme 8.3 could be attributed to the thermodynamic unfavorability of these reactions. The deprotonation of the hydroxide moiety to an oxide, in order to generate a dicopper(II) bis( $\mu$-oxo) species is disfavoured plausibly due to the +2 oxidation state hosted by the copper ions which is unsuited for such a binding mode. The inability of the potential PCET reaction to generate the ${ }^{\mathbf{S}} \mathbf{P}$ complex from its bis( $\mu$-hydroxo) counterpart, could be attributed to the BDFE of the $\mathrm{O}-\mathrm{H}$ bond of the bis( $\mu$-hydroxo) complex being higher than that of the radical substrate. ${ }^{[235]}$ Though only a few radical substrates were screened, it is worthwhile attempting alternative comparable substrates given the importance of PCET reactions within biological systems.

\subsection{Substrate reactivity exhibited by ${ }^{\mathrm{S}} \mathrm{P}$ complex of non-proton-responsive BOX ligands}

The substrate reactivity demonstrated by $\mathbf{8}$, a ${ }^{\mathbf{S}} \mathbf{P}$ motif of a non-proton responsive ligand $\mathbf{L}^{6}$ differed from systems of proton responsive ligands. Addition of DTBP-Na to a purple colored solution of $\mathbf{8}$ at $193 \mathrm{~K}$ in THF resulted in a color change to brown, and warming the solution to room temperature resulted in a color change to green. UV-vis monitoring of the reaction revealed a gradual decrease of the bands at $\sim 333 \mathrm{~nm}$ and $\sim 500 \mathrm{~nm}$ with formation of a shoulder like region at $\sim 400 \mathrm{~nm}$ (Fig. 8.4 left). A total of 2.1 equivalents of substrate was required for total decomposition of $\mathbf{8}$. On working up the reaction mixtures according to standard procedures, a yellow colored oil was obtained. Analysis of this oil by ${ }^{1} \mathrm{H}-\mathrm{NMR}$ showed $13.5 \%$ conversion of the DTBP-Na to DTBP-Q (quinone) (Scheme 8.4) with no other side products (Fig. 8.5). 

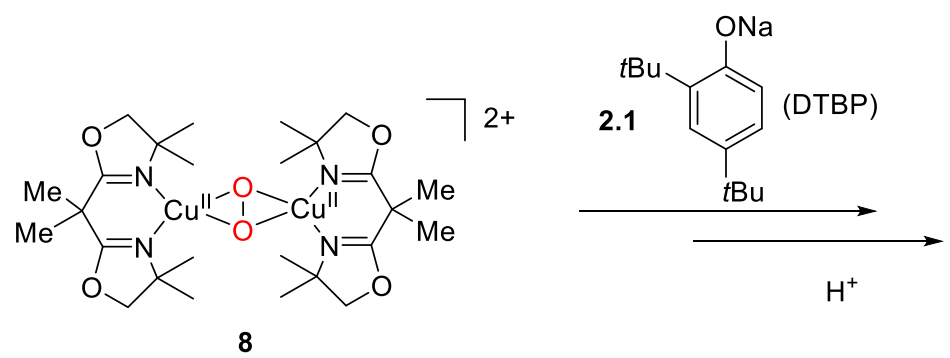<smiles>CC(C)(C)C1=CC(=O)C(=O)C(C(C)(C)C)=C1</smiles>

$13.5 \%$

Scheme 8.4 Reactivity of ${ }^{\mathbf{S}} \mathbf{P}$ complex $\mathbf{8}$ with 2.1 equivalents of DTBP at $193 \mathrm{~K}$ in THF. Acidic workup of the reaction mixture revealed $13.5 \%$ conversion of the starting material to DTBP-Q.
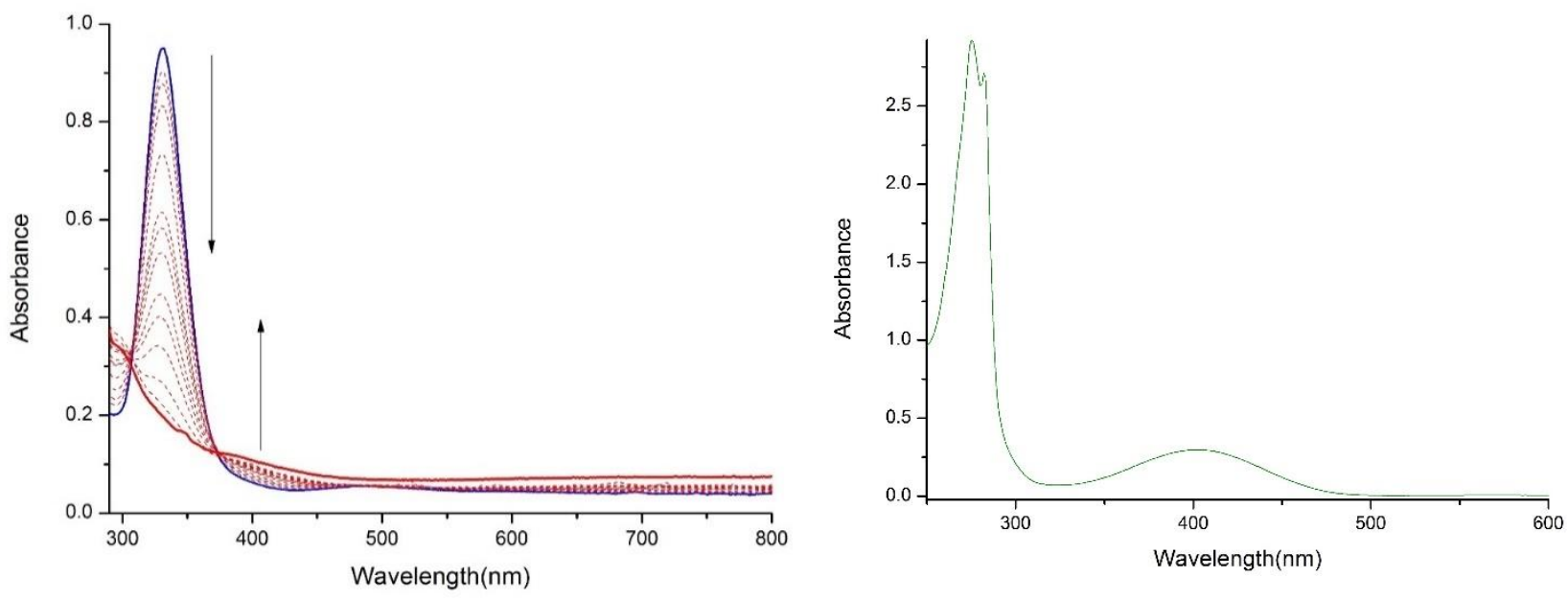

Figure 8.4 Left: Titration of ${ }^{S} \mathbf{P}$ complex 8 with 2.1 equivalents of DTBP-Na over 15 minutes, bands at $333 \mathrm{~nm}$ and $500 \mathrm{~nm}$ decrease in intensity with a slight shoulder formation $400 \mathrm{~nm}$. Right: UV-vis spectra of DTBP-Q in THF at rt with a band at $411 \mathrm{~nm}$.

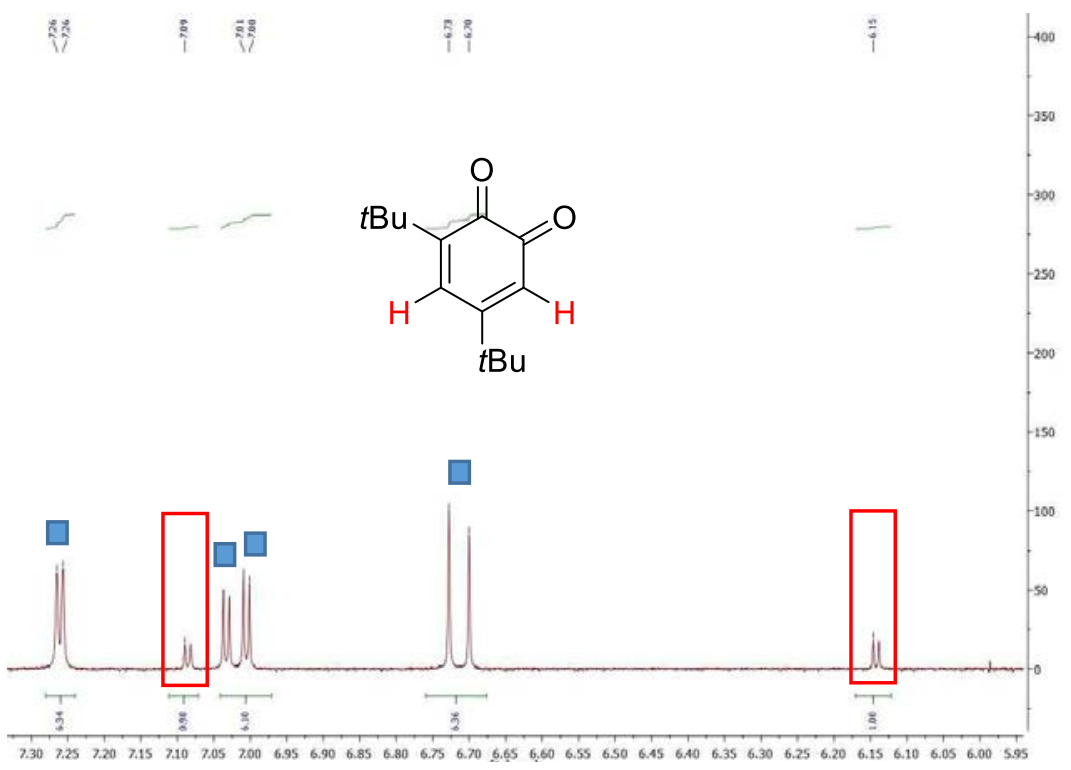

Figure 8.5 ${ }^{1} \mathrm{H}-\mathrm{NMR}$ of end product of the reaction of $\mathbf{8}$ with 2.1 eq DTBP-Na in acetone-d $\mathrm{d}_{6}$ at $293 \mathrm{~K}$ after workup. Shown in red squares are the signals corresponding to the protons of the DTBP-Q(quinone). Signals marked in $\square$ belong to the starting material after workup. 
DTBP-Q was further characterized via UV-vis spectroscopy. Measurements at room temperature in THF resulted in a band at $411 \mathrm{~nm}$ characteristic of quinones (Fig.8.4 right) in agreement to the observed shoulder like region that appeared for the eaction mixture on addition of the substrate. Analysis via HPLC-MS confirmed the product to have a mass of $221(\mathrm{~m} / \mathrm{z})^{+}$in accordance with protonated DTBP-Q. In order to gain deeper insight into the mechanism of the reaction, ESI-MS experiments using ${ }^{16} \mathrm{O}_{2}$ and ${ }^{18} \mathrm{O}_{2}$ labelled 8 were conducted. Unfortunately, the spectra showed a large mixture of products with unfeasible assignment to any potential intermediates.

Reactivity of a slightly larger amount of substrate, ( $\sim 10$ equivalents $)$ with $\mathbf{8}$ resulted in the formation of both the DTBP-Q as well as the C-C coupled product with $6 \%$ and $14 \%$ conversion respectively, whereas addition of a large excess, ( $\sim 100$ equivalents of DTBP-Na) formed exclusively the C-C coupled product with $9 \%$ conversion (Scheme 8.5). The reactions were carried out similar to that with 2.1equivalents of substrate and the products were analyzed via ${ }^{1} \mathrm{H}-\mathrm{NMR}$ spectroscopy.

In contrast to DTBP-Na, the neutral phenol DTBP-H in the presence of $\mathrm{NEt}_{3}$ did not show the same effect. A total of $\sim 150$ equivalents of DTBP-H:NEt 3 (1:2) was required for total decomposition of $\mathbf{8}$ in contrast to 2.1 equivalents of DTBP-Na. The solution was then worked up and analyzed via ${ }^{1} \mathrm{H}-\mathrm{NMR}$ spectroscopy which revealed a $10 \%$ conversion of the starting material to the $\mathrm{C}-\mathrm{C}$ coupled product and no catalytic conversion of the phenol to catechol or quinone.

This difference in the reactivity between DTBP-Na and DTBP-H:NEt 3 (1:2) can be attributed to the substrate being deprotonated before the reaction (DTBP-Na), or being deprotonated during the course of the reaction (DTBP-H:NEt ${ }_{3}$ ). In case of DTBP-Na, the deprotonated phenolate easily approaches the $\mathrm{Cu} / \mathrm{O}_{2}$ intermediate leading to reactivity favoring physiological hydroxylation. However, the neutral phenol even in the presence of a base favors unphysiological reactivity suggesting the importance of deprotonation of the phenolic substrate before the aromatic hydroxylation step.

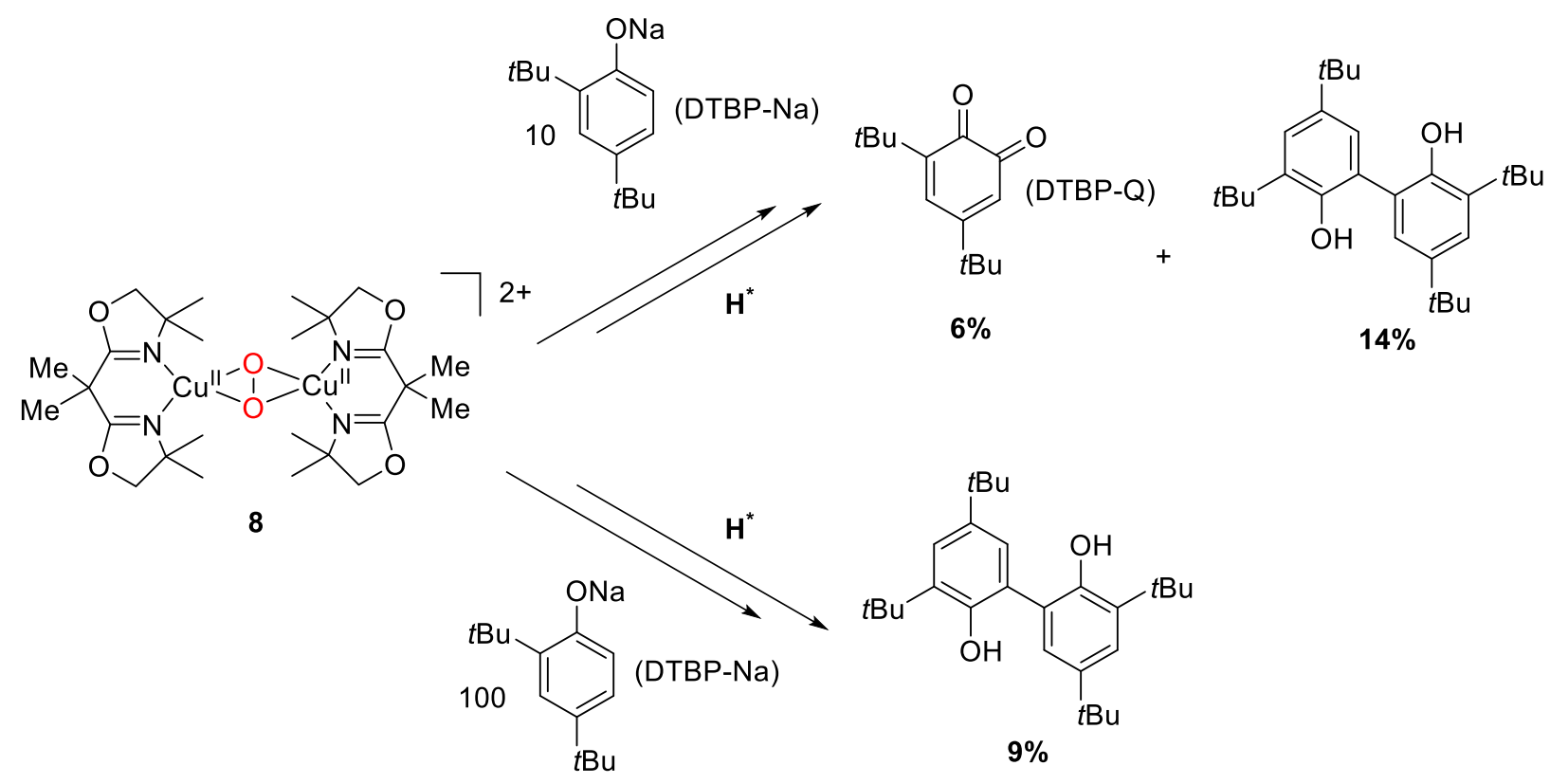

Scheme 8.5 Reactivity of ${ }^{\mathrm{S}} \mathbf{P}$ complex $\mathbf{8}$ with 10 equivalents of DTBP-Na to generate a mixture of products, and with 100 equivalents of DTBP-Na to generate only the $\mathrm{C}-\mathrm{C}$ coupled product. 
In conclusion, the nature of the ligand plays a crucial role in determining substrate reactivity. Contrary to the proton responsive ligand systems, the ${ }^{\mathbf{S}} \mathbf{P}$ complex $\mathbf{8}$ of the non-proton responsive ligand $\mathbf{L}^{\mathbf{6}}$ did not yield its $\mathbf{O}$ complex. Substoichiometric o-hydroxylation mimicking Ty reactivity was observed with deprotonated phenolates. Unfortunately, catalytic conversion of the substrate did not occur suggesting the mechanism of the reaction to be dependent on the concentration of the substrate. A detailed insight into the mechanism of this reaction is presently under investigation.

\subsection{Substrate reactivity exhibited by $O$ complexes of monoanionic BOX ligands}

The $\mathbf{O}$ complexes 10 and 11 were screened with $\mathrm{PPh}_{3}, \mathrm{PhSMe}$, DTBP-Na, and DTBP-H. $\mathrm{PPh}_{3}$ and $\mathrm{PhSMe}$ showed no reactivity with these complexes. Addition of these substrate to $\mathbf{O}$ solutions (10 and $\mathbf{1 1})$ at 193 $\mathrm{K}$ in THF resulted in no spectral changes when analyzed by UV-vis spectroscopy and analysis of the organic products by ${ }^{1} \mathrm{H}-\mathrm{NMR}$ spectroscopy after workup revealed peaks corresponding only to the starting material.

Addition of a large excess of DTBP-Na and DTBP-H:NEt 3 (1:2) ( 250 equivalents) at $193 \mathrm{~K}$ over two hours resulted in decomposition of $\mathbf{1 0}$ and $\mathbf{1 1}$ on monitoring by UV-vis spectroscopy. The reaction mixtures were worked up according to standard procedures. ${ }^{1} \mathrm{H}-\mathrm{NMR}$ spectroscopy revealed a $50 \%$ conversion of the substrate to the $\mathrm{C}-\mathrm{C}$ coupled product with respect to the starting material (Scheme 8.6).

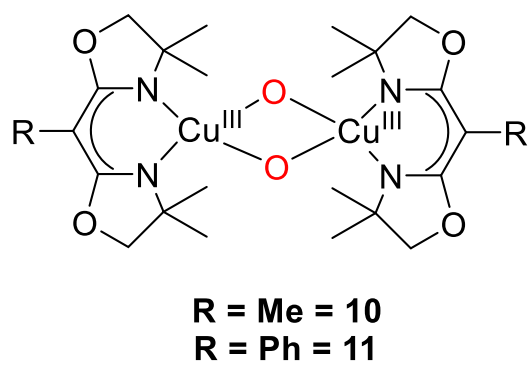

DTBP / DTBP-H:NEt 3 (1:2)<smiles>CC(C)(C)c1cc(-c2cc(C(C)(C)C)cc(C(C)(C)C)c2O)c(O)c(C(C)(C)C)c1</smiles>

$\mathrm{H}^{+}$

$50 \%$

Scheme 8.6 Reactivity of $\mathbf{O}$ complex 10 and 11 with DTBP-Na to generate the C-C coupled product. 50\% conversion occurs over 2 hours.

Unphysiological C-C coupled products are observed in a majority of cases with bis( $\mu$-oxo) dicopper(III) complexes. The $\mathbf{O}$ complexes of monoanionc BOX ligands presented herein carry out an impressive $50 \%$ conversion of the neutral phenol (in the presence of a NEt 3 ) to the $\mathrm{C}-\mathrm{C}$ coupled product. This can be attributed to the small size of the system which facilitates a fast outer-sphere one electron transfer reaction leading to the phenoxyl radical, similar to the $\mathrm{L}^{3}$ system reported by Herres-Pawlis et al. ${ }^{[208]}$ 


\subsection{Stability of ${ }^{\mathrm{S} P}$ complexes vs $\mathrm{O}$ complexes}

The new ${ }^{\mathbf{S}} \mathbf{P}$ complexes $\mathbf{7}$ and $\mathbf{8}$ as well as the $\mathbf{O}$ complexes $\mathbf{1 0}$ and $\mathbf{1 1}$ that were characterized during the course of this work showed gradual decomposition with time on warming up to room temperature. All decomposition reactions were monitored via UV-vis spectroscopy. Gradual warming of a solution of 7 from $193 \mathrm{~K}$ to rt resulted in a decrease in the intensity of the bands at $333 \mathrm{~nm}$ and $504 \mathrm{~nm}$ over a period of one hour (Fig. 8.6 left). For complexes 10 and 11, warming of the solutions to rt resulted in a decrease of the bands at $333 \mathrm{~nm}$ and $395 \mathrm{~nm}$ over one hour for $\mathbf{1 0}$ (Fig. 8.6 right) and a decrease in the bands at $337 \mathrm{~nm}$ and $397 \mathrm{~nm}$ over a period of four hours for 11 (Fig. 8.7) signifying decomposition of the complexes. Analysis of these products via ESI-MS did not yield valuable information about the nature of the decomposed products. The spectra individually showed a mixture of products comprising mainly of ligand peaks. In case of complex 8 however, the end product was isolated as a dicopper(II) bis( $\mu$-hydroxo) species. Further investigation of this process is discussed within this chapter.
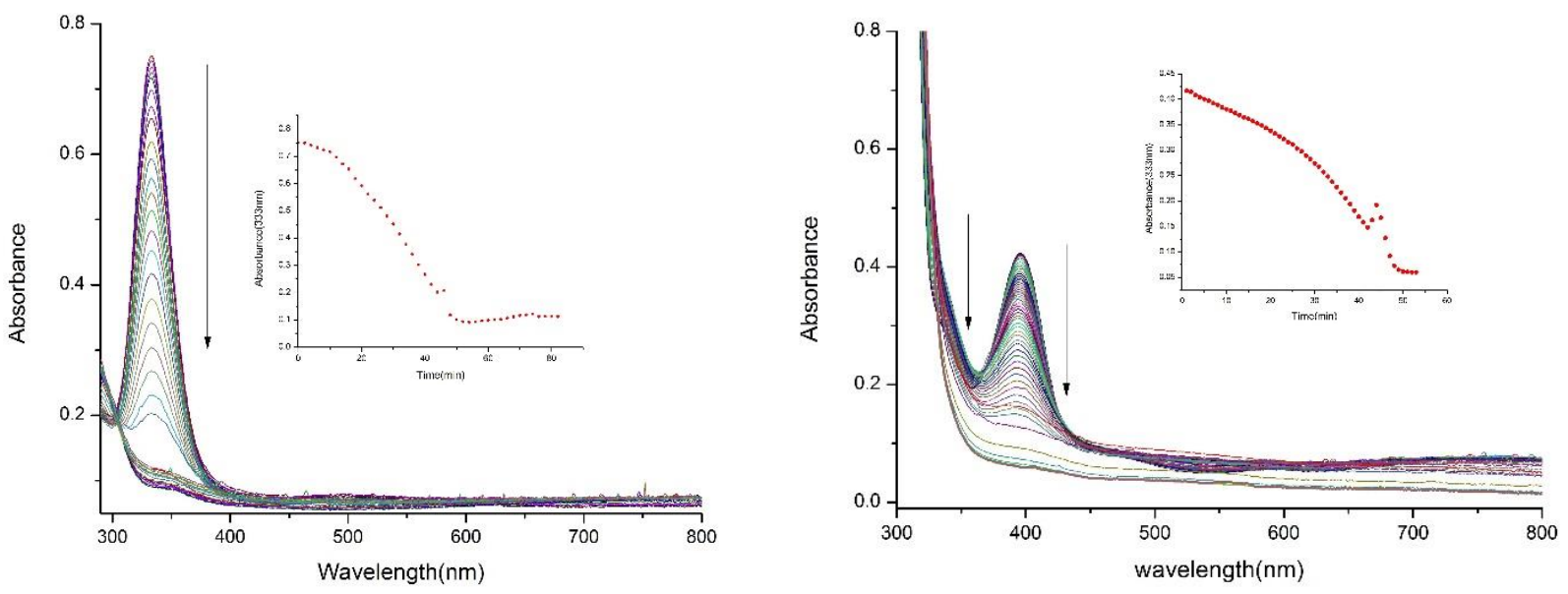

Figure 8.6 Left: Decomposition of 7 monitored by UV-vis spectroscopy depicting a decrease in bands at $333 \mathrm{~nm}$ and $504 \mathrm{~nm}$ with warming up of the solution from $193 \mathrm{~K}$ to rt. Shown in the inset is a decrease in the intensity of the band at $333 \mathrm{~nm}$ with time. Right: Decomposition of $\mathbf{1 0}$ monitored by UV-vis spectroscopy depicting a decrease in bands at $333 \mathrm{~nm}$ and $395 \mathrm{~nm}$ with warming up of the solution from $193 \mathrm{~K}$ to rt. Shown in the inset is a decrease in the intensity of the band at $333 \mathrm{~nm}$.

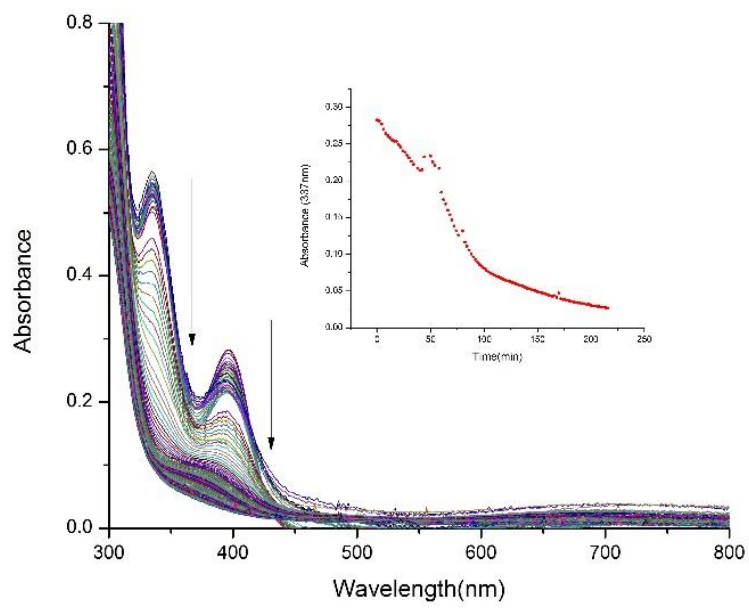

Figure 8.7 Decomposition of 11 monitored by UV-vis spectroscopy. A decrease in bands at $337 \mathrm{~nm}$ and $397 \mathrm{~nm}$ with warming up of the solution from $193 \mathrm{~K}$ to rt. Shown in the inset is a decrease in the intensity of the band at $337 \mathrm{~nm}$ with time. 


\subsubsection{Decomposition of ${ }^{\boldsymbol{S}} \boldsymbol{P}$ complex $\boldsymbol{8}$}

\subsubsection{Characterization in solution}

Warming up a solution of 8 from $193 \mathrm{~K}$ to rt resulted in a change of the purple colored solution to light blue within a span of 10 minutes. The reaction was monitored by UV-vis spectroscopy which displayed a distinct decrease in the intensity of the bands at $333 \mathrm{~nm}$ and $500 \mathrm{~nm}$ (Fig. 8.8 left). The solution was maintained at room temperature for a while for complete decomposition and the spectrum was measured again. No bands at $333 \mathrm{~nm}$ or $500 \mathrm{~nm}$ corresponding to 8 were observed, but a band with a broad shoulder like region at 680 $\mathrm{nm}\left(\varepsilon=250 \mathrm{M}^{-1} \mathrm{~cm}^{-1}\right)$ and rather low intensity appeared (Fig. $8.8 \mathrm{right}$ ). This was assigned to the dicopper(II) $\operatorname{bis}(\mu$-hydroxo) complex 16 (Scheme 8.7).

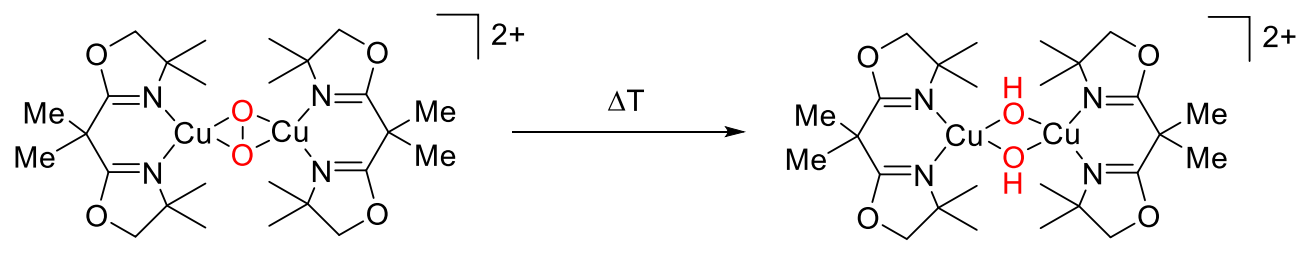

8

16

Scheme 8.7 Decomposition of ${ }^{\mathbf{S}} \mathbf{P}$ complex $\mathbf{8}$ to the bis( $\mu$-hydroxo) dicopper(II) analogue $\mathbf{1 6}$ with gradual warming up from $193 \mathrm{~K}$ to room temperature.
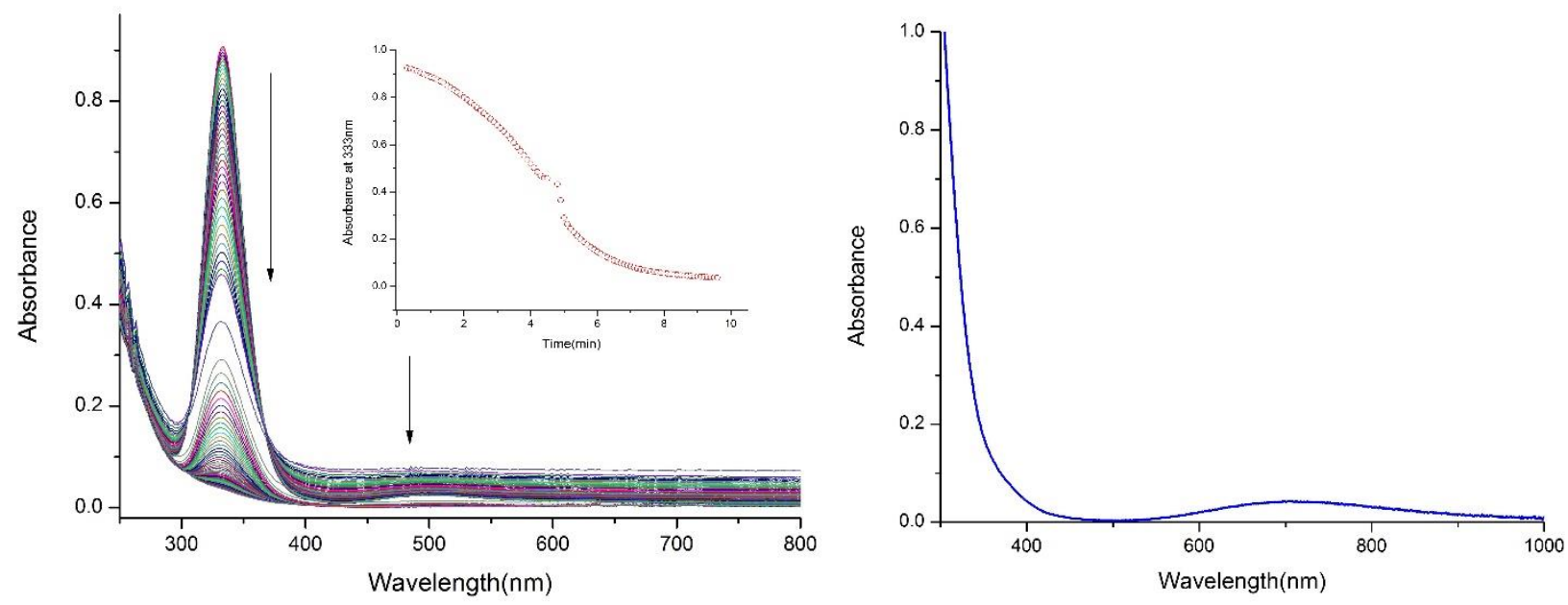

Figure 8.8 Left: Decomposition of 8 monitored by UV-vis spectroscopy depicting a decrease in bands at $333 \mathrm{~nm}$ and $500 \mathrm{~nm}$ with warming up of the solution from $193 \mathrm{~K}$ to rt over 10 minutes. Shown in the inset is a decrease in the intensity of the band at $333 \mathrm{~nm}$ with time. Right: UV-vis spectra of $\mathbf{1 6}$ in THF measured at rt. 


\subsubsection{Solid state characterization of $\mathbf{1 6}$}

The bis( $\mu$-hydroxo) dicopper(II) species (16) was also isolated in the solid state by addition of excess of $\mathrm{Et}_{2} \mathrm{O}$ to a THF solution of 16. A blue powder precipitated, which was filtered under aerobic conditions and dried under vacuum. 16 was characterized by IR spectroscopy (KBr pellet). A distinct peak at $3484 \mathrm{~cm}^{-1}$ was observed which was assigned to the hydroxo stretch of the complex (Fig. 8.9 left). The powder was also analyzed by solid state UV-vis spectroscopy whose features were similar to those of $\mathbf{1 6}$ in solution with a broad band $\sim 650 \mathrm{~nm}$ (Fig. 8.9 right)
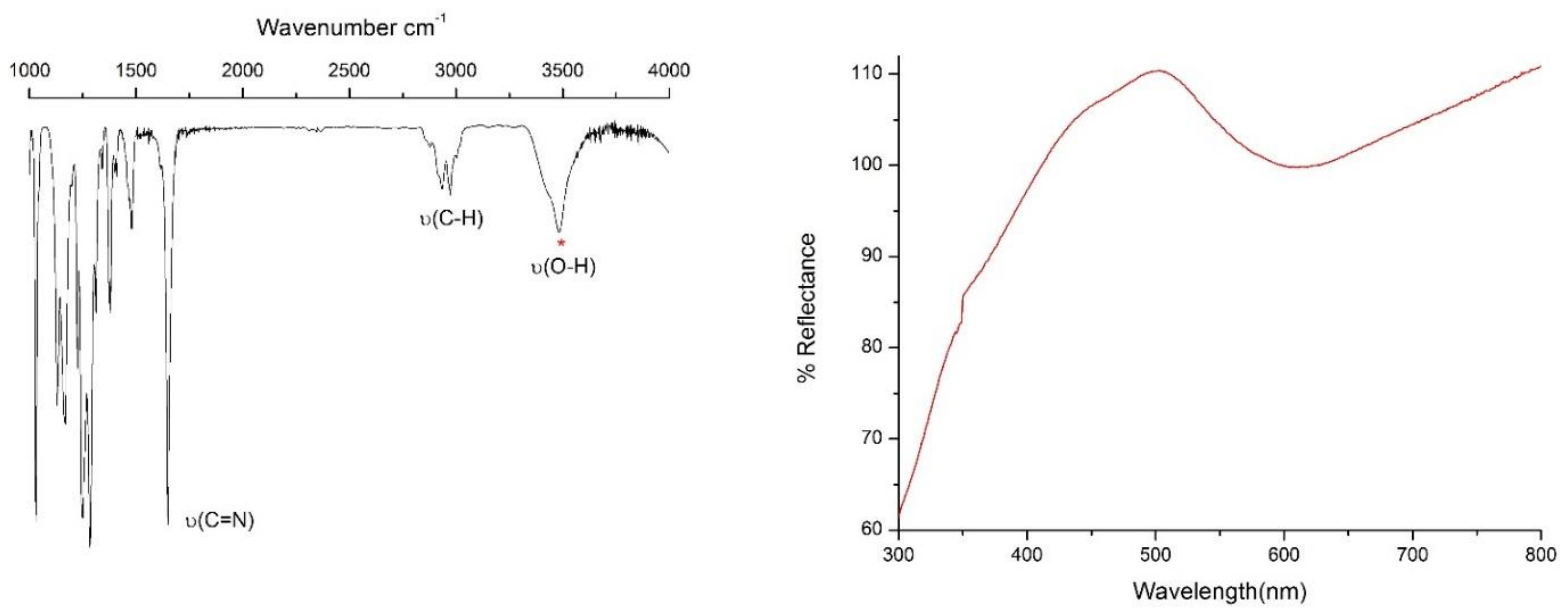

Figure 8.9 Left: IR spectra of 16 measured in a $\mathrm{KBr}$ pellet displaying the $\mathrm{OH}$ stretch at $3484 \mathrm{~cm}^{-1} \star$ Right: Solid state UVvis of 16 with a broad shoulder at $650 \mathrm{~nm}$.

\subsubsection{Structural elucidation of $\mathbf{1 6}$}

Single crystals suitable for X-ray diffraction were grown at room temperature by slow diffusion of $\mathrm{Et}_{2} \mathrm{O}$ into a THF solution of $\mathbf{1 6}$ (Fig. 8.10). X-ray diffraction analysis revealed the two cooper centers to be coordinated in a distorted square pyramidal geometry. The metal centers were coordinated to the nitrogen atoms of the ligand and oxygen atoms of the triflate counterion axially. The molecule crystallizes in a $P 2_{1}$ monoclinic space group. The $\mathrm{Cu} \cdots \mathrm{Cu}$ bond distance of $2.99 \AA$, and the $\mathrm{Cu}-\mathrm{O}$ bond length of $1.93 \AA$ were smaller compared to its peroxide analogue $8(\mathrm{Cu}-\mathrm{Cu} 3.52 \AA$ and $\mathrm{Cu}-\mathrm{O} 2.32 \AA)$. The $\mathrm{O} \cdots \mathrm{O}$ distance between the two hydroxide moieties was $2.48 \AA$, thus much larger than what was found for 8 (1.56 $)$, a result of the cleavage of the $\mathrm{O}-\mathrm{O}$ bond. In addition, the triflate ion was shown to be bound to the hydroxide units via hydrogen bonds. 


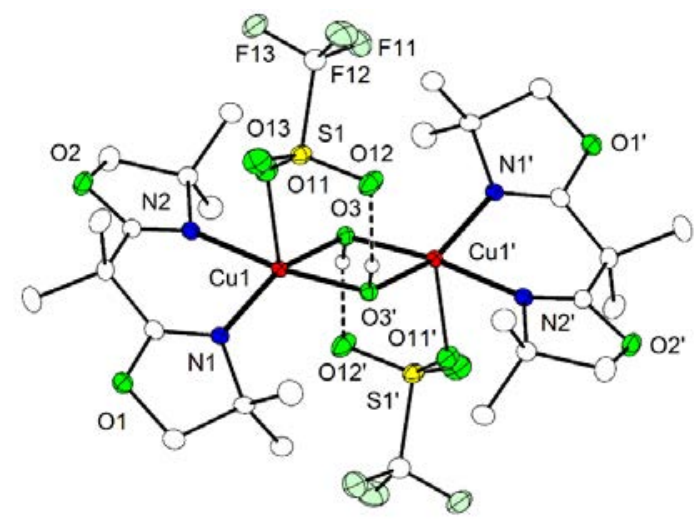

Figure 8.10. Plot (30\% probability thermal ellipsoids) of the molecular structure of $\mathbf{1 6}$ (most hydrogen atoms omitted for clarity). Selected distances $[\AA]$ and angles [ ${ }^{\circ}$ ]: $\mathrm{Cu} 1-\mathrm{O} 3$ 1.9338(14), Cu1-O3' 1.9596(14), Cu1-N1 2.0120(16), Cu1-N2

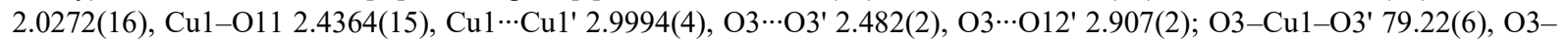
Cu1-N1 159.53(6), O3'-Cu1-N1 96.72(6), O3-Cu1-N2 98.00(6), O3'-Cu1-N2 171.57(6), N1-Cu1-N2 88.59(6), O3-Cu1O11 104.61(6), O3'-Cu1-O11 88.65(6), N1-Cu1-O11 95.28(6), N2-Cu1-O11 84.34(6), Cu1-O3-Cu1' 100.78(6), O3$\mathrm{H} 3 \cdots \mathrm{O} 12^{\prime} 170(3)$. Symmetry transformation used to generate equivalent atoms: (') $1-x, 1-y, 1-z$.

\subsection{Conclusion}

To summarize, most interesting about the $\mathrm{Cu} / \mathrm{O}_{2}$ complexes of proton responsive, non-proton responsive and monoanionic BOX ligands presented herein was the finding that all species exhibit different reactivity towards external phenolic substrates. In case of the ${ }^{\mathrm{S}} \mathbf{P}$ complexes of proton responsive BOX ligands $\mathbf{5}, \mathbf{6}$, $\mathbf{7}$ and ${ }^{\mathbf{S}} \mathbf{P H L}{ }^{3}$, reactivity with external phenolic substrates led to the formation of their $\mathbf{O}$ complexes. Comparing this to reports by Stack et al. wherein the ${ }^{\mathbf{S}} \mathbf{P}$ complex of the DBED ligand system led to formation of the $\mathbf{O}$ isomer upon coordination of the substrate, herein, deprotnation by the substrate leads to $\mathbf{O}$ formation. ${ }^{[206]}$ These corresponding $\mathbf{O}$ complexes of proton-responsive BOX ligands, upon reaction with external phenolic substrates produced the unphysiological C-C coupled product with a $33 \%$ conversion of the substrate.

Complex $\mathbf{8}$, a ${ }^{\mathbf{S}} \mathbf{P}$ system of the non-proton responsive BOX ligand $\mathbf{L}^{\mathbf{6}}$ demonstrated stoichiometric reactivity with the external phenolic substrate DTBP-Na, hydroxylating it to the quinone with $13.5 \%$ conversion. The quinone was characterized by ${ }^{1} \mathrm{H}-\mathrm{NMR}$ spectroscopy, UV-vis spectroscopy, as well as HPLC. Unfortunately, catalytic reactivity was not observed. Addition of excess substrate led to the undesired C-C coupled product. A detailed insight into the mechanism of reactivity was prevented by the high instability of the intermediates.

For $\mathbf{O}$ complexes $\mathbf{1 0}$ and 11, reaction with external phenolic subsrstrates DTBP-Na or DTBP-H produced the $\mathrm{C}-\mathrm{C}$ coupled product with $50 \%$ conversion of the substrate.

The relatively high stability of these $\mathrm{Cu} / \mathrm{O}_{2}$ intermediates was evident on warming the solutions of 7, 8, 10 and 11 to rt. UV vis monitoring of these reactions displayed a decrease of their characteristic LMCT bands over the course of one hour (for $\mathbf{7}$ and $\mathbf{8}$ ), four hours (for 11) and 10 minutes (for 8), signifying gradual decomposition of the complexes. In case of complex $\mathbf{8}$, the decomposed product was identified as the bis $(\mu$ - 
hydroxo) dicopper(II) species which was characterized by UV-vis spectroscopy, IR spectroscopy and Xray crystallography. For complexes $\mathbf{7 , 1 0}$ and 11, the nature of the decomposed product was inconclusive. This could result from a more complex mechanism of decomposition for these complexes.

The differences in reactivity of the systems mentioned above suggests that the nature of the ligand plays a crucial role in the reaction mechanism and formation of the end product. The ${ }^{\mathbf{S}} \mathbf{P}$ complexes of proton responsive BOX ligands generated their $\mathbf{O}$ congeners. In comparison to Type 3 dicopper proteins that have histidine imidazole scaffolds with backside $\mathrm{N}$-atoms amenable to deprotonation, these results speculate the reactive intermediate responsible for the catalytic behavior of Tyrosinase. Weather a ${ }^{\mathbf{S}} \mathbf{P}$ intermediate catalyzes the hxdroxylation of tyrosine, or generation of an $\mathbf{O}$ intermediate mediates reactivity is still under investigation. 
$\frac{\text { Chapter } 9}{\text { Conclusion }}$ 
In conclusion, bis-oxazoline (BOX) ligands are simple and bidentate in nature. Termed as a privileged class of ligands, they have been used extensively in asymmetric catalysis but not explored in the context of activating small molecules in bioinspired systems. During the course of this work, their use in $\mathrm{Cu} / \mathrm{O}_{2}$ chemistry has proved to be of significant benifit. A detailed investigation of neutral and monoanionic BOX ligands of $\mathrm{Cu}(\mathrm{I})$ complexes with dioxygen was conducted which suggested that the nature of the ligand was crucial in determining the $\mathrm{Cu}_{2} / \mathrm{O}_{2}$ intermediate that formed at low temperatures and their subsequent reactivity towards external substrates.

The $\mathrm{Cu}(\mathrm{I})$ complexes of neutral ligands were shown to bind dioxygen in a $\mu-\eta^{2}: \eta^{2}$ binding mode. The ${ }^{\mathbf{S}} \mathbf{P}$ complexes 5, 6, 7 and 8 were characterized in solution. Complete characterization of $\mathbf{8}$ in solution as well as solid state was possible. Moreover, structural elucidation of ${ }^{\mathbf{S}} \mathbf{P}$ complexes $\mathbf{6}$ comprising of a proton responsive ligand and $\mathbf{8}$ comprising of a non-proton responsive ligand system was achieved during this study, which emulated similar dioxygen binding modes in type III dicopper proteins. The exceptionally long $\mathrm{O}-\mathrm{O}$ bond in $\mathbf{6}$ in comparison to all other synthetic and biological systems reported so far gives it its noteworthy character. These two structures contribute to the hand-full of structurally elucidated complexes of this type characterized over the last 27 years and are rare examples of ${ }^{\mathbf{S}} \mathbf{P}$ complexes with bidentate ligands. Kinetic and activation parameters for the formation of ${ }^{\mathbf{S}} \mathbf{P}$ complexes with the new ligand systems $\mathbf{H L}^{5}$ and $\mathbf{L}^{6}$ were determined.

As mentioned earlier, the finding that certain BOX ligands exist in a tautomeric equilibrium mixture suggested that they may serve as proton responsive ligands. This was exploited in successfully tuning the equilibrium between their ${ }^{\mathbf{S}} \mathbf{P} / \mathbf{O}$ complexes. By virtue of a base the ${ }^{\mathrm{S}} \mathbf{P}$ complexes (of proton responsive ligands), 5, 6, 7, and ${ }^{\mathbf{S}} \mathbf{P} \mathbf{H L}^{3}$ generated their $\mathbf{O}$ congeners with a bis( $\mu$-oxo) dicopper(III) core. This was attributed to deprotonation of the ligand backbone, which triggered cleavage of the O-O bond shifting the equilibrium in the direction of the $\mathbf{O}$ isomer. The reactions were monitored by UV-vis spectroscopy with final confirmation of the dioxygen binding mode being attained from the dioxygen reactivity of $\mathrm{Cu}(\mathrm{I})$ complexes with new, monoanionc BOX ligands $\left[\mathbf{L}^{2}\right]^{-}$and $\left[\mathbf{L}^{3}\right]^{-}$(introduced during this study). The basic nature of the proton responsive BOX ligands gave rise to the POP (peroxo-oxo-peroxo) switch, a back and forth shift between the ${ }^{\mathbf{S}} \mathbf{P} / \mathbf{O}$ species with simple changes in stoichiometry of the ligand and copper(I) source. " $\mathrm{pH}$ " is now a new factor that now contributes to tuning the equilibrium between ${ }^{\mathbf{S}} \mathbf{P} / \mathbf{O}$ complexes.

The new monoanionic ligands $\left[\mathbf{L}^{2}\right]^{-}$and $\left[\mathbf{L}^{3}\right]^{-}$that were introduced to study the electronic effects on $\mathrm{Cu} / \mathrm{O}_{2}$ chemistry could indeed find application in this research area. Their $\mathrm{Cu}(\mathrm{I})$ analogues formed $\mathbf{O}$ complexes $\mathbf{1 0}$ and 11 with dioxygen at low temperatures. These were characterized in solution along with structural elucidation of $\mathbf{1 0}$ which unambiguously determined the dioxygen to bind in a bis( $\mu$-oxo) mode. $\mathbf{1 0}$ is the second example of a neutral bis( $\mu$-oxo) dicopper(III) species reported to date. An interesting effect of temperature variation on $\mathbf{O}$ complexes was also observed. UV-vis monitoring of the $\mathbf{O}$ complexes with temperature variation resulted in intensity changes of their characteristic LMCT bands. This was explained by the fluxional twist of the core with temperature change, an effect that has not been reported to date. Though the $\mathbf{O}$ motif has not been biologically observed, a recent proposal of the active site of the pMMO enzyme to comprise a bis( $\mu$-oxo) intermediate has been reported. This highlights the importance of this $\mathrm{Cu} / \mathrm{O}_{2}$ binding mode.

Finally, substrate reactivity of the ${ }^{\mathbf{S}} \mathbf{P}$ and $\mathbf{O}$ complexes synthesized during this study revealed differences in reactivity which were attributed to the nature of the ligand system. While ${ }^{\mathbf{S}} \mathbf{P}$ complexes of proton responsive ligands generated their $\mathbf{O}$ congeners with basic phenolic substrates, complex $\mathbf{8}$ which hosts a non-proton responsive scaffold emulated similar activity as Tyrosinase in stoichiometric amounts. The $\mathbf{O}$ complexes of the monoanionic BOX ligands demonstrated formation of the $\mathrm{C}-\mathrm{C}$ coupled product with a high conversion rate of $50 \%$. 
Though dioxygen binding in Ty is known to generate a ${ }^{\mathbf{S}} \mathbf{P}$ species, the reactive intermediate responsible for catalytic activity is still under speculation. Moreover, the existence of ${ }^{\mathbf{S}} \mathbf{P}$ complexes to exist in equilibrium with their $\mathbf{O}$ isomers has been investigated in detail in synthetic systems, though not yet biologically observed. Through this work with simple BOX ligands, key $\mathrm{Cu}_{2} / \mathrm{O}_{2}$ intermediates were isolated proving BOX ligands to be privileged not only in the field of asymmetric catalysis, but also in the field of bioinorganic chemistry. The key $\mathrm{Cu}_{2} / \mathrm{O}_{2}$ intermediates with ${ }^{\mathbf{S}} \mathbf{P}$ and $\mathbf{O}$ cores could readily be interconverted by peripheral deprotonation/protonation events at the terminal ligand backbones. Interestingly, Type 3 dicopper proteins as well as the dicopper active site of pMMO contain copper ions coordinated by histidine imidazoles. These offer a backside $\mathrm{N}$ atom amenable to potential (de)protonation equilibria in response to changes in local $\mathrm{pH}$. The work presented herein puts forward a plausible enquiry. Do changes in local $\mathrm{pH}$ deterime the active intermediate responsible for catalytic activity in novel dicopper enzymes? In fact, (de)protonation of histidine imidazole ligands in metalloproteins has widely been used for tuning redox potentials and electronic structures of the metallocofactors, ${ }^{[232-234]}$ and forms an integral part of important biological proton coupled electron transfer (PCET) reactions (such as in the Rieske proteins). ${ }^{[236][237-239]}$ It is an interesting perspective to introduce via proton-responsive ligands, PCET reactivity to $\mathrm{Cu}_{\mathrm{x}} / \mathrm{O}_{2}$ intermediates. 
[Note: This page had intentionally been left blank 


\section{Chapter 10}

An Overview of Iron in Biological and Synthetic Systems 


\section{Overview}

10.1 Diiron Enzymes activating Dioxygen

10.1.1 Hemerythrin

10.1.2 Ribonucleotide Reductase

10.1.3 Soluble Methane Monoxygenase (sMMO)

10.1.3.1 Synthetic model systems for sMMO

10.2 Nonheme Iron Enzymes and Nitric oxide

10.2.1 Synthetic model systems for FNORs

10.2.2 Synthetic model systems for DNICs

10.3 Bis(oxazoline) ligands in iron chemistry 


\subsection{Diiron Enzymes activating Dioxygen}

The high abundance of elemental iron in nature has resulted in a large number of diiron proteins performing a variety of biochemical functions. Both heme and non-heme diiron enzymes are involved in the binding and activation of dioxygen which gives rise to reactive intermediates essential for physiological processes, though the mechanism varies in each case. ${ }^{[240,241]}$. Depending on the nature of the enzyme, four electrons and four protons are required for cleavage of the O-O bond when dioxygen is finally reduced to water. ${ }^{[242]}$ Scheme 10.1 illustrates the binding mode of dioxygen at the active sites in heme iron and non-heme diiron metalloenzymes. ${ }^{[243]}$ Dioxygen binds to the metal centers in either a superoxide or a peroxide fashion, oxidizing the metal centers. Further activation of these peroxides by certain cofactors or by the diiron core itself gives rise to high-valent iron oxo compounds. These pertain a high oxidizing power and are considered to be active intermediates responsible for oxidation and oxygenation chemistry. ${ }^{[241,244-246]}$ The following sections describe three well studied diiron metalloenzymes that activate dioxygen; Hemerythrin, Ribonucleotide reductase (RNR), and soluble methane monoxygenase (sMMO). All three comprise carboxylate bridges between the iron centers resulting in these enzymes belonging to the class of carboxylate-bridged diiron proteins. ${ }^{[247,248]}$ While Hemerythrin and RNR are associated with biophysiological processes, the enzyme sMMO is associated with impressive catalytic oxygenation of organic substrates. ${ }^{[29]}$ This has attracted much attention in modern catalysis with large efforts being made to generate synthetic model systems capable of emulating the sMMO enzyme. ${ }^{[187,240]}$

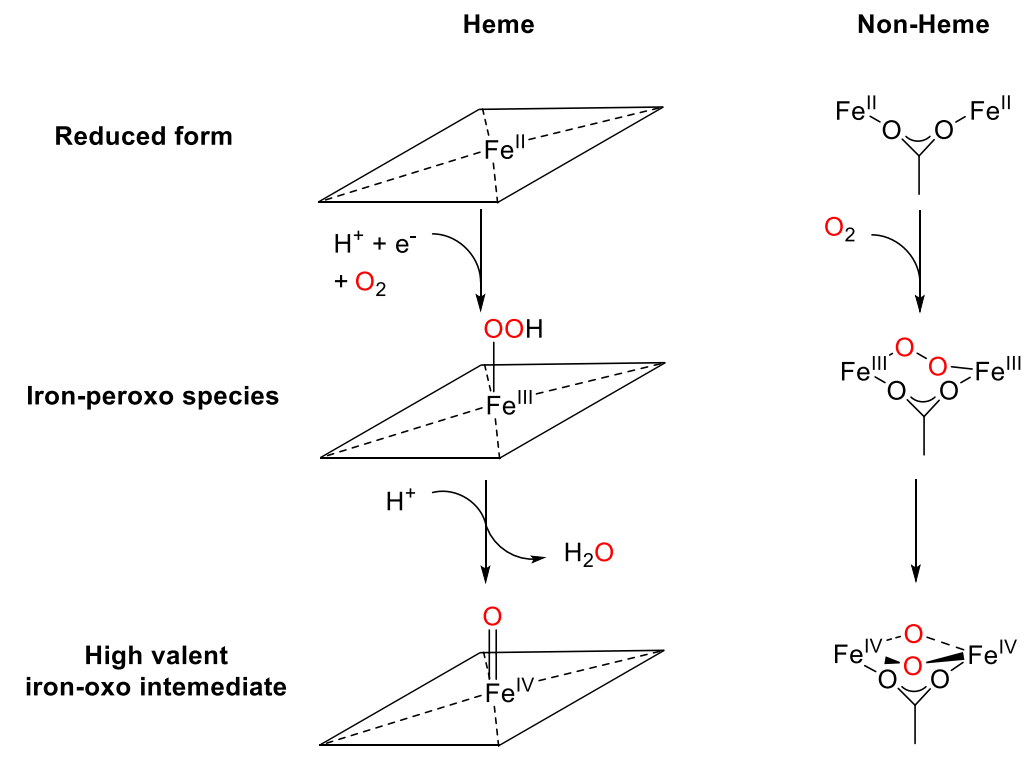

Scheme 10.1 Dioxygen activation mechanisms for heme and non-heme diiron enzymes. ${ }^{[243]}$

\subsubsection{Hemerythrin}

Similar to Hemocyanin, Hemerythrin $(\mathrm{Hr})$ which is present in several marine invertebrates reversibly binds dioxygen and carries out the function of $\mathrm{O}_{2}$ transport. ${ }^{[244]}$ The active site of deoxy Hr consists of two iron centers bridged by carboxylate groups of a glutamate and an aspartate residue and a $\mu$-hyroxo unit (Scheme 
10.2). ${ }^{[28]}$ One of the iron centers is linked to three histidine residues while the other binds to only two histidine residues making the iron centers asymmetrical in nature. The vacant coordination site present at the five coordinated iron center allows for dioxygen binding. $\mathrm{O}_{2}$ binds in a peroxide fashion with the distal hydroxo-proton being transferred to the peroxo oxygen, generating a high valent diiron(III) hydroperoxo motif which is known to be the active intermediate responsible for transport (Scheme 10.2). ${ }^{[25]}$ The final release of dioxygen with simultaneous reduction of both the metal centers along with proton transfer regenerates the $\mu$-hydroxo bridge, reforming the deoxy form of Hemerythrin.

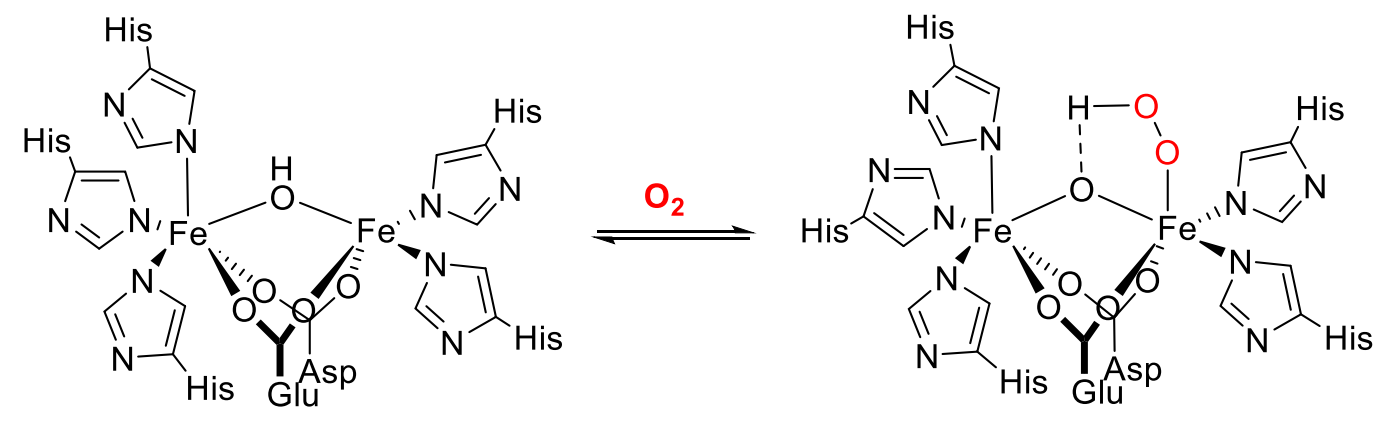

Scheme 10.2 Reversible dioxygen binding in Hemerythrin. ${ }^{[25]}$

\subsubsection{Ribonucleotide Reductase}

The enzyme ribunucleotide reductase (RNR) is essential in the transformation of RNA to DNA and is present in all living organisms. The R2 subunit of the enzyme interacts with dioxygen to form what is called a tyrosyl radical which enables conversion of ribonucleotides to deoxyribonucleotides. ${ }^{[250]}$ The active site of the reduced form of RNR contains a diiron(II) center bridged by two carboxylate ligands, a terminal histidine, and a terminal monodentate carboxylate at each iron. The pseudo tetrahedral iron centers bind $\mathrm{O}_{2}$ to form a $\mu$-oxo- $\mu$-carboxylato diiron(III) core (Scheme 10.3). ${ }^{[251]}$ The carboxylate shift of one of the glutamate residues provides a vacant site for dioxygen binding. This provides oxidizing equivalents which is essential to generate the tyrosyl radical responsible for the functioning of the enzyme. ${ }^{[252]}$

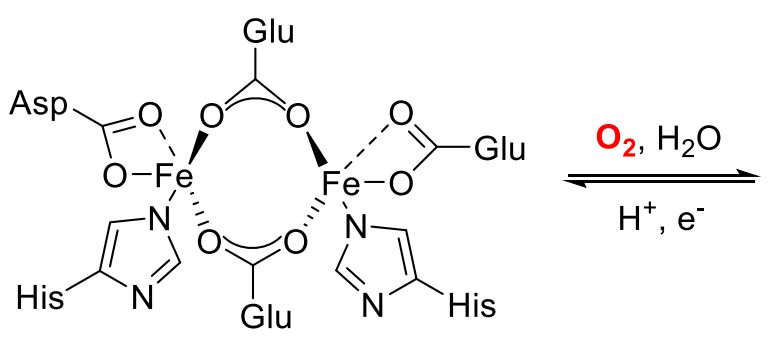

RNR R2 red

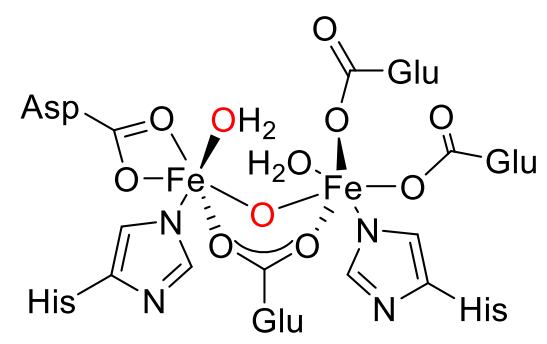

RNR R2 ox

Scheme 10.3 Active site of RNR R2 in reduced and oxidized states. ${ }^{[251]}$ 


\subsubsection{Soluble Methane Monoxygenase (sMMO)}

One of the most impressive enzymes in the family of non-heme diiron enzymes is soluble methane monoxygenase (sMMO). Present in several methanotrophic bacteria, this enzyme catalyzes the conversion of methane to methanol which requires activation of a $\mathrm{C}-\mathrm{H}$ bond, the BDE being $105 \mathrm{kcalmol}^{-1} \cdot{ }^{[253-255]} \mathrm{As}$ methane is a major component of natural gas, understanding the catalytic mechanism of sMMO under ambient conditions would benefit future fuel and feedstock generation. A significant amount of effort has been applied in synthesizing synthetic analogues capable of emulating the catalytic activity of this enzyme.

Composed of multiple components, sMMO contains two iron atoms within its active center bound to histidine and glutamate residues. ${ }^{[256]}$ In its reduced form $\mathrm{MMOH}_{\text {red, }}$, the diiron centers are bridged by two glutamates in a $\mu-\eta^{l}: \eta^{l}$ and a $\mu-\eta^{l}: \eta^{2}$ binding mode. ${ }^{[32]}$ Two additional water molecules complete the pseudo octahedral spheres of the metal centers (Scheme 10.4). Binding dioxygen eventually results in the oxidized form $\mathrm{MMOH}_{\mathrm{ox}}$, a bis( $\mu$-hydroxo) diiron(III) core. The sMMO enzyme consists of several additional subunits responsible for different reactivity. Substrate hydroxylation is carried out by the MMOH subunit, whereas electron transfer and substrate oxidation are mediated by the MMOR subunit. The regulation and control of regioselectivity and kinetics is carried out by the MMOB subunit. ${ }^{[257-259]}$ Apart from activating methane, sMMO also catalyzes the conversion of hydrocarbons, halogenated derivatives and heterocycles. ${ }^{[254,260]}$

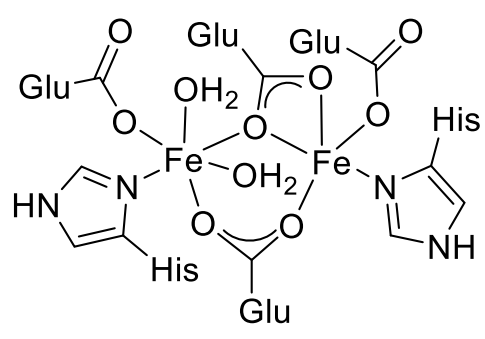

$\mathrm{MMOH}_{\text {red }}$
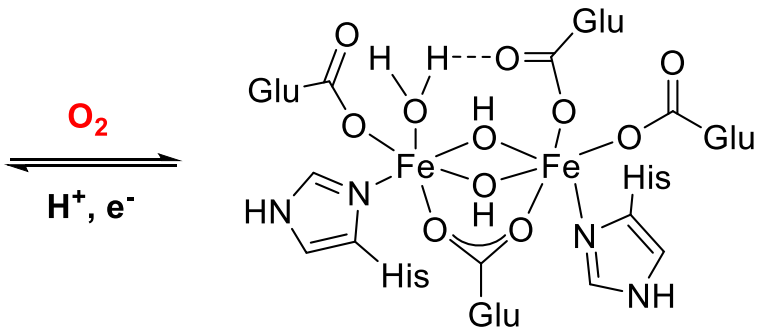

$\mathrm{MMOH}_{\text {ox }}$

Scheme 10.4 Active site of the hydroxylase unit of sMMO in its active reduced form (MMOHred), and its resting state (MMOHox).

Extensive investigations on sMMO have led to a better understanding of its catalytic cycle. ${ }^{[261-263]}$ Though the catalytic cycle comprises a total of seven intermediates, two are of special interest. The first, a $\mu-1,2-$ peroxo diiron(III) motif $\mathbf{P}$ which forms on dioxygen binding to the diiron centers in $\mathrm{MMOH}_{\text {red. }}{ }^{[187,264-267]}$ This reconfigures to form a second intermediate $\mathbf{Q}$, which has recently been characterized by extensive Raman spectroscopic studies and confirmed to be a bis( $\mu$-oxo) diiron(IV) core. ${ }^{[261,263,268]}$ Intermediate $\mathbf{Q}$ is responsible for hydroxylation reactions of the substrate. As mentioned earlier, sMMO belongs to the class of carboxylate-bridged non-heme diiron enzymes. Scheme 10.5 depicts the importance of this bridge leading up to the carboxylate shift that facilitates availability of vacant coordination sites for dioxygen binding, leading to the formation of $\mathbf{P}$ and $\mathbf{Q} .^{[269]}$ 


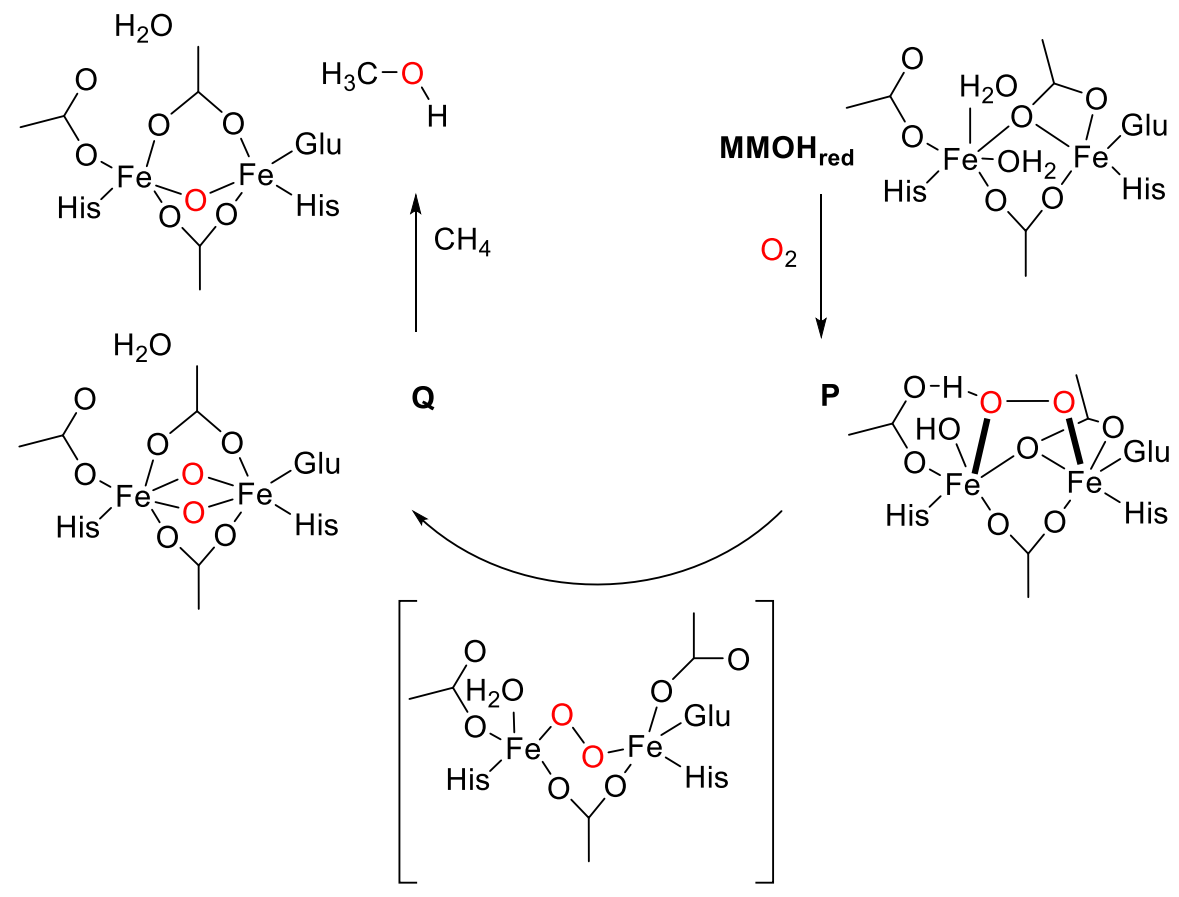

Scheme 10.5 A more detailed representation of dioxygen activation by $\mathrm{MMOH}$ with intermediates $\mathbf{P}$ and $\mathbf{Q} \cdot .^{[245]}$

\subsubsection{Synthetic model systems for sMMO}

Efforts in replicating the active site of sMMO have led to a variety of synthetic analogues which have contributed to a better understanding of the enzyme. ${ }^{[187,243,246]}$ Dating back to 1980 , the groups of Weighardt and Lippard independently reported two diferric oxo bridged complexes I and II respectively, bearing the typical $\mu$-oxo-bis( $\mu$-carboxylato)-diiron(III) core commonly found in diiron enzymes. ${ }^{[270,271]}$ Both complexes were obtained with acetate co-ligands and capping ligands triazocyclononane (tacn) (for I) and tris(pyrazole)borate ligand (for II). Analysis of these complexes via magnetic and spectroscopic measurements suggested these to be model systems for met-hemerythrin (Fig. 10.1).
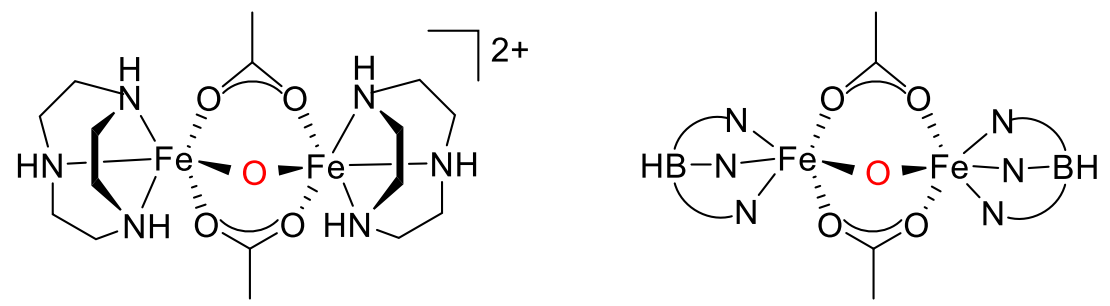

I

II

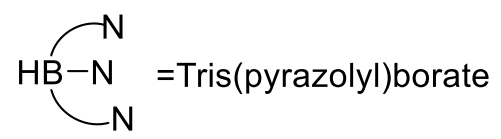

Figure 10.1 Model systems I and II featuring the $\mu$-oxo-bis( $\mu$-carboxylato)-diiron(III) core. ${ }^{[270][271]}$ 
A diferrous iron complex was later isolated with the aid of tacn and acetate co-ligands comprising a hydroxo bridge between the iron(II) cores (III). ${ }^{[272]}$ The reactivity of complex III with dioxygen led to the formation of a diiron(III) $\mu$-oxo bridged species IV (Scheme 10.6), which was the first model system reported to mimic the active site of deoxyhemerythrin. Following this, a large number of synthetic analogues have been reported in literature that target mimicking the structural and functional aspects of non-heme diiron proteins. ${ }^{[240,273,274]}$ These model systems have helped in explaining the importance of the bridging carboxylate units which have shown to switch their binding modes in order to provide vacant coordination sites for substrate binding. ${ }^{[247,248,275,276]}$ A variety of high-valent iron oxo intermediates such as peroxides, mixed-valent oxides, and high-valent diamond oxo cores have been isolated with the aim of mimicking the active intermediates of these natural enzymes. ${ }^{[240,273,277]}$

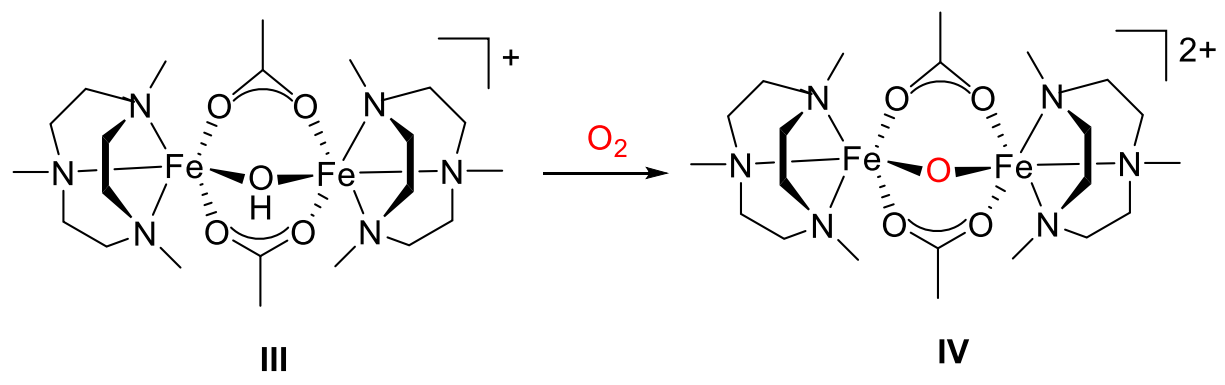

Scheme 10.6 Model system III emulating the active site of deoxyhemerythrin and its reaction with dioxygen to give the diiron complex $(\mathbf{I V}){ }^{[272]}$

Distinct synthetic diiron(III)-peroxo intermediates similar to the active intermediate $\mathbf{P}$ in sMMO were first reported in 1996 almost simultaneously and independently by Lippard, Suzuki and Que. This is regarded as a seminal achievement in search for model complexes of transient iron-oxygen intermediates. The diiron(III) peroxo species $\mathbf{V}$ reported by Lippard et al., was synthesized with a modified tris(pyrazolyl)borate ligand in combination with carboxylate ligands in a self-assembly reaction. ${ }^{[265,278]}$ The compounds VI and VII synthesized by Que and Suzuki, respectively, were obtained with pre-organized ligand systems. ${ }^{[249,266,267,279]}$ The structural and spectroscopic features of V, VI and VII (Fig. 10.2) were in good agreement with that of $\mathbf{P} .{ }^{[262,280]}$ Figure 10.2 depicts a schematic representation of the $\mu-1,2$ peroxo moieties of V, VI and VII. The O-O bond lengths of $1.42 \AA$ and resonance Raman stretching frequencies of $\sim 900 \mathrm{~cm}^{-1}$ were in the similar range of $\mathbf{P}$. Complex $\mathbf{V}$ reported by Lippard et al. beared closest resemblance to the peroxo intermediate of $\mathrm{MMOH}$ with respect to UV-vis and Mössbauer parameters. Table 10.1 gives a comparative overview of spectroscopic features of $\mathbf{V}, \mathbf{V I}$ and VII with $\mathbf{P}$.

The tris(pyridyl)amine ligand (TPA) as well as the hexa-pyridyl substituted analogue of TPA (6-HPA) have helped in developing a large number of high valent diiron model compounds (Fig. 10.3). Dating back to 1995 Que et al. developed a series of diamond core complexes with the aid of the TPA capping ligand. ${ }^{[14,268,281-287]}$ A few years later, successful structural elucidation of a mixed valent valencedelocalized system unambiguously determined the dioxygen to bind in a diamond core motif. Addition of $\mathrm{H}_{2} \mathrm{O}_{2}$ to an open core diiron(III) precursor was reported to convert intermediate $\mathbf{P}$ to $\mathbf{Q}$, an intriguing similarity in the sMMO enzyme. ${ }^{[282]}$ The usage of isotopically labelled $\mathrm{O}_{2}$ confirmed the formation of a peroxide diiron(III) intermediate. The mechanism of interconversion with ligand exchange suggested the rate of C-H cleavage to be much higher in an open core diiron(III, IV) terminal high spin ( $S=2$ ) iron (IV)oxo unit, as opposed to a bis( $\mu$-oxo) diiron(IV) low spin $S=1$ species. ${ }^{[281,283-285,287]}$ In parallel, with the aid of the 6-HPA ligand system, Kodera et al. reported a diiron compound with two iron(IV) oxo units and an $S=2$ 
spin state for both metal centers. ${ }^{[288-290]}$ This intermediate was shown to be in equilibrium with its diiron(III) peroxo motif, an interesting finding that could help provide further insights into the mechanism of interconversion of the O-O bond breakage in SMMO. ${ }^{[277]}$<smiles>[R]n1nc(C(C)C)cc1C(C)C</smiles><smiles>Cc1cc(CN(Cc2nc(-c3ccccc3)c(-c3ccccc3)n2C)Cc2nc(-c3ccccc3)c(-c3ccccc3)n2C)c([O-])c(CN(Cc2nc(-c3ccccc3)c(-c3ccccc3)n2C)Cc2nc(-c3ccccc3)c(-c3ccccc3)n2C)c1</smiles><smiles>CCn1c(CN(Cc2nc3ccccc3n2CC)CC([O-])CN(Cc2nc3ccccc3n2CC)Cc2nc3ccccc3n2CC)nc2ccccc21</smiles>

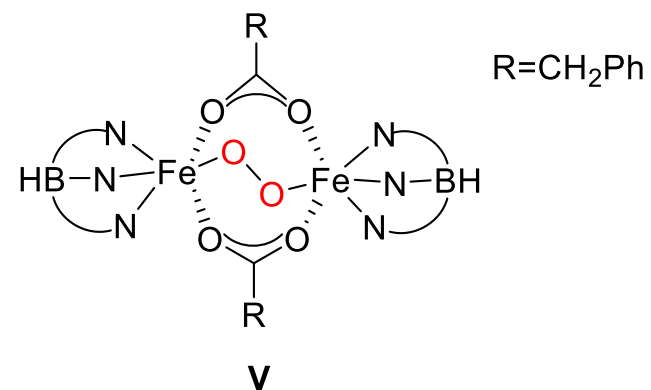

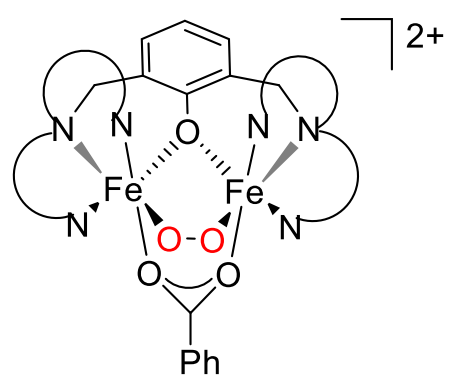

VI

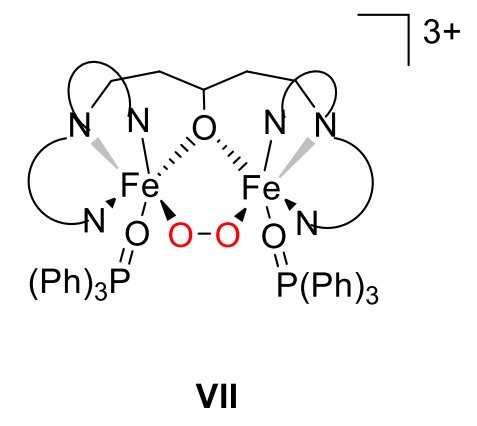

Figure 10.2 Structurally characterized diiron(III)peroxo complexes, V, VI and VII that serve as model systems for intermediate $\mathbf{P}$ in sMMO. ${ }^{[249,265-267,278,279]}$ 
<smiles>[R]c1cnc(CN(Cc2ncc([R])c([R])c2[R])Cc2ncc([R])c([R])c2[R])c([R])c1[R]</smiles>

TPA<smiles>c1ccc(CCc2cccc(CN(Cc3ccccn3)Cc3ccccn3)n2)nc1</smiles>

6-HPA

Figure 10.3 Ligands used to isolate high valent iron-oxo intermediates that have helped in elucidating the mechanism of sMMO.

Table 10.1 A comparative overview of the spectroscopic features of V, VI and VII with $\mathbf{P}$

\begin{tabular}{c|c|c|c|c|c|c|c}
\hline & $\mathrm{Fe} \cdots \mathrm{Fe}(\AA)$ & $\mathrm{O} \cdots \mathrm{O}(\AA)$ & $\lambda(\mathrm{nm})$ & $\varepsilon\left(\mathrm{M}^{-1} \mathrm{~cm}^{-1}\right)$ & $\delta\left(\mathrm{mms}^{-1}\right)$ & $\left|\Delta \mathrm{E}_{\mathrm{q}}\right|\left(\mathrm{mms}^{-1}\right)$ & $\nu(\mathrm{O}-\mathrm{O})\left(\mathrm{cm}^{-1}\right)$ \\
\hline $\mathbf{P}$ & & & $625-650$ & 1500 & 0.66 & 1.51 & 905 \\
\hline $\mathbf{V}$ & 4.00 & 1.41 & 694 & 2650 & 0.66 & 1.40 & 876 \\
\hline $\mathbf{V I}$ & 3.33 & 1.43 & $500-800$ & 1700 & $0.58 / 0.65$ & $0.74 / 1.70$ & \\
\hline VII & 3.46 & 1.42 & 588 & 1500 & 0.52 & 0.72 & 900 \\
\hline
\end{tabular}




\subsection{Nonheme iron enzymes and Nitric oxide}

The activation of small molecules by dinuclear non-heme iron enzymes is not limited to dioxygen. Nitric oxide, another small molecule with its radical nature and high affinity for $\mathrm{Fe}^{\mathrm{II}}$ has been long known to bind non-heme iron enzymes. ${ }^{[291,292]}$ Its function as a signaling molecule and an immune defense agent in mammals gives it its importance. At nanomolar concentrations, the functioning of NO is well regulated within the body. However, higher concentrations of NO can be toxic and fatal, leading to a series of harmful effects such as septic shock, the onset of cancer, and several other diseases. ${ }^{[292]}$ Proteins such as RNR, ${ }^{[34,293]}$ sMMO, ${ }^{[37]}$ and NORs (nitric-oxide reductases) ${ }^{[36,294]}$ are capable of activating NO and reducing it to nitrous oxide, a less toxic form. The former two reduce NO only in small amounts while the latter NORs, found in several bacteria, catalyze rapid reduction of NO. The c-type NOR (NorBC, $c \mathrm{NOR}$, or heme $\mathrm{b}_{3}-\mathrm{Fe}_{\mathrm{B}}$ ), present in denitrifying bacteria, catalyzes the two electron reduction of $\mathrm{NO}$ to $\mathrm{N}_{2} \mathrm{O}$. The structurally characterized NorBC comprises two subunits, NorB and NorC. ${ }^{[25]}$ The smaller NorC unit hosts a low-spin heme c redox center that transfers electrons to the NorB subunit. The NorB subunit, wherein the actual active site is located, consists of a heme $\mathrm{b}$ center that transports electrons to the active site. The actual active site of the enzyme responsible for $\mathrm{NO}$ reduction hosts a heme $\mathrm{b}_{3}$ iron center and a non heme-Fe $\mathrm{B}_{\mathrm{B}}$ iron center. X-ray characterization has shown the non heme- $\mathrm{Fe}_{\mathrm{B}}$ center to be coordinated by three histidine units, an aspartic acid, and an oxo bridge connecting it to the heme- $b_{3}$ center ${ }^{[296,297]}$ Investigations of NO binding at heme $\mathrm{b}_{3}-\mathrm{Fe}_{\mathrm{B}}$ active sites have suggested the possible formation of both heme and non-heme $\mathrm{Fe}^{\mathrm{II}} \mathrm{NO}$ adducts. Though actual intermediates for this enzyme have not yet been isolated, a mononuclear Fe-NO species for the non-heme $\mathrm{Fe}_{\mathrm{B}}$ center has been proposed.

Other than denitrifying bacteria, several pathogenic microbes such as bacteria, cyanobacteria and protozoa contain nitric oxide reducing enzymes that function as a protective mechanism against excess NO. These flavo-diiron proteins (FDPs) are subclassified into flavo-diiron dioxygen reductases $\left(\mathrm{FO}_{2} \mathrm{R}\right)$ which activate dioxygen, and flavo-diiron nitric oxide reductases (FNOR) that reduce NO. ${ }^{[35]}$ The crystallized form of Desulfovibrio gigas containing a FNOR was shown to comprise two non-heme iron centers within its active site (Scheme 10.7). ${ }^{[298]}$ One iron center is coordinated to two histidine residues and a glutamic acid, while the second is coordinated to a histidine, aspartic acid residue and a water based ligand. The two iron centers are bridged by an aspartic acid and a water based ligand completing the coordination spheres of the metal centers, making them asymmetric in nature. Crystallographic analysis of these FDPs have shown the flavin cofactor to be located in close proximity to the diiron active site. Though the mechanistic significance has not been clearly explained till date, the possibility of rapid electron transfer between the cofactor and the active site is considered important for catalysis in FDPs.

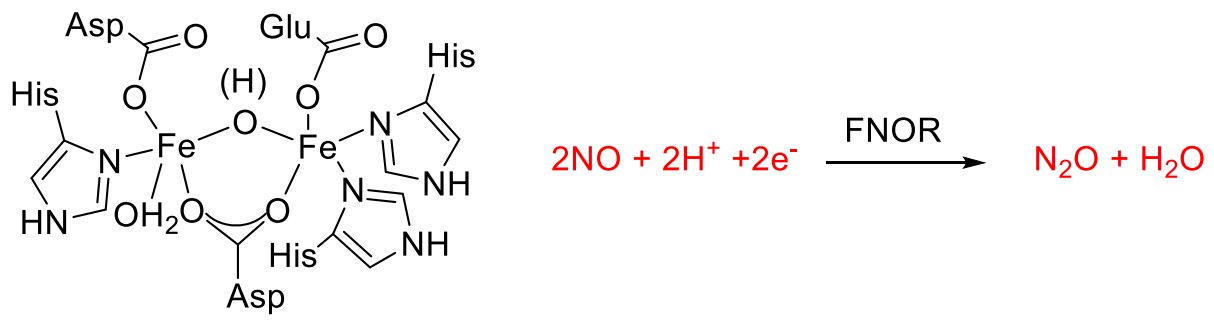

Scheme 10.7 Active site of FNOR in Desulfovibrio gigas and the catalyzed reaction in red also mediated by NORs in denitrifying bacteria. ${ }^{[298]}$ 
Besides the reduction of NO in conjunction with NOR's, NO is also known to interact with several other nonheme iron containing enzymes. The uptake of NO with the ferric uptake regulatory proteins (Fur) has attracted much attention over the last decade. ${ }^{[299-301]}$ Fur behaves as a global regulator in Gram-negative bacteria, E. coli, which controls the expression of genes and iron content within the cell. ${ }^{[302]}$ The concentration of iron within the cell is critical for its functioning and needs to be regulated in order to avoid undesirable reactions such as Fenton's reaction. In its active form, FeFur comprises a nonheme iron center bound by nitrogen and oxygen ligands. When the iron content in the cell is low, FeFur loses $\mathrm{Fe}^{2+}$, which disables further binding of the protein to DNA, increasing iron acquisition within the cell. ${ }^{[303]}$ Studies have shown NO to bind to FeFur generating an iron-nitrosyl species unable to bind DNA. This creates a link between defense against NO and iron control. However, weather binding of NO to FeFur results in a mononitrosyl (MNIC) or dinitrosyl iron complex (DNIC) is still unclear. ${ }^{[300,304,305]}$

The interaction of nitric oxide with several iron-sulfur cluster proteins leads to the formation of RSNO ( $S$ nitrosothiols) or DNICs. ${ }^{[306,307]}$ These DNICs behave as NO storage and transport units that are responsible for NO delivery to different targets within the cell. EPR characterization of these complexes in comparison to those biologically observed produces a signal at $g=2.03$, characteristic of DNICs with sulfur ligated systems. ${ }^{[308,309]}$ They are tetrahedral with the iron centers being ligated to sulfur donors such as cysteine residues or glutathionine. Though reactivity of NO with iron-sulfur clusters represents pathological targets, physiological functions such as the activation of certain transcriptional regulators, SoxR, also results from this interaction. ${ }^{[300]}$ However, a detailed analysis of DNICs in the biological system with respect to their reactivity and function is not yet well established due to the lack of suitable biomimetic model systems.

\subsubsection{Synthetic model systems for FNORs}

The chemistry of nitric oxide activation in contrast to dioxygen activation with iron and diiron model systems is still under investigation, resulting in only a handful of well established systems. The first model system replicating the binding mode of FNOR was reported by Lippard et al. in $1996 .{ }^{[310]}$ A diferrous system VIII (Fig. 10.4), coordinated by bridging alkoxide and benzoate ligands enclosed within a dinucleating benzimidazole scaffold, was shown to react with NO to form a diiron dinitrosyl complex. The NO was bound in a syn fashion to both the iron centers and the compound was characterized by Mössbaeur, UV-vis and IR spectroscopy. ${ }^{[311]}$ Further investigations with VIII in 2014 was shown to produce nitrous oxide in the presence of light, replicating the function of FNOR. ${ }^{[312]}$

A second system IX (Fig. 10.4), reported by Lehnhert et al. in 2013 with the aid of pyridine and alkoxide ligand scaffolds showed to bind NO forming a diiron dinitrosyl motif, which subsequently released NO under chemical and electrochemical reduction. ${ }^{[313,314]}$ This is reported to be the first functional biomimetic model system for FNOR. 

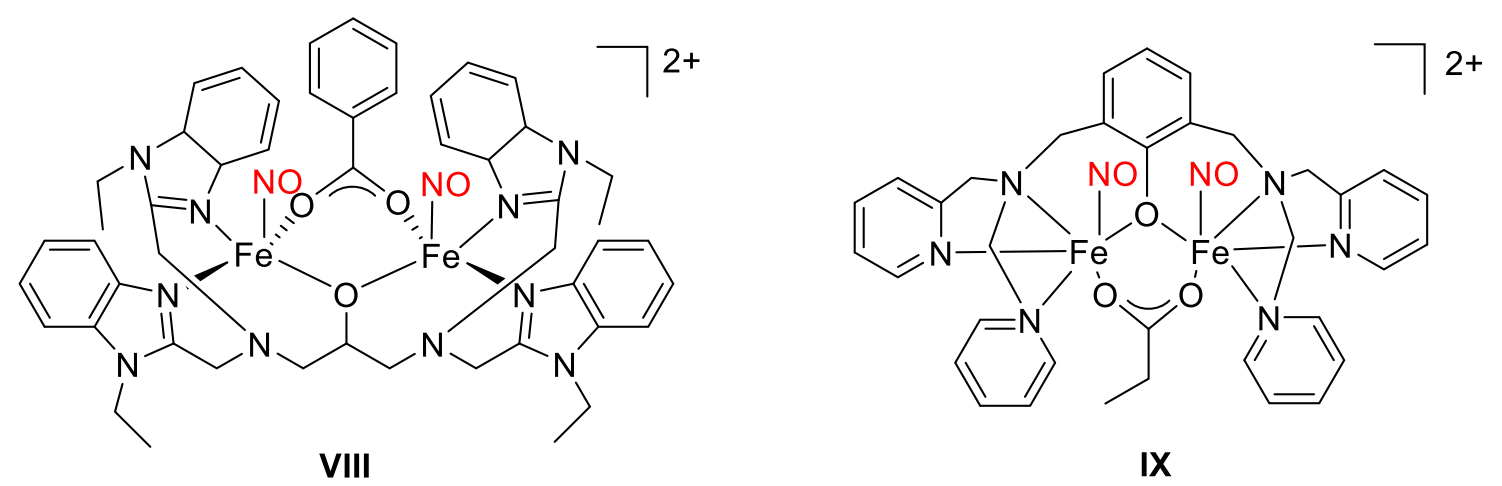

Figure 10.4 Diiron dintrosyl systems VIII and IX synthesized by Lippard and Lehnert, respectively, that function as structural and functional model systems for FNOR. [311,313]

\subsubsection{Synthetic model systems for DNICS}

The DNICs that have been synthesized and characterized so far can broadly be categorized into mononuclear or dinuclear DNICs, wherein mainly S-, N- and O- ligand donors mimicking biological scaffolds have been used. ${ }^{[315]}$ As the name suggests, mononuclear DNICs contain a single iron center with two NO groups attached to it. These have further been subclassified based on coordination number and geometry around the iron center. The classical DNICs are tetrahedral with a coordination number of 4 , whereas the nonclassical DNICs are usually square pyramidal or octahedral with coordination numbers of 5 and 6. The dinuclear DNICs contain two iron centers wherein each iron is bound to two NO molecules. The NO can either be terminally bound or bridging (Scheme 10.8).

\section{Mononuclear DNICs}

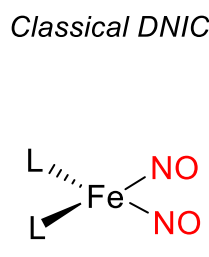

C.N. $=4$

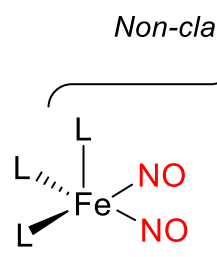

C.N. $=5$
Dinuclear DNICs

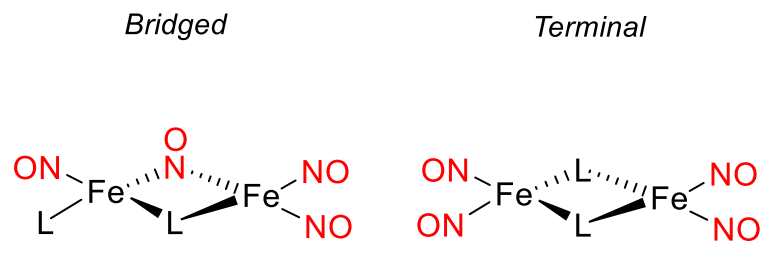

C.N. $=6$

Scheme 10.8 Schematic representation of mononuclear and dinuclear DNICs. Mononuclear DNICs are subclassified into classical and non-classical DNICs based on the coordination number of the iron center. ${ }^{[315]}$

Various chelating ligands containing thiolate, imidazolates, phenolates, carboxylates, nitrites and nitrate coordinating groups have proved to be suitable scaffolds for generating four coordinated and dinuclear DNICs. Distinct reaction pathways with the aid of suitable nucleophiles or bases, starting from a bridging 
diiron dinitrosyl iron complex yields classical mononuclear DNICs, whereas reduction of these bridging systems yields their dinuclear DNICs with different electronic states (Fig. 10.5). ${ }^{[316-323]}$

Ligand systems formining classical mononuclear DNICs

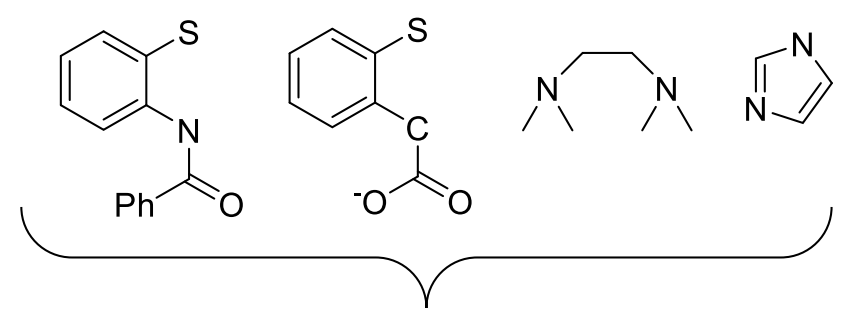

Ligand systems formining dinuclear DNICs

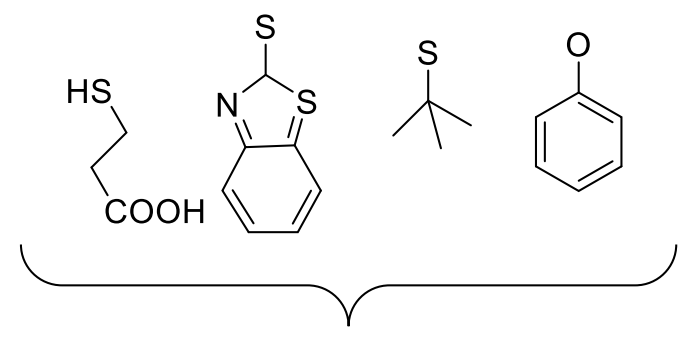

Figure 10.5 Ligand systems employed in the synthesis of classical mononuclear and dinuclear DNICs. ${ }^{[315]}$

In case of the nonclassical 5 or 6 coordinated DNICs, multidentate ligands such as bidentate $\kappa^{2}$-ONO from nitrite or $\kappa^{2}-\mathrm{O}_{2} \mathrm{NO}$ from nitrate, tridentate ligands such as iPrPDI or PyImiS and tetradentate ligands such as TPA are known to stabilize these binding modes (Fig. 10.6). ${ }^{[320,324-326]}$

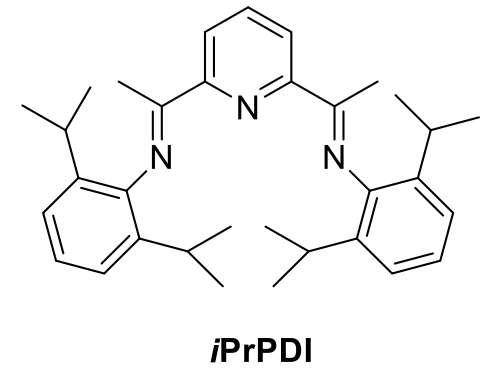

iPrPDI<smiles>C/C(=N\c1ccccc1S)c1ccccn1</smiles>

PyImiS<smiles>c1ccc(CN(Cc2ccccn2)Cc2ccccn2)nc1</smiles>

TPA

Figure 10.6 Ligand systems employed in the synthesis of non-classical mononuclear DNICs. ${ }^{[315]}$

\subsection{Bis-oxazoline ligands in Iron chemistry}

The use of chiral ligands in conjunction with transition metals has provided a large number of asymmetric catalysts for organic synthesis. ${ }^{[5,327]}$ In particular bidentate, tridentate and tetradentate N-donor ligands containing chiral oxazoline scaffolds together with their iron complexes have proved to be impressive asymmetric catalysts. ${ }^{[327]}$

Dating back to 1991 Corey et al. first reported an Fe(III) halide complex with a $C_{2}$-symmetric chiral bidentate bis(oxazoline) ligand, to behave as an efficient catalyst for enantioselective Diels Alders reactions. ${ }^{[55]}$ Following this, several systems have been developed for a variety of organic reactions. Bidentate bis(oxazoline) ligand systems such as 1,1'-bis(4,4-dimethyl-1,3-oxazolin-2-yl)ethane and Spirobox, with their $\mathrm{Fe}$ (II) and $\mathrm{Fe}$ (III) halide complexes, have proved to be catalysts for efficient atom transfer radical polymerization of styrene and $\mathrm{O}-\mathrm{H}$ bond insertion reactions, respectively. ${ }^{[328,329]}$ Recently Yoon et al. have reported high yields for regio and steriochemically controlled asymmetric oxyamination 
reactions mediated by iron(II) box complexes (Fig. 10.7). ${ }^{[330]}$ Though most of these complexes have not been structurally characterized, the system reported by Grassi et al. revealed the iron center to be coordinated in a pseudo tetrahedral environment with the ligand system. ${ }^{[328]}$<smiles>CC(C)(C1=NC(c2ccccc2)CO1)C1=NC(c2ccccc2)CO1</smiles><smiles>CC(C1=NC(C)(C)CO1)C1=NC(C)(C)CO1</smiles><smiles>[R]C1COC(C(C)(C)C2=NC([R])CO2)=N1</smiles><smiles>CC(C)(C1=N[C@@H]2c3ccccc3C[C@H]2O1)C1=N[C@@H]2c3ccccc3C[C@H]2O1</smiles>

Figure 10.7 Bidentate BOX ligand systems used in conjunction with iron for asymmetric catalysis. ${ }^{[55,328,330]}$

Tridentate Pybox ligands and their iron complexes have been reported to catalyze a series of organic reactions such as, the hydrosilylation of ketones, ${ }^{[331][332]}$ formation of carbon-heteroatom bonds (Michael addition reactions) and formation of hetero-hetero atom bonds such as sulfur-oxygen and sulfur-nitrogen bonds. ${ }^{[327]}$ In most cases, the complexes reported were coordinated solely by the ligand system. However, some groups have also utilized additional ligands such as $\mathrm{CO}$ in order to enhance catalytic reactivity. ${ }^{[333]}$ Ligands that are tetradentate in nature such as $(S, S)$-bomen and $(S, R, R, S)$-bomen have generated suitable iron complexes mediating catalysis for enantioselective epoxidation reactions (Fig. 10.8). ${ }^{[334]}$

\section{PyBOX-ligands}<smiles>[R]C1COC(c2cccc(C3=NC([R])CO3)n2)=N1</smiles>

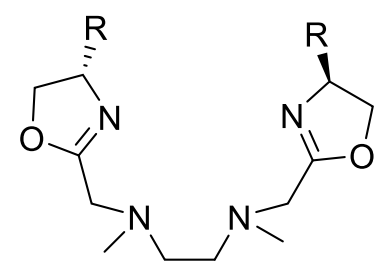

(S,S)-bomen<smiles>[R]C1COC(CN(C)C2CCCCC2N(C)CC2=N[C@@H]([R])CO2)=N1</smiles>

$(S, R, R, S)$-bomen

Figure 10.8 Tridentate PyBOX ligands and tetradentate BOX ligands used with their iron complexes in asymmetric catalysis. ${ }^{[332-334]}$ 


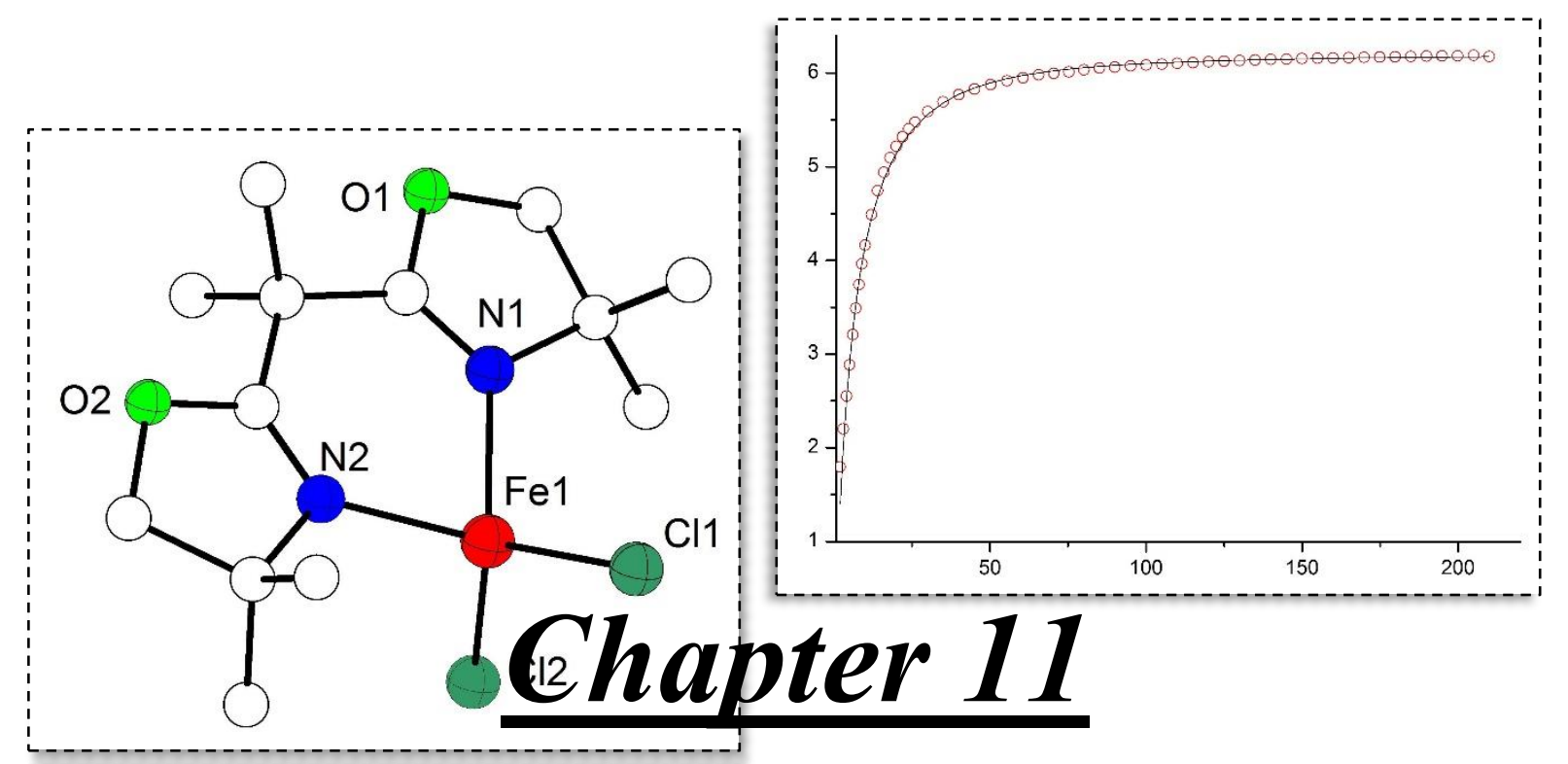

\section{Mononuclear and Dinuclear Fe(II)} Complexes of BOX.Ligands

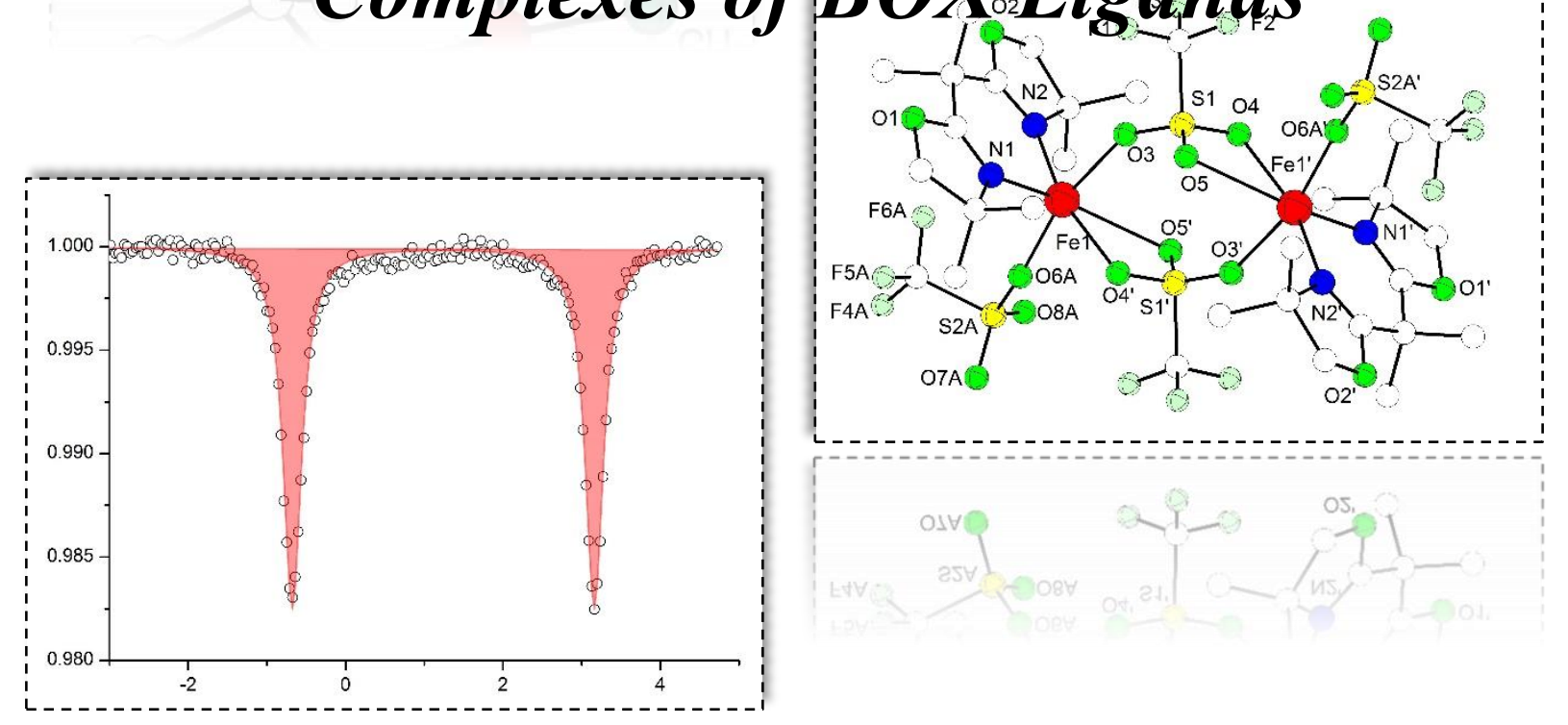




\section{Overview}

11.1 Introduction

11.2 Synthesis and characterization of mononuclear Fe(II) complexes with neutral BOX ligands 11.2.1 UV-vis and IR Spectroscopy

11.2.2 Mössbaeur Spectroscopy

11.2.3 Structural elucidation of mononuclear Fe(II)BOX complexes

11.3 Synthesis and characterization of a dinuclear Fe(II)BOX complex with ligand $\mathbf{L}^{\mathbf{6}}$

11.3.1 Properties in solid state

11.3.1.1 Structural elucidation of $\mathbf{2 2}$

11.3.1.2 Mössbaeur Spectroscopy

11.3.1.3 Magnetic Measurements

11.3.1.4 IR Spectroscopy

11.3.2 Properties in solution

11.4 Conclusion

[Note: Experimental procedures for this chapter are described in detail under Chapter 15, section 15.7] 


\subsection{Introduction}

The previous sections described the air sensitive $\mathrm{Cu}(\mathrm{I}) \mathrm{BOX}$ complexes activating dioxygen to yield $\mathrm{Cu}_{2} \mathrm{O}_{2}$ intermediates at low temperatures. Small molecule activation with Fe(II)BOX complexes was similarly investigated. Though a large number of FeBOX systems which mediate asymmetric catalysis are known in literature, a detailed study of the interaction of such metal-complexes with small molecules has not yet been explored or reported till date. The goal was thus to synthesize Fe(II) complexes of neutral BOX ligands, similar to those of their $\mathrm{Cu}(\mathrm{I})$ analogues, and investigate their reactivity towards small molecules such as $\mathrm{O}_{2}$ and $\mathrm{NO}$.

The following sections describe the synthesis and characterization of mononuclear Fe(II)BOX complexes with neutral ligands $\mathbf{H} \mathbf{L}^{1}, \mathbf{H L}^{2}, \mathbf{H L}^{3}, \mathbf{H L}^{5}$ and $\mathbf{L}^{6}$. Additionally, a dinuclear Fe(II)BOX complex with the ligand $\mathbf{L}^{6}$ was also synthesized and characterized during the course of this work. All of these complexes were successfully characterized via X-ray crystallography. Figure 11.1 illustrates the ligand systems used in the generation of Fe(II)BOX complexes.

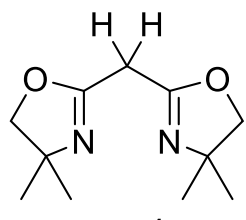

$\mathrm{HL}^{1}$

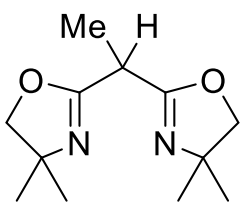

$\mathrm{HL}^{2}$

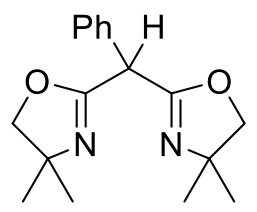

$\mathrm{HL}^{3}$

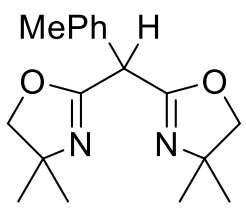

$\mathrm{HL}^{5}$

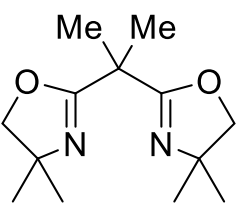

$\mathrm{L}^{6}$

Figure 11.1 Neutral ligands employed in the generation of suitable Fe(II)BOX complexes for small molecule activation.

\subsection{Synthesis and characterization of mononuclear Fe(II) complexes with neutral BOX ligands}

The general scheme employed for the generation of Fe(II) complexes with neutral BOX ligands is depicted in Scheme 11.1. Ligands $\mathbf{H L}^{1}, \mathbf{H L}^{2}, \mathbf{H L}^{3}, \mathbf{H L}^{5}$ and $\mathbf{L}^{6}$ were treated individually with 1 equivalent of $\mathrm{Fe}(\mathrm{II}) \mathrm{Cl}_{2}$ to yield yellow colored solutions of their $(\mathrm{BOX}) \mathrm{FeCl}_{2}$ complexes 17, 18, 19, 20 and 21 respectively. In case of 17, 18, 20 and $\mathbf{2 1}$, THF was used as a suitable solvent whereas for 19, DCM was preferred due to solubility reasons. ESI-MS analysis of all (BOX) $\mathrm{FeCl}_{2}$ complexes with proton responsive ligands in solution yielded a similar pattern. The yellow colored solutions of these complexes revealed two peaks belonging to the same complex. The minor peak could be assigned to the deprotonated complex $\mathbf{L}^{\mathbf{x}} \mathbf{F e C l}_{2}$ (where $\mathbf{L}^{\mathbf{x}}$ represents the deprotonated form of $\mathbf{H} \mathbf{L}^{1}, \mathbf{H} \mathbf{L}^{2}, \mathbf{H L}^{3}$ and $\mathbf{H} \mathbf{L}^{5}$ ) and the major peak could be assigned to the deprotonated complex $\mathbf{L}^{\mathbf{x}} \mathbf{F e C l}_{2}$ with additional solvent molecules $\left(\mathrm{CH}_{3} \mathrm{CN}, \mathrm{CH}_{2} \mathrm{Cl}_{2}\right.$ and $\mathrm{OH}$ ). In case of 21, the complex could not be successfully analyzed via ESI-MS. Figures 11.2 and 11.3 illustrate the ESI-MS spectra of 17 (Fig. 11.2 left), 18 (Fig. 11.2 right), 19 (Fig. 11.3 left) and 20 (Fig. 11.3 right). 


\subsubsection{UV-vis and IR Spectroscopy}

Complexes 17, 18, 19, 20 and 21 were further characterized via UV-vis and IR spectroscopy. UV-vis measurements of these $\mathrm{CH}_{3} \mathrm{CN}$ solutions revealed only a shoulder like region between 280-300 nm $(\varepsilon \approx$ $15,000 \mathrm{M}^{-1} \mathrm{~cm}^{-1}$ ) in all cases which could tentatively be assigned to a $\pi$ to $\pi^{*}$ transition originating from the ligand system. The UV-vis spectrum of the ligand itself (Fig 11.4 left inset) displayed a similar shoulder around $300 \mathrm{~nm}$ which could possibly be assigned to a $\pi$ to $\pi^{*}$ transition, which changed slightly on complexation with the metal. No MLCT or d-d transition bands were observed. IR spectroscopy of crystalline material of these complexes showed bands at $\sim 3000 \mathrm{~cm}^{-1}$ and $1500-1200 \mathrm{~cm}^{-1}$ which could be assigned to the $\mathrm{C}-\mathrm{H}$ stretching and bending modes of the ligand. In all cases, intense sharp peaks at $\sim 1650$ $\mathrm{cm}^{-1}$ and $\sim 1100 \mathrm{~cm}^{-1}$ were also observed which could be assigned to the $\mathrm{C}=\mathrm{N}$ and $\mathrm{C}-\mathrm{O}$ stretching modes of the BOX ligands, respectively. Weaker bands between $1300-1200 \mathrm{~cm}^{-1}$ were assigned to the C-N stretching modes. Figure 11.4 (left) illustrates the UV-vis spectrum of 21 in solution and figure 11.4 (right) illustrates its IR spectrum in solid state.

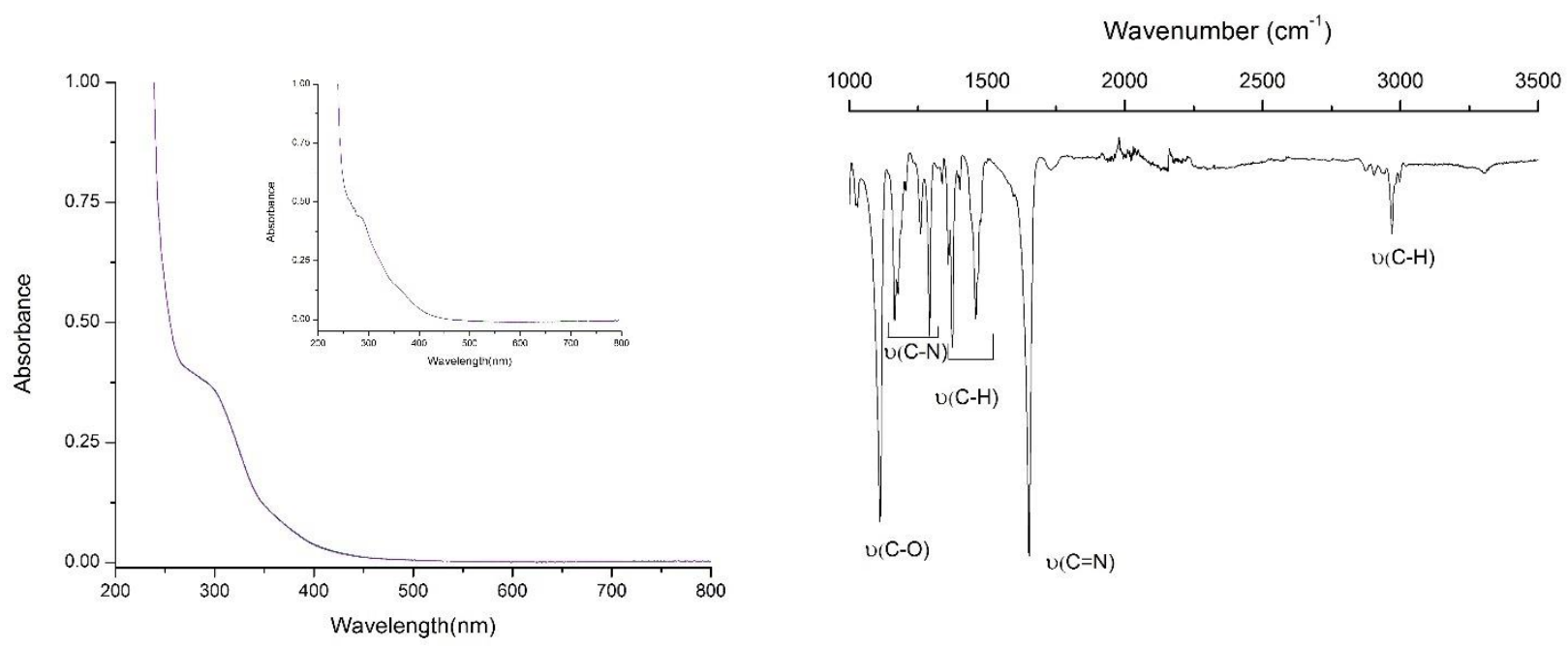

Figure 11.4 Left: UV-vis spectra of 21 measured in $\mathrm{CH}_{3} \mathrm{CN}$ at $\mathrm{rt}$. The shoulder at $\sim 300 \mathrm{~nm}\left(\varepsilon \approx 15,000 \mathrm{M}^{-1} \mathrm{~cm}^{-1}\right)$ was assigned to the $\pi$ to $\pi^{*}$ transition originating from the ligand. Sowin in the inset is the UV-vis spectrum of ligand $\mathbf{L}^{6}$. Right: IR spectroscopy of $\mathbf{2 1}$ measured from crystalline material illustrating stretching frequencies belonging to the ligand scaffold.

\subsubsection{Mössbauer Spectroscopy}

Zero field Mössbauer spectra of crystalline material of all mononuclear Fe(II)BOX complexes were measured at $80 \mathrm{~K}$. The resulting spectra revealed only an $\mathrm{Fe}(\mathrm{II})$ high spin species to be present. In all cases, the isomer shifts $(\delta)$ of $\sim 0.95 \mathrm{mms}^{-1}$, quadrupole splitting values $\left(\left|\Delta \mathrm{E}_{\mathrm{q}}\right|\right)$ of $\sim 3.14 \mathrm{mms}^{-1}$, and narrow Lorentzian profiles of $\sim 0.30 \mathrm{mms}^{-1}$ were rather similar and typical of a $\mathrm{Fe}$ (II) high spin species. These were in good agreement to the results obtained from X-ray crystallography confirming that the iron atoms were indeed present in a tetrahedral environment in the +2 oxidation state (Refer to sec. 11.2.3). Figures 11.5 to 11.7 illustrate the Mössbauer spectra of $\mathrm{Fe}(\mathrm{II}) \mathrm{BOX}$ complexes with proton and non-proton responsive ligands and Table 11.1 describes the individual isomer shifts, quadrupole splitting values and full width at half maximum values of each of these complexes. 

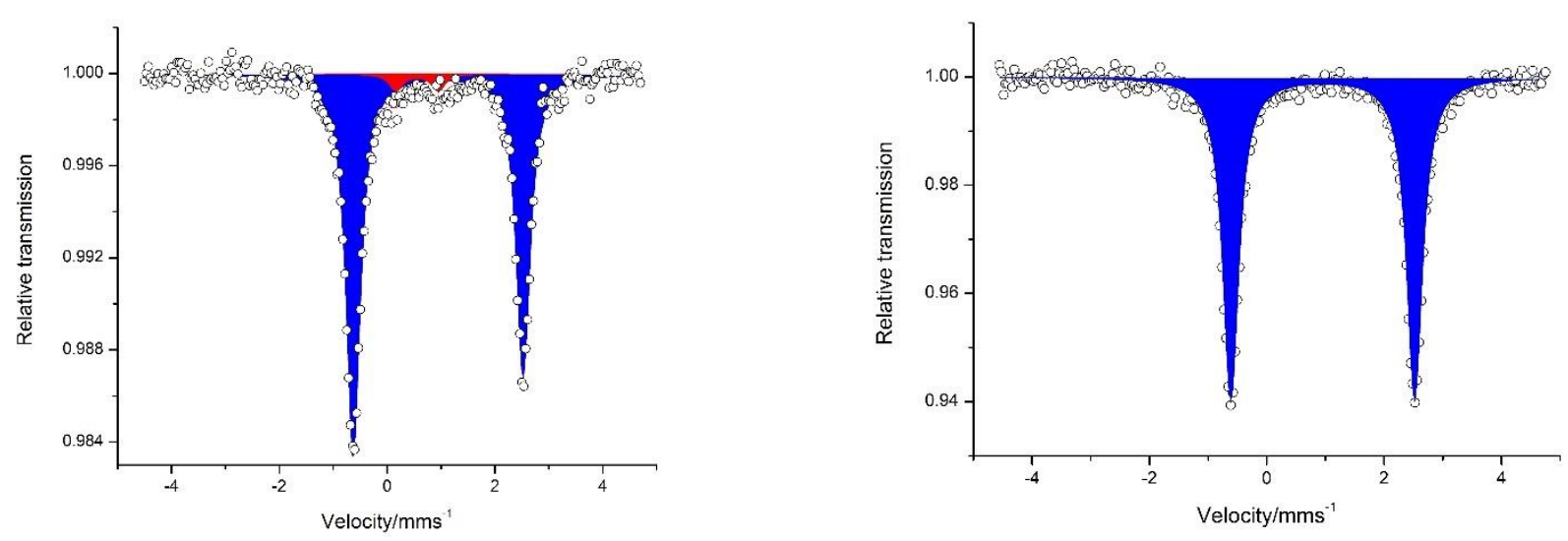

Figure 11.5 Left: Zero field Mössbauer spectrum of crystalline material of 17 measured at 80 K. Right: Zero field Mössbauer spectrum of crystalline material of $\mathbf{1 8}$ measured at $80 \mathrm{~K}$.
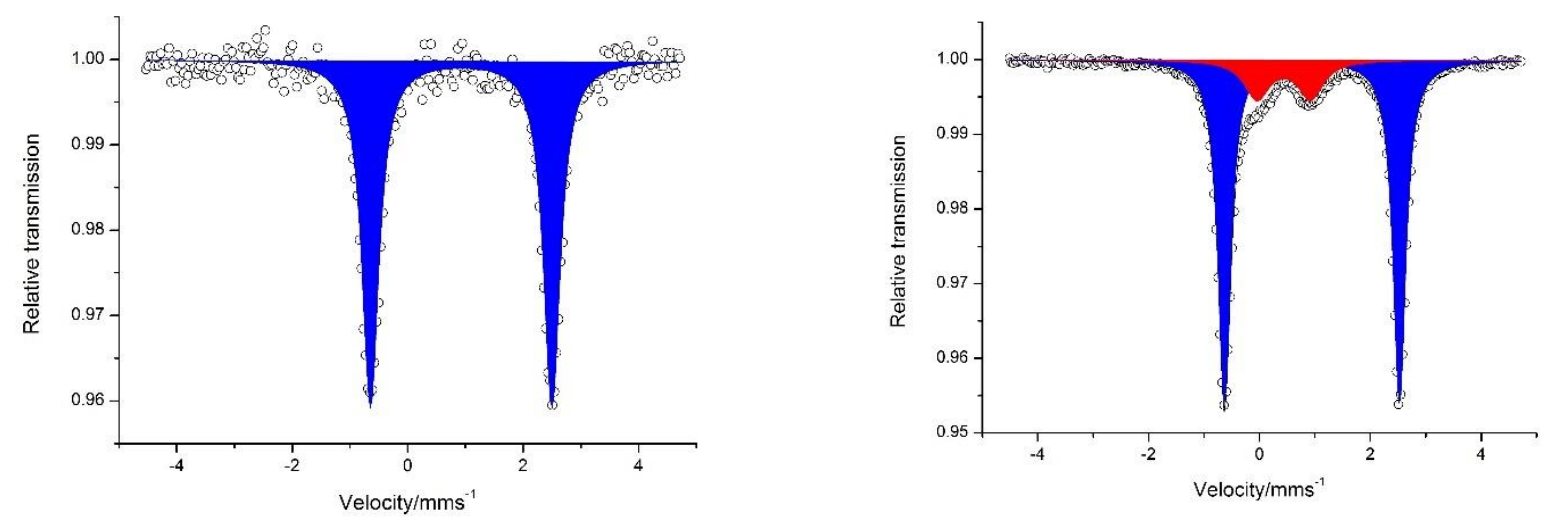

Figure 11.6 Left: Zero field Mössbauer spectrum of crystalline material of 19 measured at 80 K. Right: Zero field Mössbauer spectrum of crystalline material of $\mathbf{2 0}$ measured at $80 \mathrm{~K}$.

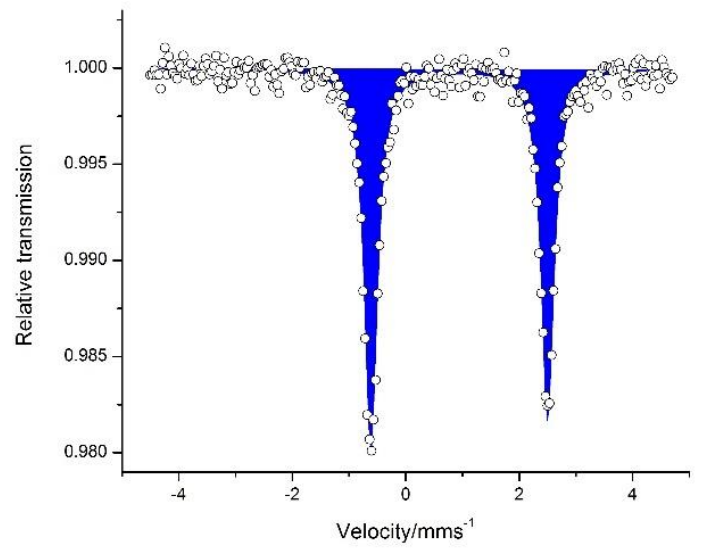

Figure 11.7 Zero field Mössbauer spectrum of crystalline material of 21 measured at $80 \mathrm{~K}$. 
Table 11.1 Mössbauer parameters of 17, 18, 19, 20 and 21 obtained at $80 \mathrm{~K}$.

\begin{tabular}{cccc}
\hline Complex & $\delta\left(\mathrm{mms}^{-1}\right)$ & $\left|\Delta \mathrm{E}_{\mathrm{q}}\right|\left(\mathrm{mms}^{-1}\right)$ & $\Gamma_{F W H M}\left(\mathrm{mms}^{-1)}\right.$ \\
\hline $\mathbf{1 7}$ & 0.95 & 3.15 & 0.32 \\
$\mathbf{1 8}$ & 0.95 & 3.13 & 0.33 \\
$\mathbf{1 9}$ & 0.93 & 3.14 & 0.36 \\
$\mathbf{2 0}$ & 0.95 & 3.16 & 0.28 \\
$\mathbf{2 1}$ & 0.94 & 3.11 & 0.28 \\
\hline
\end{tabular}

\subsubsection{Structural elucidation of mononuclear Fe(II)BOX complexes}

The Fe(II)BOX complexes of proton and non-proton responsive ligands were successfully characterized via X-ray crystallography. Layering of hexane with THF solutions of 17 (Fig. 11.8 left), 18 (Fig. 11.8 right), 20 (Fig. 11.9 right) and 21 (Fig. 11.10) yielded single crystals suitable for X-ray diffraction. In case of complex 19 (Fig. 11.9 left), layering of hexane with a DCM solution of the complex yielded suitable crystals. The molecular structures of all of these complexes reveals the iron to be coordinated in a distorted tetrahedral geometry by the bidentate BOX ligands and two chloride atoms. The angles N-Fe-N are acute $\sim 88^{\circ}$ but compensated by larger metal chloride angles $\sim 116^{\circ}$. The Fe-N bond lengths in the range of 2.07$2.08 \AA$ are, however, shorter than in other related $\alpha$-diimine complexes. ${ }^{[335]}$ Similar to what was observed with the $\mathrm{Cu}(\mathrm{I}) \mathrm{BOX}$ complexes (refer to Chapter 5), the BOX ligands take up a boat shaped coordination. The metal center along with part of the ligand scaffold comprising of the nitrogen atoms and bridging carbon, form a six membered chelate ring in an essentially single plane. The two methyl groups along with the chloride atoms and alkyl or aryl substituents present within the ligand backbone, lie above and below the plane. Contrary to the $\mathrm{Cu}(\mathrm{I}) \mathrm{BOX}$ analogues, no exogenous solvent molecules were bound to the $\mathrm{Fe}(\mathrm{II}) \mathrm{BOX}$ complexes. It should be noted that though a large number of $\mathrm{Fe}-\mathrm{BOX}$ complexes are known in literature, only two of them have been characterized via X-ray crystallography. ${ }^{[328,336]}$ Figures 11.8 to 11. 10 illustrate the structurally elucidated complexes of Fe(II)BOX ligands and Table 11.2 describes the individual bond lengths, bond angles, and space groups for each of these complexes. 

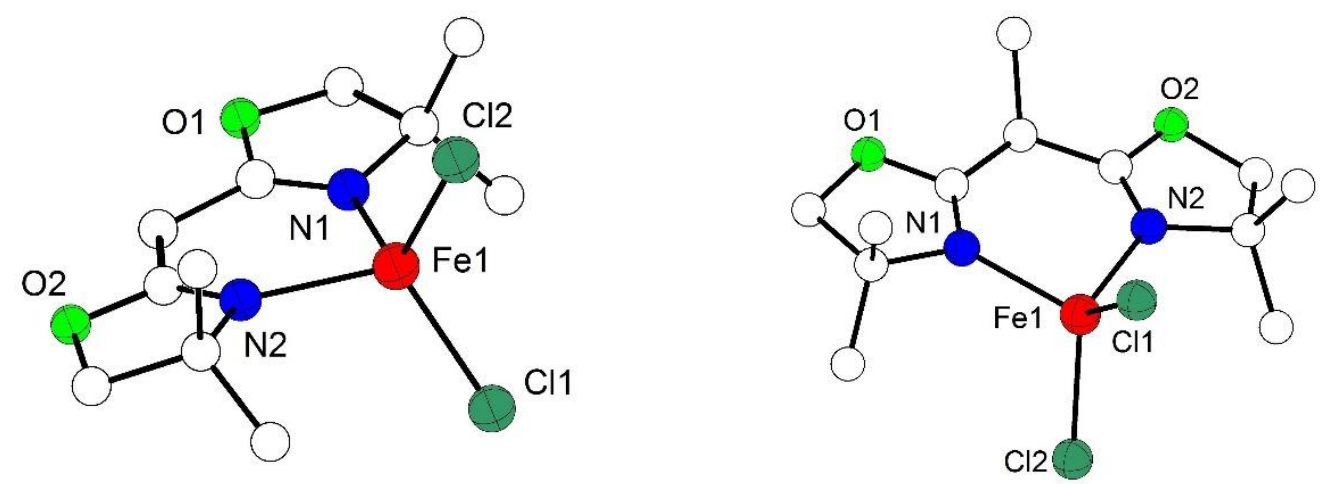

Figure 11.8 Left: Molecular structure of 17 in the crystal with partial labelling scheme. Hydrogen atoms are omitted for clarity. Selected bond lengths $[\AA]$ for 17: Fe1-N2 2.080(5), Fe1-N1 2.081(5), Fe1-Cl2 2.2529(15), Fe1-C11 2.2551(15). Right: Molecular structure of $\mathbf{1 8}$ in the crystal with partial labelling scheme. Hydrogen atoms are omitted for clarity. Selected bond lengths $[\AA]$ for 18: Fe1-N2 2.0772(14), Fe1-N1 2.0853(14), Fe1-Cl2 2.2485(5), Fe1-Cl1 2.2659(5).
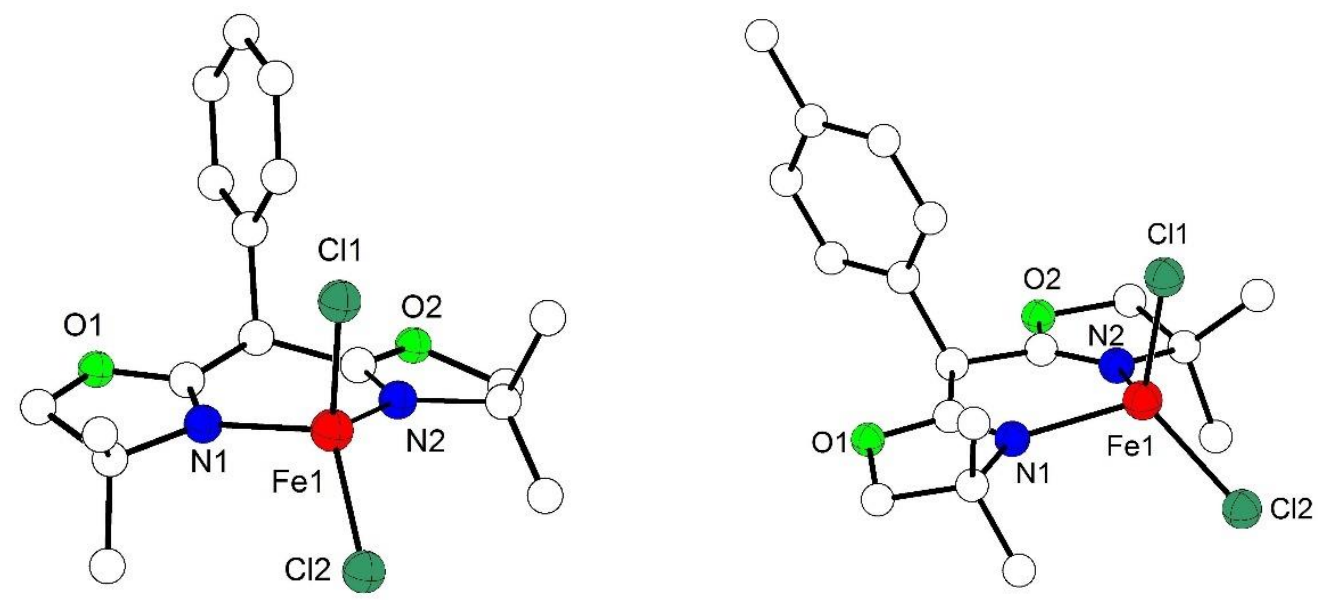

Figure 11.9 Left: Molecular structure of 19 in the crystal with partial labelling scheme. Hydrogen atoms are omitted for clarity. Selected bond lengths $[\AA]$ for 19: Fe1-N1 2.0821(18), Fe1-N2 2.0844(18), Fe1-Cl2 2.2525(6), Fe1-Cl1 2.2540(6). Right: Molecular structure of $\mathbf{2 0}$ in the crystal with partial labelling scheme. Hydrogen atoms are omitted for clarity. Selected bond lengths $[\AA]$ for 20: Fe1-N2 2.076(2), Fe1-N1 2.079(2), Fe1-Cl2 2.2499(8), Fe1-Cl1 2.2537(8).

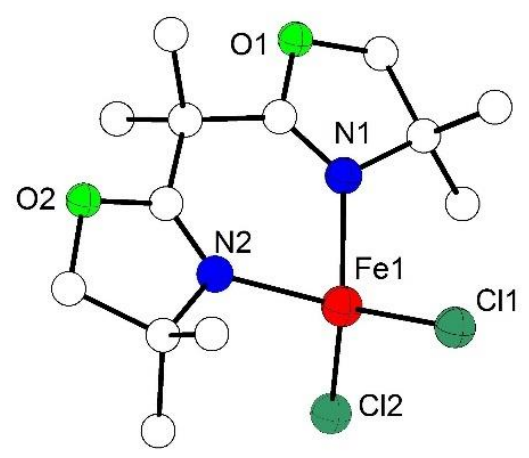

Figure 11.10 Molecular structure of 21 in the crystal with partial labelling scheme. Hydrogen atoms are omitted for clarity. Selected bond lengths $[\AA]$ for 21: Fe1-N2 2.0826(13), Fe1-N1 2.0854(15), Fe1-Cl2 2.2458(6), Fe1-Cl1 2.2556(6). 
Table 11.2 Metric parameters of Fe(II)BOX complexes 17, 18, 19, 20 and 21.

\begin{tabular}{lccccc}
\hline & $\mathbf{1 7}$ & $\mathbf{1 8}$ & $\mathbf{1 9}$ & $\mathbf{2 0}$ & $\mathbf{2 1}$ \\
\hline $\mathrm{N} 1-\mathrm{Fe}-\mathrm{N} 2\left[^{\circ}\right]$ & 88.679 & 88.214 & 87.355 & 87.949 & 87.256 \\
$\mathrm{Cl1}-\mathrm{Fe}-\mathrm{Cl} 2\left[^{\circ}\right]$ & 117.581 & 112.580 & 117.208 & 118.122 & 115.420 \\
& & & & & \\
$\mathrm{Fe}-\mathrm{N}(\AA)$ & 2.08 & 2.08 & 2.08 & 2.01 & 2.08 \\
& 2.09 & 2.07 & 2.08 & 2.07 & 2.08 \\
& & & & & \\
space group & $P c a 2_{1}$ & $P \overline{1}$ & $P 2{ }_{1}{ }_{1} 2_{1}$ & $P 2{ }_{1}{ }_{1} 2_{1}$ & $P 2_{1 / \mathrm{n}}$ \\
\hline
\end{tabular}

\subsection{Synthesis and characterization of a dinuclear $\mathrm{Fe}(\mathrm{II}) \mathrm{BOX}$ complex with $\mathrm{BOX}$ ligand $\mathrm{L}^{6}$}

In addition to the mononuclear $(\mathrm{BOX}) \mathrm{FeCl}_{2}$ complexes that have been described in the previous section, a dinuclear Fe(II) complex with ligand $\mathbf{L}^{\mathbf{6}}$ was successfully synthesized according to Scheme 11.2. The ligand was treated with 1 equivalent of $\mathrm{Fe}(\mathrm{OTf})_{2}(\mathrm{MeCN})_{2}$ under inert conditions in THF to yield a light yellow colored solution. Workup with DCM and THF, followed by layering with dry hexane yielded single crystals of 22 suitable for X-ray diffraction.

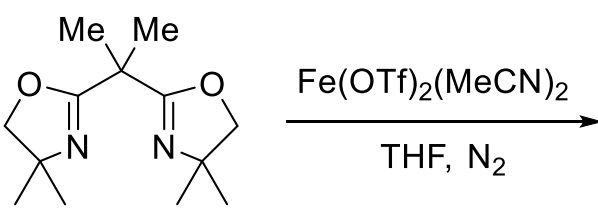

$L^{6}$

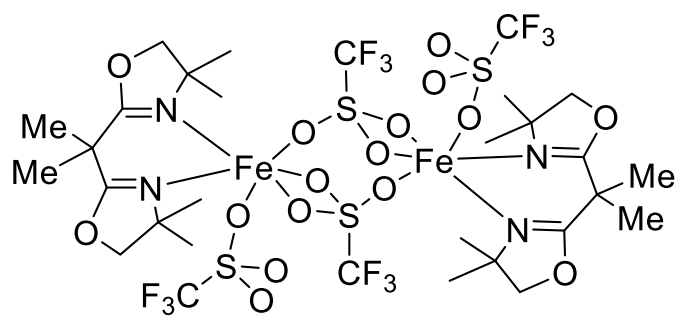

22

Scheme 11.2 Schematic representation for the synthesis of a dinuclear Fe(II)BOX complex 22 with ligand $\mathbf{L}^{6}$.

\subsubsection{Properties in solid state}

\subsubsection{Structural elucidation of 22}

Single crystals of $\mathbf{2 2}$ were successfully grown from a THF/hexane solution of the complex at rt in $10 \%$ yield revealing a dinuclear (BOX)Fe(II) species. 22 crystalizes as two crystallographically independent molecules per unit cell in the triclinic space group $P \overline{1}$ and possesses a crystallographically imposed $C_{i}$ symmetry. X-ray diffraction analysis (Fig. 11.11) showed the iron centers to be identical and coordinated in a distorted octahedral environment. Each metal atom was bound to the nitrogen donors of the BOX ligand with the metal centers being bridged by two triflate molecules. The bridging triflate moieties were bound asymmetrically to the iron centers with a single oxygen atom being coordinated to one metal while the other two oxygen atoms were coordinated to the second. An additional triflate molecule was coordinated to each 
iron center completing its octahedral coordination sphere. The Fe-N bond lengths in the dinuclear complex were similar to those of the mononuclear Fe(II) complexes in the range of 2.07-2.08 $\AA$, suggesting no change in metal to ligand coordination. The $\mathrm{Fe}-\mathrm{O}$ bond lengths of bridging triflate moieties were measured between 2.1-2.2 $\AA$ and were slightly longer compared to those of the terminally bound triflate at each iron center $(2.02 \AA)$. This can be attributed to the bridging triflate molecules having to span between the two metal centers compared to the those terminally coordinated to a single metal center. The different binding modes result in a difference of their respective Fe-O bond lengths. The $\mathrm{Fe} \cdots \mathrm{Fe}$ separation was measured at $5.32 \AA$ which was rather high compared to other dinuclear Fe(II) complexes. A plausible explanation for the formation of a dinuclear species with $\mathrm{Fe}(\mathrm{OTf})_{2}(\mathrm{MeCN})_{2}$ as opposed to mononuclear complexes with $\mathrm{FeCl}_{2}$ and $\mathrm{BOX}$ ligands could be, that the counterion in the former case being much larger with the ability to coordinate at multiple sites, prefers the formation of a dinuclear species. Moreover, as triflate is a far weaker donor than chloride, it fails to satisfy the electronic environment around the iron center, leaving the $\mathrm{Fe}$ (II) center relativiely acidic. This results in further binding of ligands to the metal center, forming a bridging dimetallic system.

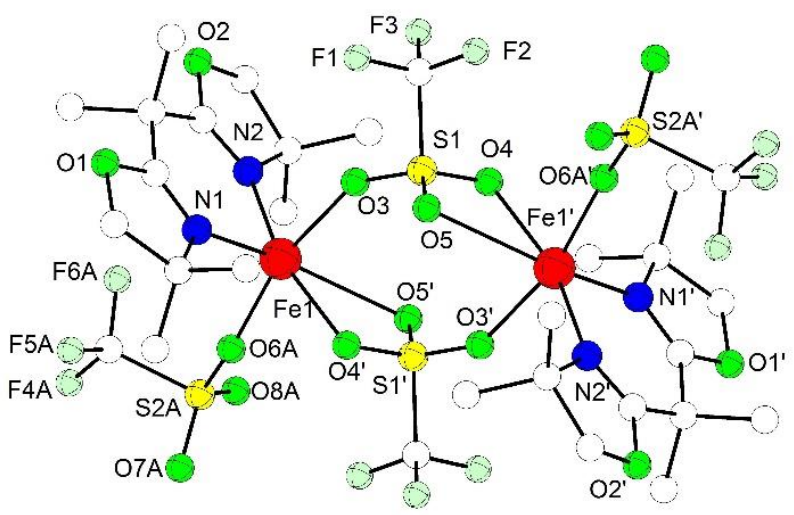

Figure 11.11 Molecular structure of 22. Hydrogen atoms are omitted for clarity. Selected bond lengths $[\AA]$ for 22: Fe1-O6A 2.020(9), Fe1-O6B 2.026(10), Fe1-N1 2.078(4), Fe1-N2 2.084(4), Fe1-O3 2.156(3), Fe1-O4' 2.219(4).

\subsubsection{Mössbauer Spectroscopy}

Zero field Mössbauer spectroscopy of crystalline material of 22 (Fig. 11.12) at $80 \mathrm{~K}$ revealed only one $\mathrm{Fe}(\mathrm{II})$ species to be present indicating both iron sites to exhibit identical Mössbauer parameters, in line with the crystallographic equivalence of the two metal sites. 22 displayed an isomer shift $(\delta)$ of $1.24 \mathrm{mms}^{-1}$ with a quadrupole splitting $\left(\left|\Delta \mathrm{E}_{\mathrm{q}}\right|\right)$ of $3.84 \mathrm{mms}^{-1}$ typical for $\mathrm{Fe}(\mathrm{II})$ high spin complexes with a high coordination number. The full-width at half maximum of the lorentzian profiles $\left(\Gamma_{F W H M}\right)$ was rather small, $\sim 0.30 \mathrm{mms}^{-1}$. 


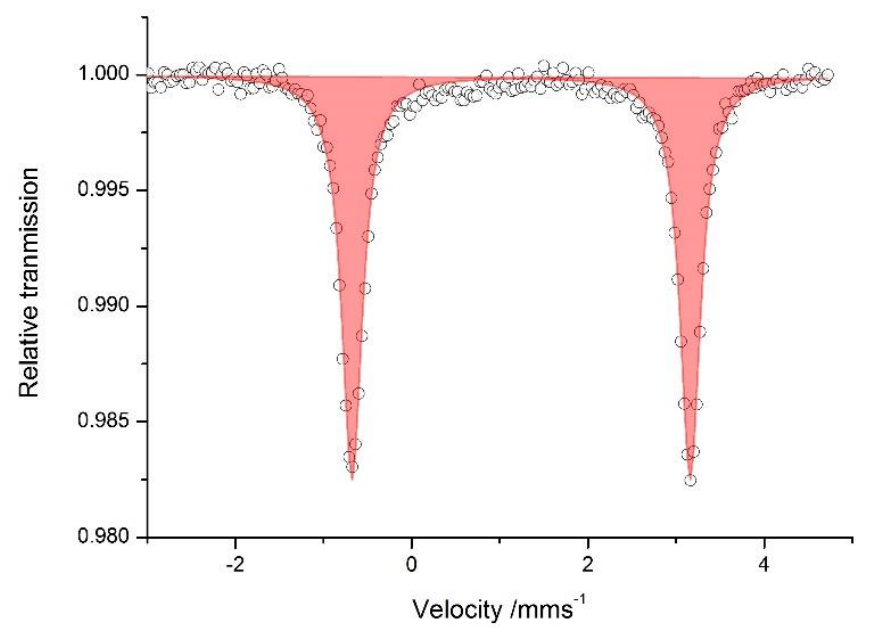

Figure 11.12 Zero field Mössbauer spectrum of crystalline material of 22 measured at $80 \mathrm{~K}$ depicting a single quadrapole doublet.

\subsubsection{Magnetic Measurements}

Since 22 represents a dinuclear high spin Fe(II) species, magnetic susceptibility measurements were carried out in order to investigate the presence of any coupling between the two metal centers. Measurements at $0.5 \mathrm{~T}$ within the range of 2 to $210 \mathrm{~K}$ generated a $\chi_{m} T$ value of $6.17 \mathrm{~cm}^{3} \mathrm{Kmol}^{-1}$ at $210 \mathrm{~K}$ which corresponded to a magnetic moment of $7.05 \mu_{B}$ (Fig. 11.13 left). This was in reasonable agreement with the spin-only value of two iron(II) ions, each with a spin state of $S=2\left(6.92 \mu_{B}\right)$. The values of $\chi_{m} T$ between $50 \mathrm{~K}$ to 210 $\mathrm{K}$ remained virtually constant signifying the absence of strong exchange interaction between the two metal centers. However, on reducing the temperature from $50 \mathrm{~K}$ to $0 \mathrm{~K}$ the magnetic susceptibly dropped indicating a possible presence of ZFS within the molecule. Variable temperature/variable field (VTVH) measurements carried out at $1 \mathrm{~T}, 3 \mathrm{~T}$ and $5 \mathrm{~T}$ indeed corroborated this assumption (Fig. 11.13 right).
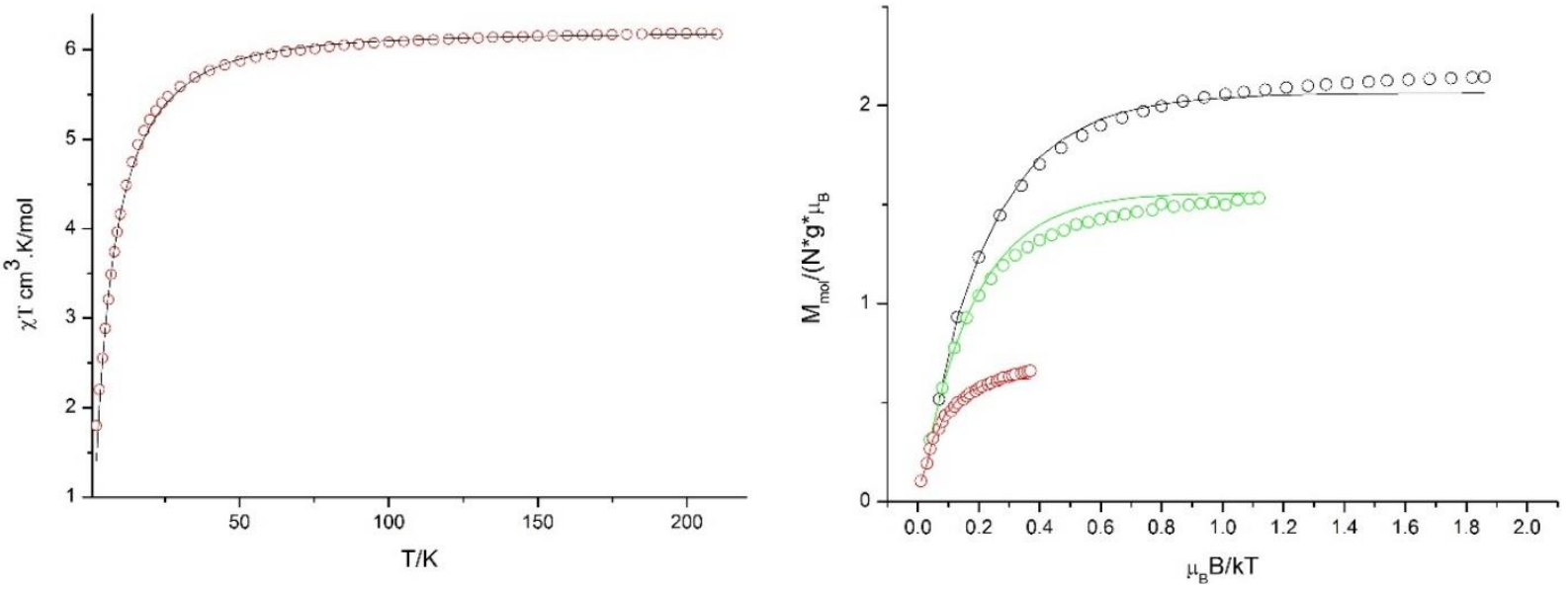

Figure 11.13 Left: Magnetic susceptibility measurements $\left(\chi_{m} T\right.$ vs $\left.T\right)$ of crystalline material of 22 measured between $2 \mathrm{~K}$ to $210 \mathrm{~K}$ at $0.5 \mathrm{~T}$. Right: Variable temperature/ variable field (VTVH) measurements at $1 \mathrm{~T}, 3 \mathrm{~T}$, and $5 \mathrm{~T}$ confirming the presence of ZFS within the molecule. Both data sets have been fitted simultaneously. 
A simulation of the susceptibility along with VTVH data sets confirmed the presence of two iron(II) centers with negligible coupling. A weak antiferromagnetic coupling of $J=-0.144 \mathrm{~cm}^{-1}$ was observed with an isotropic $g$ value of 2.04 . The ZFS value was measured at $D=+13.34 \mathrm{~cm}^{-1}$ with $E / D=0.23$.

\subsubsection{IR Spectroscopy}

IR spectra of crystalline material of $\mathbf{2 2}$ were obtained via IR-ATR. Peaks with frequencies observed at 1650 $\mathrm{cm}^{-1}$ and $1370 \mathrm{~cm}^{-1}$ could be assigned to the $\mathrm{C}=\mathrm{N}$ and $\mathrm{C}-\mathrm{N}$ stretching modes of the ligand. A large number of peaks between $1000 \mathrm{~cm}^{-1}$ to $1300 \mathrm{~cm}^{-1}$ was assigned to the $\mathrm{C}-\mathrm{H}$ modes with a sharp peak at $1024 \mathrm{~cm}^{-1}$ being assigned to the $\mathrm{C}-\mathrm{O}$ stretch of the ligand scaffold.

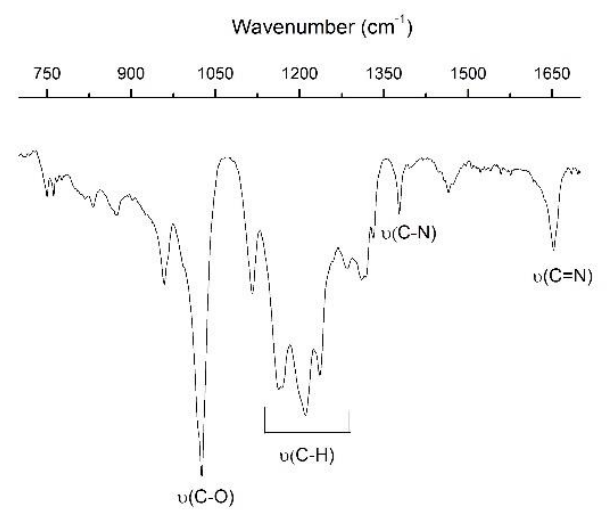

Figure 11.14 IR spectroscopy of 22 measured from crystalline material illustrating stretching frequencies belonging to the ligand scaffold.

\subsubsection{Properties in solution}

In addition to characterizing $\mathbf{2 2}$ in the solid state, UV-vis spectroscopy and ESI-MS of a solution of 22 were measured. The UV-vis spectrum of a solution of 22 in THF displayed a band at $287 \mathrm{~nm}\left(\varepsilon \approx 14,500 \mathrm{M}^{-1} \mathrm{~cm}^{-}\right.$ $\left.{ }^{1}\right)$ which was tentatively assigned to a $\pi$ to $\pi^{*}$ transition originating from the ligand (Fig. 11.15 left). No MLCT or d-d transitions bands were observed similar to mononuclear Fe(II)BOX complexes. ESI-MS analysis of 22 in $\mathrm{CH}_{3} \mathrm{CN}$ showed a large number of signals, belonging to fragmented portions of the complex, with additional solvent molecules in some cases. A peak at $\mathrm{m} / \mathrm{z}=1206.8$ could be assigned to the complex with an additional sodium ion, though the intensity was rather low (Fig. 11.15 right).
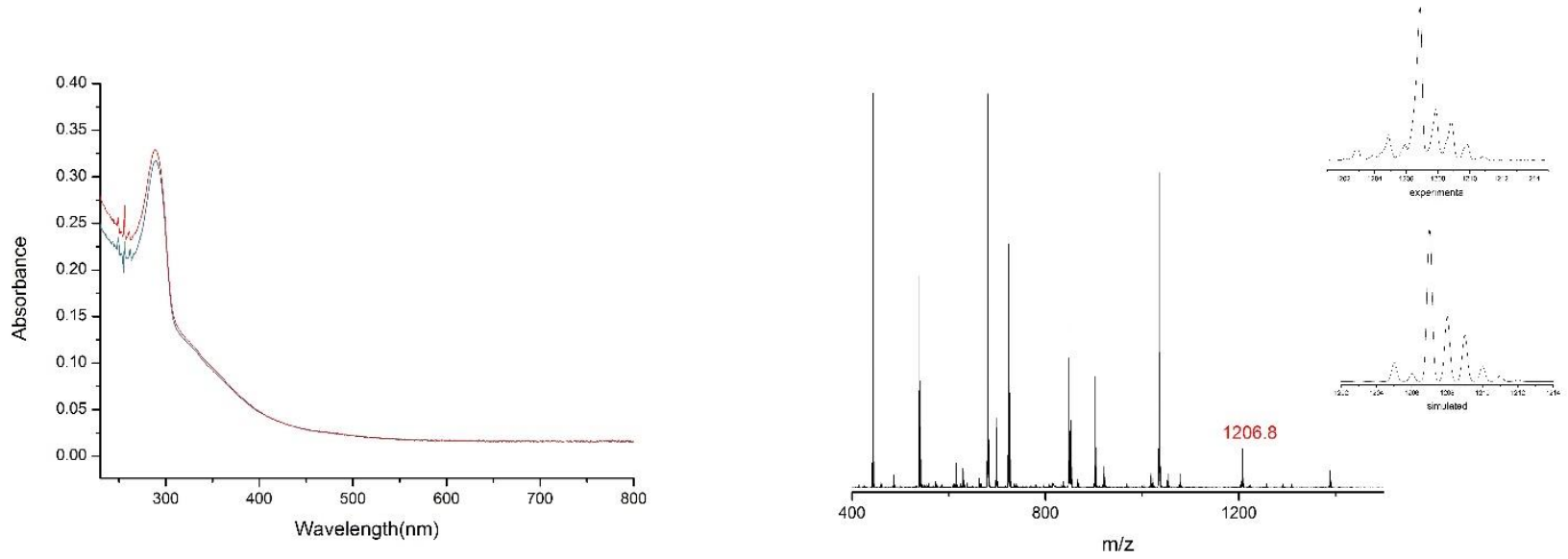

Figure 11.15 Left: UV-vis spectrum of 22 in THF with a band at $287 \mathrm{~nm}$ originating from the ligand. Right: ESI-MS of 22 in $\mathrm{CH}_{3} \mathrm{CN}$. The peak marked in red denotes $\left[\left(\mathbf{L}^{6} \mathrm{Fe}(\mathrm{OTf})_{2}\right)_{2} \mathrm{Na}\right]^{+}$. The inset shows an enlargement of this peak at $m / z=1206.8$ together with a simulation of the isotopic pattern. 


\subsection{Conclusion}

To summarize, a series of mononuclear $(\mathrm{BOX}) \mathrm{FeCl}_{2}$ complexes with neutral bidentate $\mathrm{BOX}$ ligands were successfully synthesized during the course of this work. They were all fully characterized in solution as well as in the solid state, including characterization by X-ray crystallography. Mössbauer spectroscopy suggested the iron centers to be in a +2 , high spin oxidation state which was in line with results obtained from X-ray analysis. Structural elucidation of all $5(\mathrm{BOX}) \mathrm{FeCl}_{2}$ revealed the iron centers to be coordinated in a distorted tetrahedral environment. The complexes were also characterized by UV-vis spectroscopy, IR spectroscopy and ESI-MS (except 21).

Interestingly, a dinuclear complex $\left[\left\{\mathbf{L}^{6} \mathrm{Fe}(\mathrm{OTf})\right\}_{2}(\mu-\mathrm{OTf})_{2}\right]$ was also synthesized and was fully characterized in solution and in the solid state. X-ray characterization of the complex revealed both the iron centers to be coordinated in a similar distorted octahedral environment. These results were in good agreement with the parameters obtained from Mössbauer spectroscopy which revealed the presence of a single Fe(II) high spin species. Further magnetic susceptibility measurements confirmed very weak antiferromagnetic coupling between the two iron Fe(II) ions, but substantial zero-field splitting.

Though a large number of Fe-BOX complexes are known in literature, there are only few that have been characterized via X-ray crystallography. Through this work, successful X-ray characterization of mononuclear and dinuclear Fe(II)BOX complexes adds to the list of structurally elucidated complexes belonging to this ligand class. Moreover, the feasibility to synthesize such complexes puts forward opportunities to investigate interesting chemistry of these systems. For example, small molecule activation of dioxygen or nitric oxide, similar to those investigated with $\mathrm{Cu}(\mathrm{I}) \mathrm{BOX}$ systems could potentially be explored. This shall be discussed further in the following chapter. 
[Note: This page has intentionally been left blank] 


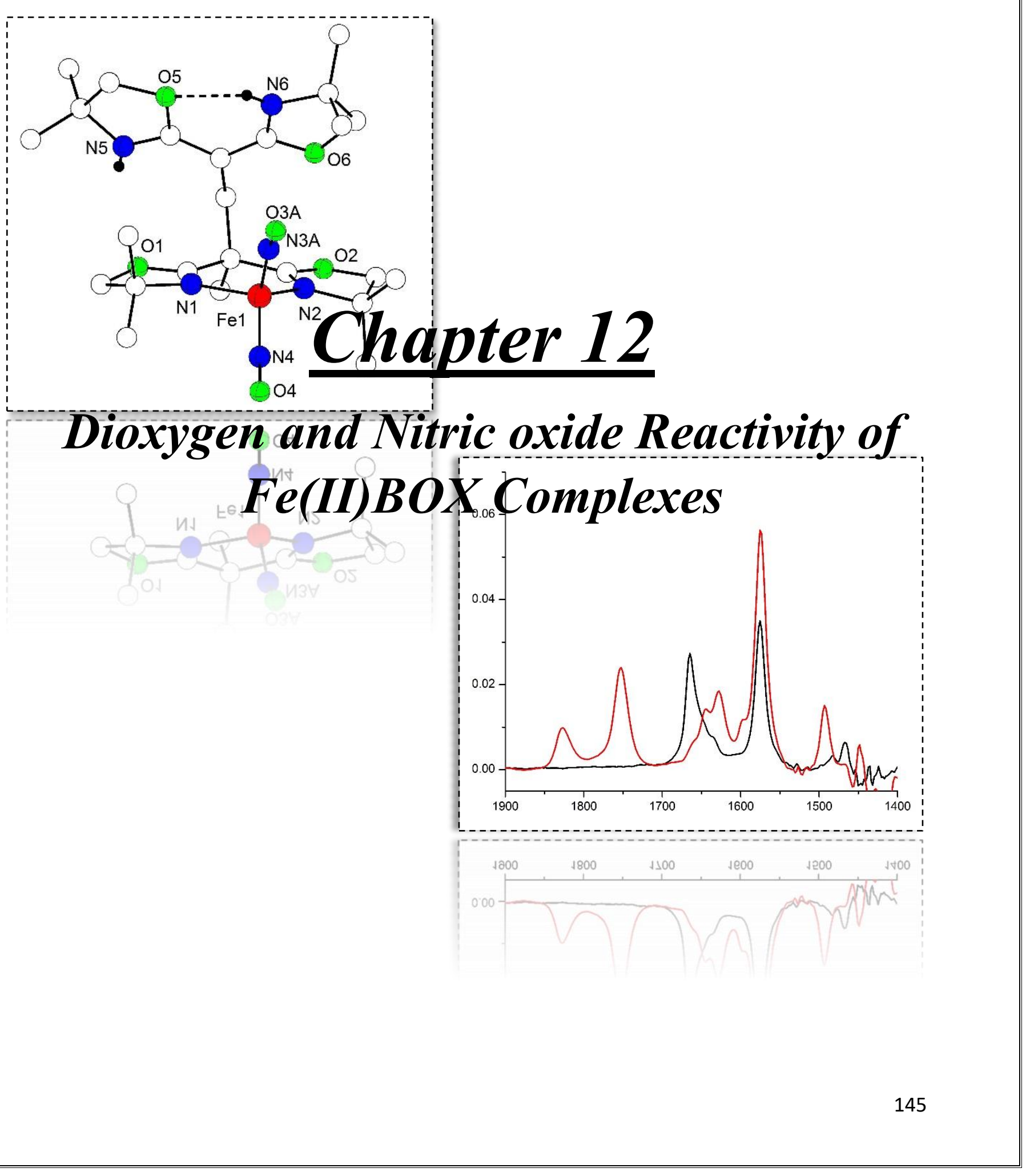




\section{Overview}

12.1 Introduction

12.2 Dioxygen reactivity of mononuclear and dinuclear Fe(II)BOX systems

12.3 Nitric oxide reactivity of a Fe(II)BOX complex with a proton responsive ligand

12.3.1 Preliminary NO reactivity of a dinuclear Fe(II) BOX complex

12.3.2 NO reactivity of a mononuclear Fe(II)BOX complex with a proton responsive ligand

12.3.2.1 Properties in solution: IR and UV-vis Spectroscopy

12.3.2.2 Properties in solid state

12.3.2.2.1 Structural elucidation

12.3.2.2.2 Mössbaeur Spectroscopy

\subsection{Conclusion}

[Note: Experimental procedures for this chapter are described in detail under Chapter 15, section 15.8] 


\subsection{Introduction}

The chemistry of mononuclear and dinuclear Fe(II) complexes with small molecules such as dioxygen and nitric oxide has attracted much attention over the last few decades. Extensive investigations with suitable ligand scaffolds capable of supporting active intermediates have helped in elucidating the mechanism and functional principles of the analogous enzymes activating such molecules. The previous sections have described small molecule activation of dioxygen with neutral and monoanionic $\mathrm{Cu}(\mathrm{I}) \mathrm{BOX}$ complexes, that yielded $\mathrm{Cu}_{2} \mathrm{O}_{2}$ intermediates. These were isolated and structurally elucidated at low temperatures which highlighted the nature of the ligand scaffold to be crucial in determining the dioxygen binding mode. Moreover, the nature of the ligand scaffold also had an effect on substrate reactivity. As a large number of mononuclear (BOX) $\mathrm{FeCl}_{2}$ complexes and a dinuclear (BOX)Fe(II) complex of a non-proton responsive ligand were successfully synthesized and characterized during the course of this work (refer to Chapter 11), their reactivity with small molecules such as dioxygen and nitric oxide was investigated for potential formation of novel intermediates.

Reactions of both mononuclear and dinuclear (BOX)Fe(II) complexes were screened with dioxygen as well as $\mathrm{PhIO}$ in various solvents at low temperatures to investigate the formation of iron-oxo intermediates. Unfortunately, unlike their $\mathrm{Cu}$ (I) analogues, the Fe(II) systems did not yield any detectable intermediates. The reactions were monitored via UV-vis spectroscopy and the end products were analyzed by Mössbuaer spectroscopy and ESI-MS. In all cases a clear oxidation of iron from the +2 to +3 oxidation state occurred. However, the nature of the decomposed product was inconclusive. In case of reactivity with nitric oxide, mononuclear (BOX)Fe(II) complexes demonstrated promising reactivity. The following sections describe the attempt to activate dioxygen with mononuclear and dinuclear (BOX)Fe(II) complexes and the nitic oxide reactivity of a mononuclear Fe(II)BOX complex with a proton responsive ligand.

\subsection{Dioxygen reactivity of mononuclear and dinuclear Fe(II)BOX systems}

The mononuclear $(\mathrm{BOX}) \mathrm{FeCl}_{2}$ complexes 17, 18, 19, 20 and 21 were treated with dioxygen in a solution of THF at $193 \mathrm{~K}$ which resulted in a gradual change of the yellow colored solutions to brown (Scheme 12.1). Monitoring the reactions via UV-vis spectroscopy displayed no major changes in the spectra of the starting material. Increasing the temperature of the reaction from $193 \mathrm{~K}$ to $233 \mathrm{~K}$ and changing the solvent to $\mathrm{MeCN}$ also resulted in no major spectral changes. In a final attempt, $\mathrm{PhIO}$ as a dioxygen source was used which too, unfortunately, did not yield any detectable iron-oxygen intermediate. Scheme 12.1 illustrates the reaction scheme pursued to form iron-oxo intermediates with $(\mathrm{BOX}) \mathrm{FeCl}_{2}$ complexes.

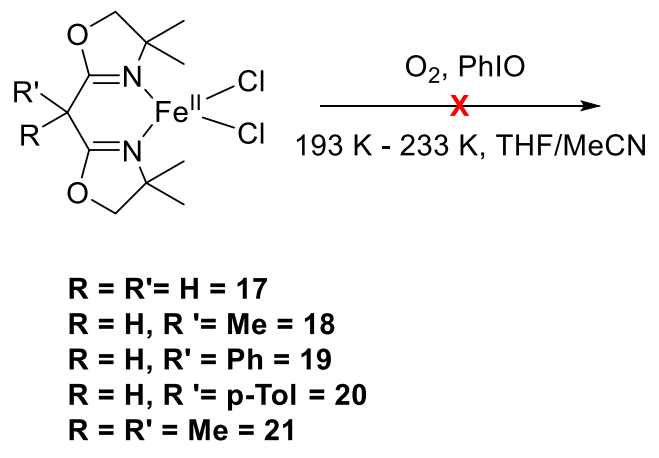

Scheme 12.1 General scheme for dioxygen activation or reaction with $\mathrm{PhIO}$ of $(\mathrm{BOX}) \mathrm{FeCl}_{2}$ complexes. 
Mössbauer spectroscopy of the crude products from the reaction of $(\mathrm{BOX}) \mathrm{FeCl}_{2}$ complexes with $\mathrm{O}_{2}$ demonstrated a clear change in the oxidation state of the metal center. Figure 12.2 illustrates the Mössbauer spectrum of the product resulting from complex 18 with $\mathrm{O}_{2}$, measured at $80 \mathrm{~K}$. The spectrum displayed a single iron species with isomer shift and quadrupole splitting values that differed from the starting material. The isomer shift value of $0.37 \mathrm{mms}^{-1}$ and a quadrupole splitting value of $1.27 \mathrm{mms}^{-1}$ are typical for a Fe(III) high spin system. ESI-MS analysis of this complex displayed peaks belonging to the ligand as well as a diiron species. However, the exact nature of the decomposed product could not be determined.

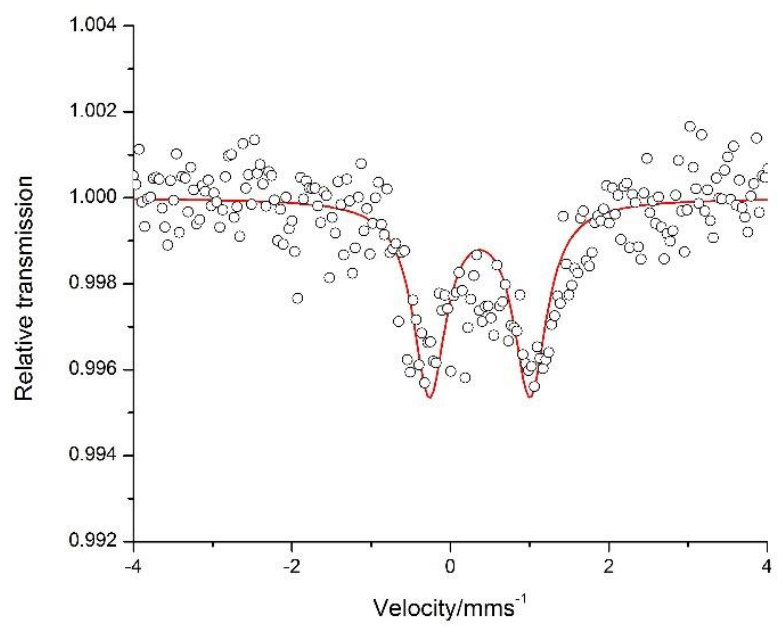

Figure 12.1: Zero field Mössbauer spectrum of $\mathbf{1 8}$ after reaction with dioxygen measured at $80 \mathrm{~K}$, depicting one iron (III) high spin species.

Though the mononuclear $(\mathrm{BOX}) \mathrm{FeCl}_{2}$ systems reacted with dioxygen, no detectable intermediates could be isolated. The same was displayed by the dinuclear (BOX)Fe(II) system (22). Reactions of 22 with PhIO in $\mathrm{MeCN}$ at $233 \mathrm{~K}$ resulted in no new bands that could be assigned to potential iron-oxo intermediates when monitored by UV-vis spectroscopy. Mössbauer spectroscopy of the sample after the reaction with PhIO displayed an iron species with isomer shift value of $0.48 \mathrm{mms}^{-1}$ and quadrupole splitting value of $0.93 \mathrm{mms}^{-}$ ${ }^{1}$, differing from those of the starting material and indicating an oxidation of the metal centers. ESI-MS analysis of the solution revealed a major peak belonging to the oxidizing agent. No peak corresponding to any iron species could be observed.

In comparison to systems wherein their Fe(II) analogues have yielded potential iron-oxo intermediates, the ligand scaffolds are far bulkier in nature with a higher denticity, ${ }^{[243,277]}$ and usually coordinate to the iron centers in an octahedral environment. Moreover, the scaffolds are designed such that a dinuclear Fe(II) complex results with the ligand system, with the $\mathrm{Fe} \cdots \mathrm{Fe}$ separation being appropriate to host a dioxygen moiety in a suitable binding mode. The tetrahedrally coordinated Fe(II) complexes of bidentate BOX ligands, despite having potential vacant sites for further coordination, do not result in the formation of detectable iron-oxo intermediates. The strong chloride donor ligands in conjunction with the simple nature of the ligand scaffold makes the formation of these intermediates a challenging task as was also observed with the dinuclear Fe(II)BOX complex. 


\subsection{Nitric oxide reactivity of a $\mathrm{Fe}(\mathrm{II}) \mathrm{BOX}$ complex with a proton responsive ligand}

\subsubsection{Preliminary NO reactivity of a dinuclear Fe(II)BOX complex}

In analogy to attempting dioxygen activation of Fe(II)BOX complexes, a second small molecule, nitricoxide, was screened to investigate potential reactivity and formation of Fe-NO intermediates. Indeed, the dinuclear Fe(II)BOX complex with bridging triflate ligands (22) demonstrated reactivity with nitric oxide. A colorless solution of $\mathbf{2 2}$ in THF was reacted with NO gas under an atmosphere of argon which resulted in an immediate color change from colorless to brown (Scheme 12.2). The reaction was monitored via UVvis spectroscopy at $193 \mathrm{~K}$ which revealed the formation of distinct bands at $334 \mathrm{~nm}\left(\varepsilon=1900 \mathrm{M}^{-1} \mathrm{~cm}^{-1}\right)$, $460 \mathrm{~nm}\left(\varepsilon=786 \mathrm{M}^{-1} \mathrm{~cm}^{-1}\right)$, and $600 \mathrm{~nm}\left(\varepsilon=373 \mathrm{M}^{-1} \mathrm{~cm}^{-1}\right)$ (Fig 12.2 left). IR analysis of the brown solution demonstrated the formation of two new stretching frequencies at $1745 \mathrm{~cm}^{-1}$ and $1823 \mathrm{~cm}^{-1}$ (Fig.12.2 right). The data obtained from UV-vis and IR spectroscopy suggested the formation of a DNIC species. ${ }^{[315]}$ However, Mössbauer spectroscopic analysis demonstrated a mixture of iron species to be present within the crude product. A comparison of the isomer shift values and quadrupole splitting values with those of the starting material indicated the reacted product to contain an iron species corresponding to the starting material, an iron-NO reacted product as well as undesired side products. ESI-MS analysis of this mixture yielded no conclusive results and X-ray characterization of any Fe-NO intermediate was challenging.

A plausible explanation for such reactivity of the dinuclear Fe(II)BOX complex with NO could be that though the labile triflate molecules allow substitution by NO, the excess of NO causes perhaps degradation of the DNIC complex formed, resulting in a mixture of products.

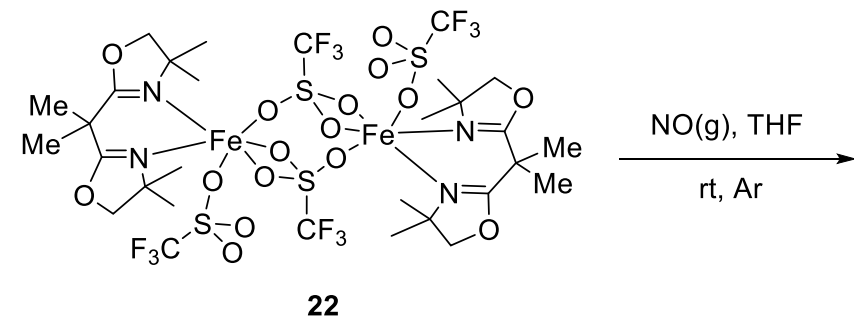

Scheme 12.2 Scheme for the reactivity of a dinuclear Fe(II)BOX complex (22) with NO at rt.
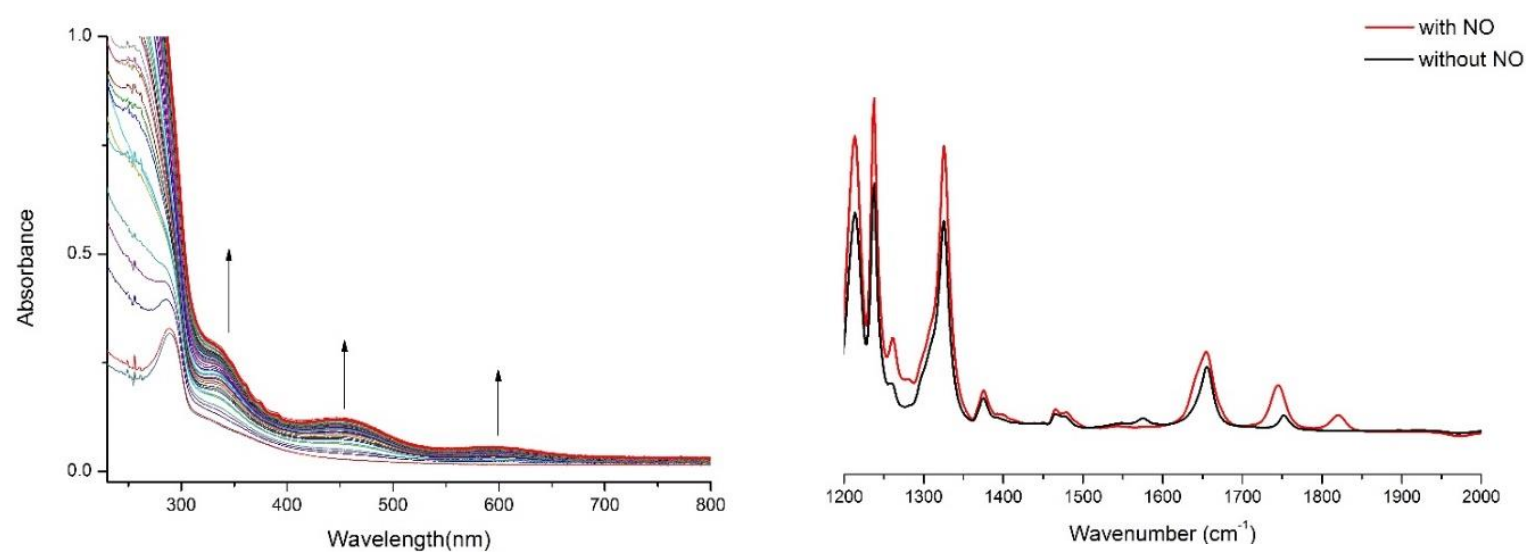

Figure 12.2 Left: UV-vis monitoring of a solution of 22 in THF at $193 \mathrm{~K}$ treated with $\mathrm{NO}(\mathrm{g})$. Shown are bands forming at $334 \mathrm{~nm}, 460 \mathrm{~nm}$ and $600 \mathrm{~nm}$. Right: IR spectra of $\mathbf{2 2}$ in THF shown in black, and a solution of 22 after NO reactivity shown in red. Stretching frequencies at $1745 \mathrm{~cm}^{-1}$ and $1823 \mathrm{~cm}^{-1}$ are tentatively assigned to a DNIC species. 
The mononuclear (BOX)FeCl 2 complexes 17-21 did not demonstrate any direct NO reactivity. This was attributed to the strong chloride ligands that have no tendency to dissociate or to undergo substitution reactions with $\mathrm{NO}$, thus resulting in no Fe-NO intermediates.

\subsubsection{NO reactivity of a mononuclear Fe(II)BOX complex with a proton responsive ligand}

In view of the results discussed above, a third route was attempted to isolate an Fe-NO intermediate. The mononuclear Fe(II)BOX complex $\mathbf{1 8}$ was treated with two equivalents of AgOTf, in order to replace the chloride ligands with more labile triflate counterions, thus facilitating substitution reactions. This solution was treated with two equivalents of $\mathrm{Ph}_{3} \mathrm{SCNO}$ instead of $\mathrm{NO}(\mathrm{g})$ in order to avoid the excess of NO. On addition of the NO source, the colorless solution gradually turned brown. The reaction mixture was stirred overnight and filtration resulted in a clean brown colored solution of a classical DNIC 23 (Scheme 12.3).

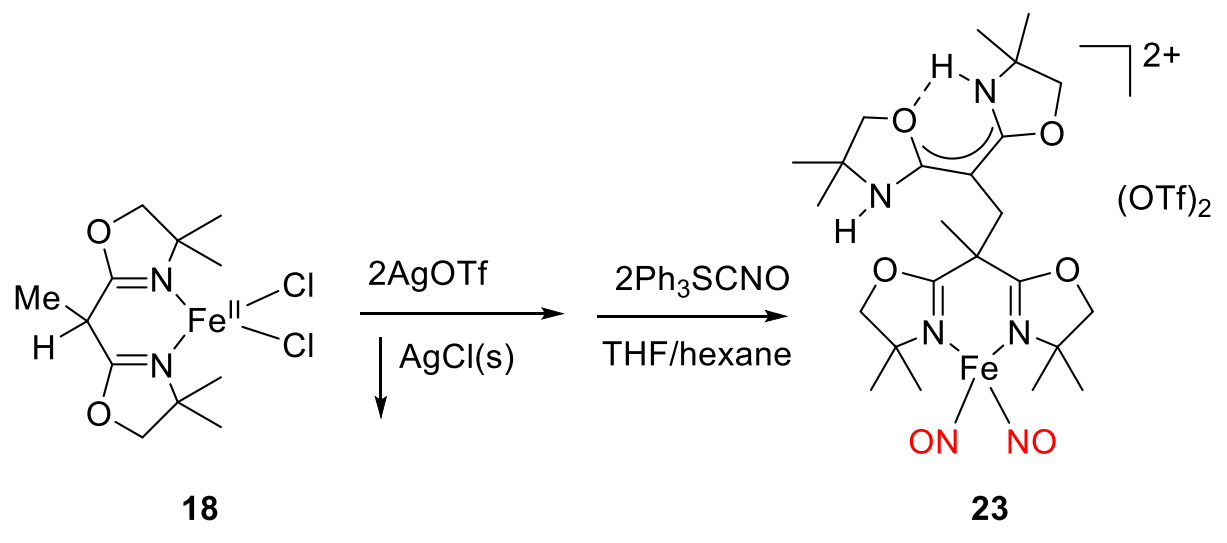

Scheme 12.3 Scheme for the generation of a DNIC (23) $\left[\mathbf{L}^{9} \mathrm{Fe}(\mathrm{NO})_{2}(\mathrm{OTf})_{2}\right]$ with a proton responsive BOX Fe(II) complex. 18, where $\mathbf{L}^{9}$ represents the modified ligand scaffold.

\subsubsection{Structural elucidation}

The first evidence of formation of a DNIC species was obtained from X-ray crystallography. Single crystals of 23 ( $\left[\mathbf{L}^{9} \mathrm{Fe}(\mathrm{NO})_{2}(\mathrm{OTf})_{2}\right]$, where $\mathbf{L}^{9}$ represents the modified ligand scaffold) suitable for X-ray diffraction were grown from a THF/hexane solution at rt (Fig. 12.3 left). The molecule crystallizes in the triclinic space group $P \overline{1}$. Structural elucidation revealed the iron center to bear a coordination number of four, in a distorted tetrahedral environment. This represents a classical DNIC and is in agreement with the $\Delta v_{\text {NO }}$ value observed by IR spectroscopy (refer to sec. 12.3.2.2). 23 was shown to be dicationic in nature with two triflate molecules. The iron center was coordinated to two NO moieties and to the nitrogen atoms of a slightly altered ligand scaffold. The simple bidentate BOX ligand $\mathbf{H L}^{2}$ was shown to undergo modification, giving rise to a scaffold that comprised the neutral ligand coupled to a second ligand moiety. This second ligand moiety which was not directly coordinated to the metal center displayed alterations within the ligand backbone, forming a $\mathrm{C}-\mathrm{C}$ bond with a neutral ligand and being twisted such that the oxygen atoms were trans to each other (Scheme 12.3). Stoichiometric analysis of complex 23 suggested a positive charge contribution from the modified ligand scaffold over the cationic portion of the complex. The geometric modification in scaffold of the second ligand moiety was in agreement with the IR spectra of the complex measured in solution, wherein the stretching frequency at $\sim 1650 \mathrm{~cm}^{-1}$ assigned to the $\mathrm{C}=\mathrm{N}$ bond was slightly altered (Fig. 12.4 left). Intramolecular hydrogen bonding between the nitrogen and a neighboring oxygen 
atom of the ligand scaffold was also observed. The two ligand moieties coupled at the backbone were oriented in a cis fashion with the NO molecules lying on the same side (Fig. 12.3 right).

The angles formed between the ligand and the metal center were acute $\sim 91.9^{\circ}$ but compensated by the metal-NO angles $\left(\sim 113^{\circ}\right)$. The Fe-NO (avg.) bond lengths of $1.69 \AA$ and N-O (avg.) bond lengths of 1.16 $\AA$ were in the range of cationic DNICs. ${ }^{[316,337]}$ The Fe-N bond lengths of 2.07-2.08 $\AA$ were similar to those of the mononuclear and dinuclear Fe(II)BOX complexes described previously (refer to Chapter 11). Though 23 represents a classical DNIC, the mechanism of its formation remains obscure.
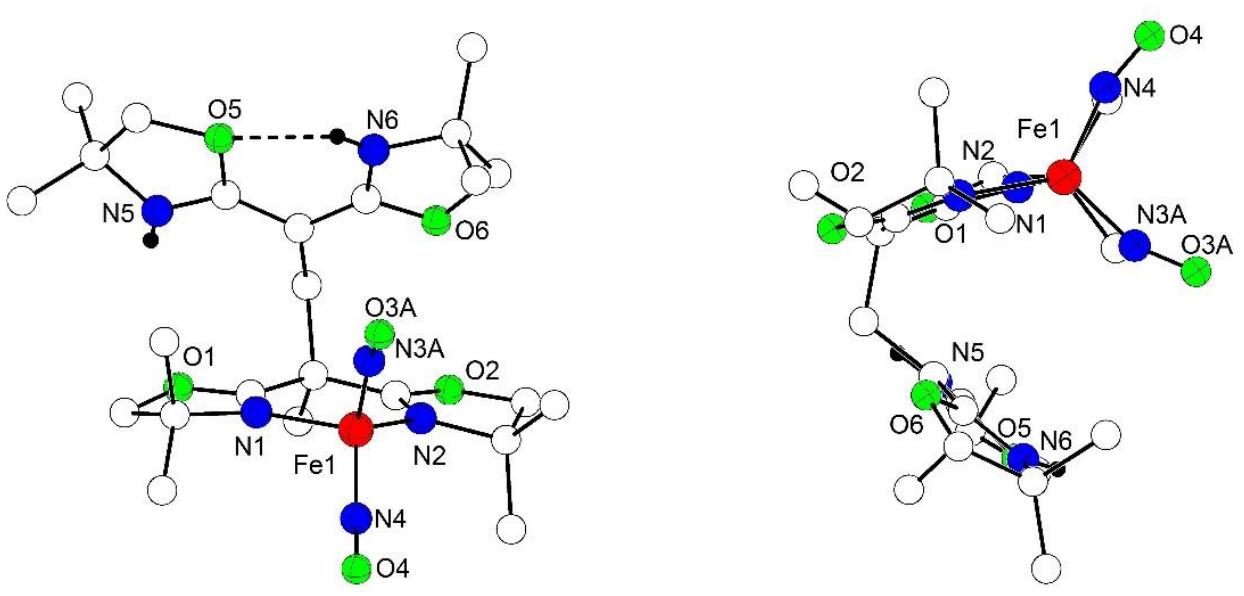

Figure 12.3: Left: Molecular structure of the cationic part of 23. Only hydrogen atoms of the protonated nitrogens are shown. Others are omitted for clarity. Right: Molecular structure of the cationic part of $\mathbf{2 3}$ depicting the two ligand moieties to be oriented in a cis fashion with the NO molecules lying on the same side. Selected bond lengths $[\AA]$ for 23: Fe1-N4 1.6905(19), Fe1-N3A 1.692(4), Fe1-N3B 1.716(15), Fe1-N1 2.0001(14), Fe1-N2 2.0078(16).

\subsubsection{Properties in solution: IR and UV-vis Spectroscopy}

Addition of the NO source to a colorless solution of $\mathbf{1 8}$ after treatment with AgOTf resulted in a gradual color change to brown, forming a classical DNIC species (23). The DNIC was characterized via IR spectroscopy in solution. Two new stretching frequencies at $1752 \mathrm{~cm}^{-1}$ and $1827 \mathrm{~cm}^{-1}$ were observed that were absent in the starting material (Fig. 12.4 left). These features are typical for N-O stretching frequencies of dinitrosyl iron complexes and are comparable to the cationic DNIC isolated with a Sparteine ligand system reported by Liaw et al.. ${ }^{[316]}$ Moreover, the $\Delta v_{\text {No }}$ of 23, defined as the separation of two NO stretching frequencies exhibited by DNICs, was measured at $\sim 70 \mathrm{~cm}^{-1}$ indicating the DNIC to be of classical nature. ${ }^{[315]}$ This was in agreement with the results obtained from X-ray crystallography. A second interesting feature that was displayed in the IR spectrum of 23 was the band at $\sim 1650 \mathrm{~cm}^{-1}$. This was assigned to the $\mathrm{C}=\mathrm{N}$ stretching frequency originating from the ligand system. However, this feature seemed slightly altered in comparison to that of the starting material, indicating a change in bonding within the ligand system. This was further highlighted on structurally elucidating the complex which displayed interesting ligand changes when bonded to the metal as is discussed in section 12.3.2.1. 
The UV-vis spectrum of a solution of 23 in THF at rt displayed two shoulders at $427 \mathrm{~nm}\left(\varepsilon=1820 \mathrm{M}^{-1} \mathrm{~cm}^{-}\right.$ $\left.{ }^{1}\right)$ and $579 \mathrm{~nm}\left(\varepsilon=432 \mathrm{M}^{-1} \mathrm{~cm}^{-1}\right)$ (Fig. 12.4 right). In comparison to the absorption features exhibited by FDPs and other synthetically characterized DNICs, these transitions are assigned to iron-nitrosyl LMCT and d-d transitions. ${ }^{[36,293,337]}$ In the case of DNICs, the unambiguous determination of the oxidation state of the iron centers remains a matter of speculation. This stems from the fact that the NO molecule itself readily switches between different redox levels of $\mathrm{NO}^{+}, \mathrm{NO}^{-}$, and $\mathrm{NO}^{-}$, with the exact nature of the $\mathrm{NO}$ binding moiety being ambiguous. ${ }^{[338,339]}$ Hence, distinctive spectroscopic features for DNICs in terms of the nature of ligand and metal orbitals remains a challenging task. ESI-MS of a solution of 23 revealed peaks belonging only to the ligand scaffold.
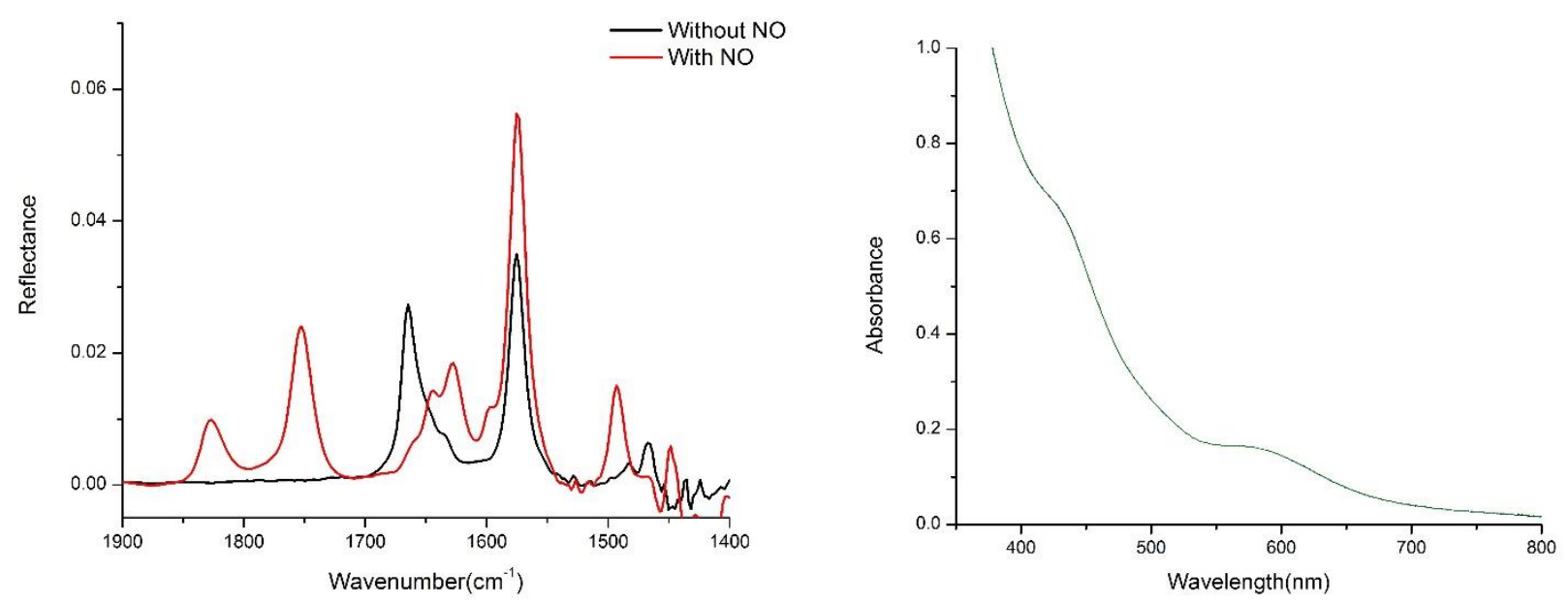

Figure 12.4 Left: IR spectrum of 23 in THF shown in red with NO stretching frequencies at $1752 \mathrm{~cm}^{-1}$ and $1827 \mathrm{~cm}^{-1}$ assigned to a DNIC species, and the spectra of the starting material shown in black. Right: UV-vis spectra of a solution of 23 in THF at rt displaying shoulder like regions at $427 \mathrm{~nm}$ and $579 \mathrm{~nm}$.

\subsubsection{Mössbauer spectroscopy}

Zero field Mössbauer spectra of crystalline material of $\mathbf{2 3}$ measured at $80 \mathrm{~K}$ revealed a single iron species to be present as the best fit with a single quadrapole doublet was obtained (Fig. 12.5). The isomer shift values of $0.33 \mathrm{mms}^{-1}$ and quadrupole splitting values of $1.43 \mathrm{mms}^{-1}$ point towards an iron species present in either a +3 oxidation state with an $S=5 / 2$ spin state or a +1 oxidation state with an $S=3 / 2$ spin state. As mentioned earlier, the metal oxidation state in $\mathrm{Fe}(\mathrm{NO})_{\mathrm{x}}$ moieties is not well defined. Since structural elucidation of the DNIC (23) revealed it to be dicationic in nature, with one cationic charge on the second ligand, and in comparison with Mössbauer paramteres of other DNICs, a tentative +1 oxidation state to the iron center was assigned. This would mean that both the NO molecules bind as neutral NO; resulting in a $\left[\mathrm{Fe}(\mathrm{NO})_{2}\right]^{9}$ system according to the Enemark-Feltham notation. The full width at half maximum obtained from the Lorentzian profile of the Mössbauer spectra was measured at $0.68 \mathrm{mms}^{-1}$.

The coupling of the ligand backbone displayed by $\mathbf{2 3}$ was investigated by replacing AgOTf with NaOTf, in order to eliminate possible oxidation. However, this yielded no valuable insights. As BOX ligands are known to display redox non-innocent behavior, ${ }^{[225]}$ one could speculate that perhaps the AgOTf oxidizes 
the ligand, which leads to $\mathrm{C}-\mathrm{C}$ coupling resulting in the formation of a modified ligand scaffold. As mentioned earlier, the exact mechanism for the formation of $\mathbf{2 3}$ still remains ambiguous.

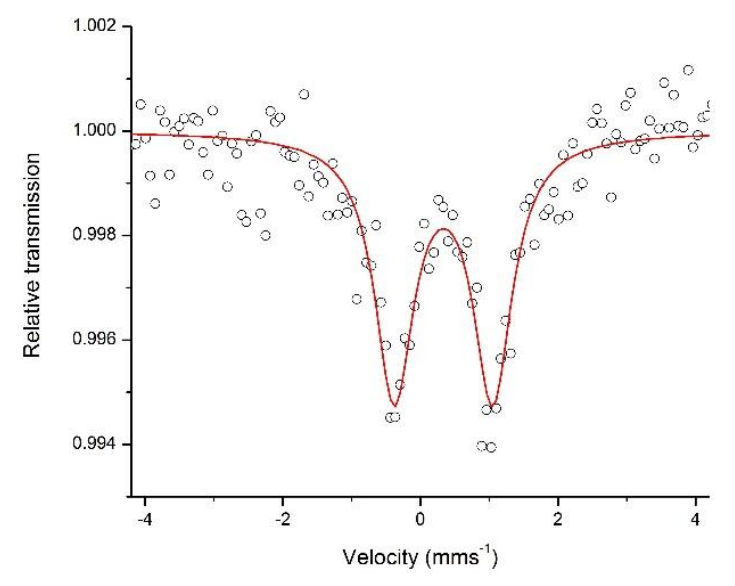

Figure 12.5: Zero field Mössbauer spectrum of 23 measured at $80 \mathrm{~K}$, showing a single quadrapole doublet with isomer shift $(\delta)=0.33 \mathrm{mms}^{-1}$ and quadrapole splitting $\left(\left|\Delta \mathrm{E}_{\mathrm{q}}\right|\right)=1.43 \mathrm{mms}^{-1}$.

\subsection{Conclusion}

Fe(II)BOX complexes have long been known to mediate asymmetric catalysis, with small molecule activation of such systems being unexplored. Through the course of this work, the reactivity of (BOX)Fe(II) complexes with dioxygen and nitric oxide was investigated. Though no detectable iron-oxo intermediates could be isolated with these complexes and dioxygen, unlike their $\mathrm{Cu}(\mathrm{I})$ analogues, the (BOX)Fe(II) complexes demonstrated promising reactivity with nitric oxide.

The Fe(II) complex of a proton responsive ligand $\mathbf{H L}^{2}$ yielded a Fe-NO complex, though the elucidation of its mechanism of formation remained challenging. The BOX ligand proved to be a suitable scaffold for generating a DNIC, which was characterized in solution and via X-ray crystallography. The stretching frequencies observed by IR spectroscopy were characteristic of classical DNICs which was in agremment to results obtained by X-ray crystallography. Structural elucidation of $\mathbf{2 3}$ exhibited a dinitrosyl iron species, ligated to a modified ligand scaffold present in a tetrahedrally coordinated environment, with measured bond lengths being in the realm of cationic DNICs. The Mössbauer parameters were suggestive of the iron existing in a +1 oxidation state, resulting in a $\left[\mathrm{Fe}(\mathrm{NO})_{2}\right]^{9}$ system according to the Enemark-Feltham notation. Through this work it was possible to successfully isolate and structurally elucidate a DNIC complex with a simple bidentate BOX ligand. This puts forward opportunities to investigate Fe-NO chemistry of structurally and electronically modified BOX ligand systems. 
[Note:This page has intentionally been left balnk] 


\section{Chapter 13 Conclusion}


In conclusion, a series of mononuclear Fe(II)BOX complexes were synthesized with proton and non-proton responsive ligand systems. These were characterized via IR, UV-vis, ESI-MS and Mössbauer spectroscopy. All of these complexes were structurally elucidated, which revealed the iron center to be coordinated in a distorted tetrahedral environment. These results were in corroboration with the parameters obtained from Mössbauer spectroscopy which revealed the iron species to be present in a +2 high spin oxidation state. Additionally, a dinuclear Fe(II)BOX complex with a non-proton responsive ligand was synthesized and structurally characterized. Mössbauer spectroscopy described the iron centers to be present in an identical environment which was finally confirmed by X-ray analysis of the complex. Structural elucidation revealed the iron centers to be coordinated in an octahedral environment with bridging triflate ligands. Magnetic measurements were suggestive of negligible coupling between the two metal centers, but revealed substantial ZFS.

Dioxygen reactivity of both the mononuclear and dinuclear (BOX)Fe(II) systems proved challenging in isolating any detectable iron-oxo intermediates. This was attributed to the simple nature of the ligand scaffold in conjunction with the strong chloride donor ligands attached to the metal center. Nitric oxide reactivity of a $\mathrm{Fe}(\mathrm{II}) \mathrm{BOX}$ system with a proton responsive ligand successfully generated a DNIC. The complex was characterized in solution by IR and UV-vis spectroscopy. The IR stretching frequencies observed were characteristic of DNICs. Analysis by Mössbaeur spectroscopy suggested the iron to host a possible +1 oxidation state which resulted in a $\left[\mathrm{Fe}(\mathrm{NO})_{2}\right]^{9}$ system according to the Enemark-Feltham notation. Through the course of this work, it was shown that bidentate BOX ligands form suitable scaffolds for DNICs. This gives an opening for further investigations of such systems in terms of modifications in the ligand skeleton or its electronic environment. 


\section{Chapter 14 \\ Outlook}


Through the course of this work, we have successfully been able to demonstrate small molecule activation by copper and iron complexes with structurally and electronically modified bis(oxazoline) ligand systems. A series of ligands comprising neutral and anionic BOX ligands were synthesized with their corresponding $\mathrm{Cu}(\mathrm{I})$ and $\mathrm{Fe}(\mathrm{II})$ complexes. Their reactivity towards dioxygen and nitric oxide were screened in order to investigate reaction pathways and the formation of potential intermediates.

$\mathrm{The} \mathrm{Cu}(\mathrm{I})$ complexes of $\mathrm{BOX}$ ligands yielded key $\mathrm{Cu}_{2} \mathrm{O}_{2}$ intermediates with dioxygen at low temperatures. The nature of the ligand scaffold was crucial in determining the dioxygen binding mode and subsequent reactivity of the intermediate. While neutral BOX ligands preferentially formed their ${ }^{\mathbf{S}} \mathbf{P}$ complexes, a dioxygen binding mode similar to those of Type 3 copper proteins, the anionic ligands preferred $\mathbf{O}$ formation. Moreover, it was possible to tune the equilibrium between the ${ }^{\mathbf{S}} \mathbf{P} / \mathbf{O}$ isomers with " $\mathrm{pH}$ ", a new factor that was previously unknown. The $\mathrm{Cu}(\mathrm{I})$ complexes of proton responsive $\left(\mathbf{H L}^{\mathbf{1}}, \mathbf{H L}^{\mathbf{5}}\right)$ and non-proton responsive ligands $\left(\mathbf{L}^{6}\right)$ were structurally elucidated, besides characterization in solution. A ${ }^{\mathbf{S}} \mathbf{P}$ complex of a non-proton responsive ligand (8) as well as a proton responsive ligand (6) was isolated and characterized by X-ray crystallography. Both the systems demonstrated a rather long $\mathrm{O}-\mathrm{O}$ bond in comparison to other reported systems, with complex 6 having the longest $\mathrm{O}-\mathrm{O}$ bond reported to date, giving it its noteworthy character. These two new structures add to the handful of ${ }^{\mathbf{S}} \mathbf{P}$ complexes that have been structurally elucidated over the last three decades.

The $\mathrm{Cu}(\mathrm{I})$ complexes of anionic BOX ligands and their corresponding $\mathbf{O}$ complexes were characterized in solution. A neutral $\mathbf{O}$ complex $\mathbf{1 0}$ was successfully characterized by X-ray crystallography, making it the second of its kind. Though this binding mode has not yet been biologically observed, recent studies have proposed the active intermediate of pMMO to bind in a similar fashion. While the $\mathbf{O}$ systems of anionic BOX ligands demonstrated unphysiological reactivity with basic phenolic substrates, the ${ }^{\mathbf{S}} \mathbf{P}$ systems of proton responsive ligands isomerized to their $\mathbf{O}$ congeners. Activity similar to that of Tyrosinase was emulated by a ${ }^{\mathbf{S}} \mathbf{P}$ system $\mathbf{8}$ hosting a non-proton responsive ligand scaffold.

Contrastingly, isolation of any detectable iron-oxo intermediates with (BOX)Fe( $\mathrm{II}) \mathrm{Cl}_{2}$ complexes and dioxygen was challenging. Reactivity of these systems with nitric oxide successfully generated a DNIC. A series of mononuclear $(\mathrm{BOX}) \mathrm{FeCl}_{2}$ complexes were structurally elucidated in addition to characterization in solution. Furthermore, a dinuclear (BOX)Fe(II) complex was also synthesized and structurally characterized. These now add to the list of Fe(II) complexes belonging to this ligand class, of which only two had previously been characterized by X-ray crystallography. The nitric oxide reactivity of a Fe(II) complex with a proton responsive ligand generated a DNIC which was characterized in solution and structurally elucidated.

In view of the results obtained from copper dioxygen reactivity with BOX systems, the ability to peripherally deprotonate the ligand systems resulting in isomerization between the ${ }^{\mathbf{S}} \mathbf{P} / \mathbf{O}$ congeners, and in conjunction with the isomerization of ${ }^{\mathbf{S}} \mathbf{P}$ systems of proton responsive ligands with substrates puts forward an interesting notion. As the analogous biological ligand scaffolds with nitrogen donors are susceptible to (de)protonation events, one could speculate whether a local $\mathrm{pH}$ change consequentially affects the active intermediate in Type 3 copper proteins responsible for catalytic activity. Given the fact that redox potentials and electronic structures of metallocofactors have been tuned by deprotonation of histidine imidazole ligands in various metalloproteins, which forms a fundamental part of PECT reactions, similar reactivity with proton-responsive ligands and $\mathrm{Cu}_{\mathrm{x}} / \mathrm{O}_{2}$ intermediates would form an interesting study. Moreover, the ability of BOX ligands to stabilize DNICs introduces opportunities to investigate these systems with structural and electronic modifications of the ligand scaffold. 
In conclusion, the ability of BOX ligands to isolate intermediates analogous to those observed in nature highlights their nobility in simplicity. Termed as a privileged class of ligands that have been used extensively in asymmetric catalysis, through the course of this work, have proved to be a privileged class in the field of bio-inorganic chemistry as well. 
[Note: This page has intentionally been left blank] 


\section{Chapter 15 \\ Experimental Section}


Overview

\subsection{General Remarks}

\subsection{Ligand Synthesis}

15.2.1 Syntheses of $\mathbf{H L}^{\mathbf{1}}, \mathbf{H L}^{2}, \mathbf{H L}^{\mathbf{3}}, \mathbf{H L}^{\mathbf{5}}$ and $\mathbf{L}^{\mathbf{6}}$

15.2.2 Synthesis of monoanionic BOX ligands $\left[\mathbf{L}^{2}\right]^{-}$and $\left[\mathbf{L}^{3}\right]^{-}$

\subsection{Experimental Section: Chapter 5}

15.3.1 Synthesis of $\mathrm{Cu}(\mathrm{I}) \mathrm{BOX}$ complexes

15.3.1.1 Synthesis of $\left[\mathrm{HL}^{1} \mathrm{Cu}(\mathrm{I}) \mathrm{MeCN} \mathrm{PF}_{6}(\mathbf{1})\right.$

15.3.1.2 Synthesis of $\left[\mathrm{HL}^{2} \mathrm{Cu}(\mathrm{I}) \mathrm{MeCN}^{2} \mathrm{ClO}_{4}(\mathbf{2})\right.$

15.3.1.3 Synthesis of $\left[\mathrm{HL}^{5} \mathrm{Cu}(\mathrm{I}) \mathrm{MeCN} \mathrm{PF}_{6}(\mathbf{3})\right.$

15.3.1.4 Synthesis of $\left[\mathrm{L}^{6} \mathrm{Cu}(\mathrm{I}) \mathrm{MeCN}^{-} \mathrm{ClO}_{4}(4)\right.$

15.3.2 Synthesis of ${ }^{\mathrm{S}} \mathbf{P}$ complexes $\mathbf{6}$ and $\mathbf{8}$

15.3.2.1 Synthesis of $\left[\left(\mathrm{HL}^{2}\right)_{2} \mathrm{Cu}_{2}\left(\mu-\eta^{2}: \eta^{2}-\mathrm{O}_{2}\right)\right]\left(\mathrm{ClO}_{4}\right)_{2}(\mathbf{6})$

15.3.2.2 Synthesis of $\left[\left(\mathrm{L}^{6}\right)_{2} \mathrm{Cu}_{2}\left(\mu-\eta^{2}: \eta^{2}-\mathrm{O}_{2}\right)\right]\left(\mathrm{ClO}_{4}\right)_{2}(\mathbf{8})$

15.3.3 UV-vis solutions

15.3.4 Raman measurements of $\mathbf{8}$

15.4 Experimental Section: Chapter 6

15.4.1 Synthesis of $\mathrm{Cu}(\mathrm{I}) \mathrm{BOX}$ complexes

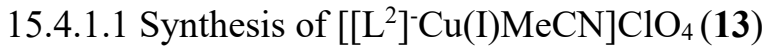

15.4.1.2 Synthesis of $\left[\left[\mathrm{L}^{3}\right]^{-\mathrm{Cu}}(\mathrm{I}) \mathrm{MeCN} \mathrm{ClO}_{4}(\mathbf{1 4})\right.$

15.4.1.3 Synthesis of $\left[\left[\left(\mathrm{L}^{2}\right)\right]_{2}{ }_{2} \mathrm{Cu}_{2}(\mu-\mathrm{O})_{2}\right](\mathbf{1 0})$

15.4.1.4 Synthesis of $\left[\left[\left(\mathrm{L}^{3}\right)\right]_{2}{ }_{2} \mathrm{Cu}_{2}(\mu-\mathrm{O})_{2}\right](\mathbf{1 1})$

15.4.2 UV-vis solutions

15.4.3 Raman measurements of $\mathbf{1 0}$ and $\mathbf{1 1}$

15.4.4 UV-vis temperature dependence measurements

\subsection{Experimental Section: Chapter 7}

15.5.1 Titration experiments of ${ }^{\mathrm{S}} \mathbf{P}$ complexes 5, 6, 7 and ${ }^{\mathrm{S}} \mathbf{P H L}{ }^{3}$ with DBU

15.5.2 POP Switch experimets

15.5.3 Oxygentation of $2: 1 \mathrm{HL}^{1}: \mathrm{Cu}(\mathrm{I})$

15.5.4 Resosnance raman measurements $2: 1 \mathrm{HL}^{1}: \mathrm{Cu}(\mathrm{I})$

\subsection{Experimental Section: Chapter 8}

15.6.1 Substrate reactivity of DTBP/DTBP-H:NEt 3 with ${ }^{\mathrm{S}} \mathbf{P}$ complexes of proton responsive ligands 15.6.2 Substrate reactivity of DTBP/DTBP-H:NEt ${ }_{3}$ with ${ }^{\mathbf{S}} \mathbf{P}$ complex $\mathbf{8}$ of non-proton responsive ligand 15.6.3 Substrate reactivity of DTBP/DTBP-H:NEt 3 with $\mathbf{O}$ complexes of monoanionic ligands

\subsection{Experimental Section: Chapeter 11}

15.7.1 Synthesis of Fe(II)BOX Complexes

15.7.1.1 Synthesis of $\mathrm{HL}^{1} \mathrm{Fe}(\mathrm{II}) \mathrm{Cl}_{2}$ (17)

15.7.1.2 Synthesis of $\mathrm{HL}^{2} \mathrm{Fe}(\mathrm{II}) \mathrm{Cl}_{2}$ (18)

15.7.1.3 Synthesis of $\mathrm{HL}^{3} \mathrm{Fe}(\mathrm{II}) \mathrm{Cl}_{2}$ (19)

15.7.1.3 Synthesis of $\mathrm{HL}^{5} \mathrm{Fe}(\mathrm{II}) \mathrm{Cl}_{2}$ (20)

15.7.1.3 Synthesis of $\mathrm{L}^{6} \mathrm{Fe}(\mathrm{II}) \mathrm{Cl}_{2}$ (21)

15.7.1.3 Synthesis of $\left[\mathrm{L}^{6} \mathrm{Fe}(\mathrm{II})(\mathrm{OTf})_{2}\right]_{2}$ (22)

\subsection{Experimental Section: Chapter 12}

15.8.1 Synthesis of $\left[\mathrm{L}^{9} \mathrm{Fe}(\mathrm{NO})_{2}\left(\mathrm{CF}_{3} \mathrm{SO}_{3}\right)_{2}\right](\mathbf{2 3})$ 


\section{Materials and Methods}

\subsection{General Remarks}

The syntheses of air or moisture sensitive ligands, building blocks or complexes were carried out using standard Schlenk technique or in a glovebox. All glassware was dried at $120{ }^{\circ} \mathrm{C}$ overnight prior to use. Solvents were purified and dried according to established procedures. They were distilled prior to use and degassed by bubbling with argon. Deuterated solvents were dried and distilled the same way as the undeuterated analogues. $\left[\mathrm{Cu}(\mathrm{MeCN})_{4}\right]\left(\mathrm{ClO}_{4}\right)$ was synthesized from commercially available copper(II) perchlorate hexahydrate and copper metal. ${ }^{[135]}$ 2,6- Lutidinium triflate was synthesized from 2,6-lutidine in pentane via addition of neat triflic acid. ${ }^{[340]}$ DBU was distilled prior to use and stored over 4A molecular sieves in a glovebox. All other chemicals were purchased and used without further purification.

${ }^{1} \mathrm{H}$ and ${ }^{13} \mathrm{C}\{1 \mathrm{H}\}$ NMR spectra were recorded on a Bruker Avance $300 \mathrm{MHz}$ spectrometer. Low Temperature ${ }^{1} \mathrm{H}$ NMR, VT- ${ }^{1} \mathrm{H}-\mathrm{NMR}$ and DOSY were recorded on a Bruker Avance $500 \mathrm{MHz}$ spectrometer. Chemical shifts are reported in ppm relative to residual proton signals of the solvents. Low resolution ESI mass spectra were recorded on a Bruker (HCT Ultra) spectrometer coupled to a glovebox. Microanalysis were performed by the "Analytic Laboratory of the Institute for Inorganic Chemistry at the University of Göttingen", using a Vario EL III.

\section{IR Spectroscopy}

IR spectra were either recorded using a Cary 630 FTIR spectrometer (Agilent) placed in a glovebox (MBRAUN UNIlab, argon atmosphere) with a DialPath and Diamond ATR accessory, or with a Bruker VERTEX 70 using KBr pellets.

\section{Resonance Raman Spectroscopy}

Raman spectra under resonant and non-resonant conditions were recorded using a HORIBA Scientific LabRAM HR 800 (400-1100 nm) spectrometer with an open-electrode CCD detector and a confocal pinhole with user controlled variable aperture in combination with a free space optical microscope. For excitation either a He: $\mathrm{Ne}(633 \mathrm{~nm})$ or a diode laser $(457 \mathrm{~nm})$ was used, as indicated for each experiment. ${ }^{18} \mathrm{O}_{2}$ labelled samples were prepared according to procedures described in the main text. Resonance Raman spectra were recorded in suitable solvents, according to description in the main text, and prepared by direct addition of ${ }^{16} \mathrm{O}_{2}$ or ${ }^{18} \mathrm{O}_{2}$, respectively, to solutions of the complexes in a Young NMR tube. Low temperature frozen solution spectra were recorded placing the tube in a liquid nitrogen bath using a transparent cold finger dewar. Solution samples were measured at Low temperature in an acetone-dry ice bath prepared in the finger dewar.

\section{Magnetic measurements}

Temperature-dependent magnetic susceptibilities were measured using a Quantum-Design MPMS XL-5 SQUID magnetometer equipped with a $5 \mathrm{~T}$ magnet within the temperature range of $300 \mathrm{~K}$ to $2 \mathrm{~K}$. The polycrystalline sample was contained in a gelatin capsule, fixed with polyfluorinated oil if necessary, and placed in a non-magnetic sample holder. Each raw data file for the magnetic moment was corrected for the 
diamagnetic contribution of the gelatin capsule according to $M^{\text {dia }}($ capsule $)=\chi_{g} \times m \times H$, with an experimentally obtained gram susceptibility of the gelatin capsule. The molar susceptibility data were corrected for the diamagnetic contribution using the Pascal constants and the increment method according to Haberditzl ${ }^{[341,342]}$ Simulation of the experimental data with full matrix diagonalization of exchange coupling was performed with the julX program. Temperature independent paramagnetism $(T I P)$ and paramagnetic impurity $(P I)$ were included according to

$$
\chi_{\text {calcd }}=\chi \cdot(1-P I)+\chi_{\text {mono }} \cdot P I+T I P
$$

The coupling constant $J_{\mathrm{AB}}$ was calculated from the Heisenberg-Dirac-vanVleck (HDvV) Hamiltonian

$$
H_{\mathrm{HDvV}}=-2 J_{\mathrm{AB}} \cdot S_{A} \cdot S_{B}
$$

\section{UV-vis Spectroscopy}

UV-vis spectra at ambient temperature were measured with a Cary 5000 Bio spectrophotometer using quartz cuvettes sealed by a rubber septum. Solid state spectra were recorded using the Cary Bio spectrophotometer but with a Praying Mantis ${ }^{\mathrm{TM}}$ diffuse reflection attachment equipped with a sample chamber with quartz windows (Harrick Scientific Products). Low temperature UV-vis spectra were recorded with an Agilent Cary 60 equipped with an Unisoku Cryostat (CoolSpek) and magnetic stirrer using quartz cuvettes attached with a screw cap and a septum.

\section{X-Ray crystallography}

X-ray data were collected on a STOE IPDS II diffractometer with an area detector (graphite monochromated MO-K radiation, $\lambda=0.71073 \AA$ ) by use of $\omega$ scans at $133 \mathrm{~K}$. The structures were solved using direct methods and refined against $F^{2}$ using all reflections with SHELX-2013. Non-hydrogen atoms were refined anisotropically, unless stated otherwise. Hydrogen atoms were placed in calculated positions and assigned to an isotropic displacement parameter of 1.2/1.5 Ueq(C) or 1.5Ueq. Face-indexed absorption corrections were performed by the program X-RED (STOE \& CIE GmbH, Darmstadt, Germany,2002). ${ }^{[343]}$ For further details on crystal data and refinement see Appendix.

\subsection{Ligand Synthesis}

15.2.1 BOX ligands $\mathbf{H L}^{1}, \mathbf{H L}^{2}, \mathbf{H L}^{3}, \mathbf{H L}^{5}$ and $\mathbf{L}^{6}$ were prepared in a three step synthesis route according to literature procedures. ${ }^{[344]}$

\section{Step 1. Synthesis of $\mathbf{X}$}

The respective diethyl malonate $(50-70 \mathrm{mmol})$ with 2.00 equivalents of aminoalcohol and 0.02 equivalents of sodium hydride ( $60 \%$ dispersion in mineral oil) were stirred together at room temperature under argon. The mixtures were heated in a round bottom Schlenk flask at $135-140{ }^{\circ} \mathrm{C}$ for $3-4$ hours. After cooling the mixtures back to room temperature, the excess pressure was first slowly released. The yellow viscous oils were put under vaccum for approximately 2 hours in order to remove ethanol. The respective malonamides were used in the next step without further purification.

Step 2. Synthesis of $\mathbf{Y}$ 
To the respective bishydroxy malonamides, 5.00 equivalents of $\mathrm{NEt}_{3}$ and $\sim 400 \mathrm{ml} \mathrm{CH}_{2} \mathrm{Cl}_{2}$ were gradually added. The solutions were stirred for $15 \mathrm{mins}$ at room temperature. To these solutions under ice-cold conditions, 2.5 equivalents of $\mathrm{MsCl}$ were added. The reaction mixtures were warmed to room temperature and stirred for 2 hours. The mixtures were then washed with a saturated solution of $\mathrm{NH}_{4} \mathrm{Cl}(3 \times 500 \mathrm{ml})$. and the solvent was removed under vaccum to yield orange/yellow oils.

Step 3. Ring closure of the Bis(oxazoline)s

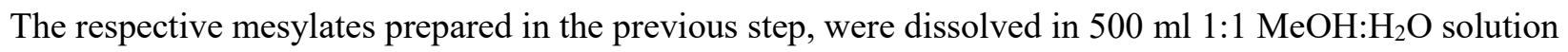
with 5.00 equivalents of $\mathrm{NaOH}$. The mixtures were refluxed for 2.5 hours. The solutions were cooled and concentrated under vacuum. The aqueous phase was extracted with $\mathrm{CH}_{2} \mathrm{Cl}_{2}(3 \times 300 \mathrm{ml})$, and the combined organic phase was dried over $\mathrm{Na}_{2} \mathrm{SO}_{4}$. The solvent was removed to yield yellow colored oils $\left(\mathrm{HL}^{1}, \mathrm{HL}^{2}\right.$ and $\left.\mathrm{L}^{6}\right)$ that were purified with Kugelrohr distillation $\left(\sim 90{ }^{\circ} \mathrm{C}, 1 \times 10^{-2}\right.$ bar $)$

\section{Analysis of ligands}

HL $^{1}$ : yield: $6.8 \mathrm{~g}, 32.2 \mathrm{mmol}, 52 \%$.

${ }^{1} \mathrm{H}-\mathrm{NMR}\left(300 \mathrm{MHz}, \mathrm{CDCl}_{3}\right): \delta(\mathrm{ppm})=3.90\left(\mathrm{~s}, 4 \mathrm{H}, 2 \mathrm{CH}_{2}\right), 3.24\left(\mathrm{~s}, 2 \mathrm{H}\right.$, bridging $\left.\mathrm{CH}_{2}\right), 1.21(\mathrm{~s}, 12 \mathrm{H}, 4 \mathrm{Me})$ ${ }^{13} \mathrm{C}\left\{{ }^{1} \mathrm{H}\right\}-\mathrm{NMR}\left(75 \mathrm{MHz}, \mathrm{CDCl}_{3}\right): \delta(\mathrm{ppm})=160.1(2 \mathrm{C}=\mathrm{N}), 79.7\left(2\right.$ oxazoline- $\left.\mathrm{CH}_{2}\right), 67.5\left(2 \mathrm{CMe}_{2}\right), 28,8$ (bridging- $\left.\mathrm{CH}_{2}\right), 28.3(4 \mathrm{Me})$

MS (ESI(+)), MeCN: $m / z=211\left([\mathrm{M}+\mathrm{H}]^{+}\right), 233\left([\mathrm{M}+\mathrm{Na}]^{+}\right)$

HL $^{2}$ : yield: $5 \mathrm{~g}, 22.3 \mathrm{mmol}, 77 \%$.

${ }^{1} \mathrm{H}-\mathrm{NMR}\left(300 \mathrm{MHz}, \mathrm{CDCl}_{3}\right): \delta(\mathrm{ppm})=3.88\left(\mathrm{~s}, 4 \mathrm{H}, \mathrm{CH}_{2}\right), 3.43(\mathrm{q}, J=7.2 \mathrm{~Hz}, 1 \mathrm{H}, \mathrm{CHMe}), 1.42(\mathrm{~d}, J=7.2$ $\mathrm{Hz}, 3 \mathrm{H}, \mathrm{CHMe}), 1.21(\mathrm{~s}, 12 \mathrm{H}, 4 M e)$

${ }^{13} \mathrm{C}\left\{{ }^{1} \mathrm{H}\right\}$-NMR $\left(75 \mathrm{MHz}, \mathrm{CDCl}_{3}\right): \delta(\mathrm{ppm})=164.4(2 \mathrm{C}=\mathrm{N}), 79.5\left(2 \mathrm{CH}_{2}\right), 67.2\left(2 \mathrm{CMe}_{2}\right), 34.1(\mathrm{CHMe})$, $28.25(2 \mathrm{Me}), 28.20(2 \mathrm{Me}), 15.31(\mathrm{CHMe})$

MS (ESI(+)), MeCN: $m / z=225\left([\mathrm{M}+\mathrm{H}]^{+}\right)$

HL $^{3}$ : yield: $7.2 \mathrm{~g}, 25.1 \mathrm{mmol}, 42 \%$.

${ }^{1} \mathrm{H}-\mathrm{NMR}\left(300 \mathrm{MHz}, \mathrm{CDCl}_{3}\right): \delta(\mathrm{ppm})=7.40-7.24(\mathrm{~m}, 5 \mathrm{H}, \mathrm{Ph}), 4.69(\mathrm{~s}, 1 \mathrm{H}$, bridging $\mathrm{CH}), 3.95(\mathrm{~d}, J=8.1$ $\mathrm{Hz}, 2 \mathrm{H}, \mathrm{CHH}), 3.90(\mathrm{~d}, J=8.1 \mathrm{~Hz}, 2 \mathrm{H}, \mathrm{CH} H), 1.25(\mathrm{~s}, 6 \mathrm{H}, 2 \mathrm{CMeMe}), 1.22(\mathrm{~s}, 6 \mathrm{H}, 2 \mathrm{CMeMe})$

${ }^{13} \mathrm{C}\left\{{ }^{1} \mathrm{H}\right\}-\mathrm{NMR}\left(75 \mathrm{MHz}, \mathrm{CDCl}_{3}\right): \delta(\mathrm{ppm})=161.5(2 \mathrm{C}=\mathrm{N}), 134.6(\mathrm{Ph}), 128.6(\mathrm{Ph}), 127.6(\mathrm{Ph}), 125.2(\mathrm{Ph})$, 79.7 (2 oxazoline- $\left.\mathrm{CH}_{2}\right), 66.8\left(2 \mathrm{CMe}_{2}\right), 44.45(\mathrm{CHPh}), 27.0(\mathrm{CMeMe}), 26.8(\mathrm{CMeMe})$

MS (ESI(+)), MeCN: $m / z=287\left([\mathrm{M}+\mathrm{H}]^{+}\right)$

HL $^{5}$ : yield: $6.8 \mathrm{~g}, 32.2 \mathrm{mmol}, 52 \%$.

${ }^{1} \mathrm{H}-\mathrm{NMR}\left(300 \mathrm{MHz}, \mathrm{CDCl}_{3}\right): \delta(\mathrm{ppm})=7.01-7.26(\mathrm{~m}, 4 \mathrm{H}, \mathrm{Ph}), 4.61(\mathrm{~s}, 1 \mathrm{H}$, bridging $\mathrm{CH}), 3.92-3.84(\mathrm{~m}$, $\left.4 \mathrm{H}, 2 \mathrm{CH}_{2}\right), 1.29(\mathrm{~s}, 6 \mathrm{H}, 2 \mathrm{Me}), 1.22(\mathrm{~d}, 6 \mathrm{H}, 2 \mathrm{Me})$

${ }^{13} \mathrm{C}\left\{{ }^{1} \mathrm{H}\right\}$-NMR $\left(75 \mathrm{MHz}, \mathrm{CDCl}_{3}\right): \delta(\mathrm{ppm})=161.5(2 \mathrm{C}=\mathrm{N}), 136.5(\mathrm{Ph}), 130.6(\mathrm{Ph}), 127.6(\mathrm{Ph}), 125.2(\mathrm{Ph})$, 79.7 (2 oxazoline- $\left.\mathrm{CH}_{2}\right), 66.2\left(2 \mathrm{CMe}_{2}\right), 44.45(\mathrm{CHPh}), 27.0(\mathrm{CMeMe}), 26.8(\mathrm{CMe} M e), 20.1(\mathrm{PhMe})$ MS (ESI(+)), MeCN: $m / z=301\left([\mathrm{M}+\mathrm{H}]^{+}\right)$

L $^{6}$ : yield: $4.2 \mathrm{~g}, 17.6 \mathrm{mmol}, 70 \%$.

${ }^{1} \mathrm{H}-\mathrm{NMR}\left(300 \mathrm{MHz}, \mathrm{CDCl}_{3}\right): \delta(\mathrm{ppm})=3.85(\mathrm{~s}, 4 \mathrm{H}, 2 \mathrm{CH}), 1.43\left(\mathrm{~s}, 6 \mathrm{H}, \mathrm{CMe}_{2}\right), 1.21(\mathrm{~s}, 12 \mathrm{H}, 4 \mathrm{Me})$ 
${ }^{13} \mathrm{C}\left\{{ }^{1} \mathrm{H}\right\}-\mathrm{NMR}\left(75 \mathrm{MHz}, \mathrm{CDCl}_{3}\right): \delta(\mathrm{ppm})=168.1(2 \mathrm{C}=\mathrm{N}), 79.9\left(2 \mathrm{CH}_{2}\right), 67.5\left(2 \mathrm{CMe}_{2}\right), 38.7(\mathrm{CHMe}), 28.5$ (4Me), $24.9\left(\mathrm{CMe}_{2}\right)$

MS (ESI(+)), MeCN: $m / z=239\left([\mathrm{M}+\mathrm{H}]^{+}\right)$

\subsubsection{Synthesis of monoanionic BOX ligands $\left[\mathbf{L}^{2}\right]^{-}$and $\left[\mathbf{L}^{3}\right]^{-}$}

The monoanionic BOX ligands were prepared according to the literature known procedure. ${ }^{[344]} 1 \mathrm{~g}$ of $\mathrm{HL}^{2}$ (4.48 mmol) was transferred into a Schlenk flask containing $15 \mathrm{ml}$ of dry pentane under a stream of argon. The reaction mixture was cooled to $233 \mathrm{~K}$ in a dry-ice/acetone bath. Under a stream of argon $2.8 \mathrm{ml}$ of $1.6 \mathrm{M} n \mathrm{BuLi}$ in Hexane (4.48 mmol) was added dropwise, which resulted in a white powder. The flask was left open under argon for 1 minute and then sealed. The reaction mixture was warmed to room temperature and stirred overnight. The white solid was filtered through a glass frit under inert conditions and washed with cold pentane $(15 \mathrm{ml} \times 3)$. The powder $\left[\mathbf{L}^{2}\right]^{-}$was dried under vacuum for one hour and then transferred into the glovebox.

$\left[\mathbf{L}^{2}\right]^{-:}$yield: $0.65 \mathrm{~g}, 13.8 \mathrm{mmol}, 63 \%$.

${ }^{1} \mathrm{H}$ NMR (300 MHz, THF-d 8 ): $\delta(\mathrm{ppm})=3.59\left(\mathrm{~s}, 4 \mathrm{H}, 2 \mathrm{CH}_{2}\right), 1.69$ (s, 3H, CMe), $1.14(\mathrm{~s}, 12 \mathrm{H}, 4 \mathrm{Me})$

${ }^{13} \mathrm{C}\left\{{ }^{1} \mathrm{H}\right\}$-NMR $\left(75 \mathrm{MHz}, \mathrm{THF}-\mathrm{d}_{8}\right): \delta(\mathrm{ppm})=170.4(2 \mathrm{C}-\mathrm{N}), 77.74\left(2 \mathrm{CH}_{2}\right), 65.2\left(2 \mathrm{CMe}_{2}\right), 56.9(\mathrm{CMe})$, $29.91(4 \mathrm{Me}), 12.27(\mathrm{CMe})$

MS (ESI(+)), $\mathrm{MeCN}: m / z=224(\mathrm{M}-\mathrm{Li}+\mathrm{H})^{+}$

The synthesis of $\left[\mathbf{L}^{3}\right]^{-}$was carried out similar to $\left[\mathbf{L}^{2}\right]^{-} .1 .5 \mathrm{~g}$ of $\mathbf{H L}^{3}(5.2 \mathrm{mmol})$ was reacted with $3.2 \mathrm{ml}$ of $1.6 \mathrm{M} n \mathrm{BuLi}$ in hexane $(5.2 \mathrm{mmol})$. A total of $20 \mathrm{ml}$ of pentane was used for the reaction, and for washing of the yellow colored powder $\left[\mathrm{L}^{3}\right]^{-}(20 \mathrm{ml} \mathrm{x} 3)$.

$\left[\mathbf{L}^{3}\right]^{-}:$yield: $0.98 \mathrm{~g}, 3.3 \mathrm{mmol}, 65 \%$.

${ }^{1} \mathrm{H}$ NMR (300 MHz, THF-d $): \delta(\mathrm{ppm})=7.14-6.65(\mathrm{~m}, 5 \mathrm{H}, \mathrm{Ph}), 3.58(\mathrm{~s}, 4 \mathrm{H}, 2 \mathrm{CH}), 1.18(\mathrm{~s}, 12 \mathrm{H}, 4 \mathrm{Me})$

${ }^{13} \mathrm{C}\left\{{ }^{1} \mathrm{H}\right\}$ NMR $\left(75 \mathrm{MHz}, \mathrm{THF}-\mathrm{d}_{8}\right): \delta(\mathrm{ppm})=133.5(\mathrm{Ph}), 126.3(\mathrm{Ph}), 122.0(\mathrm{Ph}), 121.4(\mathrm{Ph}) 77.8\left(2 \mathrm{CH}_{2}\right)$, $65.2\left(2 \mathrm{CMe}_{2}\right), 29.7(4 \mathrm{Me})$

MS (ESI(+)), MeCN: $m / z=286(\mathrm{M}-\mathrm{Li}+\mathrm{H})^{+}$

\subsection{Experimental Section: Chapter 5}

\subsubsection{Synthesis of $C u(I)$ BOX complexes}

\subsubsection{Synthesis of $\left[\mathrm{HL}^{1} \mathrm{Cu}(\mathrm{I}) \mathrm{MeCN}\right] \mathrm{PF}_{6}(1)$}

$70 \mathrm{mg}$ of $\mathrm{HL}^{1}(333.3 \mu \mathrm{mol})$ was dissolved in $10 \mathrm{ml}$ of dry THF under argon in a Schlenk flask. The flask was then transferred into the glovebox. To this solution $136.6 \mathrm{mg}$ of $\left[\mathrm{Cu}(\mathrm{I})(\mathrm{MeCN})_{4}\right] \mathrm{PF}_{6}(366 \mu \mathrm{mol})$ was added and the colorless solution was stirred for 2 hours. The mixture was then filtered and divided into two portions. Slow diffusion of $\mathrm{Et}_{2} \mathrm{O}$ into the reaction mixtures resulted in single crystals of $\mathbf{1}$.

Yield: $76 \mathrm{mg}, 166 \mu \mathrm{mol}, 50 \%$ MS (ESI(+) MeCN): $m / z=273.0\left[\mathrm{HL}^{1} \mathrm{Cu}(\mathrm{I})\right]^{+}, 314.0\left[\mathrm{HL}^{1} \mathrm{Cu}(\mathrm{I}) \mathrm{MeCN}\right]^{+}, 483.1\left[\left(\mathrm{HL}^{1}\right)_{2} \mathrm{Cu}(\mathrm{I})\right]^{+}$

${ }^{1} \mathrm{H}-\mathrm{NMR}\left(300 \mathrm{MHz}, \mathrm{CD}_{3} \mathrm{CN}\right): \delta(\mathrm{ppm})=4.34\left(\mathrm{~s}, 4 \mathrm{H}, 2 \mathrm{CH}_{2}\right), 3.38\left(\mathrm{~s}, 2 \mathrm{H}\right.$, bridging $\left.\mathrm{CH}_{2}\right), 1.34(\mathrm{~s}, 12 \mathrm{H}$, $4 M e)$ 


\subsubsection{Synthesis of $\left[\mathrm{HL}^{2} \mathrm{Cu}(\mathrm{I}) \mathrm{MeCN}\right] \mathrm{ClO}_{4}(2)$}

$55 \mathrm{mg}$ of $\mathrm{HL}^{2}(245.5 \mu \mathrm{mol})$ was dissolved in $8 \mathrm{ml}$ of dry THF under argon in a Schlenk flask. The flask was then transferred into the glovebox. To this solution $88.3 \mathrm{mg}$ of $\left[\mathrm{Cu}(\mathrm{I})(\mathrm{MeCN})_{4}\right] \mathrm{ClO}_{4}(270 \mu \mathrm{mol})$ was added and the colorless solution was stirred for 2 hours. The solvent was removed under vacuum to yield an air sensitive white powder of 2 .

Yield: $42 \mathrm{mg}, 98.2 \mu \mathrm{mol}, 40.2 \%$ MS (ESI(+) MeCN): $m / z=287.0\left[\mathrm{HL}^{2} \mathrm{Cu}(\mathrm{I})\right]^{+}, 328.1\left[\mathrm{HL}^{2} \mathrm{Cu}(\mathrm{I}) \mathrm{MeCN}\right]^{+}, 600.2\left[\left(\mathrm{HL}^{2}\right)_{2} \mathrm{Cu}_{2}(\mathrm{I}) \mathrm{CN}\right]^{+}$ ${ }^{1} \mathrm{H}-\mathrm{NMR}\left(300 \mathrm{MHz}, \mathrm{THF}-\mathrm{d}_{8}\right): \delta(\mathrm{ppm})=4.22\left(\mathrm{~s}, 4 \mathrm{H}, 2 \mathrm{CH}_{2}\right), 3.68(\mathrm{q}, 1 \mathrm{H}$, bridgingCH; weak) $1.53(\mathrm{~d}$, $\mathrm{J}=6.0 \mathrm{~Hz}, \mathrm{CMe}), 1.40(\mathrm{~d}, \mathrm{~J}=9.0 \mathrm{~Hz}, 12 \mathrm{H}, 4 \mathrm{Me}), 2.05(\mathrm{MeCN})$

\subsubsection{Synthesis of $\left[\mathrm{HL}^{5} \mathrm{Cu}(\mathrm{I}) \mathrm{MeCN}_{\mathrm{P}} \mathrm{PF}_{6}(3)\right.$}

$65 \mathrm{mg}$ of $\mathrm{HL}^{5}(216.6 \mu \mathrm{mol})$ was dissolved in $10 \mathrm{ml}$ of dry THF under argon in a Schlenk flask. The flask was then transferred into the glovebox. To this solution $88.8 \mathrm{mg}$ of $\left[\mathrm{Cu}(\mathrm{I})(\mathrm{MeCN})_{4}\right] \mathrm{PF}_{6}(238.3 \mu \mathrm{mol})$ was added and the colorless solution was stirred for 2 hours. The solvent was reduced to half the volume. $5 \mathrm{ml}$ of $\mathrm{CHCl}_{3}$ was added to this solution, and the solution was then filtered. Slow diffusion of $\mathrm{Et}_{2} \mathrm{O}$ yielded single crystals of $\mathbf{3}$.

Yield: $71 \mathrm{mg}, 130 \mu \mathrm{mol}, 60 \%$

MS (ESI(+) MeCN): $m / z=363.1\left[\mathrm{HL}^{5} \mathrm{Cu}(\mathrm{I})\right]^{+}, 404.1\left[\mathrm{HL}^{5} \mathrm{Cu}(\mathrm{I}) \mathrm{MeCN}\right]^{+}, 663.2\left[\left(\mathrm{HL}^{5}\right)_{2} \mathrm{Cu}_{2}(\mathrm{I}) \mathrm{CN}^{+}\right.$

${ }^{1} \mathrm{H}-\mathrm{NMR}\left(300 \mathrm{MHz}, \mathrm{CD}_{3} \mathrm{CN}\right): \delta(\mathrm{ppm})=7.29-7.21(\mathrm{~m}, 4 \mathrm{H}, \mathrm{Ph}), 4.58(\mathrm{~s}, 3 \mathrm{H}, \mathrm{PhMe}) 4.11(\mathrm{~d}, 2 \mathrm{H}$, bridging $\mathrm{CH}), 3.94(\mathrm{~d}, 2 \mathrm{H}$, bridging $\mathrm{CH}), 1.38(\mathrm{~s}, 6 \mathrm{H}, 2 \mathrm{Me}), 1.28(\mathrm{~s}, 6 \mathrm{H}, 2 \mathrm{Me})$

\subsubsection{Synthesis of $\left[\mathrm{L}^{6} \mathrm{Cu}(\mathrm{I}) \mathrm{MeCN}_{\mathrm{C}} \mathrm{ClO}_{4}(4)\right.$}

$25 \mathrm{mg}$ of $\mathrm{L}^{6}(105 \mu \mathrm{mol})$ was dissolved in $5 \mathrm{ml}$ of dry THF under argon in a Schlenk flask. The flask was then transferred into the glovebox. To this solution $34.3 \mathrm{mg}$ of $\left[\mathrm{Cu}(\mathrm{I}) \mathrm{MeCN}_{4}\right] \mathrm{ClO}_{4}(105 \mu \mathrm{mol})$ was added and the colorless solution was stirred for 2 hours. Slow diffusion of $\mathrm{Et}_{2} \mathrm{O}$ yielded single crystals of 4 .

Yield: $23.2 \mathrm{mg}, 52.4 \mu \mathrm{mol}, 50 \%$

MS (ESI $(+) \mathrm{MeCN}): m / z=301.0\left[\mathrm{~L}^{6} \mathrm{Cu}(\mathrm{I})\right]^{+}, 342.1\left[\mathrm{~L}^{6} \mathrm{Cu}(\mathrm{I}) \mathrm{MeCN}\right]^{+}, 628.2\left[\left(\mathrm{~L}^{6}\right)_{2} \mathrm{Cu}_{2}(\mathrm{I}) \mathrm{CN}\right]^{+}$

${ }^{1} \mathrm{H}-\mathrm{NMR}\left(300 \mathrm{MHz}, \mathrm{CD}_{3} \mathrm{CN}\right): \delta(\mathrm{ppm})=4.32\left(\mathrm{~s}, 4 \mathrm{H}, \mathrm{CH}_{2}\right), 2.30(\mathrm{MeCN}), 1.60(\mathrm{~s}, 6 \mathrm{H}, 2 \mathrm{Me}), 1.48(\mathrm{~s}, 12 \mathrm{H}$, $4 M e)$

\subsubsection{Synthesis of ${ }^{\mathrm{S}} \mathbf{P}$ complexes $\mathbf{6}$ and $\mathbf{8}$}

\subsubsection{Synthesis of $\left[\left(\mathrm{HL}^{2}\right)_{2} \mathrm{Cu}_{2}\left(\mu-\eta^{2}: \eta^{2}-\mathrm{O}_{2}\right)\right]\left(\mathrm{ClO}_{4}\right)_{2}(6)$}

$20 \mathrm{mg}$ of $2(46.7 \mu \mathrm{mol})$ was dissolved in a $6 \mathrm{ml}$ of 1:1 THF:Acetone solution in the glovebox. The reaction mixture was then filtered and transferred into a test tube that was placed within a Schlenk crystallization vessel. The set up was placed in a dry-ice acetone bath, and dry dioxygen was purged into the solution for 10 minutes. The tube was then layered with $\mathrm{Et}_{2} \mathrm{O}$ on the outside and the entire set up was flushed with argon. The crystallaization vessel was placed in a freezer at $-80{ }^{\circ} \mathrm{C}$. Single crystals of 6 were grown 
successfully after a period of 4 weeks. Due to the high sensitivity of the crystals, determining the exact yield was not possible.

Yield: $20 \%$

$\mathrm{UV} / \mathrm{vis}(\mathrm{THF}): \lambda_{\max } / \mathrm{nm}\left(\varepsilon /\left(\mathrm{Lmol}^{-1} \mathrm{~cm}^{-1}\right)\right)=330 \mathrm{~nm}\left(\varepsilon=19113 \mathrm{M}^{-1} \mathrm{~cm}^{-1}\right), 500 \mathrm{~nm}\left(\varepsilon=1350 \mathrm{M}^{-1} \mathrm{~cm}^{-1}\right) \mathrm{sh}$

\subsubsection{Synthesis of $\left[\left(\mathrm{L}^{6}\right)_{2} \mathrm{Cu}_{2}\left(\mu-\eta^{2}: \eta^{2}-\mathrm{O}_{2}\right)\right]\left(\mathrm{ClO}_{4}\right)_{2}(8)$}

$25 \mathrm{mg}$ of $4(56.5 \mu \mathrm{mol})$ was dissolved in a $8 \mathrm{ml}$ of 1:1 THF:Acetone solution in the glovebox. The reaction mixture was then filtered and transferred into a test tube that was placed within a Schlenk crystallization vessel. The set up was placed in a dry-ice acetone bath, and dry dioxygen was purged into the solution for 10 minutes. The tube was then layered with $\mathrm{Et}_{2} \mathrm{O}$ on the outside and the entire set up was flushed with argon. The crystallaization vessel was placed in a freezer at $-80{ }^{\circ} \mathrm{C}$. Single crystals of 8 were grown successfully after a period of 4 weeks. Due to the high sensitivity of the crystals, determining the exact yield was not possible.

Yield: $\sim 30 \%$

$\mathrm{UV} / \mathrm{vis}(\mathrm{THF}): \lambda_{\max } / \mathrm{nm}\left(\varepsilon /\left(\mathrm{Lmol}^{-1} \mathrm{~cm}^{-1}\right)\right)=333 \mathrm{~nm}\left(\varepsilon=21440 \mathrm{M}^{-1} \mathrm{~cm}^{-1}\right), 500 \mathrm{~nm}\left(\varepsilon=895 \mathrm{M}^{-1} \mathrm{~cm}^{-1}\right) \mathrm{sh}$

\subsubsection{UV-vis solutions}

$\left[\left(\mathbf{H L}^{1}\right)_{2} \mathbf{C u}_{2}\left(\boldsymbol{\mu}-\boldsymbol{\eta}^{2}: \boldsymbol{\eta}^{\mathbf{2}}-\mathbf{O}_{2}\right)\right]\left(\mathbf{P F}_{\mathbf{6}}\right)_{\mathbf{2}} \mathbf{( 5 )}$ : Single crystals of $\mathbf{1}(\mathbf{7} \mathrm{mg}, 0.015 \mu \mathrm{mol})$ were dissoleved in $4 \mathrm{ml}$ of dry THF in the glovebox to make a stock solution of $3.8 \mathrm{mM}$. UV-vis solutions of $0.07 \mathrm{mM} / 3 \mathrm{ml}$ were prepared in a UV-vis cuvette with a screw cap and septum, that was additionaly wrapped with parafilm. Dioxygen addition was monioted by UV-vis spectroscopy at $193 \mathrm{~K}$ to yield $\mathbf{5}$.

$\mathrm{UV} / \mathrm{vis}(\mathrm{THF}): \lambda_{\max } / \mathrm{nm}\left(\varepsilon /\left(\mathrm{Lmol}^{-1} \mathrm{~cm}^{-1}\right)\right)=330 \mathrm{~nm}\left(\varepsilon=7422 \mathrm{M}^{-1} \mathrm{~cm}^{-1}\right), 501 \mathrm{~nm}\left(\varepsilon=454 \mathrm{M}^{-1} \mathrm{~cm}^{-1}\right) \mathrm{sh}$

$\left[\left(\mathbf{H L}^{2}\right)_{2} \mathbf{C u}_{2}\left(\boldsymbol{\mu}-\boldsymbol{\eta}^{2}: \boldsymbol{\eta}^{2}-\mathbf{O}_{2}\right)\right]\left(\mathbf{C l O}_{4}\right)_{2} \mathbf{( 6 )}:$ White powder of $\mathbf{2}(9.4 \mathrm{mg}, 0.022 \mu \mathrm{mol})$ was dissolved in $5 \mathrm{ml}$ of dry THF in the glovebox to make a stock solution of $4.4 \mathrm{mM}$. UV-vis solutions of $0.05 \mathrm{mM} / \mathrm{ml}$ were prepared in a UV-vis cuvette with a screw cap and septum, that was additionaly wrapped with parafilm. Dioxygen addition was monitored by UV-vis spectroscopy at $193 \mathrm{~K}$ to yield 6.

$\left[\left(\mathbf{H L}^{5}\right)_{2} \mathbf{C u}_{2}\left(\boldsymbol{\mu}-\boldsymbol{\eta}^{2}: \boldsymbol{\eta}^{2}-\mathbf{O}_{2}\right)\right]\left(\mathbf{P F}_{6}\right)_{2} \mathbf{2}$ (7) : Single crystals of $\mathbf{3}$ (5.9 mg, $\left.0.018 \mu \mathrm{mol}\right)$ were dissoleved in $4 \mathrm{ml}$ of dry THF in the glovebox to make a stock solution of $2.7 \mathrm{mM}$. UV-vis solutions of $0.18 \mathrm{mM} / 3 \mathrm{ml}$ were prepared in a UV-vis cuvette with a screw cap and septum, that was additionaly wrapped with parafilm. Dioxygen addition was monitored by UV-vis spectroscopy at $193 \mathrm{~K}$ to yield 7.

$\mathrm{UV} / \mathrm{vis}(\mathrm{THF}): \lambda_{\max } / \mathrm{nm}\left(\varepsilon /\left(\mathrm{Lmol}^{-1} \mathrm{~cm}^{-1}\right)\right)=333 \mathrm{~nm}\left(\varepsilon=4403 \mathrm{M}^{-1} \mathrm{~cm}^{-1}\right), 504 \mathrm{~nm}\left(\varepsilon=357 \mathrm{M}^{-1} \mathrm{~cm}^{-1}\right) \mathrm{sh}$

$\left[\left(\mathbf{L}^{6}\right)_{2} \mathbf{C u}_{2}\left(\boldsymbol{\mu}-\eta^{2}: \eta^{2}-\mathbf{O}_{2}\right)\right]\left(\mathbf{C l O}_{4}\right)_{2}(\mathbf{8})$ : Single crystals of $\mathbf{4}(9.2 \mathrm{mg}, 0.018 \mu \mathrm{mol})$ were dissoleved in $6 \mathrm{ml}$ of dry THF in the glovebox to make a stock solution of $3.5 \mathrm{mM}$. UV-vis solutions of $0.1 \mathrm{mM} / 3 \mathrm{ml}$ were prepared in a UV-vis cuvette with a screw cap and septum, that was additionaly wrapped with parafilm. Dioxygen addition was monitored by UV-vis spectroscopy at $193 \mathrm{~K}$ to yield $\mathbf{8}$. 


\subsubsection{Raman measurements of $\mathbf{8}$}

Single crystals of $4(13.2 \mathrm{mg}, 30 \mathrm{mmol})$ were dissolved in $1.5 \mathrm{ml}$ of dry THF and transferred into young NMR tubes in the glovebox. The NMR tubes were dipped into dry-ice/acetone baths, and connected to the Schlenk line. Under inert conditions dry dioxygen $\left({ }^{16} \mathrm{O}_{2}\right.$ and $\left.{ }^{18} \mathrm{O}_{2}\right)$ were purged into the solutions seperatly. The solutions were gradually mixed. Measurements were made with the $633 \mathrm{~nm}$ laser in acetone/dry-ice baths within a finger dewar.

\subsection{Experimental Section: Chapter 6}

\subsubsection{Synthesis of $C u(I) B O X$ complexes}

\subsubsection{Synthesis of $\left[\left[\mathrm{L}^{2}\right]^{-} \mathrm{Cu}(\mathrm{I}) \mathrm{MeCN}\right] \mathrm{ClO}_{4}(13)$}

$20 \mathrm{mg}$ of $\left[\mathrm{L}^{2}\right]^{-}(86.9 \mu \mathrm{mol})$ was dissolved with $28.4 \mathrm{mg}\left[\mathrm{Cu}(\mathrm{I})(\mathrm{MeCN})_{4}\right] \mathrm{ClO}_{4}(86.9 \mu \mathrm{mol})$ in $5 \mathrm{ml}$ of dry THF in the glovebox to yield a yellow colored solution of $\mathbf{1 3}$. Compound $\mathbf{1 3}$ could not be characterized via $\mathrm{X}$-ray crystallography. All characterization was done in solution.

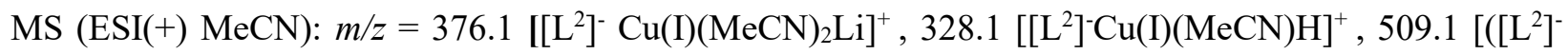
)$\left._{2} \mathrm{Cu}(\mathrm{I})\right]^{+}{ }^{1} \mathrm{H}-\mathrm{NMR}\left(300 \mathrm{MHz}, \mathrm{THF}-\mathrm{d}_{8}\right): \delta(\mathrm{ppm})=3.77\left(\mathrm{~s}, 4 \mathrm{H}, 2 \mathrm{CH}_{2}\right), 1.68(\mathrm{~s}, 3 \mathrm{H}, \mathrm{Me}), 1.21(\mathrm{~s}, 12 \mathrm{H}$, $4 \mathrm{Me}), 2.03(\mathrm{~s}, \mathrm{MeCN})$

\subsubsection{Synthesis of $\left[\left[\mathrm{L}^{3}\right]^{-} \mathrm{Cu}(\mathrm{I}) \mathrm{MeCN}^{-} \mathrm{ClO}_{4}(14)\right.$}

$25 \mathrm{mg}$ of [ $\left.\mathrm{L}^{3}\right]^{-}(85.6 \mu \mathrm{mol})$ was dissolved with $28 \mathrm{mg}\left[\mathrm{Cu}(\mathrm{I})(\mathrm{MeCN})_{4}\right] \mathrm{ClO}_{4}(85.6 \mu \mathrm{mol})$ in $5 \mathrm{ml}$ of dry THF in the glovebox to yield a yellow colored solution of $\mathbf{1 4}$. Compound 14 could not be characterized via Xray crystallography. All characterization was done in solution.

$\operatorname{MS}(\operatorname{ESI}(+) \mathrm{MeCN}): m / z=390.1\left[\left[\mathrm{~L}^{3}\right]^{-} \mathrm{Cu}(\mathrm{I})(\mathrm{MeCN}) \mathrm{H}\right]^{+}, 349.1\left[\left[\mathrm{~L}^{3}\right]^{-} \mathrm{CuH}\right]^{+}$

${ }^{1} \mathrm{H}-\mathrm{NMR}\left(300 \mathrm{MHz}, \mathrm{THF}-\mathrm{d}_{8}\right): \delta(\mathrm{ppm})=6.8-7.1(\mathrm{~m}, 5 \mathrm{H}, \mathrm{Ph}), 3.73\left(\mathrm{~s}, 4 \mathrm{H}, 2 \mathrm{CH}_{2}\right), 1.25(\mathrm{~s}, 12 \mathrm{H}, 4 \mathrm{Me}), 2.04$ $(\mathrm{s}, \mathrm{MeCN})$

\subsubsection{Synthesis of $\left[\left(\left[\mathrm{L}^{2}\right]^{-}\right)_{2} \mathrm{Cu}_{2}(\mu-\mathrm{O})_{2}\right](10)$}

A solution of compound $13\left(90 \mu \mathrm{mol}\left[\mathrm{L}^{2}\right]^{-}\right.$and $\left.90 \mu \mathrm{mol}\left[\mathrm{Cu}(\mathrm{I})(\mathrm{MeCN})_{4}\right] \mathrm{ClO}_{4}\right)$ was prepared in a 1:1 mixture of Me-THF and pentane $(4 \mathrm{ml}: 4 \mathrm{ml})$ in the glovebox. The solution was then transferred into a Schlenk crystallization vessel dipped in a dry ice/ethanol bath at $193 \mathrm{~K}$. Dioxygen was purged into the solution, and then the solution was layered with $\mathrm{Et}_{2} \mathrm{O}$ and stored at $193 \mathrm{~K}$. Single crystals of 10 suitable for X-ray diffracition were obtained over a period of 3 to 4 weeks. The high sensitivity of the crystals did not allow for precise yield determination.

Yield: $\sim 20 \%$

${ }^{1} \mathrm{H}-\mathrm{NMR}\left(500 \mathrm{MHz}, \mathrm{THF}-\mathrm{d}_{8}, 193 \mathrm{~K}\right): \delta(\mathrm{ppm})=3.94\left(\mathrm{~s}, 4 \mathrm{H}, 2 \mathrm{CH}_{2}\right), 1.58(\mathrm{~s}, 3 \mathrm{H}, \mathrm{Me}), 1.38(\mathrm{~s}, 12 \mathrm{H}, 4 \mathrm{Me})$, $2.08(\mathrm{~s}, \mathrm{MeCN})$

$\mathrm{UV} / \mathrm{vis}(\mathrm{THF}): \lambda_{\max } / \mathrm{nm}\left(\varepsilon /\left(\mathrm{Lmol}^{-1} \mathrm{~cm}^{-1}\right)\right)=297 \mathrm{~nm}\left(\varepsilon=26800 \mathrm{M}^{-1} \mathrm{~cm}^{-1}\right), 333 \mathrm{~nm}\left(\varepsilon=7440 \mathrm{M}^{-1} \mathrm{~cm}^{-1}\right), 395$ $\mathrm{nm}\left(\varepsilon=10140 \mathrm{M}^{-1} \mathrm{~cm}^{-1}\right)$ 


\subsubsection{Synthesis of $\left[\left(\left[\mathrm{L}^{3}\right]^{-}\right)_{2} \mathrm{Cu}_{2}(\mu-\mathrm{O})_{2}\right](11)$}

A solution of compound $14\left(100 \mu \mathrm{mol}\left[\mathrm{L}^{3}\right]^{-}\right.$and $\left.100 \mu \mathrm{mol}\left[\mathrm{Cu}(\mathrm{I})(\mathrm{MeCN})_{4}\right] \mathrm{ClO}_{4}\right)$ was prepared in THF (4 $\mathrm{ml}$ ) in the glovebox. Purging dioxygen at $193 \mathrm{~K}$ resulted in the formation of compound 11. X-ray characterization of $\mathbf{1 1}$ was not possible. All characterization was done in solution at $193 \mathrm{~K}$.

${ }^{1} \mathrm{H}-\mathrm{NMR}\left(500 \mathrm{MHz}, \mathrm{THF}-\mathrm{d}_{8}, 193 \mathrm{~K}\right): \delta(\mathrm{ppm})==7.15-7.03(\mathrm{~m}, 5 \mathrm{H}, \mathrm{Ph}), 3.90\left(\mathrm{~s}, 4 \mathrm{H}, 2 \mathrm{CH}_{2}\right), 1.42(\mathrm{~s}, 12 \mathrm{H}$, $4 \mathrm{Me}), 2.08(\mathrm{~s}, \mathrm{MeCN})$

$\mathrm{UV} / \mathrm{vis}(\mathrm{THF}): \lambda_{\max } / \mathrm{nm}\left(\varepsilon /\left(\mathrm{Lmol}^{-1} \mathrm{~cm}^{-1}\right)\right)=287 \mathrm{~nm}\left(\varepsilon=36720 \mathrm{M}^{-1} \mathrm{~cm}^{-1}\right), 337 \mathrm{~nm}\left(\varepsilon=13620 \mathrm{M}^{-1} \mathrm{~cm}^{-1}\right)$, $397 \mathrm{~nm}\left(\varepsilon=10740 \mathrm{M}^{-1} \mathrm{~cm}^{-1}\right)$

\subsubsection{UV-vis solutions}

$\left[\left(\left[\mathbf{L}^{2}\right]^{-}\right)_{2} \mathbf{C u}_{2}(\boldsymbol{\mu}-\mathbf{O})_{2}\right]$ (10): $5 \mathrm{mg}$ of $\left[\mathbf{L}^{2}\right]^{-}(21.4 \mu \mathrm{mmol})$ was mixed with $7.08 \mathrm{mg}$ of $\left[\mathrm{Cu}(\mathrm{I})(\mathrm{MeCN})_{4}\right] \mathrm{ClO}_{4}$ $(216.4 \mu \mathrm{mmol})$ in $2 \mathrm{ml}$ THF to produce a stock solution of $10.8 \mathrm{mM}$ in the glovebox. A UV-vis solution of $0.1 \mathrm{mM} / 3 \mathrm{ml}$ was prepared in a cuvette with a screw cap and septum. The cuvette was further sealed with paraffin. Purging dioxygen at $193 \mathrm{~K}$ yielded 10.

$\left[\left(\left[\mathbf{L}^{3}\right]^{-}\right)_{2} \mathbf{C u}_{2}(\boldsymbol{\mu}-\mathbf{O})_{2}\right]$ (11): $5 \mathrm{mg}$ of $\left[\mathbf{L}^{3}\right]^{-}(170.0 \mu \mathrm{mmol})$ was mixed with $5.5 \mathrm{mg}$ of $\left[\mathrm{Cu}(\mathrm{I})(\mathrm{MeCN})_{4}\right] \mathrm{ClO}_{4}$ $(170.0 \mu \mathrm{mmol})$ in $4 \mathrm{ml}$ THF to produce a stock solution of $4.2 \mathrm{mM}$ in the glovebox. A UV-vis solution of $0.1 \mathrm{mM} / 3 \mathrm{ml}$ was prepared in a cuvette with a screw cap and septum. The cuvette was further sealed with paraffin. Purging dioxygen at 193 K yielded 11.

\subsubsection{Raman measurements of $\mathbf{1 0}$ and $\mathbf{1 1}$}

$30 \mathrm{mM}$ solutions of $\mathbf{1 0}\left(27.7 \mathrm{mg}\right.$ of $\left[\mathbf{L}^{2}\right]^{-}(120 \mu \mathrm{mmol})$ and $39.2 \mathrm{mg}$ of $\left.\left[\mathrm{Cu}(\mathrm{MeCN})_{4}\right] \mathrm{ClO}_{4}(120 \mu \mathrm{mmol})\right)$ and $11\left(35.1 \mathrm{mg}\right.$ of $\left[\mathbf{L}^{3}\right]^{-}(120 \mu \mathrm{mmol})$ and $39.2 \mathrm{mg}$ of $\left.\left[\mathrm{Cu}(\mathrm{MeCN})_{4}\right] \mathrm{ClO}_{4}(120 \mu \mathrm{mmol})\right)$ were prepared in a $2 \mathrm{ml}$ of 1:1 THF:pentane mixture and THF respectively, and transferred into young NMR tubes in the glovebox. The NMR tubes were dipped into dry-ice/acetone baths, and connected to the Schlenk line. Under inert conditions dry dioxygen $\left({ }^{16} \mathrm{O}_{2}\right.$ and $\left.{ }^{18} \mathrm{O}_{2}\right)$ was purged into the solutions separately. The solutions were gradually mixed. Measurements were made with the $633 \mathrm{~nm}$ laser in acetone/dry-ice bath at $193 \mathrm{~K}$ for 10 and $\mathrm{N}_{2}$ bath at $77 \mathrm{~K}$ for $\mathbf{1 1}$, within a finger dewar.

\subsubsection{UV-vis temperature dependence measurements}

UV vis solutions for $\mathbf{1 0}$ and $\mathbf{1 1}$ were prepared according to the procedure mentioned above. UV-vis spectra were recorded with an Agilent Cary 60 equipped with an Unisoku Cryostat (CoolSpek), with the temperature being maintained at $193 \mathrm{~K}$. Dioxygen was purged into the solution and spectra were measured every half minute, until complete formation of $\mathbf{1 0}$ and 11. Following this, the temperature was varied by 10 degrees over a period of half an hour, and spectra were recorded at intervals of every 5 minutes. Spectra were recorded from $193 \mathrm{~K}$ to $243 \mathrm{~K}$, and again from $243 \mathrm{~K}$ to $193 \mathrm{~K}$.

In case of 9 and $12 \mathrm{UV}$-vis solutions were prepared according to procedures mentioned in Experimental section Chapter 7. DBU was titrated into these solutions until complete conversion of $\mathbf{5}$ and $\mathbf{7}$ to $\mathbf{9}$ and $\mathbf{1 2}$ respectively [Refer to titration experiments of ${ }^{\mathbf{S}} \mathbf{P}$ complexes: Experimental section chapter 7]. The temperature variation of UV-vis measurements were carried out similar to $\mathbf{1 0}$ and $\mathbf{1 1 .}$ 


\subsection{Experimental Section: Chapter 7}

\subsubsection{Titration experiments of ${ }^{S} \boldsymbol{P}$ complexes $\mathbf{5}, 6,7$ and ${ }^{S} \boldsymbol{P H L} \mathbf{L}_{3}$ with $D B U$}

Preparation of DBU stock solution: $5 \mathrm{ml}$ of $0.01 \mathrm{M} \mathrm{DBU}(7.47 \mu \mathrm{L}, 0.049 \mu \mathrm{mol})$ was prepared in THF in a glovebox

Conversion of 5 to 9 : Single crystals of $1(7 \mathrm{mg}, 0.015 \mu \mathrm{mol})$ were dissoleved in $4 \mathrm{ml}$ of dry THF in the glovebox to make a stock solution of $3.8 \mathrm{mM}$. UV-vis solutions of $0.07 \mathrm{mM} / 3 \mathrm{ml}$ were prepared in a UVvis cuvette with a screw cap and septum, that was additionaly wrapped with parafilm. Dioxygen was added via a syringe with a 3 way valve at $193 \mathrm{~K}$ unitil complete formation of 5. $0.01 \mathrm{M}$ solution of DBU was titrated via a $100 \mu \mathrm{L}$ Hamett syringe until complete conversion of 5 to 9 . The UV-vis spectra were measured at every half minute intervals.

Conversion of $\mathbf{6}$ to $\mathbf{1 0}$ : White powder of $2(9.4 \mathrm{mg}, 0.022 \mu \mathrm{mol})$ was dissolved in $5 \mathrm{ml}$ of dry THF in the glovebox to make a stock solution of $4.4 \mathrm{mM}$. UV-vis solutions of $0.05 \mathrm{mM} / \mathrm{ml}$ were prepared in a UV-vis cuvette with a screw cap and septum, that was additionaly wrapped with parafilm. Dioxygen was added via a syringe with a 3 way valve at $193 \mathrm{~K}$ unitil complete formation of 6. $0.01 \mathrm{M}$ solution of DBU was titrated via a $100 \mu \mathrm{L}$ Hamett syringe until complete conversion of $\mathbf{6}$ to 10. The UV-vis spectra were measured at every half minute intervals.

Conversion of ${ }^{{ }^{S}} \mathbf{P H L}^{3}$ to $\mathbf{1 1}: 5 \mathrm{mg}$ of $\mathrm{HL}^{3}(17.6 \mu \mathrm{mol})$ was mixed with $5.72 \mathrm{mg}$ of $\left[\mathrm{Cu}(\mathrm{I})(\mathrm{MeCN})_{4}\right] \mathrm{ClO}_{4}$ (17.6 $\mu \mathrm{mol}$ ) in $6 \mathrm{ml}$ of THF in a glovebox to form a stock solution of $2.91 \mathrm{mmol}$ of $\left[\mathrm{HL}^{3} \mathrm{Cu}(\mathrm{I})(\mathrm{MeCN})_{4}\right] \mathrm{ClO}_{4}$. Uv-vis solutions of $0.048 \mathrm{mM} / 3 \mathrm{ml}$ were prepared in a UV-vis cuvette with a screw cap and septum, that was additionaly wrapped with parafilm. Dioxygen was added via a syringe with a 3 way valve at $193 \mathrm{~K}$ unitil complete formation of ${ }^{\mathrm{S}} \mathbf{P H L}^{3}$. $0.01 \mathrm{M}$ solution of DBU was titrated via a 100 $\mu \mathrm{L}$ Hamett syringe until complete conversion of ${ }^{\mathbf{S}} \mathbf{P} \mathbf{H L}^{3}$ to 11. The UV-vis spectra were measured at every half minute intervals.

Conversion of 7 to 12 : : Single crystals of $\mathbf{3}(5.9 \mathrm{mg}, 0.018 \mu \mathrm{mol})$ were dissoleved in $4 \mathrm{ml}$ of dry THF in the glovebox to make a stock solution of $2.7 \mathrm{mM}$. UV-vis solutions of $0.18 \mathrm{mM} / 3 \mathrm{ml}$ were prepared in a UV-vis cuvette with a screw cap and septum, that was additionaly wrapped with parafilm. Dioxygen was added via a syringe with a 3 way valve at $193 \mathrm{~K}$ unitil complete formation of 7. $0.01 \mathrm{M}$ solution of DBU was titrated via a $100 \mu \mathrm{L}$ Hamett syringe until complete conversion of $\mathbf{7}$ to $\mathbf{1 2}$. The UV-vis spectra were measured at every half minute intervals.

Titration experiments with an acid: To the above solutions of $\mathbf{9 , 1 0 , 1 1}$ and 12, $0.01 \mathrm{M}$ solutions of LuOTf, $\mathrm{LuBF}_{4}$, [LuH: lituidinium], $\mathrm{HBF}_{4} \cdot \mathrm{Et}_{2} \mathrm{O},\left[\mathrm{Et}_{2} \mathrm{OH}\right] \mathrm{BArF}$ were titrated via a $100 \mu \mathrm{L}$. The UV-vis spectra were measured at every half minute intervals.

\subsubsection{POP Switch experimets}

Conversion of 5 to 9: 5 was prepared according to the procedure mentioned above. A $21 \mathrm{mM}$ ligand solution of $\mathrm{HL}^{1}(17.6 \mathrm{mg}, 84 \mu \mathrm{mol})$ in $4 \mathrm{ml}$ THF was titrated into 5 via a $100 \mu \mathrm{L}$ Hamett syringe until complete 
conversion of 5 to 9. A $15.28 \mathrm{mM}$ solution of $\left[\mathrm{Cu}(\mathrm{I})(\mathrm{MeCN})_{4}\right] \mathrm{ClO}_{4}(20 \mathrm{mg}, 61.1 \mu \mathrm{mol})$ in $4 \mathrm{ml}$ of $\mathrm{THF}$ was titrated back into the same solution via a $100 \mu \mathrm{L}$ Hamett syringe. Spectra were recorded at every 1 minute intervals. The same procedure was carried out for conversion of 6 to $10,{ }^{5} \mathbf{P H L}^{3}$ to 11 and 7 to 12 . In these cases the respective ligand solutions were prepared seperately. $21 \mathrm{mM}$ ligand solution of $\mathrm{HL}^{2}(18.8$ $\mathrm{mg}, 84 \mu \mathrm{mol})$ in $4 \mathrm{ml} \mathrm{THF} ; 21 \mathrm{mM}$ ligand solution of $\mathrm{HL}^{3}(24.0 \mathrm{mg}, 84 \mu \mathrm{mol})$ in $4 \mathrm{ml} \mathrm{THF} ; 21 \mathrm{mM}$ ligand solution of $\mathrm{HL}^{5}(25.2 \mathrm{mg}, 84 \mu \mathrm{mol})$ in $4 \mathrm{ml} \mathrm{THF}$.

15.5.3 Oxygentation of 2:1 $\mathrm{HL}^{1}: \mathrm{Cu}(\mathrm{I}): 4.2 \mathrm{mg}$ of $\mathrm{HL}^{1}(20 \mu \mathrm{mol})$, was mixed with $3.72 \mathrm{mg}$ of $\left[\mathrm{Cu}(\mathrm{I})(\mathrm{MeCN})_{4}\right] \mathrm{PF}_{6}(10 \mu \mathrm{mol})$ in $4 \mathrm{ml}$ of THF in the glovebox to form a $5 \mathrm{mM}$ stock solution. A UV-vis solution $0.1 \mathrm{mM} / 3 \mathrm{ml}$ was prepared in a UV-vis cuvette with a screw cap and septum, that was additionaly wrapped with parafilm. Dioxygen was added via a syringe with a 3 way valve at $193 \mathrm{~K}$ unitil complete formation of 9. A $2.5 \mathrm{mM}$ solution of $\left[\mathrm{Cu}(\mathrm{I})(\mathrm{MeCN})_{4}\right] \mathrm{PF}_{6}(3.72 \mathrm{mg}, 10 \mu \mathrm{mol})$ was titrated back with a 100 $\mu \mathrm{L}$ Hamett syringe until complete conversion of $\mathbf{9}$ to $\mathbf{5}$.

15.5.4 Resosnance Raman measurements $2: 1 \mathrm{HL}^{1}: \mathrm{Cu}(\mathrm{I})$ : A $30 \mathrm{mM}$ solutions of $2: 1 \mathrm{HL}^{1}{ }^{1} \mathrm{Cu}(\mathrm{I}) \mathrm{PF}_{6}(50.4$ $\mathrm{mg} \mathrm{HL}{ }^{1} 240 \mu \mathrm{mol}$ and $\left.44.7 \mathrm{mg}\left[\mathrm{Cu}(\mathrm{I})(\mathrm{MeCN})_{4}\right] \mathrm{PF}_{6} 120 \mu \mathrm{mol}\right)$ was mixed in $2 \mathrm{ml} \mathrm{THF}$ and transferred into young NMR tubes in the Glove-box. The NMR tubes were dipped into dry-ice/acetone baths, and connected to the Schlenk line. Under inert conditions dry dioxygen $\left({ }^{16} \mathrm{O}_{2}\right.$ and $\left.{ }^{18} \mathrm{O}_{2}\right)$ was purged into the solutions separately. The solutions were gradually mixed. Measurements were made with the $633 \mathrm{~nm}$ laser in liquid nitrogen in a finger dewar.

\subsection{Experimental Section: Chapter 8}

\subsubsection{Substrate reactivity of DTBP-Na/DTBP-H:NEt $t_{3}$ with ${ }^{\boldsymbol{S}} \boldsymbol{P}$ complexes of proton responsive ligands}

To a solution of $6(71 \mu \mathrm{mol})$ in $5 \mathrm{ml}$ of dry THF at $193 \mathrm{~K}$ under a stream of dioxygen, $21.3 \mathrm{mmol}$ of DTBP$\mathrm{Na}$ in dry THF was gradually added and stirred for 20 mins. Initial addition of the substrate resulted in a gradual change of color from purple to dark green ( 6 to 10). Addition of the excess equivalents resulted in a change from green to brown. The reaction mixture was warmed up to rt. The solution was then worked up by addition of $10 \mathrm{ml} 0.5 \mathrm{M} \mathrm{HCl}$. The aqueous phase was extracted three times with $20 \mathrm{ml} \mathrm{DCM}$. The solution was then dried over $\mathrm{MgSO}_{4}$, and the combined organic phase was evaporated to dryness, to yield a brown colored oil.

The product was shown by ${ }^{1} \mathrm{H}-\mathrm{NMR}$ spectroscopy to be a mixture of DTBP-H and 4,4',6,6 tetra-(tert-butyl)2,2'-biphenol (C-C coupled product) in a ratio of 67:33

$\left.{ }^{1} \mathrm{H}-\mathrm{NMR}\left(300 \mathrm{MHz}, \mathrm{CDCl}_{3}\right): \mathrm{a}\right)$ DTBP-H: $\delta(\mathrm{ppm})=7.23(\mathrm{~d}, 1 \mathrm{H}), 6.98-7.02(\mathrm{dd}, 1 \mathrm{H}), 6.54(\mathrm{~d}, 1 \mathrm{H}) 1.34$ (s, 9H, 4-tert-but), 1.22 (s, 9H, 4-tert-but)

b) 4,4',6,6 tetra-(tert-butyl)-2,2'-biphenol: $\delta(\mathrm{ppm})=7.32(\mathrm{~d}, 2 \mathrm{H}), 7.04(\mathrm{~d}, 2 \mathrm{H}), 5.15(\mathrm{~s}, 2 \mathrm{H}, \mathrm{OH}) 1.38(\mathrm{~s}$, 9H, 4-tert-but), 1.25 (s, 9H, 4-tert-but)

The reactivity of ${ }^{\mathrm{S}} \mathbf{P}$ complexes 5, 7 and ${ }^{\mathrm{S}} \mathbf{P H L}{ }^{3}$ were carried out similar to 6. Reactivity with DTBP-H:NEt ${ }_{3}$ $(1: 2)$ was also carried out similar to that of DTBP-Na. 
15.6.2 Substrate reactivity of DTBP-Na/DTBP-H:NEt 3 with ${ }^{\boldsymbol{S}} \boldsymbol{P}$ complex $\boldsymbol{8}$ of non-proton responsive ligand 2.1 equivalents of substrate: To a solution of $\mathbf{8}(10 \mu \mathrm{mol})$ in $5 \mathrm{ml}$ of dry THF at $193 \mathrm{~K}$ under a stream of dioxygen, 2.1 equivalents $(21 \mu \mathrm{mol})$ of DTBP-Na in dry THF was gradually added and stirred for 20 mins. The solution took on a brown color after substrate addition. Gradual warming of the solution to rt resulted in a color change from brown to green. The solution was then worked up by addition of $10 \mathrm{ml}$ of $0.5 \mathrm{M} \mathrm{HCl}$. The aqueous phase was extracted three times with $20 \mathrm{ml} \mathrm{DCM}$. The solution was then dried over $\mathrm{MgSO}_{4}$, and the combined organic phase was evaporated to dryness, to yield a yellow colored oil.

The product was shown by ${ }^{1} \mathrm{H}-\mathrm{NMR}$ spectroscopy to be a mixture of DTBP-H and DTBP-Q (quinone) in a ratio of $87: 13$

${ }^{1} \mathrm{H}-\mathrm{NMR}\left(300 \mathrm{MHz}\right.$, Acetone-d $\left.\left.{ }_{6}\right): \mathrm{a}\right)$ DTBP-H: $\delta(\mathrm{ppm})=7.26(\mathrm{~d}, 1 \mathrm{H}), 7.01(\mathrm{dd}, 1 \mathrm{H}), 6.73(\mathrm{~d}, 1 \mathrm{H}) 1.40(\mathrm{~s}$, 9H, 4-tert-but), 1.27 (s, 9H, 4-tert-but)

b) DTBP-Q: $\delta(\mathrm{ppm})=7.09(\mathrm{~d}, 1 \mathrm{H}), 6.15(\mathrm{~d}, 1 \mathrm{H}), 1.32(\mathrm{~s}, 9 \mathrm{H}, 4$-tert-but $), 1.25$ (s, 9H, 4-tert-but)

10 equivalents of substrate: To a solution of $8(10 \mu \mathrm{mol})$ in $5 \mathrm{ml}$ of dry THF at $193 \mathrm{~K}$ under a stream of dioxygen, 10 equivalents $(105 \mu \mathrm{mol})$ of DTBP-Na in dry THF was gradually added and stirred for 20 mins. On total addition of the substrate at $193 \mathrm{~K}$, the solution changed color from purple to light green. On warming up to rt there was no further change in color. The workup of the reaction mixture was carried out similar to the previous cases.

The product was shown by ${ }^{1} \mathrm{H}-\mathrm{NMR}$ spectroscopy to be a mixture of DTBP-H, 4,4',6,6 tetra-(tert-butyl)2,2'-biphenol, and DTBP-Q (quinone) in a ratio of 80:14:6

${ }^{1} \mathrm{H}-\mathrm{NMR}(300 \mathrm{MHz}$, Acetone-d 6 ) : a) DTBP-H: $\delta(\mathrm{ppm})=7.26(\mathrm{~d}, 1 \mathrm{H}), 7.05(\mathrm{dd}, 1 \mathrm{H}), 6.74(\mathrm{~d}, 1 \mathrm{H}) 1.40(\mathrm{~s}$, 9H, 4-tert-but), 1.27 (s, 9H, 4-tert-but)

b) 4,4',6,6'tetra-(tert-butyl)-2,2'-biphenol: $\delta(\mathrm{ppm})=7.38(\mathrm{~d}, 2 \mathrm{H}), 7.09$ (d, 2H), 1.38 (s, 9H, 4-tert-but), 1.25 (s, 9H, 4-tert-but)

c) DTBP-Q: $\delta(\mathrm{ppm})=7.09(\mathrm{~d}, 1 \mathrm{H}), 6.14(\mathrm{~d}, 1 \mathrm{H}), 1.32(\mathrm{~s}, 9 \mathrm{H}, 4$-tert-but), 1.25 (s, 9H, 4-tert-but)

100 equivalents of substrate: To a solution of $\mathbf{8}(10 \mu \mathrm{mol})$ in $5 \mathrm{ml}$ of dry THF at $193 \mathrm{~K}$ under a stream of dioxygen, 100 equivalents $(1050 \mu \mathrm{mol})$ of DTBP in dry THF was gradually added and stirred for 20 mins. On total addition of the substrate at $193 \mathrm{~K}$, the solution changed color from purple to light green. On warming up to rt there was no further change in color. The workup of the reaction mixture was carried out similar to the previous cases.

The product was shown by ${ }^{1} \mathrm{H}-\mathrm{NMR}$ spectroscopy to be a mixture of DTBP-H and 4,4',6,6 tetra-(tert-butyl)2,2'-biphenol in a ratio of 91:09

${ }^{1} \mathrm{H}-\mathrm{NMR}\left(300 \mathrm{MHz}, \mathrm{CDCl}_{3}\right)$ : a) DTBP-H: $\delta(\mathrm{ppm})=7.23(\mathrm{~d}, 1 \mathrm{H}), 6.98-7.02(\mathrm{dd}, 1 \mathrm{H}), 6.51(\mathrm{~d}, 1 \mathrm{H}) 1.34$ (s, 9H, 4-tert-but), 1.22 (s, 9H, 4-tert-but)

b) 4,4',6,6'tetra-(tert-butyl)-2,2'-biphenol: $\delta(\mathrm{ppm})=7.32(\mathrm{~d}, 2 \mathrm{H}), 7.04$ (d, 2H), 1.38 (s, 9H, 4-tert-but), 1.25 (s, 9H, 4-tert-but)

15.6.3 Substrate reactivity of DTBP-Na/DTBP-H:NEt 3 with $\boldsymbol{O}$ complexes of monoanionic ligands 
To a solution of $\mathbf{1 0}(21 \mu \mathrm{mol})$ in $5 \mathrm{ml}$ of dry THF at $193 \mathrm{~K}$ under a stream of dioxygen, $5.25 \mathrm{mmol}$ of DTBP-Na in dry THF was gradually added and stirred for 2 hours. Addition of the substrate resulted in a gradual change of color from dark green to brown. The solution was warmed to rt and worked up similar to the procedures mentioned above. After removal of solvent a yellow-brown colored oil was obtained.

The product was shown by ${ }^{1} \mathrm{H}-\mathrm{NMR}$ spectroscopy to be a mixture of DTBP-H and 4,4',6,6 tetra-(tert-butyl)2,2'-biphenol in a ratio of 50:50

${ }^{1} \mathrm{H}-\mathrm{NMR}\left(300 \mathrm{MHz}\right.$, Acetone-d $\left.{ }_{6}\right)$ : a) DTBP-H: $\delta(\mathrm{ppm})=7.23(\mathrm{~d}, 1 \mathrm{H}), 6.98-7.02(\mathrm{dd}, 1 \mathrm{H}), 6.54(\mathrm{~d}, 1 \mathrm{H})$ 1.34 (s, 9H, 4-tert-but), 1.22 (s, 9H, 4-tert-but)

b) 4,4',6,6'tetra-(tert-butyl)-2,2'-biphenol: $\delta(\mathrm{ppm})=7.32(\mathrm{~d}, 2 \mathrm{H}), 7.04(\mathrm{~d}, 2 \mathrm{H}), 5.15(\mathrm{~s}, 2 \mathrm{H}, \mathrm{OH}) 1.38(\mathrm{~s}$, 9H, 4-tert-but), 1.25 (s, 9H, 4-tert-but)

\subsection{Experimental Section: Chapter 11}

\subsubsection{Synthesis of Fe(II) BOX complexes}

\subsubsection{Synthesis of $\mathrm{HL}^{1} \mathrm{Fe}(\mathrm{II}) \mathrm{Cl}_{2}(17)$}

$31 \mathrm{mg}$ of $\mathrm{HL}^{1}(147.6 \mu \mathrm{mol})$ was dissolved in $4 \mathrm{ml}$ of dry THF in a Schlenk flask under argon. The flask was then transferred into the glovebox. To this solution $18.7 \mathrm{mg}(147.6 \mu \mathrm{mol})$ of $\mathrm{FeCl}_{2}$ was added and the yellow colored reaction mixture was allowed to stir overnight. The solution was then taken up in DCM which produced a white precipitate. After filtration, the solvent was removed and taken up again in THF. The reaction mixture was filtered to yield a light yellow colored solution. Layering with dry hexane in the glovebox yielded single crystals of 17.

Yield: $19.9 \mathrm{mg}, 59 \mu \mathrm{mol}, 40 \%$

MS (ESI(-) MeCN): $m / z=335.0\left[\mathrm{~L}^{1} \mathrm{Fe}(\mathrm{II})\right]^{-}, 478.8\left[\mathrm{~L}^{1} \mathrm{Fe}(\mathrm{II})\left(\mathrm{MeCN}^{2} \mathrm{CH}_{2} \mathrm{Cl}_{2} \mathrm{OH}\right]^{-}\right.$

IR (ATR) $\left(\mathrm{cm}^{-1}\right)=2973(\mathrm{w}), 1667(\mathrm{~s}), 1558(\mathrm{~m}), 1447(\mathrm{~m}), 1371(\mathrm{~m}), 1303(\mathrm{~m}), 1159$ (m) $1029(\mathrm{~m})$

$\mathrm{UV} / \mathrm{Vis}(\mathrm{MeCN}): \lambda_{\max } / \mathrm{nm}\left(\varepsilon / \mathrm{Lmol}^{-1} \mathrm{~cm}^{-1}\right)=380 \mathrm{~nm}(\mathrm{sh}, 13,000)$

\subsubsection{Synthesis of $\mathrm{HL}^{2} \mathrm{Fe}(\mathrm{II}) \mathrm{Cl}_{2}(18)$}

$110 \mathrm{mg}$ of $\mathrm{HL}^{2}(490 \mu \mathrm{mol})$ was dissolved in $6 \mathrm{ml}$ of dry THF in a Schlenk flask under argon. The flask was then transferred into the glovebox. To this solution $62.3 \mathrm{mg}(490 \mu \mathrm{mol})$ of $\mathrm{FeCl}_{2}$ was added and the yellow colored reaction mixture was allowed to stir overnight. The solution was then taken up in DCM which produced a heavy white precipitate. After filtration, the solvent was removed and taken up again in THF. The reaction mixture was filtered to yield a light yellow colored solution. Layering with dry hexane in the glovebox yielded single crystals of $\mathbf{1 8}$.

Yield: $103 \mathrm{mg}, 294 \mu \mathrm{mol}, 60 \%$

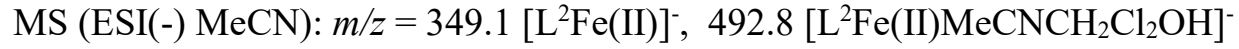

IR (ATR) $\left(\mathrm{cm}^{-1}\right)=2973(\mathrm{w}), 1665(\mathrm{~s}), 1459(\mathrm{~m}), 1372(\mathrm{~m}), 1300(\mathrm{w}), 1255(\mathrm{w}), 1088(\mathrm{~m})$

$\mathrm{UV} / \mathrm{Vis}(\mathrm{MeCN}): \lambda_{\max } / \mathrm{nm}\left(\varepsilon / \mathrm{Lmol}^{-1} \mathrm{~cm}^{-1}\right)=289 \mathrm{~nm}(\mathrm{sh}, 14,500)$

\subsubsection{Synthesis of $\mathrm{HL}^{3} \mathrm{Fe}(\mathrm{II}) \mathrm{Cl}_{2}(19)$}


$23 \mathrm{mg}$ of $\mathrm{HL}^{3}(95 \mu \mathrm{mol})$ was dissolved in $5 \mathrm{ml}$ of dry DCM in a Schlenk flask under argon. The flask was then transferred into the glovebox. To this solution $10.2 \mathrm{mg}(95 \mu \mathrm{mol})$ of $\mathrm{FeCl}_{2}$ in $4 \mathrm{ml}$ of dry THF was added and the yellow colored reaction mixture was allowed to stir overnight. The solution was filtered and then taken up in dry DCM. Layering with dry hexane in the glovebox yielded single crystals of 19.

Yield: $21.5 \mathrm{mg}, 52.2 \mu \mathrm{mol}, 55 \%$

MS (ESI(-) MeCN): $m / z=411.1\left[\mathrm{~L}^{3} \mathrm{Fe}(\mathrm{II})\right]^{-}, 554.8$ [L $\left.{ }^{3} \mathrm{Fe}(\mathrm{II}) \mathrm{MeCNCH}_{2} \mathrm{Cl}_{2} \mathrm{OH}\right]^{-}$

IR (ATR) $\left(\mathrm{cm}^{-1}\right)=2973(\mathrm{w}), 1657(\mathrm{~s}), 1454(\mathrm{~m}), 1415(\mathrm{~m}), 1373(\mathrm{w}), 1253(\mathrm{w}), 1037(\mathrm{~m})$

$\mathrm{UV} / \mathrm{Vis}(\mathrm{MeCN}): \lambda_{\max } / \mathrm{nm}\left(\varepsilon / \mathrm{Lmol}^{-1} \mathrm{~cm}^{-1}\right)=290 \mathrm{~nm}(\mathrm{sh}, 13,500)$

\subsubsection{Synthesis of $\mathrm{HL}^{5} \mathrm{Fe}(\mathrm{II}) \mathrm{Cl}_{2}(20)$}

$30 \mathrm{mg}$ of $\mathrm{HL}^{5}(100 \mu \mathrm{mol})$ was dissolved in $8 \mathrm{ml}$ of dry DCM in a Schlenk flask under argon. The flask was then transferred into the glovebox. To this solution $12.7 \mathrm{mg}(100 \mu \mathrm{mol})$ of $\mathrm{FeCl}_{2}$ was added and the yellow colored reaction mixture was allowed to stir overnight. The solution was filtered. Layering with dry hexane in the Glove-box yielded single crystals of $\mathbf{2 0}$.

Yield: $24.3 \mathrm{mg}, 57 \mu \mathrm{mol}, 57 \%$

MS (ESI(-) MeCN): $m / z=425.1\left[\mathrm{~L}^{3} \mathrm{Fe}(\mathrm{II})\right]^{-}, 568.8\left[\mathrm{~L}^{3} \mathrm{Fe}(\mathrm{II}) \mathrm{MeCNCH}_{2} \mathrm{Cl}_{2} \mathrm{OH}\right]^{-}$

IR (ATR) $\left(\mathrm{cm}^{-1}\right)=2971(\mathrm{w}), 1657(\mathrm{~s}), 1460(\mathrm{w}), 1415(\mathrm{~m}), 1373(\mathrm{w}), 1304(\mathrm{w}), 1038(\mathrm{~m})$

$\mathrm{UV} / \mathrm{Vis}(\mathrm{MeCN}): \lambda_{\max } / \mathrm{nm}\left(\varepsilon / \mathrm{Lmol}^{-1} \mathrm{~cm}^{-1}\right)=296 \mathrm{~nm}(\mathrm{sh}, 13,300)$

\subsubsection{Synthesis of $\mathrm{L}^{6} \mathrm{Fe}(\mathrm{II}) \mathrm{Cl}_{2}(21)$}

$46 \mathrm{mg}$ of $\mathrm{L}^{6}(193 \mu \mathrm{mol})$ was dissolved in $4 \mathrm{ml}$ of dry THF in a Schlenk flask under argon. The flask was then transferred into the glovebox. To this solution $24.54 \mathrm{mg}(193 \mu \mathrm{mol})$ of $\mathrm{FeCl}_{2}$ was added and the yellow colored reaction mixture was allowed to stir overnight. The solution was then taken up in DCM which produced a heavy white precipitate. After filtration, the solvent was removed and taken up again in THF. The reaction mixture was filtered to yield a light yellow colored solution. Layering with dry hexane in the glovebox yielded single crystals of 21.

Yield: $45.78 \mathrm{mg}, 125.4 \mu \mathrm{mol}, 65 \%$

IR (ATR) $\left(\mathrm{cm}^{-1}\right)=2968(\mathrm{w}), 1652(\mathrm{~s}), 1459(\mathrm{w}), 1374(\mathrm{~m}), 1291(\mathrm{w}), 1112(\mathrm{~m})$

$\mathrm{UV} / \mathrm{Vis}(\mathrm{MeCN}): \lambda_{\max } / \mathrm{nm}\left(\varepsilon / \mathrm{Lmol}^{-1} \mathrm{~cm}^{-1}\right)=300 \mathrm{~nm}(\mathrm{sh}, 15,000)$

\subsubsection{Synthesis of $\left[\left(\mathrm{L}^{6} \mathrm{Fe}(\mathrm{II})\right]_{2}\left(\mathrm{CF}_{3} \mathrm{SO}_{3}\right)_{4}(22)\right.$}

$16 \mathrm{mg}$ of $\mathrm{L}^{6}(67 \mu \mathrm{mol})$ was dissolved in $5 \mathrm{ml}$ of dry THF in a Schlenk flask under argon. The flask was then transferred into the glovebox. To this solution $29.31 \mathrm{mg}(67 \mu \mathrm{mol})$ of $\mathrm{Fe}(\mathrm{OTf})_{2}(\mathrm{MeCN})_{2}$ was added and the yellow colored reaction mixture was allowed to stir overnight. The solution was then taken up in DCM which produced a heavy white precipitate. After filtration, the solvent was removed and taken up again in THF. This process was repeated four times. The reaction mixture was filtered to yield a light yellow colored solution. Layering with dry hexane in the glovebox yielded single crystals of 22 .

Yield: $7.93 \mathrm{mg}, 6.7 \mu \mathrm{mol}, 10 \%$

MS (ESI(+) MeCN): $m / z=239\left[\mathrm{~L}_{6} \mathrm{H}\right]^{+}, 443.1\left[\mathrm{~L}^{6} \mathrm{FeOTf}\right]^{+}, 681\left[\left(\mathrm{~L}^{6}\right)_{2} \mathrm{FeOTf}\right]^{+}, 903\left[\left(\mathrm{~L}^{6} \mathrm{FeOTf}\right)_{2} \mathrm{OH}\right]^{+}, 1207$ $\left[\left(\mathrm{L}_{6} \mathrm{FeOTf}_{2}\right)_{2} \mathrm{Na}\right]^{+}, 1035\left[\left(\mathrm{~L}^{6} \mathrm{Fe}\right)_{2}(\mathrm{OTf})_{3}\right]^{+}$

IR (ATR) $\left(\mathrm{cm}^{-1}\right)=1652$ (w), $1463(\mathrm{w}), 1377$ (w), $1331(\mathrm{w}), 1236(\mathrm{~m}), 1211(\mathrm{~m}), 1163(\mathrm{~m}), 1116(\mathrm{~m}), 1025$

(s)

$\mathrm{UV} / \mathrm{Vis}(\mathrm{MeCN}): \lambda_{\max } / \mathrm{nm}\left(\varepsilon / \mathrm{Lmol}^{-1} \mathrm{~cm}^{-1}\right)=300 \mathrm{~nm}(\mathrm{sh}, 14,500)$ 


\subsection{Experimental Section: Chapter 12}

\subsubsection{Synthesis of $\left[\mathrm{L}^{9} \mathrm{Fe}(\mathrm{NO})_{2}\left(\mathrm{CF}_{3} \mathrm{SO}_{3}\right)_{2}\right](23)$}

$20 \mathrm{mg}$ of $\mathrm{HL}^{2} \mathrm{FeCl}_{2}(57 \mu \mathrm{mol})$ was reacted with $29.2 \mathrm{mg}$ of $\operatorname{AgOTf}(57 \mu \mathrm{mol})$ in $4 \mathrm{ml}$ of $\mathrm{MeCN}$. The reaction mixture was stirred for 15 minutes and then filtered. This was further reacted with $34.3 \mathrm{mg}$ of $\mathrm{Ph}_{3} \mathrm{SCNO}(57 \mu \mathrm{mol})$. The reaction was allowed to stir for one night. Filtration yielded a clear brown colored solution. The solvent was removed and taken up in $4 \mathrm{ml}$ of THF. Layering with hexane yielded single crystals of 23. ( $\mathbf{L}^{9}$ represents the modified ligand scaffold)

Yield: $3.4 \mathrm{mg}, 4 \mu \mathrm{mol}, 15 \%$

$\operatorname{MS}(\operatorname{ESI}(+) \mathrm{MeCN}): m / z=447\left[\left(\mathrm{HL}^{2}\right)-\mathrm{H}\right]^{+}$

IR $($ THF soln. $)=1826.9 \mathrm{~cm}^{-1}, 1752.3 \mathrm{~cm}^{-1}, 1642 \mathrm{~cm}^{-1}, 1627 \mathrm{~cm}^{-1}, 1575.2 \mathrm{~cm}^{-1}, 1491 \mathrm{~cm}^{-1}$

$\mathrm{UV} / \mathrm{Vis}(\mathrm{MeCN}): \lambda_{\max } / \mathrm{nm}\left(\varepsilon / \mathrm{Lmol}^{-1} \mathrm{~cm}^{-1}\right)=427 \mathrm{~nm}(\mathrm{sh}, 1280), 579 \mathrm{~nm}(\mathrm{sh}, 432)$ 
[1] K. D. Karlin, Science 1993, 261, 701-705.

[2] B. L. Valee, R. J. P. Williams, Proc. Natl. Acad. Sci. USA 1967, 59, 498-505.

[3] J. Y. Lee, K. D. Karlin, Curr Opin Chem Biol. 2015, 25, 184-193.

[4] C. J. Chang, M. C. Y. Chang, N. H. Damrauer, D. G. Nocera, Biochem. Biophys. Acta 2004, 1655, $13-28$.

[5] D. G. Nocera, M. P. Nash, Proc. Natl. Acad. Sci. USA 2007, 104, 15729-15735.

[6] Y. Surendranath, M. W. Kanan, D. G. Nocera, J. Am. Chem. Soc. 2010, 132, 16501-16509.

[7] S. Styring, L. Hammarstr, Energy Environ. Sci. 2011, 4, 2379-2388.

[8] V. Mougel, P. Horeglad, J. Pe, M. Mazzanti, J. Am. Chem. Soc. 2010, 132, 17374-17377.

[9] G. Editors, D. Nocera, D. Guldi, Chem. Soc. Rev. 2009, 38, 1-300.

[10] J. D. Megiatto, A. Antoniuk-pablant, B. D. Sherman, G. Kodis, M. Gervaldo, Proc. Natl. Acad. Sci. USA 2012, 109, 1-6.

[11] G. Knoer, Chem. Eur. J. 2009, 15, 568-578.

[12] L. Que, W. B. Tolman, Angew. Chem. Int. Ed. 2002, 41, 1114-1137.

[13] M. M. Georgiadis, H. Komiya, P. Chakrabarti, D. Woo, J. J. Kornuc, D. C. Rees, Science 1992, 257, 1653-1659.

[14] J. Kim, D. C. Rees, Science 1992, 257, 1677-1682.

[15] O. Einsle, F. A. Tezcan, S. L. A. Andrade, D. C. Rees, Science 2002, 3123, 16-21.

[16] K. C. Macleod, P. L. Holland, Nat. Chem. 2013, 5, 559-565.

[17] J. M. Modak, RESONANCE 2002, 69-77.

[18] S. Kozuch, S. Shaik, J. Phys. Chem. A 2008, 112, 6032-6041.

[19] R. Cammack, Nature 1999, 397, 214-215.

[20] A. Volbeda, M. Charon, C. Piras, E. Claude Hatchikian, M. Frey, J. C. Fontecilla-Camps, Nature 1995, 373, 580-587.

[21] A. Volbeda, E. Garcin, C. Piras, A. L. De Lacey, V. M. Fernandez, E. C. Hatchikian, M. Frey, J. C. Fontecilla-Camps, J. Am. Chem. Soc. 1996, 7863, 12989-12996.

[22] Y. Nicolet, C. Piras, P. Legrand, C. E. Hatchikian, J. C. Fontecilla-Camps, Structure 1999, 7, $13-$ 23.

[23] J. W. Peters, W. N. Lanzilotta, B. J. Lemon, L. C. Seefeldt, Science 1998, 282, 1853-1859.

[24] E. J. Lyon, I. P. Georgakaki, J. H. Reibenspies, M. Y. Darensbourg, Angew. Chem. Int. Ed. 1999, $38,3178-3180$.

[25] R. E. Stenkamp, Chem. Rev. 1994, 94, 715-726.

[26] W. H. Armstrong, A. Spool, G. C. Papaefthymiou, R. B. Frankel, S. J. Lippard, J. Am. Chem. Soc. 1984, 106, 3653-3667. 
[27] M. A. Holmes, R. E. Stenkamp, J. Mol. Biol. 1991, 220, 723-737.

[28] M. A. Holmes, I. Le Trong, S. Turley, L. C. Sieker, R. E. Stenkamp, J. Mol. Biol. 1991, 218, 583593.

[29] I. M. Klotz, T. A. Klotz, Science 1955, 121, 477-480.

[30] J. D. Lipscomb, Annu. Rev. Microbiol. 1994, 48, 371-399.

[31] M. Merkx, D. A. Kopp, M. H. Sazinsky, J. L. Blazyk, J. Müller, S. J. Lippard, Angew. Chem. Int. Ed. 2001, 40, 2787-2807.

[32] A. C. Rosenzweig, P. Nordlund, A. Frederick, P. M. Takahara, S. J. Lippard, Chem. Biol. 1995, 2, 409-418.

[33] J. Green, J. Biol. Chem. 1989, 264, 17698-17703.

[34] C. J. Haskin, N. Ravi, L. Que, Biochemistry 1995, 34, 11090-11098.

[35] D. M. Kurtz, Dalt. Trans. 2007, 4115-4121.

[36] T. Hayashi, J. D. Caranto, D. A. Wampler, D. M. Kurtz, P. Moenne-Loccoz, Biochemistry 2010, 49, 7040-7049.

[37] D. E. Coufal, P. Tavares, A. S. Pereira, B. H. Hyunh, S. J. Lippard, Biochemistry 1999, 38, 45044513.

[38] E. I. Solomon, D. E. Heppner, E. M. Johnston, J. W. Ginsbach, J. Cirera, M. F. Qayyum, M. T. Kieber-Emmons, C. H. Kjaergaard, R. G. Hadt, L. Tian, Chem. Rev. 2014, 114, 3659-3853.

[39] H. Michel, S. Iwata, C. Ostermeier, Curr Opin Str Biol. 1996, 6, 460-466.

[40] K. Brown, M. Tegoni, M. Prudêncio, A. S. Pereira, S. Besson, J. J. Moura, I. Moura, C. Cambillau, Nat. Struct. Biol. 2000, 7, 191-195.

[41] E. I. Solomon, J. W. Ginsbach, D. E. Heppner, M. T. Kieber-Emmons, C. H. Kjaergaard, P. J. Smeets, L. Tian, J. S. Woertink, Faraday Discuss. 2011, 148, 11-39.

[42] K. A. Magnus, B. H. H. Ton-that, C. Bonaventura, J. Bonaventura, W. G. J. Ho, Proteins Struct., Funct., Genet. 1994, 19, 302-309.

[43] K. E. Van Holde, K. I. Miller, J. Biol. Chem. 2001, 276, 15563-15567.

[44] C. Gerdemann, C. Eicken, B. Krebs, Acc. Chem. Res. 2002, 35, 183-191.

[45] H. Decker, R. Dillinger, F. Tuczek, Angew. Chem. Int. Ed. 2000, 39, 1591-1595.

[46] R. L. Lieberman, A. C. Rosenzweig, Nature 2005, 434, 177-182.

[47] R. L. Lieberman, D. B. Shrestha, P. E. Doan, B. M. Hoffman, T. L. Stemmler, A. C. Rosenzweig, Proc. Natl. Acad. Sci. USA 2002, 100, 3820-3825.

[48] S. I. Chan, K. H. Chen, S. S. Yu, C. Chen, S. S. Kuo, Biochemistry 2004, 43, 4421-4430.

[49] A. S. Hakemian, A. C. Rosenzweig, Annu. Rev. Biochem. 2007, 76, 223-241.

[50] S. Sirajuddin, A. C. Rosenzweig, Biochemistry 2015, 54, 2283-2294.

[51] G. Desimoni, G. Faita, K. A. Jørgensen, Chem. Rev. 2011, 111, 284-437. 
[52] M. Gomez, G. Muller, M. Rocamora, Coord. Chem. Rev. 1999, 193-195, 769-835.

[53] R. Rasappan, D. Laventine, O. Reiser, Coord. Chem. Rev. 2008, 252, 702-714.

[54] D. A. Evans, K. A. Woerpel, M. M. Hinman, M. M. Faul, J. Am. Chem. Soc. 1991, 113, 726-728.

[55] E. J. Corey, N. Imai, H. Zhang, J. Am. Chem. Soc. 1991, 113, 728-729.

[56] M. R. Netherton, G. C. Fu, Adv. Synth. Catal. 2004, 346, 1525-1532.

[57] A. Gissibl, M. G. Finn, O. Reiser, Org. Lett. 2005, 7, 2325-2328.

[58] M. P. Sibi, K. Kawashima, L. M. Stanley, Org. Lett. 2009, 11, 3894-3897.

[59] H. Fritschi, U. Leutenegger, A. Pfaltz, Helv. Chim. Acta 1988, 71, 1553-1565.

[60] U. Leutenegger, G. Umbricht, C. Fahrni, P. Von Matt, A. Pfaltz, Tetrahedron 1992, 48, $2143-$ 2156.

[61] S. Milione, V. Bertolasi, Tetrahedron Lett. 2011, 52, 3570-3574.

[62] H. Arii, F. Nakadate, K. Mochida, T. Kawashima, Organometallics 2011, 52, 4471-4474.

[63] B. Kovac, Chem. Commun., 1999, 3, 2455-2459.

[64] J.-P. Qu, Y. Liang, X. Hao, X.-L. Sun, Z.-X. Yu, Y. Tang, Chem. Eur. J. 2012, 18, 2196-2201.

[65] A. Walli, S. Dechert, F. Meyer, Eur. J. Org. Chem. 2013, 7044-7049.

[66] S. Dagorne, S. Bellemin-Laponnaz, A. Maisse-François, Eur. J. Inorg. Chem. 2007, 913-925.

[67] M. R. Douglass, M. Ogasawara, S. Hong, M. V Metz, T. J. Marks, Organometallics 2002, 21, 283-292.

[68] S. Hong, S. Tian, M. V Metz, T. J. Marks, J. Am. Chem. Soc. 2003, 125, 14768-14783.

[69] A. Alaaeddine, A. Amgoune, C. M. Thomas, S. Dagorne, S. Bellemin-Laponnaz, J. Carpentier, Eur. J. Inorg. Chem. 2006, 3652-3658.

[70] R. E. Lowenthal, A. Abiko, S. Masamune, Tetrahedron Lett. 1990, 31, 6005-6008.

[71] A. Walli, S. Dechert, M. Bauer, S. Demeshko, F. Meyer, Eur. J. Inorg. Chem. 2014, 2014, 46604676.

[72] E. I. Solomon, R. G. Hadt, Coord. Chem. Rev. 2011, 255, 774-789.

[73] D. W. Randall, D. R. Gamelin, L. B. Lacroix, E. I. Solomon, J. Biol. Inorg. Chem. 2000, 5, 16-19.

[74] E. I. Solomon, R. K. Szilagyi, S. D. George, L. Basumallick, Chem. Rev. 2004, 104, 419-458.

[75] W. H. Koppenol, D. M. Stanbury, P. L. Bounds, Free Radic. Biol. Med. 2010, 49, 317-322.

[76] E. I. Solomon, U. M. Sundaram, T. E. Machonkin, Chem. Rev. 1996, 96, 2563-2605.

[77] E. E. Chufán, S. C. Puiu, K. D. Karlin, Acc. Chem. Res. 2007, 40, 563-572.

[78] S. Ferguson-Miller, G. T. Babcock, Chem. Rev. 1996, 96, 2889-2907.

[79] E. A. Lewis, W. B. Tolman, Chem. Rev. 2004, 104, 1047-1076.

[80] E. I. Solomon, Chem. Rev. 1992, 92, 521-542. 
[81] M. Rolff, J. Schottenheim, H. Decker, F. Tuczek, Chem. Soc. Rev. 2011, 40, 4077.

[82] S. Itoh, S. Fukuzumi, Acc. Chem. Res. 2007, 40, 592-600.

[83] M. Metz, E. I. Solomon, J. Am. Chem. Soc. 2001, 123, 4938-4950.

[84] E. I. Solomon, R. Sarangi, J. S. Woertink, A. J. Augustine, Acc. Chem. Res. 2007, 40, 581-591.

[85] J. Ling, L. P. Nestor, R. S. Czernuszewicz, T. G. Spiro, R. Fraczkiewicz, K. D. Sharma, T. M. Loehr, J. S. J, J. Am. Chem. Soc. 1994, 116, 7682-7691.

[86] J. B. C. Papers, M. Doi, J.Biol. Chem. 2006, 281, 8981-8990.

[87] F. B. V. del Marmol, FEBS Lett. 1996, 381, 165-168.

[88] J. A. Halfen, S. Mahapatra, E. C. Wilkinson, S. Kaderli, V. G. Young, L. Que, A. D. Zuberbühler, W. B. Tolman, Science 1996, 271, 1397-1400.

[89] D. Maiti, J. S. Woertink, A. A. Narducci Sarjeant, E. I. Solomon, K. D. Karlin, Inorg. Chem. 2008, 47, 3787-3800.

[90] W. B. Tolman, Acc. Chem. Res. 1997, 30, 227-237.

[91] P. L. Holland, W. B. Tolman, Coord. Chem. Rev. 1999, 192, 855-869.

[92] J. Cahoy, P. L. Holland, W. B. Tolman, Inorg. Chem. 1999, 38, 2161-2168.

[93] M. J. Henson, P. Mukherjee, D. E. Root, T. D. P. Stack, E. I. Solomon, J. Am. Chem. Soc. 1999, 10332-10345.

[94] L. Tahsini, H. Kotani, Y.-M. Lee, J. Cho, W. Nam, K. D. Karlin, S. Fukuzumi, Chem. Eur. J. 2012, 18, 1084-1093.

[95] M. Rolff, J. Schottenheim, F. Tuczek, Chem. Soc. Rev. 2011, 40, 4077-4098.

[96] J. P. Klinman, Chem. Rev. 1996, 96, 2541-2561.

[97] L. M. Mirica, Science 2005, 308, 1890-1892.

[98] J. Schottenheim, C. Gernert, B. Herzigkeit, J. Krahmer, F. Tuczek, Eur. J. Inorg. Chem. 2015, 3501-3511.

[99] J. Serrano-Plana, I. Garcia-Bosch, A. Company, M. Costas, Acc. Chem. Res. 2015, 48, 2397-2406.

[100] O. Sander, A. Henß, C. Näther, C. Würtele, M. C. Holthausen, S. Schindler, F. Tuczek, Chem. Eur. J. 2008, 14, 9714-9729.

[101] M. Rolff, J. Schottenheim, G. Peters, F. Tuczek, Angew. Chem. Int. Ed. 2010, 49, 6438-6442.

[102] E. Pidcock, H. V. Obias, C. X. Zhang, K. D. Karlin, E. I. Solomon, J. Am. Chem. Soc. 1998, 120, 7841-7847.

[103] S. Palavicini, A. Granata, E. Monzani, L. Casella, J. Am. Chem. Soc. 2005, 127, 18031-18036.

[104] H. Decker, T. Schweikardt, F. Tuczek, Angew. Chem. 2006, 118, 4658-4663.

[105] S. Itoh, M. Taki, H. Nakao, P. L. Holland, W. B. Tolman, L. Que Jr., S. Fukuzumi, Angew. Chem. Int. Ed. 2000, 39, 398-400.

[106] G. Battaini, M. De Carolis, E. Monzani, F. Tuczek, L. Casella, Chem. Commun. 2003, 726-727. 
[107] P. E. M. Siegbahn, J. Biol. Inorg. Chem. 2003, 8, 567-576.

[108] N. Kitajima, K. Fujisawa, Y. Morooka, K. Toriumi, J. Am. Chem. Soc. 1989, 111, 8975-8976.

[109] K. E. Dalle, T. Gruene, S. Dechert, S. Demeshko, F. Meyer, J. Am. Chem. Soc. 2014, 136, 74287434.

[110] et al Karlin, K. D, J. Am. Chem. Soc. 1988, 110, 3690-3692.

[111] J. Zubieta, K. D. Karlin, J. Am. Chem. Soc. 1993, 115, 2677-2689.

[112] C. X. Zhang, S. Kaderli, M. Costas, E. Kim, Y. Neuhold, K. D. Karlin, A. D. Zuberbu, Inorg. Chem. 2003, 42, 1807-1824.

[113] Y. T. C. Corp, C. Abstr, C. He, J. L. Dubois, B. Hedman, K. O. Hodgson, S. J. Lippard, Angew. Chem. Int. Ed. 2001, 40, 1484-1487.

[114] D. Lee, N. Wei, N. N. Murthy, Z. Tyeklhr, K. D. Karlin, S. Kaderli, B. Jung, A. D. Zuberbiihler, J. Am. Chem. Soc. 1995, 117, 12498-12513.

[115] P. Comba, C. Katsichtis, W. Kiefer, A. Lienke, V. Nagel, H. Pritzkow, Chem. Eur. J. 1999, 5, $1716-1721$.

[116] J. A. Halfen, V. G. Young, W. B. Tolman, P. S. Se, J. Am. Chem. Soc. 1996, 118, 10920-10921.

[117] J. E. Bol, W. L. Driessen, R. Y. N. Ho, B. Maase, L. Que, J. Reedijk, Angew. Chem. Int. Ed. Int. Ed. 1997, 36, 998-1000.

[118] K. D. Karlin, N. Wei, B. Jug, S. Kaderli, P. Niklausf, A. D. Zuberbiihler, J. Am. Chem. Soc. 1993, $115,9506-9514$.

[119] N. Wei, N. N. Murthy, Z. Tyeklár, K. D. Karlin, Inorg. Chem. 1994, 33, 1177-1183.

[120] K. D. Karlin, D. Lee, S. Kaderli, Chem. Commun. 1997, 475-476.

[121] P. Comba, K. S. Hagen, M. Kerscher, H. Pritzkow, M. Schatz, S. Schindler, O. Walter, I. N. Feld, D.- Heidelberg, E. U. V, et al., Inorg. Chem. 2002, 41, 5440-5452.

[122] M. Schatz, M. Leibold, S. P. Foxon, M. Weitzer, F. W. Heinemann, F. Hampel, S. Schindler, Dalt. Trans. 2003, 1480-1487.

[123] M. Weitzer, M. Schatz, F. Hampel, W. Heinemann, S. Schindler, J. Chem. Soc., Dalt. Trans., 2002, 686-694.

[124] M. Becker, F. W. Heinemann, S. Schindler, Chem. Eur. J. 1999, 5, 3124-3129.

[125] M. Schatz, M. Becker, F. Thaler, F. Hampel, S. Schindler, R. R. Jacobson, Q. Chen, J. Zubieta, K. D. Karlin, Inorg. Chem. 2001, 40, 2312-2322.

[126] M. J. Baldwin, P. K. Ross, J. E. Pate, Z. Tyeklair, K. D. Karlin, I. Edward, J. Am. Chem. Soc. 1991, 113, 8671-8679.

[127] M. J. Henson, M. A. Vance, C. X. Zhang, H. Liang, K. D. Karlin, E. I. Solomon, J. Am. Chem. Soc. 2003, 125, 5186-5192.

[128] L. M. Berreau, J. A. Halfen, V. G. Young Jr, W. B. Tolman, Inorganica Chim. Acta 2000, 297, $115-128$.

[129] N. Wei, N. N. Murthy, K. D. Karlin, Inorg. Chem. 1994, 33, 6093-6100. 
[130] S. Kaderli, B. Jung, A. D. Zuberbuhler, Inorg. Chem. 1994, 33, 4625-4626.

[131] M. Weitzer, S. Schindler, G. Brehm, S. Schneider, E. Hoermann, B. Jung, S. Kaderli, A. D. Zuberbuehler, Inorg. Chem. 2003, 42, 1800-1806.

[132] L. M. Mirica, X. Ottenwaelder, T. D. P. Stack, Chem. Rev. 2004, 104, 1013-1045.

[133] T. Hoppe, S. Schaub, J. Becker, C. Würtele, S. Schindler, Angew. Chem. Int. Ed. 2013, 52, 870873.

[134] N. Kindermann, E. Bill, S. Dechert, S. Demeshko, E. J. Reijerse, F. Meyer, Angew. Chem. Int. Ed. 2015, 54, 1738-1743.

[135] H. Liang, K. D. Karlin, R. Dyson, S. Kaderli, B. Jung, A. D. Zuberbu, Inorg. Chem. 2000, 39, 5884-5894.

[136] N. Kitajima, K. Fujisawa, C. Fujimoto, Y. Morooka, S. Hashimoto, T. Kitagawa, K. Toriumi, K. Tatsumi, A. Nakamura, J. Am. Chem. Soc. 1992, 114, 1277-1291.

[137] L. M. Mirica, M. Vance, D. J. Rudd, B. Hedman, K. O. Hodgson, E. I. Solomon, T. D. P. Stack, J. Am. Chem. Soc. 2002, 124, 9332-3.

[138] S. Mahapatra, J. a Halfen, E. C. Wilkinson, G. Pan, X. Wang, V. G. Young Jr., C. J. Cramer, L. Que Jr., W. B. Tolman, J. Am. Chem. Soc. 1996, 118, 11555-11574.

[139] K. D. Karlin, Z. Tyeklar, A. Farooq, M. S. Haka, P. Ghosh, R. W. Cruse, Y. Gultneh, J. C. Hayes, P. J. Toscano, J. Zubieta, Inorg. Chem. 1992, 31, 1436-1451.

[140] H. V. Obias, Y. Lin, N. N. Murthy, E. Pidcock, E. I. Solomon, M. Ralle, N. J. Blackburn, Y. M. Neuhold, A. D. Zuberbuhler, K. D. Karlin, J. Am. Chem. Soc. 1998, 120, 12960-12961.

[141] Z. Hu, R. D. Williams, D. Tran, T. G. Spiro, S. M. Gorun, J. Am. Chem. Soc. 2000, 122, 35563557.

[142] M. Kodera, K. Katayama, Y. Tachi, K. Kano, S. Hirota, S. Fujinami, J. Am. Chem. Soc. 1999, 121, $11006-11007$.

[143] H. C. Liang, C. X. Zhang, M. J. Henson, R. D. Sommer, K. R. Hatwell, S. Kaderli, A. D. Zuberbühler, A. L. Rheingold, E. I. Solomon, K. D. Karlin, J. Am. Chem. Soc. 2002, 124, 41704171.

[144] B. M. Lam, J. A. Halfen, V. G. Young, J. R. Hagadorn, P. L. Holland, A. Lledós, L. CucurullSánchez, J. J. Novoa, S. Alvarez, W. B. Tolman, Inorg. Chem. 2000, 39, 4059-72.

[145] P. L. Holland, C. J. Cramer, E. C. Wilkinson, S. Mahapatra, K. R. Rodgers, S. Itoh, M. Taki, S. Fukuzumi, J. Que, Lawrence, W. B. Tolman, et al., J. Am. Chem. Soc. 2000, 122, 792-802.

[146] Z. Hu, G. N. George, S. M. Gorun, Inorg. Chem. 2001, 40, 4812-4813.

[147] K. D. Karlin, P. Ghosh, R. W. Cruse, A. Farooq, Y. Gultneh, R. R. Jacobson, N. J. Blackburn, R. W. Strange, J. Zubieta, J. Am. Chem. Soc. 1988, 110, 6769-6780.

[148] M. Cvetkovic, S. R. Batten, B. Moubaraki, K. S. Murray, L. Spiccia, Inorganica Chim. Acta 2001, $324,131-140$.

[149] K. D. Karlin, M. S. Haka, R. W. Cruse, G. J. Meyer, A. Farooq, Y. Gultneh, J. C. Hayes, J. Zubieta, J. Am. Chem. Soc. 1988, 110, 1196-1207. 
[150] K. D. Karlin, M. S. Haka, R. W. Cruse, J. Am. Chem. Soc. 1985, 107, 5828-5829.

[151] K. D. Karlin, M. S. Nasir, B. I. Cohen, R. W. Cruse, S. Kaderii, A. D. Zuberbiihlert, J. Am. Chem. Soc. 1994, 116, 1324-1336.

[152] N. Kitajima, Y. Moro-okaa, J. Chem. Soc., Chem. Comunn 1988, 1-2.

[153] N. Kitajima, I. T. Kodayt, S. Hashimoto, J. Am. Chem. Soc. 1991, 113, 5664-5671.

[154] M. S. Nasir, D. Mcgowty, J. Am. Chem. Soc. 1991, 113, 698-700.

[155] T. Osako, Y. Tachi, M. Taki, S. Fukuzumi, Inorg. Chem. 2001, 40, 6604-6609.

[156] E. Pidcock, H. V Obias, M. Abe, H. Liang, K. D. Karlin, E. I. Solomon, J. Am. Chem. Soc. 1999, $121,4263-4272$.

[157] L. Santagostini, M. Gullotti, E. Monzani, L. Casella, Á. Pavia, Chem. Eur. J. 2000, 6, 519-522.

[158] I. Sanyal, M. Mahroof-tahir, M. S. Nasir, P. Gbosh, B. I. Coben, Y. Gultneh, R. W. Cruse, A. Farooq, K. D. Karlin, S. Liu, Inorg. Chem. 1992, 31, 4322-4332.

[159] N. Tris, W. E. Lynch, D. M. Kurtz, S. Wang, R. A. Scott, J. Am. Chem. Soc. 1994, 116, 11030 11038.

[160] T. N. Sorrell, W. E. Allen, P. S. White, C. Hill, N. Carolina, Inorg. Chem. 1995, 34, 952-960.

[161] M. Taki, S. Teramae, S. Nagatomo, Y. Tachi, T. Kitagawa, S. Itoh, S. Fukuzumi, J. Am. Chem. Soc. 2002, 124, 6367-6377.

[162] Y. Funahashi, T. Nishikawa, Y. Wasada-Tsutsui, Y. Kajita, S. Yamaguchi, H. Arii, T. Ozawa, K. Jitsukawa, T. Tosha, S. Hirota, et al., J. Am. Chem. Soc. 2008, 130, 16444-16445.

[163] M. Kodera, Y. Kajita, Y. Tachi, K. Katayama, K. Kano, S. Hirota, S. Fujinami, M. Suzuki, Angew. Chem. Int. Ed. 2004, 42, 334-337.

[164] G. Y. Park, M. F. Qayyum, J. Woertink, K. O. Hodgson, B. Hedman, A. Sarjeant, E. I. Solomon, K. D. Karlin, J. Am. Chem. Soc. 2012, 2, 8513-8524.

[165] G. J. Karahalis, A. Thangavel, B. Chica, J. Bacsa, R. B. Dyer, C. C. Scarborough, Inorg. Chem. 2016, 55, 1102-1107.

[166] A. P. Cole, V. Mahadevan, L. M. Mirica, X. Ottenwaelder, T. D. P. Stack, Inorg. Chem. 2005, 44, 7345-7364.

[167] H. Hayashi, S. Fujinami, S. Nagatomo, S. Ogo, M. Suzuki, A. Uehara, Y. Watanabe, T. Kitagawa, J. Am. Chem. Soc. 2000, 122, 2124-2125.

[168] V. Mahadevan, Z. Hou, A. P. Cole, D. E. Root, T. K. Lal, E. I. Solomon, T. D. P. Stack, J. Am. Chem. Soc. 1997, 119, 11996-11997.

[169] S. Mahapatra, V. G. Young, S. Kaderli, A. D. Zuberbühler, W. B. Tolman, Angew. Chem. Int. Ed. 1997, 36, 130-133.

[170] M. Mizuno, H. Hayashi, S. Fujinami, H. Furutachi, S. Nagatomo, S. Otake, K. Uozumi, M. Suzuki, T. Kitagawa, Inorg. Chem. 2003, 42, 8534-8544.

[171] B. F. Straub, F. Rominger, P. Hofmann, Chem. Commun., 2000, 3, 1611-1612.

[172] M. Enomoto, T. Aida, J. Am. Chem. Soc. 1999, 121, 874-875. 
[173] S. Itoh, M. Taki, H. Nakao, P. L. Holland, W. B. Tolman, L. Que, S. Fukuzumi, Angew. Chem. Int. Ed. 2000, 112, 409-411.

[174] D. J. E. Spencer, N. W. Aboelella, A. M. Reynolds, P. L. Holland, W. B. Tolman, J. Am. Chem. Soc. 2002, 124, 2108-2109.

[175] S. Mahapatra, J. A. Halfen, E. C. Wilkinson, G. Pan, C. J. Cramer, L. Que, W. B. Tolman, M. Minnesorn, J. Am. Chem. Soc. 1995, 117, 8865-8866.

[176] N. W. Aboelella, E. A. Lewis, A. M. Reynolds, W. W. Brennessel, C. J. Cramer, W. B. Tolman, J. Am. Chem. Soc. 2002, 124, 10660-10661.

[177] D. J. E. Spencer, A. M. Reynolds, P. L. Holland, B. A. Jazdzewski, C. Duboc-Toia, L. Le Pape, S. Yokota, Y. Tachi, S. Itoh, W. B. Tolman, Inorg. Chem. 2002, 41, 6307-21.

[178] B. F. Straub, F. Rominger, P. Hofmann, Inorg. Chem. Commun. 2000, 3, 214-217.

[179] V. Mahadevan, J. L. DuBois, B. Hedman, K. O. Hodgson, T. D. P. Stack, J. Am. Chem. Soc. 1999, $121,5583-5584$.

[180] L. M. Berreau, S. Mahapatra, J. A. Halfen, V. G. Young, W. B. Tolman, P. S. Se, R. V March, Inorg. Chem. 1996, 35, 6339-6342.

[181] C. J. Cramer, B. A. Smith, W. B. Tolman, J. Am. Chem. Soc. 1996, 118, 11283-11287.

[182] A. Berces, Inorg. Chem. 1997, 36, 4831-4837.

[183] V. Mahadevan, M. J. Henson, E. I. Solomon, T. D. P. Stack, J. Am. Chem. Soc. 2000, 122, 1024910250.

[184] T. D. P. Stack, Dalt. Trans. 2003, 1881-1889.

[185] M. J. Henson, M. A. Vance, C. X. Zhang, H. Liang, K. D. Karlin, E. I. Solomon, J. Am. Chem. Soc. 2003, 125, 5186-5192.

[186] S. Mahapatra, J. A. Halfen, W. B. Tolman, J. Am. Chem. Soc. 1996, 7863, 11575-11586.

[187] C. E. Tinberg, S. J. Lippard, Acc. Chem. Res. 2011, 44, 280-288.

[188] M. A. Culpepper, G. E. Cutsail, B. M. Hoffman, A. C. Rosenzweig, J. Am. Chem. Soc. 2012, 134, 7640-7643.

[189] J. S. Woertink, P. J. Smeets, M. H. Groothaert, M. A. Vance, B. F. Sels, R. A. Schoonheydt, E. I. Solomon, Proc. Natl. Acad. Sci. USA 2009, 106, 18908-18913.

[190] C. Citek, J. B. Gary, E. C. Wasinger, T. D. P. Stack, J. Am. Chem. Soc. 2015, 137, 6991-6994.

[191] S. Itoyama, K. Doitomi, T. Kamachi, Y. Shiota, K. Yoshizawa, Inorg. Chem. 2016, DOI: 10.1021/acs.inorgchem.5b02603.

[192] H. Decker, T. Schweikardt, F. Tuczek, Angew. Chem. Int. Ed. 2006, 45, 4546-4550.

[193] E. W. Peterson, J. Biol. Chem. 1959, 234, 3291.

[194] J. Munoz-Munoz, F. Garcia-Molina, R. Varon, P. A. Garcia-Ruiz, J. Tudela, F. Garcia-Canovas, J. Rodriguez-Lopez, IUMBM Life 2010, 62, 539-547.

[195] H. Decker, N. Hellmann, E. Jaenicke, B. Lieb, U. Meissner, J. Markl, Integr. Comp. Biol. 2007, 47, 631-644. 
[196] L. Q. Hatcher, K. D. Karlin, J. Biol. Inorg. Chem. 2004, 9, 669-683.

[197] T. Inoue, Y. Shiota, K. Yoshizawa, J. Am. Chem. Soc. 2008, 130, 16890-16897.

[198] S. Itoh, H. Kumei, M. Taki, S. Nagatomo, T. Kitagawa, S. Fukuzumi, J. Am. Chem. Soc. 2001, 123, 6708-6709.

[199] K. D. Karlin, J. C. Hayes, Y. Gultneh, R. W. Cruse, J. W. McKown, J. P. Hutchinson, J. Zubieta, J. Am. Chem. Soc. 1984, 106, 2121-2128.

[200] L. Casella, E. Monzani, C. Generale, V. Taramelli, M. Gullotti, D. Cavagnino, G. Cerina, L. Santagostini, R. Ugo, C. Inorganica, et al., Inorg. Chem. 1996, 35, 7516-7525.

[201] A. Hoffmann, C. Citek, S. Binder, A. Goos, M. Rübhausen, E. C. Wasinger, T. D. P. Stack, O. Troeppner, I. Ivanovic, S. Herres-Pawlis, Angew. Chem. Int. Ed. 2013, 52, 5398-5401.

[202] M. Reglier, C. Jorand, B. Waegell, J. Chem. Soc. Chem. Comunn 1990, 1752-1755.

[203] L. Casella, M. Gullotti, R. Radaelli, P. Di Gennaro, J. Chem. Soc., Chem. Comunn 1991, 16111612.

[204] J. Schottenheim, C. Gernert, B. Herzigkeit, J. Krahmer, F. Tuczek, Eur. J. Inorg. Chem. 2015, 3501-3511.

[205] T. Osako, K. Ohkubo, M. Taki, Y. Tachi, S. Fukuzumi, S. Itoh, J. Am. Chem. Soc. 2003, 125, 11027-11033.

[206] L. M. Mirica, M. Vance, D. J. Rudd, B. Hedman, K. O. Hodgson, E. I. Solomon, T. D. P. Stack, Science 2005, 308, 1890-1892.

[207] A. Company, L. Que, E. V Rybak-akimova, L. Casella, X. Ribas, Chem. Eur. J. 2008, 14, 35353538.

[208] S. Herres-Pawlis, P. Verma, R. Haase, P. Kang, C. T. Lyons, E. C. Wasinger, U. Floerke, G. Henkel, T. D. P. Stack, J. Am. Chem. Soc. 2009, 131, 1154-1169.

[209] C. Wu, O. Sander, V. Lutz, T. Waitz, F. Tuczek, S. Schindler, J. Am. Chem. Soc. 2009, 131, 75447545.

[210] H. R. Lucas, L. Li, A. A. N. Sarjeant, M. A. Vance, E. I. Solomon, K. D. Karlin, J. Am. Chem. Soc. 2009, 131, 3230-3245.

[211] I. Garcia-bosch, A. Company, J. R. Frisch, M. Torrent-sucarrat, M. Cardellach, I. Gamba, M. Güell, L. Casella, L. Que, X. Ribas, et al., Angew. Chem. Int. Ed. 2010, 49, 2406-2409.

[212] M. T. Kieber-emmons, J. W. Ginsbach, P. K. Wick, H. R. Lucas, M. E. Helton, B. Lucchese, M. Suzuki, A. D. Zuberbühler, K. D. Karlin, E. I. Solomon, Angew. Chem. Int. Ed. 2014, 53, 49354939.

[213] C. Citek, S. Herres-Pawlis, T. D. P. Stack, Acc. Chem. Res. 2015, 48, 2424-2433.

[214] M. T. Kieber-Emmons, J. W. Ginsbach, P. K. Wick, H. R. Lucas, M. E. Helton, B. Lucchese, M. Suzuki, A. D. Zuberbühler, K. D. Karlin, E. I. Solomon, Angew. Chem. Int. Ed. 2014, 53, 49354939.

[215] N. Kindermann, S. Dechert, S. Demeshko, F. Meyer, J. Am. Chem. Soc. 2015, 137, 8002-8005.

[216] N. C. Eickman, R. S. Himmelwright, E. I. Solomont, Proc. Natl. Acad. Sci. USA 1979, 76, $2094-$ 
2098.

[217] L. M. Mirica, D. J. Rudd, M. a. Vance, E. I. Solomon, K. O. Hodgson, B. Hedman, T. D. P. Stack, J. Am. Chem. Soc. 2006, 128, 2654-2665.

[218] C. R. Andrew, H. Yeom, S. Valentine, B. G. Karlsson, N. Bonander, G. Van Pouderoyen, G. W. Canters, T. M. Loehr, J. Sanders-Loehr, J. Am. Chem. Soc. 1994, 116, 11489-11498.

[219] N. C. Eickman, E. I. Solomon, J. A. Larrabee, T. G. Spiro, K. Lerch, J. Am. Chem. Soc. 1978, 100, 6529-6531.

[220] K. D. Karlin, Z. Tyeklár, A. Farooq, R. R. Jacobson, E. Sinn, D. W. Lee, J. E. Bradshaw, L. J. Wilson, Inorganica Chim. Acta 1991, 182, 1-3.

[221] M. J. Baldwin, D. E. Root, J. E. Pate, K. Fujisawa, N. Kitajima, E. I. Solomon, J. Am. Chem. Soc. 1992, 114, 10421-10431.

[222] B. Hazes, K. A. Magnu, C. Bonaventura, J. Bonaventura, Z. Dauter, K. O. R. H. Kalk, W. I. M. G. J. Hol, Proteins Sci. 1993, 2, 597-619.

[223] K. A. Magnus, H. Ton-That, J. E. Carpenter, Chem. Rev. 1994, 94, 727-735.

[224] K. I. Miller, M. E. Cuff, W. F. Lang, P. Varga-weisz, K. G. Field, K. E. Van Holde, J. Mol. Biol. 1998, 278, 827-842.

[225] A. Walli, Biomimetic Copper(I)-Mediated Activation of Dioxygen and Redox Non-Innocence in Copper(II) Complexes of Bis(oxazoline)s, University of Goettingen, 2014.

[226] P. K. Ross, E. I. Solomon, J. Am. Chem. Soc. 1991, 113, 3246-3259.

[227] E. I. Solomon, F. Tuczek, D. E. Root, C. A. Brown, Chem. Rev. 1994, 94, 827-856.

[228] J. L. Dubois, P. Mukherjee, A. M. Collier, J. M. Mayer, E. I. Solomon, B. Hedman, T. D. P. Stack, K. O. Hodgson, S. U. V, S. U. V, et al., J. Am. Chem. Soc. 1997, 119, 8578-8579.

[229] B. C. Dave, R. S. Czernuszewicz, M. R. Bond, C. J. Carranot, Inorg. Chem. 1993, 32, 3593-3594.

[230] E. C. Wilkinson, Y. Dong, Y. Zang, H. Fujii, R. Fraczkiewicz, G. Fraczkiewicz, R. S. Czernuszewicz, L. Que, J. Am. Chem. Soc. 1998, 120, 955-962.

[231] Y. Dong, H. Fujii, M. P. Hendrichj, R. A. Leising, G. Pan, C. R. Randall, E. C. Wilkinson, Y. Zang, L. Que, G. F. Karl, et al., J. Am. Chem. Soc. 1995, 117, 2778-2792.

[232] N. Nakanishi, F. Takeuchi, M. Tsubaki, J.Biochem. 2007, 142, 553-560.

[233] E. Fadda, N. Chakrabarti, R. Pomes, J. Phys. Chem. B 2005, 109, 22629-22640.

[234] Y. Lin, C. Lim, J. Am. Chem. Soc. 2004, 126, 2602-2612.

[235] J. J. Warren, T. A. Tronic, J. M. Mayer, Chem. Rev. 2010, 110, 6961-7001.

[236] J. Warren, J. M. Mayer, Biochemistry 2015, 54, 1863-1878.

[237] A. Albers, S. Demeshko, S. Dechert, C. T. Saouma, J. M. Mayer, F. Meyer, J. Am. Chem. Soc. 2014, 136, 3946-3954.

[238] K. Hsueh, W. M. Westler, J. L. Markley, J. Am. Chem. Soc. 2010, 132, 7908-7918.

[239] Y. Zu, M. M. Couture, D. R. J. Kolling, A. R. Crofts, L. D. Eltis, J. A. Fee, J. Hirst, V. La, 
Biochemistry 2003, 42, 12400-12408.

[240] S. Friedle, S. J. Lippard, Chem. Soc. Rev. 2010, 39, 2768-2779.

[241] E. I. Solomon, T. C. Brunold, M. I. Davis, J. N. Kemsley, S. Lee, N. Lehnert, F. Neese, A. J. Skulan, Y. Yang, J. Zhou, Chem. Rev. 2000, 100, 235-349.

[242] E. I. Solomon, P. Chen, M. Metz, S. Lee, A. E. Palmer, Angew. Chem. Int. Ed. 2001, 40, 45704590.

[243] L. Que Jr, W. B. Tolman, Nature 2008, 455, 333-340.

[244] T. L. Poulos, Chem. Rev. 2014, 114, 3919-3962.

[245] R. Banerjee, Y. Proshlyakov, J. D. Lipscomb, D. A. Proshlyakov, Nature 2015, 518, 431-434.

[246] K. Ray, F. Felix, B. Wang, W. Nam, J. Am. Chem. Soc. 2014, 136, 13942-13958.

[247] B. Burger, S. Dechert, C. Große, S. Demeshko, F. Meyer, Chem. Commun. 2011, 47, 1042810430.

[248] M. Costas, C. W. Cady, S. V Kryatov, M. Ray, M. J. Ryan, E. V Rybak-akimova, L. Que, Inorg. Chem. 2003, 42, 7519-7530.

[249] Y. Dong, S. Menage, B. A. Brennan, T. E. Elgren, H. G. Jang, L. L. Pearce, L. Que, J. Am. Chem. Soc. 1993, 115, 1851-1859.

[250] P. Reichard, A. Ehrenberg, Science 1979, 221, 514-519.

[251] P. Nordlund, B. Sjoeberg, H. Ecklund, Nature 1990, 345, 593-598.

[252] J. M. Bollinger, D. E. Edmondson, B. H. Huynh, J. Filley, J. R. Norton, J. Stubbe, Science 1991, 253, 292-298.

[253] S. J. Blanksby, G. B. Ellison, Acc. Chem. Res. 2003, 36, 255-263.

[254] B. J. Colby, D. I. Stirling, H. Dalton, Biochem. J 1977, 165, 395-402.

[255] Y. Liu, J. C. Nesheim, K. E. Paulsen, M. T. Stankovich, J. D. Lipscomb, Biochemistry 1997, 36, 5223-5233.

[256] M. Sazinksy, S. J. Lippard, Acc. Chem. Res. 2006, 39, 558-566.

[257] J. Colby, H. Dalton, Biochem. J 1976, 157, 495-497.

[258] J. Lund, M. P. Woodland, H. Dalton, Eur. J. Biochem. 1985, 147, $297-305$.

[259] J. Green, H. Dalton, J. Biol. Chem. 1985, 29, 15795-15801.

[260] B. G. Fox, J. G. Borneman, L. P. Wackett, J. D. Lipscomb, Biochemistry 1990, 29, 6419-6427.

[261] S. Lee, B. G. Fox, W. A. Froland, J. D. Lipscomb, E. Muenck, J. Am. Chem. Soc. 1993, 115, 6450-6451.

[262] K. E. Liu, A. M. Valentine, D. Wang, B. Hanh, D. E. Edmondson, A. Salifoglou, S. J. Lippard, J. Am. Chem. Soc. 1995, 117, 10174-10185.

[263] C. E. Tinberg, S. J. Lippard, Biochemistry 2009, 48, 12145-12158.

[264] R. Banerjee, K. K. Meier, J. D. Lipscomb, Biochemistry 2013, 52, 4331-4342. 
[265] K. Kim, S. J. Lippard, J. Am. Chem. Soc. 1996, 118, 4914-4915.

[266] T. Ookubo, H. Sugimoto, J. Am. Chem. Soc. 1996, 118, 701-702.

[267] Y. Dong, S. Yan, V. G. Young, L. Que, Angew. Chem. Int. Ed. 1996, 35, 618-620.

[268] D. Wang, E. . Farquhar, A. Stbna, E. Muenck, L. Que, Nat. Chem. 2009, 1, 145-150.

[269] B. R. L. Rardin, A. Bino, P. Poganiuch, W. B. Tolman, S. Liu, S. J. Lippard, Angew. Chem. Int. Ed. 1990, 29, 812-814.

[270] W. H. Armstrong, S. J. Lippard, J. Am. Chem. Soc. 1983, 105, 4837-4838.

[271] W. Gebert, K. Pohl, K. Wieghardt, Angew. Chem. Int. Ed. 1983, 9, 727.

[272] J. R. Hartman, R. L. Rardin, P. Chaudhuri, I. K. Pohl, K. Wieghardt, I. B. Nuber, J. Weiss, G. C. Papaefthymiou, R. B. Frankel, S. J. Lippard, J. Am. Chem. Soc. 1987, 109, 7387-7396.

[273] I. Siewert, C. Limberg, Chem. Eur. J. 2009, 15, 10316-10328.

[274] E. Y. Tshuva, S. J. Lippard, Chem. Rev. 2004, 104, 987-1012.

[275] L. H. Do, S. J. Lippard, J. Am. Chem. Soc. 2011, 133, 10568-10581.

[276] B. Burger, S. Demeshko, E. Bill, S. Dechert, F. Meyer, Angew. Chem. Int. Ed. 2012, 51, 1004510049.

[277] A. R. Mcdonald, L. Que, Coord. Chem. Rev. 2013, 257, 414-428.

[278] T. C. Brunold, N. Tamura, N. Kitajima, Y. Moro-oka, E. I. Solomon, J. Am. Chem. Soc. 1998, $120,5674-5690$.

[279] S. Menage, B. A. Brennan, J. Am. Chem. Soc. 1990, 112, 6423-6425.

[280] K. E. Liu, A. M. Valentine, D. Qiu, D. E. Edmondson, E. H. Appelman, T. G. Spire, S. J. Lippard, J. Am. Chem. Soc. 1995, 117, 4997-4998.

[281] H. Zheng, S. J. Yoo, E. Muenck, L. Que Jr, J. Am. Chem. Soc. 2000, 122, 3789-3790.

[282] G. Xue, D. Wang, R. De Hont, A. T. Fiedler, X. Shan, E. Mu, L. Que, Proc. Natl. Acad. Sci. USA 2007, 104, 20713-20718.

[283] G. Xue, E. Muenck, L. Que, Proc. Natl. Acad. Sci. USA 2008, 105, 20615-20620.

[284] R. F. De Hont, G. Xue, M. P. Hendrich, L. Que, E. L. Bominaar, M. Eckard, Inorg. Chem. 2010, $49,8310-8322$.

[285] G. Xue, R. Hont, E. Muenck, L. Que, Nat. Chem. 2010, 2, 400-405.

[286] G. Xue, C. Geng, S. Ye, A. Fiedler, F. Neese, L. Que, Inorg. Chem. 2013, 52, 3976-3984.

[287] S. A. Stoian, G. Xue, E. L. Bominaar, L. Que, J. Am. Chem. Soc. 2014, 136, 1545-1558.

[288] M. Kodera, M. Itoh, K. Kano, Angew. Chem. Int. Ed. 2005, 44, 7104-7106.

[289] M. Kodera, Y. Kawahara, Y. Hitomi, T. Nomura, T. Ogura, Y. Kobayashi, J. Am. Chem. Soc. 2012, 134, 13226-13239.

[290] M. Kodera, T. Tsuji, T. Yasunaga, Y. Kawahara, T. Hirano, Y. Hitomi, Chem. Sci. 2014, 5, $2282-$ 2292. 
[291] T. C. Berto, A. L. Speelman, S. Zheng, N. Lehnert, Coord. Chem. Rev. 2013, 257, 244-259.

[292] E. Colutta, D. Koshland, Science 1992, 258, 1862-1865.

[293] S. Lu, E. Libby, L. Saleh, G. Xing, J. M. Bollinger, M. Pierre, J. Biol. Inorg. Chem. 2004, 9, 818827.

[294] J. D. Caranto, A. Weitz, M. P. Hendrich, D. M. Kurtz, J. Am. Chem. Soc. 2014, 136, 7981-7992.

[295] T. Hino, Y. Matsumoto, S. Nagano, H. Sugimoto, Y. Fukumori, T. Murata, S. Iwata, Y. Shiro, Science 2010, 330, 1666-1670.

[296] C. G. Timotoe, A. S. Pereira, C. E. Martins, S. G. Naik, G. Duarte, J. G. Moura, P. Tavares, B. H. Huynh, I. Moura, Biochemistry 2011, 50, 4251-4262.

[297] Y. Matsumoto, T. Tosha, A. V Pisliakov, T. Hino, H. Sugimoto, S. Nagano, Y. Sugita, Y. Shiro, Nat. Struct. Mol. Biol. 2012, 19, 238-245.

[298] T. A. Missall, J. K. Lodge, J. E. Mcewen, Eukaryot. Cell 2004, 3, 835-846.

[299] A. Baggf, J. B. Neilands, Biochemistry 1987, 26, 5471-5477.

[300] B. D'Autreaux, D. Touati, B. Bersch, J. Latour, I. Michaud-soret, Proc. Natl. Acad. Sci. USA 2002, 99, 16619-16624.

[301] C. Bogdan, Nat. Imunol. 2001, 2, 907-916.

[302] K. Hantke, Curr. Opin. Microbiol. 2001, 4, 172-177.

[303] V. de Lorenzo, F. Giovanninis, M. Herrero, J. . Neilands, J. Mol. Biol. 1988, 203, 875-884.

[304] M. J. Crawford, D. E. Goldberg, J. Biol. Chem. 1998, 273, 34028-34032.

[305] P. Mukhopadhyay, M. Zheng, L. A. Bedzyk, R. A. Larossa, G. Storz, Proc. Natl. Acad. Sci. USA 2004, 101, 745-750.

[306] H. Ding, B. Demple, Proc. Natl. Acad. Sci. USA 2000, 97, 5146-5150.

[307] T. C. Harrop, Z. J. Tonzetich, E. Reisner, S. J. Lippard, J. Am. Chem. Soc. 2008, 130, 1560215610 .

[308] L. Jacquamet, C. Jeandey, D. Aberdam, J. Hazemann, J. Am. Chem. Soc. 2000, 122, 394-395.

[309] B. D'Autreaux, O. Horner, J. Oddou, C. Jeandey, S. Gambarelli, C. Berthomieu, J. Latour, I. Michaud-soret, A. Ii, D. Autre, J. Am. Chem. Soc. 2004, 126, 6005-6016.

[310] A. L. Feig, M. T. Bautista, S. J. Lippard, Inorg. Chem. 1996, 35, 6892-6898.

[311] A. Majumdar, S. J. Lippard, Inorg. Chem. 2013, 52, 13292-13294.

[312] Y. Jiang, T. Hayashi, H. Matsumura, L. H. Do, A. Majumdar, S. J. Lippard, P. Moe, J. Am. Chem. Soc. 2014, 136, 12524-12527.

[313] S. Zheng, T. C. Berto, E. W. Dahl, M. B. Ho, A. L. Speelman, N. Lehnert, J. Am. Chem. Soc. 2013, 135, 4902-4905.

[314] A. L. Speelman, N. Lehnert, Acc. Chem. Res. 2014, 47, 1106-1116.

[315] M. Tsai, C. Tsou, W. Liaw, Acc. Chem. Res. 2015, 48, 1184-1193. 
[316] M. Hung, M. Tsai, G. Lee, W. Liaw, Inorg. Chem. 2006, 45, 6041-6047.

[317] M. Tsai, C. Hsieh, W. Liaw, Inorg. Chem. 2007, 46, 5110-5117.

[318] C. Tsou, T. Lu, W. Liaw, J. Am. Chem. Soc. 2007, 129, 12626-12627.

[319] Y. Chen, W. Ku, L. Feng, M. Tsai, C. Hsieh, J. Am. Chem. Soc. 2008, 130, 10929-10938.

[320] F. Tsai, T. Kuo, W. Liaw, J. Am. Chem. Soc. 2009, 131, 3426-3427.

[321] M. Tsai, F. Tsai, T. Lu, M. Tsai, Y. Wei, I. Hsu, J. Lee, Inorg. Chem. 2009, 48, 9579-9591.

[322] T. Lu, C. Chen, W. Liaw, Chem. Eur. J. 2010, 16, 8088-8095.

[323] C. Tsou, W. Liaw, Chem. Eur. J. 2011, 17, 13358-13366.

[324] F. Tsai, P. Chen, W. Liaw, J. Am. Chem. Soc. 2010, 132, 5290-5299.

[325] F. Tsai, Y. Lee, M. Chiang, W. Liaw, Inorg. Chem. 2013, 52, 464-473.

[326] C. Tsou, W. Yang, W. Liaw, J. Am. Chem. Soc. 2013, 135, 18758-18761.

[327] A. Correa, O. Garcia Mancheno, C. Bolm, Chem. Soc. Rev. 2008, 37, 1108-1117.

[328] R. Ferro, S. Milione, V. Bertolasi, C. Capacchione, A. Grassi, D. Chimica, V. Uni, F. Salerno, D. Chimica, Macromolecules 2007, 40, 8544-8546.

[329] S. Zhu, Y. Cai, H. Mao, J. Xie, Q. Zhou, Nat. Chem. 2010, 2, 546-551.

[330] K. S. Williamson, T. P. Yoon, J. Am. Chem. Soc. 2012, 134, 12370-12373.

[331] T. Inagaki, L. T. Phong, A. Furuta, J. Ito, Chem. Eur. J. 2010, 16, 3090-3096.

[332] H. Nishiyama, A. Furuta, Chem. Commun. 2007, 760-762.

[333] A. M. Tondreau, J. M. Darmon, B. M. Wile, S. K. Floyd, E. Lobkovsky, P. J. Chirik, Organometallics 2009, 28, 3928-3940.

[334] G. Guillemot, M. Neuburger, A. Pfaltz, Chem. Eur. J. 2007, 13, 8960-8970.

[335] V. C. Gibson, R. K. O. Reilly, W. Reed, D. F. Wass, J. P. White, D. J. Williams, Chem. Commun. 2002, 2, 1850-1851.

[336] S. C. Bart, E. J. Hawrelak, A. K. Schmisseur, E. Lobkovsky, P. J. Chirik, Organometallics 2004, 23, 237-246.

[337] T. Lu, S. Chiou, C. Chen, W. Liaw, Inorg. Chem. 2006, 45, 1223-1227.

[338] S. M. Brothers, M. Y. Darensbourg, M. B. Hall, Inorg. Chem. 2011, 50, 8532-8540.

[339] S. Ye, F. Neese, J. Am. Chem. Soc. 2010, 132, 3646-3647.

[340] M. M. Ti, J. J. Curley, G. Bergman, T. D. Tilley, Dalt. Trans. 2012, 41, 192-200.

[341] W. Haberditzl, Angew. Chem. Int. Ed. 1966, 5, 288-298.

[342] G. A. Bain, J. F. Berry, J. Chem. Eductaion 2008, 85, 1-5.

[343] G. M. Sheldrick, Acta Cryst. 2015, C71, 3-8.

[344] S. Dagrone, S. Bellemin-Laponnaz, R. Welter, Organometallics 2004, 23, 3053-3061. 


\section{Crystallographic Data}

Table A1: Crystal data and refinement details for 1,3 and 4

\begin{tabular}{|c|c|c|c|}
\hline compound & 1 & 3 & 4 \\
\hline empirical formula & $\mathrm{C}_{20} \mathrm{H}_{27} \mathrm{CuF}_{6} \mathrm{~N}_{3} \mathrm{O}_{2} \mathrm{P}$ & $\mathrm{C}_{13} \mathrm{H}_{21} \mathrm{CuF}_{6} \mathrm{~N}_{3} \mathrm{O}_{2} \mathrm{P}$ & $\mathrm{C}_{56} \mathrm{H}_{94} \mathrm{Cl}_{4} \mathrm{Cu}_{4} \mathrm{~N}_{10} \mathrm{O}_{24}$ \\
\hline formula weight & 549.95 & 459.84 & 1687.37 \\
\hline$T[\mathrm{~K}]$ & $133(2)$ & $133(2)$ & $133(2)$ \\
\hline crystal size $\left[\mathrm{mm}^{3}\right]$ & $0.500 \times 0.500 \times 0.090$ & $0.180 \times 0.090 \times 0.080$ & $0.500 \times 0.500 \times 0.320$ \\
\hline crystal system & monoclinic & monoclinic & triclinic \\
\hline space group & $P 2_{1} / c$ & $P 2_{1}$ & $P-1$ \\
\hline$a[\AA]$ & $9.8192(3)$ & $10.1613(14)$ & $12.2247(6)$ \\
\hline$b[\AA]$ & $15.0241(5)$ & $6.0778(10)$ & $12.3904(5)$ \\
\hline$c[\AA]$ & $16.3164(6)$ & $14.898(2)$ & $15.0274(7)$ \\
\hline$\alpha\left[^{\circ}\right]$ & 90 & 90 & $106.670(4)$ \\
\hline$\beta\left[^{\circ}\right]$ & $99.576(3)$ & $93.521(12)$ & $96.765(4)$ \\
\hline$\gamma\left[{ }^{\circ}\right]$ & 90 & 90 & $118.889(3)$ \\
\hline$V\left[\vec{\AA}^{3}\right]$ & $2373.53(14)$ & $918.3(2)$ & $1817.79(16)$ \\
\hline$Z$ & 4 & 2 & 1 \\
\hline$\rho\left[\mathrm{g} / \mathrm{cm}^{3}\right]$ & 1.539 & 1.663 & 1.541 \\
\hline$F(000)$ & 1128 & 468 & 876 \\
\hline$\mu\left[\mathrm{mm}^{-1}\right]$ & 1.057 & 1.347 & 1.381 \\
\hline$T_{\min } / T_{\max }$ & $0.5459 / 0.9119$ & $0.8345 / 0.9077$ & $0.5289 / 0.7235$ \\
\hline$\theta$-range $\left[{ }^{\circ}\right]$ & $1.855-26.737$ & $1.369-26.058$ & $1.486-26.739$ \\
\hline$h k l$-range & $\pm 12, \pm 18, \pm 20$ & $\pm 12, \pm 7, \pm 18$ & $\pm 15, \pm 15, \pm 18$ \\
\hline measured refl. & 29719 & 10755 & 25354 \\
\hline unique refl. $\left[R_{\text {int }}\right]$ & $5022[0.0354]$ & $10755[?]$ & $7693[0.0396]$ \\
\hline observed refl. $(I>2 \sigma(I))$ & 4542 & 8791 & 6079 \\
\hline data / restraints / param. & $5022 / 0 / 304$ & 10755 / 133 / 241 & $7693 / 58 / 471$ \\
\hline goodness-of-fit $\left(F^{2}\right)$ & 1.038 & 1.073 & 0.953 \\
\hline$R 1, w R 2(I>2 \sigma(I))$ & $0.0281,0.0718$ & $0.0719,0.1519$ & $0.0339,0.0758$ \\
\hline$R 1, w R 2$ (all data) & $0.0324,0.0738$ & $0.0929,0.1804$ & $0.0490,0.0799$ \\
\hline resid. el. dens. $\left[\mathrm{e} / \AA^{3}\right]$ & $-0.366 / 0.374$ & $-1.072 / 0.975$ & $-0.276 / 0.533$ \\
\hline
\end{tabular}


Table A2: Crystal data and refinement details for 6,8 and $\mathbf{1 0}$

\begin{tabular}{|c|c|c|c|}
\hline compound & 6 & 8 & 10 \\
\hline empirical formula & $\mathrm{C}_{36} \mathrm{H}_{64} \mathrm{Cl}_{2} \mathrm{Cu}_{2} \mathrm{~N}_{4} \mathrm{O}_{17}$ & $\mathrm{C}_{40} \mathrm{H}_{71} \mathrm{Cl}_{2} \mathrm{Cu}_{2} \mathrm{~N}_{5} \mathrm{O}_{17}$ & $\mathrm{C}_{34} \mathrm{H}_{58} \mathrm{Cu}_{2} \mathrm{~N}_{4} \mathrm{O}_{8}$ \\
\hline formula weight & 1022.89 & 1091.99 & 777.92 \\
\hline$T[\mathrm{~K}]$ & $133(2)$ & $133(2)$ & $133(2)$ \\
\hline crystal size $\left[\mathrm{mm}^{3}\right]$ & $0.480 \times 0.200 \times 0.180$ & $0.390 \times 0.350 \times 0.330$ & $0.500 \times 0.480 \times 0.270$ \\
\hline crystal system & monoclinic & monoclinic & orthorhombic \\
\hline space group & $P 2_{1} / c$ & $P 2_{1} / c$ & Pnna \\
\hline$a[\AA]$ & $16.6993(5)$ & $10.9869(6)$ & $14.5909(4)$ \\
\hline$b[\AA]$ & $15.6606(5)$ & $18.6852(10)$ & $12.6902(3)$ \\
\hline$c[\AA]$ & $17.7512(6)$ & $13.1285(7)$ & $20.1350(6)$ \\
\hline$\alpha\left[^{\circ}\right]$ & 90 & 90 & 90 \\
\hline$\beta\left[^{\circ}\right]$ & $92.035(2)$ & $108.876(4)$ & 90 \\
\hline$\gamma\left[^{\circ}\right]$ & 90 & 90 & 90 \\
\hline$V\left[\AA^{3}\right]$ & $4639.4(3)$ & $2550.2(2)$ & $3728.23(17)$ \\
\hline$Z$ & 4 & 2 & 4 \\
\hline$\rho\left[\mathrm{g} / \mathrm{cm}^{3}\right]$ & 1.464 & 1.422 & 1.386 \\
\hline$F(000)$ & 2144 & 1148 & 1648 \\
\hline$\mu\left[\mathrm{mm}^{-1}\right]$ & 1.104 & 1.009 & 1.194 \\
\hline$T_{\min } / T_{\max }$ & $0.7000 / 0.8400$ & $0.6323 / 0.8161$ & $0.5164 / 0.7554$ \\
\hline$\theta$-range $\left[{ }^{\circ}\right]$ & $1.735-26.811$ & $1.959-25.749$ & $1.897-25.664$ \\
\hline$h k l$-range & $-21-19, \pm 19, \pm 22$ & $\pm 13, \pm 22, \pm 15$ & $\pm 17,-13-15, \pm 24$ \\
\hline measured refl. & 64317 & 31195 & 43991 \\
\hline unique refl. $\left[R_{\text {int }}\right]$ & $9878[0.0828]$ & 4806 [0.0942] & $3522[0.0693]$ \\
\hline observed refl. $(I>2 \sigma(I))$ & 7193 & 3978 & 3312 \\
\hline data / restraints / param. & 9878 / 487 / 758 & $4806 / 257 / 374$ & $3522 / 90 / 279$ \\
\hline goodness-of-fit $\left(F^{2}\right)$ & 1.055 & 1.074 & 1.192 \\
\hline$R 1, w R 2(I>2 \sigma(I))$ & $0.0669,0.1470$ & $0.0566,0.1478$ & $0.0434,0.0924$ \\
\hline$R 1, w R 2$ (all data) & $0.0993,0.1623$ & $0.0704,0.1560$ & $0.0462,0.0938$ \\
\hline resid. el. dens. $\left[\mathrm{e} / \AA^{3}\right]$ & $-0.375 / 1.218$ & $-0.496 / 0.835$ & $-0.375 / 0.262$ \\
\hline
\end{tabular}


Table A3: Crystal data and refinement details for $\mathbf{1 6}$ and $\mathbf{1 7}$

\begin{tabular}{|c|c|c|}
\hline compound & 16 & 17 \\
\hline empirical formula & $\mathrm{C}_{40} \mathrm{H}_{71} \mathrm{Cl}_{2} \mathrm{Cu}_{2} \mathrm{~N}_{5} \mathrm{O}_{17}$ & $\mathrm{C}_{11} \mathrm{H}_{18} \mathrm{Cl}_{2} \mathrm{FeN}_{2} \mathrm{O}_{2}$ \\
\hline formula weight & 1091.99 & 337.02 \\
\hline$T[\mathrm{~K}]$ & $133(2)$ & $133(2)$ \\
\hline crystal size $\left[\mathrm{mm}^{3}\right]$ & $0.390 \times 0.350 \times 0.330$ & $0.620 \times 0.090 \times 0.040$ \\
\hline crystal system & monoclinic & orthorhombic \\
\hline space group & $P 2_{1} / c$ & $\mathrm{Pca}_{1}$ \\
\hline$a[\AA]$ & $10.9869(6)$ & $16.388(3)$ \\
\hline$b[\AA]$ & $18.6852(10)$ & $10.583(2)$ \\
\hline$c[\AA]$ & $13.1285(7)$ & $17.062(3)$ \\
\hline$\alpha\left[^{\circ}\right]$ & 90 & 90 \\
\hline$\beta\left[{ }^{\circ}\right]$ & $108.876(4)$ & 90 \\
\hline$\gamma\left[^{\circ}\right]$ & 90 & 90 \\
\hline$V\left[\AA^{3}\right]$ & $2550.2(2)$ & $2959.2(10)$ \\
\hline$Z$ & 2 & 8 \\
\hline$\rho\left[\mathrm{g} / \mathrm{cm}^{3}\right]$ & 1.422 & 1.513 \\
\hline$F(000)$ & 1148 & 1392 \\
\hline$\mu\left[\mathrm{mm}^{-1}\right]$ & 1.009 & 1.375 \\
\hline$T_{\min } / T_{\max }$ & $0.6323 / 0.8161$ & $? / ?$ \\
\hline$\theta$-range $\left[{ }^{\circ}\right]$ & $1.959-25.749$ & $1.924-26.802$ \\
\hline$h k l$-range & $\pm 13, \pm 22, \pm 15$ & $\pm 20, \pm 13, \pm 21$ \\
\hline measured refl. & 31195 & 30624 \\
\hline unique refl. $\left[R_{\text {int }}\right]$ & $4806[0.0942]$ & $6244[0.0775]$ \\
\hline observed refl. $(I>2 \sigma(I))$ & 3978 & 5389 \\
\hline data / restraints / param. & $4806 / 257 / 374$ & 6244 / 1 / 334 \\
\hline goodness-of-fit $\left(F^{2}\right)$ & 1.074 & 0.977 \\
\hline$R 1, w R 2(I>2 \sigma(I))$ & $0.0566,0.1478$ & $0.0422,0.0772$ \\
\hline$R 1, w R 2$ (all data) & $0.0704,0.1560$ & $0.0540,0.0805$ \\
\hline resid. el. dens. $\left[\mathrm{e} / \AA^{3}\right]$ & $-0.496 / 0.835$ & $-0.378 / 0.403$ \\
\hline
\end{tabular}


Table A4: Crystal data and refinement details for 18,19 and 20

\begin{tabular}{|c|c|c|c|}
\hline compound & 18 & 19 & 20 \\
\hline empirical formula & $\mathrm{C}_{12} \mathrm{H}_{20} \mathrm{Cl}_{2} \mathrm{FeN}_{2} \mathrm{O}_{2}$ & $\mathrm{C}_{17} \mathrm{H}_{22} \mathrm{Cl}_{2} \mathrm{FeN}_{2} \mathrm{O}_{2}$ & $\mathrm{C}_{18} \mathrm{H}_{24} \mathrm{Cl}_{2} \mathrm{FeN}_{2} \mathrm{O}_{2}$ \\
\hline formula weight & 351.05 & 413.11 & 427.14 \\
\hline$T[\mathrm{~K}]$ & $133(2)$ & $133(2)$ & $133(2)$ \\
\hline crystal size $\left[\mathrm{mm}^{3}\right]$ & $0.390 \times 0.310 \times 0.140$ & $0.420 \times 0.400 \times 0.340$ & $0.340 \times 0.320 \times 0.220$ \\
\hline crystal system & triclinic & orthorhombic & orthorhombic \\
\hline space group & $P-1$ & $P 2{ }_{1}{ }_{1} 2_{1}$ & $P 2{ }_{1}{ }_{1} 2_{1}$ \\
\hline$a[\AA]$ & $8.6403(3)$ & $10.2336(3)$ & $10.5991(4)$ \\
\hline$b[\AA]$ & $11.3187(4)$ & $13.5513(5)$ & $13.8361(5)$ \\
\hline$c[\AA]$ & $16.8873(6)$ & $13.9699(4)$ & $13.9419(6)$ \\
\hline$\alpha\left[^{\circ}\right]$ & $99.563(3)$ & 90 & 90 \\
\hline$\beta\left[^{\circ}\right]$ & $90.358(3)$ & 90 & 90 \\
\hline$\gamma\left[{ }^{\circ}\right]$ & $105.649(3)$ & 90 & 90 \\
\hline$V\left[\AA^{3}\right]$ & $1565.94(10)$ & $1937.33(11)$ & $2044.58(14)$ \\
\hline$Z$ & 4 & 4 & 4 \\
\hline$\rho\left[\mathrm{g} / \mathrm{cm}^{3}\right]$ & 1.489 & 1.416 & 1.388 \\
\hline$F(000)$ & 728 & 856 & 888 \\
\hline$\mu\left[\mathrm{mm}^{-1}\right]$ & 1.303 & 1.065 & 1.012 \\
\hline$T_{\min } / T_{\max }$ & $0.6168 / 0.8385$ & $0.5925 / 0.7174$ & $0.6194 / 0.8397$ \\
\hline$\theta$-range $\left[^{\circ}\right]$ & $1.898-26.720$ & $2.094-26.698$ & $2.074-26.789$ \\
\hline$h k l$-range & $\pm 10,-14-13, \pm 21$ & $\pm 12, \pm 17, \pm 17$ & $\pm 13, \pm 17, \pm 17$ \\
\hline measured refl. & 22023 & 27441 & 25119 \\
\hline unique refl. $\left[R_{\text {int }}\right]$ & $6621[0.0235]$ & $4101[0.0487]$ & $4345[0.0433]$ \\
\hline observed refl. $(I>2 \sigma(I))$ & 5889 & 3945 & 4113 \\
\hline data / restraints / param. & $6621 / 0 / 353$ & $4101 / 0 / 221$ & $4345 / 0 / 231$ \\
\hline goodness-of-fit $\left(F^{2}\right)$ & 1.024 & 1.028 & 1.040 \\
\hline$R 1, w R 2(I>2 \sigma(I))$ & $0.0280,0.0651$ & $0.0218,0.0554$ & $0.0270,0.0677$ \\
\hline$R 1, w R 2$ (all data) & $0.0336,0.0671$ & $0.0232,0.0559$ & $0.0296,0.0689$ \\
\hline resid. el. dens. $\left[\mathrm{e} / \AA^{3}\right]$ & $-0.678 / 1.124$ & $-0.244 / 0.348$ & $-0.267 / 0.469$ \\
\hline
\end{tabular}


Table A5: Crystal data and refinement details for 21, 22 and 23

\begin{tabular}{|c|c|c|c|}
\hline compound & 21 & 22 & 23 \\
\hline empirical formula & $\mathrm{C}_{13} \mathrm{H}_{22} \mathrm{Cl}_{2} \mathrm{FeN}_{2} \mathrm{O}_{2}$ & $\mathrm{C}_{30} \mathrm{H}_{44} \mathrm{~F}_{12} \mathrm{Fe}_{2} \mathrm{~N}_{4} \mathrm{O}_{16} \mathrm{~S}_{4}$ & $\mathrm{C}_{26} \mathrm{H}_{39} \mathrm{~F}_{6} \mathrm{FeN}_{6} \mathrm{O}_{12} \mathrm{~S}_{2}$ \\
\hline formula weight & 365.07 & 1184.63 & 861.60 \\
\hline$T[\mathrm{~K}]$ & $133(2)$ & $133(2)$ & $133(2)$ \\
\hline crystal size $\left[\mathrm{mm}^{3}\right]$ & $0.330 \times 0.290 \times 0.030$ & $0.440 \times 0.400 \times 0.280$ & $0.500 \times 0.500 \times 0.270$ \\
\hline crystal system & monoclinic & triclinic & triclinic \\
\hline space group & $P 2_{1} / n$ & $P-1$ & $P-1$ \\
\hline$a[\AA]$ & $8.6844(17)$ & $9.2477(5)$ & $10.5468(6)$ \\
\hline$b[\AA]$ & $17.826(4)$ & $9.6561(5)$ & $12.9991(7)$ \\
\hline$c[\AA]$ & $11.602(2)$ & $27.7493(12)$ & $15.2966(8)$ \\
\hline$\alpha\left[^{\circ}\right]$ & 90 & $83.669(4)$ & $109.598(4)$ \\
\hline$\beta\left[^{\circ}\right]$ & $108.16(3)$ & $85.631(4)$ & $104.073(4)$ \\
\hline$\gamma\left[{ }^{\circ}\right]$ & 90 & $73.513(4)$ & $98.565(4)$ \\
\hline$V\left[\AA^{3}\right]$ & $1706.6(6)$ & $2358.9(2)$ & $1854.40(18)$ \\
\hline$Z$ & 4 & 2 & 2 \\
\hline$\rho\left[\mathrm{g} / \mathrm{cm}^{3}\right]$ & 1.421 & 1.668 & 1.543 \\
\hline$F(000)$ & 760 & 1208 & 890 \\
\hline$\mu\left[\mathrm{mm}^{-1}\right]$ & 1.199 & 0.907 & 0.614 \\
\hline$T_{\min } / T_{\max }$ & $? / ?$ & $0.6557 / 0.7993$ & $0.6246 / 0.8275$ \\
\hline$\theta$-range $\left[{ }^{\circ}\right]$ & $2.172-26.732$ & $1.478-25.646$ & $1.489-26.776$ \\
\hline$h k l$-range & $\pm 10, \pm 22, \pm 14$ & $\pm 11,-11-10, \pm 33$ & $-11-13, \pm 16, \pm 19$ \\
\hline measured refl. & 17130 & 20609 & 25719 \\
\hline unique refl. $\left[R_{\text {int }}\right]$ & $3598[0.0557]$ & $8866[0.1123]$ & $7872[0.0512]$ \\
\hline observed refl. $(I>2 \sigma(I))$ & 3205 & 6892 & 6730 \\
\hline data / restraints / param. & $3598 / 0 / 187$ & $8866 / 57 / 698$ & 7872 / 174 / 606 \\
\hline goodness-of-fit $\left(F^{2}\right)$ & 0.962 & 1.020 & 1.032 \\
\hline$R 1, w R 2(I>2 \sigma(I))$ & $0.0253,0.0691$ & $0.0784,0.1978$ & $0.0391,0.1001$ \\
\hline$R 1, w R 2$ (all data) & $0.0306,0.0716$ & $0.0953,0.2143$ & $0.0480,0.1041$ \\
\hline resid. el. dens. $\left[\mathrm{e} / \AA^{3}\right]$ & $-0.216 / 0.355$ & $-1.412 / 1.549$ & $-0.328 / 0.450$ \\
\hline
\end{tabular}


Table A6: Selected bond lengths (sorted) $[\AA]$ for $\mathbf{1}$.

\begin{tabular}{ll}
\hline Atoms & Bond lengths \\
\hline Cu1-N3 & $1.8664(14)$ \\
Cu1-N2 & $1.9746(13)$ \\
Cu1-N1 & $2.0036(13)$ \\
\hline
\end{tabular}

Table A7: Selected bond angles (sorted) $\left[^{\circ}\right]$ for $\mathbf{1}$.

\begin{tabular}{ll}
\hline Atoms & Bond angles \\
\hline N2-Cu1-N1 & $94.50(5)$ \\
C9-N1-Cu1 & $123.63(11)$ \\
C14-N2-Cu1 & $123.98(11)$ \\
N3-Cu1-N1 & $126.02(6)$ \\
C16-N2-Cu1 & $128.26(10)$ \\
C11-N1-Cu1 & $128.63(10)$ \\
N3-Cu1-N2 & $139.47(6)$ \\
C19-N3-Cu1 & $170.89(14)$ \\
\hline
\end{tabular}

Table A8: Selected bond lengths (sorted) $[\AA]$ for 2.

\begin{tabular}{ll}
\hline Atoms & Bond lengths \\
\hline Cu1-N3 & $1.880(14)$ \\
Cu1-N1 & $1.969(15)$ \\
Cu1-N2 & $1.986(15)$ \\
\hline
\end{tabular}

Table A9: Selected bond angles (sorted) $\left[^{\circ}\right]$ for 2.

\begin{tabular}{ll}
\hline Atoms & Bond angles \\
\hline N1-Cu1-N2 & $95.1(5)$ \\
C7-N2-Cu1 & $122.5(12)$ \\
C2-N1-Cu1 & $123.5(13)$ \\
C4-N1-Cu1 & $128.5(12)$ \\
C9-N2-Cu1 & $129.0(11)$ \\
N3-Cu1-N1 & $131.6(8)$ \\
N3-Cu1-N2 & $133.3(8)$ \\
C12-N3-Cu1 & $172.3(17)$ \\
\hline
\end{tabular}


Table A10: Selected bond lengths (sorted) $[\AA]$ for 4.

\begin{tabular}{ll}
\hline Atoms & Bond lengths \\
\hline Cu1-N3 & $1.8540(19)$ \\
Cu2-N11 & $1.8699(17)$ \\
Cu2-N12' & $1.8738(17)$ \\
N12-Cu2' & $1.8739(17)$ \\
Cu1-N1 & $1.9469(17)$ \\
Cu1-N2 & $1.9909(18)$ \\
\hline
\end{tabular}

Table A11: Selected bond angles (sorted) $\left[{ }^{\circ}\right]$ for 4.

\begin{tabular}{ll}
\hline Atoms & \multicolumn{1}{c}{ Bond angles } \\
\hline N1-Cu1-N2 & $92.83(7)$ \\
C26-N11-Cu2 & $119.38(14)$ \\
C31-N12-Cu2' & $119.66(14)$ \\
C6-N1-Cu1 & $124.27(14)$ \\
C11-N2-Cu1 & $124.34(14)$ \\
N3-Cu1-N2 & $125.90(8)$ \\
C9-N2-Cu1 & $126.30(15)$ \\
C4-N1-Cu1 & $126.75(15)$ \\
C29-N12-Cu2' & $132.20(16)$ \\
C24-N11-Cu2 & $132.52(15)$ \\
N3-Cu1-N1 & $141.13(8)$ \\
N11-Cu2-N12' & $171.43(8)$ \\
C14-N3-Cu1 & $171.9(2)$ \\
\hline
\end{tabular}

Symmetry transformation used to generate equivalent atoms: (') 1-x, 1-y, 1-z.

Table A12: Selected bond lengths (sorted) $[\AA]$ for $\mathbf{6}$.

\begin{tabular}{ll}
\hline Atoms & Bond lengths \\
\hline $\mathrm{Cu} 1-\mathrm{O} 3$ & $1.916(3)$ \\
$\mathrm{Cu} 1-\mathrm{O} 3$ & $1.919(3)$ \\
$\mathrm{Cu} 2-\mathrm{O} 13 "$ & $1.924(3)$ \\
$\mathrm{Cu} 2-\mathrm{O} 13$ & $1.926(3)$ \\
$\mathrm{Cu} 1-\mathrm{N} 2$ & $1.942(4)$ \\
$\mathrm{Cu} 2-\mathrm{N} 11$ & $1.953(3)$ \\
$\mathrm{Cu} 2-\mathrm{N} 12$ & $1.955(4)$ \\
$\mathrm{Cu} 1-\mathrm{N} 1$ & $1.959(3)$ \\
$\mathrm{Cu} 2-\mathrm{O} 14 \mathrm{~A}$ & $2.272(15)$ \\
$\mathrm{Cu} 2-\mathrm{O} 14 \mathrm{~B}$ & $2.275(6)$ \\
$\mathrm{Cu} 1-\mathrm{O} 4 \mathrm{~A}$ & $2.328(6)$ \\
$\mathrm{Cu} 1-\mathrm{O} 4 \mathrm{~B}$ & $2.341(15)$ \\
\hline
\end{tabular}


Table A13: Selected bond angles (sorted) $\left[^{\circ}\right]$ for 6.

\begin{tabular}{ll}
\hline Atoms & Bond angles \\
\hline O13"-Cu2-O13 & $48.20(14)$ \\
O3-Cu1-O3' & $48.78(15)$ \\
O3'-O3-Cu1' & $65.51(19)$ \\
O3'-O3-Cu1 & $65.71(19)$ \\
O13"-O13-Cu2 & $65.85(19)$ \\
O13"-O13-Cu2" & $65.95(19)$ \\
O3'-Cu1-O4A & $90.0(4)$ \\
N1-Cu1-O4B & $90.5(7)$ \\
O3-Cu1-O4A & $91.4(3)$ \\
O13-Cu2-O14A & $92.5(10)$ \\
O13"-Cu2-O14B & $93.2(5)$ \\
O13"-Cu2-O14A & $93.3(13)$ \\
O13-Cu2-O14B & $93.4(3)$ \\
N11-Cu2-N12 & $94.23(15)$ \\
N2-Cu1-N1 & $94.36(15)$ \\
O3'-Cu1-O4B & $95.2(9)$ \\
N1-Cu1-O4A & $96.5(3)$ \\
N11-Cu2-O14A & $96.7(17)$ \\
N12-Cu2-O14B & $96.8(4)$ \\
N11-Cu2-O14B & $97.3(6)$ \\
N2-Cu1-O4B & $97.6(9)$ \\
N12-Cu2-O14A & $98.0(14)$ \\
O3-Cu1-O4B & $98.2(7)$ \\
N2-Cu1-O4A & $100.5(4)$ \\
O3-Cu1-N2 & $105.38(14)$ \\
O13-Cu2-N11 & $107.60(13)$ \\
O13"-Cu2-N12 & $107.74(14)$ \\
O3'-Cu1-N1 & $109.49(14)$ \\
C16B-O4B-Cu1 & $113(2)$ \\
C33A-O14A-Cu2 & $118.6(19)$ \\
C16A-O4A-Cu1 & $121.1(7)$ \\
C36B-O14B-Cu2 & $122.2(8)$ \\
C3-N1-Cu1 & $123.0(3)$ \\
C8-N2-Cu1 & $124.3(3)$ \\
C28-N12-Cu2 & $125.2(3)$ \\
C23-N11-Cu2 & $126.0(3)$ \\
C25-N11-Cu2 & $126.1(3)$ \\
C30-N12-Cu2 & $126.8(3)$ \\
C10-N2-Cu1 & $127.2(3)$ \\
C33B-O14B-Cu2 & $127.5(9)$ \\
C5-N1-Cu1 & $128.3(3)$ \\
C36A-O14A-Cu2 & $131(2)$ \\
Cu1-O3-Cu1' & $131.22(15)$ \\
Cu2"-O13-Cu2 & $131.80(14)$ \\
& \\
\hline
\end{tabular}




\begin{tabular}{ll} 
C13B-O4B-Cu1 & $133(2)$ \\
C13A-O4A-Cu1 & $134.0(7)$ \\
O3'-Cu1-N2 & $152.73(14)$ \\
O13"-Cu2-N11 & $154.34(14)$ \\
O13-Cu2-N12 & $154.48(14)$ \\
O3-Cu1-N1 & $157.01(14)$ \\
\hline
\end{tabular}

Symmetry transformations used to generate equivalent atoms: (') 1-x, 1-y, $-z$; (") $-x, 1-y, 1-z$.

Table A14: Selected bond lengths (sorted) $[\AA]$ for 8.

\begin{tabular}{ll}
\hline Atoms & Bond lengths \\
\hline $\mathrm{Cu} 1-\mathrm{O} 3$ & $1.924(3)$ \\
$\mathrm{Cu} 1-\mathrm{O} 3$ & $1.929(3)$ \\
$\mathrm{Cu} 1-\mathrm{N} 1$ & $1.942(3)$ \\
$\mathrm{Cu} 1-\mathrm{N} 2$ & $1.950(3)$ \\
$\mathrm{Cu} 1-\mathrm{O} 4 \mathrm{~A}$ & $2.318(5)$ \\
$\mathrm{Cu} 1-\mathrm{O} 4 \mathrm{~B}$ & $2.331(15)$ \\
\hline
\end{tabular}

Table A15: Selected bond angles (sorted) $\left[{ }^{\circ}\right]$ for 8 .

\begin{tabular}{ll}
\hline Atoms & Bond angles \\
\hline O3'-Cu1-O3 & $47.90(13)$ \\
O3'-O3-Cu1 & $65.86(18)$ \\
O3'-O3-Cu1' & $66.24(18)$ \\
O3-Cu1-O4A & $89.55(19)$ \\
N1-Cu1-O4B & $91.3(7)$ \\
O3'-Cu1-O4A & $92.02(18)$ \\
N1-Cu1-N2 & $93.09(14)$ \\
O3'-Cu1-O4B & $94.6(9)$ \\
N2-Cu1-O4A & $98.03(18)$ \\
O3-Cu1-O4B & $99.3(8)$ \\
N2-Cu1-O4B & $99.9(8)$ \\
N1-Cu1-O4A & $102.4(2)$ \\
O3'-Cu1-N1 & $107.96(13)$ \\
O3-Cu1-N2 & $108.16(12)$ \\
C24B-O4B-Cu1 & $113.7(16)$ \\
C21A-O4A-Cu1 & $122.2(4)$ \\
C6-N1-Cu1 & $125.0(3)$ \\
C11-N2-Cu1 & $125.2(3)$ \\
C9-N2-Cu1 & $125.9(3)$ \\
C4-N1-Cu1 & $126.1(3)$ \\
C24A-O4A-Cu1 & $126.4(5)$ \\
C21B-O4B-Cu1 & $129.0(17)$ \\
Cu1'-O3-Cu1 & $132.10(13)$ \\
O3-Cu1-N1 & $153.99(13)$ \\
O3'-Cu1-N2 & $154.15(13)$ \\
\hline
\end{tabular}

Symmetry transformation used to generate equivalent atoms: (') 1-x, 1-y, $-z$. 
Table A16: Selected bond lengths (sorted) [ $]$ for 10.

\begin{tabular}{ll}
\hline Atoms & Bond lengths \\
\hline Cu1-O3 & $1.8196(16)$ \\
O3-Cu1' & $1.8196(16)$ \\
Cu1-O4 & $1.8204(15)$ \\
O4-Cu1' & $1.8205(15)$ \\
Cu1-N1 & $1.909(2)$ \\
Cu1-N2 & $1.909(2)$ \\
Cu1-Cu1' & $2.8706(6)$ \\
\hline
\end{tabular}

Table A17: Selected bond angles (sorted) $\left[^{\circ}\right]$ for $\mathbf{1 0}$.

\begin{tabular}{lc}
\hline Atoms & Bond angles \\
\hline O3-Cu1-Cu1' & $37.93(6)$ \\
O4-Cu1-Cu1' & $37.96(6)$ \\
O3-Cu1-O4 & $75.89(9)$ \\
N1-Cu1-N2 & $93.98(9)$ \\
O4-Cu1-N2 & $97.30(9)$ \\
O3-Cu1-N1 & $97.49(9)$ \\
Cu1-O4-Cu1' & $104.08(12)$ \\
Cu1-O3-Cu1' & $104.15(13)$ \\
C3-N1-Cu1 & $124.03(19)$ \\
C8-N2-Cu1 & $124.44(18)$ \\
C10-N2-Cu1 & $126.59(17)$ \\
C5-N1-Cu1 & $126.91(17)$ \\
N2-Cu1-Cu1' & $132.92(6)$ \\
N1-Cu1-Cu1' & $133.10(7)$ \\
O3-Cu1-N2 & $161.19(7)$ \\
O4-Cu1-N1 & $161.38(8)$ \\
\hline
\end{tabular}

Symmetry transformation used to generate equivalent atoms: (') $x, 1 / 2-y, 1 / 2-z$.

Table A18: Selected bond lengths (sorted) [A] for 16.

\begin{tabular}{ll}
\hline Atoms & Bond lengths \\
\hline $\mathrm{Cu} 1-\mathrm{O} 3$ & $1.9338(14)$ \\
$\mathrm{Cu} 1-\mathrm{O} 3$ & $1.9596(14)$ \\
$\mathrm{O} 3-\mathrm{Cu} 1^{\prime}$ & $1.9597(14)$ \\
$\mathrm{Cu} 1-\mathrm{N} 1$ & $2.0120(16)$ \\
$\mathrm{Cu} 1-\mathrm{N} 2$ & $2.0272(16)$ \\
$\mathrm{Cu} 1-\mathrm{O} 11$ & $2.4364(15)$ \\
$\mathrm{Cu} 1-\mathrm{Cu} 1^{\prime}$ & $2.9994(4)$ \\
\hline
\end{tabular}

Table A19: Selected bond angles (sorted) $\left[^{\circ}\right]$ for 16.

\begin{tabular}{lc}
\hline Atoms & Bond angles \\
\hline O3'-Cu1-Cu1' & $39.30(4)$ \\
O3-Cu1-Cu1' & $39.93(4)$
\end{tabular}




\begin{tabular}{ll} 
O3-Cu1-O3' & $79.22(6)$ \\
N2-Cu1-O11 & $84.34(6)$ \\
N1-Cu1-N2 & $88.59(6)$ \\
O3'-Cu1-O11 & $88.65(6)$ \\
N1-Cu1-O11 & $95.28(6)$ \\
O3'-Cu1-N1 & $96.72(6)$ \\
O3-Cu1-N2 & $98.00(6)$ \\
O11-Cu1-Cu1' & $98.46(4)$ \\
Cu1-O3-Cu1' & $100.78(6)$ \\
O3-Cu1-O11 & $104.61(6)$ \\
C10-N2-Cu1 & $123.26(13)$ \\
C7-N1-Cu1 & $125.26(12)$ \\
C12-N2-Cu1 & $125.52(12)$ \\
C4-N1-Cu1 & $126.41(14)$ \\
S1-O11-Cu1 & $131.49(9)$ \\
N1-Cu1-Cu1' & $132.88(5)$ \\
N2-Cu1-Cu1' & $137.40(5)$ \\
O3-Cu1-N1 & $159.53(6)$ \\
O3'-Cu1-N2 & $171.57(6)$ \\
\hline
\end{tabular}

Symmetry transformation used to generate equivalent atoms: (') 1-x, 1-y, 1-z.

Table A20: Selected bond lengths (sorted) [ $[\AA]$ for 17.

\begin{tabular}{ll}
\hline Atoms & Bond lengths \\
\hline Fe2-N4 & $2.072(5)$ \\
Fe1-N2 & $2.080(5)$ \\
Fe1-N1 & $2.081(5)$ \\
Fe2-N3 & $2.088(4)$ \\
Fe1-C12 & $2.2529(15)$ \\
Fe2-C13 & $2.2550(16)$ \\
Fe1-C11 & $2.2551(15)$ \\
Fe2-Cl4 & $2.2635(16)$ \\
\hline
\end{tabular}

Table A21: Selected bond angles (sorted) [ $\left.{ }^{\circ}\right]$ for 17.

\begin{tabular}{lc}
\hline Atoms & Bond angles \\
\hline N2-Fe1-N1 & $88.66(19)$ \\
N4-Fe2-N3 & $88.95(18)$ \\
N3-Fe2-C14 & $108.04(12)$ \\
N2-Fe1-Cl2 & $108.23(13)$
\end{tabular}




\begin{tabular}{ll} 
N4-Fe2-Cl4 & $108.67(13)$ \\
N1-Fe1-C11 & $110.75(13)$ \\
N3-Fe2-Cl3 & $112.37(13)$ \\
N2-Fe1-Cl1 & $113.48(13)$ \\
N4-Fe2-Cl3 & $113.56(13)$ \\
N1-Fe1-Cl2 & $114.56(13)$ \\
C12-Fe1-Cl1 & $117.59(7)$ \\
C13-Fe2-C14 & $120.65(7)$ \\
C3-N1-Fe1 & $124.1(4)$ \\
C14-N3-Fe2 & $124.6(4)$ \\
C19-N4-Fe2 & $125.2(3)$ \\
C8-N2-Fe1 & $125.3(4)$ \\
C13-N3-Fe2 & $126.3(4)$ \\
C7-N2-Fe1 & $127.0(4)$ \\
C18-N4-Fe2 & $127.1(4)$ \\
C2-N1-Fe1 & $127.2(4)$ \\
\hline
\end{tabular}

Table A22: Selected bond lengths (sorted) $[\AA]$ for 18.

\begin{tabular}{ll}
\hline Atoms & Bond lengths \\
\hline Fe1-N2 & $2.0772(14)$ \\
Fe1-N1 & $2.0853(14)$ \\
Fe2-N12 & $2.0865(14)$ \\
Fe2-N11 & $2.0870(15)$ \\
Fe1-C12 & $2.2485(5)$ \\
Fe2-C111 & $2.2515(6)$ \\
Fe1-Cl1 & $2.2659(5)$ \\
Fe2-Cl12 & $2.2714(6)$ \\
\hline
\end{tabular}

Table A23: Selected bond angles (sorted) [ $\left.{ }^{\circ}\right]$ for 18.

\begin{tabular}{lc}
\hline Atoms & Bond angles \\
\hline N12-Fe2-N11 & $87.88(6)$ \\
N2-Fe1-N1 & $88.21(6)$ \\
N2-Fe1-Cl1 & $108.41(4)$ \\
N11-Fe2-Cl12 & $108.94(5)$ \\
N12-Fe2-Cl12 & $109.17(5)$ \\
N1-Fe1-Cl2 & $109.56(4)$ \\
C12-Fe1-Cl1 & $112.58(2)$ \\
N11-Fe2-C111 & $112.97(5)$ \\
N12-Fe2-C111 & $115.38(5)$ \\
N2-Fe1-Cl2 & $116.58(4)$ \\
C111-Fe2-C112 & $118.35(2)$ \\
N1-Fe1-Cl1 & $119.62(5)$ \\
C25-N11-Fe2 & $124.00(11)$ \\
C30-N12-Fe2 & $124.24(11)$ \\
C10-N2-Fe1 & $124.48(11)$
\end{tabular}




$\begin{array}{ll}\text { C5-N1-Fe1 } & 125.03(11) \\ \text { C28-N12-Fe2 } & 127.13(12) \\ \text { C3-N1-Fe1 } & 127.46(12) \\ \text { C23-N11-Fe2 } & 127.53(13) \\ \text { C8-N2-Fe1 } & 127.86(11)\end{array}$

Table A24: Selected bond lengths (sorted) [A] for 19.

\begin{tabular}{ll}
\hline Atoms & Bond lengths \\
\hline Fe1-N1 & $2.0821(18)$ \\
Fe1-N2 & $2.0844(18)$ \\
Fe1-Cl2 & $2.2525(6)$ \\
Fe1-Cl1 & $2.2540(6)$ \\
\hline
\end{tabular}

Table A25: Selected bond angles (sorted) $\left[{ }^{\circ}\right]$ for 19.

\begin{tabular}{lc}
\hline Atoms & Bond angles \\
\hline N1-Fe1-N2 & $87.35(7)$ \\
N1-Fe1-C11 & $107.04(5)$ \\
N2-Fe1-Cl1 & $109.01(5)$ \\
N1-Fe1-C12 & $114.65(5)$ \\
C12-Fe1-Cl1 & $117.21(2)$ \\
N2-Fe1-Cl2 & $117.43(5)$ \\
C10-N1-Fe1 & $125.32(14)$ \\
C15-N2-Fe1 & $126.04(14)$ \\
C13-N2-Fe1 & $126.43(14)$ \\
C8-N1-Fe1 & $126.45(15)$ \\
\hline
\end{tabular}

Table A26: Selected bond lengths (sorted) [ $\mathrm{A}]$ for 20.

\begin{tabular}{ll}
\hline Atoms & Bond lengths \\
\hline Fe1-N2 & $2.076(2)$ \\
Fe1-N1 & $2.079(2)$ \\
Fe1-Cl2 & $2.2499(8)$ \\
Fe1-Cl1 & $2.2537(8)$
\end{tabular}

Table A27: Selected bond angles (sorted) $\left[^{\circ}\right]$ for 20.

\begin{tabular}{ll}
\hline Atoms & \multicolumn{1}{c}{ Bond angles } \\
\hline N2-Fe1-N1 & \multicolumn{1}{c}{$87.93(10)$} \\
N2-Fe1-Cl1 & $108.88(8)$ \\
N1-Fe1-C11 & $109.55(7)$ \\
N2-Fe1-Cl2 & $112.91(7)$ \\
N1-Fe1-C12 & $115.38(7)$ \\
C12-Fe1-Cl1 & $118.12(3)$ \\
C15-N2-Fe1 & $125.6(2)$ \\
C10-N1-Fe1 & $125.81(17)$
\end{tabular}


C9-N1-Fe1 126.38(19)

C14-N2-Fe1 126.4(2)

Table A28: Selected bond lengths (sorted) [ [ ] for 21.

\begin{tabular}{ll}
\hline Atoms & Bond lengths \\
\hline Fe1-N2 & $2.0826(13)$ \\
Fe1-N1 & $2.0854(15)$ \\
Fe1-C12 & $2.2458(6)$ \\
Fe1-Cl1 & $2.2556(6)$ \\
\hline
\end{tabular}

Table A29: Selected bond angles (sorted) $\left[{ }^{\circ}\right]$ for 21.

\begin{tabular}{lc}
\hline Atoms & Bond angles \\
\hline N2-Fe1-N1 & $87.26(5)$ \\
$\mathrm{N} 1-\mathrm{Fe} 1-\mathrm{Cl} 2$ & $111.34(4)$ \\
$\mathrm{N} 2-\mathrm{Fe} 1-\mathrm{Cl} 2$ & $111.42(4)$ \\
$\mathrm{N} 1-\mathrm{Fe} 1-\mathrm{Cl1}$ & $112.28(4)$ \\
$\mathrm{C} 12-\mathrm{Fe} 1-\mathrm{Cl} 1$ & $115.42(3)$ \\
$\mathrm{N} 2-\mathrm{Fe} 1-\mathrm{Cl} 1$ & $115.86(4)$ \\
$\mathrm{C} 10-\mathrm{N} 2-\mathrm{Fe} 1$ & $123.61(9)$ \\
$\mathrm{C} 5-\mathrm{N} 1-\mathrm{Fe} 1$ & $123.87(10)$ \\
$\mathrm{C} 4-\mathrm{N} 1-\mathrm{Fe} 1$ & $128.37(10)$ \\
$\mathrm{C} 9-\mathrm{N} 2-\mathrm{Fe} 1$ & $128.60(10)$ \\
\hline
\end{tabular}

Table A30: Selected bond lengths (sorted) [ $]$ for 22.

\begin{tabular}{ll}
\hline Atoms & Bond lengths \\
\hline Fe1-O6A & $2.020(9)$ \\
Fe1-O6B & $2.026(10)$ \\
Fe2-O13 & $2.042(4)$ \\
Fe2-N12 & $2.074(4)$ \\
Fe1-N1 & $2.078(4)$ \\
Fe2-N11 & $2.081(4)$ \\
Fe1-N2 & $2.084(4)$ \\
Fe1-O3 & $2.156(3)$ \\
Fe2-O16 & $2.180(4)$ \\
Fe1-O4' & $2.219(4)$ \\
Fe2-O18" & $2.323(4)$ \\
Fe2-O17" & $2.341(3)$ \\
\hline
\end{tabular}


Table A31: Selected bond angles (sorted) $\left[{ }^{\circ}\right]$ for 22.

\begin{tabular}{ll}
\hline Atoms & Bond angles \\
\hline O18"-Fe2-O17" & $61.38(12)$ \\
O6A-Fe1-O4' & $82.4(3)$ \\
O3-Fe1-O4' & $84.45(14)$ \\
O16-Fe2-O18" & $85.94(13)$ \\
O13-Fe2-O17" & $85.96(13)$ \\
O16-Fe2-O17" & $86.21(13)$ \\
O13-Fe2-O18" & $86.62(14)$ \\
N2-Fe1-O3 & $87.41(15)$ \\
N12-Fe2-O16 & $87.57(14)$ \\
N11-Fe2-O16 & $88.44(15)$ \\
N1-Fe1-N2 & $89.26(15)$ \\
N1-Fe1-O3 & $89.95(16)$ \\
N12-Fe2-N11 & $90.13(14)$ \\
O6B-Fe1-O4' & $90.7(4)$ \\
O6B-Fe1-N2 & $91.2(5)$ \\
S12-O17-Fe2" & $93.36(17)$ \\
S12-O18-Fe2" & $94.00(18)$ \\
O13-Fe2-N11 & $97.49(16)$ \\
O6A-Fe1-N1 & $98.4(4)$ \\
O13-Fe2-N12 & $98.98(15)$ \\
N11-Fe2-O17" & $103.72(13)$ \\
O6A-Fe1-N2 & $104.0(3)$ \\
N12-Fe2-O18" & $104.17(13)$ \\
N1-Fe1-O4' & $104.96(15)$ \\
S1-O4-Fe1' & $106.9(2)$ \\
O6B-Fe1-N1 & $113.1(5)$ \\
C11-N2-Fe1 & $123.6(3)$ \\
C26-N11-Fe2 & $125.2(3)$ \\
C31-N12-Fe2 & $125.2(3)$ \\
C6-N1-Fe1 & $125.4(3)$ \\
C24-N11-Fe2 & $125.8(3)$ \\
C29-N12-Fe2 & $125.8(3)$ \\
C9-N2-Fe1 & $126.5(3)$ \\
C4-N1-Fe1 & $126.8(3)$ \\
S11-O13-Fe2 & $134.6(2)$ \\
S12-O16-Fe2 & $145.3(2)$ \\
S1-O3-Fe1 & $148.2(3)$ \\
S2B-O6B-Fe1 & $155.8(14)$ \\
S2A-O6A-Fe1 & $156.7(10)$ \\
O6B-Fe1-O3 & $156.9(5)$ \\
N2-Fe1-O4' & $163.56(15)$ \\
N11-Fe2-O18" & $164.37(14)$ \\
N12-Fe2-O17" & $164.63(13)$ \\
O6A-Fe1-O3 & $165.8(3)$ \\
&
\end{tabular}


Table A32: Selected bond lengths (sorted) $[\AA]$ for 23.

\begin{tabular}{ll}
\hline Atoms & Bond lengths \\
\hline Fe1-N4 & $1.6905(19)$ \\
Fe1-N3A & $1.692(4)$ \\
Fe1-N3B & $1.716(15)$ \\
Fe1-N1 & $2.0001(14)$ \\
Fe1-N2 & $2.0078(16)$ \\
\hline
\end{tabular}

Table A33: Selected bond angles (sorted) $\left[^{\circ}\right]$ for 23 .

\begin{tabular}{ll}
\hline Atoms & Bond angles \\
\hline N1-Fe1-N2 & \multicolumn{1}{c}{$91.92(6)$} \\
N3B-Fe1-N2 & $101.4(13)$ \\
N4-Fe1-N3A & $107.6(4)$ \\
N4-Fe1-N1 & $111.01(8)$ \\
N3A-Fe1-N1 & $111.9(3)$ \\
N3B-Fe1-N1 & $113.7(14)$ \\
N3A-Fe1-N2 & $116.8(4)$ \\
N4-Fe1-N2 & $116.93(8)$ \\
N4-Fe1-N3B & $118.6(15)$ \\
C5-N1-Fe1 & $125.47(11)$ \\
C8-N2-Fe1 & $125.92(12)$ \\
C10-N2-Fe1 & $125.99(12)$ \\
C3-N1-Fe1 & $126.18(12)$ \\
O3A-N3A-Fe1 & $159.0(7)$ \\
O4-N4-Fe1 & $164.0(2)$ \\
O3B-N3B-Fe1 & $165(3)$ \\
\hline
\end{tabular}


Overview of Ligands and Complexes

\section{Overview of Ligands}<smiles></smiles>

$\mathrm{HL}^{1}$<smiles>CC1(C)COC(C(C)(C)C2=NC(C)(C)CO2)=N1</smiles>

$\mathrm{HL}^{2}$<smiles>CC1(C)COC(C(C2=NC(C)(C)CO2)c2ccccc2)=N1</smiles>

$\mathrm{HL}^{3}$

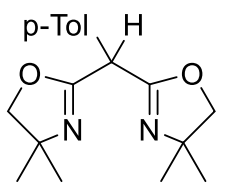

$\mathrm{HL}^{5}$

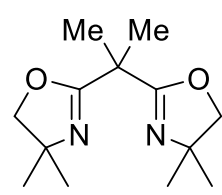

$\mathrm{IL}^{6}$<smiles>CC(=C1NC(C)(C)CO1)C1=C(C(=C2NC(C)(C)CO2)C(C)(C)C)COC1=C(C1=NC(C)(C)CO1)c1ccccc1</smiles>

$\left[L^{2}\right]^{-}$

$\left[\mathrm{L}^{3}\right]^{-}$

$\underline{\text { Overview of Complexes }}$

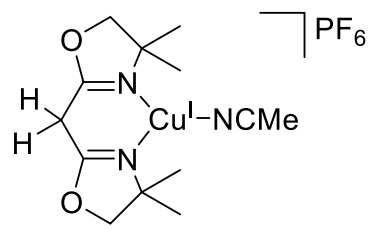

1

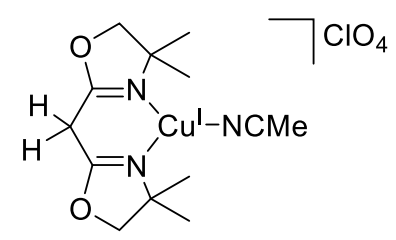

4

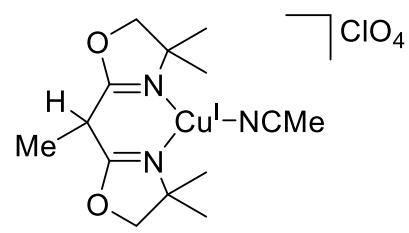

2

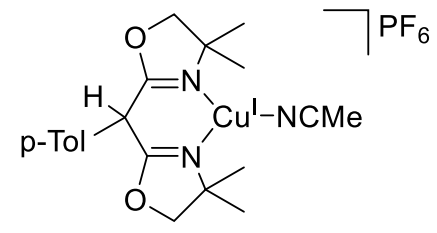

3

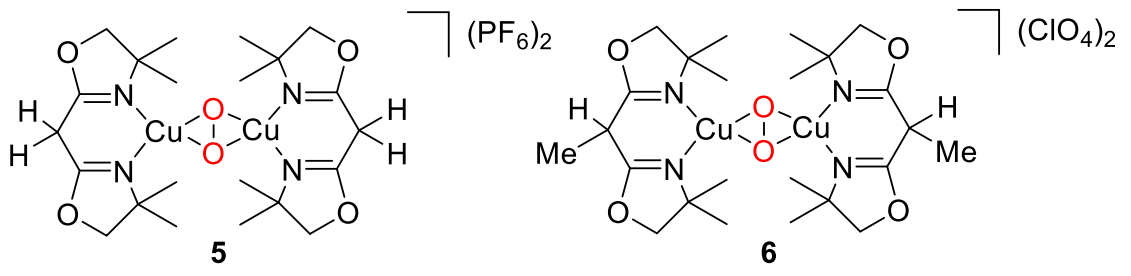

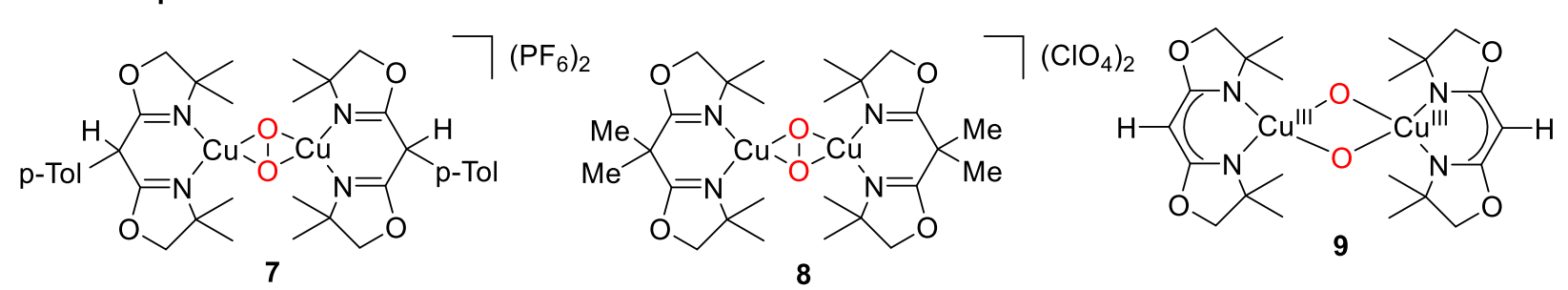



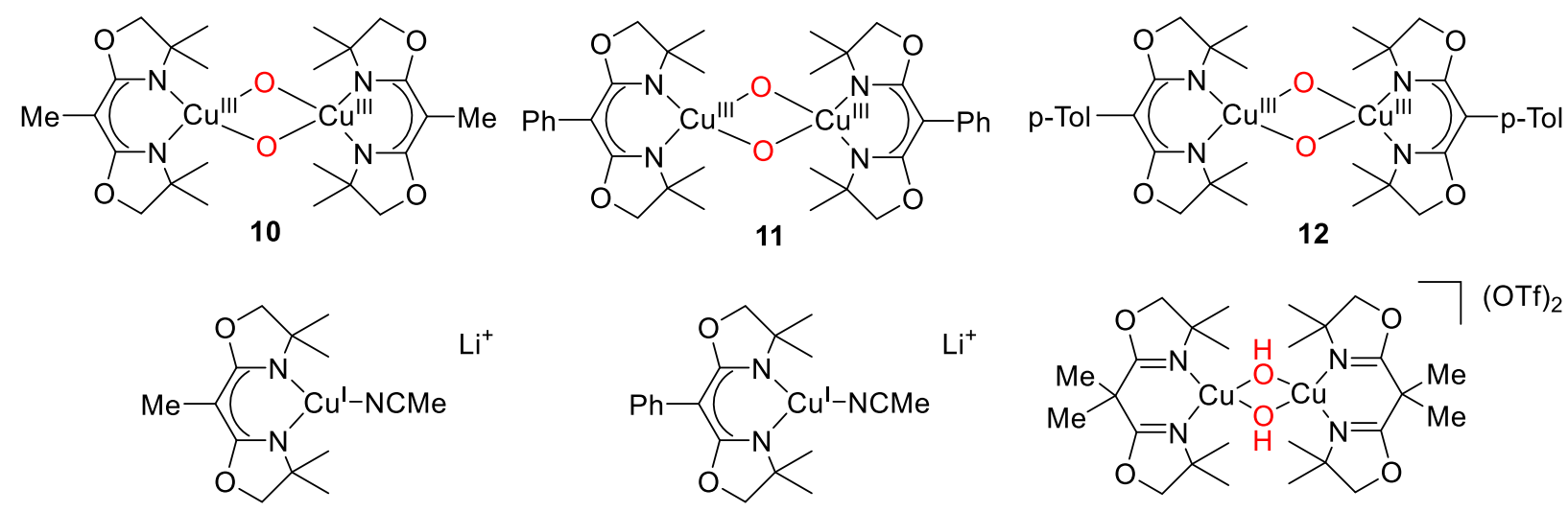

13
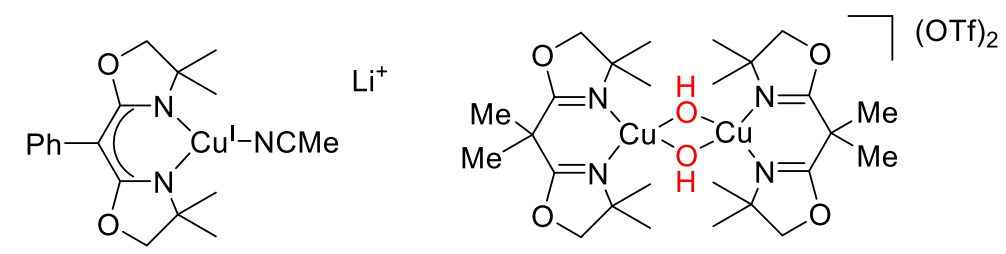

16

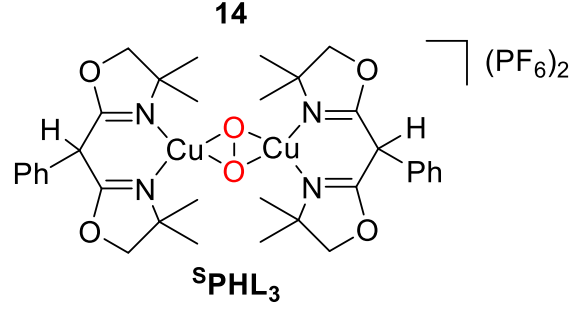

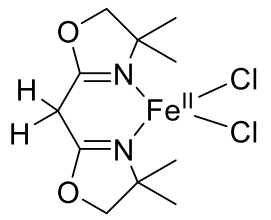

17

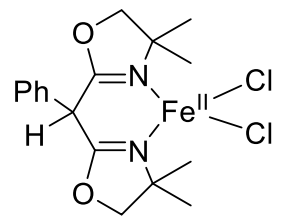

20

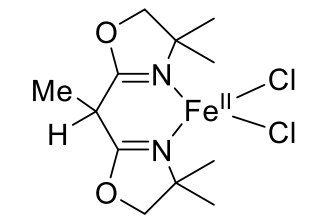

18

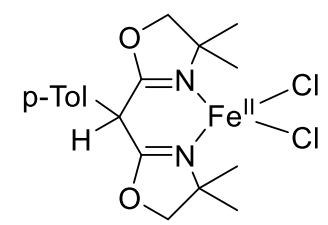

21

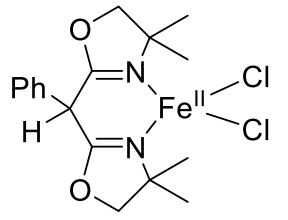

19

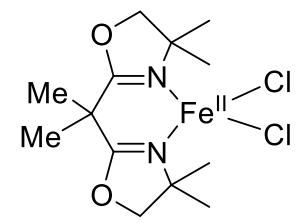

22

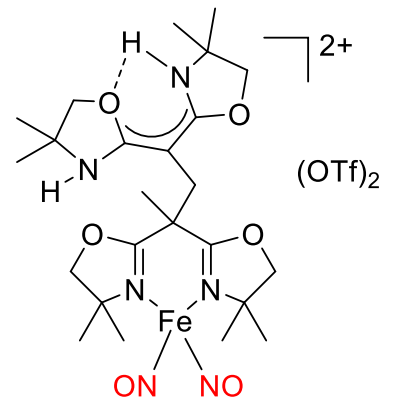

23 


$\begin{array}{ll} & \text { Abbreviations } \\ \text { tBu } & \text { tert-butyl } \\ \text { BDE } & \text { bond dissociation energy } \\ \text { CP } & \text { cis-peroxo } \\ \text { C-C } & \text { 4,4',6,6'tetra-(tert-butyl)-2,2'-biphenol } \\ \text { CHD } & \text { cyclohexadiene } \\ \text { CT } & \text { charge transfer } \\ \text { CO } & \text { Catechol Oxidase } \\ \text { DBED } & \text { N,N'-di-tert-butyl ethylenediamine } \\ \text { DCM } & \text { dichloromethane } \\ \text { DFT } & \text { density functional theory } \\ \text { DNA } & \text { deoxy ribonucleic acid } \\ \text { DNIC } & \text { dinitrosyl iron complexes } \\ \text { DTBP } & \text { 2,4-di-tert-butyl-sodium phenolate } \\ \text { DTBP-H } & 2,4 \text {-di-tert-butyl-phenol } \\ \text { DTBP-Q } & 2,4 \text {-di-tert-butyl-quinone } \\ \text { EPR } & \text { electron paramagnetic resonance } \\ \text { ESI } & \text { electrospray ionization } \\ \text { Et } & \text { ethyl } \\ \text { EXAFS } & \text { extended X-ray absorption fine structure } \\ \text { Fur } & \text { ferric uptake regulatory proteins } \\ \text { FDP } & \text { flavo diiron proteins } \\ \text { FNOR } & \text { flavo diiron nitric oxide reductase } \\ \text { Hc } & \text { Hemocyanin } \\ \text { Hr } & \text { Hemerythrin } \\ \text { HDVV } & \text { Heisenberg-Dirac-Van-Vleck } \\ \text { HOMn }\end{array}$




\begin{tabular}{|c|c|}
\hline IR & infra-red \\
\hline ISC & inter system crossing \\
\hline IVCT & intervalence charge transfer \\
\hline${ }^{3} \mathrm{~L}$ & 1,1,3,3-tertamethyl propanediamine \\
\hline LMCT & ligand-to-metal charge transfer \\
\hline Lut & 2,6-lutidine \\
\hline $\mathrm{Me}$ & methyl \\
\hline MO & molecular orbital \\
\hline MS & mass spectrometry \\
\hline MTBE & methyl tert-butyl ether \\
\hline $\mathrm{NEt}_{3}$ & triethyl amine \\
\hline NMR & nuclear magnetic resonance spectroscopy \\
\hline NOR & nitric oxide reductase \\
\hline $\mathrm{O}$ & $\operatorname{bis}(\mu$-oxo $)$ \\
\hline OTf & triflate \\
\hline pMMO & particulate methane monooxygenase \\
\hline ppm & parts per million \\
\hline $\mathrm{pz}$ & pyrazole \\
\hline PhSMe & thioanisole \\
\hline $\mathrm{PPh}_{3}$ & triphenyl amine \\
\hline RNA & ribonucleic acid \\
\hline RNR & ribonucleotide reductase \\
\hline RSNO & nitrosothiol \\
\hline $\mathrm{rR}$ & resonance Raman \\
\hline $\mathrm{rt}$ & room temperature \\
\hline $\mathrm{R}$ & residue \\
\hline sMMO & soluble methane monooxygenase \\
\hline SOC & spin-orbit coupling \\
\hline SOMO & single occupied molecular orbital \\
\hline SP & side-on peroxo \\
\hline$S_{\mathrm{p}}$ & iso(spartine) \\
\hline
\end{tabular}




$\begin{array}{ll}\text { SQUID } & \text { superconducting quantum interference device } \\ \text { tacn } & \text { triazacyclononane } \\ \text { TBA } & \text { tetrabutylammonium } \\ \text { tetb } & \text { rac-5,5,7,12,12,14-hexamethyl-1,4,8,11,tetraazacyclotetradecane } \\ \text { THF } & \text { tetrahydrofuran } \\ \text { THP } & \text { tetrahydropyran } \\ \text { MeTHF } & \text { methylated-THF } \\ \text { TP } & \text { trans-peroxo } \\ \text { Tp } & \text { tris(pyrazolyl)borate } \\ \text { TPA } & \text { tris(2-pyridylmethyl)amine } \\ \text { tren } & \text { tris(2-aminoethyl)amine } \\ \text { Ts } & \text { tosyl } \\ \text { Ty } & \text { Tyrosinase } \\ \text { UV } & \text { ultraviolet } \\ \text { vis } & \text { visible } \\ \text { VTVH } & \text { variable temperature variable field } \\ \text { ZFS } & \text { zero field splitting } \\ \end{array}$


